\title{
Creating and utilising the Wales Asthma Observatory to support health policy, health service planning and clinical research
}

\author{
Al Sallakh, Mohammad A.
}

How to cite:

Al Sallakh, Mohammad A. (2018) Creating and utilising the Wales Asthma Observatory to support health policy, health service planning and clinical research. Doctoral thesis, Swansea University. http://cronfa.swan.ac.uk/Record/cronfa48569

Use policy:

This item is brought to you by Swansea University. Any person downloading material is agreeing to abide by the terms of the repository licence: copies of full text items may be used or reproduced in any format or medium, without prior permission for personal research or study, educational or non-commercial purposes only. The copyright for any work remains with the original author unless otherwise specified. The full-text must not be sold in any format or medium without the formal permission of the copyright holder. Permission for multiple reproductions should be obtained from the original author.

Authors are personally responsible for adhering to copyright and publisher restrictions when uploading content to the repository.

Please link to the metadata record in the Swansea University repository, Cronfa (link given in the citation reference above.)

http://www.swansea.ac.uk/library/researchsupport/ris-support/ 


\section{Creating and utilising the Wales Asthma Observatory to support health policy, health service planning and clinical research}

Mohammad Anass Al Sallakh, MD, MSc

Submitted to Swansea University in fulfilment of the requirements for the Degree of Doctor of Philosophy in Medical and Health Care Studies

Swansea University 


\section{Declaration}

This work has not previously been accepted in substance for any degree and is not being concurrently submitted in candidature for any degree.

Signed (candidate)

Date

\section{STATEMENT 1}

This thesis is the result of my own investigations, except where otherwise stated. Where correction services have been used, the extent and nature of the correction is clearly marked in a footnote(s). Other sources are acknowledged by footnotes giving explicit references. A bibliography is appended.

Signed (candidate)

Date

\section{STATEMENT 2}

I hereby give consent for my thesis, if accepted, to be available for photocopying and for inter-library loan, and for the title and summary to be made available to outside organisations.

Signed (candidate)

Date 


\section{Acknowledgement}

I thank my supervisors, Professors Gwyneth Davies, Sarah Rodgers, Ronan Lyons, and Aziz Sheikh for their precious support and advice during my candidature; Professor Damon Berridge for statistical assistance in Chapter 5; Stanley Musgrave, the collaboration with whom inspired me to develop a data extraction tool and make access to the Observatory easier for researchers; the SAIL Databank team and analysts for the fruitful discussions; and, not lastly, my wife, Dana, for her encouragement, support, and sacrifice.

This thesis was funded by a studentship from Health and Care Research Wales and Abertawe Bro Morgannwg University Health Board. The studentship underwent external peer review and was awarded by the Asthma UK Centre for Applied Research [AUK-AC-2012-01]. I acknowledge the training and support from the Asthma UK Centre for Applied Research and the Farr Institute of Health Informatics Research. The Farr Institute is supported by a 10-funder consortium: Arthritis Research UK, the British Heart Foundation, Cancer Research UK, the Economic and Social Research Council, the Engineering and Physical Sciences Research Council, the Medical Research Council, the National Institute of Health Research, the National Institute for Social Care and Health Research (Welsh Assembly Government), the Chief Scientist Office (Scottish Government Health Directorates), the Wellcome Trust, (MRC Grant Nos: CIPHER MR/K006525/1, Scotland MR/K007017/1). 
Wales

Asthma

Observatory

Dedicated to those who struggle to breathe 


\section{Summary}

Asthma is a public health challenge in Wales. In order and improve its outcomes and reduce its burden, reliable evidence on disease epidemiology is needed. In this thesis, I describe the development of a platform for asthma surveillance and research in Wales using routinely collected electronic health record (EHR) data in the Secure Anonymised Information Linkage (SAIL) Databank.

To inform the development of operational definitions for asthma and its outcomes, I examine the contemporary approaches to defining asthma and assessing its outcomes using EHR data, and describe significant variations and suboptimal reporting on these approaches. I highlight the need for valid, standardised methods to study asthma, and emphasise the increasing demand for improved reporting to support research transparency and reproducibility.

Acknowledging the infeasibility of reference standards to define asthma in SAIL, I describe the development of latent class model to identify asthma patients in this databank. I assess the performance of this model in relation to other objective and self-reported measures of asthma.

I also describe other methodological aspects of the development of the Wales Asthma Observatory, including asthma data profiling and identification of important data gaps.

To demonstrate the Observatory's utility for health policy and service planning, I highlight the variations in asthma epidemiology in Wales across age groups, gender, and socioeconomic deprivation levels. I found that asthma patients living in the most deprived areas had higher healthcare utilisation for asthma, indicating worse disease control, than those in the least deprived areas.

Finally, I reflect on the experience of developing the Wales Asthma Observatory, recognising its strengths and limitations, and identify opportunities and challenges of maximising the use of routine data towards a learning health system for asthma in Wales. 


\section{Contents}

$\begin{array}{ll}\text { List of Figures } & \text { ix }\end{array}$

List of Tables $\quad$ xii

Abbreviations $\quad$ xv

1 Introduction $\quad \mathbf{3}$

1.1 Overview of asthma . . . . . . . . . . . . . 4

1.2 Asthma is a public health challenge . . . . . . . . . . . 17

1.3 Asthma epidemiology in Wales . . . . . . . . . . . . . . . 18

1.4 Asthma burden can be reduced . . . . . . . . . . . . . . . 20

1.5 Routinely collected data: an overview . . . . . . . . . . . . . 21

1.6 Routinely collected data can improve our understanding of asthma . 22

1.7 Disease registries and observatories . . . . . . . . . . . . 23

1.8 An asthma observatory for Wales: opportunities, challenges, and solutions . . . . . . . . . . . . . . . . . . . . 25

1.9 Thesis aims, research questions, and objectives $\ldots \ldots \ldots$

1.10Thesis structure . . . . . . . . . . . . . . . . . . 28

2 Defining and assessing asthma using EHR Data 33

2.1 Introduction . . . . . . . . . . . . . . 36

2.2 Methods: a systematic scoping review . . . . . . . . . . . . 38

2.3 Results . . . . . . . . . . . . . . . . . . . . 40

2.4 Discussion . . . . . . . . . . . . . . . . . 45

2.5 Conclusion .......................... 50

3 Identifying asthma patients in Wales

3.1 Introduction . . . . . . . . . . . . . . . 57

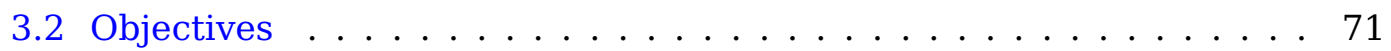

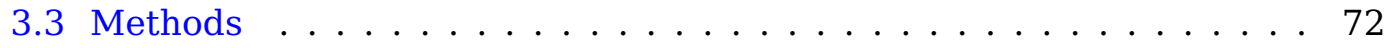

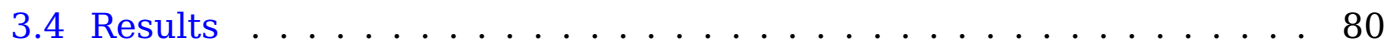

3.5 Discussion . . . . . . . . . . . . . . . . . . 97 
3.6 Conclusion . . . . . . . . . . . . . . . . . . 103

4 Development of the Wales Asthma Observatory 107

4.1 Introduction . . . . . . . . . . . . . . . . . . . . . . . . . 109

4.2 Purpose and context . . . . . . . . . . . . . . . . . 109

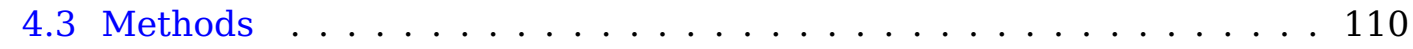

4.4 Summary statistics . . . . . . . . . . . . . . . . . . . . 125

4.5 Quality of asthma-related events in the GP database . . . . . . . . 130

4.6 Discussion . . . . . . . . . . . . . . . . . . . . . 135

4.7 Conclusion . . . . . . . . . . . . . . . . . . . . . . . 142

5 Inequalities in asthma care and outcomes in Wales 145

5.1 Introduction . . . . . . . . . . . . . . . . . . . 147

5.2 Aims and Objectives . . . . . . . . . . . . . . . . . . . . . . . . . . . . . . . . . . . . . . . . . . . . . .

5.3 Methods . . . . . . . . . . . . . . . . . . . . . . . . . . 154

5.4 Results . . . . . . . . . . . . . . . . . . . 160

5.5 Discussion . . . . . . . . . . . . . . . . . . 177

5.6 Conclusion . . . . . . . . . . . . . . . . . . . 188

6 General Discussion $\quad 191$

6.1 Summary of findings . . . . . . . . . . . . . . . . 193

6.2 Original contributions . . . . . . . . . . . . . . . . 195

6.3 Strengths and limitations . . . . . . . . . . . . . . . . 196

6.4 Interpretation of findings in the light of related literature . . . . . . 199

6.5 Challenges . . . . . . . . . . . . . . . . . . . 208

6.6 Implications and potential uses of the Observatory . . . . . . . . . 212

6.7 Towards maximising population data benefits to improve asthma outcomes . . . . . . . . . . . . . . . . . . . . . . 214

6.8 Future work . . . . . . . . . . . . . . . . . . . . . . 218

6.9 Conclusions . . . . . . . . . . . . . . . . . . 222

$\begin{array}{ll}\text { References } & \mathbf{2 2 5}\end{array}$

$\begin{array}{ll}\text { Appendices } & 255\end{array}$

A Chapter 2 Appendix $\quad \mathbf{2 5 5}$

A.1 Additional results for Chapter 2 . . . . . . . . . . . . . . . 255

A.2 Published paper related to Chapter 2 . . . . . . . . . . . . . 280

B Chapter 3 Appendix $\quad 295$

B.1 Making sense of patient-reported currently treated asthma using routinely collected data . . . . . . . . . . . . . . . . . 295 
B.2 Read Codes sets used in latent class analysis . . . . . . . . . . . . . 298

B.3 Item-response probabilities for the competing latent class models . . 308

B.4 Study protocol: identifying patients with ACOS using LCA . . . . . . . 321

C Chapter 4 Appendix

C.1 SAIL Databank Datasets used in the Observatory . . . . . . . . . 333

C.2 SAIL IGRP approval letter . . . . . . . . . . . . . . . . . . . . 364

C.3 A tool for automatic characterisation of cohorts using primary care data. . . . . . . . . . . . . . . . . . 365

C.4 Read codes used in the assessment of data quality in Chapter 4 . . 368

C.5 Density distributions of lung function test values . . . . . . . . . . . 370

D Chapter 5 Appendix $\quad 375$

D.1 Meeting abstract . . . . . . . . . . . . . 376

$\begin{array}{lr}\text { E Clinical codes } & 379\end{array}$ 


\section{List of Figures}

1.1 Trends of self-reported and GP-reported treated asthma in Wales between 1995 and 2015. . . . . . . . . . . . . . . . . . 19

$2.1 \quad$ Flowchart for study selection in this scoping review. . . . . . . . . . 41

3.1 Visual representation of a latent class model. . . . . . . . . . . . . 64

3.2 The methodology followed in Chapter 3. . . . . . . . . . . . . 73

3.3 A histogram for the sample age at the beginning of year 2014 . . . 80

3.4 Assignment of the sample individuals across the competing latent models. . . . . . . . . . . . . . . . . . . . 83

3.5 Diagnostics for the competing latent class models. . . . . . . . . . 84

3.6 Class prevalences and item-response probabilities of the eight-class model. . . . . . . . . . . . . . . . . . . . . . . 85

3.7 Visualisation of the eight-class latent class model using principal component analysis. . . . . . . . . . . . . . . . . . 88

3.8 Visualisation of the 10-fold cross-validation results of the recursive partitioning. . . . . . . . . . . . . . . . . . 90

3.9 A decision tree representation of the classification algorithm. . . . 91

4.1 Compilation of the Wales Asthma Observatory. . . . . . . . . . . . 111

4.2 Split-file approach to data anonymisation by a trusted third party. 118

4.3 Asthma case definitions as state variables in the Wales Asthma Observatory . . . . . . . . . . . . . . . . . . 120

4.4 Incidence and prevalence of asthma in Wales between 2000 and 2017. . . . . . . . . . . . . . . . . . . . 128

4.5 Asthma-related health care utilisation in patients with current asthma.129

5.1 Map of Wales showing ranks of the 2011 Welsh Index of Multiple Deprivation for Lower-level Super Output Areas. . . . . . . . . . . . 153

5.2 A flowchart of case selection. . . . . . . . . . . . . . . . 161

5.3 Distribution of age across WIMD quintiles in the study cohorts. . . 165

5.4 Histograms of outcome event counts in the study cohorts. . . . . 167 
5.5 Average counts of the outcome events between 2010 and 2014 per WIMD rank quintiles in Cohort 1 and Cohort 2. . . . . . . . . 168

5.6 Incidence rate ratios for the outcome variables in each of the deprivation quintiles. . . . . . . . . . . . . . . 170

5.7 Incidence rate ratios for the outcome variables across the age groups.171

5.8 Quantile-quantile plot for the model residuals. . . . . . . . . . . . 175

5.9 Rootograms illustrating the goodness of fit for the zero-inflated negative binomial models. . . . . . . . . . . . . . 176

C.1.1 Frequency of events per year in the SAIL datasets. . . . . . . . . 363

C.2.1 SAIL IGRP approval letter. . . . . . . . . . . . . . . . . . . . . 364

C.3.1 A tool for automatic characterisation of cohorts using primary care data . . . . . . . . . . . . . . . . . . . 367

C.5.1 Beanplots showing density distributions for lung function event values. . . . . . . . . . . . . . . . . . . . 370 


\section{List of Tables}

$1.1 \quad$ Measures of performance of asthma tests. . . . . . . . . . 9

1.2 Main clinical coding systems in the United Kingdom. . . . . . . . 21

2.1 Practices of reporting or justifying the validity of algorithms to define and assess asthma using EHR-derived data. . . . . . . . . . 45

3.1 Observed variables used in the latent class model. . . . . . . . . 75

3.2 Latent classes before and after merging. . . . . . . . . . . . . 89

3.3 Results of 10 -fold cross-validation for the recursive partitioning model. . . . . . . . . . . . . . . . . . . . . . . 89

3.4 Confusion matrix and statistics for the cross-classification of the predicted classifications against the latent class analysis (LCA)based labels. . . . . . . . . . . . . . . . . . . . . . 93

3.5 Comparison between the classification algorithm and other commonly used case definitions. . . . . . . . . . . . . . . . . . . 95

4.1 Datasets used for the development of the Wales Asthma Observatory.114

4.2 Case definitions used in the Wales Asthma Observatory. . . . . . . 119

4.3 Data tables in the asthma registry . . . . . . . . . . . . . 121

4.4 All-time number of records and unique patients for each of the case definitions . . . . . . . . . . . . . . . . . 126

4.5 Prevalence of asthma at national and health board levels in Wales in $2017 . \ldots \ldots \ldots \ldots$. . . . . . . . . . . . . . . 127

4.6 Percentages of patients with at least one recording of key asthmarelated GP events over specified periods from diagnosis. . . . . . . 132

4.7 Percentages of missing values for lung function event codes. . . . . 133

5.1 Characteristics of the source population. . . . . . . . . . 160

5.2 Characteristics of the study cohorts. . . . . . . . . . . . . 162

5.3 Outputs of the zero-inflated negative binomial models. . . . . . . 172

A.1.1 Search query used in the systematic scoping review. . . . . . . . . 256 
A.1.2 Charting table showing the data extracted from the reviewed articles. . . . . . . . . . . . . . . . . . . . . . 257

A.1.3 Geographical distribution of the reviewed studies. . . . . . . . . 259

A.1.4 Study designs of the reviewed studies. . . . . . . . . . . . . 259

A.1.5 Types of EHR-derived data sources used in the reviewed articles. . 259

A.1.6 Algorithms used to identify asthma patients. . . . . . . . . . . . . 260

A.1.7 Approaches used in identifying asthma patients. . . . . . . . . . 263

A.1.8 Age restriction approaches used in asthma patient identification. . 263

A.1.9 Co-morbidities and conditions based on which asthma patients were excluded. . . . . . . . . . . . . . . . . . . . . . 264

A.1.10 Algorithms used to ascertain asthma severity using EHR data. . . . 265

A.1.11 Algorithms used to ascertain asthma exacerbation using EHR data. 268

A.1.12 Algorithms used to assess asthma control using EHR data . . . . . 274

B.2.1 Read Codes sets used to define the observed variables in the latent class modelling in Chapter 3. . . . . . . . . . . . . . . . . . . 298

C.1.1 Data fields of SAIL datasets. . . . . . . . . . . . . . . . . . . . . 334

C.1.2 Frequency of events per year in the SAIL datasets. . . . . . . . . . . 362

C.4.1 Asthma diagnosis Read codes. . . . . . . . . . . . . . . . . . 368

C.4.3 Asthma-related event groups chosen for the coding quality analysis.369 


\section{Abbreviations}

\begin{tabular}{|c|c|}
\hline$A \& E$ & accident and emergency \\
\hline ACBS & Asthma Call-back Survey \\
\hline $\mathrm{ACOS}$ & asthma-COPD overlap syndrome \\
\hline $\mathrm{ACQ}$ & Asthma Control Questionnaire \\
\hline $\mathrm{ACT}$ & Asthma Control Test \\
\hline ADDE & Annual District Death Extract \\
\hline AHR & Airway hyperresponsiveness \\
\hline $\mathrm{AIC}$ & Akaike information criterion \\
\hline $\mathrm{ALF}$ & Anonymised Linking Field \\
\hline AQLQ & Asthma Quality of Life Questionnaire \\
\hline ARIMA & autoregressive integrated moving average \\
\hline \multirow[t]{2}{*}{ ARRISA-UK } & "At-Risk Registers Integrated into primary care to Stop Asthma crises in the United \\
\hline & Kingdom” \\
\hline ATS & American Thoracic Society \\
\hline AUC & area under the curve \\
\hline AUKCAR & Asthma UK Centre for Applied Research \\
\hline $\mathrm{BIC}$ & Bayesian information criterion \\
\hline BRFSS & Behavioral Risk Factor Surveillance System \\
\hline BSAR & Belgian Severe Asthma Registry \\
\hline BTS & British Thoracic Society \\
\hline CALIBER & Clinical research using LInked Bespoke studies and Electronic health Records \\
\hline CAMP & cyclic adenosine monophosphate \\
\hline CI & confidence interval \\
\hline COPD & chronic obstructive pulmonary disease \\
\hline CPRD & Clinical Practice Research Datalink \\
\hline DALYs & disability-adjusted life years \\
\hline ED & emergency department \\
\hline EDDS & Emergency Department Data Set \\
\hline EHR & electronic health record \\
\hline EM & expectation-maximisation \\
\hline
\end{tabular}


FeNO fractional exhaled nitric oxide

FEV1 forced expiratory volume in the first second

FVC forced vital capacity

GP general practitioner

HCRW Health and Care Research Wales

HEDIS Healthcare Effectiveness Data and Information Set

HRG Healthcare Resource Group

ICD-10 the $10^{\text {th }}$ revision of the International Classification of Disease

ICS inhaled corticosteroid

IgE immunoglobulin E

IGRP Information Governance Review Panel

IRR incidence rate ratio

ISAAC International Study of Asthma and Allergies in Childhood

ITP immune thrombocytopenic purpura

JSON JavaScript Object Notation

LABA long-acting beta adrenoceptor agonist

LAMA long-acting muscarinic antagonist

LCA latent class analysis

LHS learning health systems

LOS length of stay

LR likelihood ratio

LRTI lower respiratory tract infections

LSOA Lower Layer Super Output Area

LTA latent transition analysis

LTRA leukotriene receptor antagonist

$\mathrm{MeSH} \quad$ Medical Subject Headings

MIU minor injury unit

MRC Medical Research Council

NHS National Health Services

NICE National Institute for Health and Care Excellence

NIR no information rate

NISCHR National Institute for Social Care and Health Research

NLP natural language processing

NPV negative predictive value

NRAD National Review of Asthma Deaths

NWIS National Health Services Wales Informatics Service 
OASIS Ontario Asthma Surveillance Information System

OCS oral corticosteroids

ONS Office for National Statistics

OPD Outpatient Dataset

PEAL Population-Based Effectiveness in Asthma and Lung Diseases

PEDW Patient Episode Database for Wales

PEFR peak expiratory flow rate

PPV positive predictive value

PROM patient reported outcome measure

QOF Quality of Outcomes Framework

RALF Residential Anonymous Linking Fields

RCD routinely collected data

RECORD REporting of studies Conducted using Observational Routinely-collected health Data

ROC receiver operating characteristic

SABA short acting beta agonist

SAIL Secure Anonymised Information Linkage

SAMA short-acting muscarinic antagonist

SD standard deviation

SIGN Scottish Intercollegiate Guidelines Network

SNOMED-CT Systematized Nomenclature of Medicine-Clinical Terms

SQL Structured Query Language

STELAR Study Team for Early Life Asthma Research

STROBE Strengthening the Reporting of Observational Studies in Epidemiology

SWORD Surveillance of Work-related and Occupational Respiratory Disease

UK United Kingdom

UKAO UK Asthma Observatory

US United States

VC vital capacity

WDS Welsh Demographic Service

WHS Welsh Health Survey

WIMD Welsh Index of Multiple Deprivation

WLGP Welsh Longitudinal General Practice

WRRS Welsh Results Reports Service

WRS Weekly Returns Service

ZINB zero-inflated negative binomial 


\section{Chapter 1}

\section{Introduction}

\section{Opportunities for better understanding of asthma through routine health data}

Asthma is a public health challenge in Wales. In order to reduce asthma burden and improve its outcomes, reliable population-based evidence on the disease epidemiology is needed. In this chapter, I present an overview of asthma, its public health burden, and endeavours to understand its epidemiology in Wales. Then, I present an overview of routinely collected health data, and highlight their unique potentials for better understanding of asthma. I then introduce my thesis aim of developing the Wales Asthma Observatory, based on routinely collected data, as a platform for asthma surveillance and research. I then describe the thesis objectives, including exploring the practices and challenges of studying asthma using routinely collected data, description of the Observatory's methodology, and demonstration of its utility for health policy using inequalities in asthma outcomes as an example. I conclude with describing the thesis structure. 


\section{Chapter Contents}

1.1 Overview of asthma $\ldots \ldots \ldots \ldots \ldots \ldots \ldots \ldots \ldots$

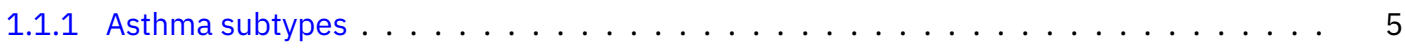

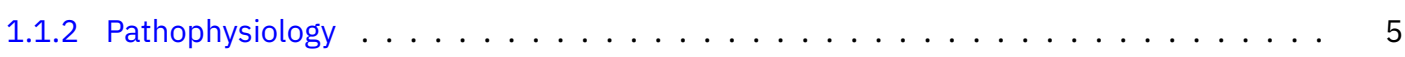

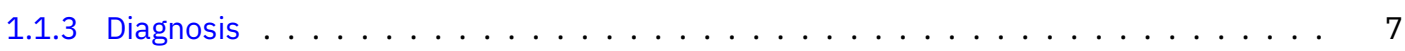

1.1.3.1 Patient medical history and clinical examination . . . . . . . . . . 7

1.1.3.2 Diagnostic testing . . . . . . . . . . . . . . . 8

1.1 .4 Management . . . . . . . . . . . . . . . . . . . 12

1.1.4.1 Pharmacological treatments .................. 13

1.2 Asthma is a public health challenge . . . . . . . . . . . . . . . . . . . 17

1.3 Asthma epidemiology in Wales . . . . . . . . . . . . . . . . . 18

1.4 Asthma burden can be reduced . . . . . . . . . . . . . . . . . . . . . . . . 20

1.5 Routinely collected data: an overview . . . . . . . . . . . . . . . . . . . . 21

1.6 Routinely collected data can improve our understanding of asthma . . . . . . . . . . . . 22

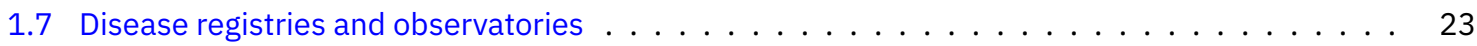

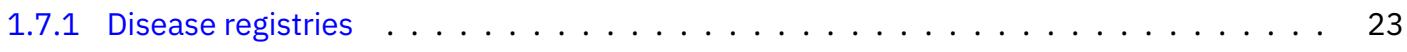

1.7.2 Health and disease observatories . . . . . . . . . . . . . . . . . . 24

1.8 An asthma observatory for Wales: opportunities, challenges, and solutions _ . . . . . . . . 25

1.9 Thesis aims, research questions, and objectives . . . . . . . . . . . . . . . . 26

1.10 Thesis structure $\ldots \ldots \ldots \ldots \ldots \ldots \ldots \ldots$

\subsection{Overview of asthma}

Asthma is a chronic respiratory disorder typically characterised by cough, dyspnoea, chest tightness, and wheeze [1]. The clinical manifestations of asthma often exhibit a recurring and remitting pattern and a variable intensity over time [1]. The disease severity varies widely among patients, ranging from mild intermittent symptoms to a severe persistent disease. Patients with any level of asthma severity may develop exacerbations - temporary periods of acute or sub-acute deterioration in symptom control that can be life-threatening [2]. 


\subsubsection{Asthma subtypes}

Asthma is increasingly considered heterogeneous rather than a single disease. There is growing evidence that "asthma" should be regarded as an umbrella term comprising distinct phenotypes with different clinical presentations potentially explained by distinct endotypes with different pathophysiological mechanisms [35]. The aetiology of asthma is thought to vary between phenotypes, with a wide range of potential risk factors related to the host, genetics, and the environment [6]. Asthma can also be classified based on other criteria such as triggerability by allergens into allergic and non-allergic, and age of onset into early- and lateonset disease [7]. In addition, other recognised phenotypes include infectious, aspirin-induced, occupational, exercise-induced, and obese asthma [8].

The heterogeneous nature of asthma contributes to the challenges of diagnosis, treatment, and developing epidemiological definitions as discussed later in Chapter 2 and Chapter 3 (Section 3.1.1).

\subsubsection{Pathophysiology}

Asthma signs and symptoms result from complex underlying pathophysiology in the airways that involves chronic inflammation, remodelling, hyperresponsiveness, and obstruction.

\section{Airway inflammation}

Airway inflammation results from an abnormal immune reaction to mostly exogenous stimuli (e.g., pollen, viruses, and bacteria). Type 2 T-helper cells are a major player in this immune reaction, which involves recruitment and activation of several other types of immune cells in the airway mucosa, including eosinophils, mast cells, basophils, neutrophils, monocytes, and macrophages [9].

Eosinophils have a unique role in asthma pathogenesis, and they are increased in number in the airway, sputum, and blood. They produce chemical mediators such as leukotrienes which cause airway smooth muscle contraction, recruitment of inflammatory cells, and an increase in mucus production [10]. 
Mast cells are increased in the airway mucosa and smooth muscle, and release histamine, leukotrienes, cytokines, chemokines, and growth factors, which target the smooth muscle, vessels, mucous glands, and sensory nerves [11].

Bradykinin, a natural vasoactive peptide and a by-product of the inflammatory process, causes bronchoconstriction and cough [12].

\section{Airway remodelling}

Chronic inflammation in the airways leads to several irreversible structural changes in the airways. These include airway wall thickening, hyperplasia and hypertrophy in the epithelium, mucous glands and smooth muscle cells, submucosal collagen deposition and basal membrane thickening, and neovascularisation and increased sizes of vessels $[9,13]$. Airway remodelling leads to irreversible narrowing of the airways and contributes to the decline in the reversibility of airway obstruction $[9,11]$.

\section{Airway hyperresponsiveness}

Airway hyperresponsiveness (AHR) is a measure of variable airflow limitation. It represents the exaggerated response of airway smooth muscle to inhaled stimuli leading to bronchoconstriction, which causes a decline in airflow. This response may occur due to nerve stimulation, mast cell mediated events, or direct effects of stimuli on the airway smooth muscle $[9,13]$. Several factors may contribute to development of AHR, including eosinophilic airway inflammation, airway epithelial damage, airway remodelling, and increased contractility of airway smooth muscle [14].

\section{Airway obstruction}

Airway obstruction in asthma results from a combination of several factors, such as increased airway thickness (due to smooth muscle cell hypertrophy and hyperplasia, and mucosal oedema), exaggerated bronchoconstriction, and excessive mucous secretion and plugs in the airway lumen [15]. Airway obstruction leads to dyspnoea, chest tightness, wheeze and a variable decline in lung function. Reversible airway obstruction is characteristic of asthma, but reversibility tends to 
decline over time with the development of fixed obstruction due to airway remodelling. The variable nature of obstruction has been attributed to AHR [13].

\subsubsection{Diagnosis}

Asthma diagnosis is mainly clinical, although reasonably certain diagnosis often requires a combination of careful medical history taking, clinical examination, and objective tests [16]. The clinical definition of asthma by the British Thoracic Society (BTS)/Scottish Intercollegiate Guidelines Network (SIGN) guidelines requires at least two of the main symptoms (cough, dyspnoea, chest tightness, and wheeze) in addition to evidence of variable airflow obstruction [16]. With high clinical suspicion of asthma, trial treatment with bronchodilators can be initiated; a good response to this treatment, assessed with objective testing, allows confirmation of asthma diagnosis [16]. An intermediate probability of asthma, based on medical history and clinical examination, warrants testing for airway obstruction (variability and hyperresponsiveness) and airway inflammation [16]. Differential diagnoses include an extensive list of disorders that can masquerade as asthma such as chronic obstructive pulmonary disease (COPD), cystic fibrosis, vocal cord dysfunction, rhinitis, chronic cough syndrome, and gastro-oesophageal reflex [1618].

\subsubsection{Patient medical history and clinical examination}

Medical history is useful to establish whether symptoms, their onset, patterns (e.g., episodic), and triggerability (e.g., association with known stimuli) are compatible with asthma. It may help to identify risk factors of asthma (e.g., atopy or a family history of asthma), assess disease severity, and to rule out alternative conditions.

On examination, the patient may show breathlessness (e.g., inability to complete sentences) and tachypnoea. Chest auscultation may reveal widespread expiratory wheeze, while chest percussion may reveal hyperresonance [13]. Non-pulmonary atopic findings such as atopic dermatitis and allergic rhinitis support the diagnosis of asthma. In moderate and severe disease, the use of accessory respiratory muscles, intercostal retractions, and pulsus paradoxus may be observed. Physical examination is useful to detect signs suggestive of alternative diagnoses (e.g., unilateral wheeze, focal lung abnormalities, and finger clubbing) [16]. 


\subsubsection{Diagnostic testing}

The diagnostic tests in asthma allow investigating airway inflammation and responsiveness, establishing variability and reversibility of airflow obstruction, and ruling out alternative diagnoses that mimic asthma. However, they can be negative during the asymptomatic periods of the disease and are therefore insufficient alone to establish or rule out asthma diagnosis $[1,16]$. Table 1.1 shows measures of performance of tests used in asthma diagnosis.

\section{Lung function tests}

Lung function tests include spirometry, plethysmography, peak flow measurement, and diffusion capacity assessment.

A spirometer is a device that measures inspired and expired air volumes. The forced vital capacity (FVC) and the forced expiratory volume in the first second (FEV1) are central to the assessment of airflow limitation. Following a full inspiration, the FVC represents the maximum possible volume of exhaled air whereas FEV1 represents only the volume of exhaled air in the first second. Both measures decrease in airflow limitation in asthma, but FEV1 usually decreases to a larger extent than FVC. The ratio of the measured to predicted FEV1 often remains above $80 \%$ in mild disease and declines below $60 \%$ in untreated severe disease [19]. The lower limit of normal FEV1/FVC ratio ranges between $85 \%$ to $70 \%$ depending on age $[16,20]$. Lower values for the respective age group are considered positive for airflow obstruction and are often present in moderate and severe asthma [16]. However, these airflow limitation criteria alone, are neither sensitive nor specific to asthma. More than half of patients with normal FEV1/FVC will have asthma [16, 21]. FEV1 is often normal in children with persistent disease and may fall in other respiratory diseases [22]. Therefore, a normal spirometry test performed when the patient is not symptomatic does not rule out asthma diagnosis. Furthermore, a single measurement of FEV1 correlates poorly with asthma severity as classified by symptoms and medications [23].

Reversibility to bronchodilators (also known as bronchodilator response) is the improvement in airflow following acute treatment with a beta-agonist bronchodilator. A positive test is defined as a $12 \%$ or more improvement, which should be at least $200 \mathrm{~mL}$, in FEV1 over the baseline [24]. However, these criteria may be 
Table 1.1: Measures of performance of asthma tests. This table is adapted from [16]. The reference tests were (spirometry and (bronchodilator reversibility or a challenge test]) with or without 'typical history of attacks', diurnal variation, physician diagnosis, documented history of wheeze on at least two occasions, and variability in FEV1 over time or during exercise testing.

\begin{tabular}{|c|c|c|c|c|c|c|}
\hline Test & Description & $\begin{array}{l}\text { Age } \\
\text { group }\end{array}$ & $\begin{array}{l}\text { Sensitivity } \\
(\%)\end{array}$ & $\begin{array}{l}\text { Specificity } \\
(\%)\end{array}$ & $\begin{array}{l}\text { Positive } \\
\text { predictive } \\
\text { value (\%) }\end{array}$ & $\begin{array}{l}\text { Negative } \\
\text { predictive } \\
\text { value (\%) }\end{array}$ \\
\hline \multirow[t]{2}{*}{ Spirometry } & FEV1/FVC < 70\% & adults & $23-47$ & $31-100$ & $45-100$ & $18-73$ \\
\hline & & children & 52 & 73 & 75 & 49 \\
\hline Bronchodilator reversibility & $\begin{array}{l}\text { Improvement in FEV1 } \\
\text { of } \geqslant 12 \% \text { (and } \geqslant 200 \mathrm{ml} \\
\text { in adults) }\end{array}$ & $\begin{array}{l}\text { adults } \\
\text { children }\end{array}$ & $\begin{array}{l}17-69 \\
50\end{array}$ & $\begin{array}{l}55-81 \\
86\end{array}$ & $53-82$ & $22-68$ \\
\hline \multirow[t]{6}{*}{$\begin{array}{l}\text { Challenge } \\
\text { tests }\end{array}$} & $\begin{array}{l}\text { Methacholine PC(20) } \\
\text { value of } \leqslant 8 \mathrm{mg} / \mathrm{ml}\end{array}$ & & $51-100$ & $39-100$ & $60-100$ & \\
\hline & & children & $47-86$ & $36-97$ & 20 & 94 \\
\hline & $\begin{array}{l}\text { Mannitol: } 635 \mathrm{mg} \\
\text { cumulative dose }\end{array}$ & & 65 & 75 & 80 & 49 \\
\hline & $\begin{array}{l}\text { causing a decrease in } \\
\text { FEV1 of } \geqslant 15 \%\end{array}$ & children & 63 & 81 & & \\
\hline & Exercise & adults & $26-80$ & 100 & 100 & 0 \\
\hline & & children & $69-72$ & $69-72$ & $90-99$ & $5-73$ \\
\hline \multirow{4}{*}{$\begin{array}{l}\text { Peak flow, mean variability } \\
\text { over } 2-4 \text { weeks }\end{array}$} & $\geqslant 20 \%$ & adults & 46 & 80 & 97 & 10 \\
\hline & $\geqslant 15 \%$ & adults & $3-5$ & $98-99$ & $60-67$ & 60 \\
\hline & $\geqslant 15 \%$ (>3 days/week) & adults & 20 & 97 & 82 & 64 \\
\hline & $\geqslant 12.3 \%$ & children & 50 & 72 & 48 & 74 \\
\hline \multirow{2}{*}{$\begin{array}{l}\text { Fractional exhaled nitric } \\
\text { oxide }\end{array}$} & $\geqslant 40$ parts per billion & adults & $43-88$ & $60-92$ & $54-95$ & $65-93$ \\
\hline & $\geqslant 35$ parts per billion & $\begin{array}{l}\text { school } \\
\text { children }\end{array}$ & 57 & 87 & 90 & 49 \\
\hline \multirow[t]{2}{*}{ Blood eosinophils } & $>4.15 \%$ & adults & $15-36$ & $39-100$ & $39-100$ & $27-65$ \\
\hline & $\geqslant 4 \%$ & children & $55-62$ & $67-84$ & $56-69$ & 73 \\
\hline \multirow[t]{2}{*}{ Immunoglobulin E } & $\begin{array}{l}\text { Any allergen-specific } \\
\text { IgE }>0.35 \mathrm{kU} / \mathrm{l}\end{array}$ & adults & $54-93$ & $67-73$ & $5-14$ & $95-99$ \\
\hline & Total IgE >100 kU/l & adults & 57 & 78 & 5 & 99 \\
\hline \multirow[t]{2}{*}{ Skin prick test } & Wheal $\geqslant 3 \mathrm{~mm}$ & adults & $61-62$ & $63-69$ & $14-81$ & $39-96$ \\
\hline & & children & $44-79$ & $56-92$ & $65-92$ & $36-79$ \\
\hline
\end{tabular}

falsely negative in many asthma patients, especially in those on treatment [15]. In addition, reversibility to bronchodilators may occur in some patients with COPD [25]. 
Peak flow (also known as peak expiratory flow rate, PEFR) is the maximum rate of expiratory flow during a short and maximally forceful expiratory effort following a full inspiration. It can be measured by the patient using a portable peak flow meter at home. Daily recording of peak flow is useful to demonstrate variability of airway obstruction. Ideally, the patient is asked to make at least two recordings (during the day and the night). A seven-day average of the diurnal differences of more than $20 \%$ is considered positive for variability in airflow obstruction [16]. However, while this criterion has high specificity and positive predictive value (PPV), it has low sensitivity and negative predictive value (NPV), which means it produces a high rate of false negative results [16]. Peak flow is also useful for asthma monitoring [16]. Limitations of peak flow measurement, compared to spirometry, include the dependency on the patient's effort and difficulty of controlling measurement quality [26].

Diffusion capacity of the lung (DL), the ability to transfer gas (such as carbon monoxide) from air to alveolar vessels, is usually normal or increased in asthma $[11,27]$.

Whole-body plethysmography may show an increase in airway resistance, total capacity of the lung, and residual volume; however, it is rarely needed in asthma [11].

\section{Airway responsiveness testing}

The exaggerated response of airways (i.e. AHR) in asthma, which is an indicator of airflow obstruction variability, can be investigated using bronchoconstrictor stimuli. These stimuli can be direct (such as methacholine or histamine) or indirect (e.g., mannitol or exercise) $[13,16]$. A decline in FEV1 of $20 \%$ or more is considered a positive result [16]. However, this test is not suitable for patients with significant decline in lung function [16].

\section{Exhaled Nitric Oxide}

Nitric oxide (NO) is produced by several types of cells in the airway, including eosinophils, during inflammation. The fractional exhaled nitric oxide (FeNO) can be therefore used as a biomarker of airway inflammation in asthma. It can be helpful in the diagnosis, disease categorisation (eosinophilic vs. non-eosinophilic), 
and determining the responsiveness to, and adjustment of, corticosteroid treatment [28]. The diagnostic accuracy measures of FeNO range between 43-95\%, depending on the studies [16].

\section{Skin prick testing}

Skin prick tests help determine whether the patient is allergic to a common allergen (e.g., house dust mite). The test involves introducing a small amount of an allergen into the superficial epidermis. A weal with diameter of 3 millimetres or more suggests the patient has specific immunoglobulin E (IgE) antibodies for the used allergen [29]. Skin prick tests can help differentiate between allergic and non-allergic asthma. However, due to its mediocre sensitivity and specificity to asthma diagnosis, it cannot be used to establish or rule out the disease on its own [16]. The current National Institute for Health and Care Excellence (NICE) guidelines on asthma (NG80) recommends that skin prick testing should not be offered for the purpose of establishing the diagnosis but rather to identify triggers after a formal asthma diagnosis is made [30].

\section{Blood tests}

A blood eosinophil count of more than $4 \%$ is suggestive to atopy. However, this threshold, alone, has a poor predictive value for asthma diagnosis [16]. It is worth noting that marked blood eosinophilia suggests alternative diagnoses (e.g., parasitic infestation or familial eosinophilia) [16].

Serum IgE can be helpful in asthma diagnosis. In adults, a serum level $>0.35 \mathrm{kU} / \mathrm{l}$ of specific IgE antibodies to seasonal and perennial allergens, or total serum IgE $>100 \mathrm{kU} / \mathrm{I}$, indicates an atopic state. However, these thresholds have a very low PPV, i.e. positive results poorly predict asthma [30]. In contrast, normal serum level of IgE substantially reduces the probability of asthma in adults with an NPV of $95-99 \%$ [16].

The current NICE guidelines on asthma, however, recommends that blood eosinophil count and total and specific IgE tests should not be offered for the purpose of establishing asthma diagnosis [30]. 


\section{Imaging}

Chest X-ray is usually normal in asthma, but it may show lung hyperinflation in severe disease and pneumothorax in exacerbations and it is useful to exclude alternative diagnoses [11].

\subsubsection{Management}

Asthma cannot be medically cured. Therefore, asthma management aims to adequately control symptoms and maintain normal activity levels (i.e. minimise asthma impairment) and minimise the risk of asthma exacerbations with the least possible treatment adverse effects $[1,16]$.

Asthma management involves key clinical concepts $[2,19]$. Asthma control is the extent to which symptoms are sufficiently eliminated or reduced through treatment to an acceptable target. Complete asthma control is defined as the absence of symptoms, activity limitation, need for rescue medication, and asthma attacks, with normal lung function and minimal side effects of treatment [16].

Responsiveness to treatment is the ease with which disease control is achieved. Impairment refers to symptom severity and the resultant functional limitation. Risk is the probability of future exacerbations, chronic morbidity, and adverse effects of medications. Lastly, asthma severity is a complex concept composed of the following components: level of control including level of impairment and exacerbations in the last 12 months, level of current prescribed treatment, responsiveness to treatment, and risk.

Management guidelines have been developed globally [1] and nationally [19, 31]. In the United Kingdom, the national guidelines recommend measures for primary and secondary prevention, pharmacological management, in addition to guided self-management [16].

Disease self-management is important in asthma and requires an adequate level of patient education. Asthma self-management includes effective trigger avoidance, adherence to treatment, appropriate inhaler technique, regular monitoring of peak flow, and following up the personalised action plan [13].

Most patients with asthma can be treated in primary care [32]. However, patients with more severe disease (e.g., requiring 'high-dose therapies' or oral steroids), 
treatment-refractory disease, comorbidities, and/or risk of exacerbations need to be referred to specialist care to review the diagnosis and management plan [13, $16]$.

\subsubsection{Pharmacological treatments}

Asthma therapies can be categorised clinically into:

- Quick relief medications (rescuers), which include bronchodilators, namely short acting beta agonists, magnesium, and short-acting muscarinic receptor antagonists (in asthma attacks), and provide quick reversal of airway obstruction;

- Controller medications (preventers), which provide long-term symptom control. These include anti-inflammatory medications (inhaled and oral corticosteroids, leukotriene receptor antagonists, and anti-immunoglobulin E antibodies) and long-acting bronchodilators (long-acting beta adrenoceptor agonists, theophyllines, and long-acting muscarinic antagonists).

\section{Pharmacological categories of therapies}

Beta 2 adrenergic agonists

Beta 2 adrenergic agonists act by increasing intracellular cyclic adenosine monophosphate (CAMP) in airway smooth muscle cells. This inhibits contractility, decreases airway hyperresponsiveness, and improves lung function [11].

Inhaled short acting beta agonists (SABAs), such as salbutamol and terbutaline, have a quick onset on action ( $<5$ minutes) [33]. They are the main choice in relieving acute symptoms in asthma [16].

Long-acting beta adrenoceptor agonists (LABAs) have a slower onset of action ( 5-30 minutes after inhalation) and their effects last longer (12 hours or more) [33]. They are usually used as additional controllers in combination with inhaled corticosteroids (ICSs), when the latter are not sufficient to control the symptoms and/or to reduce the side effects of ICS [16]. Examples of LABAs include salmeterol, formoterol, olodaterol, vilanterol, and indacaterol.

Side effects of $\beta_{2}$ adrenergic agonists may include muscle tremor, palpitations, and a mild decrease in serum potassium [33]. 


\section{Anticholinergics}

Muscarinic receptors antagonists inhibit cholinergic nerve-induced bronchoconstriction and mucus secretion [11]. Tiotropium, a long-acting muscarinic antagonist (LAMA), can be used as an additional controller if ICS and LABA combinations are not sufficient to control the symptoms [16]. Nebulised ipratropium, a shortacting muscarinic antagonist (SAMA), can be used along with a nebulised beta-2 agonist in severe acute asthma attacks to improve bronchodilation and accelerate recovery [16]. Side effects may include dry mouth, dizziness, cough, arrhythmias and, in the elderly, urinary retention and glaucoma [33].

\section{Theophyllines}

Theophyllines inhibit the metabolism of cAMP, which increase its activation of beta-adrenoreceptors, leading to relaxation of airway smooth muscles [11]. However, their narrow therapeutic window requires measuring their plasma concentration to adjust the dose. Side effects may include nausea, tachycardia, arrhythmias, and seizures [33].

\section{Corticosteroids}

Inhaled corticosteroids are the most effective controllers of asthma [16]. ICSs suppress airway inflammation mainly by suppressing the activation of the genes that produces the inflammatory mediators. They decrease the number and activity of inflammatory cells, particularly T lymphocytes, eosinophils, and mast cells, that are responsible for airway inflammation [11]. This improves the disease control by improving lung function, controlling airway hyperresponsiveness, and reducing asthma symptoms. Examples of ICSs include beclometasone, fluticasone, budesonide, mometasone, and ciclesonide [33]. Adverse effects may include oropharyngeal candidiasis, hoarseness, and, at high doses, adrenal suppression and increased bone turnover [33, 34].

Systemic corticosteroids, typically as oral prednisolone, are usually used as short courses to treat asthma exacerbations [16]. They can be also used as long-term treatment in patients with severe asthma that is uncontrolled with a high-dose ICS, a LABA, a leukotriene receptor antagonist, and theophylline [16, 33]. 
The dose of long-term corticosteroids in asthma should be carefully reviewed to ensure adequate disease control with the least possible side effects [16], which may include diabetes, cataracts, glaucoma, and osteoporosis.

\section{Leukotriene receptor antagonists}

Leukotriene receptor antagonists (LTRAs), or antileukotrienes, block either the synthesis of leukotrienes or their binding to leukotriene receptors, which suppresses bronchoconstriction, microvascular leakage, and eosinophilic inflammation [11, 13]. Examples of LTRAs include montelukast and zafirlukast [33]. LTRAs can cause modestly improve symptoms in exercise-induced asthma and in asthma patients with concomitant rhinitis $[13,15]$. Common side effects include headache and gastrointestinal disturbances [33].

\section{Biologic targeted therapy}

Omalizumab is a monoclonal antibody that binds to circulating $\operatorname{Ig} E$ and blocks its interaction with mast cells and basophils [11]. It is given as a subcutaneous injection [33]. Other biologic agents include mepolizumab and reslizumab, which are given by subcutaneous injection and intravenous infusion, respectively. These medications are very expensive and are reserved for patients with a distinct phenotype who fulfil NICE criteria; for omalizumab, patients with severe allergic asthma who have frequent exacerbations despite high doses of corticosteroids [16].

Side effects of omalizumab may include hypersensitivity reactions, leading to urticaria, hypotension, syncope, bronchospasm, and/or angioedema [33]. Rarely, eosinophilic granulomatosis with polyangiitis (EGPA, also known as Churg-Strauss syndrome) may occur due to corticosteroid withdrawal [35]. EGPA may manifest as hypereosinophilia, worsening pulmonary symptoms, vasculitic rash, eosinophilic myocarditis, and/or peripheral neuropathy [33].

\section{Cromones}

Cromones, such as cromolyn and nedocromil, inhibit mast cells and sensory nerve activation [11]. They have some benefits in adults and children aged $>5$ years, and can be used in the control of exercised induced asthma [16]. They are listed 
as alternate initial controller therapies for mild asthma in national and international guidelines, although ICSs are the preferred agents. Side effects are rare and include bronchospasm, cough, headache, eosinophilic pneumonia, rhinitis, and throat irritation [33].

\section{Magnesium sulphate}

Intravenous magnesium sulphate causes relaxation of airway smooth muscle and can be used in patients with acute severe asthma attacks who had no good initial response to inhaled bronchodilators [16].

\section{Immunosuppressants}

Immunosuppressants such as methotrexate may be initiated by specialists in patients with severe asthma to achieve disease control and reduce oral steroids, but there is no strong evidence base for their use [16, 33].

\section{Treatment approach}

The pharmacological management of asthma should follow a step-wise approach starting with the step most appropriate to the presenting disease severity. The 2016 BTS/SIGN guidelines on asthma management recommended the following treatment steps [16]:

For suspected asthma, monitored initiation of low-dose ICS treatment (very-low to low dose in children) is recommended.

For confirmed asthma:

- Regular preventer: Low-dose ICS (very low dose in children). In children < 5 years, LTRA inhalers can used instead of ICS.

- Initial add-on therapy: Add LABA to ICS, normally as a combination inhaler. In children $<5$ years, use LTRA with very low dose ICS.

- Additional add-on therapy: If no response to LABA, stop LABA, and increase ICS dose (to medium dose in adults, and low dose in children). If LABA addition was helpful but insufficient, continue LABA, but increase ICS (to medium dose in adults, and low dose in children) or try LTRA (in adults and children), theophylline or LAMA (in adults). 
- High-dose therapies: Try increasing ICS (to high-dose in adults, and medium dose in children); adding a fourth drug (in adults: LTRA, theophylline, LAMA, or an oral beta-2 agonist; in children: theophylline).

- Continuous or frequent use of oral corticosteroids: Use daily corticosteroid tablet in the lowest dose that provides adequate disease control, maintain high-dose ICS (medium dose in children), and consider steroid-sparing therapies.

Regardless of the treatment step, patients with symptomatic asthma should use a SABA inhaler as required.

The most recent BTS/SIGN guidelines emphasise the importance of supported self-management, with all those with asthma being offered education for selfmanagement which includes a written personalised asthma action plan in addition to regular review by health care professionals [16].

Treatment should be reviewed every three months until adequate disease control is achieved. Stepping up the treatment is warranted if the disease is not satisfactorily controlled with the current step (e.g., when more than two SABA inhalers are needed per week).

However, stepping up the treatment may expose the patient to increased side effects. Therefore, it should only be done after ruling out suboptimal adherence with existing therapies, poor inhaler technique, and exposure to avoidable or modifiable triggering factors [16].

\subsection{Asthma is a public health challenge}

Asthma is a worldwide public health problem affecting more than 300 million people worldwide [36]. The International Study of Asthma and Allergies in Childhood (ISAAC) was an important project for estimating and comparing asthma prevalence among children in 98 countries around the world [37]. The prevalence of clinical asthma varies between countries, ranging from around 1\% in Indonesia to more than $10 \%$ in North and South America, Australia, and the United Kingdom [38]. In many countries, asthma prevalence seems to be increasing, and the worldwide trends do not seem to be decreasing [39]. 
Although mortality from asthma is relatively low, it represents only the tip of an iceberg of a wide range of adverse outcomes and a significant public health burden of the disease. The disease is associated with expensive health care utilisation, increased morbidity, reduced quality of life, and wider societal impact such as school and work absenteeism [40-42]. The annual cost of asthma in the United Kingdom (UK) in 2011 has been estimated as £1.1 billion pounds (approximately US\$1.8 billion) [43]. Significant financial costs of asthma care are mainly driven by medication use, exacerbations, and hospitalisations [41, 43, 44]. The costs are particularly high for patients with severe refractory asthma [45]. Furthermore, 15 million disability-adjusted life years (DALYs) per year worldwide were attributed to asthma [38]. Due to its high burden, asthma has increasingly received considerable attention by health policy makers and researchers [46].

\subsection{Asthma epidemiology in Wales}

The United Kingdom has one of the highest asthma prevalences in the world across all age groups [38, 43, 47-49]. According to Asthma UK, there were estimated 5.4 million asthma patients in the UK in 2015, of whom 314,000 are in Wales [50]. The mortality rate from asthma in the UK is among the highest across Europe [51].

Asthma epidemiology in Wales has been studied over the recent decades, mainly by means of cross-sectional health surveys and, to a lesser extent, using prospective cohort studies and routinely collected health data.

Health surveys are descriptive investigations of systematically collected health determinants [52], and they are usually used for cross-sectional analyses. Health surveys have been important tools to investigate asthma epidemiology in Wales. The Welsh Health Survey (WHS) has been conducted in 1995, 1998, and annually since 2003 before it ceased in 2015 [53]. In 2014, the WHS estimated the prevalence of patient-reported currently treated asthma in children and adults as $9 \%$ and $10 \%$, respectively $[54,55]$.

The WHS had limitations for studying asthma epidemiology. Self-report has been prone to recall bias. In addition, sampling might not sufficiently represent the entire population since it was limited to people living in private households and excluded the homeless, older people, some migrant workers, and special populations (e.g., armed forces and prisoners) as well as those living in care institutions 


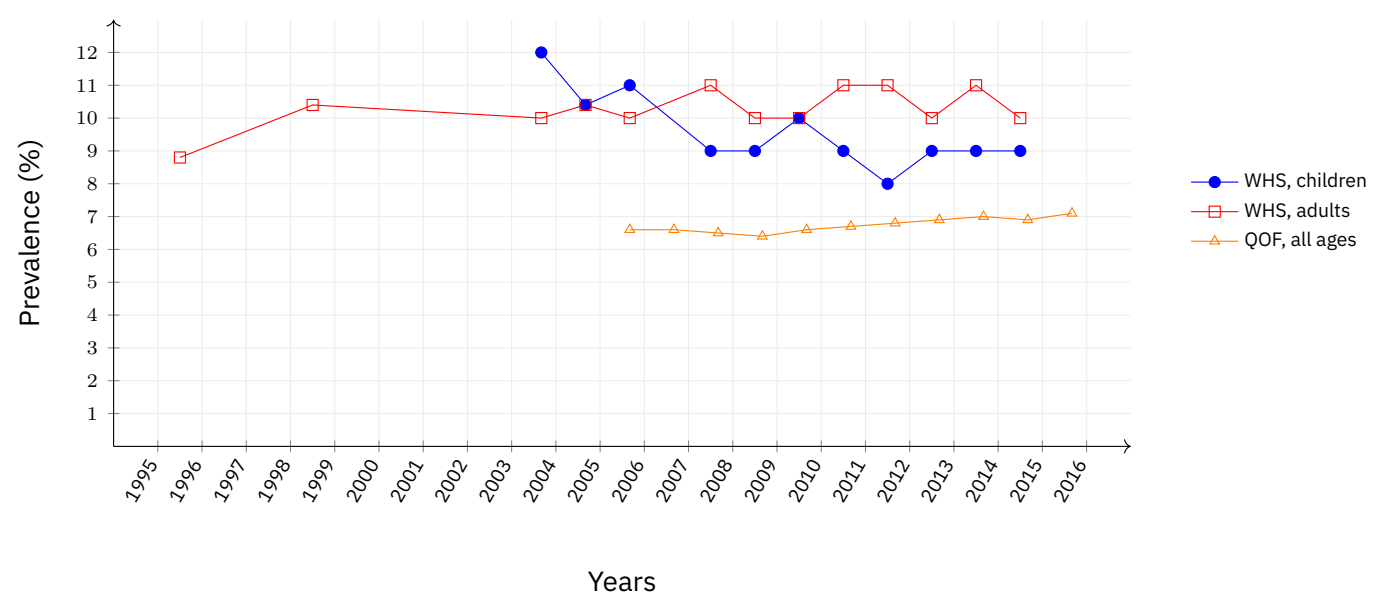

Figure 1.1: Trends of self-reported (in blue and red) and GP-reported (in orange) treated asthma in Wales between 1995 and 2015 according to the WHS [53] and the QOF [59], respectively.

and others due to language barriers [43, 56]. Being an annual study, the WHS had a limited value in providing timely disease insights. The differences in the asthma-related questions asked by the WHS and health surveys in the other UK countries hinder the comparability of self-reported asthma prevalence across the UK [43].

Electronic health record (EHR) data have been also used to estimate asthma epidemiology. Primary care data from a sample of Welsh general practitioner (GP) practices, covering around $1 \%$ of population in Wales, have been used by the Weekly Returns Service (WRS) of the Royal College of General Practitioners. However, the WRS reports did not produce separate asthma statistics for Wales [57]. Since April 2004, primary care data from more (and later all) GP practices in Wales have been used to produce clinical performance indicators as part of the Quality of Outcomes Framework (QOF) [58]. According to the QOF indicator of treated asthma (ASTHMA1 or ASTO01) the prevalence of patients with asthma diagnosis who received asthma treatment in the last 12 months, ranged from 6.4\% in 2008-09 to $7.1 \%$ in $2015-16$ [59]. These estimates were notably lower than the prevalence of patient-reported GP-diagnosed and treated asthma as estimated by the Welsh Health Surveys (see Figure 1.1).

A recent UK-wide analysis showed that Wales had a slightly higher asthma prevalence than the other member counties [43]. The annual prevalences of patientreported doctor-diagnosed-and-treated and GP-reported-diagnosed-and-treated asthma in the fiscal year 2011-2012 were 9.8\% and 6.9\% in Wales compared to UK-wide estimates of $9.2 \%$ and $6.8 \%$, respectively. That study demonstrated that asthma burden in Wales was high with estimated $£ 74.7$ million pounds being spent on 
asthma care by the Welsh public sector in the fiscal year 2011-2012 [43]. This overall cost included $£ 40.5$ million on community prescribing, £9.7 million on GP and practice nurse consultations and out-of-hour calls, £3.3 million on accident and emergency (A\&E) visits and ambulance trips, £8.4 million on hospitalisations, and £12.8 million on Disability Living Allowance.

\subsection{Asthma burden can be reduced}

Despite its high prevalence, asthma burden can be reduced by identifying modified risk factors of adverse outcomes, and improving allocation of health care resources $[60,61]$.

An investigation into asthma deaths in Wales between 1994-1996 [62] found that factors other than disease severity, such as inadequate treatment and patient factors, were identified in $70 \%$ of cases. The inquiry concluded that some of deaths that were attributed to asthma were preventable, and disease morbidity could be reduced.

The National Review of Asthma Deaths (NRAD), a UK-wide inquiry of the circumstances of deaths due to asthma, found that potentially modifiable risk factors played a significant role in the disease hospitalisation and mortality [51]. The NRAD report identified avoidable risk factor in two thirds of the reviewed asthma deaths. Adverse asthma outcomes can be avoided or ameliorated with early diagnosis, improved care, disease monitoring, patient education, personalised asthma action plan, and self-management [51, 63-65]. Exacerbations can be predicted using disease biomarkers and patient medical history [66-70].

Prevention of adverse outcomes can be boosted through better understanding of the disease epidemiology, trends, wider determinants, endotypes and phenotypes, and patterns of natural disease history. Systematic learning from health care data at the population level is crucial for improving asthma care and prevention of adverse outcomes. Effective surveillance for asthma requires a stream of realtime or near real-time data on asthma outcomes at the national level. 


\subsection{Routinely collected data: an overview}

Documentation of health and care events is an integral part of health care. It serves a variety of purposes including supporting the delivery and continuity of care as well as administrative, financial, and legal purposes [71]. However, beyond these uses, large volumes of health data accumulate over time, and can be also useful for further purposes. Routinely collected data (RCD) are data that are regularly collected without $a$ priori specific purpose into central repositories where they can be made available for secondary uses [72]. These data are usually collected in coded forms. Clinical coding systems helps standardising the documentation of clinical information and facilitates the identification of patients with a specific clinical profile. Table 1.2 shows examples of clinical coding systems used in the UK.

Common types of RCD include data on primary and secondary care (EHR data), laboratory tests, medication dispensing, medical insurance claims, vital events (e.g., births and deaths), social care, and education.

Examples of RCD uses include policy and service planning and evaluation [77], epidemiological studies, health surveillance, health technology assessment [78], comparative effectiveness research, and health economic analysis [43, 79].

Compared with traditional sources of health data, such as questionnaire surveys, medical record review, RCD have several advantages for research and surveillance. RCD are usually available in large volumes, and at relatively low costs [80].

Table 1.2: Main clinical coding systems in the United Kingdom.

\begin{tabular}{|c|c|}
\hline Coding Scheme & Description \\
\hline $\begin{array}{l}\text { International Classification of } \\
\text { Diseases, } 10^{\text {th }} \text { Revision } \\
\text { (ICD-10) }[73]\end{array}$ & $\begin{array}{l}\text { Produced and maintained by the World Health Organisation (WHO); aimed for inter- } \\
\text { national comparability of mortality and morbidity; used to classify health encounters } \\
\text { based on diagnosis and health conditions for statistical and administrative purposes; }\end{array}$ \\
\hline Read Codes [74] & $\begin{array}{l}\text { The standard medical terminology for coding clinical information in the UK general } \\
\text { practice. }\end{array}$ \\
\hline $\begin{array}{l}\text { Systematized Nomenclature of } \\
\text { Medicine - Clinical Terms } \\
\text { (SNOMED-CT) [75] }\end{array}$ & $\begin{array}{l}\text { A comprehensive medical terminology for documentation of variety of information } \\
\text { types in clinical practice. }\end{array}$ \\
\hline $\begin{array}{l}\text { Office of Population Censuses } \\
\text { and Surveys Classification of } \\
\text { Interventions and Procedures } \\
\text { (OPCS-4) [76] }\end{array}$ & $\begin{array}{l}\text { Maintained by NHS Digital; used to code operations, procedures and interventions in } \\
\text { UK secondary care. }\end{array}$ \\
\hline $\begin{array}{l}\text { British National Formulary } \\
\text { (BNF) [33] }\end{array}$ & The reference book of coded medication and prescribing in the UK. \\
\hline
\end{tabular}


Many RCD sources cover entire populations, enabling generalisability of studies, reliable epidemiological estimates, and studying rare conditions [81, 82]. The contents of many RCD resources are rich and comprehensive enough to answer a wide range of research questions. Person-level record linkage of two or more RCD sources for the same population allows further research opportunities [83]. RCD are observational, objective data that are often recorded by qualified professionals rather than patients. They are therefore less prone to biases of self-report such as recall, learning, responses biases [84, 85]. Studies using RCD are not prone to experimenter bias since collection of data is independent from their secondary uses. The often-longitudinal nature of RCD allows conducting inexpensive, complex time series analyses. RCD reflect health, morbidity, and health care in the real world rather than in idealised or artificial conditions such as in clinical trials [81]. Therefore, they have been used in the various phases of clinical trials including patient follow-up and evaluation of real-world safety and effectiveness drugs [86, 87].

Nevertheless, being originally collected for other purposes, RCD usually have limitations for use in research. While clinical codes facilitate data standardisation, they do not cover all aspects of health and care. Important non-coded narrative data are usually missing from RCD. Incomplete, inaccurate, incorrect, and inconsistent capture and coding of data is a common concern about RCD [88-93]. Confounding is an important issue in RCD-based studies, and missing residual confounders limits causality inference [94, 95]. Users of RCD should consider their provenance and the circumstances under which they are collected. For instance, with incentivised documentation of care (e.g., QOF), some data items are better recorded than others [93].

With the increasing use and quality of RCD, they have been recognised as key sources of data in the strategic plans of several health research agencies, councils, institutes, and funders across the UK [96-98].

\subsection{Routinely collected data can improve our un- derstanding of asthma}

Asthma is an exemplar of health problems in which RCD can be effectively used for surveillance and support health policy and research. Depending on their content, 
RCD data can provide information on asthma symptoms, diagnosis, laboratory tests, disease severity, disease control, treatments, monitoring, exacerbations, healthcare utilisation, and care quality [99-105].

Asthma-related RCD have been increasingly used to study different clinical and epidemiological aspects of the disease. These include incidence, prevalence, and burden and trends of asthma and its outcomes [43, 83, 106-109], risk factors [110-114], and disease prediction [66, 68, 70, 115, 116].

RCD can be also useful inform management, prevention, and compare effectiveness of interventions [117-119].

RCD have been used in several countries to create disease registries and platforms for asthma surveillance and research [120-122]. In the next section, I present an overview of those projects.

\subsection{Disease registries and observatories}

\subsubsection{Disease registries}

A disease registry is a database that systematically tracks outcomes of interest for patients who have a particular health condition and live in a defined geographic area.

Disease registries are usually set up to support health policy, service planning and/or research [123]. They have been widely used in epidemiological studies and allowed assessment of disease outcomes and understanding the natural history of chronic diseases [124]. Some disease registries can be also used to support health care of individual patients [125].

In the UK, notable examples of asthma registries are the BTS Difficult Asthma Registry [126], its successor the UK Severe Asthma Registry, ${ }^{1}$ and the UK Paediatric Difficult Asthma Network Registry. ${ }^{2}$

Traditionally, disease registries have relied on active, purpose-specific data collection. However, the growing number of EHR databases have provided inexpensive, rich, alternative sources of data. EHR-derived data are usually available in large volumes, and contain real-world data on patient care. These data have

\footnotetext{
${ }^{1}$ https://cl2.n3-dendrite.com/csp/asthma/frontpages/index.html

${ }^{2} \mathrm{http}: / / \mathrm{rs} 2 . \mathrm{e}-\mathrm{dendrite.com} / \mathrm{csp} /$ paedasthma/frontpages/index.html
} 
been increasingly used in registries of long-term conditions such as chronic kidney disease [127], cancer [128], cardiovascular disease [129], and multiple sclerosis [130].

\subsubsection{Health and disease observatories}

Health observatories are projects often run by public health or academic institutions to monitor health status of the population.

There is no consensus on the definition of a health observatory $[131,132]$. The term 'observatory' means that, unlike health authorities, health observatories observe and analyse health phenomena while 'standing back' from them [133]. This observational nature distinguishes health observatories from state-operated health surveillance systems [131-133]. They often combine academic rigour with the high quality of public health professional practice [132]. However, compared to academic research projects, observatories often seek to provide more timely answers to public health questions, supporting health service planners and policy makers' continuous need for up-to-date evidence [132].

Common core functions of health observatories include:

- highlighting health problems requiring attention and measuring their prevalences and burdens,

- conducting health surveillance ${ }^{3}$ on those problems,

- producing actionable insights,

- evaluating service delivery, and

- forecasting the population health [132]

In undertaking these functions, health observatories need to identify the various sources of data that could be used to assess health problems, such as routinely collected data sets and disease registries [43]. They can also identify important gaps in data sources, and often seek to link different data sources for better understanding of health problems [43, 132, 134].

Health observatories often target several health problems [131, 132]. However, some are dedicated to specific public health problems, such as health inequality, or specific diseases, such as asthma.

\footnotetext{
${ }^{3}$ Health surveillance is the systematic, continuous analysis, interpretation, and feedback of data related to specific health problems in a population [52]
} 
Several asthma-specific observatories and surveillance systems have been established around the world. In the UK, the Surveillance of Work-related and Occupational Respiratory Disease (SWORD) system, maintained data about workers with occupational asthma [135]. It used systematic, voluntary reporting from specialists of data about suspected new cases including demographics, occupation, and suspected causal agents. A similar system, the Observatoire National de Asthmes Professionnels, was established in France to monitor occupational asthma [136]. It was based on clinical, diagnostic and profession-related data about workers with occupational asthma, which were voluntarily reported by physicians using questionnaires. In Canada, the Ontario Asthma Surveillance Information System (OASIS) is based on a cumulative cohort of asthma patients in Ontario [134]. OASIS is based on health administrative data of physician billing, emergency department visits, and hospital admissions. It aims to monitor changes in asthma epidemiology, management, and health service use as well as variations in clinical practice and disease burden. In the United States (US), the Population-Based Effectiveness in Asthma and Lung Diseases (PEAL) Network is an asthma registry with wide research, surveillance, and public health applications [137]. The PEAL Network uses data of computerised medical billing and claims as well as pharmacy and laboratory data. Claim data for emergency department visits and hospitalisations have been also widely used for asthma surveillance elsewhere [105, 107, 120, 121 , $138,139]$.

\subsection{An asthma observatory for Wales: opportuni- ties, challenges, and solutions}

Given the high asthma burden in Wales, the motivation of this doctoral project was the pressing need for a reliable tool to monitor and study the disease and its outcomes.

In the UK, including Wales, asthma is mainly managed in primary care. Primary and secondary care data have been routinely collected with high-to-complete geographical coverage across Wales. These data, as well as various other health data source, are maintained and linked in the Secure Anonymised Information Linkage (SAIL) databank at Swansea University [140, 141]. With the high asthma prevalence in Wales, large volumes of rich, representative, real-world, longitudi- 
nal observational data on asthma patients are available in the SAIL Databank. This data-intensive environment provides a unique opportunity to develop an observatory for asthma in Wales.

Developing an observatory for asthma exploits the merits of RCD but is hindered by their limitations. Algorithms to identify patients with asthma and assess asthma outcomes using RCD data are essential in the observatory development. However, for a heterogeneous disease such as asthma, identifying cases and assessment of outcomes using RCD was a key challenge, and valid case definitions were needed. However, there were neither gold standards for the clinical definitions of asthma and its outcomes, nor consensus on their definitions using RCD.

With the above challenges, data-driven approaches can be employed to uncover patterns generated by the disease in the population. Populations health data 'speak for themselves'. Their patterns, however, need to be carefully interpreted in the light of clinical and epidemiological knowledge and hypotheses, as well as data provenance and quality [142]. Using proper design and interpretation, datadriven approaches, such as mixture models (e.g., latent class analysis (LCA), can be employed to identify asthma patients in a population.

\subsection{Thesis aims, research questions, and objectives}

The aims of this thesis are to describe the methodology and development of the Wales Asthma Observatory ${ }^{4}$ as a platform for asthma surveillance and research in Wales, and to demonstrate its utility for health policy. Throughout the thesis, I will refer to the Wales Asthma Observatory as the Observatory.

The thesis includes a systematic scoping review of approaches to define and assess asthma using RCD and their reporting quality. A particular focus of the thesis is the identification of asthma patients in Wales using RCD in the light of the absence of gold standard and inherent limitations of RCD.

\section{Research questions}

Throughout the thesis I will answer the following research questions:

\footnotetext{
${ }^{4}$ http: //www. wales-asthma-observatory.uk/
} 
1. What are the contemporary approaches and practices of identifying asthma patients and assessing asthma outcomes using RCD? How well have these approaches and their validity been described? (Chapter 2)

2. Can a latent class model based on recorded primary care data identify clinically meaningful asthma-related groups? How does this model compare with commonly used doctor-reported and self-reported measures to identify asthma patients? (Chapter 3)

3. How well have asthma-related primary care events been recorded in Wales? (Chapter 4)

4. Do asthma patients across the socioeconomic scale in Wales have equal disease outcomes? (Chapter 5)

\section{Objectives}

To address the research questions, I pursued the following objectives:

1. To review the current practices of studying asthma using routinely collected data (Chapter 2). This is performed through a systematic scoping review of the recent asthma literature with the following objectives:

a. To survey the algorithms used to define asthma and assess asthma outcomes using routinely collected EHR data;

b. To explore the practices of the reporting on the validity of those algorithms;

c. To assess the clarity of reporting on the implementation of these algorithms and other methodological aspects related to the use of RCD.

2. To discuss the challenges of identifying asthma patients using RCD (Chapter 2 and Chapter 3).

3. To develop a data-driven reference identification for asthma in Wales based on routinely collected data (Chapter 3 ). This includes:

a. Development of a mixture model, namely latent class model, to identify patients with both ever and current asthma.

b. Evaluating the concordance of this model with other routine data-based case definitions as well as with self-reported measures. 
4. To describe the purpose, context, and methodology used in the Observatory development, as well as data quality (Chapter 4). This will include the following sub-objectives:

a. To describe the purposes of the Observatory as a data-intensive platform for asthma surveillance and research.

b. To describe the logistics, technical infrastructure, and RCD databases based on which the Observatory was founded.

c. To define the Observatory's source population and the case definitions used to identifying asthma patients.

d. To describe the Observatory's data structure and the available variables.

e. To describe the approach used to improve efficiency and reproducibility of data interrogation.

f. To assess the quality of selected asthma-related primary care events in the Observatory.

5. To demonstrate the Observatory's utility for health policy by investigating inequalities in asthma outcomes (Chapter 5). This includes:

a. Investigate the variations in asthma-related outcomes across the deprivation groups in Wales, using a count regression model adjusted for age group and gender.

b. To interpret the model in the light of previous studies and strengths and limitations of routinely collected data used.

c. To reflect on the implications of the findings on health policy in Wales.

6. To reflect on the work presented in Chapters $2,3,4$, and 5, identify main strengths and weaknesses of the Observatory, interpret the findings with related literature, discuss the opportunities and challenges to maximise the potentials of asthma data, and outline further research directions.

\subsection{Thesis structure}

The rest of the thesis is organised as follows:

In Chapter 2, I present a systematic scoping review of the different approaches of defining asthma and assessing its key outcomes, and the clarity of reporting 
on their implementation and validity. The review reveals wide variations in these approaches, and highlights the challenges of developing uniform RCD-based methods to study asthma. This systematic scoping review was published in a leading respiratory journal [102].

In Chapter 3, I present an overview to approaches to develop valid methods to identify asthma patients using RCD. Recognising asthma heterogeneity, data limitations, and informed by the findings of the previous chapter, I justify using a data-driven approach to identify asthma patients. I describe a latent class model based on primary care data as a data-driven identification model for asthma in the Observatory. I also derived a reusable classification algorithm base on the latent class model. I compared the algorithm's performance with commonly used asthma case definitions based on objective and self-reported data.

In Chapter 4, I describe the Observatory's context, purpose, data structure, case definitions, variables, information governance, and approaches to improve efficiency and reproducibility of the Observatory interrogation. I also examine the quality of asthma-related primary care data and discuss their implications on the Observatory utilisation.

In Chapter 5, I demonstrate the Observatory's utility for health policy by examining the inequality gaps in asthma outcomes across the socioeconomic spectrum in Wales.

Finally, in Chapter 6 I reflect on the work presented in this chapter, present with the thesis's original contributions, and discuss the Observatory's strength and weaknesses in the wider context of asthma and RCD research. I explore opportunities and challenges towards maximising the value of RCD to improve asthma outcomes. I then propose future research directions and developments to improve the Observatory's methodology and content. 


\section{Chapter 2}

\section{Defining asthma and}

\section{assessing asthma outcomes}

using electronic health

record data: a systematic

scoping review

Same conditions, different definitions

This chapter is based on the following published paper:

Al Sallakh MA, Vasileiou E, Rodgers SE, Lyons RA, Sheikh A, and Davies GA. Defining asthma and assessing asthma outcomes using electronic health record data: a systematic scoping review. Eur Respir J 49 (6 2017) 1 (see Appendix A.2). 
In this doctoral project, I used routine data to create a registry and observatory for asthma in Wales. However, the heterogeneous nature of asthma and the limitations of routinely collected EHR data impose challenges on how these data could be used for this purpose. In addition, there is currently no consensus on approaches to defining asthma or assessing asthma outcomes using electronic health record (EHR)-derived data.

To inform the methodology of identifying of asthma patients and assessing the outcomes in this project, described in Chapter 3, in this chapter, I present a systematic scoping review of how routinely collected EHR data have been used in the recent international asthma literature.

I systematically searched for asthma-related articles published between 1-1-2014 and 31-12-2015. From the eligible studies, I extracted the algorithms used to identify asthma patients and assess severity, control and exacerbations from using different types of EHR-derived data sources. I also investigated how authors justified the validity of these algorithms, and how they reported on the aspects related to the use of EHR-derived data in their studies.

From 113 eligible articles, I found significant heterogeneity in the algorithms used to define asthma ( $n=66$ different algorithms), severity $(n=18)$, control $(n=9)$, and exacerbations $(n=24)$. For the majority of algorithms $(n=106)$, validity was not justified. In the remaining cases, approaches ranged from using algorithms validated in the same databases, to using non-validated algorithms that were based on clinical judgement or clinical guidelines. The implementation of these algorithms was sub-optimally described overall.

Although EHR-derived data are now widely used to study asthma, the approaches being used are significantly varied and are often underdescribed, rendering it difficult to assess the validity of studies and compare their findings. Given the substantial growth in this body of literature, it is crucial that scientific consensus is reached on the underlying definitions and algorithms. 


\section{Chapter Contents}

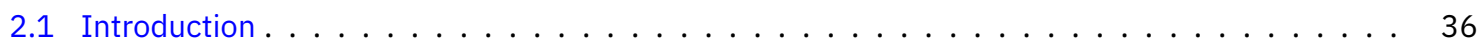

2.2 Methods: a systematic scoping review $\ldots \ldots \ldots \ldots \ldots \ldots \ldots \ldots \ldots \ldots$

2.2 .1 Identifying the research questions $\ldots \ldots \ldots \ldots \ldots \ldots \ldots$

2.2.2 Identifying relevant studies: literature search strategy . . . . . . . . . . . . . . . 38

2.2 .3 Study selection . . . . . . . . . . . . . . . . . . . . . . . . . 39

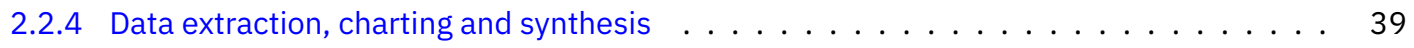

2.2.5 Collating, summarising and reporting the results $\ldots \ldots \ldots \ldots \ldots$

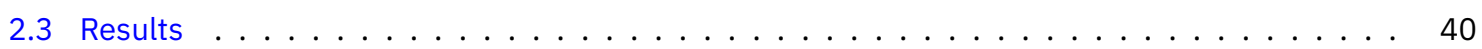

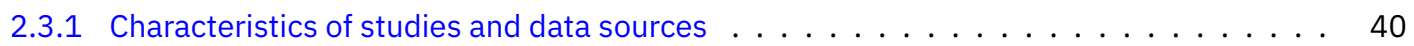

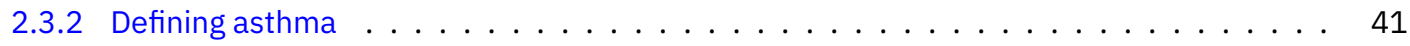

2.3.2.1 Various diagnostic labels of asthma $\ldots \ldots \ldots \ldots \ldots \ldots \ldots \ldots$

2.3.2.2 Approaches to restrict study domain . . . . . . . . . . . . . . . . . 42

2.3.3 Assessing asthma severity . . . . . . . . . . . . . . . . . . . . . . . . . 43

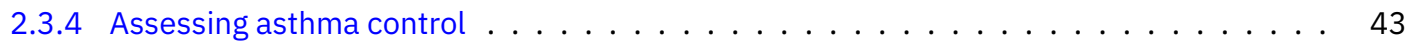

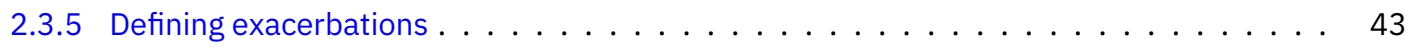

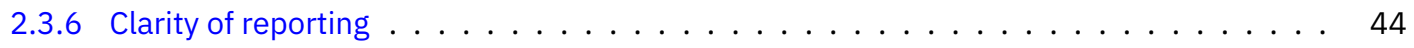

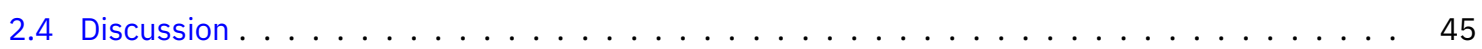

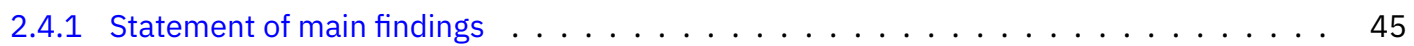

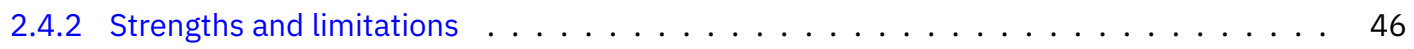

2.4.3 Interpretation in the light of previous studies $\ldots \ldots \ldots \ldots \ldots \ldots \ldots$

2.4.4 Implications for policy, practice and research . . . . . . . . . . . . . . . . . . . 49

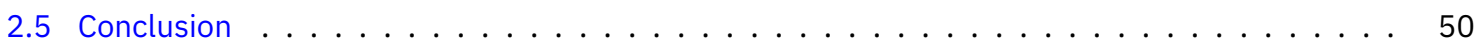




\subsection{Introduction}

Methods to define and assess asthma are key to the development of the Wales Asthma Observatory. To inform the development of Observatory, it was important to survey the contemporary methods to define and assess asthma using routinely collected electronic health record (EHR) data. This would provide insights into the different approaches and the challenges to define the disease from these data.

Asthma is in clinical practice a diagnosis based on the patient's history, examination and objective tests [143]. Typically, the diagnosis is made initially based on signs and symptoms such as wheezing, shortness of breath, tightness of chest, or cough that often follow characteristic fluctuating patterns and triggerability. Confirmation of diagnosis requires demonstrating variable expiratory obstruction of airflows over time [1]. However, asthma is not a single clinical entity. Instead, it is increasingly considered to represent a heterogeneous group of disorders with different phenotypes and endotypes [5]. In addition, several other diseases may masquerade as asthma leading to misdiagnosis [143]. Subsequently, the clinical definitions of asthma and its key outcomes, including asthma severity, control, and attacks/exacerbations have been the subject of vigorous debate with no consensus yet reached on a gold standard for diagnosis [2, 144-148].

The uncertainty in the clinical definition of asthma has significant implications for research. Particular challenges arise in the context of epidemiological studies where groups of populations rather than individual patients are compared, and in which validated, standardised, operational case definitions are needed [149, 150]. Various types of health data have been used in these studies. Traditionally, these data were often self-reported or collected by investigators specifically for the purpose of research. In addition, these studies have been increasingly conducted using data derived from EHR such as health administrative data, health insurance data, primary care data, dispensing data, and disease registries. These data usually have the advantages of being inexpensive, objective, read-world data, and are usually available in large volumes. However, they have inherent limitations such as incorrect, inconsistent, or missing recording of health care events, resulting in many key clinical variables being missing or of low quality $[82,151]$ (see Section 1.5). 
The limitations of routinely collected EHR data add a further layer of complexity and challenges to the use of these data in asthma research. Investigators often resort to indirectly assess low-quality variables using surrogate variables of higher quality or algorithms based on those surrogate variables. Although the face validity is important, formal validation of these algorithms is critical to ensure reproducibility of findings of studies conducted using routinely collected EHR data [152]. With the increasing and widespread use of EHR data in asthma literature, it is however unclear to which extent algorithms to define and assess asthma using these data have been supported by sufficient evidence of validly.

Clear reporting of methodology in studies conducted using routinely collected data (RCD) is critical not only for scientific transparency and understandability by their consumers (e.g., researchers, service providers, policy makers), but also for reproducibility and comparability of research findings as well as for evidence synthesis and meta-analysis. Guidelines to improve the reporting of observational studies have been available for several years [153]. However, adherence to these guidelines has been suboptimal [154, 155]. Furthermore, a recent assessment found that the reporting of studies conducted using routinely collected health data was often inadequate [156]. Until recently there were no clear guidelines on how to report specific methodological aspects related to the use of EHR-derived data in research. The REporting of studies Conducted using Observational Routinelycollected health Data (RECORD) Statement [157], published in September 2015, was an extension to the Strengthening the Reporting of Observational Studies in Epidemiology (STROBE) Statement which argues authors to completely and clearly communicate, in addition to the main methodology, important aspects related to the use of routinely collected health data in their study. To my knowledge, there was no existing assessment of the clarity of reporting on the use of EHR data in the recent asthma literature.

To assess the approaches and practices of using routinely collected EHR data in asthma research, I systematically interrogated the recent EHR-based asthma literature with the following objectives:

- To describe the different methods of defining asthma and assessing disease severity, control and exacerbations in EHR-based studies;

- To assess the clarity of reporting on the implementation and validity of these methods. 


\subsection{Methods: a systematic scoping review}

I conducted a systematic scoping review based on Arksey and O'Malley's five-stage framework [158]. This methodology included identifying the research question, identifying relevant studies through literature search, selection of eligible studies, data charting and collating, and summarising and reporting the results. The research questions were:

- How were asthma and its key outcomes defined using EHR data in the recent literature?

- How did authors report on the validity of their EHR-based algorithms?

- How clearly was the reporting on the implementation of these algorithms and other EHR-related methods?

\subsubsection{Identifying the research questions}

Since this framework is intended for exploratory review, research questions could be iteratively developed during the review process. The primary research question behind this scoping review is:

- How has asthma and its key outcomes been defined using RCD in the recent literature?

In addition, while reviewing the literature, two other related research questions emerged to address validation of case definitions and the clarity of reporting of methodological aspects related to routinely collected EHR data:

- How did authors report on the validity of their RCD-based case definitions?

- More generally, how clear were the RCD-related methods reported?

\subsubsection{Identifying relevant studies: literature search strategy}

I searched PubMed using a broad query (Table A.1.1) to retrieve asthma studies that used EHR-derived data and were published between January 1, 2014 and December 31, 2015. The search query was iteratively improved by adding many variations and equivalents of the keywords "EHR" and "routinely collected data" as well as named data sources found in the literature. Only articles written in English were included. 


\subsubsection{Study selection}

I excluded non-relevant articles by reviewing titles and abstracts, referring to the full-text when needed. I included only articles where asthma was a main finding. For the purpose of this review, I limited the concept of EHR-derived data to coded, objective, individual-level data that were generated as a by-product of routine health care. Therefore, I excluded studies in which only text-based medical records were used, the coded nature of EHR-derived data was unclear, the used data were aggregated, or no asthma-specific variables were measured from EHR-derived data. For example, if the only variables measured from routine data for asthma patients were related to co-morbidities or birth weight the study was excluded.

\subsubsection{Data extraction, charting and synthesis}

From each of the eligible articles, I extracted and summarised information from the full text and online supplements, including basic bibliography, setting (country) and design; names and types of EHR-derived data sources used; algorithms to identify asthma patients, assess disease severity, control, exacerbation; and how authors reported on algorithm validity. In this context, I referred to 'validation' as any attempt to assess the algorithm's concurrent ${ }^{1}$ or construct validity. ${ }^{2}$ I used the RECORD Statement's 13-items checklist [157] to assess the completeness and clarity of reporting of methodological aspects related the use of EHR-derived data in the study. I investigated whether in each study authors provided detailed information on how they identified asthma populations and assessed asthma outcomes. Ideally, the checklist requires authors to provide detailed description of the algorithms used, including complete lists of clinical codes and any validation performed previously or in the same study. In addition, information about the data sources used should be provided, including their content and validity, their catchment areas, level of access to them by authors (i.e. whether they had access to the whole or part of the dataset), explanation of any record-linkage performed, and in which date range the data used in the study were originally recorded. Authors should also explain how they prepared and cleaned the data for the purpose

\footnotetext{
${ }^{1}$ Concurrent (criterion) validity is the extent to which the algorithm agrees with a concurrent measure, the validity of which to establish the diagnosis was previously assessed.

${ }^{2}$ Construct validity is the extent to which the algorithm accurately measures the real disease state.
} 
of their study (e.g., how they processed inconsistent and invalid values). Also, they should ideally publish the programming source codes used for data extraction, preparation, and analysis. To enable the readers critically evaluate the validity of studies, authors should adequately communicate any implications of using EHR-derived data sources in their studies to assess a complex condition such as asthma. Table A.1.2 describes the data extraction and charting tool used in this review. Article screening and data extraction were performed independently by two researchers (myself and Eleftheria Vasileiou ${ }^{3}$ ) with my first supervisor, Gwyneth Davies, arbitrating.

\subsubsection{Collating, summarising and reporting the results}

I summarised the general characteristics of the reviewed articles including the country to which the study source population belonged, study design, and types of routine data sources used in the study (e.g., health insurance claims, primary or secondary care, or pharmacy dispensing data). I highlighted the clinical labels used in asthma algorithms as they appeared in the studies. I also identified the approaches used in these algorithms which aimed at improving their accuracy. I also summarised the practices of justifying the validity of algorithms (e.g., by citing a previous validation of the same algorithm in the same population) as well as aspects related to the use of EHR data.

\subsection{Results}

\subsubsection{Characteristics of studies and data sources}

I included 113 articles in the review. Figure 2.1 shows the study selection process. Most studies were conducted in the United States (US), Taiwan, and Canada (Table A.1.3), and employed longitudinal designs (Table A.1.4). The most commonly used data types were health insurance claims followed by medical record repositories and dispensing databases (Table A.1.5).

\footnotetext{
${ }^{3} \mathrm{~A}$ PhD student at Asthma UK Centre for Applied Research, University of Edinburgh.
} 


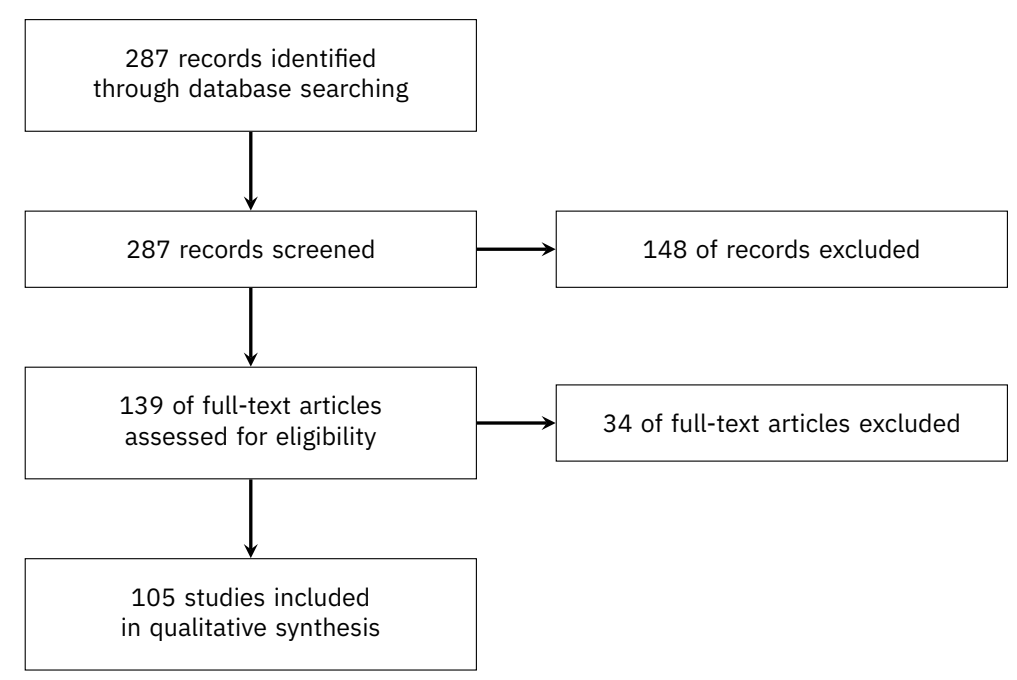

Figure 2.1: Flowchart for study selection in this scoping review.

\subsubsection{Defining asthma}

\subsubsection{Various diagnostic labels of asthma}

I identified 66 different algorithms to define asthma under seven diagnostic labels (Table A.1.6).

'Persistent asthma' was defined over 12 and 24 months using the US Healthcare Effectiveness Data and Information Set (HEDIS) criteria [159], which involved assessing for any of the following asthma-related events: (1) emergency department (ED) visit, (2) hospitalisation, (3) outpatient visit and two asthma prescriptions, or (4) four asthma prescriptions [160-163]; by HEDIS criteria except "four asthma prescriptions" [164]; and by any asthma encounter (hospitalisation or ED visit) or using oral corticosteroids (OCS) for three or more days [165].

'Current asthma' was defined by any asthma encounter in the last three years [166].

'Current general practitioner (GP)-reported and diagnosed asthma' was defined as any asthma encounter in the last 12 months, and 'current GP-reported, diagnosed and treated asthma' as the same plus any asthma prescription in the same period [167].

Patients with treated asthma were otherwise required to have at least three dispensing events of asthma treatments in three different quarters of the year [168]. 
'Acute asthma' was defined using any asthma diagnosis codes in ED or inpatient data [169].

In the remaining studies, the label 'asthma' was defined using various algorithms, some of which were similar to those of the aforementioned more specific labels.

The intervals over which asthma diagnostic/management and prescription codes where queried were specified in 31 and 8 studies, respectively. The positions of diagnostic codes in the encounter (i.e. primary or secondary) were specified in 37 studies.

I identified five approaches in these algorithms: requiring diagnostic/management events, prescription events, or both (Table A.1.7).

\subsubsection{Approaches to restrict study domain}

To reduce the risk of misclassification, some studies applied additional non-asthma selection criteria which were meant to exclude individuals who are unlikely to have asthma. These criteria were based on restricting the study population based on age and co-morbidities.

\section{Age restriction}

In 12 studies Table A.1.8, age restriction was applied to asthma definitions as an indirect way of excluding those with co-morbidities that are common at age extremes and to acknowledge the uncertainty of asthma diagnosis in these ages. The minimum age limits at were $2(\mathrm{n}=4), 3(\mathrm{n}=2), 5(\mathrm{n}=1)$ and $12(\mathrm{n}=1)$, while the maximum age limits were 40,55 and $60(\mathrm{n}=1$ for each).

\section{Excluding patients with specific co-morbidities}

Eighteen studies (Table A.1.9) applied additional criteria to exclude asthma patients who also had other conditions, most commonly cystic fibrosis and chronic obstructive pulmonary disease (COPD). The list also includes "smoker over the age of 60" as a proxy for COPD diagnosis. The complete list is shown in Table A.1.9. 


\subsubsection{Assessing asthma severity}

Eighteen studies used 20 different algorithms to assess asthma severity (Table A.1.10), as binary (i.e. severe vs. non-severe asthma) $[160,168,170-183]$ or ordinal variables (mild, moderate, and severe asthma [184]; or low, moderate, and high-risk asthma [185]). The algorithms were based on one or more of the following asthmarelated variables: number and/or dosage of prescriptions-namely short acting beta agonist (SABA), inhaled corticosteroid (ICS), OCS, and leukotriene receptor antagonist (LTRA) - and number of hospitalisations, ED and outpatient visits. Almost all algorithms (17) used prescriptions (either alone or with other variables), while one algorithm was based only on hospitalisations and ED visits [181]. The intervals over which asthma severity was assessed were three [174], six [183], $12[160,168,173,175-177,179,181,182,184,185], 24$ months [178, 180], or unclear $[171,172]$.

\subsubsection{Assessing asthma control}

Nine studies assessed asthma control using 11 algorithms, in 9 of which the interval was 12 months, in one 1-3 months, and in the remaining study this was unclear (Table A.1.12). Uncontrolled asthma was defined by a minimum number/dose of SABA prescriptions [175, 176, 184, 186, 187]; any or short-course OCS prescriptions [175, 176, 186-189]; any hospitalisation or ED visit with either diagnosis of asthma $[172,175,176,186-188,190]$ or - in already diagnosed asthma patients - diagnosis of status asthmaticus, pneumonia, dyspnoea, or respiratory insufficiency [175]; unscheduled outpatient visits for asthma or lower respiratory tract infections (LRTI) [176]; and GP consultations for LRTI requiring antibiotics in asthma patients [176]. Asthma impairment was defined based on the required SABA use, namely an average of more than two salbutamol puffs per day [176]. One study assessed asthma control based on number of OCS and SABA prescriptions per year (without giving any further details about the actual algorithm) [186].

\subsubsection{Defining exacerbations}

Twenty-four studies defined exacerbations using EHR-derived data (Table A.1.11), as a dichotomous variable (absent vs. present) [161, 162, 168, 172, 175-177, $180,182-184,187-189,191-199]$, or stratified into absent, moderate and severe 
[200]. Oral corticosteroid prescriptions were used as a marker for exacerbations in 17 studies, either alone [168, 175, 176, 180,184, 187, 192, 193, 198] or with a concurrent asthma encounter (e.g., a GP, outpatient, or ED visit, or hospitalisation within five or seven days) [161, 162, 177, 182, 183, 191, 197, 199]. In one study, exacerbations were defined by a minimum of six SABA prescriptions per year [192]. Other definitions included an outpatient code of 'asthma exacerbation' [197], asthma hospitalisation [168, 175, 177, 180, 182, 184, 188, 189, 191, 193, 195, 196, 198-200], asthma ED visit [161, 175-177, 180, 182, 183, 188, 189, 191, 193, 196-199], or hospitalisation with diagnosis of status asthmaticus, or — in already diagnosed asthma patients - diagnosis of pneumonia, dyspnoea, or respiratory insufficiency [175].

\subsubsection{Clarity of reporting}

Overall, the reporting of methodological aspects of using EHR-derived data was suboptimal. The majority of studies presented no information on the algorithms' validity. Among studies that reported on the validity, I identified 10 practices of reporting or on or justifying the validity of algorithms (Table 2.1): (1) performing validation or concordance analysis in the same study against other measures based on different data sources (e.g., medical record review or patient-reported measures); (2) referring to previous validation of similar algorithms in the same or (3) different databases; (4) referring to previous validation of similar algorithms for different diseases in the same or (5) different database (6); using algorithms 'consistent' with previous studies in the same or (7) different databases; (8) using nationally developed algorithms; (9) using algorithms based on clinical guidelines; (10) and relying on previous validation of the database content. Some studies did not provide clear algorithms for asthma severity or control, but only referred to their components [168, 180, 182, 183, 186].

Of the 113 reviewed studies, 40 studies used record-linkage, of which 17 mentioned it in the abstract, and 28 provided at least some explanation in the full text. The geographical region, time frame of data, and types or names of the data sources were mentioned in 83, 91, and 104 abstracts, respectively. Eighty-three studies reported their extent of access to the data sources. The intervals over which the algorithms were applied were often not reported. One hundred and eleven studies touched on the implications of using EHR data to study asthma. Of 
Table 2.1: Practices of reporting or justifying the validity of algorithms to define and assess asthma using EHR-derived data.

\begin{tabular}{|c|c|c|c|c|c|}
\hline \multirow[t]{2}{*}{ Algorithm validity was justified by } & \multicolumn{5}{|c|}{ Number of algorithms } \\
\hline & $\begin{array}{c}\text { Identifying } \\
\text { asthma } \\
\text { patients }\end{array}$ & $\begin{array}{l}\text { Assessing } \\
\text { severity }\end{array}$ & $\begin{array}{l}\text { Assessing } \\
\text { control }\end{array}$ & $\begin{array}{l}\text { Defining ex- } \\
\text { acerbation }\end{array}$ & $\begin{array}{l}\text { Total per } \\
\text { category }\end{array}$ \\
\hline $\begin{array}{l}\text { Validation of the same algorithm in the same } \\
\text { database }\end{array}$ & 14 & 1 & 1 & 1 & 17 \\
\hline $\begin{array}{l}\text { Validation of the same algorithm in different } \\
\text { database(s) }\end{array}$ & 2 & 6 & 3 & 2 & 13 \\
\hline $\begin{array}{l}\text { Validation of other diseases' algorithms in the } \\
\text { same database }\end{array}$ & 2 & 0 & 0 & 0 & 2 \\
\hline $\begin{array}{l}\text { Validation of other diseases' algorithms in differ- } \\
\text { ent database(s) }\end{array}$ & 1 & 0 & 0 & 0 & 1 \\
\hline $\begin{array}{l}\text { Being consistent with similar studies in the same } \\
\text { database }\end{array}$ & 1 & 0 & 1 & 0 & 2 \\
\hline $\begin{array}{l}\text { Being consistent with similar studies in different } \\
\text { database(s) }\end{array}$ & 1 & 0 & 0 & 1 & 2 \\
\hline $\begin{array}{l}\text { Validation or concordance analysis in the same } \\
\text { study }\end{array}$ & 4 & 0 & 0 & 0 & 4 \\
\hline Being based on nationally developed algorithms & 3 & 0 & 0 & 2 & 5 \\
\hline Relying on the validity of database coding & 5 & 0 & 0 & 0 & 5 \\
\hline Being based on clinical guidelines & 0 & 3 & 0 & 0 & 3 \\
\hline Not justified & 76 & 8 & 4 & 18 & 106 \\
\hline
\end{tabular}

these, 64 and 63 studies discussed the risk of misclassification bias and unmeasured confounding, respectively. Six studies acknowledged the possible changes over time in data quality and coding practices and the entailing changes in case definition eligibility and accuracy. Five studies explained their data cleansing procedures. Finally, no study shared the programming codes of data preparation and analysis.

\subsection{Discussion}

\subsubsection{Statement of main findings}

There is a considerable international activity in using EHR-derived data to study a variety of asthma populations and outcomes. This systematic analysis of the contemporaneous asthma literature provides a high-level view on how asthma and its main outcomes have been defined using routinely collected EHR data. Importantly, I found wide variations in the approaches used with limited attention being paid to the validity of the underlying algorithms used and suboptimal reporting on the 
methodology. This poses a major challenge to the interpretation and reproducibility of this important, emerging body of research inquiry.

\subsubsection{Strengths and limitations}

To my knowledge, this is the first systematic exercise to investigate the quality of reporting on EHR-based studies, especially the validity of measures, in the context of asthma. In undertaking this work, I used robust approaches which involved two people independently selecting studies and undertaking data extraction. The findings of wide variations and suboptimal reporting of methods and their validity may also apply to other chronic diseases. This review had no geographic limits, but it was confined to assessing the recent literature. Examining the most recent asthma literature is most likely to provide meaningful insights on current practices. Finding studies conducted using routinely collected EHR data in the literature was challenging as there was no standardised method to do this. A dedicated Medical Subject Headings (MeSH) term for "routinely collected health data" in MEDLINE database was previously suggested [201]. However, I believe my broad search query, including a long list of synonyms routinely collected health data and clinical codes for asthma diagnosis reasonably increased the search recall. In few studies, it was a challenge to separate criteria used to define asthma from the study-specific inclusion and exclusion criteria. A limitation was that I did not systematically check whether the references provided by a study to support the claimed validity of algorithms in question actually provided sufficient evidence of validity. For example, slight differences might exist between the algorithms used in a given study and those validated by previous studies.

\subsubsection{Interpretation in the light of previous studies}

Although EHR-derived data are convenient resources for research, they are originally collected for other purposes, and usually suffer from missing or incorrect data and potential biases [82, 151, 202]. Asthma-related EHR data potentially suffer from significant levels of uncertainty due to a set of factors. Some of these factors are inherent to asthma as a heterogeneous disease with fluctuating and variable natural history. Other factors include the wide variability in health care provision, and in the practices of documentation and coding of clinical data. In addition, EHR systems usually fail to capture complete and accurate clinical in- 
formation at the point of care due to design limitations and inefficient use of these systems by clinicians to document clinical data [203, 204]. Altogether, these factors create high levels of variability and inconsistency in the information recorded on asthma patients, even those with similar clinical profiles. Furthermore, many EHR-derived databases often lack important variables, such as lung function, indication of dispensed medications, adherence to treatment, and lifestyle, which are vital for identifying and assessing asthma patients.

The aforementioned issues impose challenges on the interpretation of asthmarelated health events in EHR-derived data and their use to identify and assess asthma patients. These issues are further discussed in Section 3.1.1. In this review, asthma diagnosis codes were commonly used solely for identification of asthma patient. However, these codes may be recorded after a trial or wrong diagnosis, and do not capture undiagnosed patients [205]. In addition, although an asthma diagnosis code may be recorded during any asthma-related health encounter, it does not necessarily imply an active or treated disease. More complex approaches for asthma patient identification included the requirement of medication codes in addition to an asthma code either to identify treated patients or to increase the specificity of "any asthma" case definition. Patients with "any asthma" were also ascertained by only medication codes where only dispensing data were available. However, this approach could exclude patients with active disease who did not receive prescriptions. In addition, it could incorrectly include non-asthma patients since some asthma medications, such as SABA and oral corticosteroids, could be prescribed in other conditions.

These challenges are however not insurmountable. In this analysis, I found several approaches, in addition to asthma-related criteria, which were intended to improve the specificity of algorithms such as age limitation, exclusion by comorbidities, and diagnosis position restriction. I was able to distinguish these approaches from study-specific patient selection criteria. Age restriction was driven by the uncertainty of asthma diagnosis in age extremes. In early childhood the clinical diagnosis of asthma is difficult to establish, while in the elderly COPD may be misdiagnosed as asthma [206]. Excluding patients, who already satisfied asthma criteria, but who also had other specific co-morbidities was a common practice. In the absence of data on respiratory symptoms, lung function, and laboratory tests, excluding adult "asthma" patients who also had COPD diagnosis and/or smoking history reflects the assumption that they were unlikely to have 
asthma, and was assumed to increase the specificity of asthma case definitions. However, although misdiagnosis between the asthma and COPD is common, they may coexist in what is known as asthma-COPD overlap syndrome (ACOS) [207], although whether this should be considered a distinct third entity is under active debate [208-211]. Many other respiratory conditions can mimic asthma. Unless excluding asthma cases with co-morbidities is required by the study's scope, it may introduce risk of diagnostic purity bias [212] which compromises the study's external validity.

Ultimately, validity of the EHR-based algorithms should be assessed. Ideally, these algorithms should be validated in the databases in which they are intended to be used. However, this was often not the case. Instead, using algorithms with only reasonable face validity based on clinical guidelines or clinical judgement is a very common practice in EHR-based studies [213, 214]. These approaches implicitly assume that clinical codes in the database accurately represent the patient's actual health care events [213], which is a questionable assumption. It is worth noting that the validity of algorithms is not necessarily portable across datasets or populations [215]. This means that a case-finding algorithm that performs well in a dataset may be inaccurate if used in another dataset. Populations may differ in demographics, health parameters, and health care. EHR-derived datasets, even those with the same type of healthcare data, may differ in their content and quality. In this review, however, I found that it is common for algorithms of asthma and its outcomes to be re-used in other populations without re-validation in the target datasets. I believe this problem is underappreciated and deserves more attention. Reproducibility and replicability are crucial issues in medical research and require complete, clear, and transparent reporting of methods. Under-reporting on the implementation details and the validity of methods compromises transparency and reproducibility. It has been previously found that in EHR-based studies, full lists of clinical codes were often not reported [216, 217]. A recent, large-scale reproducibility exercise identified similar challenges due to suboptimal reporting of EHR-based studies, particularly sharing code lists and algorithms [218]. Underreporting of a study methodology means that the time and resources invested in conducting that study is wasted [219].

For complex clinical variables such as asthma and its outcomes, the lack of standardisation of the clinical definitions and the wide variability in the EHR-based 
algorithms undermine the validity and replicability of studies [152]. The significant methodological heterogeneity I found in the EHR-based asthma assessment algorithms reflects, in addition to the content differences between the databases used, the lack of consensus on the clinical definitions in the first place despite continuous standardisation efforts [2, 146, 220,221]. The focus of this work was to examine asthma algorithms and their validity specifically in the context of EHRderived data, but this highlights the fundamental need to reach consensus on clinical asthma definitions and the appropriate validation of asthma diagnosis. For example, there is still an active debate on whether lung function is essential to establish asthma diagnosis $[147,148]$. A recent study also found significant variation in algorithms to assess asthma severity from health insurance data [222]. Unjustified inter-study variation in the operational definitions of the same clinical concepts creates challenges for comparability [121], meta-analysis and evidence synthesis. These issues have been raised for asthma [223] and other allergic conditions such as peanut allergy [224, 225] and anaphylaxis [226], where wide variations in findings were potentially attributed to inconsistent case definitions. The findings in this chapter are likely to be applicable to a wide range of chronic conditions when defined and assessed using EHRs.

\subsubsection{Implications for policy, practice and research}

This review sheds light on the opportunities offered by the increasingly ubiquitous EHRs, but also highlights considerable heterogeneity and suboptimal reporting of EHR-based asthma assessment algorithms and the implications of these practices on comparability and reproducibility of studies.

Developing reliable algorithms to identify asthma patients and assess asthma outcomes using EHR data is a non-trivial challenge. Standardising asthma algorithms used in research, where possible, is a crucial need. However, this may be impractical since databases usually differ in their content, validity may not hold across different populations [215], and no best practice currently exists [222]. Similar challenges arise when comparing asthma epidemiology between multiple populations [227], as the availability and quality of data may differ across those populations and a single case definition may not work for all of them. These methodological issues, in addition to suboptimal reporting, should be considered when interpreting and synthesising evidence from geographically dispersed studies. 
With the accelerating availability of EHR-derived data and their rapidly growing use to study asthma, I believe the global asthma research community needs to pay more attention to the methodological issues related to the use of these data in asthma research. I believe that consideration needs to be given to convening an international task force to work on the harmonisation of those algorithms under uniform and consistent clinical labels, while considering the differences between populations and databases. In addition, validation of these algorithms in the respective populations should be given a high priority [215]. Furthermore, to allow more accurate assessment of asthma from EHR-derived data, efforts are needed to improve the capture and coding of asthma-related data at the point of care [228] which requires more efficient EHR systems [203, 204]. In addition, emerging data sources such as patient-generated data and wearables need to be harnessed [229]. Finally, to improve the clarity of reporting on EHR-related methodological aspects, I strongly advocate the adoption of the RECORD Statement as an extension of the STROBE Statement by authors, journal editors, and peer reviewers $[156,157]$. Optimal reporting should include complete code lists, detailed algorithms and validity assessment. Implications of using EHR-derived data to study a complex condition such as asthma should be clearly communicated to enable judgement of internal and external validity.

\subsection{Conclusion}

This systematic scoping review showed considerable international interest in exploiting EHR-derived data to study asthma. Asthma diagnosis, exacerbation, severity and control, have been assessed from different types of EHR-derived data using various approaches. However, there were considerable variations and inconsistency in these approaches. These variations were compounded by sub-optimal reporting of methods, their validity, and other aspects concerning the use of EHRderived data for research. Reusing algorithms of asthma outcomes in new populations without re-validation, and relying only on clinical judgement and face validity were common practices. These issues make it difficult to assess the reproducibility of research and perform evidence synthesis and meta-analyses. Given the substantial investments taking place in EHRs globally, the number of EHRbased asthma studies is likely to grow substantially in the coming years. Unless addressed, these issues will aggravate the reproducibility problem and increase 
the avoidable waste in this important body of research. It is therefore important that the asthma-interested research community works to place it on a solid footing in order to ensure the quality and reproducibility of this work. Improving the reporting of these aspects using standardised guidelines such as the RECORD Statement would improve the rigour, transparency and reproducibility of asthma research.

The findings in this chapter will inform the discussion on the challenges of defining asthma using routine collected EHR data in Chapter 3. In that chapter, I will explore and demonstrate the usefulness of using data-driven methods, instead of manually developed algorithms, to identify asthma patients. 


\section{Chapter 3}

\section{Identifying asthma patients in Wales}

Defining a complex condition using real-world data

In this chapter, I discuss the challenges of developing accurate case definitions for asthma. I then highlight the common methods used in validating asthma definitions from a variety of data sources. In the absence of a gold standard for asthma definition, latent class analysis (LCA) can be used to identify hidden clusters in a population using the observed data. I describe the development of an LCA model to identify patients with asthma, particularly those with currently treated asthma, using routinely collected primary care data in the Secure Anonymised Information Linkage Databank in Wales. Based on this model, I trained a classification algorithm to identify asthma patients that can be used in this Databank and similar data settings. 


\section{Chapter Contents}

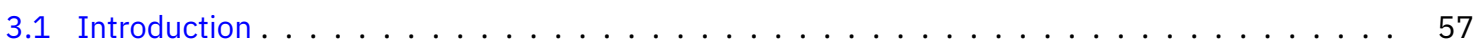

3.1.1 Challenges of developing accurate case definitions . . . . . . . . . . . . 57

3.1.1.1 Asthma is a heterogeneous condition . . . . . . . . . . . 57

3.1.1.2 Variations and changes in clinical coding practice . . . . . . . . . 58

3.1.1.3 Limitations of EHR-derived data . . . . . . . . . . . . . . . 58

3.1.2 Validity assessment of asthma case definitions is needed . . . . . . . . . . . 59

3.1.2.1 Approaches for validation of routine data-based case definitions . . . . . . . 59

3.1.3 Latent class analysis: An overview . . . . . . . . . . . . . . . . . . . 62

3.1.3.1 Concept and assumptions . . . . . . . . . . . . . . 63

3.1.3.2 Model specification . . . . . . . . . . . . . . . . . . . 64

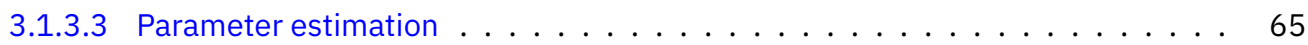

3.1.3.4 Membership probabilities . . . . . . . . . . . . . . 66

3.1.3.5 Model homogeneity and separation . . . . . . . . . . . . . 66

3.1.3.6 Model assessment and selection . . . . . . . . . . . . . . . . 67

3.1.3.7 Model interpretation . . . . . . . . . . . . . . . . . . 69

3.1.4 Using latent class analysis to identify asthma patients in the Wales Asthma Observatory 70

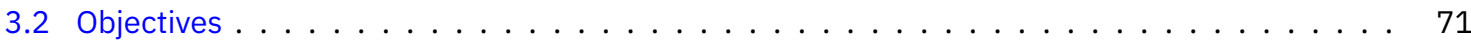

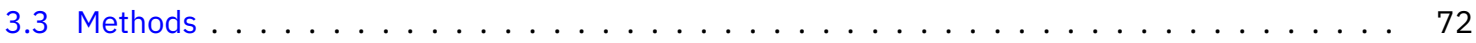

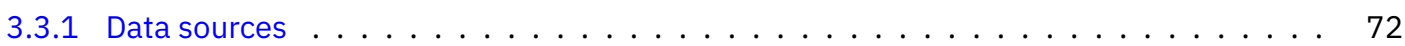

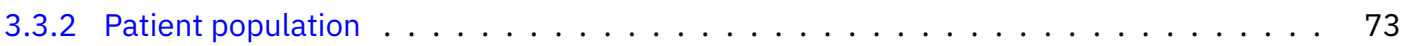

3.3 .3 Latent class modelling . . . . . . . . . . . . . . . . . . . . . . . . 74

3.3.3.1 Observed variables . . . . . . . . . . . . . . . 74

3.3.3.2 Number of classes and model selection . . . . . . . . . . . . . 75

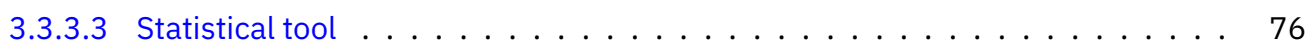

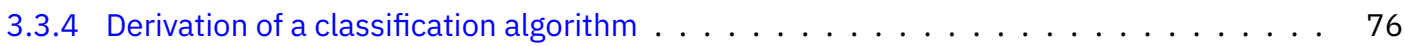

3.3.5 Comparison of the classification algorithm with other case definitions $\ldots \ldots$. . . . 78

3.3.5.1 Case definition used for comparison . . . . . . . . . . . . 78

3.3 .5 .2 statistical analysis . . . . . . . . . . . . . . . . . 79

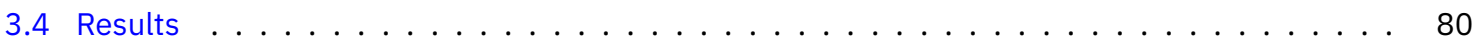

3.4.1 Sampling and sample characteristics . . . . . . . . . . . . . . . 80

3.4.2 Summary of the competing latent class models . . . . . . . . . . . . . . . 81

3.4 .3 Model selection . . . . . . . . . . . . . . . . . . . . . . . . 83

3.4 .4 Model interpretation . . . . . . . . . . . . . . . . . . . . . . 84

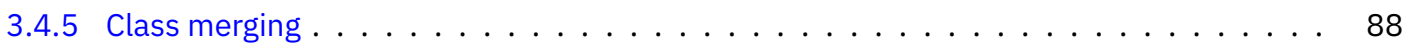

3.4.6 Derivation of classification algorithm . . . . . . . . . . . . . . 89

3.4.7 Comparing the classification algorithm with other case identification methods . . . . . . 94 


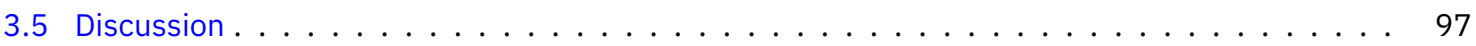

3.5.1 Summary and interpretation of the findings . . . . . . . . . . . . . . 97

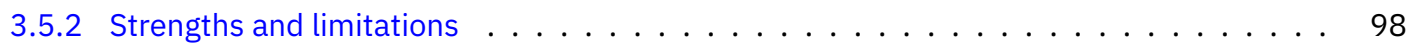

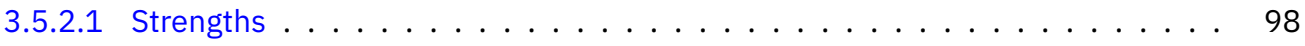

3.5 .2 .2 Limitations . . . . . . . . . . . . . . . . . . . . . 99

3.5.3 Comparison with related works . . . . . . . . . . . . . . . . . . . 101

3.5 .4 Future directions . . . . . . . . . . . . . . . . . . . . 102

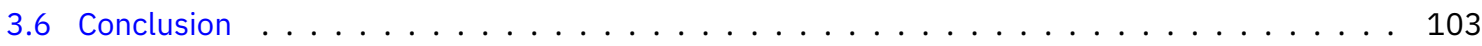

\subsection{Introduction}

In the light of the literature review findings presented in Chapter 2, I further discuss the challenges of defining and assessing asthma using routinely collected data (RCD) considering asthma heterogeneity, data limitations, and the absence of a gold standard to define asthma. I then justify the use of data-driven approaches to identify people with ever and current asthma in the Wales Asthma Observatory.

\subsubsection{Challenges of developing accurate case definitions}

Developing case definitions is an essential step in the development of disease observatories and registries [137] and is crucial to the interpretation of epidemiological estimates and research findings [213]. However, particular challenges exist when identifying patients with a complex disease such as asthma. In addition, many disease registries increasingly use electronic health record (EHR)-derived data, which adds particular challenges related to the limitations of these data [102, 151, 202]. I summarise these challenges below:

\subsubsection{Asthma is a heterogeneous condition}

Asthma is not a single disease entity; there is increasing recognition that the disease is an umbrella of heterogeneous sub-entities at the molecular, pathological and clinical levels (i.e. endotypes and phenotypes) $[4,5,230]$. This means that patients who are diagnosed with 'asthma' do not all have the same underlying disease process. Furthermore, the natural course of asthma exhibits variability within and between patients, who also differ in their response to treatment. There- 
fore, it is practically difficult to find a single, precise clinical definition of asthma that everyone agrees upon [2, 144-148].

\subsubsection{Variations and changes in clinical coding practice}

EHR-based case definitions for asthma are often based on recorded events related to patients' health, such as diagnosis, physician's visits, and prescriptions. However, physicians differ in their diagnostic skills and prescribing behaviour [231]. In addition, their practices are subject to changes by the continuous evolution of clinical guidelines, which can potentially affect clinical coding practices [109], as well as by incentives and resources. Variations in clinical practice mean that there is variation in the recorded data, even for clinically similar asthma patients. Further variations in the recorded data may result from differences in the documentation and coding of clinical data between physicians.

\subsubsection{Limitations of EHR-derived data}

Along the pathway of clinical data, from the point of care to central data repositories, several factors introduce error, uncertainty, and information loss into these data [102, 151, 202, 232]. EHRs usually capture both unstructured and structured data that are collected during clinical encounters. At the data entry stage, however, incorrect, invalid, or inaccurate data may be recorded due to both human and computer related factors. Due to design limitations of EHR systems [203, 204], not all clinical data are recorded and/or coded by physicians [228, 233]. In addition, clinical coding aims to reduce the detailed data captured during the encounter into few clinical codes on diagnosis, clinical findings, disease management events, and prescriptions. However, the commonly used clinical coding systems usually have limited granularity compared to the captured narrative data. These coding systems, while facilitating standardised coding [233], fail to codify all the details that are in the patient record. These non-codified data are important for secondary uses. Furthermore, in practice, clinical coding is a barrier to data recording [233], and physicians use only few of the available codes and under-utilise the granularity of coding schemes [228]. Finally, there is an increasing trend to link routinely collected health data from different sources, thereby introducing a further threat to data quality. Record-linkage can potentially introduce errors and biases to data analyses [234]. 


\subsubsection{Validity assessment of asthma case definitions is needed}

Due to the aforementioned challenges to define asthma, a single epidemiological case definition for asthma using routinely collected EHR data does not exist. At the same time, these challenges highlight the need to assess the validity of case definitions and clinical variables measured using these data [213, 235, 236]. Validity assessment has a particular importance in the development of disease registries and observatories as well as research databases since the validity and interpretation of epidemiological estimates and research findings depend on accurate patient identification and characterisation [236]. Reporting guidelines promote complete and transparent reporting on the methods and their validity. The REporting of studies Conducted using Observational Routinely-collected health Data (RECORD) Statement [157], which is an extension of the Strengthening the Reporting of Observational Studies in Epidemiology (STROBE) Statement [153], argues that authors of studies conducted using routine data should clarify whether the variable definitions used in their study have been validated. A comprehensive check-list of diagnostic values [201] has been proposed to be used in the reporting of validation studies including cross-tabulation, sensitivity, specificity, accuracy, positive and negative predictive values (PPV and NPV), likelihood ratios (LRs), Cohen's kappa, receiver operating characteristic (ROC) curve including the area under the curve (AUC), and case prevalence along with 95\% confidence intervals when applicable. Several approaches to validity assessment are discussed in this section.

\subsubsection{Approaches for validation of routine data-based case definitions}

In epidemiological studies, validity of case definitions can be assessed using a variety of methods. A recent systematic review identified various methods to validate asthma diagnosis in EHRs [237]. These included manual review of the narrative medical records, medication data, and questionnaire data. Since a gold standard is usually unavailable or inaccessible, acceptable reference standards are used. A reference standard can be simple or composed of multiple reference measures, in which case it is called a composite reference standard [238]. When used, a composite reference standard is thought to have a discriminatory property that is greater than those of each of its components alone [238]. 
When no accepted reference standard can is available, the researcher can perform concordance analysis or latent variable modelling. Concordance analysis evaluates the agreement between a case definition and independent measures, usually derived from other data sources [217, 236, 238]. In this method, neither the case definition under assessment, nor the independent measure, are assumed to represent the ground truth (i.e. the real disease status as confirmed by clinical diagnosis and/or laboratory tests). Instead, the level of agreement between the two identification methods can provide insights into the reliability and meaning of each method. Alternatively, labelled classes in a well-specified latent variable model can be used to train a case identification algorithm which can be used as an 'internal reference' within a particular dataset [238].

In other approaches, aggregate data are used to compare rates or distributions of two case definitions $[217,236]$. These approaches can be used when the researchers have no access to individual level data necessary to test one of the compared case definitions, but instead have access to aggregate results.

Practically, the choice of appropriate validation methods therefore depends on the available data sources, and the availability of a gold or reference standard, as well as the research questions and design.

\section{Manual review of medical records}

While routinely collected EHR data are usually coded, the source medical records, whether paper-based or computerised, usually contain more detailed data, including narrative clinical notes, about patient care. Point-of-care data are a good reference for validating case definitions because they contain more detailed data about patient care before being coded. For this purpose, medical records for a sample of individuals in a specified cohort are reviewed to confirm whether they had a confirmed asthma diagnosis at a certain point of time. Often, researchers send questionnaires to physicians [239, 240], nurses [241], or an expert panel to review patient records [242]. Clinical examination and/or laboratory tests such as lung function tests on the validation sample could be sometimes repeated to confirm or rule out the diagnosis. Ideally, the reviewer should be blind to the clinical codes to avoid confirmation bias [239, 241]. 


\section{Analysis of concordance with dispensing data}

Individual-level pharmacy dispensing datasets usually contain valuable information on asthma treatment. Analysis of concordance can be performed between case definitions based on dispensing data and other case definitions based on different data sources such as hospitalisation records, questionnaires filled by general practitioners (GPs), and self-administered questionnaires [237, 240, 243, 244].

A limitation of dispensing data is that they often lack information on diagnosis. Not all asthma medications are specific to asthma, and therefore it is difficult to ascertain whether an 'asthma medication' was actually prescribed to treat asthma or another co-morbidity. For example, inhaled corticosteroid (ICS) (in combination with bronchodilators) can be also prescribed for chronic obstructive pulmonary disease (COPD), and oral corticosteroids are indicated to a wide range of nonrespiratory conditions. It is therefore difficult to accurately find asthma patients solely from dispensing data [245, 246].

\section{Self-reported asthma measures}

Self-reported data about asthma are commonly used in research. Questions about having asthma symptoms, severity of asthma symptoms, 'ever asthma', 'current treated asthma', and/or using specific asthma medications are commonly included in national health surveys and asthma questionnaires [54, 247, 248]. Self-reported asthma measures have been used as a reference to assess the validity of asthma codes in primary care records [249] and in analysis of agreement with billing and health insurance data $[250,251]$.

Self-reported data have unique advantages and disadvantages. They represent patients' experience and understanding of their own health conditions which are not always appreciated by physicians [252]. However, some patients do not have clear understanding of their health status. For example, some asthma patients may believe they have asthma while in fact they have been diagnosed with COPD or hay fever. Self-perception of health status can be influenced by variety of other factors such as educational attainment and employment [253]. Self-reported data are usually prone to recall bias, i.e. patients with more health problems are often more likely to report previous exposures or health events. I found that, using 
the Welsh Health Survey (WHS), patient-reported currently treated asthma had suboptimal concordance with the 'ever-diagnosed currently treated asthma' ascertained from general practice data [254]. ${ }^{1}$ For these reasons, patient-reported doctor-diagnosed asthma should not be considered as a 'gold standard' case definition for asthma [255].

\section{Where no accepted reference standard exists}

The above approaches to obtain reference standards for validation of routine databased case definitions can be expensive, time-consuming, and/or labour-intensive. Where accepted reference standards based on independent data sources are unavailable or unfeasible, other approaches can be followed. For example, case definitions can be developed based on medical knowledge, clinical guidelines, and/or relevant literature, as well as knowledge of data recording and coding practices (see Section 2.3 for examples from the systemic scoping review in Chapter 2). These approaches often assume the completeness and accuracy of data recordings and may rely on heuristic techniques assumed to improve accuracy. For example, a researcher may decide to exclude from a case definition people in whom the diagnosis may not be 'certain'; e.g., those with differential diagnoses or particular morbidities (see Chapter 2).

A different approach to follow in the absence of accepted reference standards is to use data-driven methods, such as latent class analysis (LCA). Such methods use computational techniques to understand the hidden population structure in relation to specific observed characteristics [256]. In order to ensure meaningful findings, these methods should be performed in the light of the established knowledge about the disease pathophysiology, clinical course, and epidemiology as well as about data provenance and quality [142].

\subsubsection{Latent class analysis: An overview}

In the absence of accepted referenced standards for asthma case definitions, it is possible to identify asthma patients from RCD by examining the recorded events related to asthma. Examples of such variables include the following dichotomous variables:

\footnotetext{
${ }^{1}$ I presented this analysis in the British Thoracic Society Winter Meeting 2016; the poster is available in the Appendix B.1.
} 
- 'ever diagnosed with asthma'

- 'had GP attendances related to asthma in the last year', and

- 'received inhaled steroids in the last year'.

Each of these events alone may not be sensitive or specific to the 'true status' of asthma. However, analysing the patterns of correlation between them these events can help identify people groups that are likely to have the disease. Using cross-tabulation, the existing patterns of correlation between these variables can be identified along with their frequencies; these patterns of correlation represent different patient profiles. By examining these profiles, it is possible to assign, to patients, meaningful clinical labels that explain their patterns of observed characteristics. For example, if the pattern suggests asthma, it will be assigned the label 'asthma', otherwise it will be labelled as 'none' or 'no asthma'. In reality, different profiles may have the same clinical label, possibly each with different certainty. Eventually, these label-sharing patterns are merged together in common groups, some of which represent patients with the disease.

The aforementioned exercise allows us to understand the population distribution and facilitate the process of identifying patients based on their observed data patterns. However, with a high number of observed variables and their levels (i.e. high-dimensional data), this exercise becomes extremely complex and impractical. This is a typical problem where computational clustering methods, such as LCA, can be used. LCA can be useful in analysing and understanding complex patterns in high-dimensional data.

\subsubsection{Concept and assumptions}

LCA is a finite mixture modelling method, i.e. a method that models a mixture of sub-groups in a population. It aims to cluster a population into sub-groups related to a set of observed variables [257, 258]. LCA assumes that the patterns of correlation in these observed variables within the population can be explained by, in addition to measurement errors, a hidden categorical variable, called a latent variable, which has a pre-defined number of levels (Figure 3.1). The latent variable partitions the population into sub-groups called latent classes. These latent classes are qualitatively distinct although they are fuzzy in nature as individuals have probabilistic memberships in these classes (see Section 3.1.3.4: 
“Membership probabilities” below). In this chapter, the latent variable represents a patient's disease state at a given point of time.

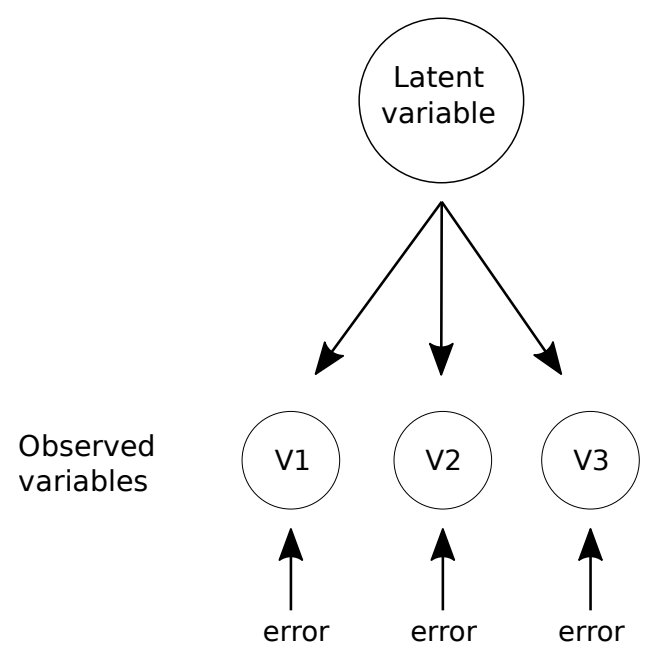

Figure 3.1: Visual representation of a latent class model. The model assumes that the observed variables are influenced by the categorical latent variable as well as measurement and random errors.

LCA is a non-parametric model which requires categorical (including dichotomous) or ordinal observed variables. Therefore, by using these types of data, assumptions about the normality, linearity, or homogeneity of data are avoided.

LCA aims to identify latent classes that explains the whole correlation between variables at the population level; that is, it assumes that any associations between the observed variables are assumed to be solely due to the influence of the latent variable. Therefore, in a perfect latent class model, the observed variables are independent of each other within each class [257, p. 44]. For example, inside the latent class of "asthma", the presence of diagnosis codes does not per se influence the probability of presence of prescription codes. This assumption is called the 'local independence' or 'conditional independence' and is important in latent class modelling, although it may not hold in a large sample.

\subsubsection{Model specification}

The construction of a simple latent class model starts with the hypothesis that certain structure exists in a population. Then a set of observed variables are chosen, which could be dichotomous or categorical. Ordinal variables can be used in the modelling but they are treated as categorical. Interval variables need to be transformed into these types of variables. The observed variables should be chosen on the basis of having strong relevance to the hypothesised population 
structure. More specifically, they should have high discriminatory power to distinguish between some of the hypothesised sub-groups in the population.

An empirical study has shown that adding a larger number of highly discriminating observed variables to the model had beneficial effects on the model estimation [259]. However, a larger number of observed variables may increase the possibility of sparseness in their contingency table, especially with a relatively small sample size. To avoid sparseness, larger sample sizes, when available, are preferred [259].

\subsubsection{Parameter estimation}

The parameters estimated by LCA include the following:

- Probabilities for any random individual to be in each latent class, i.e. prevalences of the latent classes.

- Probabilities of observing each level of each observed variable in each latent class-also known as item-response probabilities.

The model parameters are estimated using the expectation-maximisation (EM) algorithm, sometimes along with the Newton-Raphson algorithm [260], both of which iteratively search for maximum-likelihood parameter values for which the data are more likely to be observed [261]. The algorithm starts the iterations with random values for the estimated parameters and estimates the expected cell counts in the contingency table of the observed variables. Depending on how expected cell counts fit the observed ones, the model parameters are then improved in order to maximise the log-likelihood function. In each iteration, if the increase in the log-likelihood for the current solution is less than a pre-defined value, the maximum log-likelihood is considered to have been found and the current solution is chosen as the 'best solution' [257, 260]. In ideal conditions, the algorithm converges to find a best solution among all possible solutions, called the 'global maximum solution'. However, sometimes the algorithm converges to a 'local maximum' solution that is optimal only among neighbouring solutions and not among all possible solutions. This problem is more likely to happen when the number of latent classes is too high. To avoid local maxima, it is advised to repeat the estimation algorithm with different starting values so that a local maximum model is not selected by the estimation algorithm as the best solution [260]. Practically, the number of iterations is usually limited by a maximum limit, e.g. 1000 iterations, 
determined by the researcher. If the expatiation-maximisation algorithm reaches this limit before convergence, no best solution is selected.

\subsubsection{Membership probabilities}

Based on observed characteristics, individuals are assigned posterior probabilities of their membership in each of the latent classes [257, p. 67].

Thus, since each individual may belong to more than one latent class, the identified latent classes have in principle a fuzzy nature rather than being mutually exclusive. However, usually, each individual is eventually assigned to the latent class of maximum membership probability [262]. Each of the identified latent classes may contain more than one patient profile. In other words, individuals in each latent class may have different, but usually similar, observed characteristics. Item-response probabilities in each latent class represent the averaged observed characteristics in that class.

\subsubsection{Model homogeneity and separation}

The aim of LCA is to identify 'distinct' and 'homogeneous' latent groups in the population. However, since each latent class may include mixed patient profiles, especially in a model with high complexity, the best solution is that which maximises the within-class similarities and the between-classes differences.

Class homogeneity is the degree of similarity between individuals in a given latent class. It can be evaluated by examining the item-response probabilities within that latent class independently of the other latent classes. In that latent class, if most of the item-response probabilities are high or low (i.e. close to 1 or 0 ), which means that either there is a single prevalent patient profile (i.e. with a prevalence close to 1 ) or that the patient profiles in that class are highly similar to each other, then this latent class is said to be highly homogeneous. Otherwise, if most of the itemresponse probabilities are marginal (i.e. close to 0.5 ), which indicates there is no single prevalent patient profile, then this class is said to have low homogeneity. Latent class separation is the degree to which the individuals in each latent class are different from the individuals in the other latent classes. Class separation can be evaluated by comparing the item-response probabilities between the latent classes. In a model with high latent class separation, each latent class has 
item-response probabilities that are clearly distinct from those in the other latent classes.

A latent class model with a high class separation necessarily implies a high degree of homogeneity within each latent class. However, a latent class model with a high degree of homogeneity within latent classes does not necessarily imply a high degree of separation between latent class.

A useful latent class model will identify highly-differentiated, well-separated, and likely more interpretable latent classes. One way to improve homogeneity and separation is to choose observed variables with high discriminating power, i.e. thought to be strongly related, both conceptually and quantitatively, to the latent variable in question. However, a latent class model that has a good fit to the data may otherwise have poor class separation and thus poor interpretability.

\subsubsection{Model assessment and selection}

In LCA, the number of latent classes is pre-specified by the researchers based on their hypothesis and assumptions about the population structure. However, the actual data may fit lower or higher number of classes than assumed by the researchers. Therefore, they often repeat the modelling with different numbers of classes and compare them in terms of model diagnostics (to assess the absolute and relative fit of the models) and clinical plausibility.

\section{Absolute model fit}

The absolute fit of a latent class model considers its fit to the data regardless of its competing models. Since a latent class model is based on a contingency table of its observed variables, the absolute model fit can be assessed using this contingency table. Therefore, Pearson's chi-square test can be performed to test the null hypothesis that the observed counts of patient profiles in the contingency table can be produced by the estimated latent class model [257]. The likelihood ratio chi-squared statistic, $G^{2}$, is a variant of Pearson's chi-square test. In both tests, the expected counts of patient profiles, as estimated based on the model parameters, are compared to the observed counts of patient profile. The degree of freedom is the total number of observed patterns minus the number of estimated parameters minus 1 . The test statistic is then compared to the reference 
chi-square distribution to obtain a $p$-value representing the probability that the observed data can be produced by the estimated latent class model under the null hypothesis.

However, both Pearson's chi-square and the likelihood ratio chi-squared tests are not appropriate when sparseness exists in the contingency table of the observed variables, i.e. when the contingency table contains too many cells with small or zero counts. This happens in latent class models with small sample sizes and/or large number of variables. In addition, in modelling using larger samples, both tests are likely to produce smaller p-values, i.e. indicating weaker evidence of absolute model fit.

\section{Relative model fit}

The Akaike information criterion (AIC) [263] and the Schwarz's Information Criterion (often called the Bayesian information criterion, BIC) [264, 265] are two information criteria each of which provide an estimate of the information lost when a given statistical model is fitted to given data. They seek a balance between the model's goodness of fit and its parsimony. A model's parsimony is the opposite of complexity, and is represented by the number of estimated parameters, or simply by the number of latent classes. Thus, AIC and BIC are also called 'parsimony indices', as they prefer models with fewer number of estimated latent classes. To obtain the desired balance, AIC imposes a penalty on $G^{2}$ based on the number of parameters, while BIC imposes a larger penalty based on both the number of estimated parameters and the sample size.

AIC and BIC can be used to measure which one of the competing latent class models, differing by number and characteristics of classes, best fits a given dataset. The model with the lowest value represents a more optimal balance between model fit and parsimony and is preferred over its competitors. Due to the difference in imposed penalty, AIC and BIC may not agree on the 'optimal model' they suggest.

However, they do not provide a meaningful absolute measure of quality for a latent class model independently of the other competing models. This means that a competing model with the best AIC and/or BIC may still have poor absolute fit to the data. 


\section{Model interpretability}

Although the best-fit model based on the above model selection methods is often chosen by researchers, it does not always contain a clinically meaningful structure. For example, variables with lower clinical relevance to the clustering exercise (e.g., have clinically lower discriminatory power) may be used by the best-fit model in the generation of latent classes more than other more clinically relevant variables. In such cases, researchers use domain knowledge to decide whether the best-fit model is clinically and/or biologically plausible. Otherwise, the researchers may need to re-specify the model, i.e. revise the choice of observed variables and their levels.

\subsubsection{Model interpretation}

The interpretation of an LCA model is based mainly on its estimated parameters. The item-response probabilities can be used to describe the latent classes and assign meaningful labels to them [257, p. 29]. For example, a class in which individuals have high probabilities (e.g., 0.8 or more) of both of 'having asthma diagnosis' and 'receiving asthma prescriptions in the last year' can be assigned the label of 'doctor diagnosed currently treated asthma'.

If the number of classes in the best fit-model is higher than that expected by the researcher, i.e. the clustering is deeper than desired, then the researcher may choose to keep this model but manually combine similar classes to form fewer but larger 'super classes' representing the desired clinical clustering.

If the model is not clinically interpretable, then the researcher may need to choose a different competing model, or even decide to re-specify the model by modifying the observed variables.

Model interpretation particularly depends on the relevance of input variables and the quality of data. The careful choice of both input variables and number of classes (the latter is partly determined by the expected clusters as based on the predictor variables) are critical for clinically meaningful clustering. Even though, meaningful interpretation of the resultant classes could be difficult; e.g., classes may not correspond with the commonly recognised clinical definitions of the disease sub-groups. 
A latent class may contain individuals who do not match the clinical description assigned to the latent class as a whole. For example, the 'no-asthma' class, based on the model design (i.e. choice of predictor variables) may include people who can be classified by experts as people with asthma. Similarly, the 'asthma' class may include people who have conditions other than asthma, e.g., COPD, but share some of the common characteristics of asthma patients (receipt of bronchodilators). Therefore, LCA is usually considered exploratory rather than confirmatory.

\subsubsection{Using latent class analysis to identify asthma patients in the Wales Asthma Observatory}

The development of the Wales Asthma Observatory requires reliable case definitions for asthma. The Observatory is based on data in the Secure Anonymised Information Linkage (SAIL) Databank. Case identification algorithms for 'ever asthma' and 'currently treated asthma' based on diagnosis and prescription events have been validated in other datasets [102]. However, they may not necessarily retain their validity in the SAIL Databank. Assessing their validity against complete patient records is time-consuming, labour-intensive and expensive, rendering it unfeasible in this doctoral project. Data on self-reported 'currently treated asthma' were available in the results of the WHSs for the years 2013 and 2014 which were record-linked to the SAIL Databank. However, these data only contained responses to a simple question for 'currently treated asthma', without specifying a time-frame for such status. Therefore, this self-reported variable cannot be considered an accepted reference for 'currently treated asthma'.

In the absence of a feasible, accepted reference standard for asthma, LCA is an appropriate method to identify asthma patients in the SAIL Databank. This method fits the nature of both asthma and routine data held in the SAIL Databank.

Clinically, the presence of asthma is not a binary status, and the certainty of physician's diagnosis is not always perfect. In practice, asthma diagnosis could be classified into 'absent', 'possible', 'likely', or 'confirmed asthma'. Therefore, the uncertainty of asthma diagnosis can be represented probabilistically, which corresponds to the fuzzy approach followed by LCA to assign class memberships for individuals in a population. 
Asthma-related observed characteristics that can be derived from the SAIL Databank can be dichotomous (e.g., 'ever asthma diagnosis'), polytomous (e.g., 'age group at asthma diagnosis'), and continuous data (e.g., 'number of ICS prescriptions in the last year'). However, for the purpose of detecting asthma patients from such data, continuous data can be transformed into categorical (dichotomous and/or polytomous) data using appropriate cut-offs, e.g., 'no prescriptions' vs. 'one or more prescriptions'. This fits well with the types of observed variables required in LCA, namely, categorical (or ordinal) variables.

Despite guidelines on diagnosis, asthma could be clinically confused with a variety of conditions, especially COPD. Distinguishing asthma from COPD can be challenging $[266,267]$. Some patients exhibit features of both conditions in what has been recognised as the asthma-COPD overlap syndrome (ACOS) [207]. Therefore, it is worthwhile to consider the misdiagnosis and overlap between asthma and COPD in latent class models that attempt to identify asthma patients.

The output of LCA in this chapter will include individuals' class membership probabilities and their assigned classes. While such results are useful to create a reference identification for asthma in the SAIL Databank, it cannot be used for this purpose elsewhere. In order to facilitate the re-use of the resulting reference identification in data sources that are similar to the SAIL Databank, a classification algorithm in the form of a decision tree can be trained from a dataset labelled by the developed latent class model.

\subsection{Objectives}

In this chapter, I developed an identification model for asthma in the SAIL Databank, and evaluated it against other widely used physician-reported and selfreported case definitions. Specifically, the objectives were to:

- Develop a data-driven reference identification of asthma, particularly those with currently treated asthma, using LCA of RCD in the SAIL Databank.

- Derive a classification algorithm for asthma that can be used in the SAIL Databank and similar databases.

- Assess the agreement between the classification algorithm and the following commonly used case definitions:

- GP-reported ever-diagnosed asthma 
- GP-reported currently treated asthma

- GP-reported ever-diagnosed, currently treated asthma

- Self-reported currently treated asthma.

- Self-reported currently treated COPD.

These case definitions are explained below in Section 3.3.5.1. This assessment included analysis of concordance and calculating estimates of diagnostic accuracy for those case definitions using the predictions by classification algorithm as references and vice versa.

\subsection{Methods}

In a cross-sectional design, I used primary care data on asthma and COPD recorded in or before 2014 for a sample of the Welsh population to find, using LCA, clinically meaningful classes (i.e. clusters) related to the two conditions in that year. Based on the chosen latent class model, I then derived a classification algorithm to identify patients with asthma, including those with currently treated asthma, as well as those with COPD and ACOS in the primary care population. I compared that classification algorithm with other case definitions for asthma and COPD based on doctor-reported and patient-reported data.

Figure 3.2 illustrates the methodology followed in this chapter.

\subsubsection{Data sources}

I used individual-level demographic and primary care data from the SAIL Databank. The SAIL Databank contains anonymised linked data datasets derived from EHRs and several non-health datasets in Wales [140, 141]. I used the following two datasets:

- The Welsh Demographic Service (WDS): The WDS contains de-identified demographic and administrative information for National Health Services (NHS) patients in Wales. I used this dataset to identify NHS patients who satisfied the follow-up criteria described below.

- The GP dataset: The GP dataset contains de-identified health care events, such as recorded diagnoses, clinical findings, prescriptions and monitoring as well as other events codified in Read codes by GPs. I used the GP dataset as 


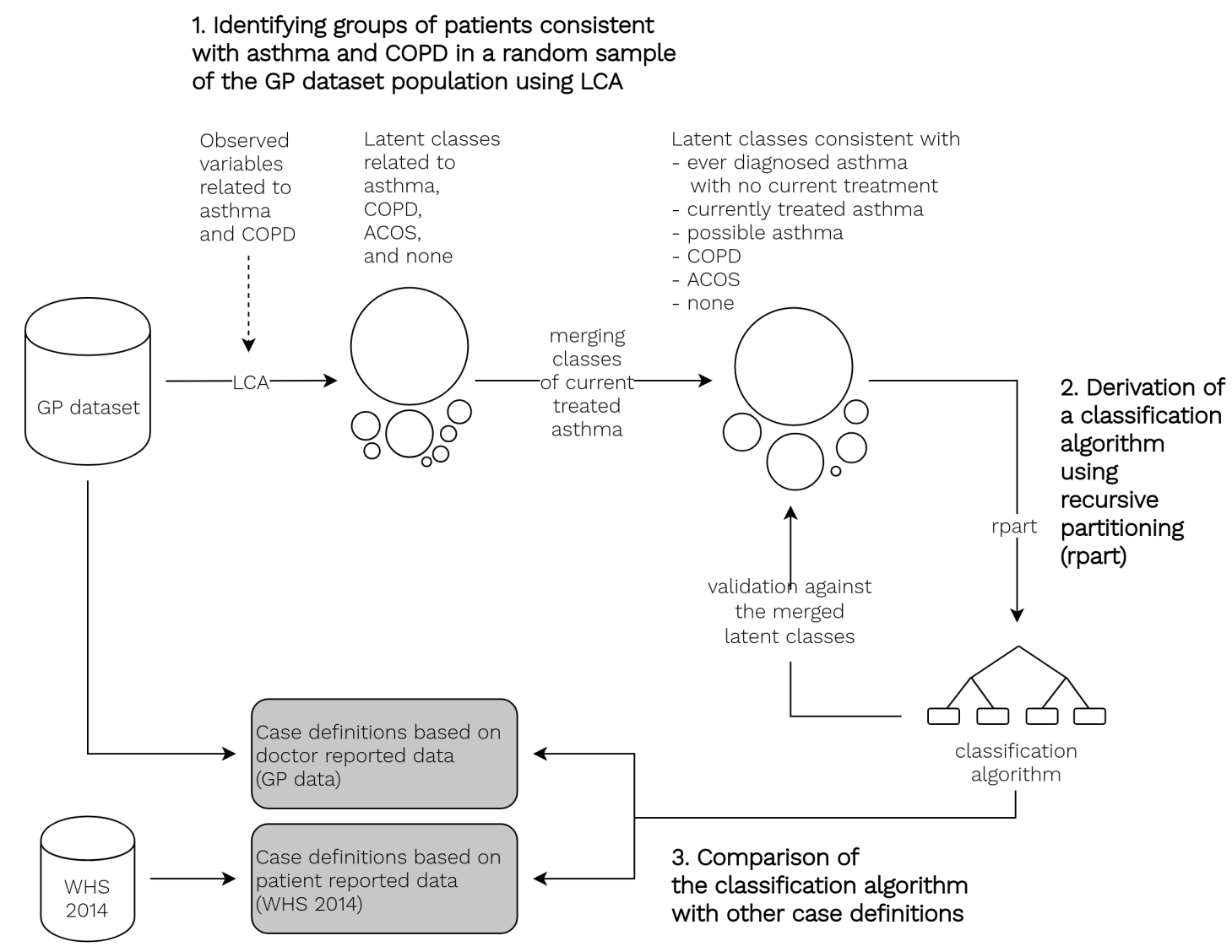

Figure 3.2: The methodology followed in Chapter 3. ACOS: asthma-COPD overlap syndrome; COPD: chronic obstructive pulmonary disease; GP: General Practice; LCA: latent class analysis; WHS: Welsh Health Survey.

a main data source in my analysis since in the United Kingdom (UK) asthma is mainly treated in primary care [32]. At the time of data extraction and analysis, the most recent extract of the GP dataset was in April 2016, covering about $80 \%$ of GP surgeries in Wales, which voluntarily sent their data to the SAIL Databank. I used the GP dataset to measure the observed variables described below.

Since I only accessed anonymised health data within the SAIL Databank and did not work with humans, no ethical approval was required. This doctoral project was covered by approval of the SAIL Information Governance Review Panel (see Appendix C.2).

\subsubsection{Patient population}

I defined the source population of the study as every individual who satisfied the following criteria: 
- Registered in at least one GP practice that contributed its data to the SAIL Databank at the time of the analysis.

- Had continuous GP registration during the analysis year, i.e. between 1-12014 and 31-12-2014. To calculate GP registration periods for individuals, I used an unpublished commonly used algorithm developed in-house by the analyst team of the SAIL Databank. This algorithm takes into account periods of registration with SAIL and non-SAIL participating practices as well as the volumes of data contributed by each practice over time. The algorithm uses that information to determine periods of continuous follow-up of patients in the primary care dataset in the SAIL Databank.

- Did not die on or before 31-12-2014.

I did not apply age restrictions to the source population. The study sample was randomly selected from the source population. The sampling was stratified by general practices to improve their representativeness. I determined the sample size for latent class modelling based on the available computational capacity in the SAIL Gateway.

\subsubsection{Latent class modelling}

\subsubsection{Observed variables}

In this modelling, the observed variables were based on GP-recorded primary care events related to asthma and COPD. The choice of these events and the dimensions of the observed variables was determined based on their usefulness, from a clinical perspective, for identifying and distinguishing between patients with asthma and/or COPD. Including observed variables of both asthma and COPD in the same latent class model allowed identification of patients with either or both conditions (i.e. ACOS). These variables included events on disease-related diagnosis, GP visits, prescriptions, and smoking. Events of GP visits and prescriptions were queried over the analysis year, while the other events were queried over any time up to the end of the analysis year. Most of the observed variables were transformed into binary variables: ' 0 ' for 'no events found' and ' 1 ' for 'one or more events found'. The 'age at asthma diagnosis' variable had three categories: '< 40 years' and '40 or more' as well as 'no diagnosis'. A full list of variables is shown in Table 3.1. The lists of Read codes used in the variable definitions are available in Table B.2.1. 
Table 3.1: Observed variables used in the latent class model. Clinical codes are listed in Table B.2.1.

\begin{tabular}{|c|c|c|}
\hline Variable & Time interval for calculation & Categories \\
\hline \multicolumn{3}{|l|}{ Asthma related } \\
\hline Asthma diagnosis codes ever & ever & $0,1+$ \\
\hline Age at asthma first diagnosis codes, if any & - & $<40, \geq 40$, no diagnosis \\
\hline Asthma GP visits codes in the last 12 months & last two years & $0,1+$ \\
\hline COPD related & ever & $0,1+$ \\
\hline \multicolumn{3}{|l|}{ COPD diagnosis codes ever } \\
\hline COPD GP visits codes in the last 12 months & last two years & $0,1+$ \\
\hline COPD-specific prescriptions codes ${ }^{\star *}$ & last two years & $0,1+$ \\
\hline \multicolumn{3}{|l|}{ Prescriptions } \\
\hline ICS codes & last two years & $0,1+$ \\
\hline SABA codes & last two years & $0,1+$ \\
\hline LABA codes & last two years & $0,1+$ \\
\hline ICS+LABA codes & last two years & $0,1+$ \\
\hline OCS codes & last two years & $0,1+$ \\
\hline LTRA codes & last two years & $0,1+$ \\
\hline \multicolumn{3}{|l|}{ Others } \\
\hline Smoking history & ever & no, yes \\
\hline
\end{tabular}

Abbreviations: COPD = chronic obstructive pulmonary disease; ICS = inhaled corticosteroids; GP = general practitioner; LTRA = leukotriene receptor antagonists; LABA = long-acting $\beta 2$ agonists; OCS = oral corticosteroids; $\mathrm{SABA}=$ short-acting $\beta 2$ agonists.

${ }^{* *}$ COPD-specific prescriptions include: glycopyrronium bromide, indacaterol, olodaterol, anticholinergic bronchodilators (ipratropium bromide, oxitropium bromide, tiotropium, aclidinium, umeclidinium), roflumilast, oxygen cylinders, and COPD rescue packs.

\subsubsection{Number of classes and model selection}

Theoretically, the expected number of latent classes is based on the observed variables used in the modelling. Since I included variables for both asthma and COPD, the minimum number of latent classes that I expected was four classes consistent 
with the following labels: 'asthma', 'COPD', 'both', and 'none'. However, the inclusion of asthma-related current GP visits and prescriptions (i.e. in the last 12 months) in the model was aimed at distinguishing groups of patients with currently treated asthma from those with ever diagnosed asthma without current treatment. Therefore, the number of expected classes could be increased to represent those different groups and possibly to also differentiate between patients with less and more severe diseases.

I started the modelling for two latent classes and then iteratively increased the numbers of latent classes. I aimed to select a model that satisfied both the following criteria:

- The BIC was minimum (compared to the competing models) or became 'stabilised' (e.g., using the elbow method, in which the researcher looks for an "elbow" in the plot of the model's BIC against the number of classes).

- The latent classes were clinically relevant.

In the selected model, I assigned to the identified classes clinical labels consistent with 'asthma' (including currently treated asthma), 'COPD', 'both', and 'none' based on the estimated item-response probabilities in each latent class. I used the class proportions as prevalence estimates of their corresponding labels in the study population in 2014 .

To simplify the model, I aimed to merge similar classes (e.g., classes of currently treated asthma differing in the probability of prescriptions) into super classes (e.g., currently treated asthma).

\subsubsection{Statistical tool}

I performed the latent class modelling using the R package poLCA (version 1.4.1, 2014) [260].

\subsubsection{Derivation of a classification algorithm}

Based on the latent class model, which was developed for the year 2014, I derived a classification algorithm which can be used to identify patients with asthma (including currently treated asthma), COPD and ACOS as well as in similar primary care datasets. To do so, I performed recursive partitioning [268] using the $\mathrm{R}$ package rpart (version 4.1-11, 2017) [269]. In this method, a decision tree is 
constructed using supervised learning from a labelled dataset, called the training dataset. A decision tree is composed of nodes and branches. The nodes include interior nodes, each of which splits the corresponding partition of the sample into two parts based on a true/false question about one of the features, and final nodes (i.e. leaves) representing the labels assigned to the corresponding branches.

The construction of a decision tree is stepwise. At each step, including that of the root node (i.e. which corresponds to the whole training dataset), the dataset is split into two subsets based on a true/false question about one of the features (i.e. a predictor variable). Each split is intended to reduce the misclassification in the resulting two children nodes compared to that in their parent node. Since more than one feature can provide a reduction in misclassification, the feature that maximises such reduction is chosen for the given split. Theoretically, while the model fit may improve with further binary splits, a split may provide only small improvement in the misclassification. Allowing such a split leads to overfitting where the accuracy of the tree is high or perfect in the training dataset but low in the validation dataset. To prevent overfitting, smaller improvements in the model fit are panellised with higher costs. In addition, any split with improvement in the model fit that is smaller than a control measure, called the complexity parameter, is considered not worth pursuing. To determine the complexity parameter, rpart fits a full tree from which it extracts all the possible sub-trees and performs on each of which 10 -fold cross-validation. It then shows the sub-trees for which the complexity parameters are greater than a set threshold, usually 0.01 or as desired by the researcher. It then determines the complexity parameter from the sub-tree that has the lowest cross-validation error. The determined complexity parameter can be then used to prune the full tree, giving a trimmed tree as a best solution, representing the best possible balance between model complexity and cost.

I used the sample previously used for LCA to perform recursive partitioning. I used the latent classes (after being merged into super classes as appropriate) as labels and the observed variables, used in the LCA model, as features. I randomly partitioned the sample into two subsets: a training subset (approximately 70\%) for the decision tree development, and a validation subset (approximately 30\%) to validate the developed decision tree. The two subsets were balanced in terms of the proportions of labels. To validate the developed decision tree, I used it to predict the labels in the validation dataset. Then, I calculated various diagnostic measures for the model using the confusionMatrix function of the caret package 
(version 6.0.77, 2017) in R. These statistics included: classification accuracy (Acc, the proportion of correct predictions in the validation dataset along with its $95 \%$ confidence interval); the no information rate (NIR, also known as the no information error rate; the proportion of the largest class, which gives an idea of how useful the predictors were in predicting the classes compared with just predicting them using class proportions); the p-value of Acc > NIR (a one-sided test to see if the model accuracy is better than just predicting the most prevalent class); Cohen's Kappa (for the agreement between the known labels and predictions); and statistics by class including sensitivity, specificity, positive and negative predictive values of class-specific prediction, class prevalence, detection rate (proportion of detected class members relative to the whole sample), detection prevalence (the prevalence of truly and falsely detected cases), and balanced accuracy ((sensitivity + specificity)/2).

\subsubsection{Comparison of the classification algorithm with other case definitions}

I compared the classification algorithm described above with other case definitions based on objective and self-reported data.

\subsubsection{Case definition used for comparison}

\section{GP-reported ever-diagnosed asthma}

The case definition 'GP-reported ever-diagnosed asthma' refers to patients who had, on a given date, asthma diagnosed by GPs and recorded using one of a set of Read codes indicating asthma diagnosis. To identify such patients in the SAIL's GP Dataset, I used the asthma diagnosis Read code set shown in Table B.2.1, which were based on the Quality of Outcomes Framework (QOF)'s AST001 indicator [270].

\section{GP-reported currently treated asthma}

The case definition 'GP-reported currently treated asthma' refers to patients who were receiving asthma prescriptions on a given date. For the purpose of this thesis, this case definition was operationalised by identifying patients who had at 
least one asthma prescription during the last 12 months before the end of the analysis year (i.e. between 1 January 2014, and 31 December 2014). The prescription codes are shown in Table B.2.1 and were based on those used in the AST001 indicator of the QOF [270].

\section{GP-reported ever-diagnosed, currently treated asthma}

The 'GP-reported ever-diagnosed, currently treated asthma' case definition requires the patients to satisfy both the aforementioned case definitions. Thus, this case definition is almost identical to the AST001 indicator (but without excluding patients with 'exception codes').

\section{Self-reported currently treated asthma}

The 'self-reported currently treated asthma' case definition was based on the WHS of 2014 [54]. The WHS 2014 collected self-reported information on a range of health and health-related lifestyles from samples of the population of Wales. The WHS 2014 results dataset was already linked to the SAIL Databank [271]. However, from that dataset, I only had access to the responses of participants who were 16-year-old and above in 2014. Those participants consented to link their responses to their medical records [272]. From this dataset, the only question related to asthma, other than asthma symptoms, asked the participant whether he or she was currently treated for a number of diseases including asthma. I used responses to this question as a case definition for 'self-reported currently treated asthma'. I considered invalid responses as negative responses.

\section{Self-reported currently treated COPD}

In the WHS 2014, participants were asked whether they were currently treated for chronic bronchitis and/or emphysema. I considered positive responses for any of these two conditions as a case definition for 'self-reported currently treated COPD'. I treated invalid responses as negative responses.

\subsubsection{Statistical analysis}

I performed the comparisons between the classification algorithm and each of the above-mentioned case definitions in the group of WHS 2014 participants whose 
responses where successfully linked to SAIL. I calculated sensitivity, specificity, PPV, NPV, and Cohen's kappa coefficient for concordance of each of the above case definitions against the classification algorithm labels as references and vice versa.

\subsection{Results}

\subsubsection{Sampling and sample characteristics}

The size of source population was 2,303,819 which equated to approximately $74.5 \%$ of the mid-year estimate of 3,092,000 for the population of Wales in mid$2014 .^{2}$ The study sample included 50,000 individuals, $50.3 \%$ of whom were females. Figure 3.3 shows a histogram for the sample age at the beginning of the year 2014.

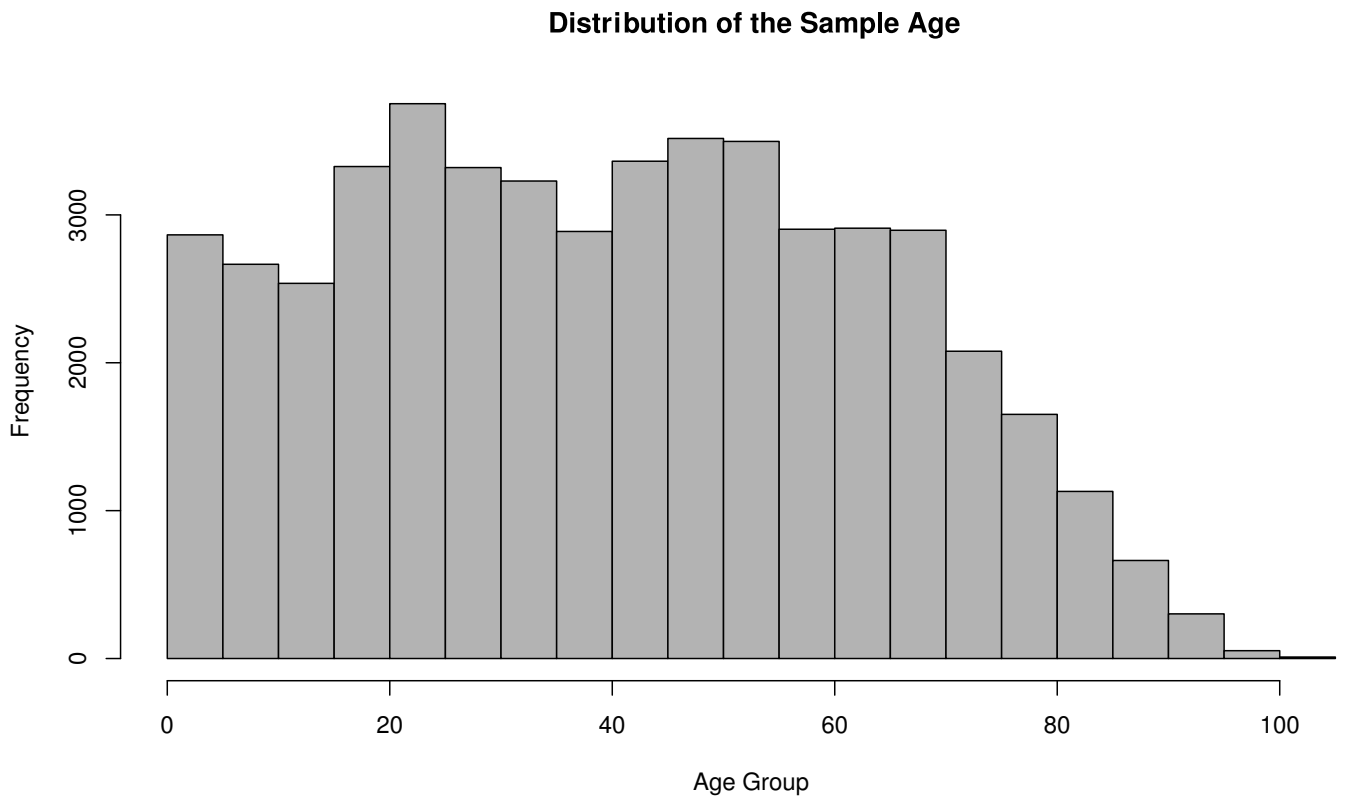

Figure 3.3: A histogram for the sample age at the beginning of year 2014 .

\footnotetext{
${ }^{2}$ Based on: Time series: Wales population mid-year estimate. Office for National Statistics. https://www.ons.gov.uk/peoplepopulationandcommunity/populationandmigration/populationestimates/timeseries/wapop/pop
} 


\subsubsection{Summary of the competing latent class models}

I started the latent class modelling for a one-class model and was able to increase the number of classes up to 12 . The item-response probabilities within the classes of each of the 12 competing models are shown in diagrams in Appendix B.3.

According to the item-response probabilities, in the two-class model, people with events related to asthma and/or COPD were aggregated in one class with a share of $16.2 \%$. In the three-class model, this class was split into two classes. One of these classes had a share of $14.1 \%$ and seemed to include mostly asthma patients, although it appeared highly heterogeneous, as indicated by the marginal probabilities for the asthma GP visits and short acting beta agonist (SABA) variables. A very small proportion of people in this class showed characteristics suggesting COPD. Another class in the two-class model, with a share of $2.4 \%$ appeared to have significant marginal probabilities (i.e., close to 0.5 ) for events related to COPD and prescriptions, suggesting high heterogeneity. A very small proportion of people in this class had characteristics related to asthma.

With higher number of classes, the models continued to reclassify people into more refined classes with higher homogeneity. In the four-class model, the asthmadominated class in the three-class model was further split into two classes both of which had 'asthma diagnosis ever'; one class with a share of $7 \%$ had also high probabilities for asthma-related current GP visits and prescriptions, while the other class had zero to very low probabilities of these events.

In the five-class model, there were one class (6.6\%) for ever diagnosed asthma with no current treatment, another class (2.4\%) appeared to be dominated by COPD characteristics with some probability for asthma-related events, and one class (84\%) with no asthma or COPD characteristics. However, there were two almostsimilar classes (3.7\% and 3.4\%) for ever-diagnosed currently treated asthma; the main differences between these two classes were that one had a very high probability for ICS and a very low probability for ICS-long-acting beta adrenoceptor agonist (LABA), while the other class had the opposite situation: a very low probability for ICS and a very high probability for ICS-LABA.

In the six-class models, those two 'ever-diagnosed currently treated asthma' were almost reunited into one class (6.9\%). There was one highly homogeneous class (1.4\%) suggesting currently treated COPD, one class (6.2\%) for ever diagnosed 
not currently treated asthma, and one class (1.0\%) that showed high probabilities for both current asthma and COPD events. However, there was one class (2.2\%) showing only variable probabilities for asthma prescription, with almost no recorded diagnosis of asthma or COPD.

In the seven-class model, the only significant refinement on the previous model was that a previously one large class with asthma or COPD characteristics become split into two classes (51.4\% and 30.9\%) with low and high probabilities of smoking history, respectively.

In the eight-class model, these two none-asthma none-COPD classes joined again. The two treated asthma classes, previously observed in the five-class model, emerged again, however, with different prevalences; the class with ICS events and no ICS-LABA combinations had a prevalence of $3.9 \%$, while the class with ICS-LABA combinations but with no sole ICS prescriptions had a prevalence of $2.7 \%$. There was an 'asthma and COPD' class (0.9\%) with high homogeneity; it showed high probabilities for both asthma and COPD diagnosis, GP visits, and prescription events. In this class, the probability of 'smoking ever' was very high (90.9\%). However, the probability of having recent asthma-related GP-recorded events visits in the last 12 months was marginal (43.0\%). These item-response probabilities indicated patients in this class potentially had ACOS. Other classes included one class (6.6\%) for 'ever diagnosed asthma without current treatment', one class (1.3\%) for 'ever diagnosed currently treated' COPD, one class (1.4\%) with high probabilities for each of SABA and ICS events and low probability for 'ever smoking', and one class $(1.0 \%)$ with low to marginal probabilities for asthma prescriptions but very high probability for 'ever smoking'.

Compared to the eight-class model, the significant refinements in the nine-class model were a split of one of the 'ever-diagnosed currently treated asthma' classes into two $(2.5 \%$ and $1.3 \%)$ which, however, were reunited in the 10 -class model. The assignment of the sample individuals into the classes across the competing models is shown in Figure 3.4, while model diagnostics for each of the competing models are shown in Figure 3.5. 


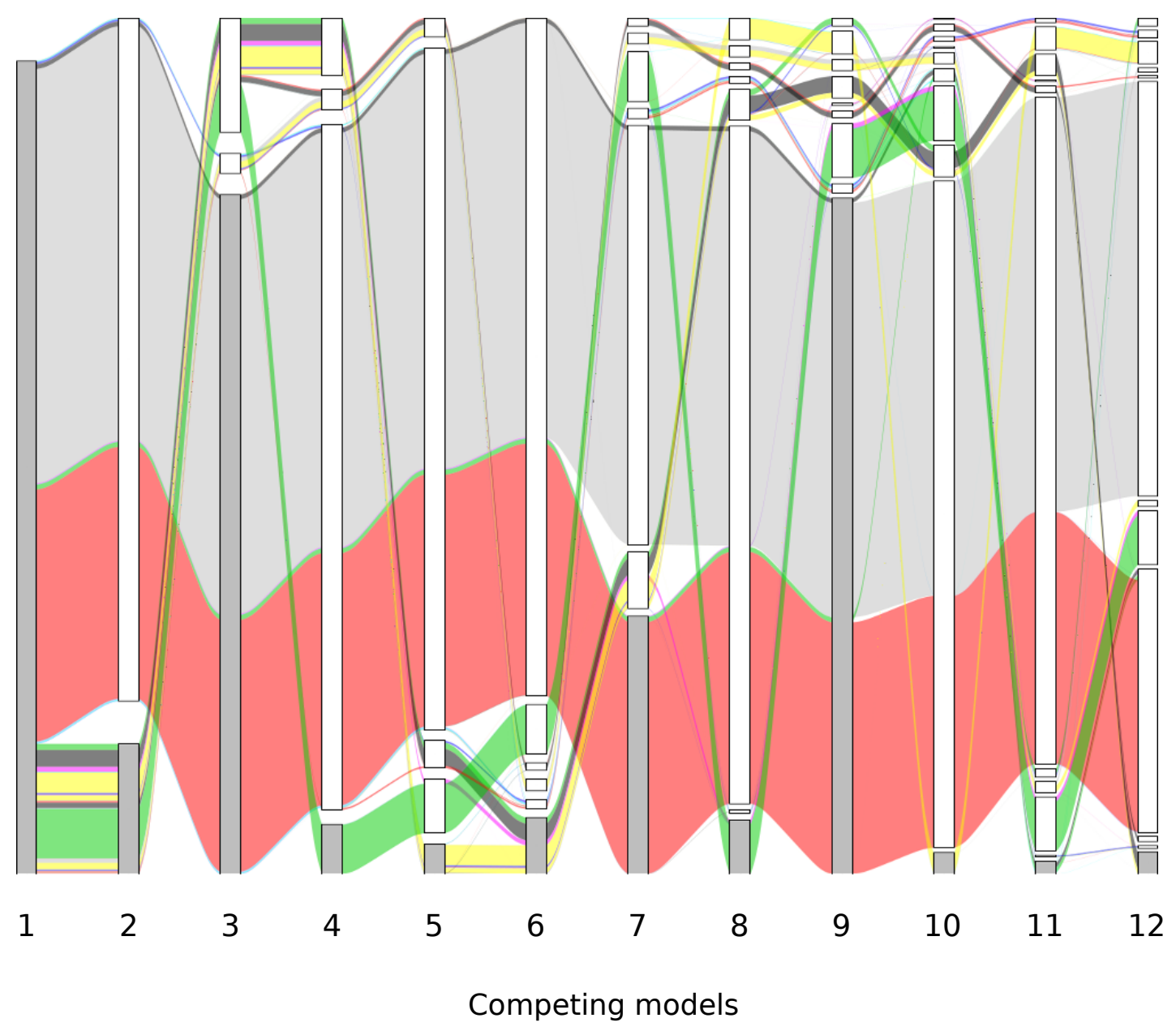

Figure 3.4: An alluvial diagram showing assignment of the sample individuals across the produced competing latent models. Each band represents a group of individuals sharing the same class in the last competing model (the 12-class model), and demonstrates how they were assigned to classes across the other competing models.

\subsubsection{Model selection}

Based on the model diagnostics diagram (Figure 3.5), the AIC, BIC, and $G^{2}$ diagnostics for the models with one to 12 classes declined significantly between the one-class model and the four-class model. Then, these three diagnostics continued to decline slightly until they stabilised at the eight-class and nine-class models. The Chi-square static declined abruptly between the one-class and the two-class models before it appeared to visually stabilise across the competing models. The nine-class model had the lowest BIC value, while the other diagnostics, AIC, Chisquare, and $G^{2}$, had their lowest values at the 12-class model. However, the decline in the BIC value between the eight-class model and the nine-class model was very small and negligible, indicating very little improvement in the information gain. In addition, as described in Section 3.4.2, the structures of these two 

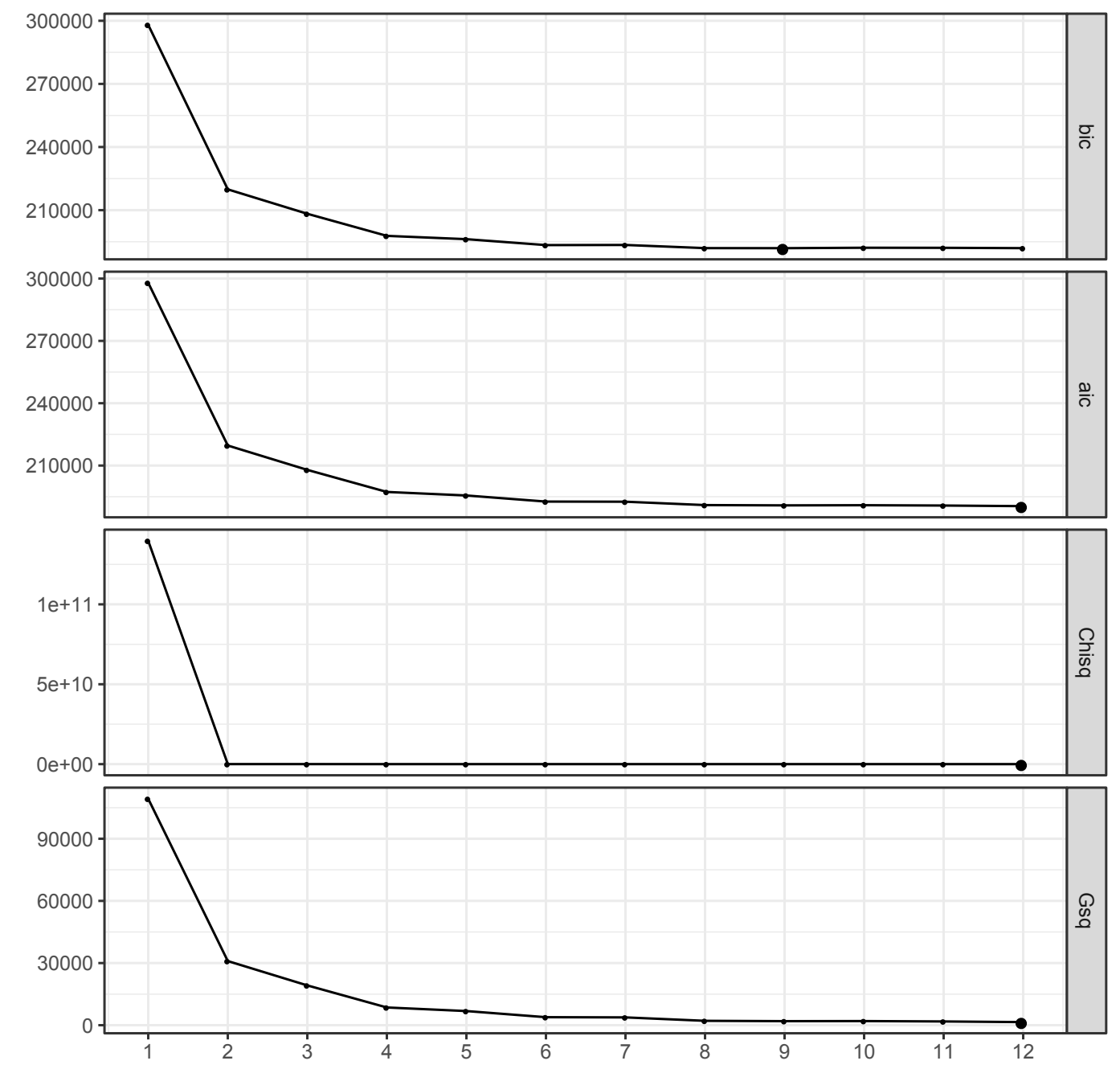

Figure 3.5: Diagnostics for the competing latent class models. For each diagnostic, the class with the minimum value was marked with a large dot. AIC = Akaike information criterion; BIC = Bayesian information criterion; Chisq = Pearson Chi-square goodness of fit statistic; Gsq = G-squared.

classes were very similar, with the eight-class model showing clinically meaningful classes. Therefore, the eight-class model appeared to be a good solution, among the other competing models, that reasonably fit with the purpose of this latent class modelling of identifying patients with asthma, including those with currently treated asthma.

\subsubsection{Model interpretation: Characterising and labelling the identified classes of the best-fit model}

Figure 3.6 shows the classes of the eight-class model along with their prevalences, item-response probabilities, and assigned labels. 


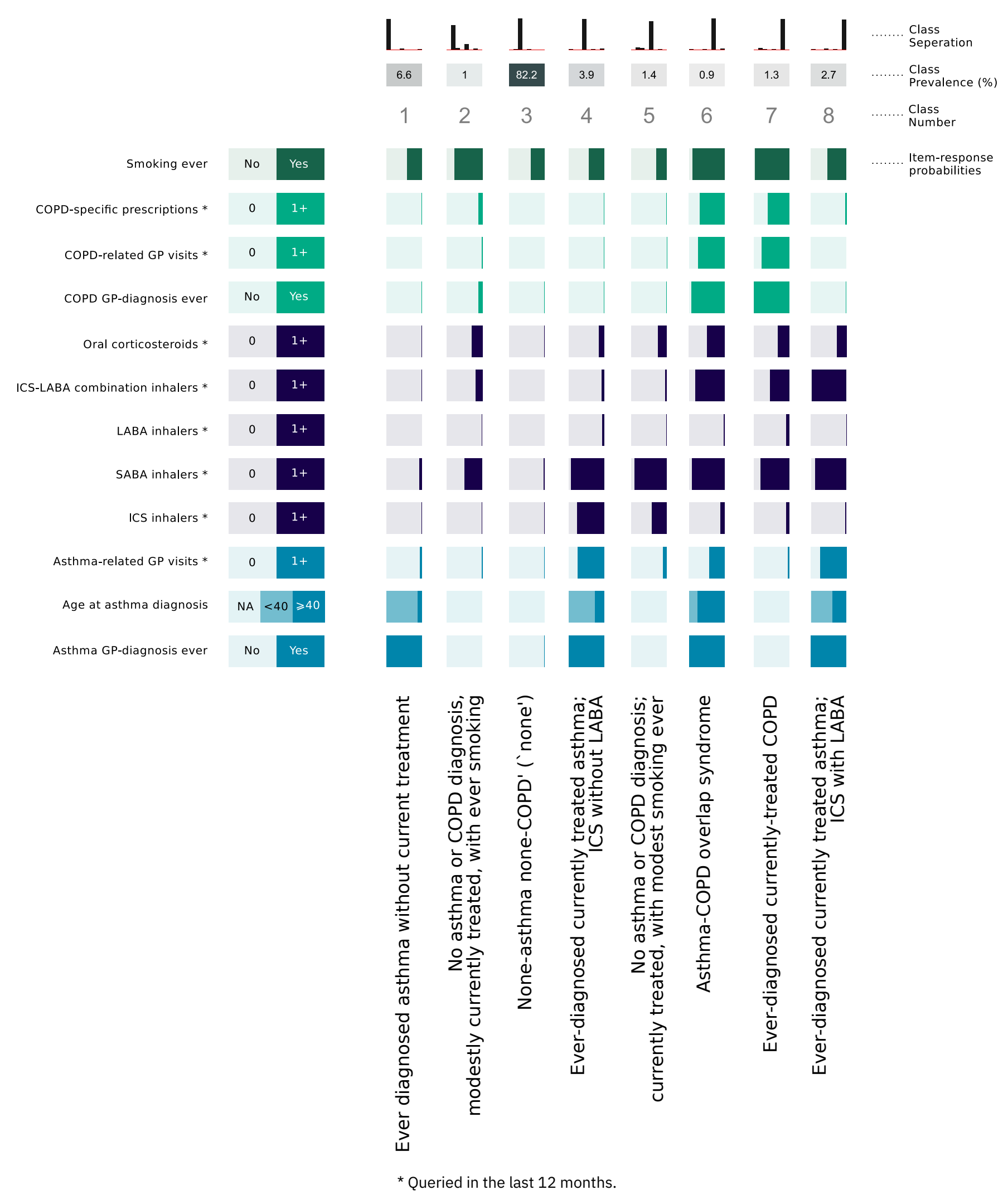

Figure 3.6: Class prevalences and item-response probabilities of the eight-class model. The left-most column shows the names and levels of the observed variables. The small bar-plot above each latent class demonstrates class separation: it shows the average probabilities, within each class, of membership in all the latent classes. 
The eight-class latent class model which I chose as a solution consisted of the following classes: ${ }^{3}$

1. 'Ever diagnosed asthma without current treatment': This class has a prevalence of $6.6 \%$. All the individuals in this class had asthma diagnosis events, recorded in $88 \%$ of the class members before the age of the 40 . Only $5.0 \%$ and $6.9 \%$ of the class members had asthma-related events and SABA prescriptions in $2014.41 .0 \%$ of the class members ever smoked on or before 2014.

\section{2. 'No asthma or COPD diagnosis, modestly currently treated, with ever} smoking': The prevalence of this class was $1.0 \%$. Its individuals had no asthma diagnosis events recorded by the end of 2014 , although $2.4 \%$ of them had non-diagnosis asthma-related GP events (excluding prescriptions) recorded in 2014 . However, $50.0 \%$ of people in this class had at least one SABA prescription, and $19.5 \%$ had ICS-LABA combination prescriptions, and 30.6\% had prescriptions for oral steroids in 2014. In addition, $11 \%$ of the class members had recorded COPD diagnosis, although only 1.8\% had COPD-related GP visits in 2014, and $11.8 \%$ had prescriptions usually prescribed for COPD. Smoking related events were found for $78.9 \%$ of the class members. The average age of the class members in 2014 was 61.7 years with a standard deviation of 18.6 years. The marginal item-response probabilities in this class suggests it includes heterogeneous sub-groups of individuals some of which might be COPD patients, while others were smokers without symptoms of COPD. For this class, I suggested the label 'possible/at risk of COPD'.

3. 'Neither asthma/COPD' (or 'none'): This class, having a prevalence of $82.2 \%$, had almost zero item-response probabilities for all the observed variables except for 'smoking ever' which had 37.8\% probability.

4. 'Ever-diagnosed currently treated asthma; ICS without LABA': This class had a prevalence of $3.9 \%$ and showed $100 \%$ probability for asthma diagnosis events recorded before the age of 40 for $73.2 \%$ of the class members. $74.6 \%$ of the class members had recorded asthma-related GP events in 2014 and had high probabilities for ICS (75.8\%) and SABA (93.9\%) prescriptions and $15.6 \%$ probability for oral corticosteroids (OCS) prescriptions. $43.3 \%$

\footnotetext{
${ }^{3}$ The numbers associated with the classes were merely numerical labels as appeared in the output of the latent class modelling, and did not imply any order.
} 
of individuals in this class had recorded smoking-related events. This class included people from all age groups (mean $=39.5$; standard deviation (SD) $=22.2$ ).

5. 'Currently treated; no recorded asthma or COPD diagnosis; with modest smoking ever': This class with $1.4 \%$ prevalence was similar to the class \#2 described above, as it had no recorded asthma diagnosis. However, it had zero-probability for COPD diagnosis events, very high probability (89.9\%) for SABA prescriptions in 2014 , and $41.8 \%$ for ICS prescriptions in that year. The class members had $24.5 \%$ probability for OCS prescriptions, and $29.8 \%$ probability of 'smoking ever'. Based on these characteristics, I assumed this class included asthma patients with no recorded diagnosis.

6. 'ACOS': This class with $0.9 \%$ prevalence showed almost total probabilities for asthma and COPD diagnosis events. For $75.3 \%$ of the class members, the earliest recorded diagnosis of asthma was before the age of 40 . While $43.5 \%$ of the class members had asthma related events in $2014,74.3 \%$ had COPD-related events in that year, and $70.2 \%$ had prescriptions specific to COPD in the same year. The class members had a very low probability for ICS prescriptions (11.0\%), but very high probabilities for SABA (91.4\%) and ICS-LABA combination prescriptions (81.7\%) in 2014. Almost half of the class members (49.7\%) had oral steroids in 2014 indicating severe and/or uncontrolled symptoms.

7. 'Ever-diagnosed currently-treated COPD': This class had a prevalence of $1.3 \%$ and showed $100 \%$ probability for COPD diagnosis events, high probabilities for COPD-related GP events (74.3\%) and COPD-specific prescriptions (70.2\%). The vast majority of people in this class (96.5\%) had recorded smoking history. There was also a high probability for SABA prescriptions (81.2\%), but a marginal probability for ICS-LABA combination prescriptions (54.4\%), and a $31.7 \%$ probability for oral steroids in 2014 . The class showed very low probabilities for ICS-alone (9.0\%) and LABA-alone (8.8\%) prescriptions.

8. 'Ever-diagnosed currently treated asthma; ICS with LABA': This class had a prevalence of $2.7 \%$. It was closely related to the class \#4 described above. All its members had asthma diagnosis events, recorded under the age of 40 for $60.2 \%$ of patients. $74.0 \%$ of the class members had asthma-related 


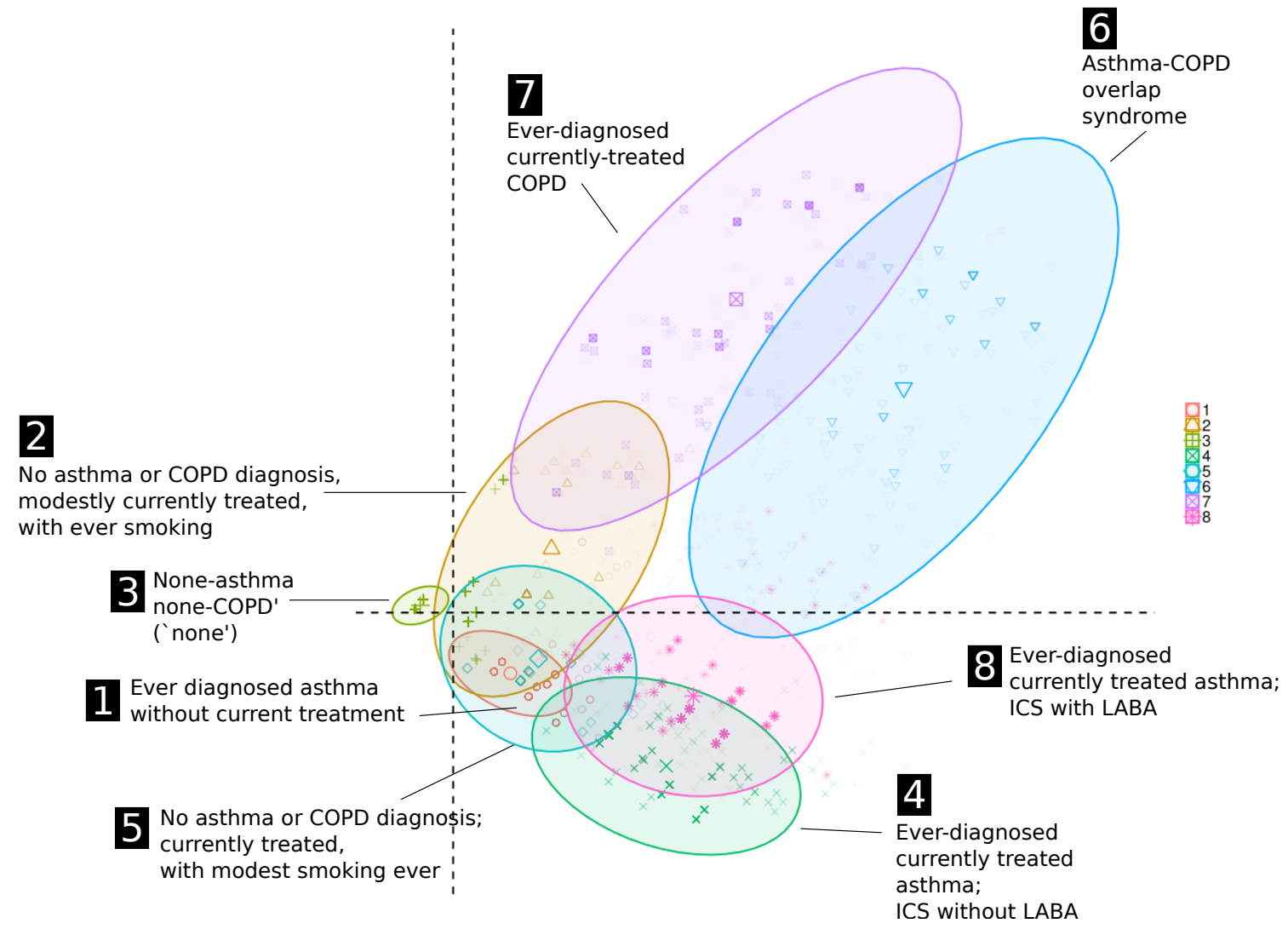

Figure 3.7: Visualisation of the eight-class latent class model using principal component analysis. ACOS: asthma-COPD overlap syndrome; COPD: chronic obstructive pulmonary disease; GP: general practitioner; ICS: inhaled corticosteroid; LABA: long-acting beta adrenoceptor agonist; SABA: short acting beta agonist.

GP events in 2014. Almost half of people in this class (53.2\%) had recorded smoking events.

Figure 3.7 visualises the classes in the eight-class model using principal component analysis (PCA). The distances and overlaps between the latent classes were consistent with their clinical interpretation overall.

\subsubsection{Class merging}

Since I was interested in identifying patients with active asthma, I merged the classes 4, 5, and 8 into a single super class labelled "currently treated asthma". Most patients (82.5\%) in that super class had recorded asthma diagnosis (Class 4 and 8 ); the remaining patients (Class 5, 17.5\%) had no recorded diagnosis of asthma or COPD but had a very high probability of using SABA inhalers, a marginal probability $(\approx 42 \%)$ of using ICS inhalers, and a low probability $(\approx 30 \%)$ of smoking history. Although some of those patients might have received those inhalers without actually having asthma, I kept them in the super class of 'currently treated 
Table 3.2: The latent classes and their prevalences before and after merging.

\begin{tabular}{|c|c|}
\hline Before merging & After merging \\
\hline $\begin{array}{l}\text { 1. Ever diagnosed asthma without current treatment } \\
(6.6 \%)\end{array}$ & No change \\
\hline $\begin{array}{l}\text { 2. possible COPD (1.0\%): No asthma or COPD } \\
\text { diagnosis, modestly currently treated, with ever } \\
\text { smoking }\end{array}$ & No change \\
\hline 3. 'None' (82.2\%): None-asthma none-COPD' & No change \\
\hline $\begin{array}{l}\text { 4. Ever-diagnosed currently treated asthma; ICS without } \\
\text { LABA }(3.9 \%)\end{array}$ & \multirow{3}{*}{ Currently treated asthma (8.0\%) } \\
\hline $\begin{array}{l}\text { 5. No asthma or COPD diagnosis; currently treated, with } \\
\text { modest smoking ever (1.4\%) }\end{array}$ & \\
\hline $\begin{array}{l}\text { 8. Ever-diagnosed currently treated asthma; ICS with } \\
\text { LABA }(2.7 \%)\end{array}$ & \\
\hline 6. Asthma-COPD overlap syndrome (0.9\%) & No change \\
\hline 7. Ever-diagnosed currently-treated COPD (1.3\%) & No change \\
\hline
\end{tabular}

ICS: inhaled corticosteroid; LABA: long-acting beta adrenoceptor agonist; COPD: chronic obstructive pulmonary disease.

asthma'. Table 3.2 shows the classes and their prevalences before and after merging. I later used the labels of the resulting simpler six-class structure in the training of a classification algorithm (see Section 3.4.6 below).

\subsubsection{Derivation of classification algorithm}

A decision tree with 11 splits (and 12 leaves) was trained using recursive partitioning. The R package rpart performed 10-fold cross-validation to select the optimal size of the decision tree. The results of this cross-validation are shown in Table 3.3 and Figure 3.8.

Table 3.3: Results of 10 -fold cross-validation for the recursive partitioning model, showing the crossvalidation error for each sub-tree.

\begin{tabular}{llllll}
\hline & $\begin{array}{l}\text { Complexity } \\
\text { parameter }\end{array}$ & Number of splits & Relative error & $\begin{array}{l}\text { Cross-validation } \\
\text { error rate }\end{array}$ & $\begin{array}{l}\text { Cross-validation } \\
\text { standard } \\
\text { deviation }\end{array}$ \\
\hline 1 & 0.39935 & 0 & 1.00000 & 1.00000 & 0.01193 \\
2 & 0.31750 & 1 & 0.60065 & 0.60065 & 0.00961 \\
3 & 0.07331 & 2 & 0.28315 & 0.28315 & 0.00679 \\
4 & 0.04409 & 3 & 0.20984 & 0.20984 & 0.00588 \\
5 & 0.03589 & 4 & 0.16576 & 0.16576 & 0.00525 \\
6 & 0.02324 & 5 & 0.12987 & 0.12987 & 0.00466 \\
7 & 0.01162 & 6 & 0.10663 & 0.10663 & 0.00423 \\
8 & 0.01025 & 7 & 0.09501 & 0.09501 & 0.00400 \\
9 & 0.01003 & 8 & 0.08476 & 0.08630 & 0.00381 \\
10 & 0.01000 & 11 & 0.05468 & 0.07536 & 0.00357 \\
\hline
\end{tabular}




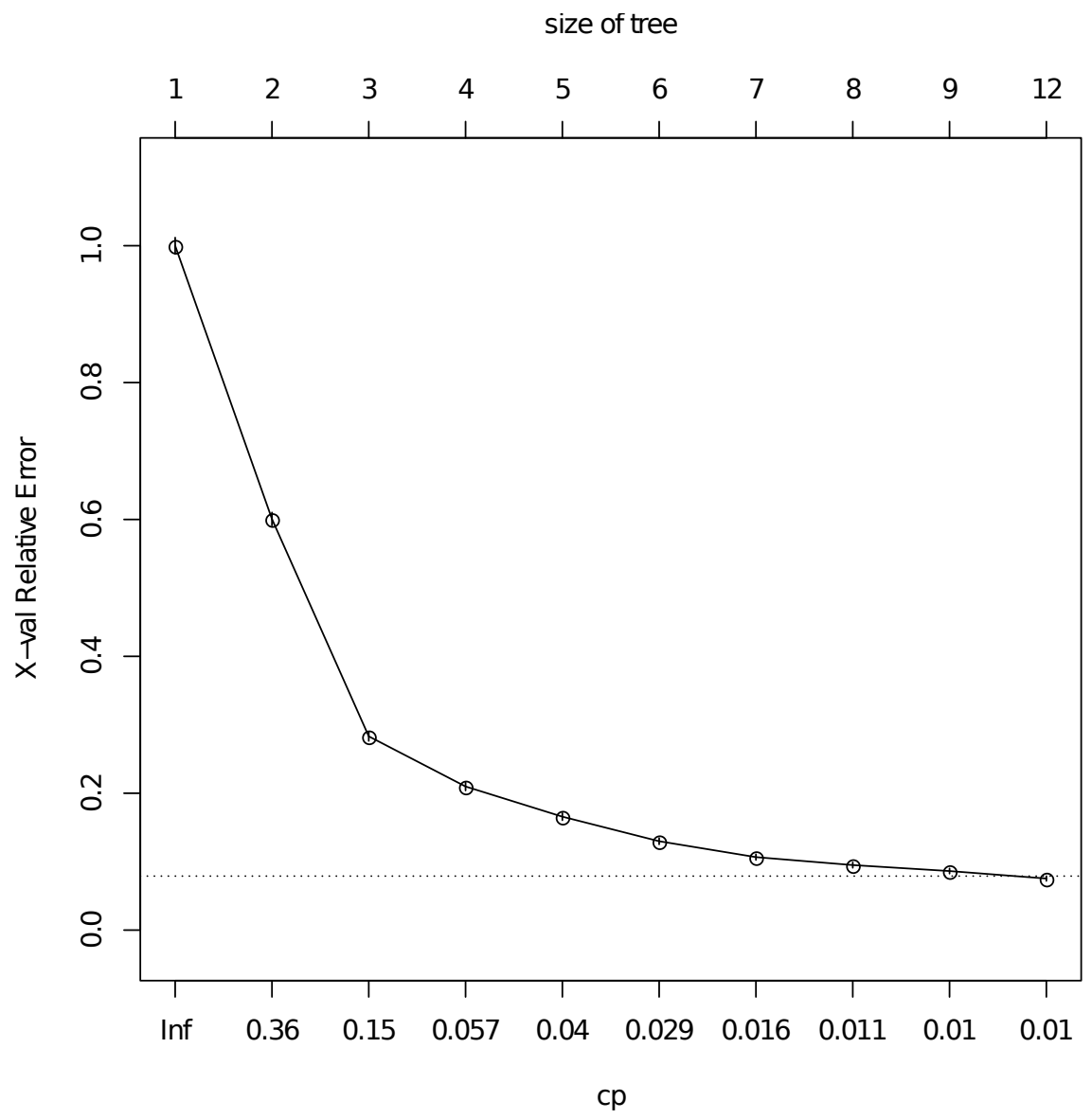

Figure 3.8: Visualisation of the 10 -fold cross-validation results of the recursive partitioning. The horizontal dotted line was drawn at 1 standard deviation above the minimal cross-validation error. The tree with 12 leaves (11 splits) had the lowest cross-validation error and was therefore the desired tree. $\mathrm{cp}$ : complexity parameter. 
In these results, the tree with 11 splits had the minimum cross-validation error rate (0.075). The complexity parameter of this tree, 0.01 , was used to further prune the full tree in an attempt to remove splits, if any, that caused over-fitting. The final pruned tree is shown in Figure 3.9.

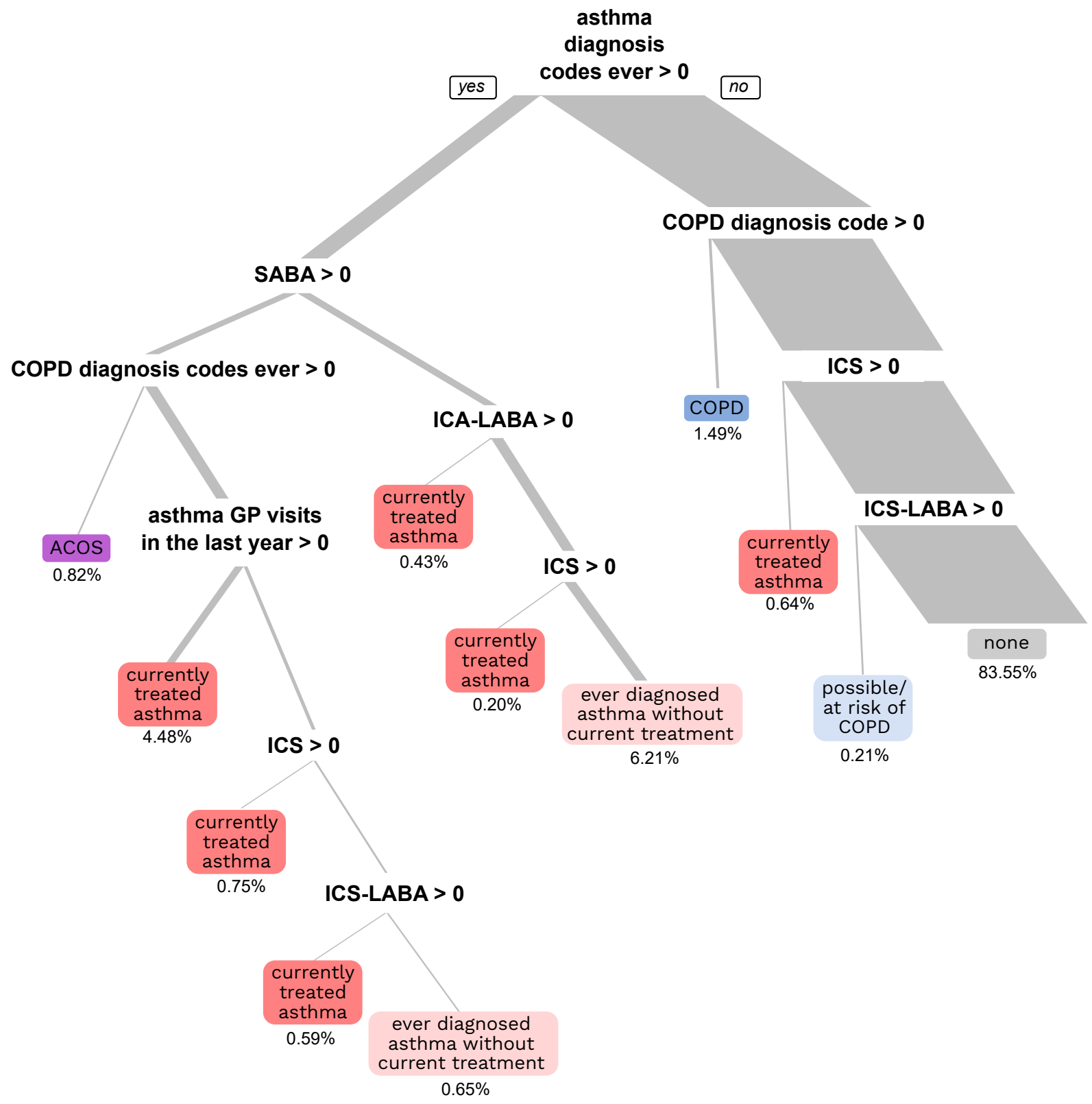

Figure 3.9: A decision tree representation of the classification algorithm. At each node, the left branch is followed when the condition is true. The width of branches is proportionate to the number of individuals in the derivation sample who followed these branches relative to the size of the entire sample. The final nodes, i.e. leaves, represent the labels to be assigned for new cases. ACOS: asthma-COPD overlap syndrome; COPD: chronic obstructive pulmonary disease; GP: general practitioner; ICS: inhaled corticosteroid; LABA: long-acting beta adrenoceptor agonist; SABA: short acting beta agonist. 
This final tree represented the classification algorithm that could be used to classify individuals in new samples into the following six categories:

- 'currently treated asthma'

- 'ever diagnosed asthma without current treatment'

- 'possible/at risk of COPD'

- 'COPD'

- 'ACOS'

- 'none'

The actual variables that were chosen by recursive partitioning for the construction of this tree were:

- "having asthma diagnosis events ever"

- "having asthma related GP visit in the last 12 months"

- "having COPD diagnosis events ever"

- "having SABA prescriptions in the last 12 months"

- "having ICS prescriptions in the last 12 months"

- "having ICS-LABA combination prescriptions in the last 12 months"

Table 3.4 shows a confusion matrix and statistics for the cross-classification of the decision tree predictions against the labels used in the training. Overall, the predictive performance of the classification algorithm was very high, which meant it could be used to accurately classify new populations based on the latent class model that I described earlier in this chapter. An exception was that the algorithm had a low sensitivity to identify people with 'possible/at risk of COPD' as it misclassified $56 \%$ of them into the 'none' class. 
Table 3.4: Confusion matrix and statistics for the cross-classification of the predicted classifications against the LCA-based labels in the validation dataset. These statistics reflected how well the classification algorithm represented the latent class model.

\section{Confusion matrix}

\begin{tabular}{|c|c|c|c|c|c|c|}
\hline & \multicolumn{6}{|c|}{ Predicted } \\
\hline & AcOS & $\begin{array}{l}\text { Ever } \\
\text { diagnosed } \\
\text { asthma } \\
\text { without } \\
\text { current } \\
\text { treatment }\end{array}$ & $\begin{array}{l}\text { Currently } \\
\text { treated } \\
\text { asthma }\end{array}$ & $\begin{array}{l}\text { Possi- } \\
\text { ble/at risk } \\
\text { of COPD }\end{array}$ & COPD & None \\
\hline \multicolumn{7}{|l|}{ LCA-based labels } \\
\hline AcOS & 116 & 6 & 10 & 0 & 0 & 0 \\
\hline $\begin{array}{l}\text { Ever diagnosed asthma } \\
\text { without current treatment }\end{array}$ & $<5^{*}$ & 1,004 & 0 & 0 & 0 & 0 \\
\hline Currently treated asthma & 9 & 8 & 1,035 & 10 & $<5^{*}$ & 37 \\
\hline Possible/at risk of COPD & 0 & 0 & 0 & 26 & $<5^{*}$ & 36 \\
\hline COPD & 0 & 0 & 0 & $<5^{\star}$ & 199 & 0 \\
\hline None & 0 & $<5^{*}$ & 11 & 0 & 25 & 12,455 \\
\hline
\end{tabular}

* Values masked to comply with the SAIL Databank policy on small numbers.

\section{Overall statistics}

Accuracy

No Information Error Rate

p-value of a one-sided test that "the Accuracy is higher than the No Information Error Rate"

Cohen's Kappa
0.9893 (95\% confidence interval (CI): $0.9875,0.9909$ )

0.8354 (i.e. if we just assigned all individuals into the largest class 'none', we would be correct in $83.5 \%$ of cases)

$<2.2^{-16}$

0.9635

\section{Class-specific statistics}

\begin{tabular}{lllllll}
\hline & \multicolumn{7}{c}{$\begin{array}{l}\text { Ever } \\
\text { diagnosed }\end{array}$} & $\begin{array}{l}\text { Currently } \\
\text { asthma } \\
\text { without } \\
\text { current } \\
\text { treatment }\end{array}$ & $\begin{array}{l}\text { Possi- } \\
\text { treated } \\
\text { asthma }\end{array}$ & $\begin{array}{l}\text { Ple/at risk } \\
\text { of COPD }\end{array}$ & COPD & None \\
\cline { 2 - 6 } & 0.921 & 0.984 & 0.980 & 0.684 & 0.873 & 0.994 \\
Sensitivity & 0.999 & 1.000 & 0.995 & 0.997 & 1.000 & 0.985 \\
Specificity & 0.879 & 0.999 & 0.940 & 0.406 & 0.990 & 0.997 \\
Positive Predictive Value & 0.999 & 0.999 & 0.998 & 0.999 & 0.998 & 0.971 \\
Negative Predictive Value & 0.008 & 0.068 & 0.070 & 0.003 & 0.015 & 0.835 \\
Prevalence & 0.008 & 0.067 & 0.069 & 0.002 & 0.013 & 0.831 \\
Detection Rate & 0.009 & 0.067 & 0.073 & 0.004 & 0.013 & 0.833 \\
Detection Prevalence & 0.960 & 0.992 & 0.988 & 0.841 & 0.936 & 0.989 \\
Balanced Accuracy & & & & & & \\
\hline
\end{tabular}

ACOS: asthma-COPD overlap syndrome; COPD: chronic obstructive pulmonary disease; CI: confidence interval; LCA: latent class analysis. 


\subsubsection{Comparing the classification algorithm with other case identification methods}

Comparisons between the classification algorithm and the case definitions described in Section 3.3.5.1 are shown in Table 3.5.

The following two comparisons had the highest Cohen's kappa value:

- The 'QOF indicator of asthma (AST001)' and the algorithm's definition of 'currently treated asthma' had a kappa value of $86.5 \%$.

- 'Asthma diagnosis code' and the union of algorithm's definitions of 'ever diagnosed asthma without current treatment' and 'currently treated asthma' (i.e. 'any asthma') had a kappa value of $94.5 \%$.

However, the self-reported definition of currently treated asthma had lower agreements with the algorithm classification, with kappa values of $61.5 \%$ and $56.4 \%$ for the concordance with the algorithm's definitions of 'currently treated asthma' and 'any asthma', respectively. Interestingly, the self-reported definition of currently treated asthma has a level of non-random agreement, although very low (kappa = 12.2), with the algorithm's definition of 'ever diagnosed asthma without current treatment'. Finally, the algorithm's definition of currently treated COPD had poor disagreement with the self-reported definition of currently treated COPD (kappa $=28.3$ ). 


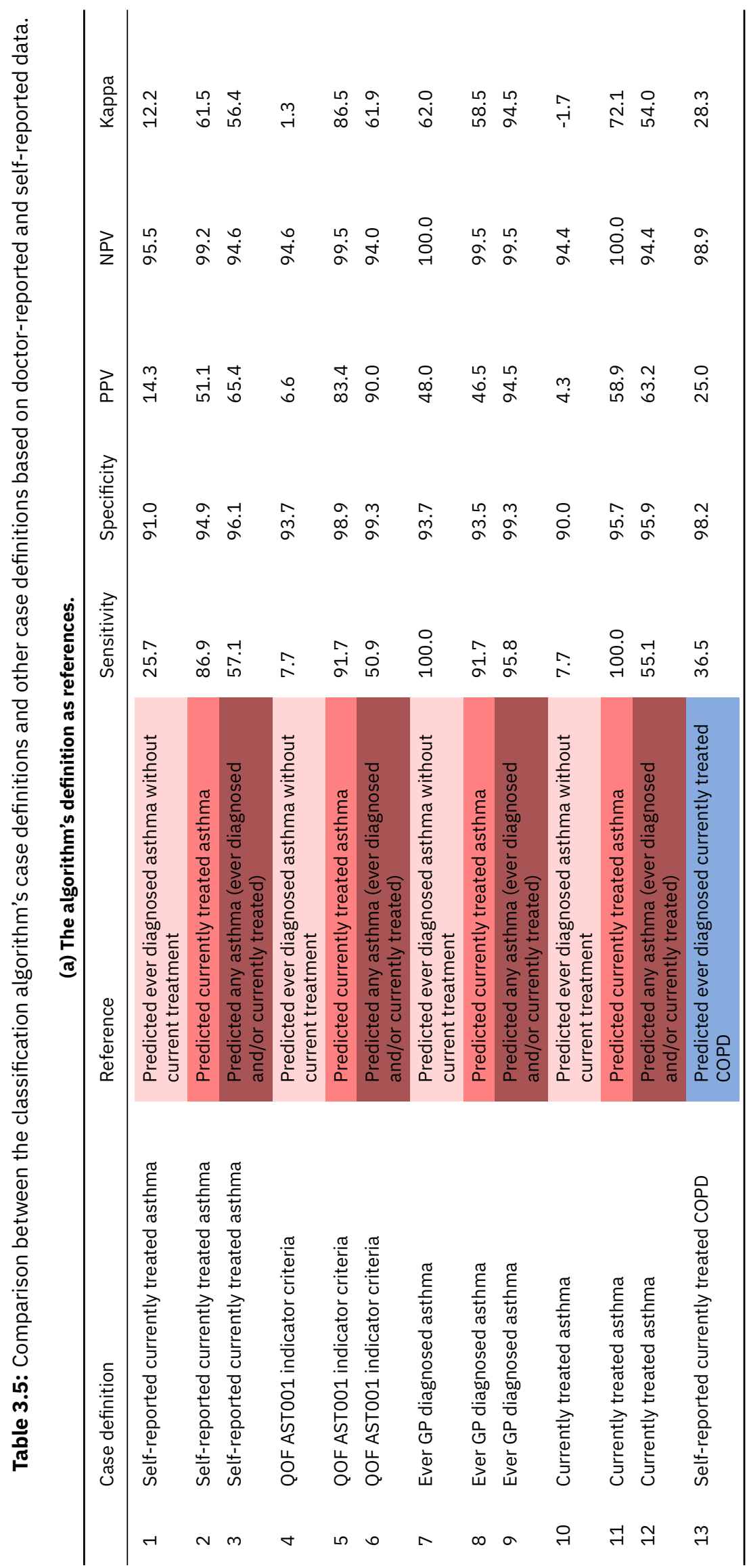




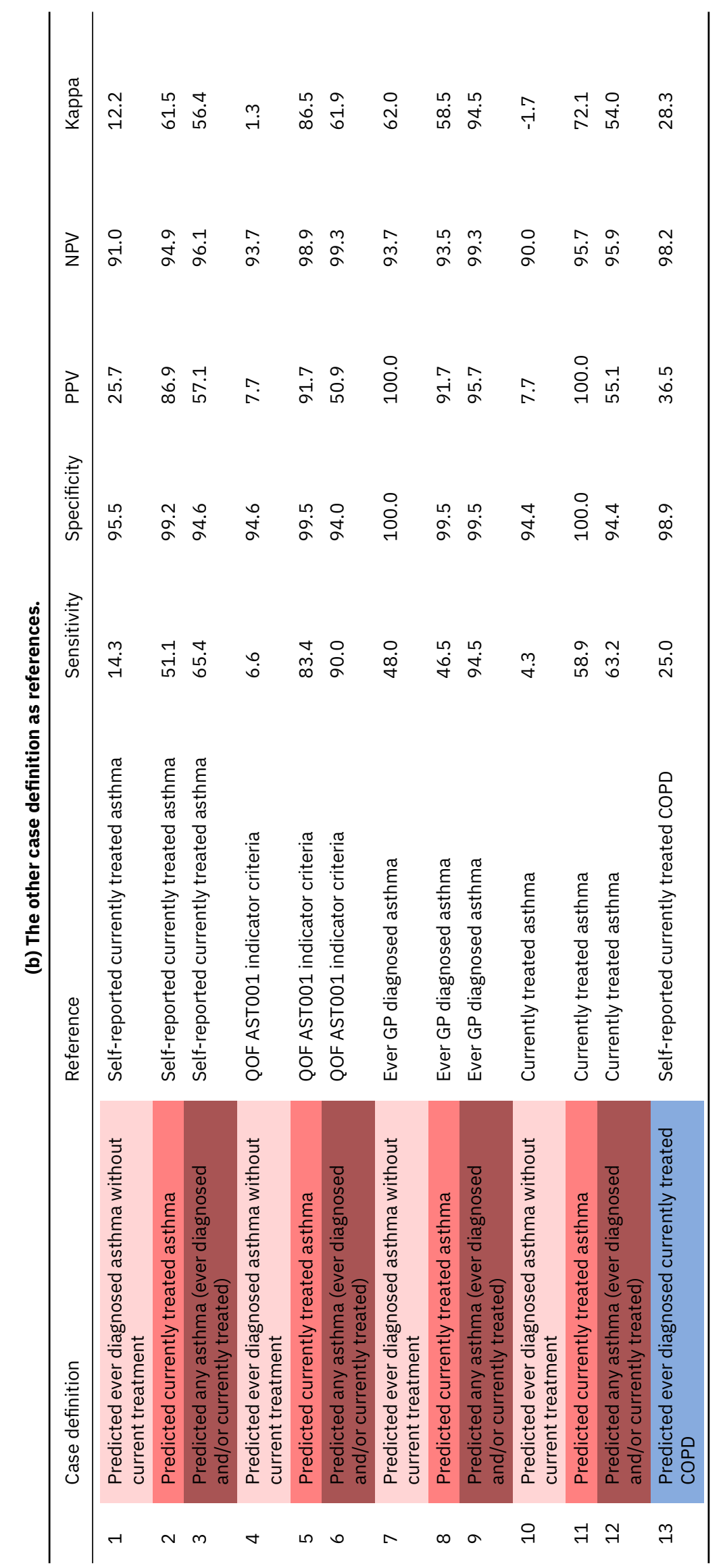




\subsection{Discussion}

\subsubsection{Summary and interpretation of the findings}

In this chapter, I described the development of a latent class model to identify patients with 'asthma' (including those currently treated asthma and those with ever diagnosed asthma without current treatment), COPD, and both conditions in Wales in 2014. Based on this model, I trained a classification tree which can be used to classify new samples into the above labels.

I performed the latent class modelling for 1-12 classes on a random sample of the Welsh population. Based on model diagnostics and clinical interpretability, I chose the eight-class model as the optimal clustering in relation to the observed variables used in the modelling. The eight-class model succeeded in clustering the population into distinct, homogeneous classes. There was one large class (82.2\%) characterised by the absence of almost all asthma and COPD related events, except having a modest probability of smoking history. There were also four classes consistent with asthma: one ever-diagnosed currently treated, two ever-diagnosed currently treated, and a smaller class with no diagnosis but with current asthma prescriptions. Only one small class had distinctive characteristics of COPD with a prevalence of 1.3\%; almost all people this class had positive smoking history. Interestingly, the overlap between asthma and COPD was clearly represented in a distinct, homogeneous class with a prevalence of $0.9 \%$. One class showed low to marginal probabilities for asthma prescriptions with very low probabilities for COPD diagnosis and prescriptions.

Following model interpretation, I merged classes consistent with 'currently treated asthma', simplifying the clustering model into six labelled groups. I then used these labelled groups along with all the observed variables, which were used in the latent class modelling, to train a classification algorithm. The best classification algorithm was a decision tree with 11 splits and 12 final nodes. This algorithm is transferable and therefore can be used in new samples in the GP dataset in the SAIL Databank and could be also tested in similar external datasets. The 'currently treated asthma' label predicted by the classification algorithm included all patients with current asthma prescriptions. This label had a high agreement 
with the QOF definition of ever diagnosed currently treated asthma (the AST001 indicator). The union of the algorithm's definitions of 'currently treated asthma' and 'ever diagnosed asthma without current treatment' had a very high agreement with having an asthma diagnosis code ever. There was a suboptimal agreement between the algorithm's definition of 'currently treated asthma' and the WHS-based definition of 'self-reported currently treated asthma'. This can be potentially explained by the possibility that some respondents thought they had asthma while they did not, while some respondents may have not received asthma prescriptions in the last 12 months or ever. In addition, the WHS did not specify a time frame when asking the respondents whether they were "currently" treated for asthma. Accordingly, respondents might have understood the word "currently" as time frames different from the 12-month interval traditionally used by researchers. These explanations were supported by the analysis I presented in Appendix B.1, in which I found discordance between the WHS definition of self-reported currently treated asthma and GP-reported asthma diagnosis and prescriptions, including the interval over which prescriptions were queried.

\subsubsection{Strengths and limitations}

\subsubsection{Strengths}

\section{Latent class analysis can reveal asthma epidemiology in routine data}

In the absence of an accepted reference standard for identifying asthma patients, mixture modelling methods such as LCA allow identifying likely patients using the available observed data. Since asthma in the UK is mainly managed in primary care, I performed the LCA based on the informed assumption that asthma epidemiology was reflected in primary care EHR data. LCA follows a top-down approach, unlike the bottom-up approach used in cluster analysis. The latter assesses the similarities between individuals in order to form clusters. LCA, however, utilises the distributions of the observed characteristics to identify distinct latent groups, and then assesses the membership probabilities of each individual into these hypothesised groups. Those probabilistic class memberships fit with the nature of asthma as a probabilistic rather than a binary condition. The identified latent classes reflected the heterogeneous nature of asthma as a condition with varying severity which overlapped with COPD. By computationally uncovering the popula- 
tion structure in relation to asthma and COPD related variables, LCA identified the likely patient groups, some of which could be otherwise overlooked in the manual researcher-led development of case definitions.

\section{Asthma and COPD were included in the same model}

The inclusion of both asthma and COPD data in the same model is a particular strength. It allowed the exploration of the overlap between the two diseases in EHR-derived data. it makes the model useful for researchers who, for example, want to study a subset of asthma or COPD patients who also have characteristics of the other disease (i.e. ACOS), or those who have one disease without the other.

\section{Derivation of a transferable classification algorithm}

A remarkable strength in this chapter is the derivation of a classification algorithm based on the best-fit LCA model. Since the LCA model was performed on a sample of 50,000 individuals and not on the entire population in the SAIL Databank, its output cannot be directly used to identify asthma patients from outside this sample. To overcome this limitation, I derived a classification algorithm that can be used on different samples and by other researchers in the SAIL Databank. This transferable classification algorithm can be also tested in other similar primary care databases. This algorithm could be used to produce more accurate estimates of the disease prevalence compared to methods based on patient-reported data from national health surveys and those based on the Quality of Outcomes Framework's AST001 indicator.

\subsubsection{Limitations}

The analysis in this chapter has some limitations.

\section{Limitations related to EHR-derived data}

The latent class modelling described in this chapter was based on relatively limited data that were usually insufficient to establish diagnosis at the point of care. Much of the information that were usually available to GPs to establish asthma and COPD diagnoses were not available in the primary care dataset of the SAIL Databank. This was mostly due to poor recording and/or coding at the point of 
care. Those low-quality data included, for example, lung function tests. Since the quality of observed variables was essential for a well-specified latent class model, I constructed these observed variables only using events that were thought to be of reasonable quality in the SAIL Databank. Such events included diagnosis, disease-related GP visits, prescriptions, and smoking history.

Despite using those observed variables which were known to be well coded in the GP dataset in the SAIL Databank, the imperfect quality of these variables could have still affected the latent class modelling. In interpreting the chosen model, I took into account the nature and limitations of EHR-derived data such as the possibility of missing or incorrect coding as well as record-linkage errors. For example, Class 5 (Figure 3.6) was characterised by current prescriptions suggesting active asthma but with no recorded diagnosis events; I assumed that patients in this class were possibly being treated for asthma as they had a low probability of ever smoking.

The clinical meanings of the latent classes were based on surrogate variables, such as GP diagnosis codes, visits, and prescriptions as well as smoking history, rather than on more direct disease markers such as clinical and laboratory findings. Nevertheless, I hypothesised that LCA of these surrogate variables can reasonably distinguish between patients with asthma, COPD, and ACOS. This provided an opportunity to assess how clustering based on these surrogate variables will perform compared with that based on asthma and COPD biomarkers [7, 273-279].

\section{Asthma-COPD overlap syndrome was treated as a separate class}

In the merging of the latent classes and the derivation of the classification algorithm, I treated ACOS as a separate label rather than merging it with asthma and/or COPD groups. This approach was in-line with the view that ACOS is a third condition, a view which was, however, subject to active debate [210, 211, 280].

\section{Limitations of latent class analysis}

Although latent class analysis was an appropriate clustering method that fit with asthma heterogeneity, it had some particular limitations.

The specification of the observed variables, model selection and interpretation all involved significant levels of subjectivity. Model interpretation and usefulness 
both depend largely on the choice and configuration of the observed variables, which thus needs careful consideration. Therefore, assessing the derived classification algorithm against other GP- and self-reported measures was needed and provided useful information to assess its meaning.

The population structure identified by LCA may not exactly represent the clinical epidemiology of asthma and COPD. It has been shown that the patient groups identified using such unsupervised statistical learning techniques may partly reflect artefact from the analysis method including transformation and encoding of the observed variables [281]. Therefore, an important future work is to compare this LCA model performance with full patient record data and/or clinical assessment for a population sample.

\section{The classification algorithm was not a superior reference}

Given limitations related to data quality and provenance as well as LCA, the classification algorithm derived in this chapter was not intended to be a superior reference against which other asthma case definitions, could be assessed. Arguably, no easily implementable, gold standard operational definition for asthma exists.

\subsubsection{Comparison with related works}

LCA has been widely used on asthma-related data. While some studies used LCA to mainly identify asthma cases [282], the more common use was to uncover phenotypes of asthma and related wheezing and atopic disorders [274, 277, 283].

The study by Prosser et al. [282] was closely related to the aim I pursued in this chapter. However, the main aim of that study was to identify only patients with treated asthma. It used slightly different configuration of the observed variables derived from health insurance claims and hospital discharge data. The model diagnostics favoured the two-class model, which estimated the prevalence of treated asthma in British Columbia in 2001 as 9.9\%. In my analysis, however, I aimed to identify treated and untreated cases of asthma, which had the prevalences of $8.0 \%$ and $6.6 \%$, respectively, and a combined prevalence of $14.5 \%$. In addition, the authors of that study did not take into consideration COPD-related data in their model specification. Conversely, I included events related to COPD as observed variables in order to allow the model to distinguish between asthma and COPD 
patients (and to identify those with ACOS). I also used the age at asthma diagnosis (before vs. after the age of 40) in order to improve distinction between asthma and COPD patients and to improve the overall interpretation of the model.

Another LCA-based study was based on questionnaire data about respiratory symptoms of 4,000 children aged 8-12 years [277]. The authors used 11 questions from the International Study of Asthma and Allergies in Childhood (ISAAC) questionnaire. They assessed their LCA model using objective asthma markers such as allergic sensitisation and bronchial hyper-responsiveness. They identified seven latent classes labelled as 'no respiratory symptoms' (with prevalence of 59.4\%), 'cough during colds' (19.1\%), 'chronic cough and phlegm' (5.3\%), 'nocturnal breathlessness' (4.9\%), 'wheeze only with colds' (4.8\%), 'wheeze without colds, with cough' (4.5\%), and 'wheeze without colds, without cough' (2.1\%). These classes were overall different from those I identified in this chapter. The authors reported that asthma diagnosis was highly reported by the parents in the 'wheeze' and 'nocturnal breathlessness' classes, leading to an $8.5 \%$ prevalence for parent-reported doctor diagnosis of asthma. In my LCA model, however, the classes consistent with 'asthma' (including every diagnosed asthma and currently treated asthma) had an aggregated prevalence of $14.5 \%$. In my modelling, I did not use objective disease markers to specify or validate the model since these data were under-recorded in the SAIL's GP dataset (see Chapter 4). Finally, that study was performed on children only, whereas my latent class modelling was based on data from all age groups. For future work, however, it would be worthwhile to perform a separate latent class modelling for each age group, or using age group as a covariate for the model in order to control its effect on the identified latent structure.

\subsubsection{Future directions}

Future developments of the latent class model described in this chapter include refinement of the observed variables and exploring new predictor variables from primary and secondary care data.

In addition, Wales-wide pathology data are expected to be linked to the SAIL Databank in 2018. These data include important asthma-related data such as peripheral eosinophil count and immunoglobulin E ( $\operatorname{IgE}$ ) levels. Such data linkage would provide an opportunity to explore asthma phenotypes in Wales in a greater depth. 
Patients with asthma exhibit different profiles in terms of the disease natural history and progression. These temporal profiles may be related to clinically recognised phenotypes and underlying endotypes. A longitudinal extension of LCA, latent transition analysis (LTA), would allow modelling the temporal profiles of asthma natural history in Wales. That extended analysis would provide better understanding of the disease's changing epidemiology, and help inform service planning, resource allocation, and support more personalised disease management.

\subsection{Conclusion}

Accurate case definitions are critical to the development of the Wales Asthma Observatory. ${ }^{4}$ However, due to various sources of uncertainty in asthma-related routine data, clear identification of asthma patients using these data is challenging. In the absence of a reliable reference standard, I used LCA of recorded primary care events in the SAIL Databank to identify clusters of likely patients with asthma and/or COPD. The model diagnostics and interpretability favoured the eight-class model which included four classes for asthma (differing by recorded asthma diagnosis and prescriptions), one for COPD, one for asthma-COPD overlap syndrome, one with scarce prescriptions probabilities, and one with no asthma or COPD related events.

Based on the latent class model, and after merging three classes of currently treated asthma, I derived a classification algorithm which could be used to classify new samples into six clinical labels: ever diagnosed asthma without treatment, currently treated asthma, COPD, ACOS, possible/at risk of COPD, and none. I assessed the classification algorithm against other objective and self-reported case definitions. The classification algorithm can be also used or tested by other researchers in similar primary care data sources.

The unsupervised machine learning approach used in this chapter relied on the assumption that despite the challenges to define asthma from RCD, these data reflected the disease clinical epidemiology. By computationally uncovering the population structure, LCA identifies all the likely patient groups that could be overlooked in the manual researcher-led development of case definitions. There-

\footnotetext{
${ }^{4}$ http: //www. wales-asthma-observatory.uk/
} 
fore, the developed LCA model could produce more reliable estimates for asthma prevalence using RCD.

Specifying the LCA model using asthma and COPD observed variables allowed identifying patients with one or both disease. This approach concurs with the current interest to understand the asthma-COPD overlap and allows defining this controversial clinical entity using RCD.

The LCA-based method to identify asthma patients from RCD that I developed in this chapter will be one of a set of asthma case definitions available in the Observatory as described in Chapter 4. 


\title{
Chapter 4
}

\section{Development of the Wales Asthma Observatory}

\author{
Purpose, design, and data quality
}

A main output of this doctoral project is to establish the foundations of the Wales Asthma Observatory based on a national asthma registry using the Secure Anonymous Information Linkage (SAIL) Databank. In this chapter, I discuss the purpose of the Wales Asthma Observatory as a platform for asthma research and surveillance, and its wider context in the United Kingdom and worldwide.

I describe the design of the Observatory and the underlying asthma registry, including source population, structure, content, technical logistics, and approaches to support reproducibility. I then identify important data gaps in asthma related events in the primary care database. Afterwards, I discuss the strengths and limitations of the Observatory, including the wide coverage, implications of data gaps and anonymisation, and specific challenges of assessing asthma outcomes using the SAIL Databank. I then present recommendations for better capture of asthma routine data in the SAIL Databank. I conclude by proposing further developments to the Observatory. 


\section{Chapter Contents}

4.1 Introduction. . . . . . . . . . . . . . . . . . . . . . . . . . . . . . . . 109

4.2 Purpose and context . . . . . . . . . . . . . . . . . . . . . . . . . . . . . 109

4.3 Methods . . . . . . . . . . . . . . . . . . . . . . . . . . . 110

4.3.1 Ethics and information governance . . . . . . . . . . . . . . . . . . . . 112

4.3.2 Logistics and technical environment: the SAIL Databank . . . . . . . . . . . . . . 113

4.3.3 Data used in the Observatory . . . . . . . . . . . . . . . . . . . . . . . . . . . . 113

4.3.3.1 Data sources . . . . . . . . . . . . . . . . . . . . . . . . . . . . . . 113

4.3.3.2 Data anonymisation and linkage . . . . . . . . . . . . . . 118

4.3.4 Eligibility criteria . . . . . . . . . . . . . . . . . . . . . . . . . . . . . . . . 119

4.3.5 Registry structure and variables . . . . . . . . . . . . . . . . . . . . . . . . . 120

4.3.6 Dealing with missing and invalid data . . . . . . . . . . . . . . . . . . . . . 122

4.3.7 Updating the Observatory data . . . . . . . . . . . . . . . . . . . . . . . . 122

4.3.8 Support for reproducible research . . . . . . . . . . . . . . . . . . . . . . 123

4.3.9 Dissemination of the Observatory output f . . . . . . . . . . . . . . . . . . 124

4.3.10 Data sharing and access to the Observatory . . . . . . . . . . . . . . . . . . . 125

4.4 summary statistics . . . . . . . . . . . . . . . . . . . . . . . . . . . . . . . . 125

4.5 Quality of asthma-related events in the GP database . . . . . . . . . . . . . . . . 130

4.5 .1 Background . . . . . . . . . . . . . . . . . . . . . . . . 130

4.5 .2 Methods . . . . . . . . . . . . . . . . . . . . . . . . . . . . . . . 131

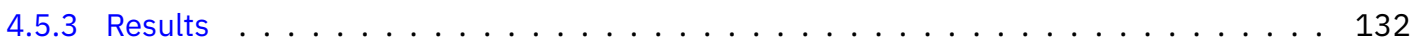

4.5.3.1 Recording of event groups . . . . . . . . . . . . . . . . . . . . . 132

4.5.3.2 Quality of values of lung function events . . . . . . . . . . . . . 132

4.5.4 Interpretation . . . . . . . . . . . . . . . . . . . . . . . . . . . . . . . . . . . 134

4.6 Discussion. . . . . . . . . . . . . . . . . . . . . . . . . . . . . . . 135

4.6.1 Summary of the Observatory design and data quality . . . . . . . . . . . . . . . 135

4.6.2 Strengths and opportunities . . . . . . . . . . . . . . . . . . . . . 136

4.6.3 Challenges and limitations . . . . . . . . . . . . . . . . . . . . . . 136

4.6.3.1 Primary care-based case definition of asthma may exclude some patients . . . 136

4.6.3.2 Inherent limitations of routine data . . . . . . . . . . . . . . 137

4.6.3.3 Traditional methods to define cases and outcomes may need reconsideration . 137

4.6.3.4 Implications of the suboptimal quality of asthma-related routinely collected data on asthma research . . . . . . . . . . . . . . . . . . . 138

4.6.3.5 Primary care coding in the United Kingdom (UK) will change . . . . . . . . . . 139

4.6.3.6 Data security and implications of anonymisation . . . . . . . . . . . . 139

4.6.4 Recommendations for better capture of asthma data . . . . . . . . . . . . . . . . . 140

4.6 .5 Future development . . . . . . . . . . . . . . . . . . . . . . . 141 


\subsection{Introduction}

A main output of this doctoral project was to establish the foundations of the Wales Asthma Observatory (http://www.wales-asthma-observatory.uk/) as a platform for asthma research and surveillance. In this chapter, I describe the Observatory's purpose, context, design and methodology. In addition, due to the known data quality issues in electronic health record (EHR) data [284, 285], I present quality assessment of selected asthma-related variables in routinely collected, primary care data in Wales. I then discuss the strengths and limitations of the Observatory as well as challenges related to asthma and routinely collected data (RCD). I also propose further developments to the Observatory, and suggest recommendations to improve the quality of asthma RCD.

\subsection{Purpose and context}

The Observatory is intended to be used as a research platform for supporting a wide range of cross-sectional and longitudinal asthma studies. It can be used as a surveillance tool, producing disease insights at both local and national levels in Wales. The Observatory includes a regularly updated, cumulative e-cohort (described in the next section), which enables near real-time disease monitoring, tracking, and forecasting. The linkage to area-based deprivation indices enables investigations into the inequalities in asthma care across Wales, as demonstrated in Chapter 5.

The Wales Asthma Observatory is closely aligned with the UK Asthma Observatory (UKAO), a UK-wide platform led by the Asthma UK Centre for Applied Research (AUKCAR) [286]. Data from the four UK countries feed into the UKAO [287]. These currently include person-level and aggregate data about primary and secondary care, community medication dispensing, ambulance services, and national health surveys [43].

In the wider context, the Wales Asthma Observatory will support the AUKCAR's research endeavours towards promoting better asthma control, maximising treatment benefits, and reducing exacerbations and adverse outcomes. 
The Observatory can play a vital role in the endeavours to bridge the gap between evidence and practice. The concept of learning health systems (LHS) has been developed over the past decade to address the crucial need to improve the reuse of health data to make continuous improvements of health care delivery [288, 289]. An LHS is a healthcare system that continuously 'learns' from care delivery. It requires an integrated, seamless cyclical process of collecting data generated as a by-product of care delivery, using these data to create new knowledge, and use the created knowledge to inform decision making and performance of everyday care delivery [290-293]. With plans for piloting an LHS for asthma in Wales, the Wales Asthma Observatory can be a building block in the foundations of such a vital system.

\subsection{Methods}

The Observatory includes a national asthma registry in Wales. I built the registry from routinely collected de-identified health data in the Secure Anonymised Information Linkage (SAIL) Databank. This registry represents a cumulative, nationwide cohort of asthma patients based on the dynamic population of Wales. It is intended to include all previous (remitted or deceased) cases of asthma in the country as well as existing and new incident cases. The cohort was assembled using variety of case definitions, mostly based on the systematic scoping review in Chapter 2, and included essential asthma outcomes such as disease severity, exacerbations, and death due to asthma as well as asthma remission.

Figure 4.1 illustrates the general process of compiling the Observatory. 


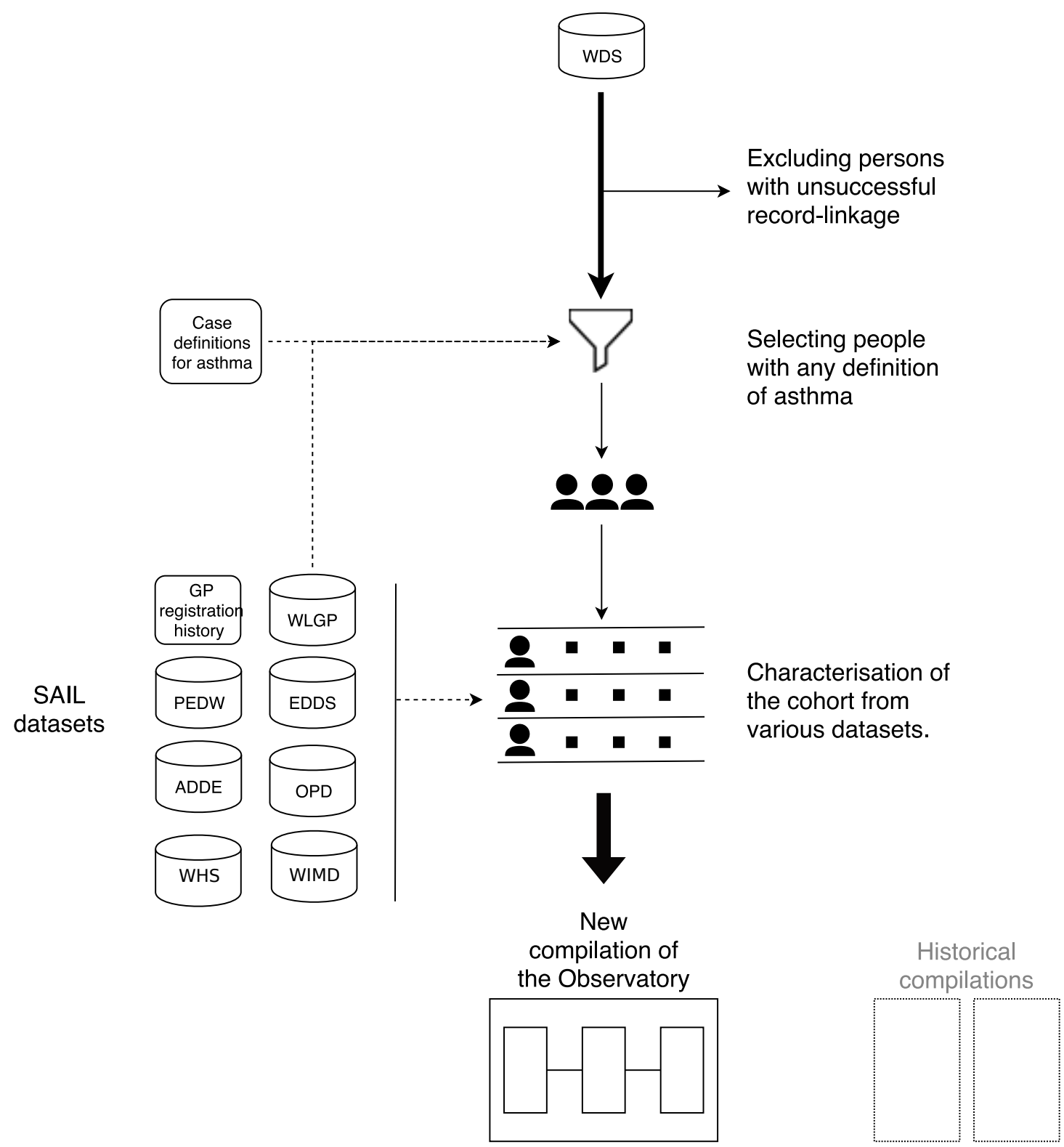

Figure 4.1: Compilation of the Wales Asthma Observatory. WDS: Welsh Demographic Service; OPD: Outpatient Dataset; WLGP: Welsh Longitudinal General Practice; PEDW: Patient Episode Database for Wales; EDDS: Emergency Department Data Set; ADDE: Annual District Death Extract.

In this section, I describe:

- the ethical and information governance requirements of the Observatory development;

- the technical environment in which the Observatory was developed;

- data sources used, including content, coverage, and data quality indicators (in addition, I consider in-depth the quality of recording of selected asthmaspecific Read codes in Section 4.5);

- the source population; 
- case definitions used to identify people with asthma;

- the Observatory's data structure and variables;

- output dissemination plan;

- approaches to support research reproducibility; and

- data sharing and requirements to access the Observatory.

\subsubsection{Ethics and information governance}

The development of the Wales Asthma Observatory was approved by the SAIL Information Governance Review Panel (IGRP), which acts as a data guardian (see Appendix C.2 for the approval letter).

Typically, an application to access SAIL data in a research project starts with a scoping discussion in which a SAIL analyst discusses with the applicants the suitability of their research projects and the required datasets. In the second stage, the applicants are required to complete a detailed application including a research proposal (objectives, methods, data required from SAIL datasets, and time periods and geographical and demographical distributions of data). The application is submitted to the IGRP which assesses the project's suitability and compliance with SAIL's information governance policy, and may raise issues and questions based on the application. After these questions are resolved and the IGRP is satisfied with the project, it sends a letter of approval to the applicants. Following approval, and before the applicants can start accessing SAIL data, they need to demonstrate appropriate information governance knowledge and skills by undertaking an accepted training course. ${ }^{1}$ In addition, users are required to sign the SAIL's Data Access Agreement which details operational and information governance rules. Detailed information about the application process can be found on the SAIL Databank website. ${ }^{2}$

An approval from a research ethics committee was not required for the Observatory development since it only used de-identified data, which was consistent with the current National Health Services (NHS) Health Research Authority guidance [294].

\footnotetext{
${ }^{1}$ Examples of accepted training course are the "Research, GDPR (General Data Protection Regulation) and confidentiality Quiz", which is run by the Medical Research Council, and the "Safe User of Research data Environments (SURE) Training" course, which is run by the Office for National Statistics (ONS), the UK Data Service, and the Administrative Data Research Network (ADRN). More details are regularly published on the SAIL Databank website: https://saildatabank.com/ application-process/following-approval/

${ }^{2}$ https: //saildatabank.com/application-process/
} 


\subsubsection{Logistics and technical environment: the SAIL Data- bank}

I developed the Observatory using data from the SAIL Databank. ${ }^{3}$ The SAIL Databank is a repository of de-identified, linked health datasets in Wales. Data providers include general practices, hospitals, and the Office for National Statistics (ONS). The Observatory was developed within the SAIL Gateway. The SAIL Gateway is a privacy-protecting safe haven for anonymised person-level data [295]. Approved users can access data through a secure remote access system.

Data in the SAIL Databank are organised in database schemas, which are logical structures that group database elements such as tables, views, and procedures. I currently maintain the Observatory data in a dedicated SAIL schema (SAILW0317V), the content of which is described below in Section 4.3.5. These data can be interrogated using the Structured Query Language (SQL). I used the SQL and R programming languages to extract data from SAIL and to organise the Observatory schema.

\subsubsection{Data used in the Observatory}

\subsubsection{Data sources}

I used the following SAIL datasets in the development of the Wales Asthma Observatory. Table 4.1 summarises the coverage and content of these datasets, while Table C.1.1 (in the Appendix) shows their data fields and Table C.1.2 and Figure C.1.1 show the frequency of events and unique patients in each dataset by calendar year. Each of these datasets cover all of Wales, except the Welsh Longitudinal General Practice (WLGP) dataset which currently covers about $80 \%$ of general practices. New extracts of these datasets are regularly received by the SAIL Databank every few months to over a year.

\section{Welsh Demographic Service dataset}

The Welsh Demographic Service (WDS) contains de-identified demographic and administrative data about people who use the NHS in Wales. These data include

\footnotetext{
${ }^{3}$ https://saildatabank.com/
} 


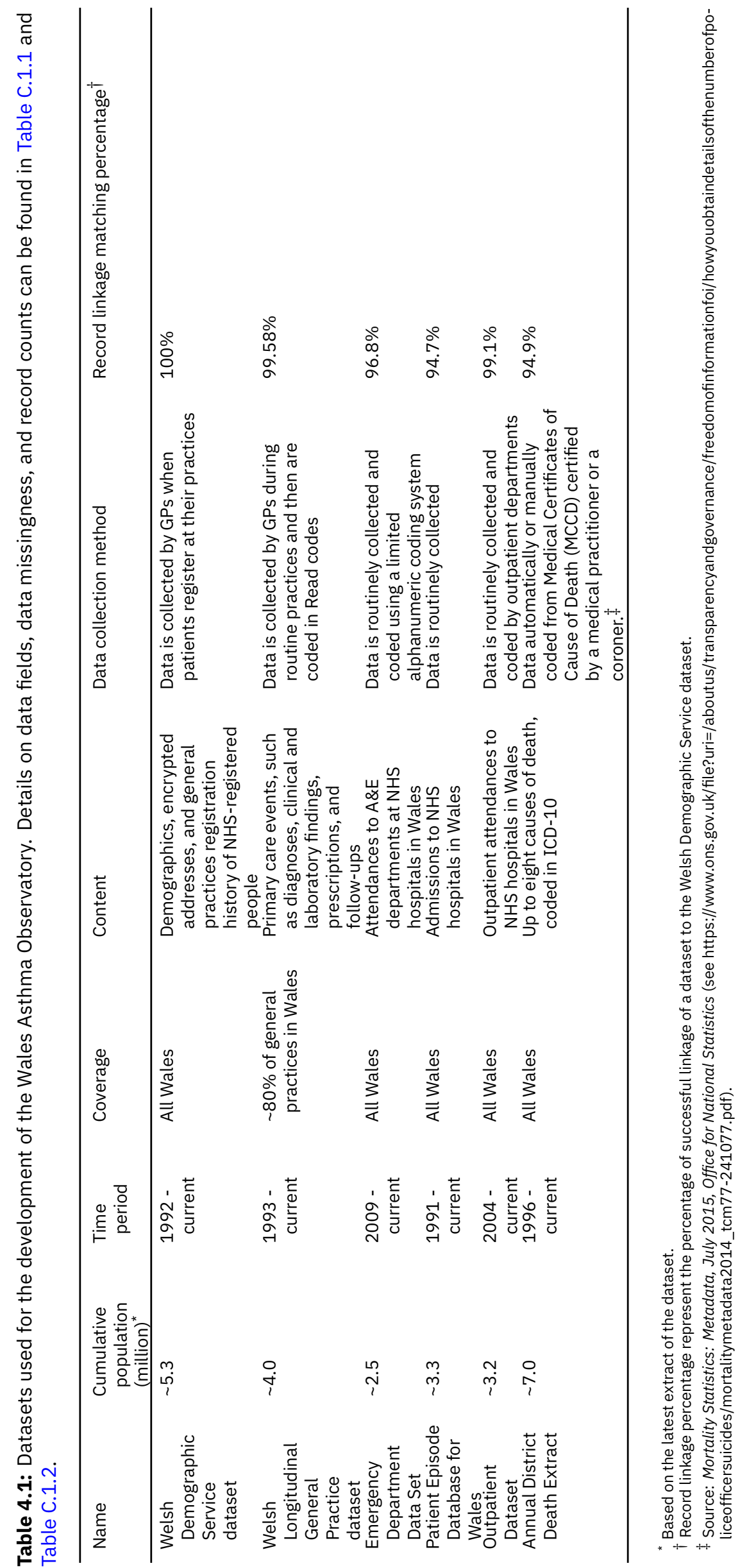


gender, week of birth (defined by date of the first Monday after birth), date of death (for dead people), registration history at general practices, and Lower Layer Super Output Areas (LSOAs) of residence as reported by the individual upon registration. The WDS data has been recorded since 1992 and covers all of Wales. The most recent extract of the WDS in SAIL was created in April 2018 and included data of a cumulative population of $\sim 5.3$ million people.

The NHS is a free to use service. However, the WDS normally does not capture persons who do not use the NHS. Those may include healthy people (particularly young men for whom there is no health screening), disengaged people who do not use the NHS unless in emergencies, some prisoners, and those who used private GPs [296].

\section{Welsh Longitudinal General Practice dataset}

The Welsh Longitudinal General Practice (WLGP) Dataset contains de-identified health care events, such as recorded diagnoses, clinical findings, prescriptions and monitoring as well as other events. Data are collected by GPs during patient visits and are then coded into Read codes by GPs or clinical coders. The most recent extract of the GP dataset was created in April 2018, covering about 80\% of GP surgeries in Wales, and including data for a cumulative population of about four million people. This dataset is of paramount importance for the Observatory since in the UK asthma is mainly treated in primary care [32].

The dataset has $99.58 \%$ matching with the WDS. The WLGP dataset shows increased recording of primary care events since the introduction of the Quality of Outcomes Framework (QOF) in 2004-2005 (see Table C.1.2). However, the lack of standardised coding practices leads to variations and inconsistencies in data recording. In Section 4.5, I consider the quality of recording of selected asthmaspecific Read codes.

\section{Emergency Department Dataset for Wales}

The Emergency Department Data Set (EDDS) was created in 2009 and captures visits to accident $\&$ emergency (A\&E) departments as well as minor injury units (MIUs) in NHS hospitals across Wales. The most recent extract of the EDDS was created in April 2018 and included data on a cumulative population of about 2.5 
million people. Data collected during each A\&E attendance include investigations performed, diagnosis made, anatomical areas involved, treatment provided, as well as other administrative data related to the attendance. Diagnosis is coded using a three-digit alphanumeric code chosen from a list of 83 possible codes representing broad diagnostic categories. In addition, to the primary diagnosis, there are further five positions to record additional or secondary diagnoses. Due to the nature of emergency attendances, recorded diagnoses may be uncertain or unconfirmed. Practices of recording and coding of data vary between the different A\&E departments and MIUs. The EDDS currently receives data on all emergency attendances in Wales. However, in the earlier years (2008-2011), some A\&E departments were not able to submit their data to the EDDS, and therefore data in that period may be incomplete (see Table C.1.2). Therefore, caution should be exercised when using this dataset for epidemiological and research analyses.

\section{Patient Episode Database for Wales}

The Patient Episode Database for Wales (PEDW) dataset was created in 1991 and includes records for all planned and emergency inpatient admissions in addition to day case admissions to all NHS Wales hospitals as well as most admissions of Welsh residents to hospitals in England. The most recent extract of the PEDW dataset was created in May 2018 and included data on a cumulative population of about 3.3 million people. Recorded data are captured during the inpatient episode and includes admission diagnosis, procedures and operations performed, as well as length of stay (LOS), Healthcare Resource Group (HRG), and other administrative data. Admission diagnosis is recorded using ICD-10. In addition to a mandatory primary diagnosis code for a hospital episode, the database allows recording of 113 secondary diagnosis codes. The PEDW is generally considered to be high quality $[297,298]$. However, it is mainly an administrative database which was created as a tool to track hospital financial activity rather than for epidemiological and research purposes. The database also suffers from between-hospital variations in practices of coding admission diagnosis in the available 14 diagnosis positions [297]. Further discussion about the quality of the PEDW is in Section 5.5.4.1. 


\section{Outpatient Dataset}

The Outpatient Dataset (OPD) includes data on outpatient appointments in all NHS hospitals in Wales. These data include attendance date, specialities of care and the treating physician, and site of treatment. In addition, fields for diagnosis and procedures performed in outpatient settings are available, but data on these items are poorly recorded. The most recent extract of the OPD was created in June 2018, including data for about 3.2 million people since 2004 across all of Wales.

\section{Annual District Death Extract}

The Annual District Death Extract (ADDE) dataset is produced and maintained by the ONS and is linked to the SAIL Databank. This dataset contains mortality data since 1996 including up to eight causes of death from Medical Certificates of Cause of Death (MCCD) certified by a medical practitioner or a coroner. ${ }^{4}$ Causes of death are automatically or manually coded in ICD-10 from the MCCD. I used it to ascertain whether death was recorded as due to or related to asthma in deceased asthma patients.

\section{Welsh Health Survey}

The Welsh Health Survey (WHS) has been conducted in 1995, 1998, and annually since 2003 before it ceased in 2015 [53]. It collected self-reported information on a range of health and health-related lifestyles from samples of the population of Wales. The WHS 2013 and 2014 results datasets for respondents aged 16-yearold and above are already linked to the SAIL Databank [271]. Those participants consented to link their responses to their medical records [272]. The only question related to asthma, other than asthma symptoms, asked the participant whether he or she was currently treated for a number of diseases including asthma. I used responses to this question as a case definition for 'self-reported currently treated asthma'. I considered invalid responses as negative responses.

\footnotetext{
${ }^{4}$ Mortality Statistics: Metadata, July 2015, Office for National Statistics (link).
} 


\section{Welsh Index of Multiple Deprivation}

The Welsh Index of Multiple Deprivation (WIMD) is the official tool to assess the level of multiple deprivation in small areas in Wales. It consists of the following weighted eight domains: Income, Employment, Health, Education, Geographical Access to Services, Housing, Physical Environment, and Community Safety. I discuss the WIMD index in depth in Section 5.1.3.2.

\subsubsection{Data anonymisation and linkage}

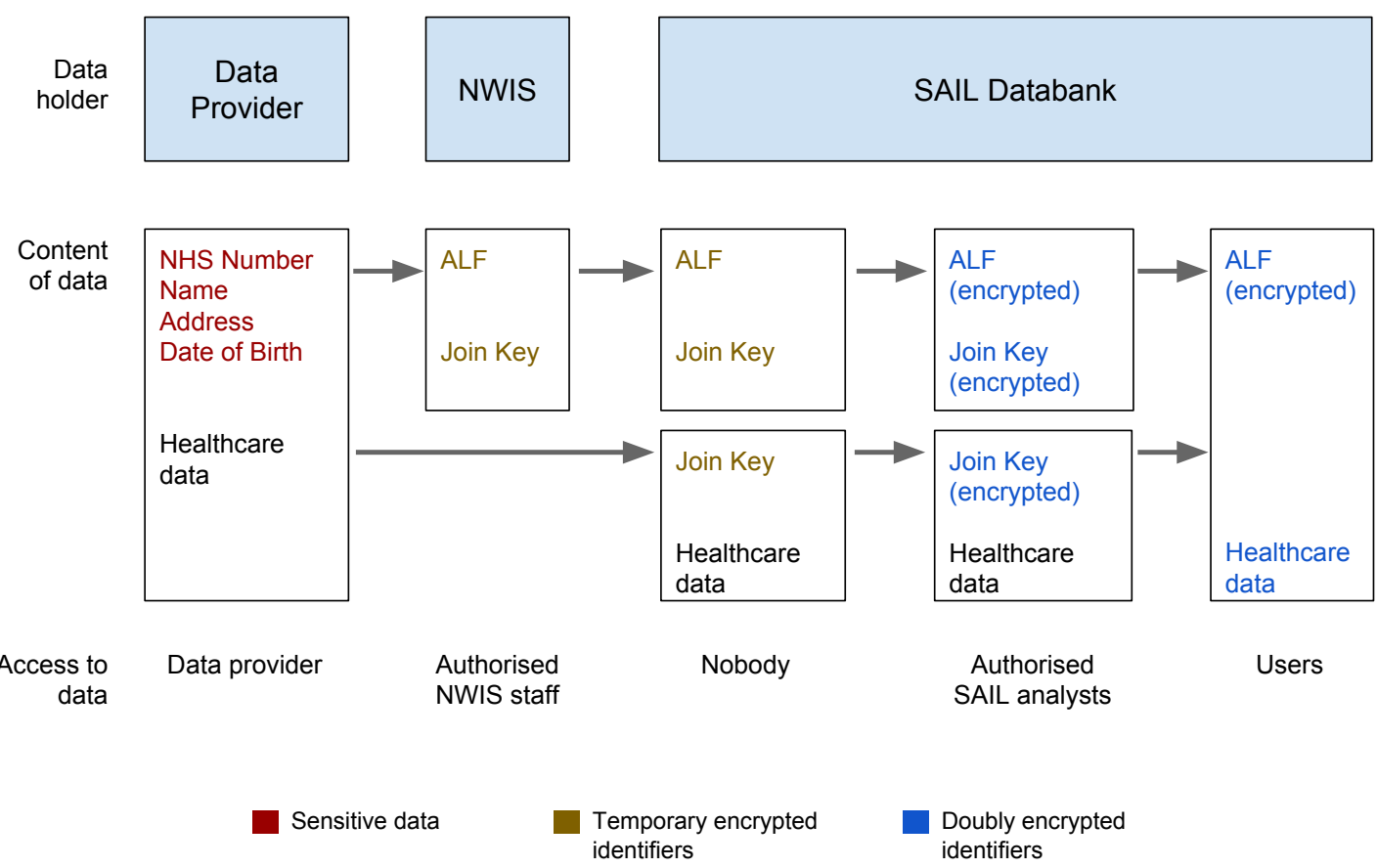

Figure 4.2: Split-file approach to data anonymisation by a trusted third party (adapted from Ford et al. [141]). ALF: Anonymised Linking Field; NWIS: National Health Services Wales Informatics Service; SAIL: Secure Anonymised Information Linkage.

Data anonymisation and linkage on the aforementioned data sources is performed by the National Health Services Wales Informatics Service (NWIS), which acts as a trusted third party (see Figure 4.2) [140, 141]. A data provider splits its data in two files: File 1 which contains demographic data, and File 2 which contains clinical data. The data provider assigns a join key for these two files. File 1 is securely transferred to NWIS. Then, NWIS replaces the demographic data with an Anonymised Linking Field (ALF), which is designed to be a unique identifier across different data providers. NWIS then creates File 3 which contains the ALF as well as the data provider's join key. Then, it sends this File 3 to the SAIL Databank. Separately, the data provider sends File 2 to the SAIL Databank. In the 
Table 4.2: Case definitions used in the Wales Asthma Observatory. Clinical codes used in the GP-databased case definitions are listed in Appendix $\mathrm{E}$

\begin{tabular}{|c|c|}
\hline Case definition & Description \\
\hline Ever-diagnosed asthma & One or more asthma diagnosis codes any time before a given date. \\
\hline $\begin{array}{l}\text { Ever-diagnosed and currently } \\
\text { treated asthma }\end{array}$ & $\begin{array}{l}\text { One or more asthma diagnosis codes any time before a given date, and one or } \\
\text { more asthma prescription codes in the last } 12 \text { months; this case definition } \\
\text { corresponded to the Quality of Outcomes Framework indicator AST001 } \\
\text { without considering exceptions. }\end{array}$ \\
\hline Currently treated asthma & One or more asthma prescription codes in the last 12 months. \\
\hline Ever-treated asthma & One or more asthma prescription codes any time before a given date. \\
\hline $\begin{array}{l}\text { Self-reported currently treated } \\
\text { asthma }\end{array}$ & $\begin{array}{l}\text { Based on the Welsh Health Surveys; only available for a small number of } \\
\text { patients. The survey question on whether the participant was 'currently being } \\
\text { treated for asthma' did not specify a time frame. However, based on my own } \\
\text { analysis (see Appendix B.1) I used a period of } 26 \text { months ending with the end } \\
\text { of the survey year. }\end{array}$ \\
\hline
\end{tabular}

SAIL Databank, File 2 and File 3 are re-joined using the join key to produce a de-identified dataset, containing encrypted ALF and clinical data, which can be linked to datasets from other data providers.

\subsubsection{Eligibility criteria}

The Observatory aims to cover the entire dynamic population of Wales. Therefore, I defined the source population as all individuals for whom records exist in the WDS dataset.

Since the Observatory aims to include all potential and confirmed asthma patients in Wales, I included in the Observatory people who met any of a set of case definitions for asthma, from the most inclusive to the most specific ones. Table 4.2 lists case definitions currently included in the Observatory. Each person in the Observatory satisfies at least one case definition ever.

The use of these multiple case definitions of asthma allows capturing most patients, ranging from those with uncertain diagnosis to those with the more strictly defined currently-treated asthma. This approach facilitates studying diverse subgroups of asthma patients differing in diagnosis certainty and disease activity. It also provides flexibility for researchers to choose the appropriate case definitions for their studies. The use of broad case definitions to capture patients with any indication of asthma was previously adopted by a similar project in the United States (US), the Population-Based Effectiveness in Asthma and Lung Diseases (PEAL) Network [137]. 
Each of the used case definition has its own meaning and uses in research. Nonetheless, I considered the 'ever GP-diagnosed currently-treated asthma' the main case definition of asthma in the Observatory as it reflects the active disease, which is often an essential criterion in many asthma studies.

The case definitions in Table 4.2 can be represented as state variables, i.e. assessed over time periods. I assessed the case definitions over patient-specific periods (Figure 4.3) rather than fixed periods (e.g., start and end of year) for all patients. This allowed an accurate start and end of disease states. For example, the definition of "currently treated asthma" ("there was at least one asthma prescription in the last 12 months") is true for any given date within the state period (see Figure 4.3).

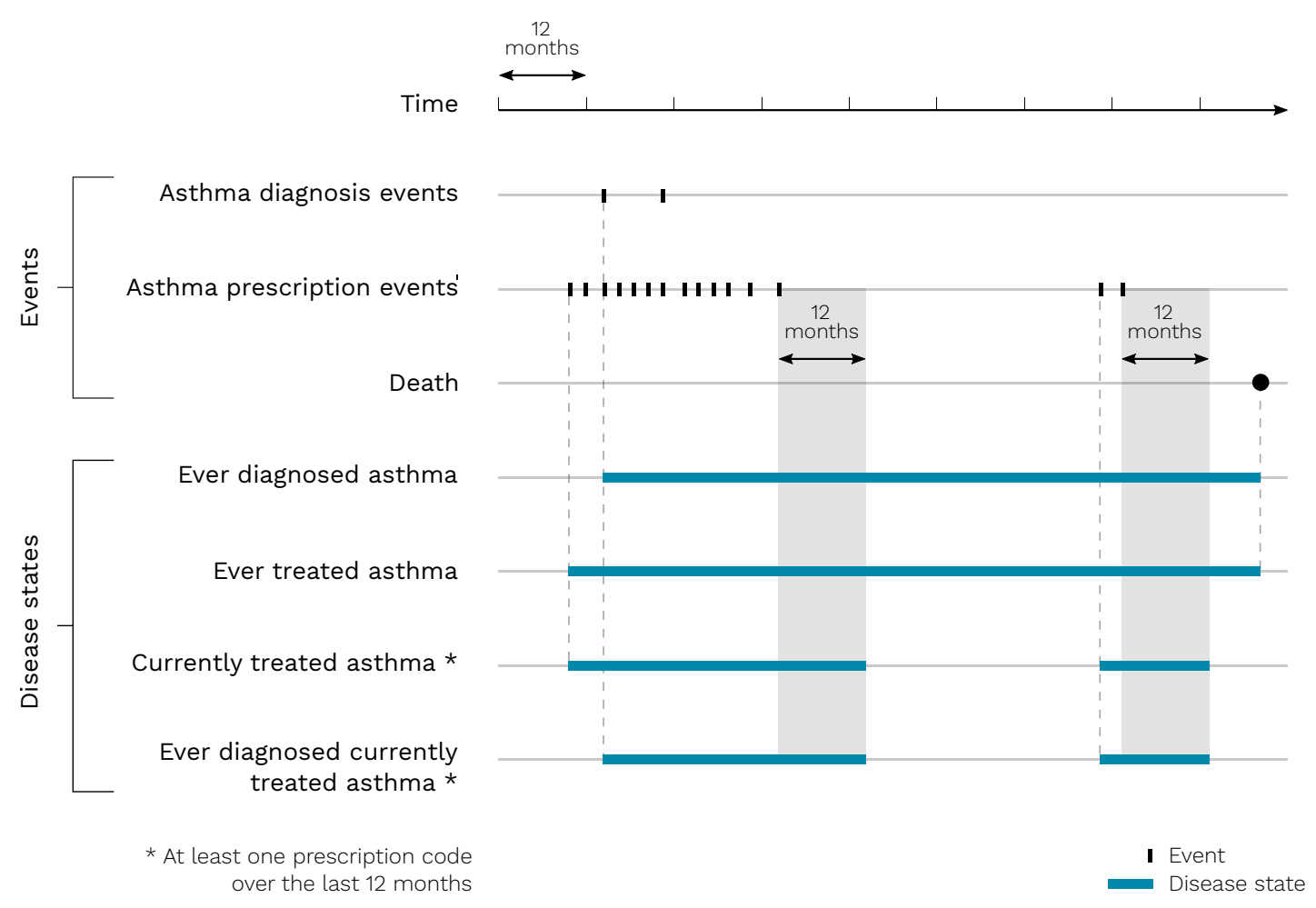

Figure 4.3: Examples of the assessment of case definitions as disease states (state variables) in the Wales Asthma Observatory. The diagram shows asthma diagnosis and prescription events for an imaginary patient in addition to four case definitions assessed over patient-specific time intervals based on those events.

\subsubsection{Registry structure and variables}

Data for each person in the Observatory currently include demographics, which asthma case definitions ("asthma states") were satisfied over which periods, as well as asthma-related outcomes and variables. These outcomes and variables 
include asthma treatment step, disease severity, and exacerbations, and periods of follow-up based on GP registration history (see Table 4.3). Metadata about the Observatory compilations and dataset versions used in each compilation are stored in a separate database table (WAO_data_sources_versions).

Table 4.3: Data tables in the asthma registry

\begin{tabular}{|c|c|c|}
\hline Name & Description & Fields \\
\hline Demographics & Basic demographic data & $\begin{array}{l}\text { ALF } \\
\text { WOB } \\
\text { DOD }\end{array}$ \\
\hline $\begin{array}{l}\text { Patient } \\
\text { follow-up }\end{array}$ & $\begin{array}{l}\text { Periods of follow-up for patients based on GP registrations from } \\
\text { the WDS dataset. Those GP registration records were filtered } \\
\text { using an in-house algorithm (FNC.CLEAN_GP_REG, developed } \\
\text { by the SAIL Analytical Services Team) that excludes periods } \\
\text { over which some GP practices, despite being participating in } \\
\text { SAIL, did not send reasonably adequate amounts of data to } \\
\text { SAIL. }\end{array}$ & $\begin{array}{l}\text { ALF } \\
\text { start date } \\
\text { end date }\end{array}$ \\
\hline Asthma State & $\begin{array}{l}\text { Whether an individual satisfied a case definition of asthma over } \\
\text { a specific period or a calendar year. Examples of asthma case } \\
\text { definitions include "ever diagnosed asthma" and "currently } \\
\text { treated asthma" (see Table 4.2). }\end{array}$ & $\begin{array}{l}\text { ALF } \\
\text { case definition/state } \\
\text { start date } \\
\text { end date }\end{array}$ \\
\hline Treatment Step & $\begin{array}{l}\text { Treatment step from } 1 \text { to } 5 \text { based on the } 2016 \text { BTS/SIGN } \\
\text { asthma guidelines, in addition to 'SABA as needed', over a } \\
\text { period of up to six months. }\end{array}$ & $\begin{array}{l}\text { ALF } \\
\text { start date } \\
\text { start end } \\
\text { treatment step }\end{array}$ \\
\hline $\begin{array}{l}\text { Asthma } \\
\text { Severity }\end{array}$ & $\begin{array}{l}\text { Disease severity classified as intermittent, mild, moderate, or } \\
\text { severe based on prescriptions [299] over a period of up to six } \\
\text { months. Intermittent = SABA as needed; mild: low-dose ICS or } \\
\text { other low-intensity therapies; moderate: low/moderate-dose } \\
\text { ICS with LABA (or other additional therapies); severe: } \\
\text { high-intensity therapies (high-dose ICS with LABA, oral } \\
\text { corticosteroids, or other additional therapies. }\end{array}$ & $\begin{array}{l}\text { ALF } \\
\text { start date } \\
\text { end date } \\
\text { asthma severity }\end{array}$ \\
\hline $\begin{array}{l}\text { Asthma } \\
\text { Exacerbation }\end{array}$ & $\begin{array}{l}\text { Records for asthma exacerbation defined based on primary and } \\
\text { secondary care utilisation. An asthma exacerbation is defined } \\
\text { by short course of oral corticosteroids, asthma-related } \\
\text { emergency admission, or asthma-related hospitalisation, with } \\
\text { periods less than } 4 \text { weeks apart being merged into single } \\
\text { exacerbation period. }\end{array}$ & $\begin{array}{l}\text { ALF } \\
\text { start date } \\
\text { end date }\end{array}$ \\
\hline $\begin{array}{l}\text { Asthma-related } \\
\text { death }\end{array}$ & $\begin{array}{l}\text { Record of death in which asthma was an underlying cause of } \\
\text { death from the ONS's ADDE dataset. }\end{array}$ & $\begin{array}{l}\text { ALF } \\
\text { DOD } \\
\text { position of asthma code in death record }\end{array}$ \\
\hline $\begin{array}{l}\text { Data source } \\
\text { versions }\end{array}$ & $\begin{array}{l}\text { Shows the available compilations of the Observatory and the } \\
\text { names and versions/extracts of SAIL datasets used in each } \\
\text { compilation (table name: WAO_data_sources_versions). }\end{array}$ & $\begin{array}{l}\text { Observatory compilation number and } \\
\text { date (e.g., WAO_2_20181005) Source } \\
\text { data table name and version (e.g., } \\
\text { SAILWLGPV.ALF_GP_EVENTS_ } \\
\text { CLEANSED_20180820) }\end{array}$ \\
\hline
\end{tabular}

ALF: Anonymous Linking Field; BTS: British Thoracic Society; DOD: date of death; GP: general practitioner; LABA: long-acting beta adrenoceptor agonist; SABA: short acting beta agonist; SIGN: Scottish Intercollegiate Guidelines Network; WOB: week of birth.

Additional data that could be added in the future include:

- Timeline of laboratory test results such as total immunoglobulin E (IgE), blood eosinophil count, as well as lung function measurements.

- Asthma phenotypes (e.g., eosinophilic asthma, adult-onset asthma, asthma with fixed airflow limitation, and poorly steroid-responsive asthma); pheno- 
types can be identified using clustering methods guided by the relevant literature $[4,5,7]$.

- Environmental data including air pollution, housing quality, calculated for small areas and linked through the Residential Anonymous Linking Fields (RALF) [300].

- Patient reported outcome measures (PROMs), such as Asthma Control Questionnaire (ACQ) responses.

\subsubsection{Dealing with missing and invalid data}

In the Observatory compilation script, I excluded persons with an invalid ALF (i.e. had a value of NULL). In addition, the Observatory includes periods of follow-up based on GP registration history, which can be used for censoring in time-to-event analyses.

Non-existence of a health event in an event-based dataset, such as most SAIL datasets, does not imply non-occurrence of that event; it may rather due to nonrecording of such an event in categorical codes and may have been recorded in narrative fields not available within SAIL. The nature of such event-based datasets means that it was impossible to identify such unrecorded events. However, in the Observatory development, the case definitions were based on events that are assumed to be well recorded.

\subsubsection{Updating the Observatory data}

The Observatory data are based on the SAIL Databank. Data in the SAIL Databank are not collected in real-time but are rather collected and updated with a variable lag time ranging from few months to over a year. Subsequently, the Observatory data are not real-time, but are intended to be updated following updates to any of the source SAIL datasets.

The updating process can be performed using the same data extraction and programming script used in the initial compilation of the dynamic cohort. For each update, names of newer dataset extracts should be used as input in that script. This process will create a new version of the Observatory, including an updated patient cohort and variables (see Figure 4.1). 


\subsubsection{Support for reproducible research}

Reproducibility is important for epidemiological studies [157, 218]. It requires full and clear documentation of the methods used, including algorithms to define health variables and extract data as well as programming code used for analysis.

For studies using routine data, certain considerations are needed to address reproducibility. In such studies, interpretation of findings largely depends on the clinical codes and data extraction methods used to identify patients and define outcomes. Therefore, these codes should be accessible and reusable by the wider research community in order to support transparency, reproducibility, and comparability of findings [102, 157, 301].

A key part of an EHR-based disease registry is a library of clinical code lists used by studies. In the Wales Asthma Observatory, I developed a technical platform where clinical code lists could be collaboratively maintained, shared, and reused by researchers and analysts (see Figure C.3.1). Interrogation of routine databases often involves repetitive programming tasks, such as manually constructing and modifying complex database queries. These tasks generally require significant time from an experienced data analyst. The above-mentioned platform enables users to collaboratively develop and reuse study-specific data extraction procedures. To minimise the need to write manual and repetitive queries, the platform automates significant parts of data extraction from the Observatory and the GP dataset. It automatically generates and executes the required SQL queries. Automating data extraction is aimed to support scalability, save significant time by analysts, and reduce human error. In addition, the platform has a graphical user interface which allows researchers with no programming skills to develop code to subsequently interrogate the data.

At the time of writing, the platform is maintained inside the SAIL Gateway at the address http://gpact.chi.swan.ac.uk. However, requirements are being discussed with the SAIL Databank team to make the query building platform available for the public outside the SAIL Gateway. A similar public repository of clinical codes is being maintained by the University of Manchester [216]. ${ }^{5}$ Compared to that repository, the platform that I developed will enable public sharing not only of clinical code lists, but also of data extraction procedures that are used inside

\footnotetext{
${ }^{5}$ http://ClinicalCodes.org
} 
the SAIL Gateway, but not the underlying patient level data. When the platform will be publicly available, each code set and data extraction procedure will have a permanent citable Internet address. This platform is intended to support research transparency and reproducibility as stated in the Strengthening the Reporting of Observational Studies in Epidemiology (STROBE) [153] and the REporting of studies Conducted using Observational Routinely collected health Data (RECORD) statements [157].

Another threat to reproducibility is that the data sources used by the asthma registry are regularly updated. A new data extract (i.e. version) usually contains more recent data captured since the previous extract. However, it is possible that a new data extract also includes additional historical data not sent before from data providers to SAIL. Reproducibility of analyses is not guaranteed if repeated using different extracts of data. To minimise this limitation, the registry data tables are versioned based on the updates of the source dataset. This allows epidemiological estimates to be reproduced using the same data used in the previous calculations.

\subsubsection{Dissemination of the Observatory output}

The Observatory can be used to perform epidemiological analyses on a regular basis or on demand. Examples of these analyses include basic epidemiological parameters for asthma such as disease incidence, life-time and annual disease prevalences, incidence of exacerbations, emergency visits, hospitalisation, as well as disease burden. In addition, prevalence of asthma phenotypes and temporal profiles of disease activity can be explored.

Consumers of the Observatory output are intended to include several user groups such as service planners and managers, policy makers, scientists and academics, health care professionals, asthma patients, and other members of the public. It is therefore important for the published output to consider the needs of this wide spectrum of users. The Observatory output will be published on a dedicated public website using appropriate format and state-of-the-art visualisation techniques. Users will be able to subscribe with newsletters and alerts about output of their interests. Dissemination will also utilise the infrastructure of AUKCAR as well as Asthma UK's dissemination channels, allowing wider reach to people with asthma. 


\subsubsection{Data sharing and access to the Observatory}

Since the Observatory is based on the SAIL Databank, researchers who wish to access the Observatory need to seek approval from the SAIL's IGRP (see 4.3.1). The Observatory can be queried by approved SAIL projects and can be linked to internal SAIL data (e.g., about other health conditions) or external data (e.g., for a bespoke cohort) that are linked into SAIL.

\subsection{Summary statistics}

This section presents statistics from the Observatory describing the database records of asthma case definitions, incidence and prevalence of selected case definitions, and asthma-related health care utilisation.

Table 4.4 shows the all-time number of records and unique patients for each of the case definitions defined in Table 4.2 based the most recent versions of SAIL datasets. The current version of the Observatory data includes a cumulative cohort of 541,159 patients with ever diagnosed asthma for whom there are $6,456,786.3$ years of follow-up data available in the primary care dataset (WLGP, 2018-08-20 extract). 
Table 4.4: All-time number of records and unique patients for each of the case definitions. These records belong to all patients in the WDS, WLGP, and WHS datasets, including living and deceased patients.

\begin{tabular}{lccc}
\hline Case definition & Number of records & $\begin{array}{l}\text { Number of unique } \\
\text { patients }\end{array}$ & $\begin{array}{c}\text { Patient-years of } \\
\text { follow-up in SAIL* }\end{array}$ \\
\hline $\begin{array}{l}\text { Ever-diagnosed asthma } \\
\text { Ever-treated asthma }\end{array}$ & 541,159 & 541,159 & $6,456,786.3$ \\
Currently treated asthma & $1,174,389$ & $1,174,389$ & $11,531,260.3$ \\
$\begin{array}{l}\text { Ever-diagnosed, currently treated } \\
\text { asthma }\end{array}$ & $1,220,979$ & $1,175,621$ & $5,518,568.2$ \\
Self-reported currently treated & $1,046,819$ & 476,546 & $3,594,309.5$ \\
asthma (based on the WHS) & 1,199 & 1,173 & \\
\hline
\end{tabular}

GP = general practitioner; WDS = Welsh Demographic Service (WDS); WHS = Welsh Health Survey (WHS); WLGP = Welsh Longitudinal General Practice (WLGP). * Only available for case definitions based on the GP dataset (WLGP).

Using these records, Table 4.5 shows the period prevalences of asthma case definitions in the calendar year 2017 at national and health board levels.

In addition, cumulative incidences and period prevalences of the asthma case definitions between 2000 and 2017 are shown in Figure 4.4. For cumulative incidence of each of the case definitions in each year, the denominator was the number of people with continuous registration at GP practices and complete data in the WLGP (extract 2018-08-20) in the respective year, excluding people who already satisfied the case definition at the beginning of the year. The numerator included people in the denominator who satisfied the case definition during the respective year for the first time in their life. For period prevalence, I defined the denominator was the same used for incidence without excluding people with the condition at the beginning of the year. The numerator was the number of people in the denominator who satisfied the case definition for any period in that year.

Prevalences of lifetime and current asthma showed a steady although slow increase between 2000 and 2017, except for the prevalence of lifetime asthma treatment which showed a steeper increase from $15 \%$ to $30 \%$. However, incidences of asthma diagnosis asthma treatment showed an overall decreasing trend, starting in 2000 at $7.4 \%$ and $18.8 \%$ for diagnosis and treatment, respectively, with a slight 


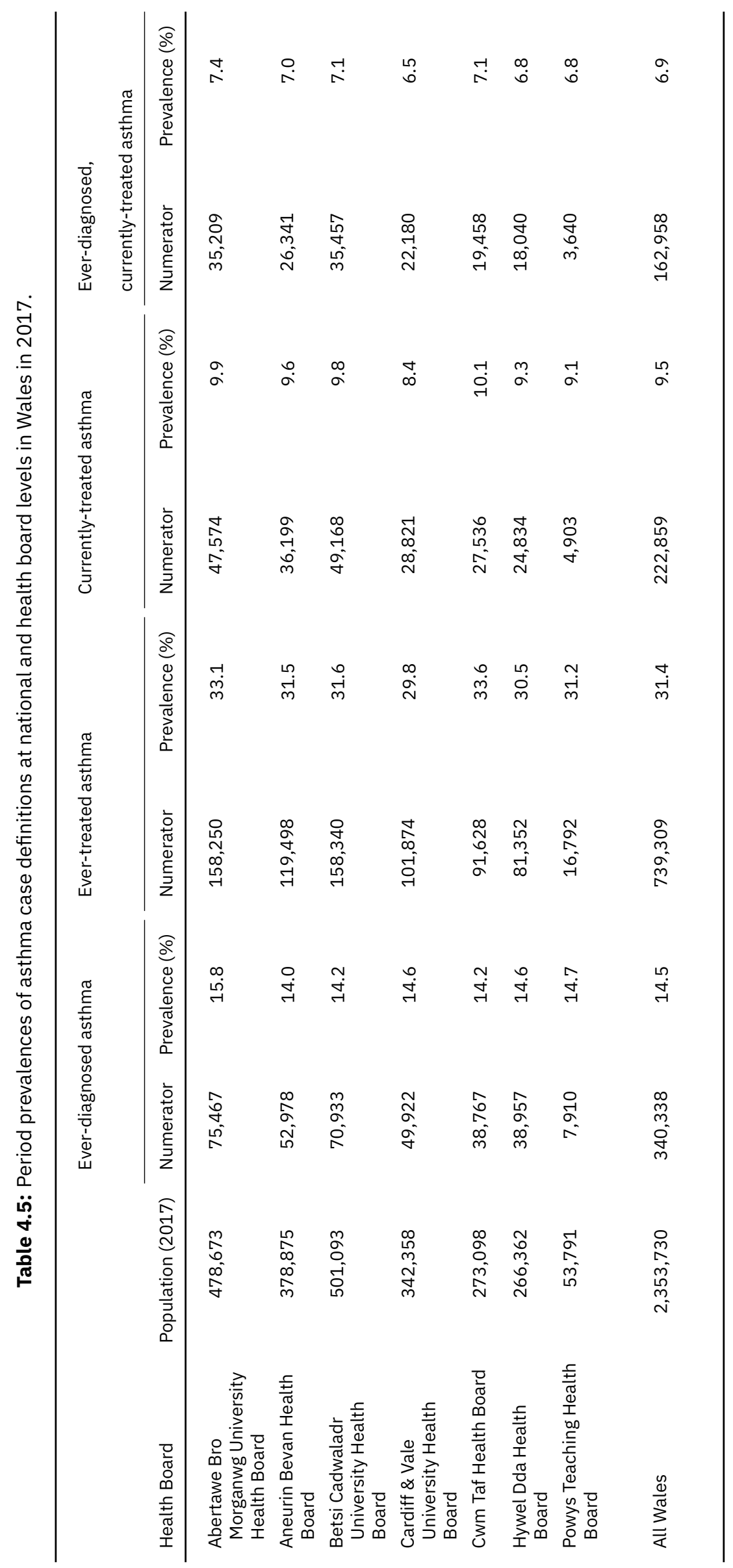


increase between up to 2002, before declining significantly between 2003 and 2006-2007 (which might be, in part, due to a change in the recording of asthma diagnosis during that period), followed by stabilisation at 3.1-3.4\% and 15.0-16.6\% for the incidence of diagnosis and treatment, respectively.

Figure 4.5 shows statistics about asthma-related primary and secondary care utilisation by patients with GP diagnosed asthma patients who received at least one asthma prescription in 2017. The figure shows percentages of those patients who had specific asthma-related events including specific asthma prescriptions, A\&E events, and hospitalisations in the same year (2017).
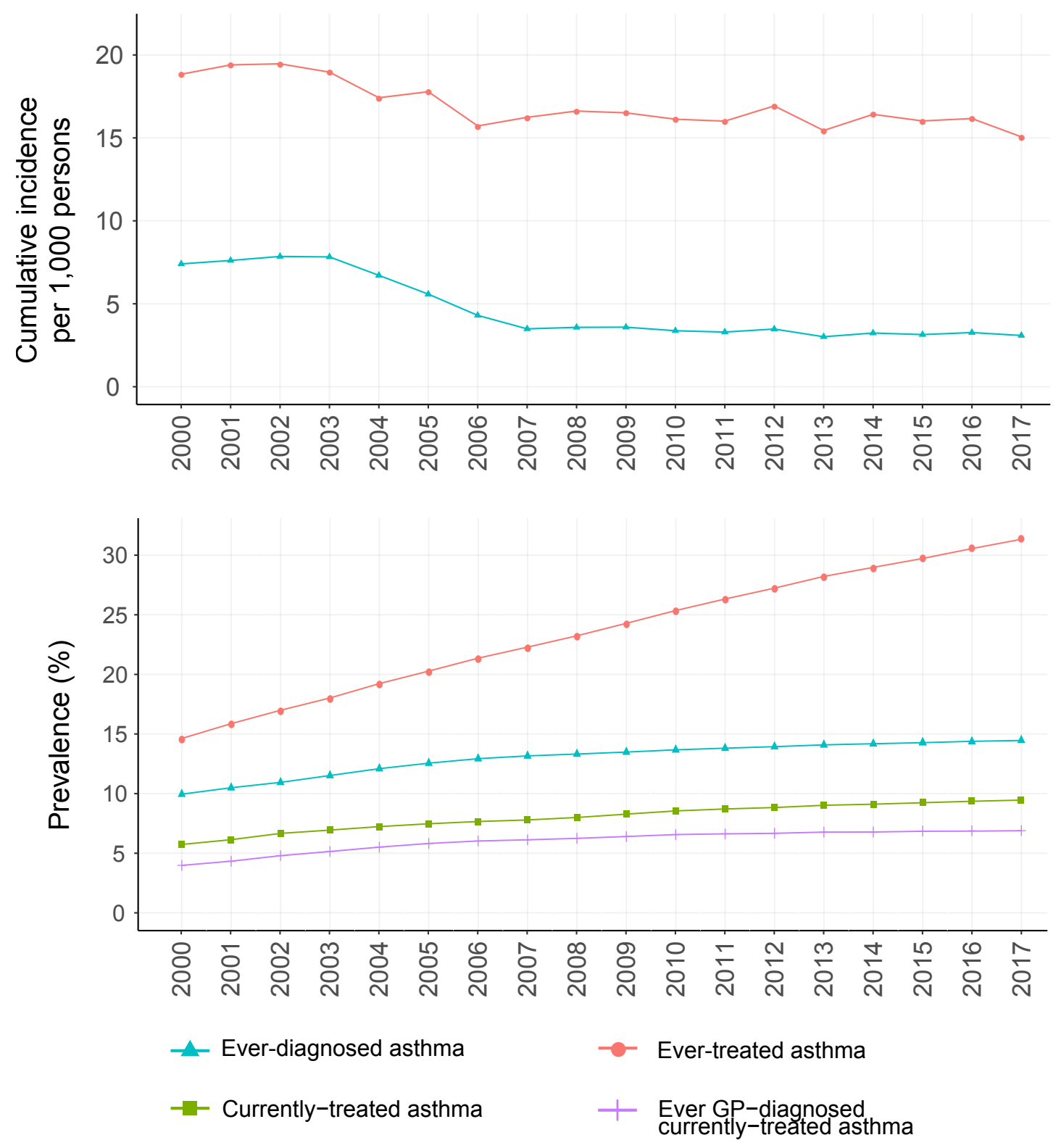

Figure 4.4: Cumulative incidences and period prevalences of asthma case definitions in Wales between 2000 and 2017. 

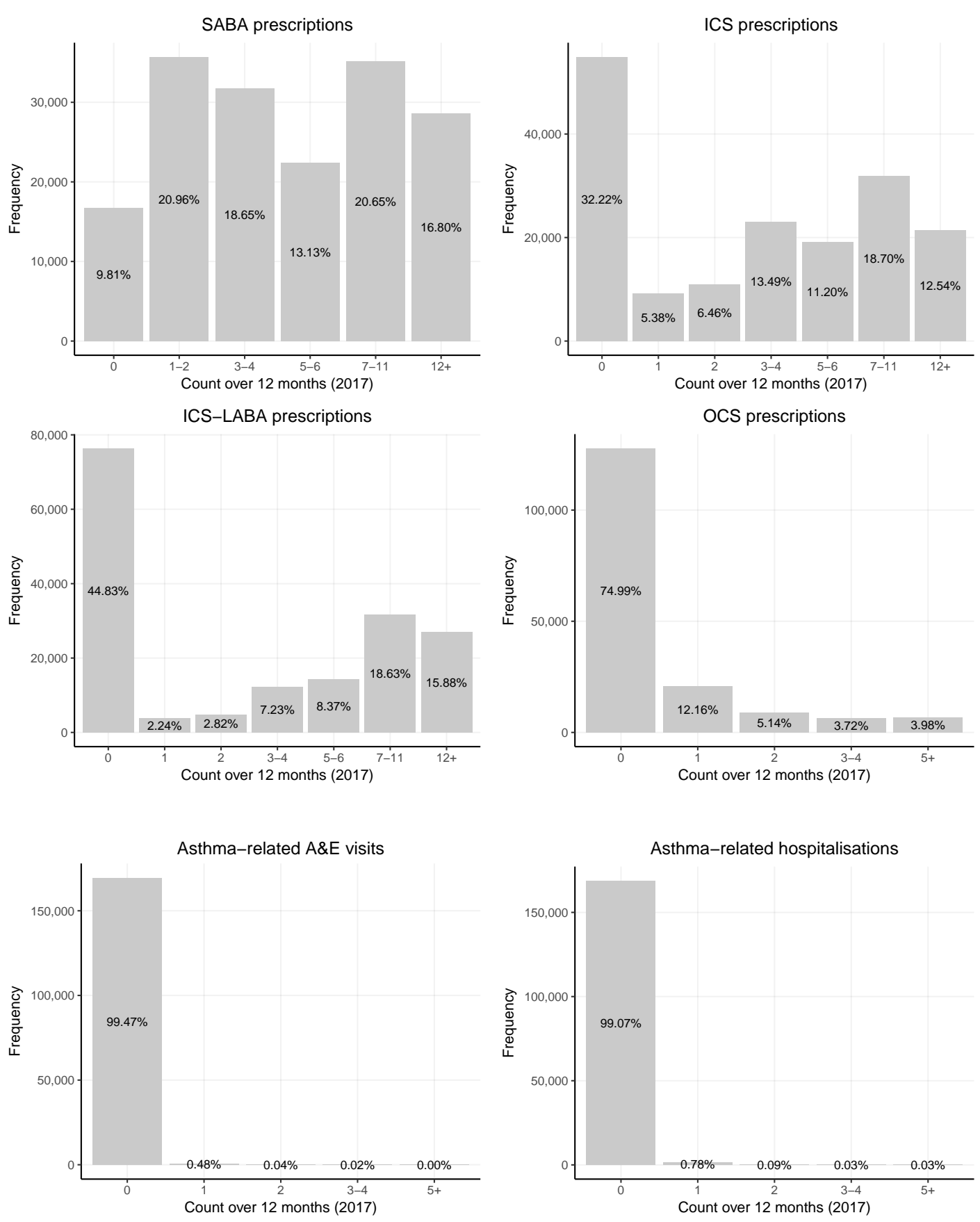

Figure 4.5: Distributions of asthma-related primary and secondary care utilisation events by patients with GP diagnosed asthma patients who received at least one asthma prescription in 2017.

Lastly, in the most recent extraction of the ADDE dataset, there were 3,180 people for which asthma was recorded among the conditions related to death. Among those people, 1,273 (40.0\%) had no GP-recorded asthma diagnosis, 859 (27.0\%) had no GP-recorded asthma prescriptions ever, and 823 (25.9\%) had neither diagnosis nor prescription records for asthma. 


\subsection{Quality of asthma-related events in the GP database}

\subsubsection{Background}

Health care data are mainly event-based and are mostly captured in a narrative format [302]. Clinical coding is often motivated by clinical and administrative purposes. However, even when perfect, clinical coding often involves information loss since coding schemes do not cover all aspects of health and health care. In practice, both electronic capture and coding of patient data are suboptimal [285]. Examples of barriers include cost, training needs, inefficient design and negative attitudes to EHR systems as well as lengthy lists of codes to choose from [233, 302]. Subsequently, many healthcare events may not be captured or only partially recorded or coded at the point of care. Since only coded data are usually routinely collected, those healthcare events would be missing in central repositories.

For most primary care events, minimal event attributes include code and date. This also applies to events involving measurement of health parameters such as body mass index (BMI) and blood eosinophil count. For such measurement events, a GP can use an informative code (e.g., 42K1. : Eosinophil count normal) to describe the measurement. Alternatively, he or she can use a declarative code (e.g., $42 \mathrm{~K}$. . : Eosinophil count) with the measurement value recorded in a separate field.

The incidence of non-recording may differ between different types of events; for example, while some primary care events are known to be well-recorded (e.g., diagnosis codes that are required for the QOF indicators), others are less frequently coded, partially coded, or are even completely not coded [284]. Regarding events for which numerical values are expected besides the codes, it is possible to directly calculate the frequency of missing or invalid values for the recorded codes. However, where the code itself is absent, it is impossible to ascertain, at the individual level, whether the event did not happen at all or it was simply not coded. Nonetheless, some insights into the levels of missingness of these events could be potentially still obtained by calculating their recording frequency in the asthma 
population. This is particularly feasible for essential health care events that are required by national guidelines (e.g., lung function testing to confirm diagnosis), which are expected to be recorded for large proportions of patients.

To investigate the quality of recording of asthma-related events in the GP dataset of the SAIL Databank, I examined the percentage frequency of recording for selected asthma-related event codes and event values for a sub-cohort of the asthma population.

\subsubsection{Methods}

I included in the sub-cohort all patients who had an asthma diagnosis Read code between 1-1-2006 and 1-1-2012, with no "asthma resolved" in the four years following the diagnosis date. I did not included patients diagnosed before 1-1-2016 (one and nine months after the introduction of the QOF in April 2004) to ensure adequate level of recording of GP data. The Read codes used for patient inclusion are listed in Table C.4.1.

I chose the following six groups of asthma-related events: Triggers of asthma in the patient, disease severity, steps undertaken by GPs to manage asthma control, spirometry tests to assess lung function, serum eosinophil count, and level of total IgE. The Read code definitions of these events are shown in Table C.4.3. For each event group, I calculated the proportion of patients in the sub-cohort who had at least one code over their follow-up period. For the first three groups, the follow-up period was four years from the diagnosis date. This was an appropriate period to allow equal follow-up for all patients in the cohort within the date range of the available GP data at the time of data extraction. For the events of lung function, eosinophil count, and total IgE, the follow-up period was similar except that it also included three months before the diagnosis date. This was because these diagnostic procedures could have been performed before the diagnosis was confirmed and recorded.

I also examined the recorded values for 54 Read codes for lung function testing (see Table 4.7). I calculated the percentage of missing values and inspected the distributions of the recorded values. Usually, event values only include numerical data. For values represented as percentage, there was no percent sign (\%) and 
therefore they were not directly distinguishable from other data formats. Nonetheless, the intended values can be often easily inferred from the event description.

\subsubsection{Results}

The sub-cohort included 127,303 asthma patients, 55.1\% of whom were females.

\subsubsection{Recording of event groups}

Table 4.6 shows percentages of patients with at least one recording of key asthmarelated GP events over the follow-up period. $81.6 \%$ of the patients had at least one event code for lung function testing. In addition, $52.5 \%$ of the patients had at least one event code for serum eosinophil count. However, for the other event groups, the proportion were smaller: $9.7 \%$ for asthma control steps, $4.8 \%$ for asthma triggers, $1.8 \%$ for asthma severity, and $1.2 \%$ for total serum IgE.

Table 4.6: Percentages of patients with at least one recording of key asthma-related GP events over specified periods from diagnosis.

\begin{tabular}{ll}
\hline GP Event & \% of patients $(95 \%$ confidence interval) \\
\hline Asthma triggers $^{*}$ & $4.8(4.7,4.9)$ \\
Asthma severity $^{*}$ & $1.8(1.8,1.9)$ \\
Asthma control steps $^{*}$ & $9.7(9.6,9.9)$ \\
Lung function test $^{* *}$ & $81.6(81.4,81.8)$ \\
Serum eosinophil count $^{* *}$ & $52.5(52.2,52.7)$ \\
Serum total IgE $^{* *}$ & $1.2(1.1,1.2)$ \\
\hline
\end{tabular}

$\mathrm{GP}=$ general practitioner; IgE $=$ immunoglobulin $\mathrm{E}$.

* In the four years after diagnosis date.

${ }^{\star *}$ From three months before to four years after diagnosis date.

\subsubsection{Quality of values of lung function events}

For the recorded lung function events, values were missing in $11.4 \%$ of these events. The lowest proportion of missingness was for predicted peak expiratory flow rate (PEFR) using the 13826 European Standard ${ }^{6}$ (0.3\% [95\% confidence interval (CI): $0.2-0.4 \%]$ ), while the highest proportion was for percentage of predicted vital capacity (93.5\% [81.1-98.3\%]). Results for the rest of the codes are shown in Table 4.7).

Figure C.5.1 includes visualisation of lung function event values using beanplots [304] which show distribution density and makes it easy to spot anomalies in data

\footnotetext{
${ }^{6}$ This standard specifies the requirements for peak expiratory flow meters which is designed to be used to evaluate lung function in humans with spontaneous breathing [303].
} 
Table 4.7: Percentages of missing values for lung function event codes.

\begin{tabular}{|c|c|c|c|c|c|}
\hline \multirow[t]{2}{*}{$\begin{array}{l}\text { Event } \\
\text { code }\end{array}$} & \multirow[t]{2}{*}{ Event description } & \multirow[t]{2}{*}{$\begin{array}{l}\text { Number of } \\
\text { all events }\end{array}$} & \multirow{2}{*}{$\begin{array}{l}\text { Number of } \\
\text { events with } \\
\text { missing values }\end{array}$} & \multicolumn{2}{|c|}{$\begin{array}{l}\text { Percentage of events } \\
\text { with missing values }\end{array}$} \\
\hline & & & & $\begin{array}{l}\text { Percent- } \\
\text { age }\end{array}$ & $\begin{array}{l}95 \% \\
\text { confidence } \\
\text { interval }\end{array}$ \\
\hline 3395. & $\begin{array}{l}\text { Peak exp. flow rate: } \\
\text { PEFR/PFR }\end{array}$ & 320,621 & 5,869 & 1.8 & $(1.8-1.9)$ \\
\hline 339A. & PFR - before bronchodilation & 45,657 & 750 & 1.6 & $(1.5-1.8)$ \\
\hline $339 \mathrm{H}$. & Predicted peak flow & 35,741 & 127 & 0.4 & $(0.3-0.4)$ \\
\hline 339p. & $\begin{array}{l}\text { Predict PEFR using } \\
\text { EN13826 std }\end{array}$ & 25,121 & 73 & 0.3 & $(0.2-0.4)$ \\
\hline $339 g$. & $\begin{array}{l}\text { Serial peak expiratory flow } \\
\text { rate }\end{array}$ & 23,325 & 2,371 & 10.2 & $(9.8-10.6)$ \\
\hline 339M. & FEV1/FVC ratio & 19,962 & 361 & 1.8 & $(1.6-2.0)$ \\
\hline 339R. & FEV1/FVC percent & 18,974 & 1,503 & 7.9 & $(7.5-8.3)$ \\
\hline $339 \mathrm{~S}$. & Percent predicted FEV1 & 14,866 & 1,920 & 12.9 & $(12.4-13.5)$ \\
\hline 3390. & PEFR using EN 13826 device & 14,206 & 112 & 0.8 & $(0.7-1.0)$ \\
\hline 339B. & PFR - after bronchodilation & 13,140 & 437 & 3.3 & $(3.0-3.7)$ \\
\hline 339P. & Expected FEV1 & 7,186 & 55 & 0.8 & $(0.6-1.0)$ \\
\hline $339 \mathrm{C}$. & PFR - expected & 6,436 & 144 & 2.2 & $(1.9-2.6)$ \\
\hline 339D. & PFR - best ever & 6,284 & 65 & 1.0 & $(0.8-1.3)$ \\
\hline $339 Q$. & Expected FVC & 5,086 & 31 & 0.6 & $(0.4-0.9)$ \\
\hline 339i. & FVC/Expected FVC percent & 4,735 & 1,655 & 35.0 & $(33.6-36.3)$ \\
\hline 339b. & FEV1 after bronchodilation & 3,843 & 148 & 3.9 & $(3.3-4.5)$ \\
\hline $339 n$. & Serial PEFR abnormal & 3,570 & 2,349 & 65.8 & $(64.2-67.4)$ \\
\hline $339 \mathrm{~N}$. & Expected FEV1/FVC ratio & 3,212 & 105 & 3.3 & $(2.7-4.0)$ \\
\hline 745D4 & $\begin{array}{l}\text { Post bronchodilator } \\
\text { spirometry }\end{array}$ & 3,137 & 2,668 & 85.0 & $(83.7-86.3)$ \\
\hline 339a. & FEV1 before bronchodilation & 2,680 & 114 & 4.3 & $(3.5-5.1)$ \\
\hline $339 \mathrm{E}$. & PFR $>80 \%$ of predicted & 2,405 & 1,174 & 48.8 & $(46.8-50.8)$ \\
\hline $339 \mathrm{~m}$. & $\begin{array}{l}\text { FEV1/FVC ratio after } \\
\text { bronchodilator }\end{array}$ & 2,344 & 74 & 3.2 & $(2.5-4)$ \\
\hline 339T. & $\begin{array}{l}\text { FEV1/FVC }>70 \% \text { of } \\
\text { predicted }\end{array}$ & 1,811 & 810 & 44.7 & $(42.4-47.1)$ \\
\hline $339 \mathrm{~V}$. & $\begin{array}{l}\text { Recorded/predicted PEFR } \\
\text { ratio }\end{array}$ & 1,392 & $<5$ & $<0.4$ & * \\
\hline $339 d$. & PEFR post steroids & 1,307 & 338 & 25.9 & $(23.5-28.3)$ \\
\hline 339c. & PEFR pre steroids & 1,262 & 169 & 13.4 & $(11.6-15.4)$ \\
\hline $339 \mathrm{~F}$. & PFR $60-80 \%$ of predicted & 1,171 & 553 & 47.2 & $(44.3-50.1)$ \\
\hline 339l. & $\begin{array}{l}\text { FEV1/FVC ratio before } \\
\text { bronchodilator }\end{array}$ & 1,083 & 59 & 5.4 & $(4.2-7.0)$ \\
\hline $66 \mathrm{Yc}$. & $\begin{array}{l}\text { Num consecutive days } \\
<80 \% \text { PEFR }\end{array}$ & 956 & 83 & 8.7 & $(7.0-10.7)$ \\
\hline 3390. & $\begin{array}{l}\text { FEV1/FVC }<70 \% \text { of } \\
\text { predicted }\end{array}$ & 838 & 314 & 37.5 & $(34.2-40.9)$ \\
\hline $339 u$. & Peak inspiratory flow rate & 799 & 642 & 80.4 & $(77.4-83)$ \\
\hline 33950 & Diurnal variation of PEFR & 753 & 302 & 40.1 & $(36.6-43.7)$ \\
\hline 339G. & PFR $<60 \%$ of predicted & 640 & 251 & 39.2 & $(35.4-43.1)$ \\
\hline 33901 & FEV1/vital capacity ratio & 344 & 7 & 2.0 & $(0.9-4.3)$ \\
\hline 339L. & $\begin{array}{l}\text { Expected peak flow rate } x \\
80 \%\end{array}$ & 329 & $<5$ & $<1.5$ & * \\
\hline 339I. & $\begin{array}{l}\text { Expected peak flow rate } x \\
50 \%\end{array}$ & 327 & $<5$ & $<1.5$ & * \\
\hline $339 \mathrm{~K}$. & $\begin{array}{l}\text { Expected peak flow rate } x \\
30 \%\end{array}$ & 325 & $<5$ & $<1.5$ & * \\
\hline 33900 & FEV1 reversibility & 170 & 7 & 4.1 & $(1.8-8.6)$ \\
\hline $339 x$ & $\begin{array}{l}\text { Percentage of best ever } \\
\text { PEFR }\end{array}$ & 162 & $<5$ & $<3.1$ & \\
\hline 339Y. & $\begin{array}{l}\text { Percentage of PEFR } \\
\text { variability }\end{array}$ & 155 & 23 & 14.8 & $(9.8-21.6)$ \\
\hline $339 r$. & FEV1/VC percent & 118 & 86 & 72.9 & $(63.8-80.5)$ \\
\hline $339 f$. & FEV1 post steroids & 69 & $<5$ & $<7.2$ & * \\
\hline 33950 & $\begin{array}{l}\text { Percentage predicted FEV1 } \\
\text { after bronchodilation }\end{array}$ & 69 & 8 & 11.6 & $(5.5-22.1)$ \\
\hline $339 e$. & FEV1 pre steroids & 48 & $<5$ & $<10.4$ & * \\
\hline
\end{tabular}

* Value masked to comply with the SAIL Databank's disclosure policy. 
Table 4.7: Percentages of missing values for lung function event codes. (cont'd)

\begin{tabular}{|c|c|c|c|c|c|}
\hline \multirow[t]{2}{*}{$\begin{array}{l}\text { Event } \\
\text { code }\end{array}$} & \multirow[t]{2}{*}{ Event description } & \multirow[t]{2}{*}{$\begin{array}{l}\text { Number of } \\
\text { all events }\end{array}$} & \multirow{2}{*}{$\begin{array}{l}\text { Number of } \\
\text { events with } \\
\text { missing values }\end{array}$} & \multicolumn{2}{|c|}{$\begin{array}{l}\text { Percentage of events } \\
\text { with missing values }\end{array}$} \\
\hline & & & & $\begin{array}{l}\text { Percent- } \\
\text { age }\end{array}$ & $\begin{array}{l}95 \% \\
\text { confidence } \\
\text { interval }\end{array}$ \\
\hline $339 t$. & Percentage of predicted VC & 46 & 43 & 93.5 & $(81.1-98.3)$ \\
\hline $339 Z$. & Respiratory flow rates NOS & 39 & 27 & 69.2 & $(52.3-82.5)$ \\
\hline 339s. & FVC before bronchodilation & 34 & 16 & 47.1 & $(30.2-64.6)$ \\
\hline $339 \mathrm{k}$. & FEV1/FVC ratio post steroids & 21 & $<5$ & $<23.8$ & * \\
\hline $339 J$. & Optimal peak flow rate & 21 & $<5$ & $<23.8$ & * \\
\hline $339 \mathrm{~W}$. & Worst peak flow rate & 12 & $<5$ & $<41.7$ & * \\
\hline 33972 & $\begin{array}{l}\text { FEV1 after change of } \\
\text { bronchodilator }\end{array}$ & 7 & $<5$ & $<71.4$ & * \\
\hline $339 j$. & FEV1/FVC ratio pre steroids & 5 & $<5$ & * & * \\
\hline 33951 & PEFR after exercise & $<5$ & $<5$ & * & * \\
\hline
\end{tabular}

and multimodal distributions. For most of the lung function testing events, the distribution of the recorded values appeared to be consistent with the expected units and ranges. For example, event values such as the forced expiratory volume in the first second (FEV1) and expected forced vital capacity (FVC) appeared to be recorded mostly in litres as expected, but with few apparent percentage values. For event values that were expected to be recorded as percentages, most of the values appeared to be percentages, with few values, for some event types, recorded as simple ratios. Examples included percent predicted FEV1, percent of actual to expected FVC, FEV1/FVC ratios, FEV1/vital capacity (VC) ratio, recorded/predicted PEFR ratio, and percentage of best ever PEFR. FEV1 reversibility had a large peak between 1 and 10, with a small peak at 100. Post-bronchodilator spirometry (745D4) values distributed mostly between 200 and 500, likely representing the change in FEV1 in millilitres from before and after bronchodilator administration.

\subsubsection{Interpretation}

Based on the above-studied asthma-related events, the recording of events and their values varied widely between event groups. Events that document asthma triggers, severity, and steps to manage the disease control were occasionally recorded. These events are important for asthma studies concerned in disease activity and management. Blood eosinophil count is usually a part of the full blood count test which can be performed for many indications other than asthma (e.g., for women in pregnancy). Blood eosinophil count can be used to predict severe exacerbations 
and poor asthma control [305]. However, this test was only available in the GP dataset for about half of the studied patients. Future developments in automatic reporting of results may change this.

Lung function tests, particularly PEFR, were relatively better recorded. However, codes for airway obstruction reversibility tests were underrecorded. They are examples of events for which numerical measurements are supposed to be recorded along with the event code. However, this analysis demonstrated that the values of these events showed variable levels of missingness and inconsistency. Bimodal distributions were common among these event values. One apparent reason is the different ways test results were recorded by healthcare professionals. Many of the values that were supposed to be recorded as either percentages or simple ratios were recorded in both formats, one of which was often dominant. For events such as FEV1 before bronchodilation, a possible explanation of the bimodal distribution is that GPs had different understanding of the unit in which the event values should be recorded (e.g., litres vs. percent or change). A longitudinal between-GP practice analysis of these values could be helpful in evidencing these potential explanations.

\subsection{Discussion}

\subsubsection{Summary of the Observatory design and data quality}

The Wales Asthma Observatory represents a regularly updated asthma registry. It also offers a platform for various types of asthma epidemiological research and a surveillance tool to inform health policy and service planning. While traditional disease registries use de novo data collection, the Observatory represents an untraditional approach to disease registry as it mainly uses RCD to identify and describe cases.

The Observatory included patients who satisfied one or more of multiple case definitions for asthma, including the one developed in Chapter 3. Patients were longitudinally characterised using a number of key asthma outcomes. Improving efficiency and reproducibility of data extraction was considered in the Observatory structure and user interface. The Observatory data are versioned, and the user interface allows rapid, reproducible, reusable, and shareable data extraction. 
However, I demonstrated a traditional problem of using RCD: suboptimal quality of data. There were various patterns of missingness and inconsistency in asthma data in Wales. Many lung function tests were recorded without measurements. When recorded, measurements of some tests were inconsistent.

\subsubsection{Strengths and opportunities}

The Wales Asthma Observatory project has several strengths. It relies on inexpensive, sustainable, and regularly updated sources of routine data in the SAIL Databank. The wide-to-complete national coverage of the SAIL datasets enables performing representative, population-based studies with large number of patients. This opportunity is usually not available through other traditional sources of data such as national surveys and primary data collected by researchers. The availability of several case definitions to identify asthma patients provides researchers with flexibility and ability to compare their findings with studies performed elsewhere using various case definition. In addition, the Observatory benefits from a collaborative platform to share clinical code lists and data extraction procedures within research teams and, in the future, with the wider research community and the public as well. This collaborative platform is intended to save analyst time, to improve collaboration and sharing of methods, as well as to support research documentation, reproducibility and transparency.

\subsubsection{Challenges and limitations}

\subsubsection{Primary care-based case definition of asthma may exclude some patients}

The asthma case definitions used in the Observatory, including the inclusive and strict ones, were based on primary care data only. This was justified as asthma in the UK is managed mostly in primary care [32].

However, it is possible that some people with asthma may not be captured by the primary care dataset (WLGP). The WLGP dataset currently covers only $\sim 80 \%$ of GP practices (see Section 4.3.3). This means that people with asthma who never registered at those participating practices were not included in the Observatory. In addition, it is possible that some people had presented with acute asthma symp- 
toms at $A \& E$ departments and/or were hospitalised for asthma without being captured by GP data-based case definitions of asthma used in the Observatory.

Therefore, the Observatory would benefit from using secondary care data in identifying asthma patients, which will be considered in future developments.

\subsubsection{Inherent limitations of routine data}

Limitations of routine data for use in research are discussed in Chapters 2 and 3. The use of these data in disease registries is challenged by a range of limitations. For example, these data are usually collected for clinical and administrative purposes in mind and therefore may not be readily appropriate for secondary uses such as disease surveillance, service planning, or research. Despite the wide use of standardised clinical coding schemes such as ICD-10 or Read codes, EHRderived data collected over many years often lack standardisation as the same piece of clinical information may be recorded in different forms [233, 306].

Case definitions are a core part of a disease registry or observatory. However, developing accurate case definitions based on routine data is challenging. Disease registries that are built using active reporting of individual cases have the advantage of individual-level assessment of eligibility, often using confirmed diagnosis by clinicians. In contrast, a routine data-based disease registry is populated by applying the same eligibility criteria en masse to all people in a large population in a database [213], which can introduce high risk of misclassification. In addition, it has been shown than methods to estimate asthma prevalence from routine data may be inaccurate [307].

\subsubsection{Traditional methods to define cases and outcomes may need re- consideration}

In Chapter 2, I found significant heterogeneity in the definitions of asthma and asthma outcomes. There were variations, not only in the types of health events used to assess the disease, but also in the time interval over which these health events are queried. These query intervals should be chosen based on stability of disease statuses over time [251]. Longer intervals may conceal important temporal variations of the measured disease status. Conversely, shorter intervals may introduce unrealistic temporal variations. The case definitions currently supported 
by the Observatory use 12-month intervals. This has been traditionally the most frequently used interval in research and for clinical and administrative purposes (e.g., for the QOF). However, evidence is lacking about whether 12 months is the best interval over which asthma activity, severity, and control are assessed. Therefore, further studies are needed to choose the most appropriate and meaningful interval for each of these variables.

\subsubsection{Implications of the suboptimal quality of asthma-related routinely collected data on asthma research}

Data gaps undermine the ability of routinely collected EHR data to inform asthma care [308] and support research. Significance and implications of data gaps are specific to how data are used. For example, gaps in lung function data make it difficult to assess asthma severity, which could be alternatively assessed by asthma medications [309]. Similarly, unavailability of medication dispensing data is a significant limitation in adherence studies. In contrast, such gaps are unlikely to be an issue in prevalence studies that rely on physician's diagnosis codes.

Guidelines in the UK recommend performing airway obstruction reversibility tests when asthma diagnosis is uncertain [16]. An essential objective part of the clinical diagnosis of asthma is to confirm the airway obstruction reversibility, which often strongly indicates an asthma diagnosis and rules out chronic obstructive pulmonary disease (COPD). Reversibility data would allow identification of a patient subgroup with very high certainty of asthma diagnosis. However, I found that codes of these tests were under-recorded in the GP dataset. One explanation is that a significant number of lung function tests were performed in secondary care settings. A similar gap between the number of diagnosis codes and spirometry codes were observed in Alberta, Canada [308].

The QOF, arguably, appeared to improve the recording of healthcare events that are required by its quality indicators (see Table C.1.2). However, the asthma indicators AST001 and AST002, for example, only require event codes to be recorded; they do not assess the quality and completeness of recorded event measurements per se [310]. 


\subsubsection{Primary care coding in the UK will change}

The primary care data used in the Observatory are coded using Read codes. Read codes are a clinical coding scheme which covers wide aspects of primary care encounters. It is the main coding scheme used in primary care in the UK. However, GP practices in the UK are expected to transition from Read codes to Systematized Nomenclature of Medicine-Clinical Terms (SNOMED-CT) in 2018 [311]. Therefore, to ensure sustainability of the Observatory, the methods used to identify patients and assess disease outcomes need to be modified to support SNOMED-CT coding in due time.

\subsubsection{Data security and implications of anonymisation}

There are important considerations when using de-identified data, such as those used in the Observatory. Despite replacing people's identifiers in the SAIL Databank with multiply encrypted unique identifiers (i.e. ALFs), re-identifiability of patient data is possible unless additional steps are taken. For example, very small groups of patients with rare combinations of characteristics related to their health or health care usage may be re-identified if the data are presented in particular formats. This may be an issue for less common events, such as hospital admissions or day cases, or complex combinations of multiple broad criteria. For instance, there could be fewer than five patients who were in a specific age group at a specific date, lived in a small city, had asthma diagnosis made in a specific year, and received a very high number of inhalers in a specific year; these combinations may increase the risk of patient re-identifiability.

To avoid the risk re-identifiability of patients, all outputs from the Observatory must conform to the SAIL information governance policy [295]. For outputs to be available outside the Gateway, they must be first reviewed by senior analysts who assess the output for re-identifiability risk and compliance with the approved project proposals. In the output data, groups with frequencies smaller than a certain limit, usually five, must be suppressed from reporting or aggregated with other groups, and dates are aggregated into time periods.

Risk of patient re-identifiability can be high when person-level information from difference sources is publicly available. However, SAIL controls all individual link- 
ages within the databank and does not export individual level data to avoid this possibility.

Although the use of anonymised data in the Observatory protects patient identities, it places limits on how these data can be used. For example, a prediction model can be developed within the anonymised registry using the power of multiple, linked, large volume datasets. Within such an anonymised setting, individual predictions for anonymous persons can be obtained. Due to anonymisation, however, it is impossible to link these predictions back to patient records at the point of care, e.g., a GP surgery. Instead, to use that prediction model at the point of care, ideally all predictor variables from other care settings (e.g., emergency, inpatient, and/or outpatient care) that are used in the model development need to be available at the point of care. However, this is often not feasible. A possible workaround approach is to develop a prediction model, within the anonymised registry, using only the types of data that will be available at the point of care. Then, using sensitivity analysis, the performance of this prediction model can be compared with a model that is based on all the data sources available in the registry.

\subsubsection{Recommendations for better capture of asthma data}

Based on the analysis of data quality that I presented earlier in this chapter, I recommend that effort needs to be made to ensure that better data on asthma care are captured and recorded in EHRs. Wider standardisation of the ways health events are recorded is particularly needed. This could be achieved though better clinical coding training of healthcare professionals. Motivating GPs to improve coding when their time is very short could be potentially achieved by demonstrating the value of projects using complete coding (e.g., those already performed using the SAIL Databank ${ }^{7}$ ). EHR systems should facilitate standardisation of clinical coding by incorporating on-screen coding advice and better validation rules, which insure the right data are recorded in the right place and in the expected format. Natural language processing (NLP) techniques, which are increasingly implemented in EHR systems to codify narrative data, should consider validity of the produced coded data. Capture of more accurate and complete data at the point of care will result in better value and utility of the asthma registry and the Observatory. Pro-

\footnotetext{
${ }^{7}$ Examples of studies that used the SAIL Databank can be found in the following links: https://saildatabank.com/saildata/uses-for-sail-data/ and https://saildatabank.com/saildata/sail-publications/
} 
fessional societies representing primary and secondary care respiratory medicine could be the most appropriate groups to lead efforts to improve the quality of captured data. In addition, implication of data quality and the lack thereof on patient care, service planning, and research needs to have more presence in venues of continued professional education and research meetings to make clinicians more data conscious. Furthermore, payment for performance schemes such as the QOF need to consider the quality and completeness of the recorded data in addition to their quantity [284] as added incentives.

\subsubsection{Future development}

Although the Wales Asthma Observatory benefits from rich sources of routine data in the SAIL Databank, data about several aspects of healthcare are still missing. These include clinical, laboratory, and medication prescribing data from secondary care, as well as community medication dispensing data. However, endeavours are currently under way to link the all-Wales pathology data to the SAIL Databank as well as to increase the depth of coding of clinical correspondences using NLP techniques. Using these data in the Observatory would enable highly useful research applications such as improving accuracy of asthma case definitions and disease phenotyping. Environmental data including data on housing quality, pollution, greenness, use of outdoor spaces, commuting routes, and modes of transport can be also linked to the Observatory in order to answer questions about the effect of various environmental factors on asthma outcomes [300, 312, 313].

Emerging sources of data such as data from smart inhalers and wearable technologies, despite being currently of limited use, can be later used to enrich the asthma registry with important variables about disease activity and medication usage and adherence over time. Asthma-related PROMs can be of high significance to clinical care and research [314]. Future development of the asthma registry can include developing platforms to collect asthma-related PROMs and link them to asthma-related routine data. This will enable investigating the relationship between doctor-reported and self-reported asthma outcomes and will allow assessing the association of PROMs with each of health services utilisation patterns, health care quality, and health care inequalities.

An LHS of asthma in Wales is needed to close the gap between evidence and practice. By facilitating near real-time disease surveillance, the Wales Asthma 
Observatory can be a building block which would help this ambitious project to materialise.

\subsection{Conclusion}

The Wales Asthma Observatory represents an untraditional approach to a disease registry, and a platform for research and surveillance. In this chapter, I described development of the Observatory, including purpose, source population, structure, content, and technical logistics.

The quality of asthma related data in Wales is suboptimal. There were various patterns of missingness and inconsistency in these data. Many lung function tests were recorded without measurements. When recorded, measurements of some tests were inconsistent. To improve the capture of asthma data, I proposed enhanced EHR data entry quality checks, data quality awareness training for healthcare professionals, and data quality based incentivisation of health care providers. I described approaches to improve efficiency and reproducibility of studies that will use the Observatory. I developed an easy-to-use user interface that supports shareable, reusable, and scalable data extraction from the Observatory and the GP dataset.

Further developments to the Observatory will provide linkage to additional RCD sources and PROMs, and adaptation to the upcoming clinical coding system, SNOMEDCT. 


\section{Chapter 5}

\section{Inequalities in asthma care and outcomes in Wales}

In the previous chapters, I have discussed the development of the Wales Asthma Observatory. In this chapter, I demonstrate an example of utilising the Observatory to inform health policy. Variations in asthma outcomes between population groups have been widely reported worldwide. These inequalities can be assessed using area-based deprivation indices. An important application of the Wales Asthma Observatory in supporting health policy is to investigate whether inequalities in asthma care and outcomes exist between socioeconomic groups. In this chapter, I investigated the variations in the incidence of asthma-related healthcare utilisation in primary and secondary care among asthma patients in Wales across the quintiles of the Welsh Index of Multiple Deprivation. I found wide social gradient in asthma where patients in the most deprived areas had remarkably more asthmarelated hospitalisations indicating poorer outcomes. I also discuss the implications of these findings on health policy. 


\section{Chapter Contents}

5.1 Introduction. . . . . . . . . . . . . . . . . . . . . . . . 147

5.1.1 Asthma variations are common . . . . . . . . . . . . . . . . . . . . . . . . 147

5.1.2 Inequality and inequity in health and health care . . . . . . . . . . . . . . 148

5.1.3 Area-based socioeconomic measures . . . . . . . . . . . . . . . . . . . . . . . 149

5.1.3.1 Overview . . . . . . . . . . . . . . . . . . . . . . . . . . . 149

5.1.3.2 The Welsh Index of Multiple Deprivation . . . . . . . . . . . . . . . . 150

5.2 Aims and Objectives . . . . . . . . . . . . . . . . . . . . . . . . . . 154

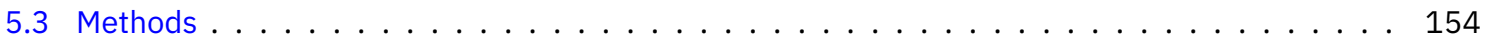

5.3 .1 Data sources . . . . . . . . . . . . . . . . . . . . . . . . . . . . . . . . . 154

5.3.2 The source population and study cohorts $\ldots \ldots \ldots \ldots \ldots$

5.3 .3 Socioeconomic status . . . . . . . . . . . . . . . . . . . . . . . . . 156

5.3 .4 Outcome variables . . . . . . . . . . . . . . . . . . . . . . 157

5.3.4.1 Number of asthma-related GP visits . . . . . . . . . . . . . . . . . 157

5.3.4.2 Number of asthma reviews . . . . . . . . . . . . . . . . . . . . . . 157

5.3.4.3 Asthma-related emergency department visits . . . . . . . . . . . . . . . 157

5.3.4.4 Asthma-related hospital admissions . . . . . . . . . . . . . . . . . . . 157

5.3 .5 Statistical analysis . . . . . . . . . . . . . . . . . . . . . . 158

5.3.5.1 Descriptive statistics . . . . . . . . . . . . . . . . . . . . 158

5.3.5.2 Variation of age between deprivation quintiles $\ldots \ldots \ldots \ldots$

5.3 .5 .3 Count regression . . . . . . . . . . . . . . . . . . . . . . 158

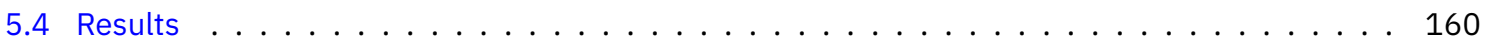

5.4.1 Descriptive statistics of the source population and study cohorts _ . . . . . . . . . 160

5.4.2 Zero-inflated negative binomial regression models . . . . . . . . . . . . . . . 168

5.4.2.1 Incidence rate ratios of study outcomes across deprivation quintiles . . . . . 169

5.4.2.2 Incidence rate ratios of study outcomes for age groups and gender . . . . . . 171

5.4 .2 .3 Model fit . . . . . . . . . . . . . . . . . . . . . . 174

5.4.2.4 Sensitivity analysis . . . . . . . . . . . . . . . . . . 175

5.5 Discussion . . . . . . . . . . . . . . . . . . . . . . . . . . . . 177

5.5 .1 Summary of findings . . . . . . . . . . . . . . . . . . . . . . . 177

5.5.2 Interpretation in the light of previous studies . . . . . . . . . . . . . . 178

5.5.2.1 Comparison with previous studies $\ldots \ldots \ldots \ldots \ldots \ldots$

5.5.2.2 WIMD mainly describes areas, and to a lesser extent, individuals . . . . . . . 179

5.5.2.3 Asthma-related emergency department visits and hospitalisations usually indicate worse asthma severity and control . . . . . . . . . . . . . . 179

5.5.2.4 Why did the most deprived asthma patients have more GP visits? . . . . . . 180

5.5 .3 Study strengths . . . . . . . . . . . . . . . . . . . . . . . . . . . . . . 181 
5.5 .4 Study limitations . . . . . . . . . . . . . . . . . . . . . . . . . . . . 182

5.5.4.1 Case definitions for asthma-related A\&E visits and hospitalisations were not

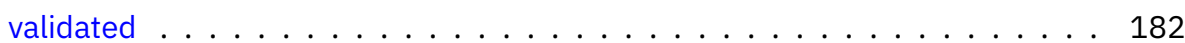

5.5.4.2 Possible residual confounders . . . . . . . . . . . . 183

5.5.4.3 WIMD overall index and asthma exacerbations: a possible circular relationship 184

5.5 .5 Implications for health policy . . . . . . . . . . . . . . . . . . . 185

5.5 .6 Future work . . . . . . . . . . . . . . . . . . . . . 187

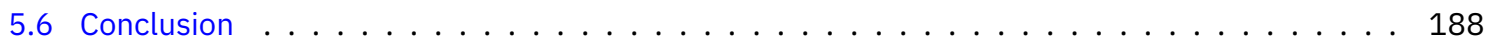

\subsection{Introduction}

\subsubsection{Asthma variations are common}

The epidemiology of asthma and asthma outcomes exhibits variations around the world. The International Study of Asthma and Allergies in Childhood (ISAAC) revealed wide geographical variations in the prevalence of self-reported asthma diagnosis. There were twenty-fold variations in the prevalence of self-reported asthma between the study centres around the world [48, 315]. The variations were not only seen between countries but also within countries and within cities such as Mexico City and New York city [37]. In the United Kingdom (UK), moderate variations have existed between its member countries in the prevalence and incidence of asthma, based on self-reported and doctor-reported data [43]. However, there were limited variations between UK metropolitan and non-metropolitan areas, with the latter having slightly higher prevalences [316].

Studying the variations in asthma epidemiology can potentially help understand the aetiological factors and determinants of the disease. It has been suggested that the geographical variations in asthma epidemiology result from complex interaction of numerous factors. The effect of environmental determinants on asthma has been extensively studied. Air pollution has been linked to asthma epidemiology. There is contradictory evidence on whether air pollution is associated with increased asthma incidence and prevalence [317, 318], with a suggestion that adverse effects of traffic-related air pollution tend to be close to major roads [318]. It is more evident, however, that air pollution increases the incidence of asthma exacerbations among people who already have the disease [318, 319]. Climate has been also suggested to influence the prevalence of asthma symptoms. Data from 
146 centres of the ISAAC study showed that in Western Europe the prevalence of asthma symptoms in school children was positively associated with indoor humidity, and negatively associated with temperature, outdoor humidity, and altitude [320].

Socioeconomic determinants have been also found to influence asthma epidemiology. In 1973, Mitchell et al. found that in Scotland, severe asthma was more often observed in children of semi-skilled and unskilled manual worker parents and children of larger families [321]. A systematic review has found that lower socioeconomic status is associated with higher asthma prevalence [322]. In England, a study in the early 1990s found that asthma hospital admissions rates were higher in areas with high deprivation where most admissions came via Accidents and Emergency departments rather than referrals from general practitioners (GPs) [323]. Another study in Cardiff found that hospital admission rates for asthma were correlated with the level of social deprivation [324]. Those hospital admission rates were, however, not correlated with the prevalence of asthma or wheezing but with the prevalence of chronic phlegm and the exposure to second-hand smoke at home. Low socioeconomic status was associated with less treatment in wheezy children [325] and poorer asthma control and persistent airway obstruction in adults [326].

Variations in asthma epidemiology, especially those ascribed to socioeconomic factors, highlight inequalities in health and health care and represent important challenges to health policy.

\subsubsection{Inequality and inequity in health and health care}

Health inequalities have been defined by the World Health Organisation [327] as: "differences in health status or in the distribution of health determinants between different population groups". Uneven distributions of health status and its determinants may result from numerous factors creating advantages and disadvantages that accumulate over the course of life [328]. The term health inequalities is a descriptive term that is often used to describe those uneven distributions in health status and determinants and that do not per se imply moral judgement [329]. Some forms of health inequalities are practically unavoidable and do not represent injustice. Examples include health disparities that result from biological differences between population groups or external factors, such as natural 
environment, over which they usually do not have control. Arguably, individuals' 'free choices' may also contribute to health inequalities [327], although this is debatable since individuals' 'free choices' can be influenced by the environment in which they live [330]. However, other disparities in health result from unnecessary, unfair, and unjust variations in health determinants, in which case they are called health inequities [327, 331]. The concept of health inequity has attracted hot debate and controversy [329], and since it is based on value judgement, it is not easy to determine which health inequalities are universally inequitable. Braveman et al. (2003) presented an operationalised definition of health equity: "Equity in health is the absence of systematic disparities in health (or in the major social determinants of health) between groups with different levels of underlying social advantage/disadvantage-that is, wealth, power, or prestige" [332].

In epidemiological studies, health inequalities are often assessed by comparing health status and determinants between social groups in the population. In large epidemiological studies, however, individual-level data on socioeconomic status may not be available, and collecting these data from the study individuals is impractical. In these studies, area-based measures of socioeconomic status and deprivation have been widely used to study health inequalities.

\subsubsection{Area-based socioeconomic measures}

\subsubsection{Overview}

The different socioeconomic factors can have accumulating, interactive effects on the individual's health over the course of life [328, 333]. Some of these factors, such as income, employment, and educational attainment, act on the individual level. On the other hand, factors related to the community and environment act on the group and area levels and may have effects on the person's health status independent from individual health determinants [334, 335]. To account for those complex and interacting factors, area-based socioeconomic measures have been developed, usually using census data, to provide 'simple' socioeconomic profiles and ranks for geographic areas [336]. These measures can be based on a single or, more commonly, multiple components representing different socioeconomic factors [333]. Area-based socioeconomic measures have been widely used in epidemi- 
ological studies to assess the effect of socioeconomic status on health, although they are most commonly used as control variables and confounders [333].

In the UK, examples of area-based socioeconomic measures include:

- Townsend's Index [337],

- Index of Multiple Deprivation (England) [338],

- the Scottish Index of Multiple Deprivation [339], and

- the Welsh Index of Multiple Deprivation [340].

\subsubsection{The Welsh Index of Multiple Deprivation}

The Welsh Index of Multiple Deprivation (WIMD) is the official area-based measure of relative socioeconomic deprivation in Wales. The WIMD is based on socioeconomic indicators that represent aggregate characteristics of residents in the area and/or describe the area itself. The WIMD was commissioned by the Welsh Government to create a measure to understand relative differences in deprivation, based on several domains measured at a small area level across Wales. The WIMD was designed a tool to inform the development of policies and allocation of funding so that they target the most disadvantaged communities [341]. The WIMD index is updated every few years, with versions released in the years 2000, 2005, 2008, 2011, and 2014.

The WIMD 2011 index is constructed from weighted sum of eight deprivation domains, each is composed of several deprivation related indicators. According to the WIMD 2011 Technical Report [342], those deprivation domains include the following, ordered by weighting: Income, Employment, Health, Education, Geographical Access to Services, Housing, Physical Environment, and Community Safety. I provide an overview for these deprivation domains including how they were constructed.

1. Income domain This domain is based on the proportion of residents in a given area with low income or those who claim income-related benefits, and has a $23.5 \%$ weighting in the overall WIMD 2011 index.

2. Employment domain This domain represents the proportion of residents in the working age in a given area who have employment-related deprivation (i.e. receiving benefits related to employment). This domain has a $23.5 \%$ weighting in the overall WIMD 2011 index. 
3. Health domain This domain captures the health-related deprivation, and is constructed from four indicators including limiting long-term illness, death rate in the area from all causes, incidence of cancer, and low birth weight. This domain has a weighting of 14\% in the overall WIMD 2011 index.

4. Education domain This domain reflects the deprivation relating to educational attainment in a given area among children and young residents as well as the lack of educational qualifications and skills among adults. It is constructed from average school scores of children, proportion of residents not in higher education at the age of 18 or 19, proportion of residents aged 25 or above with no educational qualifications, and proportions of half day absence among children in primary and secondary schools. This domain has a weighting of $14 \%$ in the overall WIMD 2011 index.

5. Geographical Access to Services domain This domain captures the deprivation relating to inaccessibility of necessary services to each household in a given area. Inaccessibility to a service is measured by the average time needed to reach it using the shortest trips by bus and/or by walking. The services include National Health Service dentists, food shops, GPs, Post Office, primary and secondary schools, leisure centres, and transport nodes. This domain has a weighting of 10\% in the overall WIMD 2011 index.

6. Housing domain This domain represents the level of disadvantage due to lack of adequate housing, and is constructed from indicators including proportion of residents who lack central heating in their households, and proportion of residents who live in overcrowded households. This domain has a weighting of 5\% in the overall WIMD 2011 index.

7. Physical Environment domain This domain represents the disadvantage from environmental factors in a given area that can affect the quality of life. These factors include air quality and pollution, emissions, risk of flooding, and distance to waste disposal and industrial sites. This domain has a weighting of 5\% in the overall WIMD 2011 index.

8. Community Safety domain This domain reflects the level of safety and protection from crimes in a given area. It is constructed from indicators including the proportions of offenders among adults and young people, numbers of 
burglaries, criminal damages, thefts, violent crimes, and fire incidents. This domain has a weighting of 5\% in the overall WIMD 2011 index.

The WIMD index is produced for all Lower Layer Super Output Areas (LSOAs) in Wales. LSOAs were outlined by the Office for National Statistics of the UK for census related purposes [343]. LSOAs vary widely in spatial size but they are intended to have comparable population sizes; according to the WIMD 2011 index, the average population in those small areas was $\approx 1,600$ people in that year [341]. The WIMD index gives a rank from 1 (most deprived) to 1,896 (least deprived) to the 1,896 LSOAs in Wales.

The most deprived areas in Wales are distributed mostly in the southern areas such as Rhondda Cynon Taf, Blaenau Gwent, as well as the east and north of Swansea, pockets in Newport, and the south and east of Cardiff (Figure 5.1).

\section{Limitations of the WIMD index}

A relative ranking measure

WIMD is a ranking system in which areas are ordered according to their sum of weighted deprivation scores. However, it does not quantify the level of deprivation, and therefore it does not quantify the differences in deprivation between areas [341]. That is, the WIMD index can tell that an area has a higher or lower multiple deprivation than another area, but it does not tell by how much.

\section{It describes areas, not residents}

Being an area-based measure, the WIMD is intended to describe the relative multiple deprivation in the area as a whole based on average scores of individuals. Therefore, it does not imply that all the residents have the same multiple deprivation. For example, it is possible that different residents in an area have different types and levels of deprivation. A consequence of this limitation is that not all deprived individuals live in the most deprived areas, and not all least deprived individuals live in the least deprived areas [341]. Rather, it is possible that a number of individuals with very low deprivation live in areas with overall high deprivation. This limitation mainly concerns the deprivation domains that are based on individual-level data. However, it almost does not apply to the other deprivation 


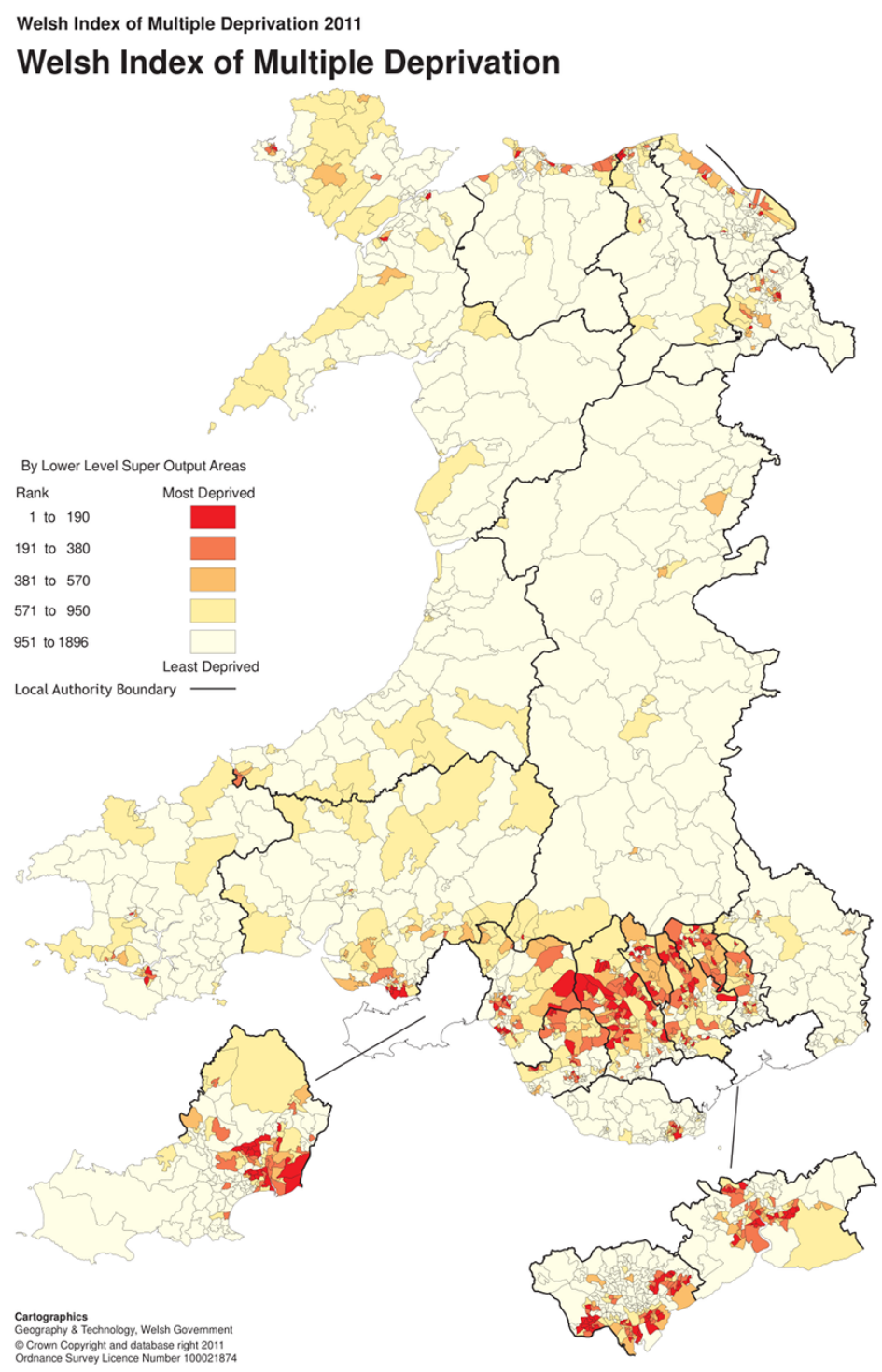

Figure 5.1: Map of Wales showing ranks of the 2011 Welsh Index of Multiple Deprivation for Lower-level Super Output Areas. Source: StatsWales, Welsh Government (https://statswales.gov.wales/ Download/File?fileId=91). (c Crown Copyright and database right 2011.

domains that use data on the areas themselves, namely the Geographical Access to Services, Physical Environment, and the Community Safety domains.

It is incomparable with indices in other UK countries

Other UK countries have their own multiple deprivation indices. However, it is not possible to directly compare these indices with the WIMD index due to the differences in the deprivation domains and the ways they are calculated [341]. 


\subsection{Aims and Objectives}

Variations in asthma outcomes between socioeconomic groups represent a significant challenge to health policy. While such variations were previously reported on small, localised populations in Wales [324], a country-wide analysis is needed to assess the scale of these inequalities. The aim in this chapter is to investigate the variations of asthma outcomes across the socioeconomic deprivation spectrum in Wales.

The objectives were as follows:

- To develop count regression models for asthma-related outcomes against the WIMD index quintiles, adjusted for age group and gender. The count regression models will be performed in a cohort of ever-diagnosed asthma (regardless of current treatment) and in a cohort of ever-diagnosed currently-treated asthma ('current asthma').

The asthma-related outcomes included the following:

- Asthma-related GP events: Any asthma-related visits to GPs

- Asthma routine reviews

- Asthma-related visits to Accident and Emergency departments

- Asthma-related hospital admissions

- To interpret the models in the light of previous studies and strengths and limitations of the routinely collected data used.

- To reflect on the implications of the findings on health policy in Wales.

\subsection{Methods}

Using the Wales-wide Secure Anonymised Information Linkage (SAIL) Databank, I accessed anonymised data on patients with a GP diagnosis of asthma in or before 2009 and continuous GP registration between 2010 and 2014 and linked those data to the quintiles of the 2011 version of the WIMD. I define the follow-up period as five calendar years from 2010-1-1 to 2014-12-31.

\subsubsection{Data sources}

In this analysis, I used the following datasets in the SAIL databank: 
- The Welsh Demographic Service (WDS): The WDS contained de-identified demographic and administrative information for National Health Services (NHS) patient in Wales.

- The WIMD 2011 dataset: I used the 2011 version of the WIMD index which was the latest version available in the SAIL Databank. This dataset included rank quintiles of the overall WIMD index for all small areas (i.e. LSOAs) in Wales.

- The Welsh Longitudinal General Practice dataset: I described the Welsh Longitudinal General Practice (WLGP) dataset in Section 3.3.1. I used the “2018-08-20" version of the dataset.

- The Emergency Department Data Set (EDDS) for Wales: The EDDS dataset was created in 2009 and captured visits to Accident \& Emergency (A\&E) departments as well as minor injury units (MIUs) in NHS hospitals in Wales. Recorded data for each attendance include investigations performed, diagnosis made, anatomical areas involved, treatment provided, as well as other administrative data related to the attendance. Diagnosis is coded using a three-digit code chosen from a list of 83 possible codes representing broad diagnostic categories. In addition, to the primary diagnosis, there are five further positions to record additional or secondary diagnoses. Due to the nature of emergency attendances, recorded diagnoses may be uncertain or unconfirmed. Practices of recording and coding of data vary between the different A\&E departments and MIUs. The EDDS currently receives data on all emergency attendances in Wales. However, in the earlier years, some A\&E departments were not able to submit their data to the EDDS, and therefore data in that period may be incomplete. Therefore, caution should be exercised when using this dataset for epidemiological and research analyses.

- Patient Episode Database for Wales: The Patient Episode Database for Wales (PEDW) database was created in 1991 and includes records for all planned and emergency inpatient admissions in addition to day case admissions to NHS Wales hospitals as well as most admissions of Welsh residents to hospitals in England. Recorded data include admission diagnoses, procedures and operations performed during admissions, as well as length of stay (LOS), Healthcare Resource Groups (HRGs), and other administrative data. Admission diagnoses are recorded using the $10^{\text {th }}$ revision of the International Classification of Disease (ICD-10). In addition to a mandatory 
primary diagnosis code for a hospital episode, the database allows recording of an additional subsidiary code and up to 12 secondary diagnosis codes. The PEDW database is considered of a high quality. ${ }^{1}$ However, it is mainly an administrative database which was created as a tool to track hospital financial activity rather than for epidemiological or research purposes. The database also suffers from between-hospital variations in practices of coding of admission diagnosis in the available fourteen diagnosis positions.

\subsubsection{The source population and study cohorts}

The source population included people who met all the following criteria:

- Had records in the WDS dataset (version 2018-04-10).

- Had records in the WLGP dataset (version 2018-08-20).

- Lived at least to 2014-12-31.

- Were successfully linked to a valid WIMD 2011 ranking.

- Had continuous GP registration in the period between 2010-1-1 and 2014-1231 , which includes the period over which the outcome events are queried in addition to one year before it. To calculate GP registration periods for individuals, I used an unpublished algorithm developed in-house by the analyst team of the SAIL Databank. I assessed the effect of requiring continuous GP registration in a sensitivity analysis in Section 5.4.2.4.

From the source population defined above, I created the following two cohorts:

- Cohort 1 included people with asthma diagnosis recorded before 2010-01-01.

- Cohort 2 was a sub-cohort of Cohort 1 in which people received at least one asthma prescription in any year between 2010 and 2014 (i.e. the follow-up period), in addition to having asthma diagnosis before 2010-01-01.

Asthma diagnosis was defined using the Read codes "H33\%", "H3120", "102. .". Asthma prescriptions were defined using the Read code sets in Appendix E.

\subsubsection{Socioeconomic status}

I linked each patient to the WIMD quintile of their area of residence during the follow-up period of 2010-2014. Where a patient had more than one address during

\footnotetext{
${ }^{1}$ See http://www.publichealthwalesobservatory.wales.nhs.uk/PEDW
} 
the follow-up period, I selected the address with the longest duration within that period. This WIMD quintile variable was coded with 1 (the most deprived) to 5 (the least deprived).

\subsubsection{Outcome variables}

The outcome variables were counts of asthma-related events in primary and secondary care in the period from 2010-1-1 to 2014-12-31. The code lists used in the construction of these variables are shown in Appendix E. The following are description of each of the outcome variables:

\subsubsection{Number of asthma-related GP visits}

For this analysis, I defined an 'asthma-related GP visit' by any Read code that indicates an asthma-related contact with a GP. Where more than one relevant code occurred on the same date, I treated them as a single visit.

\subsubsection{Number of asthma reviews}

An asthma review is a special, scheduled visit to GP, in which disease control is assessed and management plan including prescriptions and self-management advice is reviewed. Asthma reviews are ideally arranged regularly on at least an annual basis [16]. To identify asthma reviews from the GP dataset, I used a list of codes for annual review, medication review, follow-up, monitoring by nurse, and review using the Royal College of Physicians' three questions [344].

\subsubsection{Asthma-related emergency department visits}

I identified asthma-related emergency department (ED) visits from the EDDS dataset using the code 14A ("asthma"). I treated ED visits as asthma-related if it contained this code in any of the primary or secondary diagnosis positions.

\subsubsection{Asthma-related hospital admissions}

I identified asthma-related hospital admissions from the PEDW dataset by looking for episode records in which an ICD-10 code for asthma (J45) or status asthmaticus 
(J46) was in the primary diagnosis position or any of the remaining 13 secondary diagnosis positions.

\subsubsection{Statistical analysis}

I performed the statistical analyses described below for each of the two study cohorts.

\subsubsection{Descriptive statistics}

The descriptive statistics described the source population and the two asthma cohorts. For the source population, I calculated statistics about age and gender in addition to the prevalence of ever-diagnosed asthma at 2010-01-01 and the prevalence of ever-diagnosed currently treated asthma over 2010.

For the two asthma cohorts, I calculated the distributions of specific characteristics in relation to the WIMD quintiles. These characteristics included age, gender, receiving specific types of asthma prescriptions over the follow-up period, and the four outcomes variables (see above). In addition, I calculated asthma medication ratio, which represents the ratio of controller to controller-and-rescuer asthma medications [345]. I included inhaled corticosteroids (ICSs) and ICS-LABA (long-acting beta adrenoceptor agonist) combination inhalers as controller prescriptions, and included short acting beta agonist (SABA) inhalers as the rescuer medications. The formula was $\frac{I C S+I C S_{-} L A B A}{I C S+I C S_{-} L A B A+S A B A}$ calculated over the five follow-up years. In averaging the ratio, $\bar{I}$ excluded those who received none of these three inhaler categories over that period.

\subsubsection{Variation of age between deprivation quintiles}

I used the Kruskal-Wallis rank sum test to test the differences in age distribution between quintiles of the overall WIMD index.

\subsubsection{Count regression}

To test the effect of multiple deprivation on each of the four outcome asthma variables, which were count variables, I developed a count regression model for each of them. The independent variable was the quintile of the overall WIMD index. I 
tested the distribution of counts for each of the outcome variables and found that their variances were larger than their means. In addition, there were excessive numbers of patients with zero counts for each of the outcome variables. Therefore, I used zero-inflated negative binomial (ZINB) regression, which allows for over dispersion and models the excess in the zero counts.

A ZINB model assumes that count data are generated by two processes. One of these is a Bernoulli process which determines whether an individual is theoretically eligible to have a non-zero count [346]. Accordingly, there are individuals who are not eligible to have non-zero counts and therefore should have no events. In my study, those individuals were asthma patients in whom the disease was mild or remitted and therefore they needed no visits to GPs. Those patients would also have had no need for asthma-related ED visits or hospital admissions. Patients with more severe but well-controlled disease would also have no asthma-related ED visits or hospital admissions. On the other hand, for individuals who are eligible to have non-zero counts, the counts are assumed to be determined by a negative binomial distribution which expects some individuals to have no events and others to have one or more events. In my study, this applied to asthma patients who had active disease and, depending on disease severity and control as well as other non-asthma-related factors, might or might not need to have contact with primary and/or secondary care. To model the above described two processes, a ZINB model fits two regressions: a logistic regression to model the probability of having non-zero count, and a negative binomial regression to model the magnitude of counts.

In this chapter, I used ZINB regression models to model the counts of the abovementioned outcome variables in relation to the WIMD index quintile. I considered the least deprived areas (i.e. the fifth quintile) the reference group. Therefore, in the resulting model, the exponentiated coefficients for each of the other four quintiles ( 1 to 4 ) represented the incidence rate ratio (IRR) of the relevant events for that quintile compared with the least deprived areas. I also calculated the 95\% confidence intervals (CIs) for these IRRs. I adjusted the model for 5-year age groups and gender.

I used the zeroinfl function from the R package pscl version 1.4 .9 to perform the zero-inflated negative binomial modelling [347]. 
I examined the model fit with quantile-quantile (Q-Q) plots of the raw residuals as well as with rootograms. Rootograms are graphical representation of both the observed counts as bars, and the expected counts, which are predicted by the model, as a curve [348]. The axis that represents the counts (i.e. usually the vertical axis) has a square-root scale. By including both the predicted and observed values in the same graph, a rootogram helped show the deviation of the predicted counts from the observed counts. A hanging rootogram has the bars of observed counts "hanged" on the curve of predicted counts. The deviations of the predicted counts from the observed counts were shown as deviations from the horizontal axis, and provided a visualisation of the goodness of fit of the model.

\subsection{Results}

\subsubsection{Descriptive statistics of the source population and study cohorts}

Table 5.1 shows characteristics of the source population. A flowchart of case selection for both asthma cohorts is shown in Figure 5.2.

Table 5.1: Characteristics of the source population in the year 2010 across the WIMD quintiles.

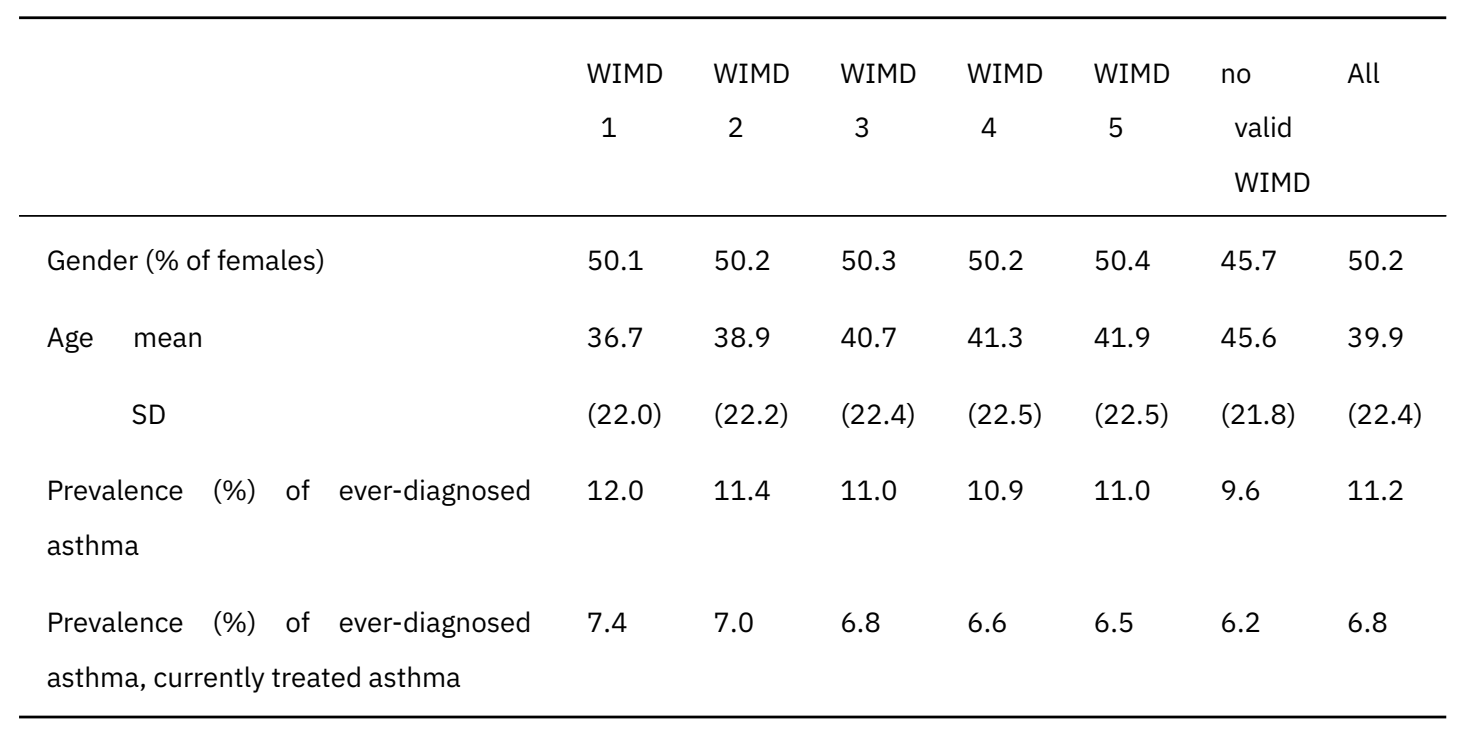




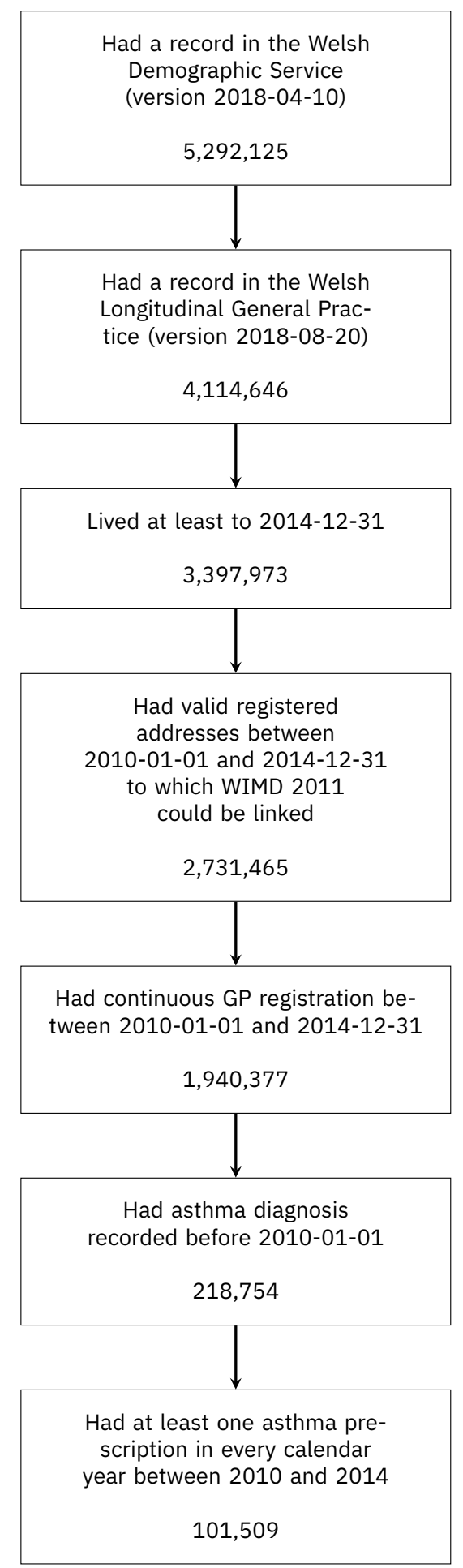

Cohort 1:

People with

ever-diagnosed asthma

\section{Cohort 2:}

People with

ever-diagnosed

currently treated asthma

Figure 5.2: A flowchart of case selection.

The first cohort included 218,754 patients with ever-diagnosed asthma, while the second cohort included 101,509 patients with ever-diagnosed currently treated asthma. Table 5.2 shows basic characteristics of both cohorts. 
Table 5.2: Characteristics of the study cohorts: Cohort 1.

\begin{tabular}{llrrrrrr}
\hline & & WIMD 1 & WIMD 2 & WIMD 3 & WIMD 4 & WIMD 5 & All \\
\hline $\begin{array}{llrrrr}\text { Number of } \\
\text { patients }\end{array}$ & $\mathrm{N}$ & 49,597 & 43,681 & 43,570 & 37,228 & 44,678 & 218,754 \\
\multirow{2}{*}{ Gender } & $\%$ & 22.7 & 20.0 & 19.9 & 17.0 & 20.4 & 100.0 \\
Age & \% Females & 53.6 & 52.5 & 51.8 & 51.2 & 50.5 & 52.0 \\
& mean & 36.7 & 38.1 & 39.3 & 39.8 & 40.4 & 38.8 \\
& SD & 20.1 & 20.4 & 20.7 & 20.7 & 20.6 & 20.5 \\
& median & 33.9 & 35.9 & 37.4 & 38.1 & 39.2 & 37.0 \\
& IQR & $19.8-51.5$ & $20.7-53.7$ & $21.6-55.4$ & $22.2-56.0$ & $22.8-56.1$ & $21.3-54.4$
\end{tabular}

Study outcomes

\begin{tabular}{|c|c|c|c|c|c|c|c|}
\hline \multirow{3}{*}{$\begin{array}{l}\text { Asthma-related } \\
\text { GP visits }\end{array}$} & $\mathrm{N}$ & 179,149 & 165,094 & 198,431 & 176,769 & 150,756 & 870,199 \\
\hline & mean count & 4.00 & 4.05 & 4.11 & 4.05 & 3.70 & 3.98 \\
\hline & $\%$ with count $\geq 1$ & 68.6 & 69.0 & 69.3 & 68.8 & 67.3 & 68.6 \\
\hline \multirow[t]{3}{*}{ Asthma reviews } & $\mathrm{N}$ & 111,065 & 97,277 & 97,656 & 82,053 & 98,721 & 486,772 \\
\hline & mean count & 2.24 & 2.21 & 2.24 & 2.23 & 2.20 & 2.25 \\
\hline & $\%$ with count $\geq 1$ & 62.4 & 63.1 & 63.3 & 63.0 & 62.6 & 62.9 \\
\hline \multirow{3}{*}{$\begin{array}{l}\text { Asthma related } \\
\text { A\&E visits }\end{array}$} & $\mathrm{N}$ & 1,011 & 848 & 808 & 702 & 621 & 3,990 \\
\hline & mean count & 0.020 & 0.019 & 0.019 & 0.019 & 0.014 & 0.018 \\
\hline & $\%$ with count $\geq 1$ & 1.5 & 1.4 & 1.4 & 1.4 & 1.1 & 1.3 \\
\hline \multirow{3}{*}{$\begin{array}{l}\text { Asthma related } \\
\text { hospitalisations }\end{array}$} & $\mathrm{N}$ & 2,390 & 1,568 & 1,351 & 1,037 & 980 & 7,326 \\
\hline & mean count & 0.048 & 0.036 & 0.031 & 0.028 & 0.022 & 0.033 \\
\hline & $\%$ with count $\geq 1$ & 2.4 & 2.1 & 1.8 & 1.6 & 1.4 & 1.9 \\
\hline \multicolumn{8}{|l|}{ Prescriptions } \\
\hline \multirow[t]{2}{*}{ SABA inhalers } & mean count & 19.8 & 17.1 & 15.2 & 13.9 & 11.8 & 15.7 \\
\hline & $\%$ with count $\geq 1$ & 71.8 & 71.4 & 71.1 & 70.6 & 69.6 & 70.9 \\
\hline \multirow[t]{2}{*}{ ICS inhalers } & mean count & 6.5 & 5.8 & 5.5 & 5.4 & 5.0 & 5.7 \\
\hline & $\%$ with count $\geq 1$ & 38.4 & 38.0 & 37.8 & 38.6 & 37.8 & 38.1 \\
\hline \multirow{2}{*}{$\begin{array}{l}\text { ICS-LABA } \\
\text { combination } \\
\text { inhalers }\end{array}$} & mean count & 11.7 & 10.8 & 10.0 & 9.1 & 8.2 & 10.0 \\
\hline & $\%$ with count $\geq 1$ & 35.9 & 34.9 & 34.3 & 32.7 & 31.1 & 33.8 \\
\hline $\begin{array}{l}\text { Asthma } \\
\text { medication ratio }\end{array}$ & mean & 0.42 & 0.43 & 0.44 & 0.44 & 0.45 & 0.43 \\
\hline \multirow[t]{2}{*}{ Theophylline } & mean count & 0.7 & 0.6 & 0.5 & 0.4 & 0.3 & 0.5 \\
\hline & $\%$ with count $\geq 1$ & 1.9 & 1.7 & 1.4 & 1.3 & 0.8 & 1.4 \\
\hline \multirow{2}{*}{$\begin{array}{l}\text { Leukotriene } \\
\text { receptor } \\
\text { antagonists }\end{array}$} & mean count & 2.2 & 2.1 & 1.8 & 1.6 & 1.5 & 1.8 \\
\hline & $\%$ with count $\geq 1$ & 8.4 & 8.0 & 7.6 & 7.0 & 6.6 & 7.6 \\
\hline \multirow{2}{*}{$\begin{array}{l}\text { Oral } \\
\text { corticosteroids }\end{array}$} & mean count & 1.9 & 1.8 & 1.8 & 1.7 & 1.4 & 1.7 \\
\hline & $\%$ with count $\geq 1$ & 31.8 & 30.6 & 30.7 & 29.7 & 26.6 & 29.9 \\
\hline \multirow{6}{*}{$\begin{array}{l}\% \text { of patients } \\
\text { with } \geq 1 \text { asthma } \\
\text { prescriptions in } \\
\text { every } N \text { years of } \\
\text { the } 5 \text {-year } \\
\text { follow-up period }\end{array}$} & 0 years & 28.5 & 28.6 & 28.4 & 28.9 & 30.0 & 28.9 \\
\hline & 1 year & 6.0 & 6.1 & 6.2 & 6.2 & 6.5 & 6.2 \\
\hline & 2 years & 5.4 & 5.5 & 5.6 & 5.8 & 5.7 & 5.6 \\
\hline & 3 years & 5.5 & 5.5 & 5.6 & 5.7 & 5.9 & 5.6 \\
\hline & 4 years & 5.1 & 5.1 & 5.3 & 5.7 & 5.7 & 5.4 \\
\hline & 5 years * & 49.4 & 49.1 & 49.0 & 47.8 & 46.3 & 48.3 \\
\hline
\end{tabular}

* Patients in this row represent Cohort 2 (patients with ever-diagnosed currently treated asthma). 
Table 5.2: Characteristics of the study cohorts (continued): Cohort 2.

\begin{tabular}{|c|c|c|c|c|c|c|c|}
\hline & & WIMD 1 & WIMD 2 & WIMD 3 & WIMD 4 & WIMD 5 & All \\
\hline \multirow{2}{*}{$\begin{array}{l}\text { Number of } \\
\text { patients }\end{array}$} & $\mathrm{N}$ & 19,760 & 23,574 & 20,657 & 20,471 & 17,047 & 101,509 \\
\hline & $\%$ & 19.5 & 23.2 & 20.3 & 20.2 & 16.8 & 100 \\
\hline Gender & $\%$ Females & 59.0 & 57.2 & 55.4 & 54.7 & 53.7 & 56.1 \\
\hline \multirow[t]{4}{*}{ Age } & mean & 45.1 & 46.3 & 47.0 & 47.6 & 47.6 & 46.6 \\
\hline & SD & 20.0 & 20.2 & 20.6 & 20.5 & 20.6 & 20.4 \\
\hline & median & 46.5 & 47.8 & 48.6 & 48.7 & 48.8 & 48.0 \\
\hline & IQR & $30.4-60.7$ & $31.6-62.3$ & $32.2-63.3$ & $33.4-63.7$ & $33.6-63.5$ & $32.1-62.7$ \\
\hline \multicolumn{8}{|l|}{ Study outcomes } \\
\hline \multirow{3}{*}{$\begin{array}{l}\text { Asthma-related } \\
\text { GP visits }\end{array}$} & $\mathrm{N}$ & 155,206 & 137,387 & 138,218 & 114,315 & 125,401 & 670,527 \\
\hline & mean count & 6.58 & 6.65 & 6.75 & 6.71 & 6.35 & 6.61 \\
\hline & $\%$ with count $\geq 1$ & 98.0 & 98.0 & 98.3 & 98.2 & 98.7 & 98.2 \\
\hline \multirow[t]{3}{*}{ Asthma reviews } & $N$ & 90,765 & 79,034 & 78,574 & 65,497 & 78,302 & 392,172 \\
\hline & mean count & 3.9 & 3.8 & 3.8 & 3.8 & 4.0 & 3.9 \\
\hline & $\%$ with count $\geq 1$ & 93.6 & 94.5 & 94.6 & 95.0 & 96.3 & 94.7 \\
\hline \multirow{3}{*}{$\begin{array}{l}\text { Asthma related } \\
\text { A\&E visits }\end{array}$} & $\mathrm{N}$ & 825 & 694 & 694 & 552 & 506 & 3,271 \\
\hline & mean count & 0.035 & 0.034 & 0.034 & 0.032 & 0.026 & 0.032 \\
\hline & $\%$ with count $\geq 1$ & 2.4 & 2.3 & 2.4 & 2.3 & 1.9 & 2.3 \\
\hline \multirow{3}{*}{$\begin{array}{l}\text { Asthma related } \\
\text { hospitalisations }\end{array}$} & $\mathrm{N}$ & 2,193 & 1,381 & 1,214 & 895 & 874 & 6,557 \\
\hline & mean count & 0.093 & 0.067 & 0.059 & 0.053 & 0.044 & 0.065 \\
\hline & $\%$ with count $\geq 1$ & 4.3 & 3.8 & 3.3 & 3.0 & 2.6 & 3.4 \\
\hline \multicolumn{8}{|l|}{ Prescriptions } \\
\hline \multirow[t]{2}{*}{ SABA inhalers } & mean count & 38.1 & 33.0 & 29.3 & 27.3 & 23.7 & 30.7 \\
\hline & $\%$ with count $\geq 1$ & 98.6 & 98.1 & 97.4 & 97.5 & 97.5 & 97.8 \\
\hline \multirow[t]{2}{*}{ ICS inhalers } & mean count & 12.2 & 10.9 & 10.4 & 10.4 & 9.9 & 10.8 \\
\hline & $\%$ with count $\geq 1$ & 53.9 & 53.1 & 53.8 & 55.4 & 55.2 & 54.2 \\
\hline \multirow{2}{*}{$\begin{array}{l}\text { ICS-LABA } \\
\text { combination } \\
\text { inhalers }\end{array}$} & mean count & 23.7 & 21.9 & 20.5 & 19.0 & 17.7 & 20.7 \\
\hline & $\%$ with count $\geq 1$ & 64.9 & 63.5 & 62.3 & 60.3 & 59.3 & 62.2 \\
\hline $\begin{array}{l}\text { Asthma } \\
\text { medication ratio }\end{array}$ & mean & 0.48 & 0.50 & 0.52 & 0.52 & 0.54 & 0.51 \\
\hline \multirow[t]{2}{*}{ Theophylline } & mean count & 1.5 & 1.3 & 1.0 & 0.9 & 0.6 & 1.1 \\
\hline & $\%$ with count $\geq 1$ & 3.8 & 3.5 & 2.9 & 2.7 & 1.8 & 3.0 \\
\hline \multirow{2}{*}{$\begin{array}{l}\text { Leukotriene } \\
\text { receptor } \\
\text { antagonists }\end{array}$} & mean count & 4.5 & 4.3 & 3.6 & 3.4 & 3.2 & 3.8 \\
\hline & $\%$ with count $\geq 1$ & 15.9 & 15.2 & 14.3 & 13.4 & 13.1 & 14.4 \\
\hline \multirow{2}{*}{$\begin{array}{l}\text { Oral } \\
\text { corticosteroids }\end{array}$} & mean count & 3.5 & 3.3 & 3.2 & 3.1 & 2.5 & 3.1 \\
\hline & $\%$ with count $\geq 1$ & 52.0 & 49.7 & 49.1 & 48.6 & 44.7 & 49.0 \\
\hline
\end{tabular}

* All patients in this cohort had one or more prescriptions in every year between 2010-2014. 


\section{Asthma prevalences in the source population across WIMD quintiles}

The prevalence of ever-diagnosed asthma in the source population at the beginning of 2010 was $11.2 \%$, ranging from 10.9 in the next least deprived areas (WIMD 4) and 11.0 in the least deprived areas (WIMD 5) to $12.0 \%$ in the most deprived areas (WIMD 1). The prevalence of ever-diagnosed, currently treated asthma during the same year was 6.8\%, ranging from 6.2 in the least deprived areas (WIMD 5) to $7.4 \%$ in the most deprived areas (WIMD 1).

\section{Distribution of WIMD quintiles}

In both cohorts, the quintiles of the WIMD rank had comparable shares (Table 5.2), with the next least deprived quintile (WIMD 4) having the least proportion of individuals (17.0\% and $16.8 \%$ in Cohorts 1 and 2 ) and the most deprived quintile (WIMD 1) having the highest proportion (22.7\% and 23.2\% in Cohorts 1 and 2).

\section{Distribution of gender}

Females were $52.0 \%$ of Cohort 1 and $56.1 \%$ of Cohort 2 . In both cohorts, the more deprived areas had higher proportions of females - the gradient in Cohort 1 ranged from 50.5\% in WIMD 5 areas to 53.6\% in WIMD 1 areas and in Cohort 2 from $53.7 \%$ to $59.0 \%$ in those areas, respectively.

\section{Distribution of age}

Cohort 1 was younger than Cohort 2. The mean age in Cohort 1 was 38.8 years with a standard deviation (SD) of 20.5, a median of 37.0, and an inter-quartile range of 21.3-54.4 years. In Cohort 2 , the mean age was 46.6 years (SD $=20.4$ ), and the median was 48.0 with an inter-quartile range of 32.1-62.7 years.

There were more young people in areas of higher deprivation. The mean age ranged in Cohort 1 from 36.7 in the most deprived areas (WIMD 1) to 40.4 in the least deprived areas (WIMD 5) and in Cohort 2 from 45.1 in WIMD 1 areas to 47.6 in WIMD 5 areas. Kruskal-Wallis rank sum test confirmed the unequal distribution of age ranks across the five deprivation groups in both cohorts $\left(\chi^{2}=\right.$ 923.8 and 231.0 for Cohorts 1 and 2, with p-values $<0.0001$ ). Figure 5.3 shows the distribution of age for each of the WIMD quintiles. 
Cohort 1

WIMD 5

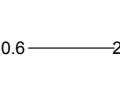

WIMD 4
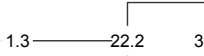

(a)

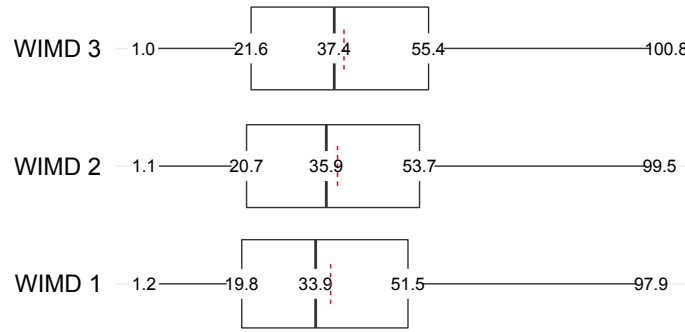

(b)

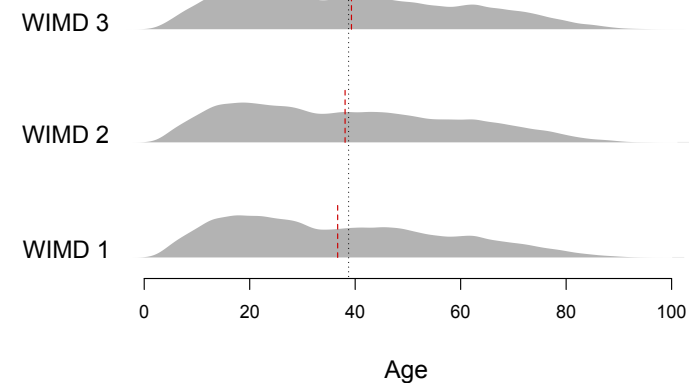

Cohort 2
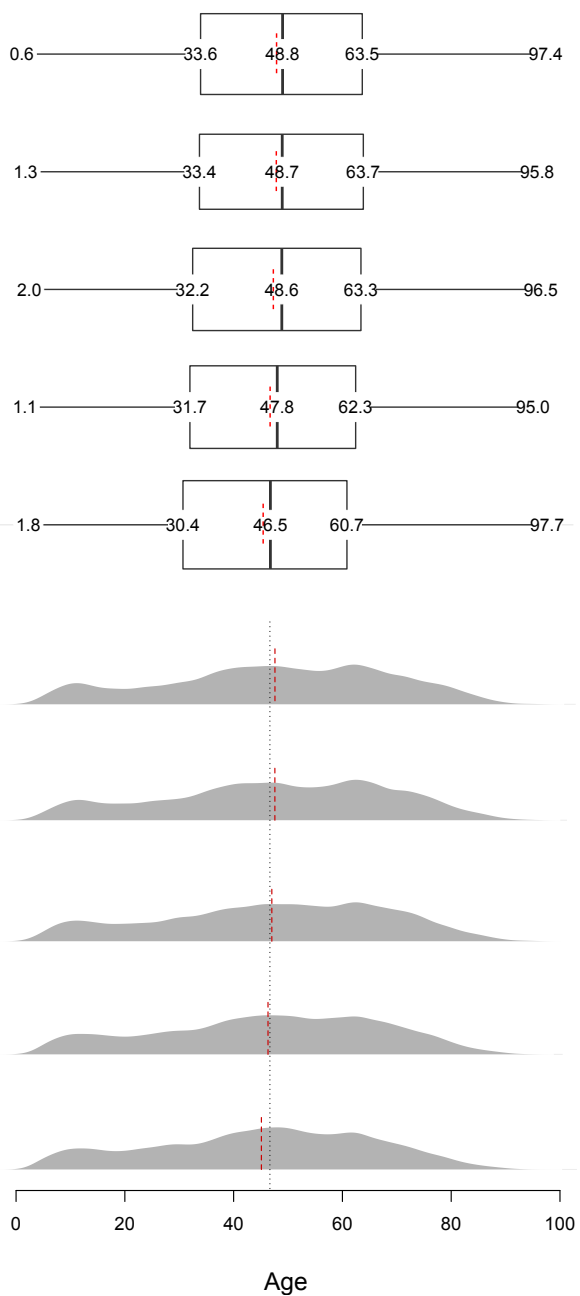

Figure 5.3: Distribution of age across WIMD overall rank quintiles in the study cohorts. (a) Boxplots showing the minimum, maximum, first and third quartiles, median (black lines), and mean (red dotted lines) of age for each WIMD quintile. (b) Beanplots showing the density of age distribution for each WIMD quintile. The large black dotted line represents the overall mean, and the smaller red dotted lines represent the per WIMD quintile means.

\section{Distribution of asthma prescriptions across the deprivation quintiles}

In Cohort 1, the more affluent areas had more patients with intermittent or no asthma treatment over the five follow-up years (Table 5.2). Conversely, the higher the deprivation, the higher the percentage of patients with continuous asthma prescriptions.

In Cohort 2, where all patients received asthma prescriptions in each of the followup years, there was a remarkable gradient of more prescriptions with higher deprivation. For example, in the most deprived areas the average number of SABA inhalers over five years per patient was 38.1 compared with 23.7 in the most affluent areas, while the gap for ICS inhalers was 12.1 to 9.9, for ICS-LABA combi- 
nation inhalers was 23.7 to 17.7 , and for oral corticosteroids (OCS) was 3.5 to 2.5, respectively, between those areas. It is worth noting that the average controller to controller-and-rescuer medication ratio in the most deprived areas (0.48) was lower than that in the least deprived areas (0.54).

\section{Percentages of patients with outcome events overall}

$68.6 \%$ and $62.9 \%$ of Cohort 1 patients had recorded asthma GP visits and asthma reviews, respectively, in the 5-year follow-up period. In comparison, 98.2\% and 94.7\% of patients in Cohort 2 had no recorded asthma GP visits and asthma reviews, respectively. In both cohorts, however, only very few patients had recorded asthma-related A\&E visits and hospital admissions (1.3\% and 1.9\% in Cohort 1, and $2.3 \%$ and $3.4 \%$ in Cohort 2). Histograms of the outcome variable counts illustrate the skewed data in both cohorts (Figure 5.4).

\section{Average number of outcome events overall}

The average counts of outcome events over the five-year follow-up period were significantly higher in Cohort 2 than in Cohort 1; on averages there were 4.0 and 6.6 asthma-related GP visits, 2.2 and 3.9 asthma reviews, 0.018 and 0.032 asthmarelated A\&E visits, and 0.033 and 0.065 asthma-related hospital admissions in Cohorts 1 and 2, respectively.

\section{Average counts of outcome events in each WIMD quintile}

Without adjustment to age group and gender, in Cohort 1, the most deprived areas had on average more outcome events per patient than the least deprived areas (Table 5.2): 4.00 vs. 3.70 asthma-related visits to GPs, 2.24 vs. 2.21 asthma reviews, 0.020 vs. 0.014 asthma-related visits to $A \& E$, and 0.048 vs. 0.022 asthma-related hospital admissions.

In Cohort 2, on average, the most deprived areas had also more asthma-related visits to GPs (6.58 vs. 6.35), more asthma-related visits to A\&E (0.035 vs. 0.026), more asthma-related hospital admissions (0.093 vs. 0.044), but less asthma reviews (3.85 vs. 3.96) per patient than the least deprived areas. 
Cohort 1

Asthma GP Visits

Asthma Reviews

Asthma A\&E Visits

Asthma Hospitalisations
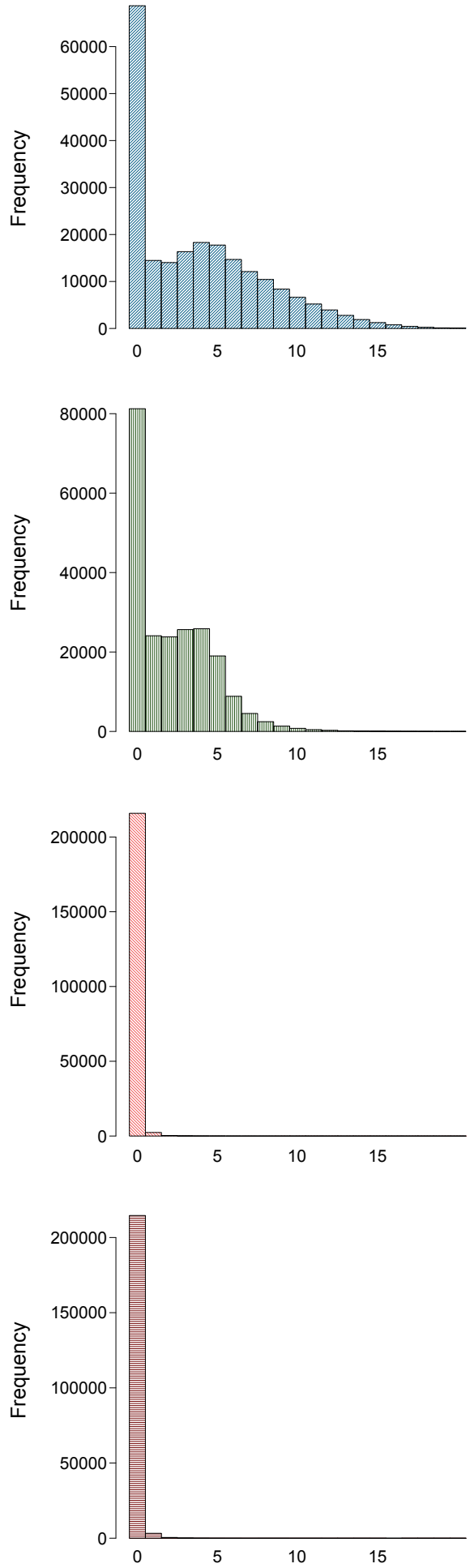

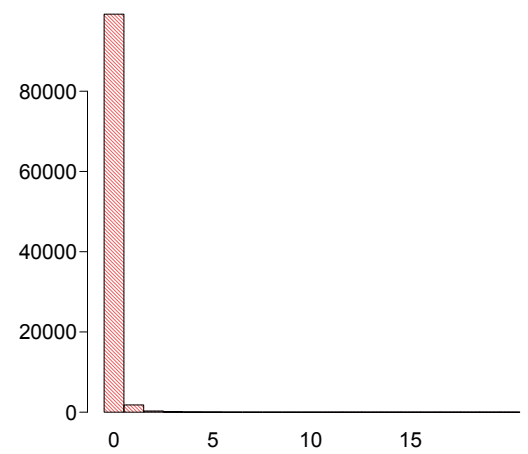

Cohort 2
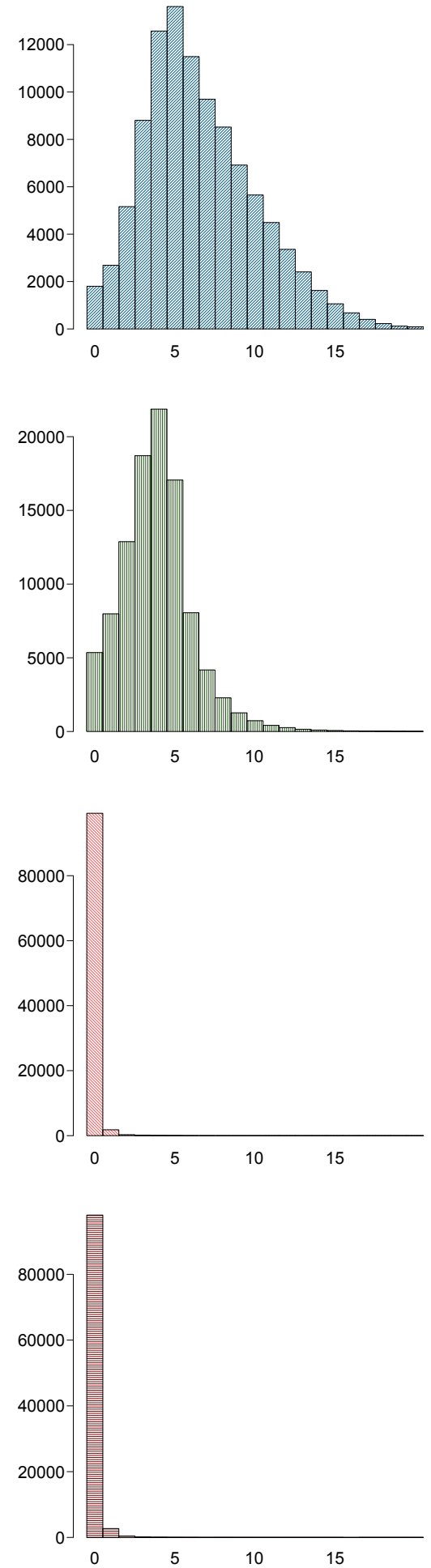

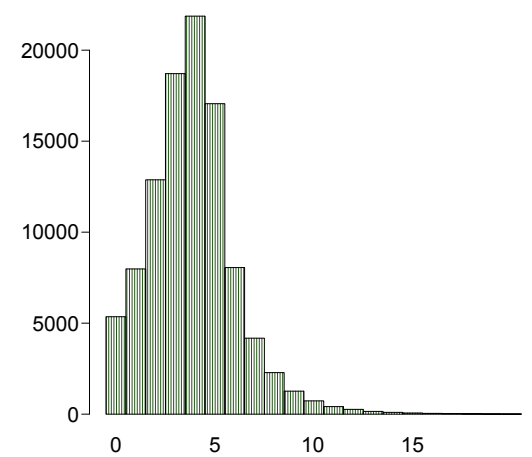

Figure 5.4: Histograms of outcome event counts in the study cohorts. 

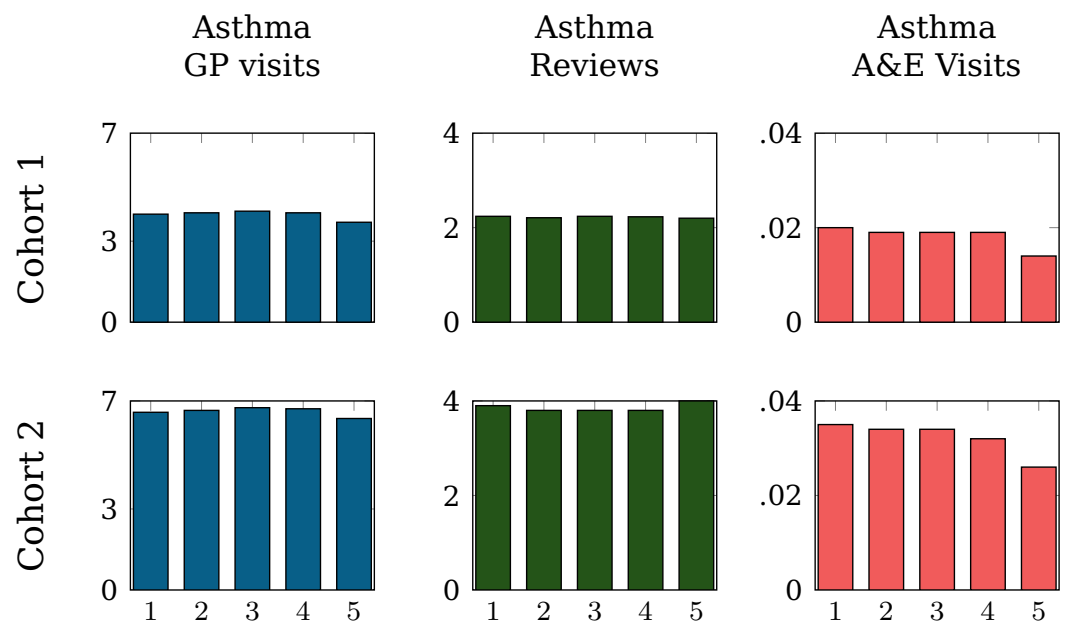

Asthma

Hospitalisations

Figure 5.5: Average counts of the outcome events between 2010 and 2014 per WIMD rank quintiles in Cohort 1 and Cohort 2.

\section{Gradients}

In both cohorts, despite the gap in asthma GP visits and reviews on the two sides of the deprivation scale, there were no consistent gradients across the whole deprivation scale (Figure 5.5). The three middle deprivation areas (WIMD 2, 3, and 4) had on average more asthma GP visits than both the most (WIMD 1) and least deprivation (WIMD 5) areas, with the middle deprivation areas having the highest averages in both cohorts. In contrast, for asthma reviews, the WIMD 2, 3 and 4 areas had smaller average counts than WIMD 1 and 5 areas in Cohort 2, with no clear pattern in Cohort 1.

In both cohorts, although the average counts of asthma A\&E visits decreased with less deprivation, there was no gradient since the least deprived areas (WIMD 5) had significantly smaller average counts than the other four more deprived areas. Finally, there were clear gradients of more asthma hospitalisations with higher deprivation in both cohorts.

\subsubsection{Zero-inflated negative binomial regression (ZINB) mod- els}

The outputs of the four ZINB models for each study cohort are shown in Table 5.3. 


\subsubsection{Incidence rate ratios of study outcomes across deprivation quin- tiles}

Following adjustments for age group and gender, I found statistically significant differences in incidence rates of the outcome events between the WIMD quintiles. For asthma-related GP visits, all the first four WIMD quintiles had statistically significant IRRs compared to the fifth quintile. Over the five-year follow-up period, there were $7.8 \%$ more predicted events per patient in the most deprived areas (WIMD 1) than in the least deprived areas (WIMD 5), with predicted counts of 3.94 and 3.65, respectively. In Cohort 2, the difference in predicted events between the WIMD 1 and 5 areas was smaller (3.6\%), with predicted counts of 6.37 and 6.15 in those areas, respectively. The WIMD 2, 3, and 4 areas had slightly higher IRRs than WIMD 1 areas in both cohorts.

For asthma reviews, the only statistically significant IRR at the 0.05 level in Cohort 1 was 1.037 (1.025-1.048) for WIMD 1; there were 3.7\% more predicted events in the least deprived areas (WIMD 1, 2.34 events) compared to the least deprived areas (WIMD 5,2.25). In Cohort 2, all the IRRs were statistically significant and slightly less than 1 , with an IRR of 0.99 (0.978-0.999) for the most deprived areas compared to the least deprived areas (with predicted number of asthma reviews of 3.81 and 3.86, respectively).

For asthma A\&E visits, the IRRs for WIMD 1 to 4 areas in Cohort 1 were all statistically significant, ranging from 1.368 for the most deprived areas (WIMD 1) to 1.298 for the middle deprivation areas (WIMD 3). There were $36.8 \%$ more predicted asthma-related $A \& E$ visits in the most deprived areas than in the least deprived areas (0.023 vs. 0.016 visits). In Cohort 2 , the IRRs were slightly lower than those in Cohort 1, ranging from 1.229 for the most deprived areas (WIMD 1) to 1.257 for the middle deprivation areas (WIMD 3). There were $22.9 \%$ more predicted asthma-related $A \& E$ visits in the most deprived areas than in the least deprived areas (0.043 vs. 0.035 visits).

In both cohorts, the clear gaps in the predicted asthma-related GP visits and A\&E visits were between the four more deprived quintiles (WIMD 1-4) together, which showed relatively similar estimates, and the least deprived quintile (WIMD 5).

Lastly, a clear and steep social gradient existed for asthma-related hospital admissions. In Cohort 1, in the most deprived areas, there were $123.2 \%$ more pre- 
Cohort 1

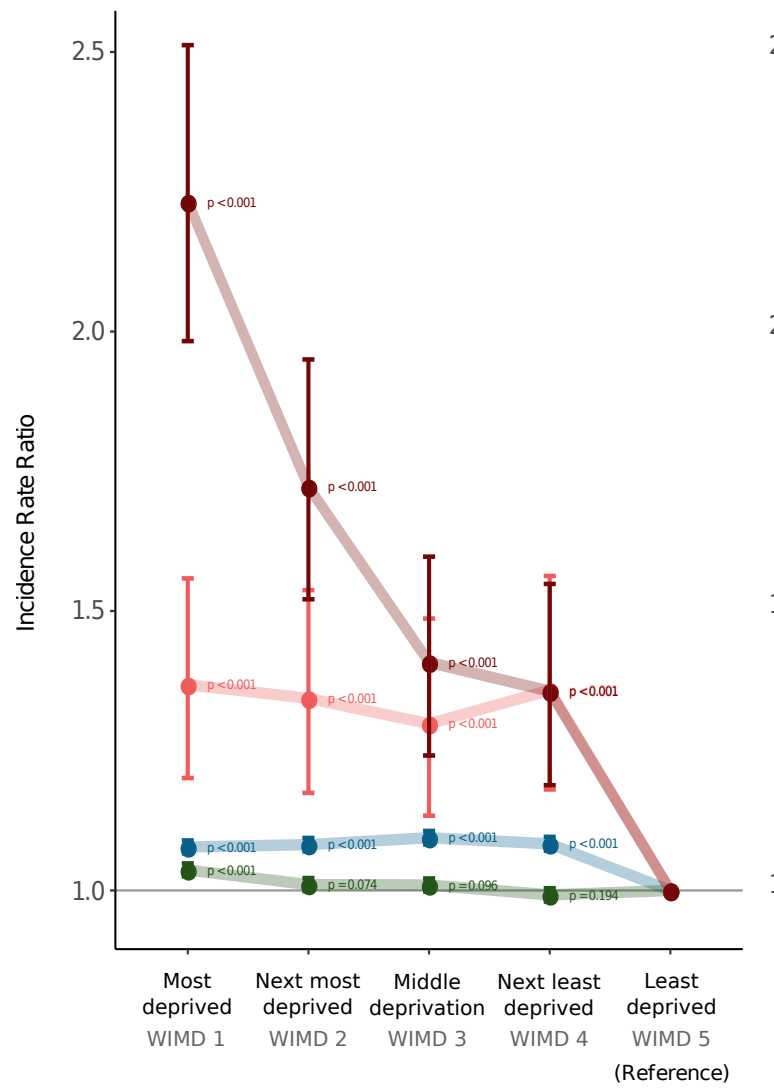

Cohort 2

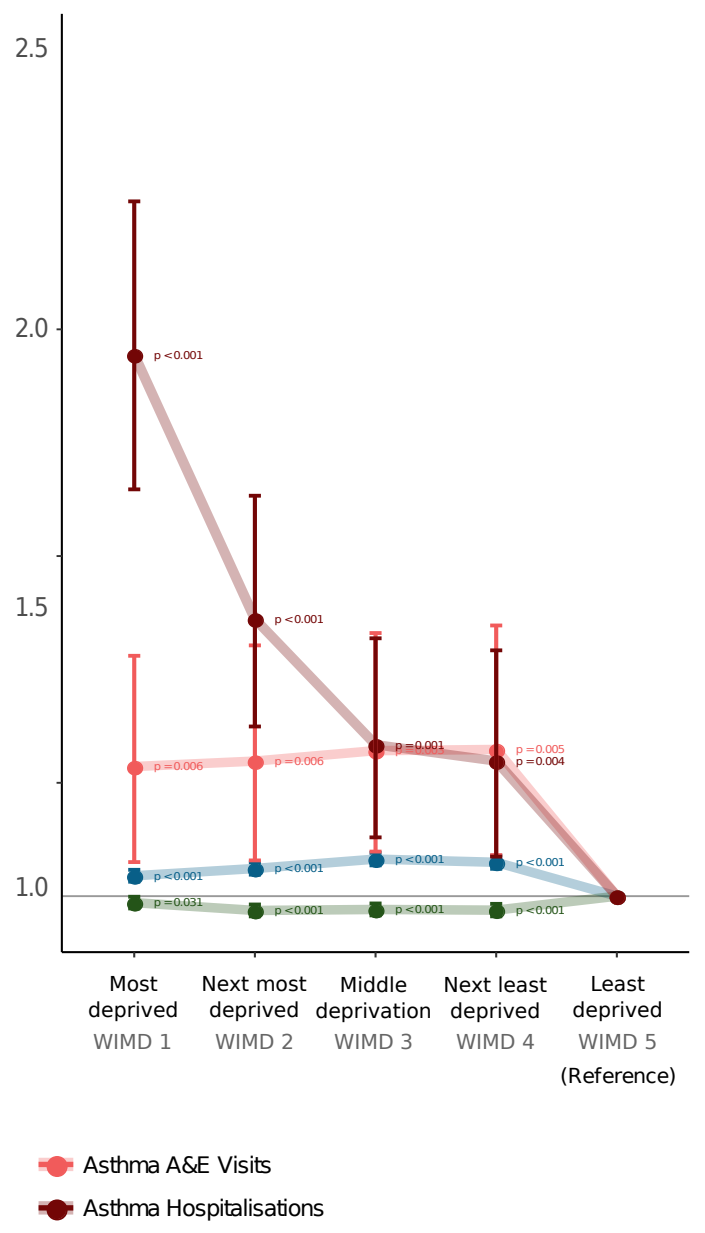

Figure 5.6: Incidence rate ratios with $95 \%$ confidence intervals in both study cohorts for each of the outcome variables in each of the deprivation quintiles relative to the least deprived quintile, controlled for age group and gender.

dicted asthma-related hospital admissions than in the least deprived areas $(0.074$ vs 0.033 predicted asthma admissions, IRR $=2.232$ [1.983-2.512]). The gap was slightly smaller in Cohort 2, where there were 95.5\% more predicted asthmarelated hospital admissions in the most deprived areas compared to the least deprived areas ( 0.139 vs. 0.071 predicted asthma admissions, $I R R=1.955[1.718$ 2.226]).

For Cohort 1, all p-values for the IRRs of the outcome variables were less than 0.0001, except those for in the asthma reviews model which were above 0.05. For Cohort 2, most of the p-values were less than 0.001; the IRRs of asthma reviews and A\&E visits between WIMD 1 vs. WIMD 5 had p-values of 0.031 and 0.006, respectively.

A visualisation of IRRs of the outcome variables for the WIMD rank quintiles is shown in Figure 5.6. 


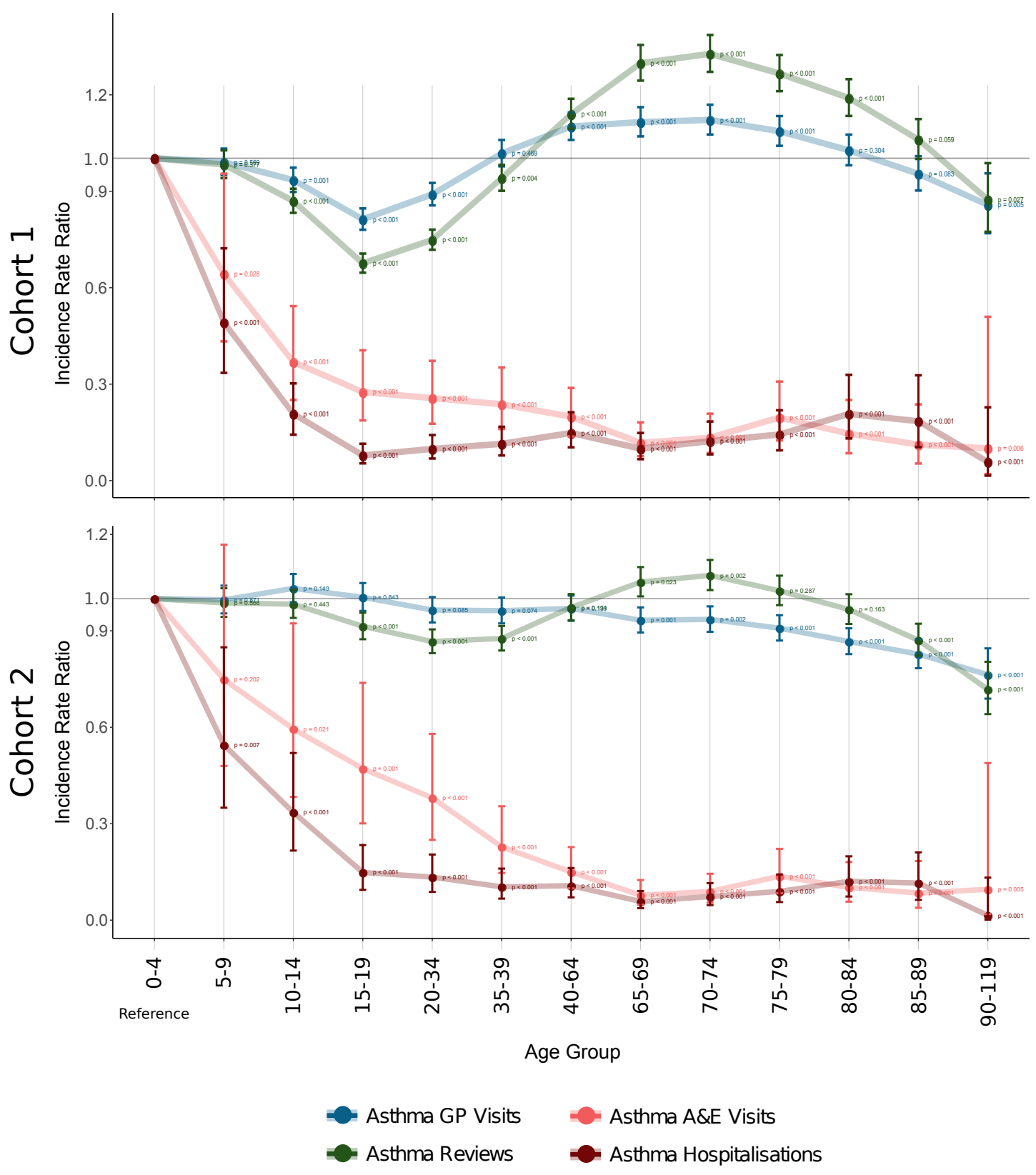

Figure 5.7: Incidence rate ratios with $95 \%$ confidence intervals for each of the outcome variables in age groups relative to the youngest age group (0-4 years), controlled for deprivation quintile and gender.

\subsubsection{Incidence rate ratios of study outcomes for age groups and gender}

IRRs of the outcome variables across age groups are shown in Figure $5.7 .^{2}$

In both study cohorts, the variations of IRRs, relative to the reference age group 04 years, between age groups were relatively small for asthma GP visits and asthma reviews but were large for $A \& E$ visits and admissions.

\footnotetext{
${ }^{2}$ It is worth noting that since age was calculated at the beginning of the follow-up period, IRRs for patients in a given age group covered their next five years of life; e.g., for patients in the 15-19 age group, the IRRs covered periods starting at the age of 15 to 19 and ending at the age of 20 to 24 , depending on patient's age at the beginning of the follow-up period.
} 
Table 5.3: Outputs of the zero-inflated negative binomial models. The values shown include the incidence rate ratios and their $95 \%$ confidence intervals.

\section{Cohort 1}

\begin{tabular}{|c|c|c|c|c|c|c|c|c|c|c|}
\hline & \multicolumn{10}{|c|}{ Outcome Variable $\sim$ WIMD Quintile + Age Group + Gender | 1} \\
\hline & \multicolumn{10}{|c|}{ Outcome variables } \\
\hline & \multicolumn{2}{|c|}{$\begin{array}{l}\text { Asthma-related } \\
\text { GP visits }\end{array}$} & \multicolumn{3}{|c|}{ Asthma reviews } & \multicolumn{3}{|c|}{$\begin{array}{c}\text { Asthma-related } \\
\text { A\&E visits }\end{array}$} & \multicolumn{2}{|c|}{$\begin{array}{l}\text { Asthma-related } \\
\text { hospitalisations }\end{array}$} \\
\hline & IRR sig & $95 \% \mathrm{CI}$ & IRR & sig & $95 \% \mathrm{CI}$ & IRR & $\operatorname{sig}$ & $95 \% \mathrm{CI}$ & IRR sig & $95 \% \mathrm{CI}$ \\
\hline \multirow{2}{*}{\multicolumn{11}{|c|}{$\begin{array}{l}\text { Deprivation Quintile } \\
\text { (reference level: WIMD 5) }\end{array}$}} \\
\hline & & & & & & & & & & \\
\hline WIMD1 & $1.078^{* * *}$ & $1.066-1.090$ & 1.037 & *** 1 & $1.025-1.048$ & 1.368 & *** & $1.201-1.558$ & $2.232 * \star \star$ & $1.983-2.512$ \\
\hline WIMD2 & $1.082 * * *$ & 1.070-1.094 & 1.011 & & $0.999-1.022$ & 1.344 & *** & $1.175-1.537$ & $1.722 * \star \star$ & $1.521-1.950$ \\
\hline WIMD3 & $1.094^{* * *}$ & 1.082-1.107 & 1.010 & & $0.998-1.022$ & 1.298 & $* \star *$ & $1.134-1.487$ & $1.408^{* * *}$ & $1.241-1.597$ \\
\hline WIMD4 & $1.083^{* * *}$ & $1.071-1.096$ & 0.992 & & $0.980-1.004$ & 1.358 & $* \star *$ & $1.181-1.563$ & 1.357 *** & $1.188-1.548$ \\
\hline \multicolumn{11}{|c|}{$\begin{array}{l}\text { Age Group } \\
\text { (reference level: 0-4 years) }\end{array}$} \\
\hline $5-9$ & 0.988 & $0.948-1.030$ & 0.980 & & $0.938-1.024$ & 0.641 & * & $0.431-0.952$ & 0.490 *** & $0.334-0.720$ \\
\hline $10-14$ & $0.932 * \star *$ & $0.895-0.971$ & 0.867 & $* * *$ & $0.830-0.905$ & 0.367 & $\star \star \star *$ & $0.249-0.541$ & $0.206 * \star \star$ & $0.142-0.301$ \\
\hline $15-19$ & $0.810 * * *$ & $0.778-0.844$ & 0.674 & $* * *$ & $0.645-0.704$ & 0.274 & $\star \star \star *$ & $0.186-0.404$ & $0.077^{\star \star *}$ & $0.053-0.113$ \\
\hline $20-34$ & $0.888^{* * *}$ & $0.854-0.923$ & 0.746 & $* * *$ & $0.716-0.778$ & 0.255 & 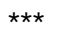 & $0.176-0.371$ & $0.097^{* \star *}$ & $0.068-0.140$ \\
\hline $35-39$ & 1.015 & $0.975-1.057$ & 0.938 & $* *$ & 0.899-0.979 & 0.236 & 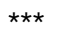 & $0.159-0.350$ & $0.113^{* * *}$ & $0.077-0.166$ \\
\hline $40-64$ & $1.098^{* * *}$ & 1.057-1.142 & 1.137 & $* * * 1$ & $1.091-1.185$ & 0.198 & 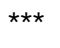 & $0.136-0.287$ & $0.147^{* \star *}$ & $0.102-0.211$ \\
\hline $65-69$ & $1.112 * * *$ & $1.068-1.159$ & 1.295 & $* * * 1$ & $1.241-1.352$ & 0.116 & 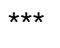 & $0.075-0.180$ & $0.098^{* * *}$ & $0.066-0.147$ \\
\hline $70-74$ & 1.119 *** & $1.074-1.166$ & 1.324 & $* * * 1$ & $1.268-1.383$ & 0.132 & $\star \star * \star$ & $0.085-0.207$ & $0.121 * * *$ & $0.081-0.183$ \\
\hline $75-79$ & $1.084^{* * *}$ & $1.039-1.131$ & 1.263 & $* * * 1$ & $1.208-1.321$ & 0.195 & $\star \star * \star$ & $0.124-0.307$ & 0.142 *** & $0.093-0.217$ \\
\hline $80-84$ & 1.025 & $0.978-1.073$ & 1.187 & $* * * 1$ & $1.131-1.246$ & 0.145 & $* * *$ & $0.084-0.249$ & 0.206 *** & $0.130-0.327$ \\
\hline $85-89$ & 0.952 & $0.900-1.007$ & 1.058 & . & $0.998-1.122$ & 0.111 & $\star * *$ & $0.052-0.236$ & $0.184 * \star \star$ & $0.103-0.326$ \\
\hline $90-119$ & $0.855 * \star$ & $0.767-0.953$ & 0.872 & * & $0.772-0.985$ & 0.099 & $\star \star$ & $0.019-0.508$ & $0.057^{\star * *}$ & $0.014-0.227$ \\
\hline \multirow{2}{*}{\multicolumn{11}{|c|}{$\begin{array}{l}\text { Gender } \\
\text { (reference level: male) }\end{array}$}} \\
\hline & & & & & & & & & & \\
\hline Female & $1.042 * * *$ & $1.034-1.049$ & 1.100 & $* * * 1$ & $1.092-1.109$ & 1.708 & $* * *$ & $1.568-1.860$ & $2.310 * * *$ & $2.132-2.503$ \\
\hline Intercept & $5.051 * * *$ & $4.857-5.252$ & 2.994 & $* * * 2$ & $2.873-3.121$ & 0.042 & $* \star \star$ & $0.029-0.061$ & $0.088 * * *$ & $0.061-0.127$ \\
\hline $\begin{array}{l}\text { Log } \\
\text { likelihood }\end{array}$ & -52 & 20934.8 & & -415 & 5837.7 & & & 7497.8 & & 4249.9 \\
\hline
\end{tabular}

Significance codes: $\cdot p<0.1 ;{ }^{*} p<0.05 ;{ }^{* *} p<0.01 ;{ }^{* *} p<0.001$; IRR: incidence rate ratio. 
Table 5.3: Outputs of the zero-inflated negative binomial models (continued). The values shown include the incidence rate ratios, statistical significance, and their $95 \%$ confidence intervals.

\section{Cohort 2}

\begin{tabular}{|c|c|c|c|c|c|c|c|c|c|}
\hline & \multicolumn{9}{|c|}{ Outcome Variable WIMD Quintile + Age Group + Gender | 1} \\
\hline & \multicolumn{9}{|c|}{ Outcome variables } \\
\hline & \multicolumn{2}{|c|}{$\begin{array}{l}\text { Asthma-related } \\
\text { GP visits }\end{array}$} & \multicolumn{2}{|c|}{ Asthma reviews } & \multicolumn{3}{|c|}{$\begin{array}{c}\text { Asthma-related } \\
\text { A\&E visits }\end{array}$} & \multicolumn{2}{|c|}{$\begin{array}{l}\text { Asthma-related } \\
\text { hospitalisations }\end{array}$} \\
\hline & IRR sig & $95 \% \mathrm{CI}$ & IRR sig & $95 \% \mathrm{CI}$ & IRR & sig & $95 \% \mathrm{CI}$ & IRR sig & $95 \% \mathrm{CI}$ \\
\hline \multicolumn{10}{|c|}{$\begin{array}{l}\text { Deprivation Quintile } \\
\text { (reference level: WIMD 5) }\end{array}$} \\
\hline WIMD1 & $1.036 * * *$ & $1.025-1.046$ & $0.989 *$ & $0.978-0.999$ & 1.229 & ** & $1.060-1.424$ & $1.955^{\star * \star}$ & $1.718-2.226$ \\
\hline WIMD2 & $1.049 * * *$ & $1.038-1.060$ & $0.975^{* * *}$ & $0.964-0.985$ & 1.238 & ** & $1.063-1.442$ & $1.489^{\star * *}$ & 1.299-1.706 \\
\hline WIMD3 & $1.065 * * \star$ & $1.054-1.076$ & $0.977^{\star \star \star}$ & $0.966-0.987$ & 1.257 & $\star \star$ & $1.079-1.464$ & $1.267^{\star \star \star}$ & $1.104-1.455$ \\
\hline WIMD4 & $1.060 * \star \star$ & $1.048-1.071$ & $0.975^{* \star *}$ & $0.964-0.986$ & 1.259 & $\star \star$ & $1.072-1.478$ & $1.238 * \star$ & $1.070-1.434$ \\
\hline \multicolumn{10}{|l|}{ Age Group } \\
\hline \multicolumn{10}{|c|}{ (reference level: 0-4 years) } \\
\hline $5-9$ & 0.996 & $0.954-1.041$ & 0.987 & $0.943-1.032$ & 0.749 & & $0.480-1.168$ & $0.545 * *$ & $0.350-0.849$ \\
\hline $10-14$ & 1.032 & 0.989-1.077 & 0.983 & $0.940-1.027$ & 0.595 & * & $0.383-0.923$ & $0.335^{* * *}$ & $0.216-0.520$ \\
\hline $15-19$ & 1.004 & $0.962-1.049$ & $0.914 * * \star$ & $0.874-0.956$ & 0.471 & $\star \star$ & $0.301-0.738$ & $0.148^{* \star *}$ & $0.094-0.234$ \\
\hline $20-34$ & 0.965 & $0.926-1.005$ & $0.867^{\star \star *}$ & $0.830-0.904$ & 0.380 & $* * *$ & $0.250-0.579$ & $0.134^{\star * \star}$ & $0.088-0.204$ \\
\hline $35-39$ & 0.963 & $0.923-1.004$ & $0.876^{* * *}$ & $0.839-0.915$ & 0.228 & $* * *$ & $0.147-0.354$ & $0.104 * * *$ & $0.067-0.160$ \\
\hline $40-64$ & 0.969 & 0.931-1.009 & 0.973 & $0.933-1.014$ & 0.150 & 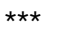 & $0.099-0.227$ & $0.107^{\star * \star}$ & $0.071-0.162$ \\
\hline $65-69$ & $0.933 * *$ & $0.895-0.973$ & $1.052 *$ & $1.007-1.098$ & 0.078 & $\star \star \star ~$ & $0.048-0.125$ & $0.058^{* * *}$ & $0.037-0.091$ \\
\hline $70-74$ & $0.936 * *$ & $0.897-0.976$ & $1.072 * \star$ & $1.027-1.120$ & 0.089 & $\star \star \star ~$ & $0.055-0.144$ & $0.073^{* * *}$ & $0.047-0.115$ \\
\hline $75-79$ & $0.908 * * *$ & $0.870-0.949$ & 1.025 & $0.980-1.072$ & 0.137 & $\star \star \star *$ & $0.084-0.222$ & $0.089 * \star \star$ & $0.056-0.142$ \\
\hline $80-84$ & $0.867 * \star \star *$ & $0.828-0.908$ & 0.966 & $0.921-1.014$ & 0.102 & $\star \star \star *$ & $0.057-0.181$ & $0.121 * * \star$ & $0.074-0.199$ \\
\hline $85-89$ & $0.828^{* \star *}$ & $0.784-0.874$ & $0.871 * \star \star *$ & $0.822-0.922$ & 0.084 & $\star \star \star ~$ & $0.039-0.184$ & $0.116^{* * *}$ & $0.063-0.211$ \\
\hline $90-119$ & $0.763 * * *$ & $0.689-0.845$ & $0.718^{\star \star \star}$ & $0.641-0.804$ & 0.096 & $\star \star$ & $0.019-0.489$ & $0.015^{* * *}$ & $0.002-0.132$ \\
\hline \multirow{2}{*}{\multicolumn{10}{|c|}{$\begin{array}{l}\text { Gender } \\
\text { (reference level: male) }\end{array}$}} \\
\hline & & & & & & & & & \\
\hline Female & 1.031 *** & $1.025-1.038$ & $1.061^{* * *}$ & $1.054-1.069$ & 1.597 & 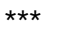 & $1.448-1.760$ & $2.135 * * \star$ & $1.954-2.334$ \\
\hline Intercept & $6.536^{* * *}$ & $6.275-6.808$ & 4.068 *** & $3.900-4.244$ & 0.085 & $* \star *$ & $0.056-0.129$ & $0.208 * \star *$ & $0.137-0.315$ \\
\hline $\begin{array}{l}\text { Log } \\
\text { likelihood }\end{array}$ & -26 & 67255.4 & -21 & 8843.2 & & & 2495.4 & & 8616.1 \\
\hline
\end{tabular}

Significance codes: $\cdot p<0.1 ;{ }^{*} p<0.05 ;{ }^{* *} p<0.01 ;{ }^{* * *} p<0.001$; IRR: incidence rate ratio. 
The IRRs patterns across age groups for asthma reviews, A\&E visits, and admissions were similar between the two cohorts, which, however, differed in the patterns of asthma GP visit IRRs.

In Cohort 1, the rates of asthma GP visits gradually decreased with age in the youngest age groups with a minimum of 0.81 in the 15-19 group (relative to the reference age group 0-4) year before increasing again towards a maximum of 1.12 in the 70-74 group and decreasing again to 0.85 in the 90-119 group. In contrast, in Cohort 2 the estimates peaked at 1.03 in the 10-14 before decreasing slightly and steadily with older age, reaching 0.76 for the age group 90-119.

In Cohort 1, asthma review IRRs pattern across age groups was similar to that of asthma GP visits, although it had a higher magnitude of variation. In Cohort 2, the pattern was similar to that in Cohort 1 but with less variation between age groups.

For asthma-related A\&E visits, there was a general gradient of decreasing IRRs with older age in both cohorts. In Cohort 1, the gradient was steep in the younger age groups, i.e. under 19-year old, before flattening between the age groups 15-18 and 35-39 years and decreasing slightly further in the older age groups. However, in Cohort 2, the gradient was less steep in the younger groups and steadily decreased up to the 65-68 age group.

The two cohorts had almost similar patterns of asthma-related hospitalisation IRRs, showing steeper, decreasing gradients with age in children, before stabilising at the 15-18 age group with slight fluctuations over the older groups.

Females had overall 4\% more predicted asthma-related GP visits, 10\% more asthma reviews, $71 \%$ more asthma related A\&E visits, and 131\% more asthma-related hospitalisations - p-values for gender differences were all less than 0.001 .

\subsubsection{Model fit}

In both study cohorts, the quantile-quantile plots for the raw residuals for the four ZINB models showed that the raw residuals overall followed a normal distribution (Figure 5.8). However, while there was a little right skewness for the asthmarelated GP visit models, the right skewness was clearer in the models of the other three outcome variables-asthma reviews, asthma-related A\&E visits and asthmarelated hospitalisations-especially for hospitalisations. 


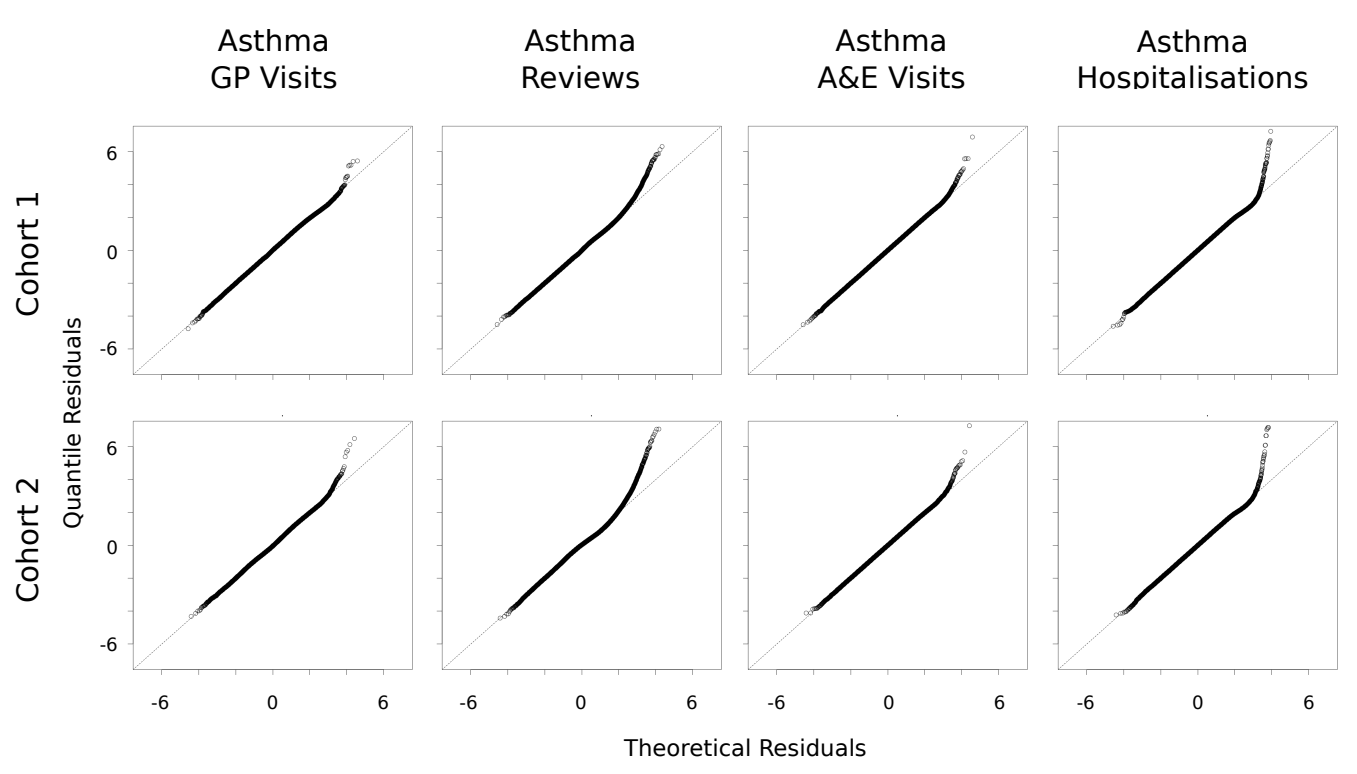

Figure 5.8: Quantile-quantile plot for the model residuals.

Rootograms showed an alternative visualisation of the model fit, focusing of the deviation between the observed and predicted frequencies for the counts of the outcome variables (Figure 5.9). The deviations between the observed and predicted frequencies were relatively small, indicating a good overall fit for each of the four models in both cohorts.

\subsubsection{Sensitivity analysis}

The findings presented above in both cohorts were produced for patients with complete GP registration in Wales between 2010 and 2014. However, among 297,976 and 111,253 patients with "ever-diagnosed asthma" and "ever-diagnosed currently treated asthma over the follow-up period" in the source population, 79,222 (26.6\%) and 9,744 (8.8\%) patients, respectively, had incomplete GP registration over the five-year follow-up period.

By including those previously excluded patients to Cohort 1 and Cohort 2, the overall patterns of IRRs across the WIMD quintiles did not change significantly in both cohorts. Between the most and least deprived areas, most IRRs became slightly higher than but still close to those in the original cohorts with complete GP registrations. The IRRs of asthma GP visits, reviews, A\&E visits and hospitalisations became 1.102 [1.090-1.114], 1.064 [1.052-1.075], 1.542 [1.377-1.726], and 2.254 [2.033-2.498] in the ever diagnosed asthma cohort (Cohort 1), and 1.032 [1.022-1.042], 0.987 [0.977-0.997], 1.242 [1.081-1.427], and 1.889 [1.671-2.135] in the ever-diagnosed, currently-treated asthma cohort (Cohort 2), respectively. 


\section{Cohort 1}
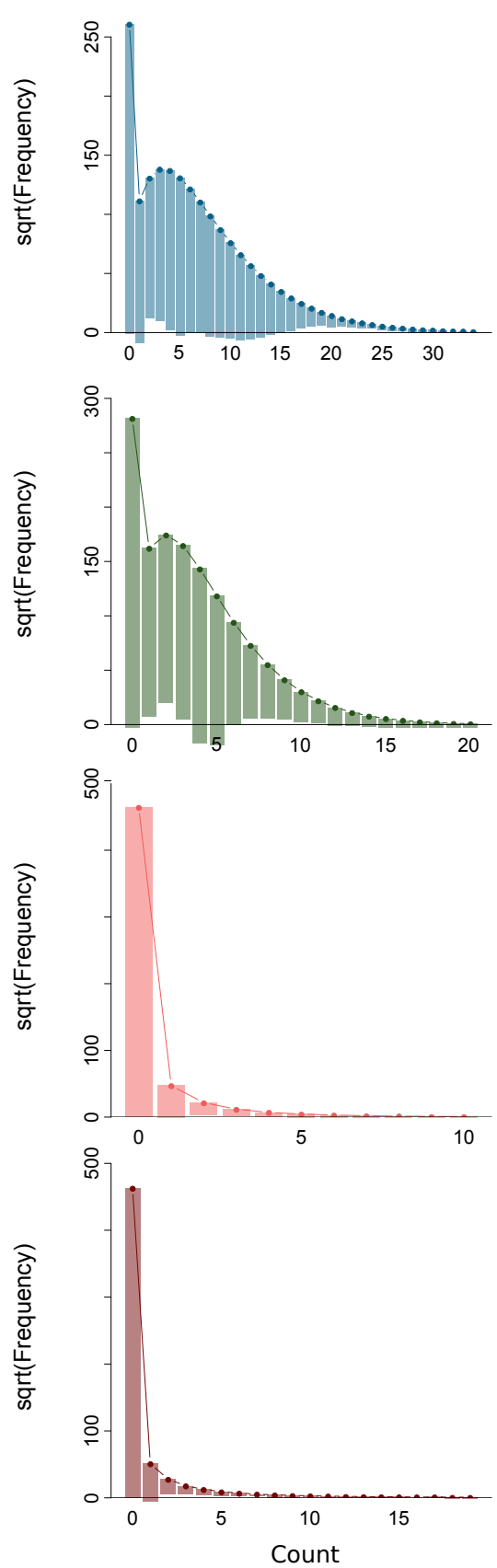

Cohort 2

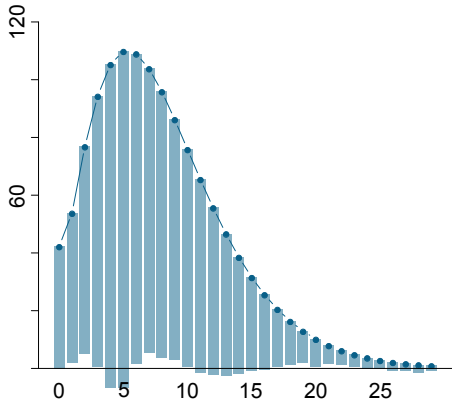

Asthma

GP Visits

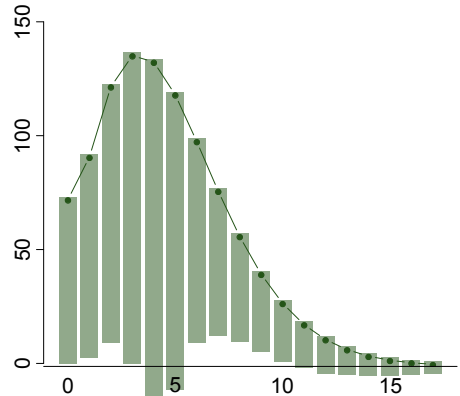

Asthma

Reviews

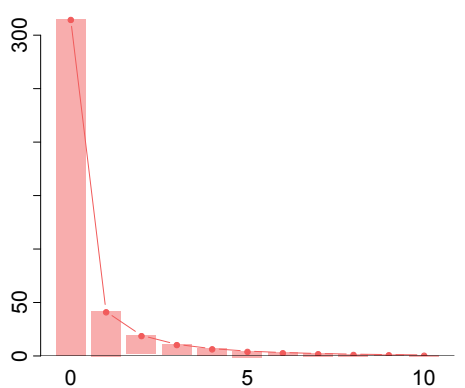

Asthma

A\&E Visits

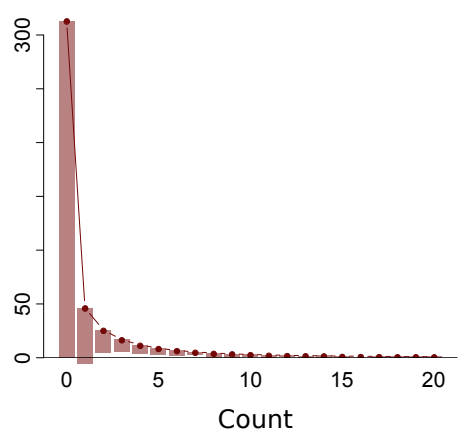

Asthma

Hospitalisations

Figure 5.9: Rootograms illustrating the goodness of fit for the zero-inflated negative binomial models for the four outcome variables in both study cohorts.

The sensitivity analysis confirmed that the observed differences in asthma-related primary and secondary care utilisation between the most and the least deprived areas existed regardless of the continuity of GP registrations. 


\subsection{Discussion}

\subsubsection{Summary of findings}

I identified patterns of variation in asthma-related healthcare utilisation across the deprivation scale as well as between age and gender groups in Wales.

Compared to the least deprived areas, there were wide gaps in asthma-related ED visits (36.8\%) and hospital admissions (123.2\%) in the most deprived areas despite a small excess in asthma-related primary care use, including asthma reviews, in these areas. However, these gaps were slightly smaller $(22.9 \%$ and $95.5 \%$, respectively) among asthma patients with continuous asthma prescriptions throughout the study period.

There was a gradient of increasing asthma-related hospital admissions by increased deprivation. However, there were no consistent gradients for asthma-related GP visits, reviews, and A\&E department visits across the deprivation scale. Instead, for asthma-related GP visits and A\&E department visits, the contrast was between the more affluent areas, which had the lowest incidence rates, and the other more deprived areas together, which had higher incidence rates.

Notably, there were also significant differences by gender in asthma-related healthcare utilisation. Although females with asthma had a modest excess in diseaserelated primary care contacts including asthma reviews, they were far more likely to attend $A \& E$ departments and to be admitted to hospitals due to asthma.

Age groups also showed wide variations in asthma-related healthcare utilisation. Among patients who continuously received asthma treatments, asthma-related GP visits decreased with older ages. However, asthma patients between the age of 10 and 35 were less likely to undergo asthma reviews compared to those in the age groups between 40 and 85. Finally, younger patients were more likely to visit $A \& E$ departments and to be admitted for asthma than older patients.

There was a remarkable gradient of more prescriptions but lower proportion of asthma controller medications with higher deprivation.

The above findings were not affected by whether patients were treated for asthma throughout the follow-up period or by their continuity of GP registrations. 


\subsubsection{Interpretation in the light of previous studies}

\subsubsection{Comparison with previous studies}

The associations that I found between socioeconomic status and asthma outcomes in Wales were consistent with other studies in Wales, England, and elsewhere. Asthma outcomes were shown to be associated with both area-based and individuallevel deprivation [349, 350].

Asthma severity has been widely linked to socioeconomic status, where severe disease was found to be more prevalent in people with lower socioeconomic status [351]. Lower socioeconomic status also increased the risk of poor asthma control and the incidence of exacerbation, independent of disease severity [349, 352,353 ]. Also, asthma patients with low socioeconomic groups, measured mainly through education level, had higher asthma morbidity, including higher incidence of exacerbations [352] and worse asthma control independently from the disease severity. One explanation of the poorer asthma control among patients with low socioeconomic status is that this group has poorer medication adherence [354].

In my study, the prevalences of both ever-diagnosed asthma and ever-diagnosed, currently treated asthma increased in the more deprived areas. Similar findings were reported in England [350]. However, the poorer asthma outcomes associated with higher deprivation were contrasted, in other studies, with the reports of increased disease incidence with lower deprivation $[324,355]$. In one study, asthma-related hospitalisation rates were correlated with the prevalence of chronic phlegm and indoor exposure of second-hand smoke but the variations of these rates between socioeconomic groups were not explained by variations in the prevalences of asthma or wheezing [324].

Another study, however, reported contradicting findings that no statistically significant difference was found in the number of asthma exacerbations between patients in low and medium/high socioeconomic status groups [356]. That study reported that the less deprived people had lower secondary care utilisation after exacerbations, which might suggest they received better management and had better self-management than the most deprived groups [356]. 


\subsubsection{WIMD mainly describes areas, and to a lesser extent, individuals}

The Welsh Index of Multiple Deprivation is an area-based measure, i.e. an ecological variable. Therefore, associations studied using the WIMD index may not necessarily hold at the individual level [357, 358]. Essentially, the findings in this chapter highlighted variations between small areas of different deprivation ranks. While deprivation indicators for a given small area represent the average levels of disadvantage of persons living there, those persons may widely differ in their own characteristics. Therefore, depending on the extent of those variations, grouplevel associations might not be valid for individuals who were far from the average characteristics.

While the Income, Employment, Health, Education, and Housing domains of the WIMD are constructed based on average scores of individuals living in a given area, the other domains, i.e. Access to Services, Community Safety, Physical Environment domains, are by definition roughly equal for all residents in that area. Therefore, the findings in this chapter could be carefully extended to the individual level, i.e. asthma patients living in the most deprived areas had worse asthma control despite having slightly more primary care contact.

\subsubsection{Asthma-related emergency department visits and hospitalisations usually indicate worse asthma severity and control}

Asthma-related ED visits and hospital admissions have been widely used as proxy measures for asthma severity, control, and exacerbations [102]. Asthma control is the extent to which the disease symptoms and future risks are sufficiently eliminated or reduced through treatment to an acceptable target $[1,2,16]$. Suboptimal asthma control may result from inadequate secondary and tertiary prevention for asthma, such as inappropriate prescribing, inadequate asthma review, and suboptimal self-management and patient education. Accordingly, the findings in this chapter suggest that asthma patients in the least deprived areas could have not only more severe disease (i.e. requiring a higher level of medication to achieve control) but also that disease control (i.e., achieving satisfactory symptom control) was poorer overall (e.g., due to inadequate treatment). The difference in the average asthma medication ratio and asthma reviews between the least and the 
most deprived areas may partly explain the gap in asthma-related A\&E visits and admissions between those areas.

However, not all the asthma-related visits to A\&E departments indicate real medical emergencies or worsening in the disease control. Instead, in areas of higher deprivation levels in particular, the higher number of such visits might not be completely due to genuine need to visit these facilities. There are a wide range of reasons why patients may bypass their GPs to visit A\&E departments, i.e. using A\&E departments as primacy care facilities [359]. Inaccessibility to GP practices and pharmacies, which were accounted for in the Access to Services domain of the WIMD index, might play a role in the increased A\&E visits in the more deprived areas.

A study in the United States found that patients with insufficient health literacy made more return visits in 14 days to $A \& E$ departments than those with adequate health literary [360]. Subsequently, inadequate health literacy in areas of higher deprivation could be contributing to the higher asthma-related A\&E visits found in this chapter.

Overcrowded GP practices in the more deprived areas may also contribute to the increased $A \& E$ visits in these areas. A recent report investigating the pressure on general practices in the UK had found that general practices in areas with higher levels of deprivations faced higher pressure than those in lower deprivation areas [361]. In that report, residents of higher deprivation areas used health services more likely and frequently than those in lower deprivation areas. In East London, for a given age group after the third decade of age, areas with higher deprivation had higher rates of GP consultations per patient compared to areas with lower deprivation [362]. This suggests that the IRR of 1.31 in asthma A\&E visits between the most deprived and the least deprived areas may overestimate the differences in asthma control between them.

\subsubsection{Why did the most deprived asthma patients have more GP visits?}

The small excess of $8 \%$ in primary care contacts in the most deprived groups compared to the least deprived might be due to several factors. The intrinsic disease severity might be contributing to this gap. It has been found that asthma patients in the most deprived areas often had more severe disease than those in the least derived areas [351, 355]. 
The excess could be also, in part, due to poorer disease control in the most deprived areas, leading to higher use of primary and secondary care. Unscheduled GP visits for asthma often indicate suboptimal disease control and/or exacerbations. However, in the SAIL Databank, it was not possible to directly distinguish between scheduled and unscheduled GP visits.

Another potential factor for the excess in asthma-related GP visits in the most deprived areas is lower health literacy. This might have hindered successful asthma self-management, and reduced patient's adherence to treatment and engagement in decision making [363]. Asthma patients with lower levels of education were found to use more short-acting bronchodilators and less controller medications, leading to poorer asthma control [364]. Lower health literacy can therefore lead to higher dependency on GPs and an increasing tendency to request appointments.

\subsubsection{Study strengths}

The main strength of this work was the use of objective real-world data at the individual patient level. The GP dataset extract that I used had a coverage of almost $80 \%$ of practices in Wales at the time of analysis. Through this dataset, I was able to identify and study a very large proportion of all asthma patients living in Wales between 2010 and 2014. In addition, I used an in-house developed algorithm that identified patients with gaps of more than 30 days that might result from either deregistration by the patient or the practice not sending data to the SAIL Databank. By excluding those patients from the study population, I minimised the possibility of missing GP events due to these reasons. However, it was possible that patients with gaps in their GP registrations were less concerned about their health, which could be due to inadequate health literacy which was in turn associated with lower education attainment and worse health outcomes [365]—characteristics suggesting higher deprivation levels. Alternatively, it was possible that those patients could have had milder asthma, or simply did not reside in Wales for the whole follow-up period. I demonstrated through a sensitivity analysis that the exclusion of those patients led to only small reductions to the IRRs that did not alter the conclusions.

Another strength is that the 2011 WIMD index incorporated a comprehensive range of deprivation domains for small areas in Wales, representing a helpful tool for multifaceted measurement of socioeconomic status. 
In Wales, the National Health Services provides free-of-charge medical services, including prescriptions, to Welsh residents. This could be an advantage in this study by avoiding possible bias that could have happened if those services where paid for, completely or partly, by patients. Direct contributions to health care costs required by patients, especially with low income, may lead to less healthcare utilisation in order to avoid costs [366, 367]. For example, a study in the United States (US) suggested that higher proportions of consumed out-of-pocket asthma medication costs to household income were associated with higher exacerbation risk in children [353].

\subsubsection{Study limitations}

\subsubsection{Case definitions for asthma-related A\&E visits and hospitalisa- tions were not validated}

To my knowledge, there are currently no validated algorithms that would have allowed me to accurately identify asthma-related A\&E visits and hospitalisations in the corresponding datasets in the SAIL Databank. Data recording and coding practices at inpatient and A\&E departments may vary across Wales [297, 368]. For ED visits, the recorded diagnosis may not be final, and may not always correspond to the reason of the visit [368]. In the PEDW dataset, hospital episodes had 14 available fields for recording admission diagnoses: one primary diagnosis, one subsidiary diagnosis, and 12 secondary diagnoses [369].

In my analysis, asthma-related hospital admissions included any hospital episodes in which asthma was recorded as an admission diagnosis in any of the 14 available diagnosis positions. However, this definition of asthma-related hospital admission may have overestimated the number of actual events. In some cases, it was possible that asthma was recorded as a secondary indication for admission. Alternatively, asthma could have been recorded despite not being a reason for admission (e.g., it was well controlled prior or during the admission, and did not need specialist care hospital stay). Therefore, it is possible that some counted hospital admissions in my analysis were not related to asthma and therefore should have been excluded. Nonetheless, I assumed that the algorithm that I used to identify asthma-related hospitalisations equally inflated, if any, the estimated counts 
across the five WIMD rank quintiles and therefore would not significantly affect the estimated IRRs.

In contrast, querying asthma diagnosis codes from the first position only may exclude relevant hospital episodes in which asthma was a main or a contributing reason for admission. In the PEDW episode data, primary diagnosis field was inconsistently used. Diagnosis codes were sometime found only following nondiagnosis codes. ${ }^{3}$ This means that the actual main medical diagnosis was not always recorded in the primary diagnosis field. Such a data quality issue was addressed in a recent study were asthma-related hospital episodes in Wales were identified using an asthma code being recorded in the first diagnosis position after ignoring the aforementioned non-diagnosis codes [43]. In my own analysis, I found that this approach increased the number of identified asthma-related hospital episodes by about $64 \%$ compared with using the primary diagnosis field only. Further work is needed to develop and validate accurate case definitions for asthma-related $A \& E$ visits and hospitalisations, possibly using a machine learning approach.

\subsubsection{Possible residual confounders}

Several variables that might have affected both the deprivation level and asthma outcomes were not included in the regression models presented in this Chapter. These variables were not available or were sub-optimally coded in routine data.

For example, I did not adjust the regression models for smoking status. Nor did I investigate the effect of smoking status on the outcome variables. Cigarette smoke is a known and strong trigger of asthma exacerbations among first-hand or second-hand smoker asthma patients. A systematic review and meta-analysis found that exposure to second-hand tobacco smoke among children with asthma almost doubled the risk of hospitalisation for asthma exacerbation and worsened pulmonary function [370].

Smoking was also shown to be positively associated with deprivation [371]. In England, an analysis by the Office of National Statistics found a strong association between the proportion of current smokers and the level of deprivation [372]. In that analysis, people living in the areas at the highest deprivation quintile were

\footnotetext{
${ }^{3}$ Such codes include symptoms, signs, abnormal clinical or laboratory findings, medical, surgical and allergy history, and other miscellaneous health-related statuses or events that exist in the $\mathrm{R}$ and $\mathrm{Z}$ chapters of the ICD-10 classification.
} 
more than twice likely to smoke than those living in areas categorised as the lowest deprivation quintile. Part of the effect of the level of multiple deprivation on the number of asthma-related ED visits and hospital admissions might be influenced by differential smoking status.

On the other hand, smoking is a leading cause of premature death, and a risk factor for numerous health problems, including cancer, low birth weight, and limiting health conditions [373], all of which are part of the Health domain of the WIMD index. Accordingly, smoking status might have, to some extent, confounded the relationship between multiple deprivation level and the four asthma-related events that I studied. Therefore, if recorded in high quality, smoking status should have been added to the regression models used in my analysis to reduce the potential confounding bias.

Health literacy is also a potential confounder. The Institute of Medicine [374] defined health literacy as "the degree to which individuals have the capacity to obtain, process and understand basic health information and services needed to make appropriate health decisions". Health literacy affects asthma outcomes both directly and indirectly, though inadequate patient knowledge of asthma, suboptimal asthma self-management and improper use of inhalers [375, 376]. Health literacy also affects the WIMD through the Education domain [377]. However, data on health literacy were not available in the routinely collected electronic health record (EHR) data in the SAIL Databank.

\subsubsection{WIMD overall index and asthma exacerbations: a possible circular relationship}

An important consideration when studying the associations between area-based socioeconomic measures and health outcomes is when the former include a health component.

The overall WIMD index includes the Health domain, which includes limiting longterm illness, death rate in the area from all causes, incidence of cancer, and low birth weight. Asthma mortality in Wales in 2011 was 1.8 per 100,000 population, a small contribution to the all-cause crude death rate of 990 per 100,000 population [378, 379]. However, asthma, especially when uncontrolled, is likely to be a limiting condition with significant and profound effects on patients' quality of life [380-382]. A recent survey of 4,650 asthma patients in the UK by Asthma UK 
found that $46.4 \%$ of the respondents reported sleep difficulties due to asthma, and $45.2 \%$ reported that asthma interfered with their daily activities [383]. Low birth weight and other adverse perinatal outcomes, such as pre-term delivery and small for gestational age infants, have been linked to the severity of maternal asthma and/or asthma medications used by mothers during pregnancy [384-386].

Asthma could also affect the Education domain of the WIMD index. Suboptimal asthma control among children, as well as urgent or emergent asthma-related healthcare utilisation, have been shown to be associated with school absenteeism [387]. In addition, asthma could affect the Employment domain of the WIMD index by increasing job absenteeism and hindering job retention. A Danish study found that people with current asthma were more likely to miss work and lose their jobs [388].

With the above links between asthma and the Health, Education, and Employment domains of the overall WIMD index, using the latter as a predictor of asthma outcomes is challenging, and caution should be exercised in the interpretation of results. However, the effect of such circular relationship could be limited. A study In England found that removing the health domain from the Index of Multiple Deprivation had small, practically unimportant effect on the measured socioeconomic disparities in census measures of health [389].

\subsubsection{Implications for health policy}

Health inequalities are of paramount importance to health policy. The work presented in this chapter is an example of the utility of routinely collected, linked data from EHRs and the WIMD in Wales in assessing health inequalities, namely those in asthma outcomes, and in informing health policy.

The presented findings could improve our understanding of the social gradient in asthma in Wales and inform the development and redesign of policies to reduce inequalities in asthma outcomes. These findings suggest that several aspects of health care services for asthma patients in the most deprived areas in Wales could be targeted for improvement. These aspects may include quality of primary care services, including early diagnosis and optimal prescribing, the lack of which could lead to poor disease control. 
To reduce health literacy gap and the associated gaps in asthma outcomes, the most deprived asthma population requires active efforts and further resources to ensure effective education on asthma and asthma self-management. This includes training in proper inhaler technique, adequate adherence to medications, and avoiding exacerbation triggers. The variation in quality of secondary care for asthma patients across the socioeconomic spectrum could also identify further potential to reduce the gap in asthma outcomes.

Avoidable health inequalities at any level create concerns and are socially and politically unacceptable. They may also result in wasted resources. With the high prevalence of asthma in Wales, even modest inequalities in asthma outcomes are likely to result in likely avoidable, significant disease costs at the country level. The overall cost of asthma in Wales in the fiscal year 2011-2012 was estimated as $£ 74.7$ million pounds (approximately US\$104.7 million) [43]. An IRR of 2.23 for asthma hospitalisations between the extreme WIMD quintiles means that there were 1,340 hospitalisations ${ }^{4}$ in the most deprived areas that would have not happened if the hospitalisation rate there was equal to that in the most affluent areas. Assuming a conservatively estimated average of $£ 1,000$ for the cost of an asthma hospital episode (approximated from cost data presented by Mukherjee et al. [43]), those extra 1,340 asthma hospitalisations in the most deprived areas costed NHS Wales at least $£ 1,340,000$ over the five-year follow-up. This calculation demonstrates the potential for avoiding significant, unnecessary costs of asthma healthcare utilisation in the most deprived areas. However, it does not take into consideration the costs of increased asthma prescriptions, A\&E visits, and GP visits in the more deprived areas. In fact, while hospital admissions accounted for $13.1 \%$ of asthma-related costs to the NHS in Wales in 2011-2012, prescriptions accounted for two third and asthma-related GP visits, ambulance trips, and A\&E visits together accounted for around 16\% [43]. A comprehensive cost analysis is therefore needed to estimate the variations in the overall asthma financial burden across deprivation levels in Wales and the potential savings by reducing these variations. Such an analysis should include the costs of asthma-related visits to GPs, A\&E and outpatient departments, ambulance trips, and prescriptions and hospital admissions as well as other wider societal costs such as Disability Living Allowance and costs resulting from school and work absenteeism.

\footnotetext{
$4(2.23-1) \times 0.022 \times 49,597$; where 0.022 is the hospitalisation rate in WIMD 5 , and 49,597 is the number of patients in WIMD 1 areas based on Cohort 1 characteristics (Table 5.2).
} 
Identifying inequalities in asthma outcomes and identifying potential targets to reduce them aligns with the Welsh Respiratory Health Implementation Group's vision towards reducing inappropriate variations in respiratory outcomes across Wales [390]. Reducing health inequalities is also a key objective of the Welsh Government. The use of routinely collected data in this exercise to explore asthma outcomes aligns with political landscape in Wales to maximise the use of these data to support respiratory health policy and care delivery [96, 391].

\subsubsection{Future work}

The limitations and methodological challenges encountered in this study warrant further work. For example, algorithms to identify asthma-related A\&E visits and hospital admissions in the SAIL databank need to be externally validated. If available in high quality, measures of potential confounders such as smoking status and health literacy could be added to the statistical model.

Further analysis would be needed to improve the understanding of the asthma social gradient in Wales and explore possible explanations to the observed inequalities, taking into account the complex inter-relationships between the relevant variables. Including additional data on healthcare utilisation and asthma outcomes across the deprivation levels would provide additional insights into the differential severity of asthma exacerbations and the associated cost of avoidable outcomes. Such data may include detailed hospitalisation data, such as LOS and HRG, as well as death due to asthma.

Further work on associations between asthma outcomes and each of the eight domains of the overall WIMD index would be useful to understand the individual contribution of each deprivation domain to asthma inequalities.

Asthma is associated with a wide range of comorbidities such as rhinitis, sinusitis, obstructive sleep apnoea, gastroesophageal reflux disease, obesity, anxiety, and depression [392-394]. More comorbidities are in general associated with lower socioeconomic status [395]. Therefore, the impact of comorbidities on asthma inequalities and the associated avoidable cost and burden of these comorbidities is worth exploring.

Ethnicity may in general influence both socioeconomic status and health outcomes [396-398]. Wales is generally a homogeneous country with about $5 \%$ of the pop- 
ulation identified themselves as being from non-white background [399]. However, the percentages of non-white people are higher in large urban areas such as Cardiff (17.2\%), Swansea (10.6\%), and Newport (7.7\%) [400]. In these areas, the individual role of ethnicity in the social gradient of asthma is worth investigating. Finally, future work will explore the trends and costs of asthma inequalities over the last two decades to see whether they are changing over time.

\subsection{Conclusion}

This chapter demonstrated an important application of the Wales Asthma Observatory in supporting health policy regarding equality in health care. I found wide inequality gaps in asthma outcomes between the extremes of socioeconomic deprivation spectrum in Wales and showed a social gradient in asthma-related hospital admissions. Compared to the least deprived areas, the most deprived areas had slightly more primary care contacts, including annual asthma reviews, per asthma patient. However, asthma patients in the most deprived areas were $37 \%$ more likely to have asthma-related ED visits and more than twice as likely to be admitted to hospitals due to asthma than those in the least deprived areas, although those gaps decreased slightly among patients with continuous asthma prescriptions throughout the study period.

These wide gaps in asthma healthcare utilisation were possibly due to higher severity and poorer control of the disease in the least deprived areas. Possible underlying factors such as suboptimal asthma prescribing, inadequate health literacy, poor asthma self-management, and wider non-health related socioeconomic determinants, such as income, employment, education, air pollution, might be contributing to the observed gaps and require further investigation. 


\section{Chapter 6}

\section{General Discussion}

\section{Reflection and future directions}

In this chapter, I summarise the work I performed in this thesis about the development and utilisation of the Wales Asthma Observatory, highlighting my original contributions. I critically review the strengths and limitations of the Observatory as a platform for asthma research and surveillance, and as a tool to improve our understanding of asthma in Wales and to inform health policy, service planning and delivery. I then compare my findings with related works and studies. I then discuss the opportunities and challenges encountered during the Observatory development and utilisation. I reassert the high potential of using asthma-related routinely collected data to improve asthma patients' lives, and the pressing need to reduce avoidable harm and waste from the suboptimal re-use of these data. I discuss the potential role of the Observatory in the national efforts to improve asthma outcomes. I then propose a future research agenda to improve the Observatory's methodology and to answer further questions about the social gradient of asthma in Wales, I also propose further technical and content developments to the Observatory. 


\section{Chapter Contents}

6.1 Summary of findings . . . . . . . . . . . . . . . . . . . . . . 193

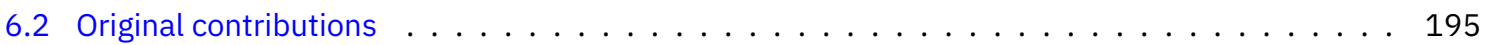

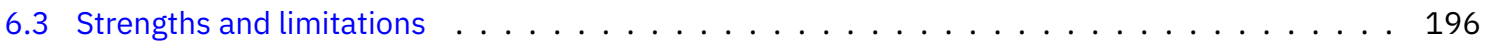

6.3.1 Data sources: pros and cons . . . . . . . . . . . . . . . . . . . 196

6.3.2 Case definitions: Flexibility and data driven approach . . . . . . . . . . . . . . 197

6.3.3 Longitudinally assessed disease outcomes . . . . . . . . . . . . . . . . . . . . 198

6.3.4 Supporting research reproducibility $\ldots \ldots$. . . . . . . . . . . . . . . . . . . . . 199

6.4 Interpretation of findings in the light of related literature . . . . . . . . . . . . . . . . . 199

6.4.1 Methods to define complex disease entities using routinely collected data . . . . . . . 199

6.4.2 Various approaches to asthma registries, surveillance systems, and research platforms 200

6.4.2.1 Asthma surveillance systems used routinely collected and/or self-reported data 201

6.4.2.2 Asthma registries generally target the problematic cases . . . . . . . . . 202

6.4.2.3 Routinely collected data (RCD) for disease registries . . . . . . . . . . . 203

6.4.2.4 Data acquisition, management, and quality . . . . . . . . . . . . . . . 204

6.4.2.5 Facilitating data interrogation is an increasingly recognised need . . . . . . . 205

6.4.3 Social gradient of asthma: consistent findings and methodological challenges . . . . . 207

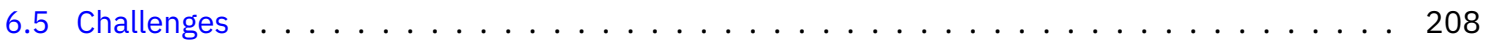

6.5.1 Asthma heterogeneity complicates case identification and comparability of studies . . 208

6.5.2 Data from important care domains are still missing . . . . . . . . . . . . . . . . . . 209

6.5.3 Quality of routinely collected data is imperfect . . . . . . . . . . . . . . . . . 209

6.5.4 Routinely collected data suffer from time lags . . . . . . . . . . . . . . . . . . . 210

6.5.5 Routinely collected data does not reflect precise disease timeline . . . . . . . . . 211

6.5.6 Lack of valid methods to assess outcomes . . . . . . . . . . . . . . . . . . . . . 211

6.5.7 Public attitudes to data re-use are mixed . . . . . . . . . . . . . . . . . . . . . . 211

6.6 Implications and potential uses of the Observatory . . . . . . . . . . . . . . . . . . 212

6.6.1 Implications on health policy and wider societal impact . . . . . . . . . . . . 212

6.6.2 Implications on service planning and delivery . . . . . . . . . . . . . . . . . 212

6.6.3 Implications on clinical practice and patient outcomes . . . . . . . . . . . . . . . 213

6.6.4 Implications on asthma research . . . . . . . . . . . . . . . . . . . . . . . . 214

6.7 Towards maximising population data benefits to improve asthma outcomes . . . . . . . . . 214

6.7.1 Improving data capture . . . . . . . . . . . . . . . . . . . . . . 214

6.7.2 Reducing waste from underuse of data . . . . . . . . . . . . . . . . . . . . . . . . 215

6.7.3 Supportive data-intensive research environment . . . . . . . . . . . . . . . . . . . 216

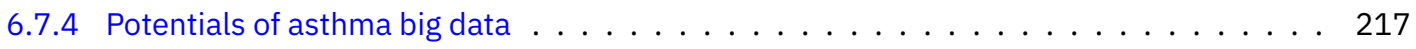

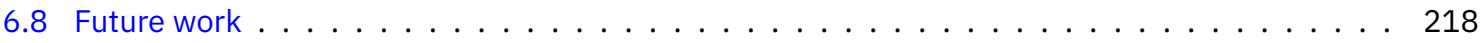

6.8.1 Improving methods to define asthma patients and assess disease outcomes . . . . . 218 
6.8.2 Understanding inequalities in asthma outcomes . . . . . . . . . . . . . . . 219

6.8.3 Monitoring and forecasting asthma trends . . . . . . . . . . . . . . . . . . . 219

6.8 .4 Linking additional data sources . . . . . . . . . . . . . . . . . . . 220

6.8.5 Improving the Observatory's technical platform . . . . . . . . . . . . . . . . . . 221

6.8 .6 Data quality reports . . . . . . . . . . . . . . . . . . . . 221

6.8 .7 Getting ready for SNOMED-CT . . . . . . . . . . . . . . . . . . . . 221

6.9 Conclusions . . . . . . . . . . . . . . . . . . . . . . . . . 222

\subsection{Summary of findings}

In this doctoral project, I demonstrated how routinely collected electronic health record (EHR) data can be used to develop a data-intensive platform for asthma research and surveillance-the Wales Asthma Observatory. The Observatory aims to maximise the benefit of these data and is based on a regularly updated, national cohort for asthma.

Due to the inherent limitations of routinely collected data (RCD) [88-93], special attention should be paid to methods of defining diseases and health outcomes using these data. Therefore, to inform the Observatory development, I systematically reviewed the contemporary methods to define and assess asthma using routinely collected data and the ways these methods have been described (Chapter 2). I found a wide variation in these methods and suboptimal reporting on their implementation and validity. I highlighted the challenges of standardising methods to define and assess asthma, and the need to develop and validate database-specific methods.

In Chapter 3, in the light of the literature review findings, and considering asthma heterogeneity, data limitations, and the absence of a gold standard to define asthma, I justified the use of data-driven approaches to identify people with asthma. I demonstrated the appropriateness and benefits of using latent class analysis (LCA) on recorded asthma-related primary care data to identify clusters of asthma patients, including those with current asthma. My latent class modelling was based on healthcare utilisation data related to asthma and chronic obstructive pulmonary disease (COPD) for a large, random sample of the population of Wales. I chose the eight-class model as the best-fit model based on its model diagnostics and clinical interpretability. I assigned clinical labels to the latent classes. I then 
reduced the model complexity by merging the 'asthma' classes into two classes representing 'ever diagnosed asthma without current treatment' and 'currently treated asthma'. I then applied recursive partitioning (a supervised machine learning technique) to derive a decision tree which could identify patients with asthma, including whether they were currently treated, as well as those with COPD and asthma-COPD overlap syndrome (ACOS) in primary care data.

I used this case identification algorithm, in addition to several other case definitions for asthma, in the development of the Observatory, described in Chapter 4. I included in the Observatory a number of essential disease outcomes and variables such as disease severity, treatment step, and asthma exacerbations. I also developed and described a technical platform to improve the efficiency, reporting, and reproducibility of data extraction of studies that use the Observatory.

I investigated the quality of selected asthma-related event groups, and described variable patterns and levels of data missingness. Notably, many lung function tests were recorded without their measurements. When recorded, measurements were inconsistent for many of the lung function event codes. To improve the capture of asthma data, I recommended improved data entry quality checks by EHR systems, data-quality awareness training for clinicians, and the inclusion of data quality to receive a greater focus in payment-for-performance schemes.

In Chapter 5, to demonstrate the Observatory's utility for health policy, I investigated the inequalities of asthma outcomes across the socioeconomic spectrum in Wales. I used count regression models to compare asthma-related primary and secondary care events between asthma patients living in areas with different deprivation levels. I found that, compared to asthma patients who lived in the least deprived areas, those in the most deprived areas had slightly more primary care contact (7.8\% more general practitioner (GP) visits per patient), yet they had significantly more asthma-related emergency department visits (31.1\%) and hospitalisations (123.2\%). There was a clear gradient of more asthma-related hospital admissions in the more deprived areas in Wales. The inequality gaps were slightly smaller among patients who continuously received asthma prescriptions over the five-year follow-up period. I discussed the implications of these inequality gaps, and outlined future research directions to improve the modelling and account for possible confounders. I then proposed potential measures to reduce and bridge the inequality gaps in asthma outcomes. 


\subsection{Original contributions}

I summarise the original contributions in this thesis as follows:

\section{Chapter 2}

1. I found a wide variation in the contemporary methods to define asthma and assess asthma outcomes in observational studies conducted using routinely collected EHR data.

2. I found that reporting on the implementation and validity of these methods was poor overall.

3. I identified 10 practices of reporting or justifying the validity of these methods (Table 2.1). These practices varied widely from performing validity assessment in the same study, to relying on clinical guidelines or the validity of database coding. The majority of EHR-based asthma studies reported no information on the methods' validity.

\section{Chapter 3}

4. I described a probabilistic approach, using LCA of primary care data of a large population sample, to identify groups of people with asthma (including those with current asthma), distinguish them from those with COPD, and to identify people with asthma-COPD overlap.

5. I described the groups of people with asthma based on their asthma-related health care utilization.

\section{Chapter 4}

6. To identify people with asthma in the Wales Asthma Observatory, I used the above-mentioned data-driven probabilistic model as well as commonly used deterministic case definitions.

7. I described different levels and patterns of missingness and inconsistencies in asthma-recorded data, apparently due to different approaches used by GPs to record similar data items and using the same clinical codes for different purposes.

\section{Chapter 5}


8. I demonstrated the extent of inequality gaps in asthma health care utilisation in Wales: Compared to the least deprived areas, the most deprived areas had $8 \%$ more asthma related GP contact, but $123.3 \%$ more asthma hospitalisations; the inequality gaps were slightly smaller among patients who continuously received asthma prescriptions over the five-year follow-up period. I also found wide variations in these outcomes across age and gender groups.

\subsection{Strengths and limitations}

The Wales Asthma Observatory is the first of its kind in Wales as a platform for asthma research and surveillance using routinely collected data. The work presented in this thesis has a number of strengths and limitations related to the data used in the Observatory, the methods used to identify asthma patients and assess disease outcomes, and the Observatory design and structure.

\subsubsection{Data sources: pros and cons}

The type and sources of data used in the Observatory are one of its major strengths. The Observatory currently utilises key nationwide clinical datasets in Wales, including data on primary care, secondary care, area-based deprivation as well as causes of death. These data are already collected from EHRs, anonymised, and linked in the Secure Anonymised Information Linkage (SAIL) Databank. This offers a unique opportunity to answer a wide range of research questions that is not feasible with de novo data collection.

Throughout the previous chapters, I highlighted the advantages of EHR-based routinely collected data over purpose-specific data collection. Briefly, RCD are inexpensive, person-level streams of data that reflect the real-world picture of people' health status and clinical care. They are mainly recorded from the perspective of healthcare professionals rather than patients. Thus, their validity does not rely on patients' memory or health literacy. These data are routinely collected in huge volumes across Wales. This allows obtaining nationally representative epidemiological estimates, and enables conducting high-power studies and investigating rare outcomes. These opportunities are usually not present with small-sized primary data collected first hand by investigators. 
However, RCD suffer from a wide range of problems such as missingness, miscoding, under-recording, and linkage errors. There was no information about the version of coding system used to codify primary care data. Primary care events in the United Kingdom (UK) are usually coded with the Read code vocabulary, namely the second and third versions. The second version is a hierarchical vocabulary, while the third version is a radically developed version which, for example, supports poly-hierarchy (i.e., a code can have multiple parent codes), includes additional concepts, and has the codes changed for some of the existing concepts. It was difficult within the SAIL Databank to ascertain the vocabulary version in which a GP event was coded. The vast majority of GP practices in Wales, however, use the second version of Read Code vocabulary [401]. Therefore, in the Observatory development, I used only the second version for data extraction. However, this might have introduced a misclassification bias. This may happen, for example, if the GP practices that used Read Code version 3 differed from the rest of practices in their population characteristics or in the quality of care. Nonetheless, given the small number of those GP practices, this limitation is unlikely to undermine the national representativeness of the Observatory.

\subsubsection{Case definitions: Flexibility and data driven approach}

The Observatory is empowered by the availability of multiple case dentitions of asthma. Thereby, it allows capturing most cases of asthma including those with uncertain diagnosis. At the same time, it includes more strict case definitions such as currently treated asthma. This flexible approach facilitates studying diverse groups of asthma patients. It also allows researchers to choose, for their studies, the appropriate case definitions that are comparable with other particular studies.

Those case definitions, whether they are based on a single diagnosis code or more complex deterministic algorithms, are based on clinical guidelines, clinical knowledge, or epidemiological judgement. Each of those case definitions has a specific meaning and is intended to be used to identify a specific group of people with 'asthma'. In particular, the case definition of ever-diagnosed currently-treated asthma is the most useful one as it allows selecting people with active asthma at a certain point in time. This case definition has been commonly used as an essential eligibility criterion in the contemporary EHR-based asthma studies [102] and is also the basis of the main asthma indicator in the Quality of Outcomes Frame- 
work (QOF). Therefore, the Observatory will commit to use the ever-diagnosed currently-treated asthma as the main case definition of asthma for surveillance and research.

The Observatory also benefits from a latent class model which can be used as an 'internal' data-driven reference to identify asthma patients. Unlike cluster analysis, which uses distances between individuals, LCA uses a top-down approach to understand the population structure: it utilises the distributions of the observed data to identify the likely population latent classes. By computationally uncovering the population structure, LCA identifies all the likely patient groups, some of which could be overlooked in the manual researcher-led development of case definitions. LCA probabilistically determines to which latent class each person belongs. This probabilistic approach fits well with the nature of asthma as a heterogeneous condition represented by a continuous spectrum of various pathogeneses, overlapping phenotypes, and variable severity and natural history, which may coexist with other conditions (COPD, for example). This approach to patient identification allows researchers to select patients, not only based on their most likely classes, but also by preferred probability thresholds, or based on overlap patterns of interest (e.g., asthma-COPD overlap). The decision tree that I derived from the LCA model allows researchers to use this model to identify asthma patients in the SAIL Databank and similar databases.

However, unsupervised approaches such as LCA has limitations. The output of LCA depends on the quality of the input data and their relevance to the desired classification. It also involves a level of subjectivity in the model specification (i.e. choosing features), selection of the best-fit model, and interpretation of the latent classes. Therefore, while data-driven approaches can be useful to understand the population structure, they need to be coupled by knowledge about the disease pathophysiology, clinical course, and epidemiology as well as about data provenance and quality [142].

\subsubsection{Longitudinally assessed disease outcomes}

The Observatory includes longitudinally calculated key disease outcomes. For each patient in the Observatory, key disease states and outcomes such as treatment step and disease severity and exacerbations are ascertained longitudinally as state variables along the patient's follow-up period. This allows both cross- 
sectional and longitudinal analysis of these variables. The definition of these variables was informed by the algorithms to assess asthma outcomes that I found in the systematic scoping review in Chapter 2 . While the validity of those algorithms was assessed elsewhere, it should not be assumed to hold in the SAIL Databank. However, assessing the validity of these definitions was not feasible within the time-frame of this doctoral project.

\subsubsection{Supporting research reproducibility}

In the Observatory's design and implementation, I took into consideration the challenges of data extraction reproducibility. Therefore, I equipped the Observatory with a clinical code set library and data extraction platform, with an easy-to-use graphical interface, which allows researchers with no programming skills to interrogate the Observatory and the SAIL's primary care dataset. This platform is also intended to save time for experienced analysts by reducing unnecessarily repetitive programming code writing and database query development. This platform is aimed to support and promote research transparency and reproducibility as well as sharing and re-use of clinical code sets and data extraction procedures.

I designed the Observatory data structure in such a way that it can be seamlessly updated when the source datasets in the SAIL Databank are updated, using a programming script I built for this purpose. Since updated data may include historical changes, versioning of the Observatory data allows reproducing studies performed on historical versions.

\subsection{Interpretation of findings in the light of related literature}

\subsubsection{Methods to define complex disease entities using rou- tinely collected data}

The findings in the Chapter 2 were in line with previous studies. A related systematic review identified wide variation in the categorisation of asthma severity using health insurance claim data [222]. Similar variations in case definitions and the need for standardisation have been recognised in other conditions such as heart 
diseases [402], osteoarthritis [403], and immune thrombocytopenic purpura (ITP) $[404,405]$.

Standardisation of methods to define and assess asthma from RCD has been faced with a number of challenges including the disease heterogeneity, lack of consensus on its clinical definitions, variations in populations' characteristics, and crosscountry differences and limitations of RCD resources.

Clinical coding systems help standardise the documentation of health and health care concepts in EHRs. However, the clinical meaning of those concepts should be standardised in the first place. Standardisation of asthma terminology requires clear understanding of the disease's aetiology, genetic and molecular pathogenesis including gene-environment interaction, and how the underlying disease mechanisms manifest in different pathophysiological and clinical phenotypes. However, from a precision medicine perspective, a fixed terminology to describe a heterogeneous continuum of diseases may be insufficient to provide personalised diagnosis and management. Instead, Agusti et al. have suggested a label-free precision medicine strategy for chronic lung diseases in which treatable traits, rather than encompassing labels such as asthma and COPD, form the basis for diagnosis and management [406]. This contemporary clinical perspective has been epitomised in a recent editorial with Oscar Wilde's quote "to define is to limit" [208].

Wherever the debate on clinical definitions of asthma and asthma outcomes might move, standardisation and harmonisation of the corresponding operationalised RCD-based definitions are needed. Algorithms to define particular clinical concepts (e.g., 'asthma', asthma endotypes or phenotypes, or treatable traits) should be ideally validated wherever they are used. Subsequently, the optimal method to measure the same clinical concept may differ across databases and populations.

\subsubsection{Various approaches to asthma registries, surveillance systems, and research platforms}

Asthma surveillance systems and registries around the world have various purposes. They have been also established using different approaches to defining source populations and cases of interest. They also differ in their data sources, content, and usability, and data security models. 


\subsubsection{Asthma surveillance systems used routinely collected and/or self- reported data}

To be used for surveillance, data sources should have high geographical coverage and representation, sufficient data quality, and sustainability. RCD usually satisfy these requirements and are widely used for asthma surveillance. These include data on asthma-related accident and emergency (A\&E) visits, urgent care, and hospital admissions, medication dispensing, and health insurance claims [134, 407-409].

The Ontario Asthma Surveillance Information System (OASIS) is closely related to the Observatory in terms of the purpose and the use of RCD [134, 242, 410]. It was established as a platform for asthma surveillance and epidemiological research in the Canadian province of Ontario. OASIS uses administrative and health insurance data about out-of-hospital, emergency, and inpatient asthma care. The Observatory, however, use EHR data which are richer and more comprehensive than administrative and health insurance data.

Self-reported data are commonly used in the United States (US) asthma surveillance systems to estimate the disease prevalence [407-409]. These data are collected as part of the Behavioral Risk Factor Surveillance System (BRFSS) [411] telephone survey. The BRFSS survey contains questions about whether the respondent ever had and still has asthma, the age at diagnosis, symptoms frequency, exacerbation history, routine asthma check-up, number of preventer and rescuer asthma medications used, and the impact of asthma on the quality of life [412]. People who report having asthma are invited to the Asthma Call-back Survey (ACBS) [413]. The ACBS collects more detailed information on the disease history, healthcare utilisation, knowledge of asthma and management plan, patient's behaviour towards environmental risk factors, medication use, medical self-management, personal cost of asthma, asthma effects on work and/or school attendance, workrelated asthma, asthma comorbidities, and use of complementary and alternative therapy $[414,415]$.

Compared to the rich asthma-related self-reported data collected in the US, asthmarelated self-reported data in the UK nations' annual health surveys are much more limited. In particular, the Welsh Health Survey (WHS) only asked whether the respondent (or their child) was currently being treated for asthma or wheezing or 
had recent shortness of breath, tightness of chest, or wheezing [416]. In addition to their scarce asthma-related details, the WHS data had small sample sizes and suboptimal geographical representation, let alone the biases of self-report. Therefore, their role in the Observatory development was limited to being used as an external data source for the evaluation of the RCD-based case identification model.

\subsubsection{Asthma registries generally target the problematic cases}

Unlike asthma surveillance systems which generally target the whole asthma population, asthma registries are mainly dedicated to the more severe or complicated cases. One example is the British Thoracic Society (BTS) Difficult Asthma Network (DAN) registry [126], which was succeeded by the UK Severe Asthma Registry. ${ }^{1}$ The DAN registry included people who fulfilled the American Thoracic Society (ATS) definition of refractory asthma [417]. The UK Paediatric Difficult Asthma Network Registry ${ }^{2}$ comprises four specialist asthma specialist centres in the UK and aligns with efforts to incentivise the appropriate identification and management of problematic asthma cases. Both registries receive data entered by health professionals into secure portals as well as routinely collected data.

The Belgian Severe Asthma Registry (BSAR) is dedicated to difficult asthma cases [418]. It collects data on asthma diagnostics, such as lung functions, fractional exhaled nitric oxide (FeNO), blood eosinophil count, serum immunoglobulin E (IgE), sputum inflammatory cell profile, skin prick test, medication use, comorbidities, as well as smoking status. It also collects asthma-related patient reported outcome measures (PROMs) including Asthma Quality of Life Questionnaire (AQLQ), Asthma Control Questionnaire (ACQ), and Asthma Control Test (ACT).

The Italian Registry of Severe and Uncontrolled Asthma (abbreviated in Italian as RItA) offers secure web-based access to a database of asthma-related clinical data, risk factors, and exacerbations for patient with severe and/or uncontrolled asthma [419].

In comparison to those asthma registries, the Observatory is designed to be both a surveillance and research platform and a disease registry. Thanks to its nationwide

\footnotetext{
${ }^{1}$ https://cl2.n3-dendrite.com/csp/asthma/frontpages/index.html

${ }^{2}$ http://rs2.e-dendrite.com/csp/paedasthma/frontpages/index.html
} 
data sources, the Observatory is a comprehensive disease registry that targets the whole asthma population across Wales, regardless of disease severity.

Algorithms based on domain expert knowledge (e.g., researcher's clinico-epidemiological judgement and/or clinical guidelines) to identify eligible cases have been the conventional approach in asthma registries. Those algorithms are often validated against clinical reassessment or review of the full medical record, neither of which is a universal gold standard for asthma diagnosis.

The Observatory's multi-approach to case identification, based on domain expert knowledge and data-driven methods, offers the flexibility needed to study different asthma populations. LCA helped see the likely population structure behind the recorded data, while recursive partitioning produced a corresponding transferable algorithm to identify patients with asthma and/or COPD. Deriving a simple classification algorithm from a complex clustering model was previously described by Moore et al. [273]. They identified asthma phenotypes from cluster analysis of 34 variables, and derived a simpler three-variable classification tree which later identified similar clusters in a different population [420].

\subsubsection{Routinely collected data (RCD) for disease registries}

For disease registries, the approach of using RCD to develop a disease registry is relatively new. Instead, traditional disease registries are set up to include cases of interest that are managed in health care facilities within particular geographical areas. Cases are usually included in a registry by healthcare professionals based on defined criteria which are assessed on a case by case basis. Inclusion of cases in the registry is often carried out using detailed clinical information available in the doctor-patient encounter and the full patient record. However, there are a number of disadvantages of the traditional approach of asthma registries:

- the denominator is often not defined [124];

- the number of patients in the registry is relatively small due to the oftenlimited geographical coverage and the strict case definitions;

- case ascertainment is labour-intensive (which makes traditional disease registries more suitable for rare conditions);

- the inclusion in the registry often require the patient's consent; and,

- data collection is subject to the experimenter bias. 
For a prevalent condition such as asthma, individual case identification at a national scale would consume significant time and resources.

RCD offer inexpensive, accessible, wide-coverage, and rich alternative data sources for disease registry development. In this approach, cases of interest are automatically identified and characterised en masse from large datasets [127]. Larger numbers of cases can be identified. RCD usually have defined denominators, allowing estimation of the condition's epidemiology and burden. RCD are usually de-identified, and therefore no individual patient consents are needed [421]. However, misclassification of cases and missing variables are among the disadvantages of using RCD to create a disease registry.

\subsubsection{Data acquisition, management, and quality}

Sariyar et al. proposed a framework to evaluate medical registries purposes, data acquisition and management processes, and data quality [422]. The framework included assessment of data accuracy, trustworthiness, consistency, granularity, timeliness, completeness, security, and privacy. These criteria should be assessed along the flow of data in the registry: from acquisition, through storage, to presentation [422]. The authors argued that a registry should only include high quality variables that fit with its purpose(s). The Observatory, however, is built for generic purposes of asthma research and surveillance. This requires continuously expanding sets of case definitions and research-ready variables to satisfy the growing surveillance needs and the emerging research questions. However, RCD used in the Observatory inherently suffer from accuracy, trustworthiness, consistency, and completeness issues (see Section 4.5). Therefore, further assessment of case definitions validity and variables quality should be high priority in the future developments of the Observatory.

Modern implementations of registries for asthma and other conditions are increasingly web-based, where data are entered by patients and/or healthcare professionals through secure online user interfaces [126, 130,418]. Patient consent is normally needed before their data are included in a registry. The stored data are usually de-identified. In contrast, the Observatory uses already-linked deidentified data from the SAIL Databank. The Observatory's person-level data are maintained within the secure environment of the SAIL Gateway. In the future, 
however, secure online or mobile-based data collection could be implemented to capture asthma-related PROMs and link them to RCD in the Observatory.

\subsubsection{Facilitating data interrogation is an increasingly recognised need}

I designed the Observatory's user interface so that it improves the workflow of data interrogation including automation, reproducibility, reusability, and shareability. With the growing use of EHR-derived data for research elsewhere, the need for data extraction automation and code set engineering has been recognised in many EHR-derived data resources [301]. Facilitating data interrogation from EHR data resources can be achieved by variety of approaches such as providing 'research-ready' variables, developing tools to automate common data extraction tasks, and maintaining clinical code set libraries.

The Observatory provides essential asthma-related 'research-ready' variables including disease state (i.e. current case defintion) and key outcomes and variables such as treatment steps, asthma severity, and exacerbations. 'Research-ready' variables have been provided in EHR-derived data resources elsewhere. An example is the Clinical research using LInked Bespoke studies and Electronic health Records (CALIBER), a UK-based platform that provides access to 'research-ready' variables derived from data linked across EHRs, disease registries, bespoke cohort studies, and other routine data sources through a common data model [129].

The Observatory also provides an easy-to-use platform to design, share, and reuse complex data extraction procedures as well as manage clinical code sets. With this platform, researchers can create additional study-specific Read code sets and complex variables derived from the SAIL's GP dataset. The graphical interface mimics the process of creating a data table and populating its fields in the Structured Query Language (SQL). It can be used by users with no programming skills. In addition, the visual interface accepts inserting SQL pieces of codes so that users with programming skills can design more advanced data extraction procedures. A related tool is $\mathrm{rEHR}$, an $\mathrm{R}$ package which provides functions for advanced data interrogation from the Clinical Practice Research Datalink (CPRD) [423]. However, as a programming library, rEHR can only be used by members of a research team who have programming skills. In contrast, the Observatory interface allows collaborative development of code sets and data extraction procedures by anyone in a multidisciplinary research team. Another difference is that rEHR works on data 
files exported from the CPRD, whereas data extraction procedures designed with the Observatory's platform can be exported to be run on a database connection, such as the case in the SAIL Gateway. Lastly, an rEHR-based data extraction code can be shared and published as a computer file. By contrast, a data extraction procedure designed in the Observatory is maintained centrally on a web address where it can be (re)used, shared, cited, and exported as an SQL or an interoperable JavaScript Object Notation (JSON) file. ${ }^{3}$

The need for supporting the collaboration in data interrogation has been previously recognised. eLab is a web-based environment which allows multidisciplinary research teams including researchers and healthcare professionals to access health dataset and collaboratively develop methods to analyse and visualise the results [424].

eLab is based on the concept of Research Objects. Research Objects have been proposed as a generic, comprehensive approach to representing the research process and outcomes as semantically linked, reusable, shareable, entities [425, 426]. eLab has been used as 'Asthma eLab' in the Study Team for Early Life Asthma Research (STELAR) consortium [427]. Asthma eLab provides web-based platform for collaborative management and analysis of asthma-related data from five birth cohorts in the UK. It allows research teams to model relationships between pathological and physiological processes in graphical and computable forms.

The Observatory's approach of using simpler variable types to specify complex data extraction procedures ('building blocks' approach) that are reusable, extensible, and shareable roughly corresponds to the concept of Research Objects.

ClinicalCodes.org is another related tool which provides repository for clinical codes used in EHR studies [216]. This public web-based repository is similar to the code set library provided in the Observatory. Whereas it only archives already used code sets, the Observatory's platform allows users to collaboratively create, edit, and revise code sets and then easily use them in data extraction procedures hosted in the same platform.

In summary, an efficient and effective user interface for an EHR-based data resource such as the Observatory should ideally satisfy a number of principles [426] including methods versioning, repeatability, auditability, reusability, repurposeabil-

\footnotetext{
${ }^{3} \mathrm{JSON}$ is an open-standard file format to exchange human-readable data in the form of arrays and attribute-value pairs.
} 
ity, shareability, referenceability, and interoperability, as well as results reproducibility.

\subsubsection{Social gradient of asthma: consistent findings and methodological challenges}

The findings in Chapter 5 were consistent with local and international studies. While some studies found that societal affluence was associate with higher asthma prevalence, others found the disease more severe in poorer areas [428]. Low socioeconomic status was associated with less treatment in wheezy children [325], poorer asthma control and persistent airway obstruction in adults [326], and higher asthma hospitalisation rates [323, 324]. Watson et al. found that higher asthma hospitalisation rates among the most deprived could not be explained solely by readmissions; instead, more asthma patients from the poorer areas were hospitalised [323]. The route of admission to hospitals for asthma was not considered in my study, but were previously found to differ by deprivation level. In the West Midland, England, the proportion of asthma admissions through A\&E departments was higher in the poorest than in the richest areas [323], but rates of GP referrals for asthma were not associated with deprivation level. The environment plays a role in inequalities. Air pollution induces asthma exacerbations [318, 319], whereas persistent asthma was associated with poor housing [326, 429-431].

Health inequalities including those in asthma outcomes has been traditionally ecologically assessed. For example, Watson et al. assessed the association between asthma age-standardised admission rate in the whole population of a geographical area with the area's Townsend Deprivation Index [323]. Theoretically, an ecological variable such as asthma prevalence in an area might have affected the admission rate in that area. Yet, the authors ruled out an increased asthma prevalence in poorer communities based on previous surveys. In my study, I assessed the person-level association between area-based deprivation level and asthma hospitalisations, among other outcomes. Therefore, my findings were independent from asthma prevalence. That study used the deprivation level of the hospital area rather than that of the person's address. In my analysis, I used the deprivation index associated with the patient address, which eliminated the bias from admissions in hospitals located in areas with different deprivation levels. 
When both the explanatory and outcome variables being measured at the group or area levels, associations are threatened by ecological fallacy. Replacing aggregated data, person-level RCD are increasingly used to measure health outcomes in health inequality studies [432]. In this thesis, the availability of nation-wide person-level data on asthma outcomes in the SAIL Databank reduced the risk of ecological bias. This bias was further reduced by using a deprivation index, the Welsh Index of Multiple Deprivation (WIMD), that was calculated for relatively small areas (average population $\approx 1,600$ ).

Researching health inequalities is challenging. It is important to calculate the pure effects of socioeconomic factors on health outcomes and to determine causality direction [433]. However, these are not straightforward exercises in an indeterminate space of complexly interrelated factors. Important social determinants of health as well as confounders are often missing or indirectly assessed. RCD often lack these variables; EHRs usually do not capture sufficient data on health literacy, disease self-management, and wider social determinants of health. In order to advance health inequality research, those vital data need to be routinely collected [434].

\subsection{Challenges}

I identified several challenges towards the development of the Observatory. These were mainly related to the complex nature of asthma, data limitations, lack of locally validated methods to assess asthma outcomes, and the public's attitudes to reusing health data.

\subsubsection{Asthma heterogeneity complicates case identification and comparability of studies}

There is an increasing recognition that asthma is a heterogeneous condition, comprising distinct phenotypes and endotypes [4,5]. In addition, there is no consensus on the clinical definitions of asthma and its key outcomes such as disease severity, control, exacerbations $[147,148]$. This is probably reflected in the wide heterogeneity in the methods in which asthma and asthma outcomes have been defined from routinely collected data, as I found in Chapter 2. The lack of standardisation of methods to define and assess asthma hinders the comparability of studies and 
evidence of synthesis. For flexibility, however, the Observatory users can choose from several case definitions of asthma, in addition to the one I developed locally using LCA and recursive partitioning. This enable researchers who use the Observatory to choose a method to identify people with asthma so that their study can be compared to studies that used the same case definition.

\subsubsection{Data from important care domains are still missing}

Despite the availability of various datasets in the SAIL Databank, data from important healthcare domains are still not available. Dispensing data contain information needed to assess medication adherence. However, they are yet to be linked into the SAIL Databank. In addition, treatments and prescriptions given to asthma patients during hospital episodes are not collected into the SAIL Databank. These data can be potentially useful to improve the sensitivity and specificity of methods to identify asthma-related hospitalisations. These data, especially pathology test results, could also improve methods of asthma phenotyping in the Observatory.

In Chapter 5, I demonstrated the utility of the Observatory to support health policy by investigating inequalities in asthma outcomes across the socioeconomic groups. The study had high statistical power and provided useful insights into the magnitude of the asthma social gradient in Wales. However, as with all observational studies, there were limitations with potentially residual confounders, many of which were not readily available or directly measurable in the SAIL Databank. An example of such confounders was health literacy which has significant effects on health status, disease prevention, early diagnosis, adherence to treatment, and disease self-management. Direct, patient-level data on health literacy was not available in the SAIL Databank. Instead, possible proxies include individual or area-based data on education attainment.

\subsubsection{Quality of routinely collected data is imperfect}

Data quality has implications in almost all uses of data. Quality of RCD can significantly influence the internal validity of studies using these data. Data quality is potentially compromised by a variety of factors at different stages of their flow from points of care to data safe havens. These factors include, for example, poor capture and record linkage errors. 
At the point of care, recording and coding of clinical data is often incentivised by payment-for-performance schemes such as the UK's QOF. This means that only a small set of essential health events are well recorded. I showed in Section 4.5 examples of data quality issues where many asthma related data were missing from the SAIL's GP dataset, including lung functions recordings, disease severity stratification, and measures to manage disease control.

Practices of data recording and coding into EHRs differ between health organisations and potentially between healthcare professionals in the same organisation. These practices have been influenced by the type of EHR systems used [435, 436]. They may also change over time due to administrative requirements (e.g., introduction and changes in the QOF indicators) and in response to changes in clinical guidelines and practice protocols.

Record linkage errors also compromise quality of linked data. Despite the high matching rate in the SAIL Databank [140], linkage error may still happen if identifiers are incorrectly recorded or missing [234]. Record linkage errors have been associated with several individual and population factors such as gender, race, geographical location, health status, and socioeconomic status [437]. If not properly addressed, record linkage errors may introduce random and/or systematic errors to study findings $[234,438]$.

\subsubsection{Routinely collected data suffer from time lags}

Many routinely collected data are not available for secondary uses in real-time [80, $439,440]$. Rather, considerable lead time is usually needed before they are made available in usable form [80]. This time, ranging from weeks to several months or years [441], is needed for preparation, transfer, anonymisation, record linkage and encryption as well as quality checks of data [141]. This time lag limits the usability of routinely collected data for applications that require timely data such as producing real-time epidemiological estimates and follow-up of outcomes in prospective studies and clinical trials [442]. Therefore, advances in data collection and transfer operations as well as in infrastructures are needed for seamless and faster production of usable RCD in order to facilitate applications that need timely access to data [440]. 


\subsubsection{Routinely collected data does not reflect precise dis- ease timeline}

Data derived from EHRs often do not reflect precise timeline of chronic disease development. For example, it is practically impossible to accurately determine the date on which a disease starts to develop without intensive follow-up [443, p. 11]. Instead, EHRs usually record the dates at which patients report symptoms to their physicians and when physicians make and record the diagnosis. This leaves implications on epidemiological studies and timely surveillance of asthma. For instance, asthma prevalence at a certain date or during a certain period, may be underestimated unless future data for the denominator population are considered in the estimation.

\subsubsection{Lack of valid methods to assess outcomes}

There were no standardised operational definitions for asthma outcomes in the SAIL Databank. For example, to my knowledge, there were no validated and standardised methods to identify asthma-related emergency and secondary care use. Using different diagnosis positions in hospital admission records to ascertain asthma-related hospitalisation may have significant impact on sensitivity and specificity. Lack of standardisation hinders comparability of studies and evidence synthesis.

\subsubsection{Public attitudes to data re-use are mixed}

The Wales Asthma Observatory is based on linked, anonymised routinely collected data held in the SAIL Databank. The public's awareness of secondary use, anonymisation, and linkage of person level health data is currently limited [444]. In addition, attitudes towards these important concepts are mixed, although a minority of people in the United Kingdom are thought to have concerns about them [444, 445]. Public and patient involvement and partnership, strict information security and governance, and transparency [446] are all needed to win and maintain the trust of data safe haven stakeholders including the public, patients, and data providing organisations. Data safe havens should satisfy high level of competency in safe-guarding data and must have strict protocols to ensure the use of data for the 
public's good [447]. These requirements are fulfilled in the operation model of the SAIL Databank [295]. The mixed public's attitudes toward reusing health data for research may have implications on the plan to collect PROMs into the Observatory from the asthma population in Wales.

\subsection{Implications and potential uses of the Obser- vatory}

The Observatory can be utilised for asthma research as well as to support asthma care in Wales at national, organisational, and patient levels. The design of the Observatory facilitates answering a wide range of questions about asthma in Wales, ranging from prevalence studies to incidence and retrospective longitudinal studies as well as assessment of quality and equality of care.

\subsubsection{Implications on health policy and wider societal impact}

With near complete geographical coverage, the Observatory can support health policy and service planning across Wales. For example, the Observatory can be used to identify variations in the asthma outcomes between patient groups differing by socioeconomic status or age as demonstrated in Chapter 5 . The Observatory can also be used to analyse the trends of these variations and their implication on the disease burden. Linking the Observatory to data on air pollution and housing quality would allow generating insights about the effects of environmental factors data on asthma outcomes. These insights could be used to support healthy urban planning and assess housing regeneration interventions [312, 448, 449].

\subsubsection{Implications on service planning and delivery}

The Observatory can be used to monitor the trends of asthma incidence, prevalence, and estimating the disease burden on the National Health Services (NHS) at regional and national levels and across patient groups on a regular basis. By including linked data on asthma management and disease outcomes across the levels of care, the Observatory provides an ideal platform for Health Boards to assess their performance in asthma care and evaluate the impact of asthma ser- 
vice level interventions. The quality of primary care services, including asthma prescribing and reviews, can be assessed against national guidelines.

\subsubsection{Implications on clinical practice and patient outcomes}

The Observatory has potential applications in clinical practice to improve the outcomes of patients with asthma. The availability of longitudinally assessed asthma outcomes, which are linkable to person level EHR-derived data in the SAIL Databank, facilitates research aiming to improve patient outcomes.

The Observatory can be used in the identification of risk factors, including modifiable ones, of asthma and asthma adverse outcomes. This allows development of algorithms to predict the risk of asthma exacerbations and assess asthma prognosis [70]. I used the Observatory data and its technical platform in the validation of the asthma risk prediction algorithm that has been developed in the "At-Risk Registers Integrated into primary care to Stop Asthma crises in the United Kingdom" (ARRISA-UK) study [450].

The Observatory can also facilitate pharmacovigilance research and studies on how the effectiveness of different interventions differs based on patient characteristics. Such interventions include therapeutic regimens; primary care interventions such as routine and proactive review of asthma status, medications, and action plans; asthma self-management approaches.

The outputs of asthma studies that will use the Observatory can be translated into clinical decision support tools that can be used by healthcare professionals to improve patient care. For example, the ARRISA-UK risk-finding algorithm will be used in GP practice EHR systems to flag records of high-risk asthma patients so that they receive the appropriate attention, disease management, and prevention [451]. In addition, comparative effectiveness studies can be used to develop EHRtools that provide clinicians with patient-tailored asthma prescribing recommendations. The development and validation of such clinical decision support tools is an important potential application of the Observatory in providing stratified and personalised care.

The Observatory can also contribute to improvement of patient outcomes by facilitating implementation research and informing care pathway development. Linking the Observatory data to asthma-related healthcare utilisation from primary 
and secondary care in the SAIL Databank can be useful for the assessment and auditing of guideline implementation and in the improvement of asthma care pathways in primary and secondary care settings.

\subsubsection{Implications on asthma research}

The Observatory can be used as a platform to conduct various types of personlevel observational studies including cross-sectional and longitudinal studies. By linking the Observatory to other data sources in the SAIL Databank, such as education or pollution data, using the linking field, many more research questions about patients in the Observatory can be answered. The Observatory facilitates interrogation of data on asthma patients in a way that increases research efficiency and reproducibility.

\subsection{Towards maximising benefits from population- based data to improve asthma outcomes}

\subsubsection{Improving data capture}

In Chapter 4, I proposed recommendations to improve the capture of asthma data in routine health care. These data should not only include clinical data but also wider societal determinants of health. It has been argued that "every doctor writing in the medical record is an information designer and is responsible for making the data recorded easy to find and interpret" [452]. There are increasing calls to include health informatics education in medical curricula [453], which could improve data quality awareness among health professionals. However, with short clinical encounters, doctors have limited time to spend on data recording. Facilitating valid, accessible, and timely recording of data is one of the core functions of EHR systems. Informatics approaches including natural language processing (NLP), machine learning, and medical knowledge engineering promise automated capture of data that are locked in narrative clinical documentation [454]. EHR design should consider secondary uses of data such as research, service planning, and health policy $[455,456]$. 


\subsubsection{Reducing waste from underuse of data}

Huge volumes of asthma-related RCD are collected every day across Wales. However, a small amount of these data is actually utilised to advance medical knowledge and improve health care delivery and patient outcomes. It has been argued that "the biggest waste in the healthcare system is not unnecessary treatment or duplicated test results; it is that we collect data and never use it again." ${ }^{4}$ Suboptimal utilisation of these data can arguably lead to unnecessary waste in resources, repeated care mistakes and, most importantly, avoidable adverse outcomes for patients [457].

Despite being a preventable disease, asthma adverse outcomes such as exacerbations and deaths still unnecessarily happen. The National Review of Asthma Deaths (NRAD) report "Why asthma still kills" which was published in 2014, found that over two-thirds of asthma deaths were potentially avoidable by better health care and adoption of clinical guidelines as well as better patient adherence to medical advice and treatment [51]. Among its recommendations, the report called for a national audit of asthma. It recommended that asthma audits should be performed on an ongoing basis with involvement and collaboration of patient organisations and commissioners as well as clinicians. A national audit for asthma for Wales and England is currently being scoped and developed in a project led by the Royal College of Physicians [458]. It will focus on helping clinicians improve the documentation of asthma reviews in order to improve patient outcomes. It may also cover several aspects of asthma care such as diagnosis, prescribing, personalised action plan, disease triggers, emergency and secondary care, patient monitoring [459]. Many of these care events can be assessed through the Wales Asthma Observatory on an ongoing basis. Therefore, with its aforementioned strengths, the Observatory is well positioned to play a vital role to support this forthcoming audit programme [458]. Bringing additional asthma-related data such as community prescribing and pathology data to the Observatory will further augment its capability for regularly performed asthma audit and surveillance.

The NRAD was a crucial inquiry into reasons of asthma deaths, which received publicity and attention among respiratory health professional societies and patient organisations. Nonetheless, it is important to evaluate the report's impact since

\footnotetext{
${ }^{4}$ Chris Lehmann, MD, Vanderbilt University Medical Center. https://www.healthcare-informatics.com/blogs/david-raths/promise-structured-data-capture
} 
its publication in 2014. Asthma continues to kill and exacerbate. Similar repeated inquiries are therefore likely to be needed in order to explore the avoidable factors. The NRAD was based mainly on manual review of clinical records from health care providers-a burdensome, expensive and time-consuming process. Such an inquiry can potentially be instead performed using routinely collected data. This will allow rapid and timely investigation into asthma adverse outcomes, which can be regularly repeated, possibly as a part of the forthcoming national asthma audit. The overriding and growing need to bridge the gap between research and care has led to developing the concept of learning health systems (LHS). An LHS aims to maximise learning from delivered care on an ongoing basis which can seamlessly inform future care delivery [291]. Ideally, in a cyclical process, data is converted into knowledge, which in turn informs performance, from which new data is generated and feedback to create new knowledge [292]. The opposite case of a learning health system has been dubbed as a 'forgetting health system' in which "today's mistakes are forgotten quickly and are repeated tomorrow" [460]. In the UK, an initiative to develop a learning health system for asthma has started in Scotland [461]. In Wales, the Wales Asthma Observatory is well-suited to be a building block in a future LHS for asthma.

\subsubsection{Supportive data-intensive research environment}

The Observatory benefits from a supportive, unique research environment and atmosphere in Wales. This doctoral project was funded by Welsh Government's Health and Care Research Wales (HCRW) ${ }^{5}$ and the Abertawe Bro Morgannwg University Health Board. This funding came in line with the Welsh Government's vision to extend the investment in novel research applications of routinely collected data [462]. The Government's report titled "Maximising the Use of Routine Data for Research in Wales" described its plans to support research based on routinely collected data that can be translated into actionable knowledge and direct benefits to the residents of Wales [96].

Partnerships involving government bodies, healthcare providers, research community, and funders are vital to maximise benefits from routinely collected data [77]. The SAIL Databank is funded mainly by the Welsh Government's Health and Care Research Wales and receives support from Farr Institute of Health Infor-

\footnotetext{
${ }^{5}$ Previously named as the National Institute for Social Care and Health Research (NISCHR).
} 
matics Research which is in turn funded by the Medical Research Council (MRC). Governmental and political support and the cooperation of data providing organisations are all crucial for sustainable routine data collection [77]. This facilitates disease surveillance and enables undertaking up-to-date studies. Continuous collection of evidence is also a core requirement of a successful learning health system $[289,291]$.

In the SAIL Databank, anonymisation and record linkage of routine health data are performed with support of the National Health Services Wales Informatics Service (NWIS) $[140,141]$. The SAIL Databank regularly seeks to link new datasets to the existing ones. For example, the Welsh Result Report Service dataset, which includes pathology data, is expected to be available in the SAIL Databank in 2018. Medication dispensing data is another vital dataset which are expected to be linked to the SAIL Databank. Linked data from various domains of health care in the SAIL Databank are key to study and monitor a chronic condition that is managed at various levels of care such as asthma. The prospect of the Observatory, including further developments and wider utilisation, is highly contingent on the continuous support of routinely collected data research in Wales.

\subsubsection{Potentials of asthma big data}

Successful experiences of learning from big data to offer personalised services and insights for organisations have been already happening in non-health care sectors. These sectors include, for example, personal banking, marketing, retailing, social networking, and digital personal assistants. Health care is already in a significant lag behind other industries towards unlocking the full potentials of linkable big data [463]. Utilisation of asthma big data is still at a nascent stage. Patient data are scattered across multiple healthcare providers (e.g., GP practices, hospitals, and pharmacies). While medical record linkage in the UK is more than half a century old, not all patient data are currently linked together. Despite being a single organisation, the UK National Health Services effectively has been acting as disconnected providers. Experience of US healthcare providers such as Kaiser Permanente demonstrates promising case of rapid learning from patient data [288].

Asthma data are rapidly expanding in volumes and complexity including data from emerging and non-clinical paradigms. Advanced biomedical technologies such as 
smart inhalers, wearable sensors, and internet-enabled devices, and gene analysis enable the collection of rich, granular information about symptoms, breathomics, disease self-management, medication use and adherence, environmental factors, and PROMs [464-468]. Large volume of these data could enable better understanding of the disease aetiology and mechanism, and developing precise risk prediction and decision support tools. Publicly available data on people's internet information seeking behaviour and online behaviour can be used to forecast asthma epidemiology and health care resource utilisation [469-472]. Ubiquitous collection, linkage, analysis of asthma-related big data can unlock substantial advantages for individuals and populations.

\subsection{Future work}

\subsubsection{Improving methods to define asthma patients and as- sess disease outcomes}

The latent class model to identify asthma patients from the GP data, described in Chapter 3, was based on a single calendar year (2014). However, due to the change in data capture practices over time, this model may not be valid for different years. It is therefore important to compare this model with similar models developed in different years.

The change in disease status (e.g., new diagnosis, change in severity, and development of comorbidities) can be tracked over time. Latent transition analysis (LTA) can be used for this purpose. It aims to identify latent statuses of individuals, defined over multiple time points or intervals, which explain the changes in the observed characteristics in the population. LTA can be thought of as an LCA repeated for the same cohort of patients over several intervals. LTA will allow exploration of common disease trajectories of asthma patients and their transition, if any, between different disease subgroups.

In Chapter 2, I found that, in the contemporary asthma literature, asthma severity and control were most often assessed over a 12-month interval. An expert report proposed that asthma control should be assessed over an interval of 2 to 4 weeks for adults and at least 4 weeks for children [2]. A 12-month interval for assessing a disease state is arguably a traditional artefact that makes assessment easier for 
investigators. However, this interval may not be the optimal one for assessment of asthma severity and control. For example, a high disease severity inferred from the number of high-dose prescriptions over 12 months does not necessarily mean the disease was 'severe' all over the year. An unjustified interval may lead to misclassification of disease statuses and may undermine the study validity. Therefore, it is important to explore the optimal intervals for disease statuses, possibly using event sequence analysis and related visualisation techniques [473, 474].

\subsubsection{Understanding inequalities in asthma outcomes}

The analysis of inequalities of asthma outcomes, presented in Chapter 5, revealed a wide gradient in asthma outcomes over five-year follow-up across the socioeconomic groups in Wales. In that analysis, I used the WIMD index quintiles as an explanatory variable. However, it is important to investigate the distribution of asthma outcomes across each of the individual domains that make up the overall deprivation index.

An extension of the study should also investigate whether the inequality gaps can be partly explained by the individual deprivation domains and a number of potential factors. These include patient's health literacy, education attainment, selfmanagement, inhaler technique, environmental smoke exposure, housing conditions, air pollution, comorbidities, ethnicity, as well as quality of primary care and prescribing and proximity to GP practices and emergency departments. The time trends of the social gradient of asthma should be also assessed at national and regional levels.

\subsubsection{Monitoring and forecasting asthma trends}

The availability of longitudinal data from about two decades in the SAIL Databank allows understanding and forecasting the seasonal and annual trends of asthma. This can be performed by time series analysis and forecasting techniques such as the autoregressive integrated moving average (ARIMA) of counts of healthcare events over time intervals. 


\subsubsection{Linking additional data sources}

Linking additional healthcare datasets to the Observatory will allow answering more research questions. Among such person-level datasets is the Welsh Results Reports Service (WRRS) dataset [475]. This dataset includes laboratory test results across all Wales, and is currently in the process of being transferred into the SAIL Databank. While laboratory test results can be recorded in the general practice EHR system, they suffer from missingness and inconsistency across general practices. Linking the WRRS data to the Observatory will potentially allow improvement in the identification and phenotyping of asthma patients. For example, more accurate data on peripheral eosinophil counts, total and specific IgE can help in the identification of patients with eosinophilic and atopic asthma.

Person-level medication dispensing data are also available in Wales, but are not currently available in the SAIL Databank. These data can be used to complement and crosscheck prescription data in the General Practice (GP) dataset. Prescription data in the GP dataset currently do not include the quantity of the total prescribed dose prescribed to the patient. Dispensing data ideally contain these pieces of information, which can be used, for example, to assess asthma severity and control (e.g., using a more accurate number of actually used short-acting beta agonist inhalers) and identify exacerbations (e.g., by calculating the total supplied dose and duration of administration of oral corticosteroids). Dispensing data also contain the evidence that prescriptions issued by GPs are taken by patients to pharmacies where they are dispensed. This information would provide an indicative picture, although not certain, about patient's adherence to medications. It is not currently known, however, when dispensing data will be available in the SAIL Databank.

Collecting asthma-related PROMs is important to understand patient's perspective about the disease, treatment, disease control, and quality of life. These data include standardised tools such as the AQLQ, ACQ, and ACT, which can be collected during the clinical encounter or at patients' home. The incentives and barriers towards collecting such data need to be explored. Linking those data to RCD in the SAIL Databank will enable identifying healthcare interventions that are most important from patients' perspectives. It will also allow the assessment 
of the concurrent validity of objective measures of asthma outcomes against the correspondent PROMs (e.g., RCD-based asthma control definition vs. ACQ).

\subsubsection{Improving the Observatory's technical platform}

The Observatory's data interrogation interface needs further work to improve the workflow of users. Planned improvements include support to additional data extraction methods, and better documentation of data extraction. I also work with the SAIL technical team towards making this interface available to the public. ${ }^{6}$ This will promote transparency, sharing, and reproducibility of studies that use the Observatory.

\subsubsection{Data quality reports}

The Observatory could provide reports for the quality of its data. Examples of these reports are those on the quality of recorded lung function data presented in Chapter 4. It is important for users of the Observatory to be aware of data quality issues beforehand. This will inform their study design and analysis, and makes it easier for them to communicate data quality issues in their reports [157].

\subsubsection{Getting ready for SNOMED-CT}

In 2018, the Systematized Nomenclature of Medicine-Clinical Terms (SNOMEDCT) will replace Read code vocabulary as the terminology of primary care in the UK [311]. This transition is intended to support standardisation of clinical data capture across the National Health Service. It is unclear how long this transition will take across Wales. However, it will be crucial to ensure compatibility of the Observatory with the new data that will feed into the SAIL Databank from general practices. This requires updating the methods used to identify asthma patients and assess disease outcomes to capture data coded in SNOMED-CT. This can be performed with the help of the Data Migration package, originally provided by the NHS's Health and Social Care Information Centre (NHS Digital) to guide the transition. In addition to recognising the new data coded with SNOMED-CT, the Observatory will still support backward compatibility with the historical Read Code data held in the SAIL Databank.

\footnotetext{
${ }^{6}$ The interrogation interface outside the SAIL Gateway does not allow access to patient data.
} 


\subsection{Conclusions}

In this thesis, I described the establishment of the Wales Asthma Observatory using routinely collected data in Wales. The Observatory represents a non-traditional, cost-effective approach to a patient registry and a platform for asthma surveillance and research.

RCD offer unique opportunities to understand asthma, inform health policy and service planning, and improve patient outcomes. However, the inherent limitations of these data impose challenges on those endeavours.

Among these challenges, defining a heterogeneous disease such as asthma using RCD is fraught with pitfalls. In a systematic scoping review of the contemporary literature, I found wide variation in methods to define asthma and its key outcomes using RCD, let alone suboptimal reporting on implementation and validity of those methods. These findings reflected the lack of consensus on the clinical definitions of asthma and its outcomes as well as the wide differences in data resources. The findings highlight the need to reach a consensus on clinical definition of asthma and its outcomes and to harmonise operational definitions in RCD studies.

With the absence of a gold standard for asthma definition, unsupervised analysis of asthma-related RCD coupled with clinico-epidemiological knowledge can identify likely asthma patients. Clustering methods seek to identify the most likely population structure behind the recorded data. Using latent class analysis, I identified fuzzy clusters of asthma and COPD patients, based on which I derived a classification algorithm to identify patients with any or both diseases. The probabilistic case definition of asthma using RCD fits with the probabilistic approach to diagnose asthma in clinical practice. In addition to the LCA-based case definition, the Observatory offers other commonly used asthma case definitions.

Quality of asthma-related RCD in Wales is suboptimal. I described various patterns of missingness and inconsistencies in the recorded asthma data. I recommended measures to improve the capture of asthma data including data quality awareness training of healthcare professionals, improved data entry checks, and data qualitybased incentives. NLP promises to capture clinical data that are otherwise locked in narrative documentations. 
Facilitating data interrogation is a growing requirement in RCD resources. The Observatory supports shareable, reusable, and scalable data extraction, which promotes research efficiency and reproducibility.

Health inequalities indicate unjust outcome variations within the population and lead to wasted resources. They are a key challenge to health policy. I demonstrated the Observatory's value to health policy by exploring the social gradient of asthma in Wales. I described a wide social gradient in asthma outcomes; despite an excess in asthma-related primary care contact in the most deprived areas, asthma patients there were more than twice likely to be hospitalised for asthma than those in the least deprived areas. This suggested a wide gap in asthma control, that should be further investigated to identify avoidable contributing factors. There is a growing attention to the waste and harm caused by the underuse of health data. The Observatory is a promising endeavour to maximise the use of asthma data in Wales in research and surveillance. It is well-positioned to play a vital role in the upcoming national asthma audit programme in Wales. Learning health systems effectively learn from experience in order to improve services. The Observatory could be a building block in a future learning health system for asthma in Wales. 


\section{References}

[1] Global Initiative for Asthma. Global Strategy for Asthma Management and Prevention (2017 update). 2017.

[2] Bousquet J, Mantzouranis E, Cruz AA, Alt-Khaled N, Baena-Cagnani CE, et al. Uniform definition of asthma severity, control, and exacerbations: document presented for the World Health Organization Consultation on Severe Asthma. J Allergy Clin Immunol 126.5 (2010), 926938.

[3] Wenzel SE. Asthma: defining of the persistent adult phenotypes. Lancet 368.9537 (2006), 804813.

[4] Lötvall J, Akdis CA, Bacharier LB, Bjermer L, Casale TB, et al. Asthma endotypes: a new approach to classification of disease entities within the asthma syndrome. J Allergy Clin Immunol 127.2 (2011), 355-360.

[5] Wenzel SE. Asthma phenotypes: the evolution from clinical to molecular approaches. Nat Med 18.5 (2012), 716-725.

[6] Subbarao P, Mandhane PJ, and Sears MR. Asthma: epidemiology, etiology and risk factors. Can Med Assoc J 181.9 (2009), E181-E190.

[7] Haldar P, Pavord ID, Shaw DE, Berry MA, Thomas M, et al. Cluster analysis and clinical asthma phenotypes. Am J Respir Crit Care Med 178.3 (2008), 218-224.

[8] Hekking PPW and Bel EH. Developing and emerging clinical asthma phenotypes. J Allergy Clin Immunol Pract 2.6 (2014), 671-80, quiz 681.

[9] Holgate ST, Wenzel S, Postma DS, Weiss ST, Renz H, et al. Asthma. Nature Reviews Disease Primers (2015), 15025.

[10] Maskell N and Millar A. Oxford Desk Reference: Respiratory Medicine. Oxford University Press, 2009. 471 pp. ISBN: 0199239126.

[11] Kasper DL, Fauci AS, Hauser SL, Longo DL, and Jameson JL. Harrison's Principles of Internal Medicine, 19 ed. McGraw-Hill Education, LLC CoreSource, 2015.

[12] Ricciardolo FL, Folkerts G, Folino A, and Mognetti B. Bradykinin in asthma: Modulation of airway inflammation and remodelling. European Journal of Pharmacology 827 (2018), 181188.

[13] Spiro S. Clinical Respiratory Medicine. Elsevier Health Sciences, 2012. 1000 pp.

[14] Chapman DG and Irvin CG. Mechanisms of airway hyper-responsiveness in asthma: the past, present and yet to come. Clinical \& Experimental Allergy 45.4 (2015), 706-719.

[15] JA Bernstein and ML Levy, eds. Clinical Asthma: Theory and Practice. CRC Press, 2014. 337 pp.

[16] British Thoracic Society, Scottish Intercollegiate Guidelines Network. British guideline on the management of asthma : A national clinical guideline. 2016. 
[17] Weinberger $M$ and Fischer A. Differential diagnosis of chronic cough in children. Allergy Asthma Proc 35.2 (2014), 95-103.

[18] Miravitlles M, Andreu I, Romero Y, Sitjar S, Altés A, et al. Difficulties in differential diagnosis of COPD and asthma in primary care. Br J Gen Pract 62.595 (2012), e68-e75.

[19] Third Expert Panel on the Diagnosis and Management of Asthma. Expert Panel Report 3: Guidelines for the Diagnosis and Management of Asthma. National Asthma Education, Prevention Program. National Heart, Lung, and Blood Institute (US), Bethesda (MD), 2007.

[20] Quanjer PH, Stanojevic S, Cole TJ, Baur X, Hall GL, et al. Multi-ethnic reference values for spirometry for the 3-95-yr age range: the global lung function 2012 equations. European Res piratory Journal 40.6 (2012), 1324-1343.

[21] Melbye H, Drivenes, Dalbak, Leinan, Ostrem, et al. Asthma, chronic obstructive pulmonary disease, or both? Diagnostic labeling and spirometry in primary care patients aged 40 years or more. International Journal of Chronic Obstructive Pulmonary Disease (2011), 597.

[22] Brouwer AFJ, Roorda RJ, and Brand PLP. Home spirometry and asthma severity in children. European Respiratory Journal 28.6 (2006), 1131-1137.

[23] Bacharier LB, Strunk RC, Mauger D, White D, Lemanske RF, et al. Classifying Asthma Severity in Children. American Journal of Respiratory and Critical Care Medicine 170.4 (2004), 426-432.

[24] Irvin CG. Pulmonary function testing in asthma. UpToDate. 2018. URL: https : / / www . uptodate.com/contents/pulmonary-function-testing-in-asthma.

[25] Calverley PMA. Bronchodilator reversibility testing in chronic obstructive pulmonary disease. Thorax 58.8 (2003), 659-664.

[26] A Harver and H Kotses, eds. Asthma, Health and Society. Springer-Verlag GmbH, 2010. ISBN: 0387782842.

[27] Saydain G, Beck KC, Decker PA, Cowl CT, and Scanlon PD. Clinical Significance of Elevated Diffusing Capacity. Chest 125.2 (2004), 446-452.

[28] Dweik RA. Exhaled nitric oxide analysis and applications. UpToDate. 2018. URL: https : / / www. uptodate.com/contents/exhaled-nitric-oxide-analysis-and-applications.

[29] Rees J, Kanabar D, and Pattani S. ABC of Asthma. BMJ Books, 2010. ISBN: 978-1-4051-8596-7.

[30] Asthma: diagnosis, monitoring and chronic asthma management [NG80]. 2017. URL: https: //www.nice.org.uk/guidance/ng80/chapter/recommendations.

[31] British Thoracic Society, Scottish Intercollegiate Guidelines Network. British guideline on the management of asthma : A national clinical guideline. 2014.

[32] Lenney W, Clayton S, Gilchrist FJ, Price D, Small I, et al. Lessons learnt from a primary care asthma improvement project. npj Primary Care Respiratory Medicine 26.1 (2016).

[33] British National Formulary. URL: https: //www. bnf.org (visited on 02/07/2018).

[34] Chee C, Sellahewa L, and Pappachan JM. Inhaled Corticosteroids and Bone Health. The Open Respiratory Medicine Journal 8.1 (2015), 85-92.

[35] Wechsler ME, Wong DA, Miller MK, and Lawrence-Miyasaki L. Churg-Strauss Syndrome in Patients Treated With Omalizumab. Chest 136.2 (2009), 507-518.

[36] The Global Asthma Report 2014. The Global Asthma Network, 2014.

[37] Mallol J, Crane J, von Mutius E, Odhiambo J, Keil U, et al. The International Study of Asthma and Allergies in Childhood (ISAAC) Phase Three: A global synthesis. Allergol Immunopathol (Madr ) 41.2 (2013), 73-85. 
[38] Masoli M, Fabian D, Holt S, and Beasley R. The global burden of asthma: executive summary of the GINA Dissemination Committee Report. Allergy 59.5 (2004), 469-478.

[39] Anandan C, Nurmatov U, Van Schayck O, and Sheikh A. Is the prevalence of asthma declining? Systematic review of epidemiological studies. Allergy 65.2 (2010), 152-167.

[40] Bousquet J, Bousquet PJ, Godard P, and Daures JP. The public health implications of asthma. Bull World Health Organ 83.7 (2005), 548-554.

[41] Bahadori K, Doyle-Waters MM, Marra C, Lynd L, Alasaly K, et al. Economic burden of asthma: a systematic review. BMC Pulm Med 9.1 (2009), 24.

[42] Fletcher M, Jha A, Dunlop W, Heron L, Wolfram V, et al. Patient Reported Burden of Asthma on Resource Use and Productivity Across 11 Countries in Europe. Adv Ther 32.4 (2015), 370-380.

[43] Mukherjee M, Stoddart A, Gupta RP, Nwaru BI, Farr A, et al. The epidemiology, healthcare and societal burden and costs of asthma in the UK and its member nations: analyses of standalone and linked national databases. BMC Med 14.1 (2016).

[44] Ivanova JI, Bergman R, Birnbaum HG, Colice GL, Silverman RA, et al. Effect of asthma exacerbations on health care costs among asthmatic patients with moderate and severe persistent asthma. J Allergy Clin Immunol 129.5 (2012), 1229-1235.

[45] OŃeill S, Sweeney J, Patterson CC, Menzies-Gow A, Niven R, et al. The cost of treating severe refractory asthma in the UK: an economic analysis from the British Thoracic Society Difficult Asthma Registry. Thorax 70.4 (2015), 376-378.

[46] Pawankar R. Allergic diseases and asthma: a global public health concern and a call to action. World Allergy Organ J 7.1 (2014), 12.

[47] Asher MI, Montefort S, Björkstén B, Lai CK, Strachan DP, et al. Worldwide time trends in the prevalence of symptoms of asthma, allergic rhinoconjunctivitis, and eczema in childhood: ISAAC Phases One and Three repeat multicountry cross-sectional surveys. Lancet 368.9537 (2006), 733-743.

[48] Patel SP, Järvelin MR, and Little MP. Systematic review of worldwide variations of the prevalence of wheezing symptoms in children. Environ Health 7 (2008), 57.

[49] Lai CKW, Beasley R, Crane J, Foliaki S, Shah J, et al. Global variation in the prevalence and severity of asthma symptoms: Phase Three of the International Study of Asthma and Allergies in Childhood (ISAAC). Thorax 64.6 (2009), 476-483.

[50] Asthma UK. URL: http: //www.asthma.org.uk/cymru (visited on 03/31/2016).

[51] Levy M, Andrews R, Buckingham R, Evans H, Francis C, et al. Why asthma still kills: The National Review of Asthma Deaths (NRAD). Royal College of Physcians, 2014.

[52] Porta M, Greenland S, Hernán M, Santos Silva I dos, and Last JM. A dictionary of epidemiology. 6th Edition. Oxford University Press, USA, 2014.

[53] Welsh Assembly Government. Welsh Health Survey. URL: http : / gov . wales/statistics and-research/welsh-health-survey/ (visited on 02/06/2018).

[54] Welsh Assembly Government. Welsh Health Survey 2014: Health status, illnesses, and other conditions. 2015.

[55] Welsh Assembly Government. Welsh Health Survey 2014: Health of children. 2015.

[56] Doyle M, Dixon J, and Sadler K. Welsh Health Survey Evaluation. National Centre for Social Research, 2010.

[57] Weekly Returns Service Annual Report 2011. Royal College of General Practitioners - Research \& Surveillance Centre, 2011. 
[58] GPOne. QOF. URL: http://www. gpone.wales.nhs.uk/qof (visited on 02/06/2018).

[59] GMS Contract. StatsWales. URL: https://statswales.wales.gov.uk/Catalogue/Healthand - Social - Care / NHS - Primary - and - Community - Activity / GMS - Contract (visited on 05/09/2016).

[60] Kupczyk M, Haahtela T, Cruz AA, and Kuna P. Reduction of asthma burden is possible through National Asthma Plans. Allergy 65.4 (2010), 415-419.

[61] Haahtela T. A 10 year asthma programme in Finland: major change for the better. Thorax 61.8 (2006), 663-670.

[62] Burr ML, Davies BH, Hoare A, Jones A, Williamson IJ, et al. A confidential inquiry into asthma deaths in Wales. Thorax 54.11 (1999), 985-989.

[63] Cowie RL, Revitt SG, Underwood MF, and Field SK. The Effect of a Peak Flow-Based Action Plan in the Prevention of Exacerbations of Asthma. Chest 112.6 (1997), 1534-1538.

[64] Ducharme FM, Zemek RL, Chalut D, McGillivray D, Noya FJD, et al. Written Action Plan in Pediatric Emergency Room Improves Asthma Prescribing, Adherence, and Control. Am J Respir Crit Care Med 183.2 (2011), 195-203.

[65] Honkoop PJ, Taylor DR, Smith AD, Snoeck-Stroband JB, and Sont JK. Early detection of asthma exacerbations by using action points in self-management plans. Eur Respir J 41.1 (2012), 5359.

[66] Turner S, Burden A, Thomas M, Murray C, and Price D. Predicting asthma exacerbations in children - A real life observational study. Vol. 46. suppl 59. European Respiratory Society (ERS), 2015, PA4511.

[67] Pinart M, Smit HA, Keil T, Bousquet J, Antó JM, et al. Systematic review of childhood asthma prediction models. Eur Respir J 46.suppl 59 (2015), PA4513.

[68] OĆonnor RD, Bleecker ER, Long A, Tashkin D, Peters S, et al. Subacute lack of asthma control and acute asthma exacerbation history as predictors of subsequent acute asthma exacerbations: evidence from managed care data. J Asthma 47.4 (2010), 422-428.

[69] van der Mark LB, van Wonderen KE, Mohrs J, van Aalderen WM, ter Riet G, et al. Predicting asthma in preschool children at high risk presenting in primary care: development of a clinical asthma prediction score. Prim Care Respir J 23.1 (2014), 52-59.

[70] Blakey JD, Price DB, Pizzichini E, Popov TA, Dimitrov BD, et al. Identifying Risk of Future Asthma Attacks Using UK Medical Record Data: A Respiratory Effectiveness Group Initiative. J Allergy Clin Immunol Pract 5.4 (2017), 1015-1024.e8.

[71] Dimond B. Exploring the legal status of healthcare documentation in the UK. Br J Nurs 14 (9 2005), 517-518.

[72] Hansell A and Aylin P. Routine Data and Health Impact Assessment: A Review of Epidemiological Studies of Socio-economic Influence on Health and Evaluation of Outcome Indicators Derived from Routine Health Data for Health Impact Assessment. 2000.

[73] NHS Wales Informatics Service. ICD-10. URL: http : / / www . nwisinformationstandards . wales.nhs.uk/icd-10 (visited on 02/07/2018).

[74] NHS Digital. Read Codes. URL: https://digital.nhs.uk/article/1104/Read-Codes (visited on $02 / 07 / 2018)$.

[75] NHS Digital. SNOMED-CT. URL: https : / / digital . nhs . uk / snomed - ct (visited on 02/07/2018).

[76] NHS Digital. National Clinical Coding Standards OPCS-4 (2017). (Visited on 02/07/2018). 
[77] Morrato EH, Elias M, and Gericke CA. Using population-based routine data for evidence-based health policy decisions: lessons from three examples of setting and evaluating national health policy in Australia, the UK and the USA. J Public Health (Oxf) 29.4 (2007), 463-471.

[78] Raftery J, Roderick P, and Stevens A. Potential use of routine databases in health technology assessment. Health Technol Assess 9.20 (2005), 1-92, iii-iv.

[79] Husain MJ, Brophy S, Macey S, Pinder LM, Atkinson MD, et al. HERALD (health economics using routine anonymised linked data). BMC Med Inform Decis Mak 12 (2012), 24.

[80] Kane R, Wellings K, Free C, and Goodrich J. Uses of routine data sets in the evaluation of health promotion interventions: opportunities and limitations. Health Educ 100.1 (2000), 33-41.

[81] Hemkens LG, Langan SM, and Benchimol EI. Better research reporting to improve the utility of routine data for making better treatment decisions. J Comp Eff Res (2016).

[82] Hemkens LG, Contopoulos-Ioannidis DG, and Ioannidis JPA. Routinely collected data and comparative effectiveness evidence: promises and limitations. Can Med Assoc J (2016).

[83] Anandan C, Simpson CR, Fischbacher C, and Sheikh A. Exploiting the potential of routine data to better understand the disease burden posed by allergic disorders. Clin Exp Allergy 36.7 (2006), 866-871.

[84] Furnham A. Response bias, social desirability and dissimulation. Pers Individ Dif 7.3 (1986), 385-400.

[85] Choi BC and Pak AW. A Catalog of Biases in Questionnaires. Prev Chronic Dis 2.1 (2005).

[86] Cord KAM, Salman RAS, Treweek S, Gardner H, Strech D, et al. Routinely collected data for randomized trials: promises, barriers, and implications. Trials 19.1 (2018).

[87] Fralick M, Kesselheim AS, Avorn J, and Schneeweiss S. Use of Health Care Databases to Support Supplemental Indications of Approved Medications. JAMA Internal Medicine 178.1 (2018), 55.

[88] Whitelaw FG, Nevin SL, Milne RM, Taylor RJ, Taylor MW, et al. Completeness and accuracy of morbidity and repeat prescribing records held on general practice computers in Scotland. $B r$ J Gen Pract 46 (404 1996), 181-186.

[89] Gray J. Use of Read codes in diabetes management in a south London primary care group: implications for establishing disease registers. BMJ 326.7399 (2003), 1130.

[90] Majeed A, Car J, and Sheikh A. Accuracy and completeness of electronic patient records in primary care. Fam Pract 25.4 (2008), 213-214.

[91] Quan H and Williamson T. Guiding the reporting of studies that use routinely collected health data. Can Med Assoc J (2016).

[92] Loke YK. Use of databases for clinical research. Arch Dis Child 99.6 (2014), 587-589.

[93] de Lusignan S. The use of routinely collected computer data for research in primary care: opportunities and challenges. Fam Pract 23.2 (2005), 253-263.

[94] Stammers JG, Kuo A, Hart AJ, Smeeth L, and Skinner JA. Registry Data-Valuable Lessons But Beware the Confounders. J Arthroplasty 32.9 (2017), S63-S67.

[95] Nathan H and Pawlik TM. Limitations of Claims and Registry Data in Surgical Oncology Research. Ann Surg Oncol 15.2 (2007), 415-423.

[96] The National Institute for Social Care and Health Research (NISCHR). Maximising the Use of Routine Data for Research in Wales. 2013.

[97] Enabling Data Linkage to Maximise the Value of Public Health Research Data: Summary. Wellcome Trust, 2015. 
[98] Linking and sharing routine health data for research in England. PHG Foundation, 2017.

[99] Schatz $M$ and Zeiger RS. Improving asthma outcomes in large populations. J Allergy Clin Immunol 128.2 (2011), 273-277.

[100] Stempel DA, McLaughin TP, Stanford RH, and Fuhlbrigge AL. Patterns of asthma control: a 3-year analysis of patient claims. J Allergy Clin Immunol 115.5 (2005), 935-939.

[101] Schatz M, Nakahiro R, Crawford W, Mendoza G, Mosen D, et al. Asthma quality-of-care markers using administrative data. Chest 128.4 (2005), 1968-1973.

[102] Al Sallakh MA, Vasileiou E, Rodgers SE, Lyons RA, Sheikh A, et al. Defining asthma and assessing asthma outcomes using electronic health record data: a systematic scoping review. Eur Respir J 49 (6 2017).

[103] Schatz M, Zeiger RS, Yang SJ, Chen W, Crawford W, et al. Change in asthma control over time: predictors and outcomes. J Allergy Clin Immunol Pract 2.1 (2014), 59-64.

[104] Klomp H, Lawson JA, Cockcroft DW, Chan BT, Cascagnette P, et al. Examining asthma quality of care using a population-based approach. CMAJ 178.8 (2008), 1013-1021.

[105] Peled R, Tal A, Pliskin JS, and Reuveni H. A computerized surveillance system for the quality of care in childhood asthma. J Healthc Qual 27.5 (2005), 28-33.

[106] Hsiao HJ, Wang LC, Yang YH, Lee JH, Yu HH, et al. A nationwide survey of the severity, comorbidity, and mortality of hospitalized patients with asthma in Taiwan. Pediatr Neonatol 54.4 (2013), 254-260.

[107] Dombkowski KJ, Wasilevich EA, and Lyon-Callo SK. Pediatric asthma surveillance using Medicaid claims. Public Health Rep 120.5 (2005), 515-524.

[108] Anandan C, Gupta R, Simpson C, Fischbacher C, and Sheikh A. Epidemiology and disease burden from allergic disease in Scotland: analyses of national databases. J R Soc Med 102.10 (2009), 431-442. eprint: http://jrs.sagepub.com/content/102/10/431. full.pdf+html.

[109] Simpson CR and Sheikh A. Trends in the epidemiology of asthma in England: a national study of 333,294 patients. J R Soc Med 103.3 (2010), 98-106.

[110] Almqvist C, Wettermark B, Hedlin G, Ye W, and Lundholm C. Antibiotics and asthma medication in a large register-based cohort study - confounding, cause and effect. Clin Exp Allergy 42.1 (2012), 104-111.

[111] Metsälä J, Lundqvist A, Virta LJ, Kaila M, Gissler M, et al. Prenatal and post-natal exposure to antibiotics and risk of asthma in childhood. Clin Exp Allergy 45.1 (2015), 137-145.

[112] Decker WW, Campbell RL, Manivannan V, Luke A, St Sauver JL, et al. The etiology and incidence of anaphylaxis in Rochester, Minnesota: a report from the Rochester Epidemiology Project. J Allergy Clin Immunol 122.6 (2008), 1161-1165.

[113] Watson L, Turk F, James P, and Holgate ST. Factors associated with mortality after an asthma admission: A national United Kingdom database analysis. Respir Med 101.8 (2007), 16591664.

[114] Lee T, Kim J, Kim S, Kim K, Park Y, et al. Risk factors for asthma-related healthcare use: longitudinal analysis using the NHI claims database in a Korean asthma cohort. PLoS One 9.11 (2014), e112844.

[115] Peters D, Chen C, Markson LE, Allen-Ramey FC, and Vollmer WM. Using an asthma control questionnaire and administrative data to predict health-care utilization. Chest 129.4 (2006), 918-924. 
[116] Dombkowski KJ, Leung SW, and Gurney JG. Prematurity as a predictor of childhood asthma among low-income children. Ann Epidemiol 18.4 (2008), 290-297.

[117] Thomas M, Cleland J, and Price D. Database studies in asthma pharmacoeconomics: uses, limitations and quality markers. Expert Opin Pharmacother 4.3 (2003), 351-358.

[118] Price D, Chisholm A, van der Molen T, Roche N, Hillyer EV, et al. Reassessing the evidence hierarchy in asthma: evaluating comparative effectiveness. Curr Allergy Asthma Rep 11.6 (2011), 526-538.

[119] Krishnan JA, Schatz M, and Apter AJ. A call for action: Comparative effectiveness research in asthma. J Allergy Clin Immunol 127.1 (2011), 123-127.

[120] Labrèche F, Kosatsky T, and Przybysz R. Childhood asthma surveillance using administrative data: consistency between medical billing and hospital discharge diagnoses. Can Respir J 15.4 (2008), 188-192.

[121] Dombkowski KJ, Wasilevich EA, Lyon-Callo S, Nguyen TQ, Medvesky MG, et al. Asthma surveillance using Medicaid administrative data: a call for a national framework. J Public Health Manag Pract 15.6 (2009), 485-493.

[122] Travers D, Lich KH, Lippmann SJ, Weinberger M, Yeatts KB, et al. Defining emergency department asthma visits for public health surveillance, North Carolina, 2008-2009. Prev Chronic Dis 11 (2014), E100.

[123] Larsson S, Lawyer P, Garellick G, Lindahl B, and Lundstrom M. Use Of 13 Disease Registries In 5 Countries Demonstrates The Potential To Use Outcome Data To Improve Health Cares Value. Health Aff (Millwood) 31.1 (2011), 220-227.

[124] Newton J and Garner S. Disease registers in England. Institute of Health Sciences, University of Oxford, 2002.

[125] Metzger J. Using computerized registries in chronic disease care. California HealthCare Foundation, 2004.

[126] Heaney LG, Brightling CE, Menzies-Gow A, Stevenson M, Niven RM, et al. Refractory asthma in the UK: cross-sectional findings from a UK multicentre registry. Thorax 65.9 (2010), 787-794.

[127] Navaneethan SD, Jolly SE, Schold JD, Arrigain S, Saupe W, et al. Development and validation of an electronic health record-based chronic kidney disease registry. Clin J Am Soc Nephrol 6.1 (2011), 40-49.

[128] Mowat F, Lau, Whyte, Kelsh, Legg, et al. Use of electronic medical records (EMR) for oncology outcomes research: assessing the comparability of EMR information to patient registry and health claims data. Clinical Epidemiology (2011), 259.

[129] Denaxas SC, George J, Herrett E, Shah AD, Kalra D, et al. Data Resource Profile: Cardiovascular disease research using linked bespoke studies and electronic health records (CALIBER). Int J Epidemiol 41.6 (2012), 1625-1638.

[130] Ford DV, Jones KH, Middleton RM, Lockhart-Jones H, Maramba ID, et al. The feasibility of collecting information from people with Multiple Sclerosis for the UK MS Register via a web portal: characterising a cohort of people with MS. BMC Med Inform Decis Mak 12.1 (2012).

[131] Hemmings J and Wilkinson J. What is a public health observatory? J Epidemiol Community Health 57.5 (2003), 324-326.

[132] Ashton JR. Public Health Observatories-the key to timely public health intelligence in the new century. J Epidemiol Community Health 54.10 (2000), 724-725. 
[133] Liverpool Public Health Observatory. URL: https : / / www . liverpool . ac . uk/psychology health-and-society/research/public-health-observatory/about/.

[134] The Ontario Asthma Surveillance Information System (OASIS). URL: http://lab. research . sickkids.ca/oasis (visited on 05/12/2016).

[135] Meredith S, Taylor V, and McDonald J. Occupational respiratory disease in the United Kingdom 1989: a report to the British Thoracic Society and the Society of Occupational Medicine by the SWORD project group. Occup Environ Med 48.5 (1991), 292-298.

[136] Kopferschmitt-Kubler M, Ameille J, Popin E, Calastreng-Crinquand A, Vervloet D, et al. Occupational asthma in France: a 1-yr report of the Observatoire National de Asthmes Professionnels project. Eur Respir J 19.1 (2002), 84-89.

[137] Desai JR, Wu P, Nichols GA, Lieu TA, and OĆonnor PJ. Diabetes and asthma case identification, validation, and representativeness when using electronic health data to construct registries for comparative effectiveness and epidemiologic research. Med Care 50 Suppl (2012), S30-S35.

[138] Morris RD, Naumova EN, Goldring J, Hersch M, Munasinghe RL, et al. Childhood asthma surveillance using computerized billing records: a pilot study. Public Health Rep 112.6 (1997), 506-512.

[139] Karakis I, Blumenfeld M, Yegev Y, Goldfarb D, Bolotin A, et al. A computerized surveillance system for asthma. Int J Health Care Qual Assur 24.4 (2011), 308-313.

[140] Lyons RA, Jones KH, John G, Brooks CJ, Verplancke JP, et al. The SAIL databank: linking multiple health and social care datasets. BMC Med Inform Decis Mak 9 (2009), 3.

[141] Ford DV, Jones KH, Verplancke JP, Lyons RA, John G, et al. The SAIL Databank: building a national architecture for e-health research and evaluation. BMC Health Serv Res 9.1 (2009), 157.

[142] Belgrave D, Henderson J, Simpson A, Buchan I, Bishop C, et al. Disaggregating asthma: Big investigation versus big data. J Allergy Clin Immunol 139.2 (2017), 400-407.

[143] Global Initiative for Asthma. Global Strategy for Asthma Management and Prevention (2015 update). 2015.

[144] Hargreave FE and Nair P. The definition and diagnosis of asthma. Clin Exp Allergy 39.11 (2009), 1652-1658.

[145] A plea to abandon asthma as a disease concept. Lancet 368.9537 (2006), 705.

[146] Reddel HK, Bateman ED, Becker A, Boulet LP, Cruz AA, et al. A summary of the new GINA strategy: a roadmap to asthma control. Eur Respir J 46.3 (2015), 622-639.

[147] van den Akker IL, van der Zeijden H, and Verheij TJ. Is spirometry essential in diagnosing asthma? Yes. Br J Gen Pract 66.650 (2016), 484-484.

[148] Levy ML. Is spirometry essential in diagnosing asthma? No. Br J Gen Pract 66.650 (2016), 485-485.

[149] Toelle BG, Peat JK, Salome CM, Mellis CM, and Woolcock AJ. Toward a Definition of Asthma for Epidemiology. Am Rev Respir Dis 146.3 (1992), 633-637.

[150] Pekkanen J and Pearce N. Defining asthma in epidemiological studies. Eur Respir J 14.4 (1999), 951-957.

[151] Jorm L. Routinely collected data as a strategic resource for research: priorities for methods and workforce. Public Health Res Pract 25.4 (2015).

[152] Ioannidis JP. Why Most Published Research Findings Are False. PLoS Med 2.8 (2005), e124. 
[153] von Elm E, Altman DG, Egger M, Pocock SJ, Gøtzsche PC, et al. The Strengthening the Reporting of Observational Studies in Epidemiology (STROBE) statement: guidelines for reporting observational studies. J Clin Epidemiol 61.4 (2008), 344-349.

[154] Samaan Z, Mbuagbaw L, Kosa D, Borg Debono V, Dillenburg R, et al. A systematic scoping review of adherence to reporting guidelines in health care literature. J Multidiscip Healthc 6 (2013), 169-188.

[155] Pouwels KB, Widyakusuma NN, Groenwold RHH, and Hak E. Quality of reporting of confounding remained suboptimal after the STROBE guideline. J Clin Epidemiol 69 (2016), 217-224.

[156] Hemkens LG, Benchimol EI, Langan SM, Briel M, Kasenda B, et al. The reporting of studies using routinely collected health data was often insufficient. J Clin Epidemiol 79 (2016), 104111.

[157] Benchimol EI, Smeeth L, Guttmann A, Harron K, Moher D, et al. The REporting of studies Conducted using Observational Routinely-collected health Data (RECORD) Statement. PLoS Med 12.10 (2015), e1001885.

[158] Arksey H and OMalley L. Scoping studies: towards a methodological framework. Int J Soc Res Methodol 8.1 (2005), 19-32.

[159] Use of appropriate medications for people with asthma. HEDIS 2003. Vol. 2. Washington, DC: National Committee for Quality Assurance, 2003, 25-28.

[160] Wu CL, Andrews AL, Teufel RJ, and Basco WT. Demographic predictors of leukotriene antagonist monotherapy among children with persistent asthma. J. Pediatr. 164.4 (2014), 827831.e1.

[161] Zeiger RS, Schatz M, Li Q, Chen W, Khatry DB, et al. High blood eosinophil count is a risk factor for future asthma exacerbations in adult persistent asthma. J Allergy Clin Immunol Pract 2.6 (2014), 741-50.

[162] Schatz M, Zeiger RS, Yang SJ, Chen W, Crawford W, et al. Change in asthma control over time: predictors and outcomes. J Allergy Clin Immunol Pract 2.1 (), 59-64.

[163] Jena AB, Ho O, Goldman DP, and Karaca-Mandic P. The Impact of the US Food and Drug Administration Chlorofluorocarbon Ban on Out-of-pocket Costs and Use of Albuterol Inhalers Among Individuals With Asthma. JAMA Intern Med 175.7 (2015), 1171-9.

[164] McRoy L, Weech-Maldonado R, and Kilgore M. The relationship between direct to consumer advertising (DTCA) and asthma-related emergency department use among Medicaid-enrolled children. J Asthma 51.9 (2014), 922-6.

[165] Wu AC, Butler MG, Li L, Fung V, Kharbanda EO, et al. Primary adherence to controller medications for asthma is poor. Ann Am Thorac Soc 12.2 (2015), 161-6.

[166] Tomasallo CD, Hanrahan LP, Tandias A, Chang TS, Cowan KJ, et al. Estimating Wisconsin asthma prevalence using clinical electronic health records and public health data. Am J Public Health 104.1 (2014), e65-73.

[167] Mukherjee M, Gupta R, Farr A, Heaven M, Stoddart A, et al. Estimating the incidence, prevalence and true cost of asthma in the UK: secondary analysis of national stand-alone and linked databases in England, Northern Ireland, Scotland and Wales-a study protocol. BMJ Open 4.11 (2014), e006647.

[168] Laforest L, Licaj I, Devouassoux G, Chatte G, Martin J, et al. Asthma drug ratios and exacerbations: claims data from universal health coverage systems. Eur. Respir. J. 43.5 (2014), 1378-86. 
[169] Lemke LD, Lamerato LE, Xu X, Booza JC, Reiners JJ, et al. Geospatial relationships of air pollution and acute asthma events across the Detroit-Windsor international border: study design and preliminary results. J Expo Sci Environ Epidemiol 24.4 (2014), 346-57.

[170] Jian ZH, Huang JY, Lin FCF, Nfor ON, Jhang KM, et al. The use of corticosteroids in patients with COPD or asthma does not decrease lung squamous cell carcinoma. BMC Pulm Med 15 (2015), 154.

[171] Garne E, Hansen AV, Morris J, Zaupper L, Addor MC, et al. Use of asthma medication during pregnancy and risk of specific congenital anomalies: A European case-malformed control study. J Allergy Clin Immunol 136 (6 2015), 1496-502.e1-7.

[172] Tan NC, Nadkarni NV, Lye WK, Sankari U, et al. Ten-year longitudinal study of factors influencing nocturnal asthma symptoms among Asian patients in primary care. NPJ Prim Care Respir Med 25 (2015), 15064.

[173] Kenyon CC, Rubin DM, Zorc JJ, Mohamad Z, Faerber JA, et al. Childhood Asthma Hospital Discharge Medication Fills and Risk of Subsequent Readmission. J. Pediatr. 166.5 (2015), 1121-7.

[174] Rust G, Zhang S, Holloway K, and Tyler-Hill Y. Timing of emergency department visits for childhood asthma after initial inhaled corticosteroid use. Popul Health Manag 18.1 (2015), 5460.

[175] Bülow A von, Kriegbaum M, Backer V, and Porsbjerg C. The prevalence of severe asthma and low asthma control among Danish adults. J Allergy Clin Immunol Pract2.6 (2014), 759-67.

[176] Martin RJ, Price D, Roche N, Israel E, Aalderen WMC van, et al. Cost-effectiveness of initiating extrafine- or standard size-particle inhaled corticosteroid for asthma in two health-care systems: a retrospective matched cohort study. NPJ Prim Care Respir Med 24 (2014), 14081.

[177] Schatz M, Meckley LM, Kim M, Stockwell BT, and Castro M. Asthma exacerbation rates in adults are unchanged over a 5-year period despite high-intensity therapy. $\mathrm{J}$ Allergy Clin Immunol Pract 2.5 (2014), 570-4.e1.

[178] Capo-Ramos DE, Duran C, Simon AE, Akinbami LJ, and Schoendorf KC. Preventive asthma medication discontinuation among children enrolled in fee-for-service Medicaid. J Asthma 51.6 (2014), 618-26.

[179] Nordlund B, Melén E, Schultz ES, Grönlund H, Hedlin G, et al. Prevalence of severe childhood asthma according to the WHO. Respir Med 108.8 (2014), 1234-7.

[180] Ismaila A, Corriveau D, Vaillancourt J, Parsons D, Stanford R, et al. Impact of adherence to treatment with fluticasone propionate/salmeterol in asthma patients. Curr Med Res Opin 30.7 (2014), 1417-25.

[181] Fung V, Graetz I, Galbraith A, Hamity C, Huang J, et al. Financial barriers to care among low-income children with asthma: health care reform implications. JAMA Pediatr 168.7 (2014), 649-56.

[182] Dilokthornsakul P, Chaiyakunapruk N, Schumock GT, and Lee TA. Calendar time-specific propensity score analysis for observational data: a case study estimating the effectiveness of inhaled long-acting beta-agonist on asthma exacerbations. Pharmacoepidemiol Drug Saf 23.2 (2014), 152-64.

[183] Adimadhyam S, Schumock GT, Walton S, Joo M, McKell J, et al. Risk of arrhythmias associated with ipratropium bromide in children, adolescents, and young adults with asthma: a nested case-control study. Pharmacotherapy 34.4 (2014), 315-23. 
[184] Blais L, Kettani FZ, and Forget A. Associations of maternal asthma severity and control with pregnancy complications. J Asthma 51.4 (2014), 391-8.

[185] Chang J, Freed GL, Prosser LA, Patel I, Erickson SR, et al. Comparisons of health care utilization outcomes in children with asthma enrolled in private insurance plans versus medicaid. $J$ Pediatr Health Care 28.1 (2014), 71-9.

[186] Sullivan PW, Campbell JD, Ghushchyan VH, and Globe G. Outcomes before and after treatment escalation to Global Initiative for Asthma steps 4 and 5 in severe asthma. Ann. Allergy Asthma Immunol. 114.6 (2015), 462-9.

[187] Ali AK, Hartzema AG, Winterstein AG, Segal R, Lu X, et al. Application of multicategory exposure marginal structural models to investigate the association between long-acting betaagonists and prescribing of oral corticosteroids for asthma exacerbations in the Clinical Practice Research Datalink. Value Health 18.2 (2015), 260-70.

[188] Wu AC, Li L, Fung V, Kharbanda EO, Larkin EK, et al. Use of leukotriene receptor antagonists are associated with a similar risk of asthma exacerbations as inhaled corticosteroids. $J$ Allergy Clin Immunol Pract 2.5 (), 607-13.

[189] Tan CC, McDowell KM, Fenchel M, Szczesniak R, and Kercsmar CM. Spirometry use in children hospitalized with asthma. Pediatr. Pulmonol. 49.5 (2014), 451-7.

[190] Keast SL, Thompson D, Farmer K, Smith M, Nesser N, et al. Impact of a prior authorization policy for montelukast on clinical outcomes for asthma and allergic rhinitis among children and adolescents in a state Medicaid program. J Manag Care Spec Pharm 20.6 (2014), 612-21.

[191] Kim S, Kim J, Park SY, Um HY, Kim K, et al. Effect of pregnancy in asthma on health care use and perinatal outcomes. J Allergy Clin Immunol 136 (5 2015), 1215-23.e1-6.

[192] Confino-Cohen R, Brufman I, Goldberg A, and Feldman BS. Vitamin D, asthma prevalence and asthma exacerbations: a large adult population-based study. Allergy 69.12 (2014), 1673-80.

[193] Tunceli O, Williams SA, Kern DM, Elhefni H, Pethick N, et al. Comparative effectiveness of budesonide-formoterol combination and fluticasone-salmeterol combination for asthma management: a United States retrospective database analysis. J Allergy Clin Immunol Pract 2.6 (2014), 719-26.

[194] Bhattacharjee R, Choi BH, Gozal D, and Mokhlesi B. Association of adenotonsillectomy with asthma outcomes in children: a longitudinal database analysis. PLoS Med. 11.11 (2014), e1001753.

[195] Nanchal R, Kumar G, Majumdar T, Taneja A, Patel J, et al. Utilization of mechanical ventilation for asthma exacerbations: analysis of a national database. Respir Care 59.5 (2014), 644-53.

[196] Tse SM, Charland SL, Stanek E, Herrera V, Goldfarb S, et al. Statin use in asthmatics on inhaled corticosteroids is associated with decreased risk of emergency department visits. Curr Med Res Opin 30.4 (2014), 685-93.

[197] Sumino K, O’Brian K, Bartle B, Au DH, Castro M, et al. Coexisting chronic conditions associated with mortality and morbidity in adult patients with asthma. J Asthma 51.3 (2014), 306-14.

[198] Li L, Vollmer WM, Butler MG, Wu P, Kharbanda EO, et al. A comparison of confounding adjustment methods for assessment of asthma controller medication effectiveness. Am. J. Epidemiol. 179.5 (2014), 648-59.

[199] Hagiwara M, Delea TE, and Stanford RH. Health-care utilization and costs with fluticasone propionate and fluticasone propionate/salmeterol in asthma patients at risk for exacerbations.

Allergy Asthma Proc 35.1 (2014), 54-62. 
[200] Blais L, Kettani FZ, Forget A, Beauchesne MF, and Lemière C. Asthma exacerbations during the first trimester of pregnancy and congenital malformations: revisiting the association in a large representative cohort. Thorax 70.7 (2015), 647-52.

[201] Benchimol EI, Manuel DG, To T, Griffiths AM, Rabeneck L, et al. Development and use of reporting guidelines for assessing the quality of validation studies of health administrative data. J Clin Epidemiol 64.8 (2011), 821-829.

[202] Schneeweiss S and Avorn J. A review of uses of health care utilization databases for epidemiologic research on therapeutics. J Clin Epidemiol 58.4 (2005), 323-337.

[203] Sheikh A, Cornford T, Barber N, Avery A, Takian A, et al. Implementation and adoption of nationwide electronic health records in secondary care in England: final qualitative results from prospective national evaluation in "early adopter" hospitals. BMJ 343.oct17 1 (2011), d6054d6054.

[204] Frenkel LD. Electronic health records-Applications for the allergist/immunologist: All that glitters is not gold. Allergy Asthma Proc 37.4 (2016), 273-278.

[205] Huzel L, Roos LL, Anthonisen NR, and Manfreda J. Diagnosing asthma: the fit between survey and administrative database. Can Respir J 9.6 (2002), 407-412.

[206] Tinkelman DG, Price DB, Nordyke RJ, and Halbert RJ. Misdiagnosis of COPD and Asthma in Primary Care Patients 40 Years of Age and Over. J Asthma 43.1 (2006), 75-80.

[207] Postma DS and Rabe KF. The Asthma-COPD Overlap Syndrome. N Engl J Med 373.13 (2015), 1241-1249.

[208] McDonald VM and Gibson PG. To define is to limit: perspectives on asthma-COPD overlap syndrome and personalised medicine. Eur Respir J 49.5 (2017), 1700336.

[209] Miravitlles M. Diagnosis of asthma-COPD overlap: the five commandments. Eur Respir J 49.5 (2017), 1700506.

[210] Bateman ED, Reddel HK, Zyl-Smit RN van, and Agusti A. The asthma-COPD overlap syndrome: towards a revised taxonomy of chronic airways diseases? Lancet Respir Med 3.9 (2015), 719728.

[211] Benfante A, Sorino C, and Scichilone N. The asthma-COPD overlap syndrome (ACOS): hype or reality? That is, a curiosity for the media or an opportunity for physicians? Shortness of Breath 3.4 (2014), 165-174.

[212] Delgado-Rodriguez M. Bias. J Epidemiol Community Health 58.8 (2004), 635-641.

[213] Manuel DG, Rosella LC, and Stukel TA. Importance of accurately identifying disease in studies using electronic health records. BMJ 341 (2010), c4226.

[214] Shivade C, Raghavan P, Fosler-Lussier E, Embi PJ, Elhadad N, et al. A review of approaches to identifying patient phenotype cohorts using electronic health records. $J$ Am Med Inform Assoc 21 (2 2014), 221-230.

[215] Ehrenstein V, Petersen I, Smeeth L, Jick S, Benchimol EI, et al. Helping everyone do better: a call for validation studies of routinely recorded health data. Clinical Epidemiology (2016), 49.

[216] Springate DA, Kontopantelis E, Ashcroft DM, Olier I, Parisi R, et al. ClinicalCodes: an online clinical codes repository to improve the validity and reproducibility of research using electronic medical records. PLoS One 9 (6 2014), e99825. 
[217] Herrett E, Thomas SL, Schoonen WM, Smeeth L, and Hall AJ. Validation and validity of diagnoses in the General Practice Research Database: a systematic review. Br J Clin Pharmacol 69.1 (2010), 4-14.

[218] Wang SV, Verpillat P, Rassen JA, Patrick A, Garry EM, et al. Transparency and Reproducibility of Observational Cohort Studies Using Large Healthcare Databases. Clin Pharmacol Ther 99.3 (2016), 325-332.

[219] Glasziou P, Altman DG, Bossuyt P, Boutron I, Clarke M, et al. Reducing waste from incomplete or unusable reports of biomedical research. Lancet (London, England) 383 (9913 2014), 267276.

[220] Bel EH, Sousa A, Fleming L, Bush A, Chung KF, et al. Diagnosis and definition of severe refractory asthma: an international consensus statement from the Innovative Medicine Initiative (IMI). Thorax 66.10 (2011), 910-917.

[221] Chung KF, Wenzel SE, Brozek JL, Bush A, Castro M, et al. International ERS/ATS guidelines on definition, evaluation and treatment of severe asthma. Eur Respir J 43.2 (2014), 343-373.

[222] Jacob C, Haas JS, Bechtel B, Kardos P, and Braun S. Assessing asthma severity based on claims data: a systematic review. Eur J Health Econ (2016).

[223] Ford ES. The epidemiology of obesity and asthma. J Allergy Clin Immunol Pract 115 (5 2005), 897-909, quiz 910.

[224] Kotz D, Simpson CR, and Sheikh A. Incidence, prevalence, and trends of general practitionerrecorded diagnosis of peanut allergy in England, 2001 to 2005. J Allergy Clin Immunol 127.3 (2011), 623-630.e1.

[225] Custovic A and Nicolaou N. Peanut allergy: overestimated in epidemiology or underdiagnosed in primary care? J Allergy Clin Immunol: In Practice 127 (3 2011), 631-632.

[226] Panesar S, Javad S, Silva Dd, Nwaru B, Hickstein L, et al. The epidemiology of anaphylaxis in Europe: a systematic review. Allergy 68.11 (2013), 1353-1361.

[227] Nwaru BI, Mukherjee M, Gupta RP, Farr A, Heaven M, et al. Challenges of harmonising data from UK national health surveys: a case study of attempts to estimate the UK prevalence of asthma. J R Soc Med (2015).

[228] Mukherjee M, Wyatt JC, Simpson CR, and Sheikh A. Usage of allergy codes in primary care electronic health records: a national evaluation in Scotland. Allergy (2016).

[229] Howie L, Hirsch B, Locklear T, and Abernethy AP. Assessing The Value Of Patient-Generated Data To Comparative Effectiveness Research. Health Aff (Millwood) 33.7 (2014), 12201228.

[230] Corren J. Asthma phenotypes and endotypes: an evolving paradigm for classification. Discov Med 15.83 (2013), 243-249.

[231] Naish J, Sturdy P, and Toon P. Appropriate prescribing in asthma and its related cost in east London. BMJ 310.6972 (1995), 97-100.

[232] Coleman N, Halas G, Peeler W, Casaclang N, Williamson T, et al. From patient care to research: a validation study examining the factors contributing to data quality in a primary care electronic medical record database. BMC Family Practice 16.1 (2015).

[233] Lusignan S de. The optimum granularity for coding diagnostic data in primary care: report of a workshop of the EFMI Primary Care Informatics Working Group at MIE 2005. Inform Prim Care 14 (2 2006), 133-137. 
[234] Harron K, Wade A, Gilbert R, Muller-Pebody B, and Goldstein H. Evaluating bias due to data linkage error in electronic healthcare records. BMC Med Res Methodol 14.1 (2014), 36.

[235] Denney MJ, Long DM, Armistead MG, Anderson JL, and Conway BN. Validating the extract, transform, load process used to populate a large clinical research database. Int $\mathrm{J}$ Med Informatics 94 (2016), 271-274.

[236] Weiskopf NG and Weng C. Methods and dimensions of electronic health record data quality assessment: enabling reuse for clinical research. J Am Med Inform Assoc 20.1 (2013), 144151.

[237] Nissen F, Quint J, Wilkinson S, Müllerova H, Smeeth L, et al. Validation of asthma recording in electronic health records: a systematic review. Clinical Epidemiology Volume 9 (2017), 643656.

[238] Reitsma JB, Rutjes AWS, Khan KS, Coomarasamy A, and Bossuyt PM. A review of solutions for diagnostic accuracy studies with an imperfect or missing reference standard. J Clin Epidemiol 62.8 (2009), 797-806.

[239] Nissen F, Morales DR, Mullerova H, Smeeth L, Douglas IJ, et al. Validation of asthma recording in the Clinical Practice Research Datalink (CPRD). BMJ open 7 (8 2017), e017474.

[240] Hansen S, Strøm M, Maslova E, Mortensen EL, Granström C, et al. A comparison of three methods to measure asthma in epidemiologic studies: results from the Danish National Birth Cohort. PLoS One 7.5 (2012), e36328.

[241] Blais L, Lemière C, Menzies D, and Berbiche D. Validity of asthma diagnoses recorded in the Medical Services database of Quebec. Pharmacoepidemiol Drug Saf 15.4 (2006), 245-252.

[242] Gershon AS, Wang C, Guan J, Vasilevska-Ristovska J, Cicutto L, et al. Identifying patients with physician-diagnosed asthma in health administrative databases. Can Respir J 16.6 (2009), 183-188.

[243] Moth G, Vedsted P, and Schiøtz P. Identification of asthmatic children using prescription data and diagnosis. Eur J Clin Pharmacol 63.6 (2007), 605-611.

[244] Bai JR, Mukherjee DV, Befus M, Apa Z, Lowy FD, et al. Concordance between medical records and interview data in correctional facilities. BMC Med Res Methodol 14.1 (2014).

[245] Hoffmann F and Glaeske G. Prescriptions as a proxy for asthma in children: a good choice? Eur J Clin Pharmacol 66.3 (2010), 307-313.

[246] Veninga C, Denig P, Pont LG, and Haaijer-Ruskamp FM. Comparison of indicators assessing the quality of drug prescribing for asthma. Health Serv Res 36 (1 Pt 1 2001), 143-161.

[247] Barry DM, Burr ML, and Limb ES. Prevalence of asthma among 12 year old children in New Zealand and South Wales: a comparative survey. Thorax 46.6 (1991), 405-409.

[248] Weiland SK, Björkstén B, Brunekreef B, Cookson WOC, Mutius E von, et al. Phase II of the International Study of Asthma and Allergies in Childhood (ISAAC II): rationale and methods. Eur Respir J 24.3 (2004), 406-412.

[249] Ward DG, Halpin DM, and Seamark DA. How accurate is diagnosis of asthma in a general practice database? A review of patients notes and questionnaire-reported symptoms. $B r \mathrm{~J}$ Gen Pract 54.507 (2004), 753-758.

[250] Muggah E, Graves E, Bennett C, and Manuel DG. Ascertainment of chronic diseases using population health data: a comparison of health administrative data and patient self-report. BMC Public Health 13 (2013), 16. 
[251] Schatz M, Zeiger RS, Yang SJT, Chen W, Crawford WW, et al. Persistent asthma defined using HEDIS versus survey criteria. Am J Manag Care 16.11 (2010), e281-e288.

[252] Nelson EC, Eftimovska E, Lind C, Hager A, Wasson JH, et al. Patient reported outcome measures in practice. BMJ 350 (2015), g7818.

[253] Kaleta D, Polańska K, Dziankowska-Zaborszczyk E, Hanke W, and Drygas W. Factors influencing self-perception of health status. Cent Eur J Public Health 17 (3 2009), 122-127.

[254] Al Sallakh M, Rodgers S, Lyons R, Sheikh A, and Davies G. P148 Making sense of patientreported currently treated asthma using routinely collected data. Thorax 71.Suppl 3 (2016), A163.2-A164.

[255] Peat JK, Toelle BG, Marks GB, and Mellis CM. Continuing the debate about measuring asthma in population studies. Thorax 56 (5 2001), 406-411.

[256] Rutjes AWS, Reitsma JB, Coomarasamy A, Khan KS, and Bossuyt PMM. Evaluation of diagnostic tests when there is no gold standard. A review of methods. Health Technol Assess 11.50 (2007), iii, ix-iii, 51.

[257] Linda M. Collins STL. Latent Class and Latent Transition Analysis: With Applications in the Social, Behavioral, and Health Sciences. John Wiley \& Sons Inc, 2010. 285 Seiten. ISBN: 0470228393.

[258] Howard R, Rattray M, Prosperi M, and Custovic A. Distinguishing Asthma Phenotypes Using Machine Learning Approaches. Curr Allergy Asthma Rep 15.7 (2015), 38.

[259] Wurpts IC and Geiser C. Is adding more indicators to a latent class analysis beneficial or detrimental? Results of a Monte-Carlo study. Front Psychol 5 (2014).

[260] Linzer DA and Lewis JB. poLCA: An R package for polytomous variable latent class analysis. J Stat Softw 42.10 (2011), 1-29.

[261] Dempster AP, Laird NM, and Rubin DB. Maximum likelihood from incomplete data via the EM algorithm. J R Stat Soc Series B Stat Methodol (1977), 1-38.

[262] McLachlan G and Peel D. Finite Mixture Models. 1st ed. Wiley Series in Probability and Statistics. Wiley-Interscience, 2000. ISBN: 9780471006268.

[263] Akaike H. A new look at the statistical model identification. IEEE Trans Automat Contr 19.6 (1974), 716-723.

[264] Schwarz G. Estimating the dimension of a model. Ann Stat 6.2 (1978), 461-464.

[265] Nylund KL, Asparouhov T, and Muthén BO. Deciding on the number of classes in latent class analysis and growth mixture modeling: A Monte Carlo simulation study. Struct Equ Modeling 14.4 (2007), 535-569.

[266] Global Initiative for Asthma. Global Strategy for Asthma Management and Prevention (2016 update). Accessed: Jun 19, 2017. 2016. URL: http://ginasthma.org.

[267] Fattahi F, Vonk JM, Bulkmans N, Fleischeuer R, Gouw A, et al. Old dilemma: asthma with irreversible airway obstruction or COPD. Virchows Arch 467.5 (2015), 583-593.

[268] Strobl C, Malley J, and Tutz G. An introduction to recursive partitioning: rationale, application, and characteristics of classification and regression trees, bagging, and random forests. Psychol Methods 14 (4 2009), 323-348.

[269] Therneau TM and Atkinson EJ. An Introduction to Recursive Partitioning Using the RPART Routines (2015).

[270] HSCIC - QOF Business Rules team. New GMS Contract QOF Implementation Dataset and Business Rules - Asthma Indicator Set. 2014. 
[271] Atkinson MD, Kennedy JI, John A, Lewis KE, Lyons RA, et al. Development of an algorithm for determining smoking status and behaviour over the life course from UK electronic primary care records. BMC Med Inform Decis Mak 17.1 (2017).

[272] Consent to link. URL: http://gov . wales/statistics-and-research/making-better-useexisting-data/consent-link/?lang=en.

[273] Moore WC, Meyers DA, Wenzel SE, Teague WG, Li H, et al. Identification of asthma phenotypes using cluster analysis in the Severe Asthma Research Program. Am J Respir Crit Care Med 181.4 (2010), 315-323.

[274] Garcia-Aymerich J, Benet M, Saeys Y, Pinart M, Basagaña X, et al. Phenotyping asthma, rhinitis and eczema in MeDALL population-based birth cohorts: an allergic comorbidity cluster. Allergy (2015).

[275] Weatherall M, Travers J, Shirtcliffe PM, Marsh SE, Williams MV, et al. Distinct clinical phenotypes of airways disease defined by cluster analysis. Eur Respir J 34.4 (2009), 812-818.

[276] Mäkikyrö EMS, Jaakkola MS, and Jaakkola JJK. Subtypes of asthma based on asthma control and severity: a latent class analysis. Respir Res 18.1 (2017).

[277] Weinmayr G, Keller F, Kleiner A, Prel JB du, Garcia-Marcos L, et al. Asthma phenotypes identified by latent class analysis in the ISAAC phase II Spain study. Clin Exp Allergy 43.2 (2013), 223-232.

[278] Burgel PR, Paillasseur JL, Caillaud D, Tillie-Leblond I, Chanez P, et al. Clinical COPD phenotypes: a novel approach using principal component and cluster analyses. Eur Respir J 36.3 (2010), 531-539.

[279] Ghebre MA, Bafadhel M, Desai D, Cohen SE, Newbold P, et al. Biological clustering supports both Dutch and British hypotheses of asthma and chronic obstructive pulmonary disease. J Allergy Clin Immunol 135.1 (2015), 63-72.e10.

[280] Rodrigo GJ, Neffen H, and Plaza V. Asthma-chronic obstructive pulmonary disease overlap syndrome: a controversial concept. Curr Opin Allergy Clin Immunol 17 (1 2017), 36-41.

[281] Prosperi MCF, Sahiner UM, Belgrave D, Sackesen C, Buchan IE, et al. Challenges in Identifying Asthma Subgroups Using Unsupervised Statistical Learning Techniques. Am J Respir Crit Care Med 188.11 (2013), 1303-1312.

[282] Prosser RJ, Carleton BC, and Smith MA. Identifying persons with treated asthma using administrative data via latent class modelling. Health Serv Res 43.2 (2008), 733-754.

[283] Depner M, Fuchs O, Genuneit J, Karvonen AM, Hyvärinen A, et al. Clinical and epidemiologic phenotypes of childhood asthma. Am J Respir Crit Care Med 189.2 (2014), 129-138.

[284] Strong M, South G, and Carlisle R. The UK Quality and Outcomes Framework pay-forperformance scheme and spirometry: rewarding quality or just quantity? A cross-sectional study in Rotherham, UK. BMC Health Serv Res 9.1 (2009).

[285] Jordan K. Quality of morbidity coding in general practice computerized medical records: a systematic review. Fam Pract 21.4 (2004), 396-412.

[286] Asthma UK Centre for Applied Research (AUKCAR). URL: http://www .aukcar.ac.uk/ (visited on 04/15/2015).

[287] UK Asthma Observatory. URL: https://www.aukcar.ac.uk/asthma-observatory (visited on 01/09/2018).

[288] Etheredge LM. A Rapid-Learning Health System. Health Aff (Millwood) 26.2 (2007), w107w118. 
[289] Friedman C, Rubin J, Brown J, Buntin M, Corn M, et al. Toward a science of learning systems: a research agenda for the high-functioning Learning Health System. J Am Med Inform Assoc (2014).

[290] Ovretveit J, Nelson E, and James B. Building a learning health system using clinical registers: a non-technical introduction. J Health Organ Manag 30.7 (2016), 1105-1118.

[291] Potts J, Thompson R, Merchant R, Ciemins EL, Bush RW, et al. Learning: Contemplating the unexamined core of Learning Health Systems. Learn Health Syst 1.4 (2017), e10036.

[292] Nwaru BI, Friedman C, Halamka J, and Sheikh A. Can learning health systems help organisations deliver personalised care? BMC Med 15.1 (2017).

[293] Friedman CP, Wong AK, and Blumenthal D. Achieving a nationwide learning health system. Sci Transl Med 2.57 (2010), 57cm29-57cm29.

[294] UK Health Departments Research Ethics Service NHRA. Standard Operating Procedures for Research Ethics Committees, Version 7.2 January 2017. 2017. URL: https : / www. hra.nhs . uk/documents/7/standard-operating-procedures-version-7-2.pdf.

[295] Jones KH, Ford DV, Jones C, Dsilva R, Thompson S, et al. A case study of the Secure Anonymous Information Linkage (SAIL) Gateway: a privacy-protecting remote access system for healthrelated research and evaluation. J Biomed Inform 50 (2014), 196-204.

[296] Office for National Statistics. Patient Register: quality assurance of administrative data used in population statistics, Dec 2016. 2016. URL: https : / / www . ons . gov . uk / peoplepopulationandcommunity / populationandmigration / populationestimates / methodologies / patientregisterqualityassuranceofadministrativedatausedinpopulationstatisticsdec2016.

[297] Public Health Wales Observatory. Patient Episode Database for Wales (PEDW). URL: http : //www. publichealthwalesobservatory.wales.nhs.uk/pedw.

[298] NHS Wales Informatics Service. Annual PEDW Data Tables - Notes \& Definitions. URL: http: //www. infoandstats. wales.nhs.uk/docopen. cfm?orgid=869\&id=142683.

[299] Taylor DR, Bateman ED, Boulet LP, Boushey HA, Busse WW, et al. A new perspective on concepts of asthma severity and control. Eur Respir J 32.3 (2008), 545-554.

[300] Rodgers SE, Lyons RA, Dsilva R, Jones KH, Brooks CJ, et al. Residential Anonymous Linking Fields (RALFs): a novel information infrastructure to study the interaction between the environment and individuals' health. J Public Health (2009), fdp041.

[301] Williams R, Kontopantelis E, Buchan I, and Peek N. Clinical code set engineering for reusing EHR data for research: A review. J Biomed Inform 70 (2017), 1-13.

[302] Walsh SH. The clinicians perspective on electronic health records and how they can affect patient care. BMJ 328.7449 (2004), 1184-1187.

[303] Standard: DS/EN 13826 Peak Expiratory Flow Meters. URL: http://standards.globalspec. com/std/498042/ds-en-13826.

[304] Kampstra P et al. Beanplot: A boxplot alternative for visual comparison of distributions (2008).

[305] Price DB, Rigazio A, Campbell JD, Bleecker ER, Corrigan CJ, et al. Blood eosinophil count and prospective annual asthma disease burden: a UK cohort study. Lancet Respir Med 3.11 (2015), 849-858.

[306] Andrews JE, Richesson RL, and Krischer J. Variation of SNOMED CT Coding of Clinical Research Concepts among Coding Experts. Journal of the American Medical Informatics Association 14.4 (2007), 497-506. 
[307] Yang CL, Simons E, Foty RG, To T, and Dell SD. Administrative Databases Useful For Asthma Surveillance But Overestimate Asthma Prevalence. B61. PEDIATRIC ASTHMA. American Thoracic Society, 2012.

[308] LaBranche J, Crossley J, Gara CD, and Vethanayagam D. Failure of Administrative Data to Guide Asthma Care. American Thoracic Society International Conference Abstracts. doi:10.1164/ajrccm-conference.2015.191.1MeetingAbstracts.A4196. American Thoracic Society, 2015, A4196-A4196.

[309] Yu TH, Fu PK, and Tung YC. Using medication utilization information to develop an asthma severity classification model. BMC Med Inform Decis Mak 17.1 (2017).

[310] NHS Wales Informatics Service. New GMS Contract QOF Implementation Dataset and Business Rules - Asthma Indicator Set - Wales. 2015.

[311] NHS Digital, ed. SNOMED CT implementation in primary care. URL: https://digital.nhs. uk/SNOMED-CT-implementation-in-primary-care.

[312] Rodgers SE, Heaven M, Lacey A, Poortinga W, Dunstan FD, et al. Cohort Profile: The Housing Regeneration and Health Study. Int J Epidemiol 43.1 (2012), 52-60.

[313] Rodgers SE. Green-blue space exposure changes and impact on individual-level wellbeing and mental health: a population-wide record-linked natural experiment. 2017. URL: https: //www . journalslibrary.nihr.ac.uk/programmes/phr/160707/非/.

[314] Worth A, Hammersley V, Knibb R, Flokstra-de-Blok B, DunnGalvin A, et al. Patient-reported outcome measures for asthma: a systematic review. NPJ Prim Care Respir Med 24.1 (2014).

[315] Beasley R, Asthma TIS of, et al. Worldwide variation in prevalence of symptoms of asthma, allergic rhinoconjunctivitis, and atopic eczema: ISAAC. The International Study of Asthma and Allergies in Childhood (ISAAC) Steering Committee. Lancet 351.9111 (1998), 1225-1232.

[316] Kaur B, Anderson HR, Austin J, Burr M, Harkins LS, et al. Prevalence of asthma symptoms, diagnosis, and treatment in 12-14 year old children across Great Britain (international study of asthma and allergies in childhood, ISAAC UK). BMJ 316.7125 (1998), 118-124.

[317] Bowatte G, Lodge C, Lowe AJ, Erbas B, Perret J, et al. The influence of childhood traffic-related air pollution exposure on asthma, allergy and sensitization: a systematic review and a metaanalysis of birth cohort studies. Allergy 70.3 (2014), 245-256.

[318] GOWERS AM, CULLINAN P, AYRES JG, ANDERSON HR, STRACHAN DP, et al. Does outdoor air pollution induce new cases of asthma? Biological plausibility and evidence: a review. Respirology 17.6 (2012), 887-898.

[319] Sofianopoulou E, Rushton SP, Diggle PJ, and Pless-Mulloli T. Association between respiratory prescribing, air pollution and deprivation, in primary health care. J Public Health (Oxf) 35.4 (2013), 502-509.

[320] Weiland SK, Hüsing A, Strachan DP, Rzehak P, and Pearce N. Climate and the prevalence of symptoms of asthma, allergic rhinitis, and atopic eczema in children. Occup Environ Med 61.7 (2004), 609-615.

[321] Mitchell RG and Dawson B. Educational and social characteristics of children with asthma. Arch Dis Child 48.6 (1973), 467.

[322] Uphoff E, Cabieses B, Pinart M, Valdés M, Antó JM, et al. A systematic review of socioeconomic position in relation to asthma and allergic diseases. Eur Respir J 46.2 (2014), 364-374.

[323] Watson JP, Cowen P, and Lewis RA. The relationship between asthma admission rates, routes of admission, and socioeconomic deprivation. Eur Respir J 9.10 (1996), 2087-2093. 
[324] Burr M, Verrall C, and Kaur B. Social deprivation and asthma. Respir Med 91.10 (1997), 603608.

[325] Anderson H, Cooper J, Bailey P, and Palmer J. INFLUENCE OF MORBIDITY, ILLNESS LABEL, AND SOCIAL, FAMILY, AND HEALTH SERVICE FACTORS ON DRUG TREATMENT OF CHILDHOOD ASTHMA. Lancet 318.8254 (1981), 1030-1032.

[326] Connolly CK, Chan NS, and Prescott RJ. The influence of social factors on the control of asthma. Postgrad Med J 65 (763 1989), 282-285.

[327] World Health Organisation. Health Impact Assessment (HIA). Glossary of terms used. URL: http://www. who.int/hia/about/glos/en/index1.html.

[328] Marmot M and Bell R. Social inequalities in health: a proper concern of epidemiology. Ann Epidemiol 26.4 (2016), 238-240.

[329] Kawachi I, Subramanian SV, and Almeida-Filho N. A glossary for health inequalities. J Epidemiol Community Health 56.9 (2002), 647-652.

[330] Woodward A. Why reduce health inequalities? J Epidemiol Community Health 54.12 (2000), 923-929.

[331] Whitehead M. The Concepts and Principles of Equity and Health. Int J Health Serv 22.3 (1992), 429-445.

[332] Braveman P and Gruskin S. Defining equity in health. J Epidemiol Community Health 57.4 (2003), 254-258.

[333] Braveman PA, Cubbin C, Egerter S, Chideya S, Marchi KS, et al. Socioeconomic Status in Health Research. JAMA 294.22 (2005), 2879.

[334] Yen IH and Syme SL. The Social Environment and Health: A Discussion of the Epidemiologic Literature. Annu Rev Public Health 20.1 (1999), 287-308.

[335] Robert SA. SOCIOECONOMIC POSITION AND HEALTH: The Independent Contribution of Community Socioeconomic Context. Annu Rev Sociol 25.1 (1999), 489-516.

[336] Denny K and Davidson MJ. Area-based socio-economic measures as tools for health disparities research, policy and planning. Can J Public Health 103 (8 Suppl 2 2012), S4-S6.

[337] Townsend P, Phillimore P, and Beattie A. Health and Deprivation: Inequality and the North. Croom Helm, 1988. ISBN: 9780709943518.

[338] Department for Communities and Local Government. English indices of deprivation 2015. URL: https://www.gov.uk/government/statistics/english-indices-of-deprivation-2015.

[339] Scottish Government. The Scottish Index of Multiple Deprivation. URL: http : / / www . gov . scot/Topics/Statistics/SIMD.

[340] Welsh Assembly Government. The Welsh Index of Multiple Deprivation (WIMD).

[341] Welsh Assembly Government. The Welsh Index of Multiple Deprivation (WIMD) 2011 - Summary Report. Cardiff, 2011.

[342] Welsh Assembly Government. The Welsh Index of Multiple Deprivation (WIMD) 2011: Technical Report. Cardiff, 2011.

[343] Office for National Statistics. Census geography: An overview of the various geographies used in the production of statistics collected via the UK census. URL: https: / /www. ons.gov.uk/ methodology/geography/ukgeographies/censusgeography.

[344] Pearson M. Measuring clinical outcome in asthma: a patient-focused approach. Royal College of Physicians. 2000 
[345] Schatz M, Zeiger RS, Vollmer WM, Mosen D, Mendoza G, et al. The Controller-to-Total Asthma Medication Ratio Is Associated With Patient-Centered As Well As Utilization Outcomes. Chest 130.1 (2006), 43-50.

[346] Lord D, Washington SP, and Ivan JN. Poisson, Poisson-gamma and zero-inflated regression models of motor vehicle crashes: balancing statistical fit and theory. Accid Anal Prev 37.1 (2005), 35-46.

[347] Zeileis A, Kleiber C, and Jackman S. Regression Models for Count Data in R. J Stat Softw 27.8 (2008).

[348] Kleiber C and Zeileis A. Visualizing count data regressions using rootograms. Am Stat 70.3 (2016), 296-303.

[349] Bacon SL, Bouchard A, Loucks EB, and Lavoie KL. Individual-level socioeconomic status is associated with worse asthma morbidity in patients with asthma. Respir Res 10.1 (2009).

[350] Gupta RP, Mukherjee M, Sheikh A, and Strachan DP. Persistent variations in national asthma mortality, hospital admissions and prevalence by socioeconomic status and region in England. Thorax 73.8 (2018), 706-712. eprint: https ://thorax. bmj . com/content/73/8/706. full. pdf.

[351] Mielck A, Reitmeir P, and Wjst M. Severity of childhood asthma by socioeconomic status. Int J Epidemiol 25.2 (1996), 388-393.

[352] Laurent O, Filleul L, Havard S, Deguen S, Declercq C, et al. Asthma attacks and deprivation: gradients in use of mobile emergency medical services. J Epidemiol Community Health 62.11 (2008), 1014-1016.

[353] Ungar WJ, Paterson JM, Gomes T, Bikangaga P, Gold M, et al. Relationship of asthma management, socioeconomic status, and medication insurance characteristics to exacerbation frequency in children with asthma. Ann Allergy Asthma Immunol 106.1 (2011), 17-23.

[354] Barr RG, Somers SC, Speizer FE, and Camargo CA. Patient Factors and Medication Guideline Adherence Among Older Women With Asthma. Arch Intern Med 162.15 (2002), 1761.

[355] Poyser M, Nelson H, Ehrlich R, Bateman E, Parnell S, et al. Socioeconomic deprivation and asthma prevalence and severity in young adolescents. Eur Respir J 19.5 (2002), 892-898.

[356] Mazalovic K, Jacoud F, Dima AL, Ganse EV, Nolin M, et al. Asthma exacerbations and socioeconomic status in French adults with persistent asthma: A prospective cohort study. J Asthma (2017), 1-9.

[357] Piantadosi S, Byar DP, and Green SB. The ecological fallacy. Am J Epidemiol 127.5 (1988), 893-904.

[358] Piantadosi S. Invited commentary: ecologic biases. Am J Epidemiol 139.8 (1994), 761-764.

[359] Rieffe C, Oosterveld P, Wijkel D, and Wiefferink C. Reasons why patients bypass their GP to visit a hospital emergency department. Accid Emerg Nurs 7 (4 1999), 217-225.

[360] Griffey RT, Kennedy SK, McGownan L, Goodman M, and Kaphingst KA. Is low health literacy associated with increased emergency department utilization and recidivism? Acad Emerg Med 21.10 (2014), 1109-1115.

[361] Baird B, Charles A, Honeyman M, Maguire D, and Das P. Understanding pressures in general practice. The Kingś Fund, 2016.

[362] Boomla K, Hull S, and Robson J. GP funding formula masks major inequalities for practices in deprived areas. BMJ (Clinical research ed.) 349 (2014), g7648. 
[363] Thai AL and George M. The effects of health literacy on asthma self-management. J Asthma Allergy Educ 1.2 (2010), 50-55.

[364] Arnlind MH, Wettermark B, Sjöborg B, Dahlén E, Loikas D, et al. Socioeconomic status and the quality of prescribing asthma drugs in Sweden. J Asthma 50.8 (2013), 842-849.

[365] ND B, SL S, KE D, DJ H, and K C. Low health literacy and health outcomes: An updated systematic review. Ann Intern Med 155.2 (2011), 97-107. eprint: /data/journals/aim/20235/

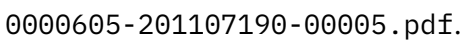

[366] Bender BG and Bender SE. Patient-identified barriers to asthma treatment adherence: responses to interviews, focus groups, and questionnaires. Immunol Allergy Clin North Am 25.1 (2005), 107-130.

[367] Soumerai SB, Ross-Degnan D, Avorn J, McLaughlin TJ, and Choodnovskiy I. Effects of Medicaid Drug-Payment Limits on Admission to Hospitals and Nursing Homes. N Engl J Med 325.15 (1991), 1072-1077.

[368] Public Health Wales Observatory. Emergency Department Data Set (EDDS). URL: http : / / www. publichealthwalesobservatory.wales.nhs.uk/edds.

[369] NHS Wales Data Dictionary - Admitted Patient Care Data Set (APC Ds) - Data Set Structure. Accessed: 21.11.2017. URL: http: //www. datadictionary . wales.nhs.uk/非!WordDocuments/ datasetstructure.htm.

[370] Wang Z, May SM, Charoenlap S, Pyle R, Ott NL, et al. Effects of secondhand smoke exposure on asthma morbidity and health care utilization in children: a systematic review and metaanalysis. Ann Allergy Asthma Immunol 115.5 (2015), 396-401.e2.

[371] Dolman R, Gibbon R, and Roberts C. Smoking in Wales: current facts. Wales Centre for Health, 2007.

[372] Do smoking rates vary between more and less advantaged areas? Office for National Statistics. 2014. URL: http: //webarchive nationalarchives.gov. uk/20160105204521/http: //www . ons . gov . uk / ons / rel / disability - and - health - measurement / do - smoking - rates - vary between-more-and-less-advantaged-areas-/2012/sty-smoking-rates.html.

[373] Surgeon General. The health consequences of smoking-50 years of progress: a report of the Surgeon General. US Department of Health and Human Services. 2014.

[374] Health Literacy: A Prescription to End Confusion (2004).

[375] Mancuso CA and Rincon M. Impact of health literacy on longitudinal asthma outcomes. J Gen Intern Med 21.8 (2006), 813-817.

[376] Apter AJ, Wan F, Reisine S, Bender B, Rand C, et al. The association of health literacy with adherence and outcomes in moderate-severe asthma. J Allergy Clin Immunol 132.2 (2013), 321327.

[377] van der Heide I, Wang J, Droomers M, Spreeuwenberg P, Rademakers J, et al. The relationship between health, education, and health literacy: results from the Dutch Adult Literacy and Life Skills Survey. J Health Commun 18.sup1 (2013), 172-184.

[378] Office for National Statistics. Asthma deaths in England and Wales, 2001 to 2015 occurrences. 2016. URL: https : / / www . ons . gov . uk / peoplepopulationandcommunity / birthsdeathsandmarriages / deaths / adhocs / 005955asthmadeathsinenglandandwales2001to2015occurrences.

[379] Danielis J, Clarke L, Swain K, and Studley R. Mortality Statistics in Wales. Welsh Government, 2013. 
[380] Newacheck PW and Halfon N. Prevalence, impact, and trends in childhood disability due to asthma. Arch Pediatr Adolesc Med 154 (3 2000), 287-293.

[381] Lloyd A, Price D, and Brown R. The impact of asthma exacerbations on health-related quality of life in moderate to severe asthma patients in the UK. Prim Care Respir J 16 (1 2007), 22-27.

[382] GBD 2015 Chronic Respiratory Disease Collaborators. Global, regional, and national deaths, prevalence, disability-adjusted life years, and years lived with disability for chronic obstructive pulmonary disease and asthma, 1990-2015: a systematic analysis for the Global Burden of Disease Study 2015. Lancet Respir Med 5 (9 2017), 691-706.

[383] Annual Asthma Survey 2016 report. Asthma UK, 2016.

[384] Stenius-Aarniala BS, Hedman J, and Teramo KA. Acute asthma during pregnancy. Thorax 51.4 (1996), 411-414. eprint: http://thorax.bmj.com/content/51/4/411.full.pdf.

[385] Murphy VE, Gibson P, Talbot PI, and Clifton VL. Severe asthma exacerbations during pregnancy. Obstet Gynecol 106 (5 Pt 1 2005), 1046-1054.

[386] Namazy JA, Murphy VE, Powell H, Gibson PG, Chambers C, et al. Effects of asthma severity, exacerbations and oral corticosteroids on perinatal outcomes. Eur Respir J 41.5 (2013), 10821090. eprint: http://erj.ersjournals.com/content/41/5/1082. full.pdf.

[387] Hsu J, Qin X, Beavers SF, and Mirabelli MC. Asthma-related school absenteeism, morbidity, and modifiable factors. Am J Prev Med 51.1 (2016), 23-32.

[388] Hansen CL, Baelum J, Skadhauge L, Thomsen G, Omland Ø, et al. Consequences of asthma on job absenteeism and job retention. Scand J Soc Med 40.4 (2012), 377-384.

[389] Adams J and White M. Removing the health domain from the Index of Multiple Deprivation 2004-effect on measured inequalities in census measure of health. J Public Health 28.4 (2006), 379-383.

[390] Group RHI. Respiratory Health Delivery Plan 2018-2020: Reducing inappropriate variation and sharing best practice. Welsh Government.

[391] Davies G, Akbari A, Sallakh MA, Barry S, Boycott K, et al. Consensus Position Statement: Harnessing Big Data to improve Respiratory Health. 2017.

[392] Soriano JB, Visick GT, Muellerova H, Payvandi N, and Hansell AL. Patterns of Comorbidities in Newly Diagnosed COPD and Asthma in Primary Care. Chest 128.4 (2005), 2099-2107.

[393] Boulet LP and Boulay MÈ. Asthma-related comorbidities. Expert Rev Respir Med 5.3 (2011), 377-393.

[394] Cazzola M, Segreti A, Calzetta L, and Rogliani P. Comorbidities of asthma: current knowledge and future research needs. Curr Opin Pulm Med 19.1 (2013), 36-41.

[395] Macleod U, Mitchell E, Black M, and Spence G. Comorbidity and socioeconomic deprivation: an observational study of the prevalence of comorbidity in general practice. Eur $\mathrm{J}$ Gen Pract 10.1 (2004), 24-26.

[396] Marmot M. Social determinants of health inequalities. Lancet 365.9464 (2005), 1099-1104.

[397] Campbell C and McLean C. Ethnic identities, social capital and health inequalities: factors shaping African-Caribbean participation in local community networks in the UK. Social Science \& Medicine 55.4 (2002), 643-657.

[398] Evandrou M, Falkingham J, Feng Z, and Vlachantoni A. Ethnic inequalities in limiting health and self-reported health in later life revisited. J Epidemiol Community Health 70.7 (2016), 653662 . 
[399] Office for National Statititcs. Ethnicity and National Identity in England and Wales: 2011. URL: https://www.ons.gov.uk/peoplepopulationandcommunity/culturalidentity/ethnicity/ articles/ethnicityandnationalidentityinenglandandwales/2012-12-11.

[400] StatsWales. Ethnicity by area and ethnic group (2017). URL: https: //statswales.gov . wales/ Catalogue/Equality-and-Diversity/Ethnicity/ethnicity-by-area-ethnicgroup.

[401] Migration to consistent use of coding and terminology in NHS Wales. Informing Healthcare National Architecture Design Board. NHS Wales.

[402] Rumsfeld JS, Joynt KE, and Maddox TM. Big data analytics to improve cardiovascular care: promise and challenges. Nat Rev Cardiol 13.6 (2016), 350-359.

[403] Kraus V, Blanco F, Englund M, Karsdal M, and Lohmander L. Call for standardized definitions of osteoarthritis and risk stratification for clinical trials and clinical use. Osteoarthritis Cartilage 23.8 (2015), 1233-1241.

[404] Ruggeri M, Fortuna S, and Rodeghiero F. Heterogeneity of terminology and clinical definitions in adult idiopathic thrombocytopenic purpura: a critical appraisal from a systematic review of the literature. Haematologica 93.1 (2008), 98-103.

[405] Rodeghiero F, Stasi R, Gernsheimer T, Michel M, Provan D, et al. Standardization of terminology, definitions and outcome criteria in immune thrombocytopenic purpura of adults and children: report from an international working group. Blood 113.11 (2008), 2386-2393.

[406] Agusti A, Bel E, Thomas M, Vogelmeier C, Brusselle G, et al. Treatable traits: toward precision medicine of chronic airway diseases. Eur Respir J 47.2 (2016), 410-419.

[407] Malomo M. Pennsylvania Asthma Prevalence Report 2015. Bureau of Epidemiology, Pennsylvania Department of Health, 2015.

[408] Milet M, Lutzker L, Flattery J, and Wohl-Sanchez L. Asthma in California: A surveillance report. $2013,47$.

[409] The Burden of Asthma in Mississippi. Office of Health Data and Research, Mississippi State Department of Health, 2014.

[410] To T, Dell S, Dick PT, Cicutto L, Harris JK, et al. Case verification of children with asthma in Ontario. Pediatr Allergy Immunol 17.1 (2006), 69-76.

[411] Behavioral Risk Factor Surveillance System. Accessed: 21.3.2018. URL: https : //www . cdc . gov/brfss/.

[412] Behavioral Risk Factor Surveillance System Questionnaire (2017).

[413] BRFSS Asthma Call-back Survey. Accessed: 21.3.2018. URL: https: / /www . cdc.gov/brfss / acbs.

[414] BRFSS Asthma Survey Adult Questionnaire 2013. URL: https : / / www . cdc . gov/brfss/acbs (visited on 03/12/2018).

[415] BRFSS Asthma Survey Child Questionnaire 2013.

[416] Welsh Health Survey 2014 - Variable List. 2014.

[417] Proceedings of the ATS Workshop on Refractory Asthma. Am J Respir Crit Care Med 162.6 (2000), 2341-2351.

[418] Schleich F, Brusselle G, Louis R, Vandenplas O, Michils A, et al. Heterogeneity of phenotypes in severe asthmatics. The Belgian Severe Asthma Registry (BSAR). Respir Med 108.12 (2014), 1723-1732.

[419] Maio S, Baldacci S, Bresciani M, Simoni M, Latorre M, et al. RItA: The Italian severe/uncontrolled asthma registry. Allergy (2017). 
[420] Patrawalla P, Kazeros A, Rogers L, Shao Y, Liu M, et al. Application of the asthma phenotype algorithm from the Severe Asthma Research Program to an urban population. PLoS One 7.9 (2012), e44540.

[421] Data Protection \& Medical Research. Parliamentary Office of Science and Technology. UK Parliament., 2005.

[422] Sariyar M, Borg A, Heidinger O, and Pommerening K. A practical framework for data management processes and their evaluation in population-based medical registries. Inform Health Soc Care 38.2 (2013), 104-119.

[423] Springate DA, Parisi R, Olier I, Reeves D, and Kontopantelis E. rEHR: An R package for manipulating and analysing Electronic Health Record data. PLOS ONE 12.2 (2017). Ed. by J Harezlak, e0171784.

[424] Ainsworth J, Cunningham J, and Buchan I. eLab: Bringing Together People, Data and Methods to Enhance Knowledge Discovery in Healthcare Settings. Stud Health Technol Inform 175. HealthGrid Applications and Technologies Meet Science Gateways for Life Sciences (2012), 39-48.

[425] Bechhofer S, De Roure D, Gamble M, Goble C, and Buchan I. Research objects: Towards exchange and reuse of digital knowledge (2010).

[426] Bechhofer S, Buchan I, Roure DD, Missier P, Ainsworth J, et al. Why linked data is not enough for scientists. Future Gener Comput Syst 29.2 (2013), 599-611.

[427] Custovic A, Ainsworth J, Arshad H, Bishop C, Buchan I, et al. The Study Team for Early Life Asthma Research (STELAR) consortium 'Asthma e-lab: team science bringing data, methods and investigators together: Figure 1. Thorax 70.8 (2015), 799-801.

[428] Strachan DP, Anderson HR, Limb ES, O’Neill A, and Wells N. A national survey of asthma prevalence, severity, and treatment in Great Britain. Arch Dis Child 70.3 (1994), 174-178.

[429] Zock JP, Jarvis D, Luczynska C, Sunyer J, and Burney P. Housing characteristics, reported mold exposure, and asthma in the European Community Respiratory Health Survey. J Allergy Clin Immunol 110.2 (2002), 285-292.

[430] Williamson IJ, Martin CJ, McGill G, Monie RD, and Fennerty AG. Damp housing and asthma: a case-control study. Thorax 52.3 (1997), 229-234.

[431] Hughes HK, Matsui EC, Tschudy MM, Pollack CE, and Keet CA. Pediatric Asthma Health Disparities: Race, Hardship, Housing, and Asthma in a National Survey. Academic Pediatrics 17.2 (2017), 127-134.

[432] Cookson R, Mondor L, Asaria M, Kringos DS, Klazinga NS, et al. Primary care and health inequality: Difference-in-difference study comparing England and Ontario. PLoS One 12.11 (2017). Ed. by H Zeeb, e0188560.

[433] FRANK J and HAW S. Best Practice Guidelines for Monitoring Socioeconomic Inequalities in Health Status: Lessons from Scotland. Milbank Q 89.4 (2011), 658-693.

[434] Carney TJ and Kong AY. Leveraging health informatics to foster a smart systems response to health disparities and health equity challenges. J Biomed Inform 68 (2017), 184-189.

[435] Waize T, Anandarajah S, Dhoul N, and de Lusignan S. Variation in clinical coding lists in UK general practice: a barrier to consistent data entry? J Innov Health Inform 15.3 (2007), 143150. 
[436] Kontopantelis E, Buchan I, Reeves D, Checkland K, and Doran T. Relationship between quality of care and choice of clinical computing system: retrospective analysis of family practice performance under the UKs quality and outcomes framework. BMJ Open 3.8 (2013), e003190.

[437] Bohensky MA, Jolley D, Sundararajan V, Evans S, Pilcher DV, et al. Data Linkage: A powerful research tool with potential problems. BMC Health Serv Res 10.1 (2010).

[438] Lim FJ, Blyth CC, Klerk N de, Valenti B, Rouhiainen OJ, et al. Optimization is required when using linked hospital and laboratory data to investigate respiratory infections. J Clin Epidemiol 69 (2016), 23-31.

[439] Bottle A, Jarman B, and Aylin P. Strengths and weaknesses of hospital standardised mortality ratios. BMJ 342.jan21 1 (2010), c7116-c7116.

[440] Powell GA, Bonnett LJ, Tudur-Smith C, Hughes DA, Williamson PR, et al. Using routinely recorded data in the UK to assess outcomes in a randomised controlled trial: The Trials of Access. Trials 18.1 (2017).

[441] Steenkamp M, Frazier L, Lipskiy N, DeBerry M, Thomas S, et al. The National Violent Death Reporting System: an exciting new tool for public health surveillance. Inj Prev 12.suppl2 (2006), ii3-ii5.

[442] Appleyard S and Gilbert D. Innovative Solutions for Clinical Trial Follow-up: Adding Value from Nationally Held UK Data. Clin Oncol 29.12 (2017), 789-795.

[443] Pearce N, Beasley R, Burgess C, and Crane J. Asthma Epidemiology: Principles and Methods. Oxford University Press, 1998, 11. ISBN: 0195080165.

[444] Riordan F, Papoutsi C, Reed JE, Marston C, Bell D, et al. Patient and public attitudes towards informed consent models and levels of awareness of Electronic Health Records in the UK. Int J Med Informatics 84.4 (2015), 237-247.

[445] Haddow G, Bruce A, Sathanandam S, and Wyatt JC. 'Nothing is really safe': a focus group study on the processes of anonymizing and sharing of health data for research purposes. $J$ Eval Clin Pract 17.6 (2010), 1140-1146.

[446] Lea NC, Nicholls J, Dobbs C, Sethi N, Cunningham J, et al. Data Safe Havens and Trust: Toward a Common Understanding of Trusted Research Platforms for Governing Secure and Ethical Health Research. JMIR Medical Informatics 4.2 (2016), e22.

[447] Stockdale J, Cassell J, and Ford E. Giving something back: A systematic review and ethical enquiry of public opinions on the use of patient data for research in the United Kingdom and the Republic of Ireland. Wellcome Open Res 3 (2018), 6.

[448] Corburn J. Urban planning and health disparities: Implications for research and practice. Plan Pract Res 20.2 (2005), 111-126.

[449] Barton H, Grant M, Mitcham C, and Tsourou C. Healthy urban planning in European cities. Health Promotion International 24.Supplement 1 (2009), i91-i99.

[450] Smith JR, Noble MJ, Musgrave S, Murdoch J, Price GM, et al. The at-risk registers in severe asthma (ARRISA) study: a cluster-randomised controlled trial examining effectiveness and costs in primary care. Thorax 67.12 (2012), 1052-1060.

[451] Smith JR, Musgrave S, Payerne E, Noble M, Sims EJ, et al. At-risk registers integrated into primary care to stop asthma crises in the UK (ARRISA-UK): study protocol for a pragmatic, cluster randomised trial with nested health economic and process evaluations. Trials 19.1 (2018). 
[452] WyattJC and Wright P. Design should help use of patients' data. Lancet 352.9137 (1998), 13751378.

[453] Moskowitz A, McSparron J, Stone DJ, and Celi LA. Preparing a new generation of clinicians for the era of big data. Harv Med Stud Rev 2.1 (2015), 24.

[454] Friedman C, Rindflesch TC, and Corn M. Natural language processing: State of the art and prospects for significant progress, a workshop sponsored by the National Library of Medicine. J Biomed Inform 46.5 (2013), 765-773.

[455] Kukafka R, Ancker JS, Chan C, Chelico J, Khan S, et al. Redesigning electronic health record systems to support public health. J Biomed Inform 40.4 (2007), 398-409.

[456] Chalmers DJ, Deakyne SJ, Payan ML, Torok MR, Kahn MG, et al. Feasibility of Integrating Research Data Collection into Routine Clinical Practice Using the Electronic Health Record. J Urol 192.4 (2014), 1215-1220.

[457] Jones KH, Laurie G, Stevens L, Dobbs C, Ford DV, et al. The other side of the coin: Harm due to the non-use of health-related data. International Journal of Medical Informatics 97 (2017), 43-51.

[458] Asthma Audit Development Project. URL: https://www.rcplondon.ac.uk/projects/asthmaaudit-development-project.

[459] Asthma Audit Feasibility Study Team. National Asthma Audit Feasibility Study: Phase 1 Ful Report. Royal Collage of Physicians, 2017.

[460] Coiera E. The Forgetting Health System. URL: https : / / coiera . com / 2015/10/ 07 / the forgetting-health-system/.

[461] Learning Health System for Asthma (NCT00287391). 2017. URL: https://clinicaltrials. gov/ct2/show/NCT03000491.

[462] Use of Routine Data in Research. URL: https : / www . healthandcareresearch.gov . wales / use-of-routine-data-in-research/.

[463] Sacristán JA and Dilla T. No big data without small data: learning health care systems begin and end with the individual patient. J Eval Clin Pract 21.6 (2015), 1014-1017.

[464] Howard S, Lang A, Patel M, Sharples S, and Shaw D. Electronic monitoring of adherence to inhaled medication in asthma. Curr Respir Med Rev 10.1 (2014), 50-63.

[465] Ryan D, Blakey J, Chisholm A, Price D, Thomas M, et al. Use of electronic medical records and biomarkers to manage risk and resource efficiencies. Eur Clin Respir J 4.1 (2017), 1293386.

[466] van der Schee MP, Paff T, Brinkman P, Aalderen WMC van, Haarman EG, et al. Breathomics in Lung Disease. Chest 147.1 (2015), 224-231.

[467] Bos LD, Sterk PJ, and Fowler SJ. Breathomics in the setting of asthma and chronic obstructive pulmonary disease. J Allergy Clin Immunol 138.4 (2016), 970-976.

[468] Turner SW, Ayres JG, Macfarlane TV, Mehta A, Mehta G, et al. A methodology to establish a database to study gene environment interactions for childhood asthma. BMC Med Res Methodol 10 (2010), 107.

[469] Ram S, Zhang W, Williams M, and Pengetnze Y. Predicting Asthma-Related Emergency Department Visits Using Big Data. IEEE J Biomed Health Inform 19.4 (2015), 1216-1223.

[470] Dai H, Lee BR, and Hao J. Predicting Asthma Prevalence by Linking Social Media Data and Traditional Surveys. Ann Am Acad Pol Soc Sci 669.1 (2016), 75-92. 
[471] Mavragani A, Sampri A, Sypsa K, and Tsagarakis KP. Integrating Smart Health in the US Health Care System: Infodemiology Study of Asthma Monitoring in the Google Era. JMIR Public Health Surveill 4.1 (2018), e24.

[472] Bian J, Topaloglu U, and Yu F. Towards large-scale twitter mining for drug-related adverse events. Proceedings of the 2012 international workshop on Smart health and wellbeing - SHB '12. ACM Press, 2012.

[473] Wongsuphasawat K, Guerra Gómez JA, Plaisant C, Wang TD, Taieb-Maimon M, et al. LifeFlow: visualizing an overview of event sequences. Proceedings of the SIGCHI conference on human factors in computing systems. ACM. 2011, 1747-1756.

[474] Monroe M, Lan R, Lee H, Plaisant C, and Shneiderman B. Temporal event sequence simplification. IEEE Trans Vis Comput Graph 19.12 (2013), 2227-2236.

[475] Welsh Results Reports Service (WRRS). URL: http://www.wales.nhs.uk/nwis/page/53509/.

[476] Scott PJ, Cornet R, McCowan C, Peek N, Fraccaro P, et al. Informatics for Health 2017: Advancing both science and practice. Journal of Innovation in Health Informatics 24.1 (2017), 1 . 
Appendices 


\section{Appendix A}

\section{Chapter 2 Appendix}

A.1 Additional results for Chapter 2 
Table A.1.1: Search query used in the systematic scoping review.

\begin{tabular}{|c|c|}
\hline $\begin{array}{l}\text { Search } \\
\text { ID }\end{array}$ & Query \\
\hline \#1 & $\begin{array}{l}\text { Search ("humans"[mh] AND English[lang] AND ("2012/11/19"[PDat] : "2015/11/18"[PDat]) AND "loattrfull text"[sb] AND ( Asthma[mh] OR "Anti-Asthmatic Agents"[mh] ) AND ( Asthr } \\
\text { Asthmatic[tiab] OR Asthmatics[tiab] ) NOT "Comment" [pt] NOT "Editorial"[pt] NOT "Letter" [pt] NOT "review"[pt] NOT "Meta-Analysis" [pt] NOT "clinical trial"[pt] NOT "Randomized } \\
\text { [pt] NOT "Clinical Trial, Phase I" [pt] NOT "Clinical Trial, Phase II" [pt] NOT "Clinical Trial, Phase III" [pt] NOT "Clinical Trial, Phase IV" [pt] NOT "Controlled Clinical Trial" [pt] NOT "C } \\
\text { Topic" [Mesh] NOT "double-blind" [All] NOT "placebo-controlled" [All] NOT "case reports" [pt] NOT "pilot study" [All] NOT "pilot projects" [Mesh] NOT "Prospective Studies" [Mesh]) }\end{array}$ \\
\hline \#2 & 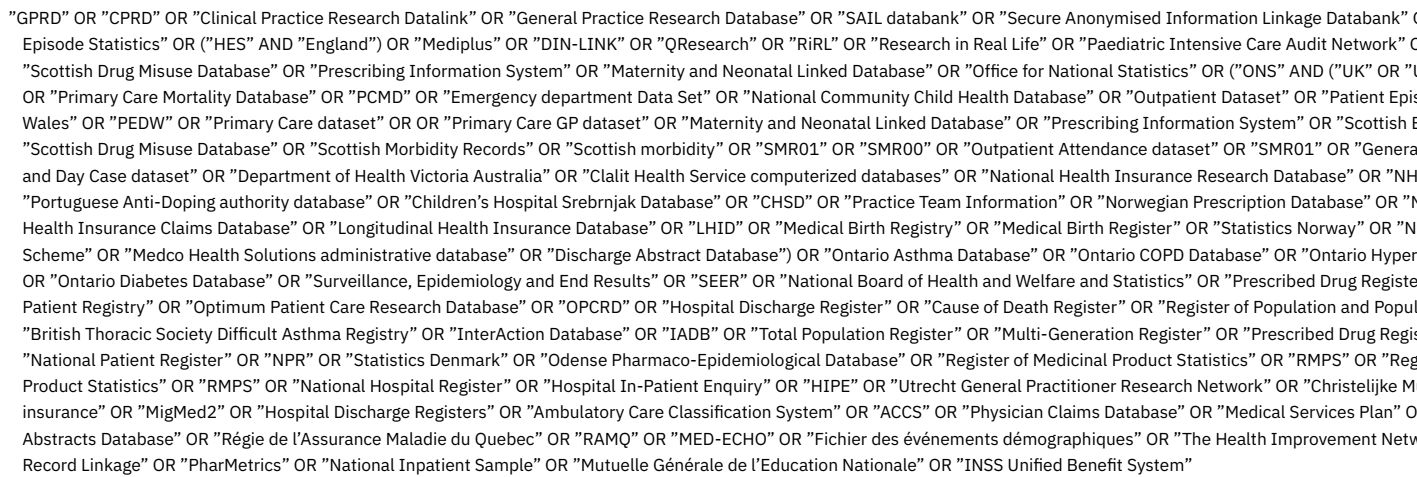 \\
\hline
\end{tabular}

("Premier" [All] OR "Solucient" [All] OR "Cerner" [All] OR "Ingenix" [All] OR "LabRx" [All] OR "IHCIS" [All] OR "marketscan" [All] OR "market scan" [All] OR "Medstat" [All] OR "Thoms "pharmetrics" [All] OR "healthcore" [All] OR "united healthcare" [All] OR "UnitedHealthcare" [All] OR "UHC" [All] OR "Research Database" [All] OR "Group Health" [All] OR "HCUP" [A Cost" [All] AND "Utilization Project" [All]) OR ("Health Care Cost" [All] AND "Utilization Project" [All]) OR "MEPS" [All] OR "Medical Expenditure Panel Survey" [All] OR "NAMCS" [All] Hospital Ambulatory Medical Care Survey" [All] OR "National Ambulatory Medical Care Survey" [All] OR "NHIS" [All] OR "National Health Interview Survey" [All] OR "Kaiser" [All] OR Hospital Ambulatory Medical Care Survey" [All] OR "National Ambulatory Medical Care Survey" [All] OR "NHIS" [All] OR "National Health Interview Survey" [All] OR "Kaiser" [All] OR
"Kaiser-Permanente" [All] OR "Kaiser Permanente" [All] OR "HMO Research" [All] OR "Health Maintenance Organization" [All] OR "HMO" [All] OR "Cleveland Clinic" [All] OR "Lovelac "Kaiser-Permanente" [All] OR "Kaiser Permanente "All] OR "HMO Research" [All] OR "Health Maintenance Organization" [All] OR "HMO" [All] OR "Cleveland Clinic" [All] OR "Lovelac
"Department of Defense" [All] OR "Henry Ford" [All] OR "i3 Drug Safety" [All] OR "i3" [All] OR "Aetna" [All] OR "Humana" [All] OR "Wellpoint" [All] OR "IMS" [All] OR "Intercontinenta Services" [All] OR "IMS Health" [All] OR "Geisinger" [All] OR "GE Healthcare" [All] OR "MQIC" [All] OR "PHARMO" [All] OR "Institute for Drug Outcome Research" [All] OR "Pilgrim" [A Sound" [All] OR "Regenstrief" [All] OR "Saskatchewan" [All] OR "Tayside" [All] OR "MEMO" [All] OR "Veterans Affairs" [All] OR "Partners Healthcare" [All] OR "Mayo Clinic" [All] OR " Epidemiology" [All] OR "Indiana Health Information Exchange" [All] OR "Indiana Health" [All] OR "Intermountain" [All] OR "blue cross" [All] OR "health partners" [All] OR "health pla services" [All] OR "Nationwide Inpatient Sample" [All] OR "National Inpatient Sample" [All] OR "medicaid" [All] OR "medicare" [All] OR "MediPlus" [All] OR "Outcome Assessment" [ [tiab]) OR (RAMQ [tiab]) OR (Cigna [tiab]) OR ((british columbia [tiab]) AND ((health [tiab]) OR (data [tiab]) OR (database [tiab]) OR (population [tiab])) OR (CIHI [All Fields]) OR ((mani ((center for health policy [all fields]) OR (population [tiab]) OR (health insurance [tiab])) OR ((ontario [tiab]) AND ((population [tiab]) OR (OHIP [tiab]) OR (registered persons database insurance [tiab]) OR (ICES [All Fields]) OR (Institute for Clinical Evaluative Sciences [All Fields])) OR ((Alberta [tiab]) AND ((health [tiab]) OR (data [tiab]) OR (database [tiab]) OR (pop (Alberta Health and Wellness [All Fields]))) OR "ICD-9-CM" [All Fields] OR "ICD-10-CM" [All Fields] OR "ICD-9" [All] OR "ICD-10" [All] OR "international statistical classification" [All classification of diseases" [All] OR "Database Management Systems" [Mesh] OR "Medical Records Systems, Computerized" [Mesh] OR "CPT" [All] OR "Current procedural terminolog OR "OPCS-4" OR "Read code" OR "SNOMED-CT" OR "J45*" OR "H33" OR "insurance database" [All] OR "insurance databases" [All] OR "health insurance claim*" OR "health insul "claim data" OR "claims data" OR ("claims" [tw] AND "administrative" [tw]) OR "Insurance Claim Review" [mh] OR ((medical OR pharmacy) AND claim) OR ((medical OR pharmacy) AR "Insurance Claim Reporting" [mh] OR "routine data" OR "routine health data" OR "routine clinical data" OR "routine electronic data" OR "routinely collected data" OR "routinely-colle "routinely-collected health data" OR "drug surveillance" [All] OR "pharmacy data" OR "dispensing data" OR "administrative data" OR "administrative health data" OR "health adminis ("data" [tw] AND "administrative" [tw]) OR "database analysis" OR "register" OR "registry" OR "Databases, Factual" [Mesh] OR "Databases as topic" [Mesh] OR "Data Warehouse" [A Record Linkage" [Mesh] OR "record-linkage" OR "record linkage") 
Table A.1.2: Charting table showing the data extracted from the reviewed articles.

\begin{tabular}{|c|c|}
\hline Variable & Notes \\
\hline \multicolumn{2}{|l|}{ General } \\
\hline \multicolumn{2}{|l|}{ Title/Year } \\
\hline \multicolumn{2}{|l|}{ Country } \\
\hline \multicolumn{2}{|l|}{ Study design } \\
\hline Routine data sources used & $\begin{array}{l}\text { We extracted the } \\
\text { routine datasets } \\
\text { are measured or de }\end{array}$ \\
\hline \multicolumn{2}{|l|}{ Algorithms and case definitions } \\
\hline Asthma & $\begin{array}{l}\text { Includes asthma } \\
\text { comorbidity and } \\
\text { validity reporting. } \\
\text { study between the } \\
\text { and the study-s } \\
\text { selection which cal }\end{array}$ \\
\hline Asthma severity & Also includes valid \\
\hline Asthma control & Also includes valid \\
\hline Asthma exacerbation & Also includes valid \\
\hline \multicolumn{2}{|l|}{ Clarity of reporting routine data-related methods } \\
\hline \multicolumn{2}{|l|}{ Title and abstract } \\
\hline \multicolumn{2}{|l|}{ RECORD 1.1: Types or names of routine data sources used are mentioned } \\
\hline \multicolumn{2}{|l|}{ RECORD 1.2: Geographical regions covered by the routine data sources used are mentioned } \\
\hline \multicolumn{2}{|l|}{ RECORD 1.2: Study-time frame is mentioned } \\
\hline \multicolumn{2}{|l|}{ RECORD 1.3: Record linkage is mentioned (if used) } \\
\hline \multicolumn{2}{|l|}{ Methods } \\
\hline $\begin{array}{l}\text { RECORD 6.1: Selection process of study population is mentioned in detail; clinical codes for asthma case } \\
\text { definitions are reported }\end{array}$ & $\begin{array}{l}\text { Clinical codes cou } \\
\text { section or in suppl }\end{array}$ \\
\hline \multicolumn{2}{|l|}{ RECORD 6.2: Validation for case definitions } \\
\hline \multicolumn{2}{|l|}{ RECORD 6.3 and 12.3: Record-linkage, if used, is sufficiently explained } \\
\hline \multicolumn{2}{|l|}{ RECORD 7.1: List of codes used in study variables } \\
\hline \multicolumn{2}{|l|}{ RECORD 12.1: Authors explained their level of access to database population } \\
\hline \multicolumn{2}{|l|}{ RECORD 12.2: Data cleaning is explained } \\
\hline \multicolumn{2}{|l|}{ Results } \\
\hline \multicolumn{2}{|l|}{ RECORD 13.1: Details of study population selection } \\
\hline \multicolumn{2}{|l|}{ Discussion } \\
\hline $\begin{array}{l}\text { RECORD 19.1: Implications of using routine data for asthma research (e.g. misclassification bias, un- } \\
\text { measured confounding, missing data, and changing eligibility over time) }\end{array}$ & \\
\hline
\end{tabular}


RECORD 22.1: Information on how to access study protocol, raw data, and programming code is men- 
Table A.1.3: Geographical distribution of the reviewed studies.

\begin{tabular}{ll}
\hline Country & Number of studies \\
\hline US & 52 \\
Taiwan & 20 \\
Canada & 12 \\
Sweden & 4 \\
Denmark & 4 \\
UK & 3 \\
Republic of Korea & 2 \\
Israel & 2 \\
France & 2 \\
Finland & 2 \\
Europe & 2 \\
USA, UK & 2 \\
Spain & 1 \\
Singapore & 1 \\
Portugal & 1 \\
Netherlands & 1 \\
Korea & 1 \\
Italy & 1 \\
Iran & 1 \\
Australia & 1 \\
& 1 \\
\hline
\end{tabular}

Table A.1.4: Study designs of the reviewed studies.

\begin{tabular}{ll}
\hline Study design & Number of studies \\
\hline cohort study, retrospective, using routine database(s) & 62 \\
cross-sectional / prevalence study & 27 \\
nested case-control & 5 \\
cohort study, prospective, using routine database(s) & 5 \\
validation study & 3 \\
time series analysis & 3 \\
population based cross-sectional ecological study & 2 \\
cohort study, retrospective, linked to self-reported data & 1 \\
cohort study, retrospective, linked to medical charts & 1 \\
cohort study, retrospective, linked to death registry & 1 \\
case-crossover study & 1 \\
case-control study & 1 \\
case-control & 1 \\
\hline
\end{tabular}

Table A.1.5: Types of EHR-derived data sources used in the reviewed articles.

\begin{tabular}{lc}
\hline Type & Number of studies \\
\hline health insurance claim & 72 \\
medical records or medical administrative data & 39 \\
dispensing & 13 \\
mortality with causes of death & 2 \\
public health surveillance database & 1 \\
medical birth register & 1 \\
health insurance claim + medical records & 1 \\
drug adverse effect surveillance & 1 \\
disease register & 1 \\
\hline
\end{tabular}


Table A.1.6: Algorithms used to identify asthma patients.

\begin{tabular}{|c|c|}
\hline Label & Algorithm \\
\hline asthma & 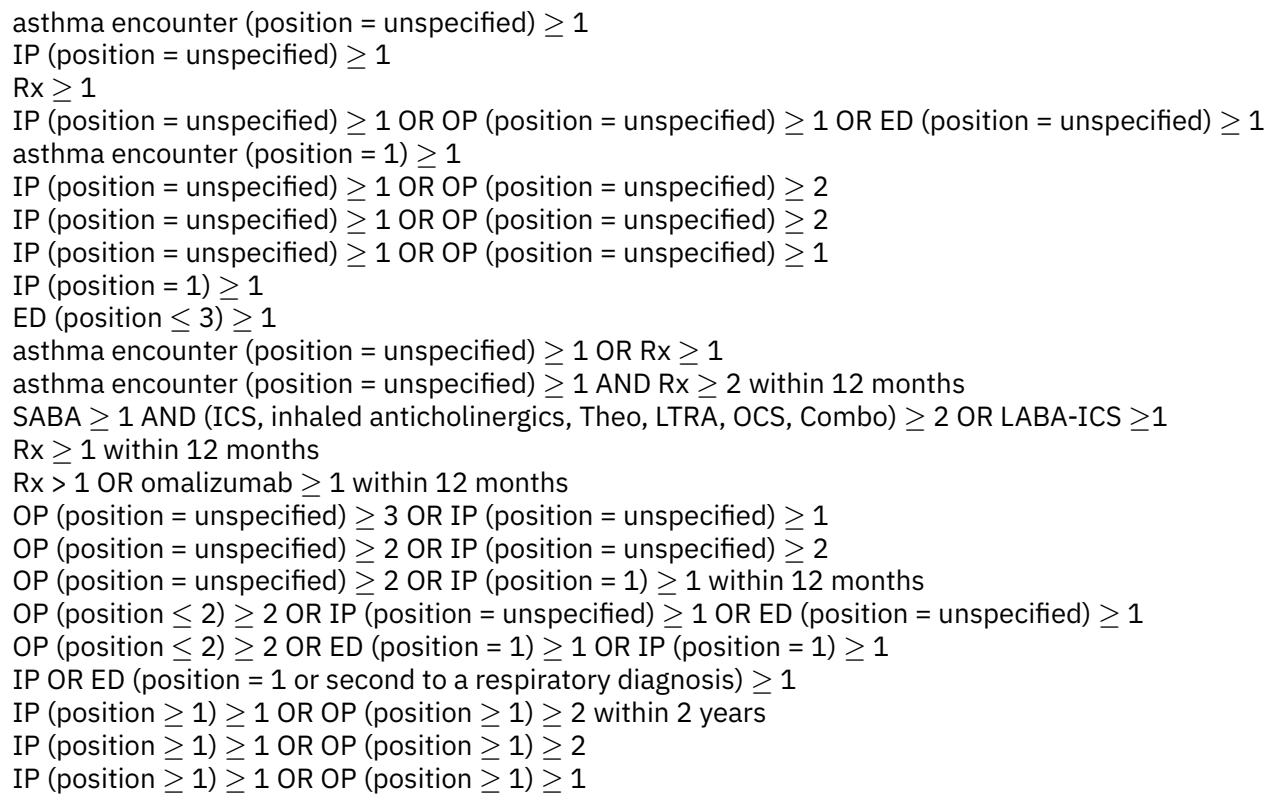 \\
\hline
\end{tabular}

$\mathrm{ED}=$ emergency department visit; GP = general practitioner visit; ICS = inhaled corticosteroid; IP = inpatient hospitalisation; LABA = long-acting beta agonist; oral corticosteroids; OP = outpatient visit; RSV = respiratory syncytial virus; SABA = short-acting beta-2 agonists 
Table A.1.6: Algorithms used to identify asthma patients.(cont'd)

\begin{tabular}{|c|c|}
\hline Label & Algorithm \\
\hline & 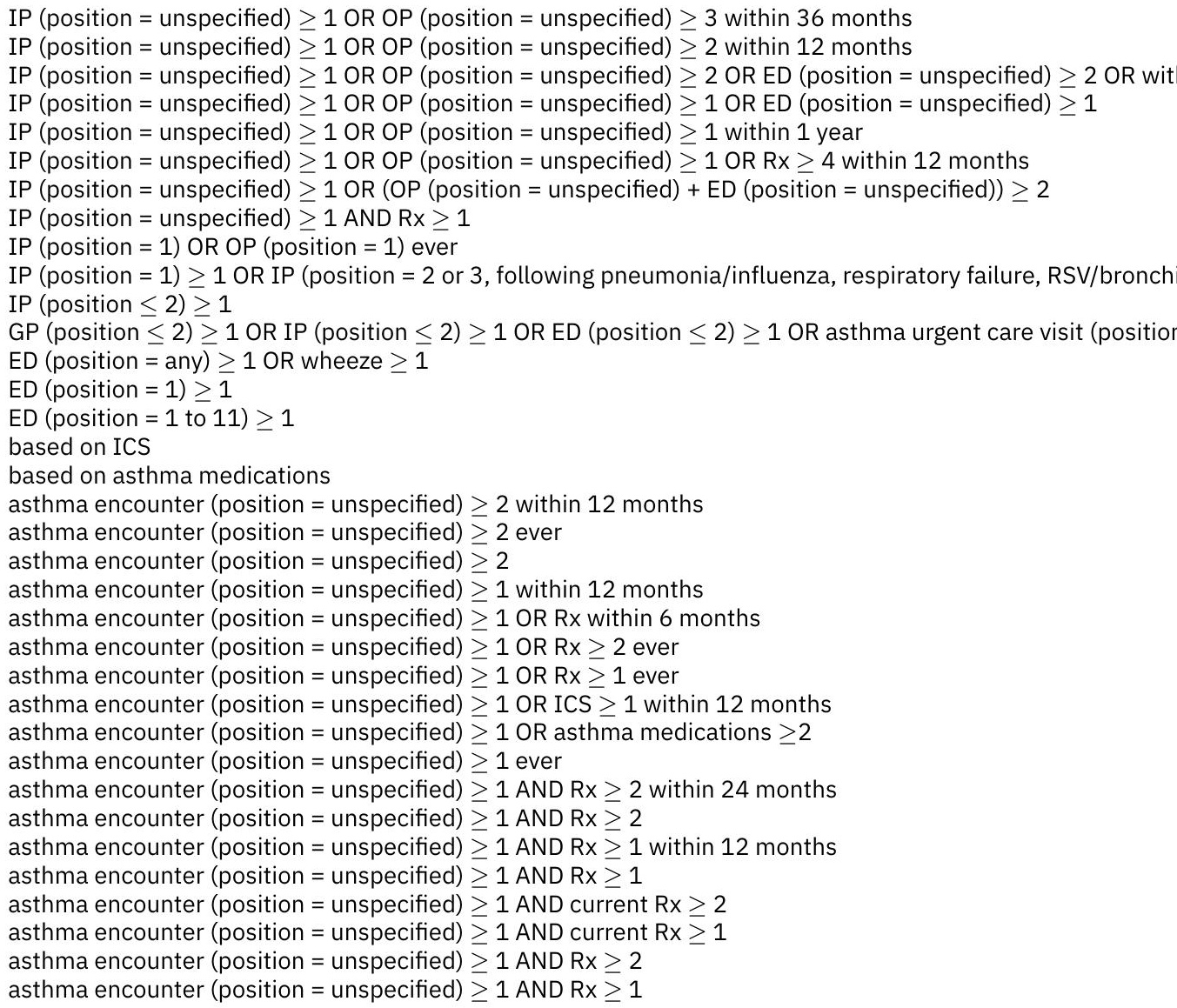 \\
\hline
\end{tabular}

ED = emergency department visit; GP = general practitioner visit; ICS = inhaled corticosteroid; IP = inpatient hospitalisation; LABA = long-acting beta agonist oral corticosteroids; $\mathrm{OP}=$ outpatient visit; $\mathrm{RSV}$ = respiratory syncytial virus; $\mathrm{SABA}=$ short-acting beta-2 agonists 
Table A.1.6: Algorithms used to identify asthma patients.(cont'd)

\begin{tabular}{|c|c|}
\hline Label & Algorithm \\
\hline current asthma & $\begin{array}{l}\text { (asthma encounter (position }=1) \geq 1 \text { OR asthma encounter (position } \geq 1) \geq 4 \text { ) AND (asthma prescriptions } \geq \text {. } \\
\text { within } 5 \text { years } \\
\text { asthma encounter }(\text { position }=\text { unspecified }) \geq 1\end{array}$ \\
\hline $\begin{array}{l}\text { current GP-reported } \\
\text { asthma }\end{array}$ & asthma encounter (position $=$ unspecified) $\geq 1$ within 12 months \\
\hline current treated asthma & asthma encounter (position $=$ unspecified) $\geq 1$ AND $R x \geq 1$ within 12 months \\
\hline treated asthma & $\mathrm{Rx}>=3$ within 12 months \\
\hline persistent asthma & $\begin{array}{l}\mathrm{Rx} \geq 4 \text { OR IP } \geq 1 \text { OR ED (position = } 1) \geq 1 \text { OR (OP (position = any) } \geq 1 \text { AND Rx } \geq 2 \text { ) within } 12 \text { months } \\
\mathrm{Rx} \geq 4 \text { OR IP } \geq 1 \text { OR ED (position = 1) } \geq 1 \text { OR (OP (position = any) } \geq 1 \text { AND Rx } \geq 2 \text { ) within } 24 \text { months } \\
\mathrm{Rx} \geq 4 \text { OR IP } \geq 1 \text { OR ED (position = 1) } \geq 1 \text { OR (OP (position = any) } \geq 1 \text { AND Rx } \geq 2 \text { ) within } 12 \text { months } \\
\mathrm{Rx} \geq 4 \text { OR IP } \geq 1 \text { OR ED (position = } 1) \geq 1 \text { OR (OP (position = any) } \geq 1 \text { AND Rx } \geq 2 \text { ) } \\
\mathrm{IP}(\text { position = unspecified) } \geq 1 \text { OR ED (position = unspecified) } \geq 1 \text { OR OCS } \geq 3 \text { ) within } 12 \text { months }\end{array}$ \\
\hline
\end{tabular}

ED = emergency department visit; GP = general practitioner visit; ICS = inhaled corticosteroid; IP = inpatient hospitalisation; LABA = long-acting beta agonist; oral corticosteroids; OP = outpatient visit; RSV = respiratory syncytial virus; SABA = short-acting beta-2 agonists 
Table A.1.7: Approaches used in identifying asthma patients.

\begin{tabular}{|c|c|c|}
\hline Criteria base on & $\begin{array}{l}\text { Diagnostic label } \\
\text { used }\end{array}$ & Number of studies \\
\hline & 'asthma' & 68 \\
\hline \multicolumn{2}{|c|}{ Asthma diagnostic/management codespersistent asthma } & 1 \\
\hline & acute asthma & 1 \\
\hline & current asthma & 1 \\
\hline & $\begin{array}{l}\text { current GP-reported } \\
\text { and diagnosed } \\
\text { asthma }\end{array}$ & 1 \\
\hline \multicolumn{2}{|c|}{$\begin{array}{l}\text { Asthma diagnostic/management codes 'asthma' } \\
\text { AND asthma prescription codes }\end{array}$} & 11 \\
\hline AND asthma prescription codes & $\begin{array}{l}\text { current treated } \\
\text { asthma }\end{array}$ & 1 \\
\hline & persistent asthma & 2 \\
\hline \multirow{4}{*}{ Asthma prescription codes } & asthma & 22 \\
\hline & & \\
\hline & treated asthma & 1 \\
\hline & persistent asthma & 4 \\
\hline
\end{tabular}

Table A.1.8: Age restriction approaches used in asthma patient identification.

\begin{tabular}{lll}
\hline Age limits & Studies & Number of studies \\
\hline Minimum age limits & & \\
6 months & A1 & 1 \\
2 years & A2-A7 & 6 \\
3 years & A8-A10 & 3 \\
5 years & A11-A13 & 3 \\
Maximum age limits & & \\
44 years & A14 & 1 \\
55 years & A15 & 1 \\
60 years & A16 & 1 \\
64 years & A17 & 1 \\
\hline
\end{tabular}


Table A.1.9: Co-morbidities and conditions based on which asthma patients were excluded.

\begin{tabular}{ll}
\hline Condition & Number of studies \\
\hline COPD & 11 \\
Cystic fibrosis & 13 \\
Pulmonary embolism & 3 \\
Bronchiectasis & 4 \\
Pulmonary hypertension & 4 \\
Congestive heart failure & 3 \\
Emphysema & 3 \\
Chronic bronchitis & 2 \\
Immunodeficiency & 2 \\
Churg Strauss syndrome & 1 \\
Wegener syndrome & 1 \\
Sarcoidosis & 1 \\
Smoker over age of 60 & 1 \\
Pneumonia & 1 \\
Anti-cholinergic prescription as a proxy of COPD & 1 \\
Chronic respiratory failure & 1 \\
Achondroplasia & 1 \\
Bronchopulmonary dysplasia & 1 \\
Respiratory cancer & 1 \\
Active or past tobacco use & 1 \\
Primary ciliary dyskinesia & 1 \\
Tracheomalacia & 1 \\
Bronchiolitis/RSV infection & 2 \\
Pneumoconiosis & 1 \\
Other lung diseases due to external agents & 1 \\
Psychosis & 1 \\
"Perinatal respiratory condition" & 1 \\
Tracheostomy & 1 \\
Gastrostomy & 1 \\
\hline
\end{tabular}


Table A.1.10: Algorithms used to ascertain asthma severity using EHR data.

\begin{tabular}{|c|c|c|c|}
\hline Variable & Algorithm & $\begin{array}{l}\text { Interval } \\
\text { (months) }\end{array}$ & Appears in \\
\hline Mild asthma & $\begin{array}{l}\text { either } \\
500 \mathrm{mg} / \text { day of ICS monotherapy (in beclomethasone } \\
\text { chlorofluorocarbon equivalents) } \\
\text { OR } \\
250 \mathrm{mg} / \text { day of ICS + additional controller } \\
\text { AND } \\
\text { either } \\
\leq 3 \text { SABA doses per week on average (each = } 2 \\
\text { salbutamol 100mg puffs) } \\
\text { OR } \\
\text { both } \\
4-10 \text { doses of SABA per week on average } \\
\text { AND } \\
\text { no moderate to severe asthma exacerbation (defined } \\
\text { as asthma ED visit OR asthma hospitalisation OR } \\
\text { short-course OCS) }\end{array}$ & 12 & [A18] \\
\hline Moderate asthma & $\begin{array}{l}\text { NOT mild asthma NOR severe asthma as defined in the } \\
\text { same study }\end{array}$ & 12 & [A18] \\
\hline \multirow[t]{3}{*}{ Severe asthma } & $\begin{array}{l}>1000 \mathrm{mg} / \text { day of ICS } \\
\text { AND } \\
\text { one of } \\
>3 \text { SABA per week on average } \\
\text { OR } \\
\geq 1 \text { moderate to severe asthma exacerbation } \\
\text { OR } \\
\text { both } \\
\text { lower doses of ICS with }>10 \text { SABA doses per week on } \\
\text { average } \\
\text { AND } \\
1 \text { moderate to severe asthma exacerbation }\end{array}$ & 12 & [A18] \\
\hline & $>6$ albuterol refills per year & 12 & {$[\mathrm{~A} 2]$} \\
\hline & GINA step 4 or higher & unclear & [A19] \\
\hline
\end{tabular}

ED = emergency department; GINA = Global Initiative for Asthma; HEDIS = Healthcare Effectiveness Data and Information Set; ICS = inhaled corticosteroid corticosteroids; $\mathrm{OP}=$ outpatient; $\mathrm{SABA}=$ short-acting $\beta 2$ agonists. 
Table A.1.10: Algorithms used to ascertain asthma severity using EHR data. (cont'd).

\begin{tabular}{|c|c|c|c|}
\hline Variable & Algorithm & $\begin{array}{l}\text { Interval } \\
\text { (months) }\end{array}$ & Appears in \\
\hline & $\begin{array}{l}\text { continuous treatment with ICS (at least } 800 \mathrm{mg} \\
\text { budesonide daily or equivalent [500 mg fluticasone]) } \\
\text { and (LABA) }\end{array}$ & 12 & {$[\mathrm{~A} 20]$} \\
\hline & $\begin{array}{l}\text { presence of persistent asthma according to the HEDIS } \\
\text { criteria associated with readmission }\end{array}$ & 12 & [A4] \\
\hline & $\begin{array}{l}\text { OR } \\
\text { presence of complex chronic condition within the prior } \\
\text { year associated with readmission }\end{array}$ & & \\
\hline & based on number of ICS, LABA, and OCS prescriptions & 24 & [A21] \\
\hline & $\begin{array}{l}\text { based on number of asthma prescriptions (including } \\
\text { OCS) }\end{array}$ & 12 & {$[\mathrm{~A} 22]$} \\
\hline & $\begin{array}{l}\text { based on asthma hospitalisation, asthma Ed visits, } \\
\text { outpatient visits for asthma exacerbation, number of } \\
\text { SABA dispensings, number of OCS dispensings }\end{array}$ & 12 & {$[\mathrm{~A} 23]$} \\
\hline & $\begin{array}{l}\text { based on number of asthma hospitalisations, asthma } \\
\text { ED visits, SABA prescriptions, OCS prescriptions, and } \\
\text { asthma exacerbations over } 6 \text { months }\end{array}$ & 6 & [A13] \\
\hline & $\begin{array}{l}\text { based on acute OCS course, mean daily SABA dose, } \\
\text { number of asthma consultations with no acute OCS }\end{array}$ & 12 & {$[\mathrm{~A} 16]$} \\
\hline & $\begin{array}{l}\text { ICS (>800 mg } \\
\text { budesonide daily) AND second controller } \\
\text { OR } \\
\text { ICS-LABA } \\
\text { OR } \\
\text { omalizumab }\end{array}$ & 12 & [A14] \\
\hline & According to GINA 2006 classification of severity & unclear & {$[\mathrm{A} 24]$} \\
\hline & Based on OCS prescriptions & unclear & {$[\mathrm{A} 25]$} \\
\hline & Number of OP over variable follow-up periods & variable & {$[\mathrm{A} 26]$} \\
\hline ‘More severe asthma’ & $\begin{array}{l}\geq 2 \text { SABA prescriptions within } 90 \text { days of ICS } \\
\text { prescriptions }\end{array}$ & 3 & {$[\mathrm{~A} 27]$} \\
\hline
\end{tabular}

ED = emergency department; GINA = Global Initiative for Asthma; HEDIS = Healthcare Effectiveness Data and Information Set; ICS = inhaled corticosteroid corticosteroids; OP = outpatient; SABA = short-acting $\beta 2$ agonists. 
Table A.1.10: Algorithms used to ascertain asthma severity using EHR data. (cont'd).

\begin{tabular}{|c|c|c|c|}
\hline Variable & Algorithm & $\begin{array}{l}\text { Interval } \\
\text { (months) }\end{array}$ & Appears in \\
\hline & $\begin{array}{l}\text { HEDIS criteria for persistent asthma: } \\
\geq 1 \text { asthma hospitalisation } \\
\text { OR } \\
\geq 1 \text { asthma ED visit } \\
\text { OR } \\
\geq 4 \text { asthma prescriptions } \\
\text { OR } \\
\text { both } \\
\geq 4 \text { asthma outpatient visits } \\
\text { AND } \\
\geq 2 \text { asthma prescriptions }\end{array}$ & 24 & {$[\mathrm{~A} 6]$} \\
\hline & $\geq 1$ asthma hospitalisations or ED visits & 12 & [A28] \\
\hline Low-risk asthma & $\begin{array}{l}\text { no asthma ED visits } \\
\text { AND } \\
\text { no asthma hospitalisations } \\
\text { AND } \\
<15 \beta \text {-agonist canisters dispensed } \\
\text { AND } \\
\text { no OCS dispensed }\end{array}$ & 12 & [A9] \\
\hline $\begin{array}{l}\text { Moderate-risk } \\
\text { asthma }\end{array}$ & $\begin{array}{l}\text { no asthma ED visits } \\
\text { AND } \\
\text { no asthma hospitalisations } \\
\text { AND } \\
\text { only one of: } \\
\geq 15 \beta \text {-agonist canisters dispensed } \\
\text { OR } \\
\geq 1 \text { OCS dispensings }\end{array}$ & 12 & [A9] \\
\hline High-risk asthma & $\begin{array}{l}\geq 1 \text { asthma ED visits } \\
\text { OR } \\
\geq 1 \text { asthma hospitalisations } \\
\text { OR } \\
\text { both: } \\
\geq 15 \beta \text {-agonist canisters dispensed } \\
\text { AND } \\
\geq 1 \text { OCS dispensings }\end{array}$ & 12 & [A9] \\
\hline
\end{tabular}

ED = emergency department; GINA = Global Initiative for Asthma; HEDIS = Healthcare Effectiveness Data and Information Set; ICS = inhaled corticosteroid corticosteroids; $\mathrm{OP}=$ outpatient; $\mathrm{SABA}=$ short-acting $\beta 2$ agonists . 
Table A.1.11: Algorithms used to ascertain asthma exacerbation using EHR data.

\begin{tabular}{|c|c|c|c|c|c|c|c|c|}
\hline \multirow[t]{2}{*}{ Variable } & \multirow[t]{2}{*}{ Study } & \multirow[t]{2}{*}{ Algorithm } & \multicolumn{4}{|c|}{ OCS } & \multirow[t]{2}{*}{ IP } & \multirow[t]{2}{*}{ ED } \\
\hline & & & alone & $+\mathrm{OP}$ & $\begin{array}{c}\text { + IP, } \\
\text { ED, OP } \\
\text { or GP }\end{array}$ & $\begin{array}{l}+ \text { IP or } \\
\text { ED }\end{array}$ & & \\
\hline \multirow[t]{5}{*}{$\begin{array}{l}\text { Exacerba- } \\
\text { tion }\end{array}$} & [A29] & $\begin{array}{l}\geq 1 \text { OCS prescription for }<21 \text { days } \\
\text { OR } \\
\geq 4 \text { asthma GP visits per year } \\
\text { OR } \\
\geq 5 \text { SABA prescriptions per year }\end{array}$ & $\begin{array}{l}<21 \\
\text { days }\end{array}$ & & & & & \\
\hline & [A14] & $\begin{array}{l}\geq 1 \text { OCS prescription } \\
\text { OR } \\
\text { Hospitalisation or ED visit for asthma, } \\
\text { status asthmaticus, pneumonia, } \\
\text { dyspnoea, or respiratory insufficiency }\end{array}$ & $\geq 1$ & & & & $\mathrm{p}$ & $\mathrm{p}$ \\
\hline & [A31] & $\begin{array}{l}\text { asthma hospitalisation } \\
\text { OR } \\
\text { asthma ED visit } \\
\text { OR } \\
\text { OCS pharmacy claim }\end{array}$ & $p$ & & & & $\mathrm{p}$ & $\mathrm{p}$ \\
\hline & [A19] & $\begin{array}{l}\text { OCS prescription within } 7 \text { days of any } \\
\text { asthma encounter (which may } \\
\text { include hospitalisation, ED, } \\
\text { outpatient, or GP visit, ascertained } \\
\text { with the ICD-9 code } 493 \text { as a } \\
\text { primary diagnosis or as a secondary } \\
\text { diagnosis provided the primary } \\
\text { diagnosis is another respiratory } \\
\text { condition) }\end{array}$ & & & $\begin{array}{l}\text { within } \\
7 \text { days }\end{array}$ & $\begin{array}{l}\text { within } \\
7 \text { days }\end{array}$ & & \\
\hline & & $\begin{array}{l}\text { Variation: asthma encounter = asthma } \\
\text { hospitalisation or ED visit only }\end{array}$ & & & & & & \\
\hline
\end{tabular}

$\mathrm{p}=$ present; $\mathrm{a}=$ absent; $\mathrm{OCS}$ = oral corticosteroids; $\mathrm{AE}=$ asthma exacerbation; $\mathrm{SABA}=$ short-acting $\beta 2$ agonists; $\mathrm{ED}=$ emergency department; $\mathrm{ICD}=\mathrm{International}$ Clas outpatient; GP = general practitioner. 
Table A.1.11: Algorithms used to ascertain asthma exacerbation using EHR data. (cont'

\begin{tabular}{|c|c|c|c|c|c|c|c|c|}
\hline \multirow[t]{2}{*}{ Variable } & \multirow[t]{2}{*}{ Study } & \multirow[t]{2}{*}{ Algorithm } & \multicolumn{4}{|c|}{ OCS } & \multirow[t]{2}{*}{ IP } & \multirow[t]{2}{*}{ ED } \\
\hline & & & alone & $+\mathrm{OP}$ & $\begin{array}{l}\text { + IP, } \\
\text { ED, OP } \\
\text { or GP }\end{array}$ & $\begin{array}{l}+ \text { IP or } \\
\text { ED }\end{array}$ & & \\
\hline & [A21] & $\begin{array}{l}\text { OCS with asthma as indication } \\
\text { OR } \\
\text { asthma ED visit } \\
\text { OR } \\
\text { asthma hospitalisation }\end{array}$ & $\begin{array}{l}\text { indica- } \\
\text { tion is } \\
\text { asthma }\end{array}$ & & & & $\mathrm{p}$ & $p$ \\
\hline & [A22] & $\begin{array}{l}\text { OCS prescription } \\
\text { OR } \\
\text { number of asthma GP visits } \\
\text { OR } \\
\text { hospitalisation for asthma (as a } \\
\text { primary diagnosis; variation: as a } \\
\text { primary or secondary diagnosis) }\end{array}$ & $\mathrm{p}$ & & & & $\mathrm{p}$ & \\
\hline & [A32] & $\begin{array}{l}\text { Occurrence, after } 3 \text { months from } \\
\text { previous asthma hospitalisation, if } \\
\text { any, of: } \\
\text { OCS short-course } \\
\text { OR } \\
\text { asthma ED visit (ICD-9-CM = } \\
\text { 493) } \\
\text { OR } \\
\text { asthma hospitalisation } \\
\text { (ICD-9-CM = 493) }\end{array}$ & $p$ & & & & $\mathrm{p}$ & $\mathrm{p}$ \\
\hline & [A33] & $\begin{array}{l}\text { Primary hospital discharge diagnosis of } \\
\text { asthma exacerbation }\end{array}$ & & & & & $\mathrm{p}$ & \\
\hline
\end{tabular}

$\mathrm{p}=$ present; $\mathrm{a}=$ absent; $\mathrm{OCS}$ = oral corticosteroids; $\mathrm{AE}$ = asthma exacerbation; SABA = short-acting $\beta 2$ agonists; $\mathrm{ED}=$ emergency department; ICD = International Clas outpatient; GP = general practitioner. 
Table A.1.11: Algorithms used to ascertain asthma exacerbation using EHR data. (cont'

\begin{tabular}{|c|c|c|c|c|c|c|c|c|}
\hline \multirow[t]{2}{*}{ Variable } & \multirow[t]{2}{*}{ Study } & \multirow[t]{2}{*}{ Algorithm } & \multicolumn{4}{|c|}{ OCS } & \multirow[t]{2}{*}{ IP } & \multirow[t]{2}{*}{ ED } \\
\hline & & & alone & $+\mathrm{OP}$ & $\begin{array}{c}\text { + IP, } \\
\text { ED, OP } \\
\text { or GP }\end{array}$ & $\begin{array}{c}+ \text { IP or } \\
\quad \text { ED }\end{array}$ & & \\
\hline & {$[\mathrm{A} 34]$} & $\begin{array}{l}\text { ED visit with primary diagnosis of } \\
\text { asthma } \\
\text { OR } \\
\text { outpatient visit with } \\
\text { diagnosis of asthma exacerbation } \\
\text { OR } \\
\text { diagnosis of asthma with OCS } \\
\quad \text { prescription (<14-day supply) } \\
\quad \text { within } 5 \text { days } \\
\text { OR } \\
\text { hospitalisation with diagnosis of } \\
\text { asthma (primary) or asthma } \\
\text { exacerbation (any position) }\end{array}$ & & $\begin{array}{c}<14- \\
\text { day } \\
\text { sup- } \\
\text { ply; } \\
\text { within } \\
5 \text { days }\end{array}$ & & & $\mathrm{p}$ & $\mathrm{p}$ \\
\hline & [A35] & $\begin{array}{l}\text { OCS use } \\
\text { OR } \\
\text { asthma ED visit } \\
\text { OR } \\
\text { asthma hospitalisation }\end{array}$ & $\mathrm{p}$ & & & & $\mathrm{p}$ & $p$ \\
\hline & [A23] & $\begin{array}{l}\text { outpatient visit with primary diagnosis } \\
\text { of asthma (ICD-9-CM }=493 \text { ) and } \\
\text { OCS dispensing within } 5 \text { days } \\
\text { OR } \\
\text { asthma ED visit (ICD-9-CM }=493 . x x \text { ) } \\
\text { OR } \\
\text { asthma hospitalisation (ICD-9-CM = } \\
493 . x x \text { ) }\end{array}$ & & $\begin{array}{l}\text { within } \\
5 \text { days }\end{array}$ & & & $\mathrm{p}$ & $\mathrm{p}$ \\
\hline
\end{tabular}

$\mathrm{p}=$ present; $\mathrm{a}=$ absent; $\mathrm{OCS}$ = oral corticosteroids; $\mathrm{AE}=$ asthma exacerbation; SABA = short-acting $\beta 2$ agonists; $\mathrm{ED}=$ emergency department; ICD = International Cla outpatient; GP = general practitioner. 
Table A.1.11: Algorithms used to ascertain asthma exacerbation using EHR data. (cont'

\begin{tabular}{|c|c|c|c|c|c|c|c|c|}
\hline \multirow[t]{2}{*}{ Variable } & \multirow[t]{2}{*}{ Study } & \multirow[t]{2}{*}{ Algorithm } & \multicolumn{4}{|c|}{ OCS } & \multirow[t]{2}{*}{ IP } & \multirow[t]{2}{*}{ ED } \\
\hline & & & alone & $+\mathrm{OP}$ & $\begin{array}{l}\text { + IP, } \\
\text { ED, OP } \\
\text { or GP }\end{array}$ & $\begin{array}{l}+ \text { IP or } \\
\text { ED }\end{array}$ & & \\
\hline & [A36] & $\begin{array}{l}\text { ED visit with any asthma diagnosis } \\
\text { OR } \\
\text { hospitalisation with primary diagnosis } \\
\text { asthma } \\
\text { OR } \\
\text { OCS with asthma claim within } 7 \text { days }\end{array}$ & & & $\begin{array}{l}\text { within } \\
7 \text { days }\end{array}$ & & $p$ & $\mathrm{p}$ \\
\hline & [A37] & $\begin{array}{l}\text { one-off OCS prescription } \\
\text { (short-course) }\end{array}$ & $\mathrm{p}$ & & & & & \\
\hline & [A38] & $\begin{array}{l}\text { OCS within } 7 \text { days of an encounter with } \\
\text { diagnosis of exacerbation or } \\
\text { uncontrolled asthma }\end{array}$ & & & $P$ & & & \\
\hline & [A39] & $\begin{array}{l}\geq 1 \text { asthma ED visits } \\
\text { OR } \\
\geq 1 \text { asthma hospitalisations } \\
\text { OR } \\
\text { OCS prescriptions }\end{array}$ & $p$ & & & & $\mathrm{p}$ & $\mathrm{p}$ \\
\hline & [A40] & $\begin{array}{l}\text { asthma ED visit }(\mathrm{ICD}-9-\mathrm{CM}=493) \\
\text { AND/OR } \\
\text { asthma hospitalisation }(\mathrm{ICD}-9-\mathrm{CM}= \\
493 \text { ) }\end{array}$ & & & & & $\mathrm{p}$ & $p$ \\
\hline & [A8] & $\begin{array}{l}\text { Encounter with asthma exacerbation } \\
\text { code }\end{array}$ & & & & & & \\
\hline & [A16] & $\begin{array}{l}\text { acute OCS } \\
\text { OR } \\
\text { unscheduled asthma hospitalisation } \\
\text { OR } \\
\text { ED visit }\end{array}$ & $p$ & & & & $p$ & $\mathrm{p}$ \\
\hline
\end{tabular}

$\mathrm{p}=$ present; $\mathrm{a}=$ absent; $\mathrm{OCS}$ = oral corticosteroids; $\mathrm{AE}=$ asthma exacerbation; SABA = short-acting $\beta 2$ agonists; $\mathrm{ED}=$ emergency department; ICD = International Clas outpatient; $\mathrm{GP}=$ general practitioner. 
Table A.1.11: Algorithms used to ascertain asthma exacerbation using EHR data. (cont'

\begin{tabular}{|c|c|c|c|c|c|c|c|c|}
\hline \multirow[t]{2}{*}{ Variable } & \multirow[t]{2}{*}{ Study } & \multirow[t]{2}{*}{ Algorithm } & \multicolumn{4}{|c|}{ OCS } & \multirow[t]{2}{*}{ IP } & \multirow[t]{2}{*}{ ED } \\
\hline & & & alone & $+\mathrm{OP}$ & $\begin{array}{c}+\mathrm{IP}, \\
\mathrm{ED}, \mathrm{OP} \\
\text { or GP }\end{array}$ & $\begin{array}{c}+ \text { IP or } \\
\quad \text { ED }\end{array}$ & & \\
\hline & {$[\mathrm{A} 17]$} & $\begin{array}{l}\text { new occurrence (after }>=8-\text { day } \\
\text { wash-up period) of: } \\
\text { Both } \\
\text { Asthma outpatient visit (with a } \\
\text { code for acute exacerbation, } \\
\text { status asthmaticus, acute } \\
\text { asthma attack, uncontrolled } \\
\text { asthma, asthmatic bronchitis) } \\
\text { AND } \\
\text { OCS dispensing within } 7 \text { days } \\
\text { OR } \\
\text { Asthma ED visit or hospitalisation } \\
\text { (asthma diagnosis position = } 1 \\
\text { OR position = } 2 \text { following a } \\
\text { primary respiratory diagnosis) }\end{array}$ & & $p$ & & & $\mathrm{p}$ & $\mathrm{p}$ \\
\hline & {$[A 41]$} & $\begin{array}{l}\text { Asthma hospitalisation } \\
\text { OR } \\
\text { Asthma ED visit } \\
\text { OR } \\
\text { Asthma OP visit with OCS prescription }\end{array}$ & & $\mathrm{p}$ & & & $\mathrm{p}$ & $\mathrm{p}$ \\
\hline & {$[\mathrm{A} 24]$} & Based on rescue medications & & & & & & \\
\hline $\begin{array}{l}\text { Moderate- } \\
\text { to-severe } \\
\text { exacerba- } \\
\text { tion }\end{array}$ & [A18] & $\begin{array}{l}\text { OCS short-course } \\
\text { OR } \\
\text { asthma ED visit } \\
\text { OR } \\
\text { asthma hospitalisation }\end{array}$ & $\mathrm{p}$ & & & & $\mathrm{p}$ & $p$ \\
\hline
\end{tabular}

$\mathrm{p}=$ present; $\mathrm{a}=$ absent $\mathrm{OCS}=$ oral corticosteroids; $\mathrm{AE}=$ asthma exacerbation; SABA = short-acting $\beta 2$ agonists; $\mathrm{ED}=$ emergency department; $\mathrm{ICD}=\mathrm{International}$ Clas outpatient; GP = general practitioner. 
Table A.1.11: Algorithms used to ascertain asthma exacerbation using EHR data. (cont'

\begin{tabular}{|c|c|c|c|c|c|c|c|c|}
\hline \multirow[t]{2}{*}{ Variable } & \multirow[t]{2}{*}{ Study } & \multirow[t]{2}{*}{ Algorithm } & \multicolumn{4}{|c|}{ OCS } & \multirow[t]{2}{*}{ IP } & \multirow[t]{2}{*}{ ED } \\
\hline & & & alone & $+\mathrm{OP}$ & $\begin{array}{l}+\mathrm{IP} \\
\mathrm{ED}, \mathrm{OP} \\
\text { or GP }\end{array}$ & $\begin{array}{c}+ \text { IP or } \\
\text { ED }\end{array}$ & & \\
\hline & [A13] & $\begin{array}{l}\text { OCS within } 7 \text { days of asthma } \\
\text { outpatient visit } \\
\text { OR } \\
\text { Asthma ED visit }\end{array}$ & & $\begin{array}{l}\text { within } \\
7 \text { days }\end{array}$ & & & & $p$ \\
\hline $\begin{array}{l}\text { Moderate } \\
\text { exacerba- } \\
\text { tion }\end{array}$ & [A42] & $\begin{array}{l}\geq 1 \text { ED visits for asthma } \\
\text { AND } \\
\text { no hospitalisation for asthma }\end{array}$ & & & & & $a$ & $p$ \\
\hline
\end{tabular}


Table A.1.12: Algorithms used to assess asthma control using EHR data

\begin{tabular}{|c|c|c|}
\hline Variable & Algorithm & Interval \\
\hline \multirow[t]{5}{*}{$\begin{array}{l}\text { Low control/ } \\
\text { uncontrolled asthma }\end{array}$} & $\begin{array}{l}\geq 600 \text { doses ( } 1 \text { dose }=1 \text { puff) of SABA in the recent year } \\
\text { OR } \\
\geq 1 \text { exacerbation in the recent year, defined as: } \\
\geq 1 \text { hospitalisation or ED visit associated with ICD-10 code for asthma, } \\
\text { status asthmaticus, pneumonia, dyspnoea, or respiratory insufficiency } \\
\text { OR } \\
\quad \geq 1 \text { OCS prescription }\end{array}$ & 12 months \\
\hline & $\begin{array}{l}\geq 1 \text { hospitalisation or ED visit } \\
\text { OR } \\
\text { dispensing of OCS for } \geq 3 \text { days }\end{array}$ & 12 months \\
\hline & $\begin{array}{l}\geq 1 \text { ED or OP visit for asthma } \\
\text { OR } \\
\geq 1 \text { antibiotic prescriptions }\end{array}$ & unclear \\
\hline & $\begin{array}{l}\geq 1 \text { moderate to severe asthma exacerbation } \\
\text { AND } \\
>3 \text { and } 10 \text { SABA doses per week on average for mild and moderate/severe } \\
\text { asthma, respectively }\end{array}$ & 12 months \\
\hline & $\begin{array}{l}\geq 2 \text { acute care contact within } 1 \text { month } \\
\text { OR } \\
\geq 3 \text { reliever inhaler uses per week } \\
\text { OR } \\
\text { severe exacerbation requiring ICU/intubation in the last } 3 \text { months } \\
\text { OR } \\
\text { asthma hospitalisation in the last } 3 \text { months }\end{array}$ & 1-3 months \\
\hline
\end{tabular}

$\overline{\mathrm{LRTI}}=$ lower respiratory tract infection; SABA = short-acting $\beta$ agonists; $\mathrm{OCS}=$ oral corticosteroids; $\mathrm{GP}$ = general practitioner; $\mathrm{ED}=$ emergency department; ICl 
Table A.1.12: Algorithms used to assess asthma control using EHR data (cont'd)

\begin{tabular}{|c|c|c|}
\hline Variable & Algorithm & Interval \\
\hline & $\begin{array}{l}\text { at the assessment date } \\
>2 \text { asthma drug classes } \\
\text { OR } \\
\geq 1 \text { SABA } \\
\text { OR } \\
\text { in } 12 \text { months } \\
\geq 1 \text { OCS } \\
\text { OR } \\
\geq 6 \text { SABA } \\
\text { OR } \\
\geq 1 \text { asthma ED visits } \\
\text { OR } \\
\geq 1 \text { asthma hospitalisations }\end{array}$ & 12 months \\
\hline \multirow[t]{2}{*}{$\begin{array}{l}\text { Low-risk asthma } \\
\text { control }\end{array}$} & $\begin{array}{l}\text { Absence of all the following: } \\
\text { hospitalisation, ED, and unscheduled outpatient visits for asthma } \\
\text { (ascertained by any asthma or LRTI codes) } \\
\text { GP consultation for LRTI requiring antibiotics } \\
\text { acute course of OCS }\end{array}$ & 12 months \\
\hline & based on number of OCS prescriptions per year & 12 months \\
\hline \multirow[t]{2}{*}{$\begin{array}{l}\text { Impairment-domain } \\
\text { asthma control }\end{array}$} & based on number of $\beta$-agonists prescriptions per year & 12 months \\
\hline & $>2$ salbutamol puffs per day ( $>200 \mu \mathrm{g}$ in the UK and $>180 \mu \mathrm{g}$ in the US) & 12 months \\
\hline
\end{tabular}

$\overline{\mathrm{LRTI}}=$ lower respiratory tract infection; SABA = short-acting $\beta$ agonists; $\mathrm{OCS}$ = oral corticosteroids; GP = general practitioner; $\mathrm{ED}=$ emergency department; IC 
Table A.1.12: Algorithms used to assess asthma control using EHR data (cont'd)

\begin{tabular}{lll}
\hline Variable & Algorithm & Apterval \\
\hline Overall asthma control & $\begin{array}{c}\text { based on impairment-domain and risk-domain asthma control algorithms } \\
\text { used by the same study }\end{array}$ & 12 months
\end{tabular}

LRTI = lower respiratory tract infection; SABA = short-acting $\beta$ agonists; OCS = oral corticosteroids; GP = general practitioner; ED = emergency department; IC 


\section{References for Chapter 2 appendix}

[A1] Parikh K, Davis AB, and Pavuluri P. Do we need this blood culture? Hosp Pediatr 4.2 (2014), 7884.

[A2] Wu CL, Andrews AL, Teufel RJ, and Basco WT. Demographic predictors of leukotriene antagonist monotherapy among children with persistent asthma. J. Pediatr. 164.4 (2014), 827831.e1.

[A3] Kaiser SV, Bakel LA, Okumura MJ, Auerbach AD, Rosenthal J, et al. Risk Factors for Prolonged Length of Stay or Complications During Pediatric Respiratory Hospitalizations. Hosp Pediatr 5.9 (2015), 461-73.

[A4] Kenyon CC, Rubin DM, Zorc JJ, Mohamad Z, Faerber JA, et al. Childhood Asthma Hospital Discharge Medication Fills and Risk of Subsequent Readmission. J. Pediatr. 166.5 (2015), 11217.

[A5] Parikh K, Hall M, Mittal V, Montalbano A, Mussman GM, et al. Establishing benchmarks for the hospitalized care of children with asthma, bronchiolitis, and pneumonia. Pediatrics 134.3 (2014), 555-62.

[A6] Capo-Ramos DE, Duran C, Simon AE, Akinbami LJ, and Schoendorf KC. Preventive asthma medication discontinuation among children enrolled in fee-for-service Medicaid. J Asthma 51.6 (2014), 618-26.

[A7] Lachance L, Benedict MB, Doctor LJ, Gilmore LA, Kelly C, et al. Asthma coalition effects on vulnerable sub groups of children: the most frequent users of health care and the youngest. $J$ Asthma 51.5 (2014), 474-9.

[A8] Bhattacharjee R, Choi BH, Gozal D, and Mokhlesi B. Association of adenotonsillectomy with asthma outcomes in children: a longitudinal database analysis. PLoS Med. 11.11 (2014), e1001753.

[A9] Chang J, Freed GL, Prosser LA, Patel I, Erickson SR, et al. Comparisons of health care utilization outcomes in children with asthma enrolled in private insurance plans versus medicaid. $J$ Pediatr Health Care 28.1 (2014), 71-9. 
[A10] Liu X, Olsen J, Pedersen LH, Agerbo E, Yuan W, et al. Antidepressant use during pregnancy and asthma in the offspring. Pediatrics 135.4 (2015), e911-7.

[A11] Jena AB, Ho O, Goldman DP, and Karaca-Mandic P. The Impact of the US Food and Drug Administration Chlorofluorocarbon Ban on Out-of-pocket Costs and Use of Albuterol Inhalers Among Individuals With Asthma. JAMA Intern Med 175.7 (2015), 1171-9.

[A12] Malhotra K, Baltrus P, Zhang S, McRoy L, Immergluck LC, et al. Geographic and racial variation in asthma prevalence and emergency department use among Medicaid-enrolled children in 14 southern states. J Asthma 51.9 (2014), 913-21.

[A13] Adimadhyam S, Schumock GT, Walton S, Joo M, McKell J, et al. Risk of arrhythmias associated with ipratropium bromide in children, adolescents, and young adults with asthma: a nested case-control study. Pharmacotherapy 34.4 (2014), 315-23.

[A14] Bülow A von, Kriegbaum M, Backer V, and Porsbjerg C. The prevalence of severe asthma and low asthma control among Danish adults. J Allergy Clin Immunol Pract 2.6 (2014), 759-67.

[A15] Hasegawa K, Tsugawa Y, Brown DFM, and Camargo CA. A population-based study of adults who frequently visit the emergency department for acute asthma. California and Florida, 20092010. Ann Am Thorac Soc 11.2 (2014), 158-66.

[A16] Martin RJ, Price D, Roche N, Israel E, Aalderen WMC van, et al. Cost-effectiveness of initiating extrafine- or standard size-particle inhaled corticosteroid for asthma in two health-care systems: a retrospective matched cohort study. NPJ Prim Care Respir Med 24 (2014), 14081.

[A17] Zeiger RS, Schatz M, Li Q, Chen W, Khatry DB, et al. High blood eosinophil count is a risk factor for future asthma exacerbations in adult persistent asthma. J Allergy Clin Immunol Pract 2.6 (2014), 741-50.

[A18] Blais L, Kettani FZ, and Forget A. Associations of maternal asthma severity and control with pregnancy complications. J Asthma 51.4 (2014), 391-8.

[A19] Schatz M, Meckley LM, Kim M, Stockwell BT, and Castro M. Asthma exacerbation rates in adults are unchanged over a 5-year period despite high-intensity therapy. $\mathrm{J}$ Allergy Clin Immunol Pract 2.5 (2014), 570-4.e1.

[A20] Nordlund B, Melén E, Schultz ES, Grönlund H, Hedlin G, et al. Prevalence of severe childhood asthma according to the WHO. Respir Med 108.8 (2014), 1234-7.

[A21] Ismaila A, Corriveau D, Vaillancourt J, Parsons D, Stanford R, et al. Impact of adherence to treatment with fluticasone propionate/salmeterol in asthma patients. Curr Med Res Opin 30.7 (2014), 1417-25.

[A22] Laforest L, Licaj I, Devouassoux G, Chatte G, Martin J, et al. Asthma drug ratios and exacerbations: claims data from universal health coverage systems. Eur. Respir. J. 43.5 (2014), 137886.

[A23] Dilokthornsakul P, Chaiyakunapruk N, Schumock GT, and Lee TA. Calendar time-specific propensity score analysis for observational data: a case study estimating the effectiveness of inhaled long-acting beta-agonist on asthma exacerbations. Pharmacoepidemiol Drug Saf 23.2 (2014), 152-64.

[A24] Tan NC, Nadkarni NV, Lye WK, Sankari U, et al. Ten-year longitudinal study of factors influencing nocturnal asthma symptoms among Asian patients in primary care. NPJ Prim Care Respir Med 25 (2015), 15064.

[A25] Garne E, Hansen AV, Morris J, Zaupper L, Addor MC, et al. Use of asthma medication during pregnancy and risk of specific congenital anomalies: A European case-malformed control study. J Allergy Clin Immunol 136 (6 2015), 1496-502.e1-7.

[A26] Jian ZH, Huang JY, Lin FCF, Nfor ON, Jhang KM, et al. The use of corticosteroids in patients with COPD or asthma does not decrease lung squamous cell carcinoma. BMC Pulm Med 15 (2015), 154.

[A27] Rust G, Zhang S, Holloway K, and Tyler-Hill Y. Timing of emergency department visits for childhood asthma after initial inhaled corticosteroid use. Popul Health Manag 18.1 (2015), 5460.

[A28] Fung V, Graetz I, Galbraith A, Hamity C, Huang J, et al. Financial barriers to care among lowincome children with asthma: health care reform implications. JAMA Pediatr 168.7 (2014), 64956.

[A29] Confino-Cohen R, Brufman I, Goldberg A, and Feldman BS. Vitamin D, asthma prevalence and asthma exacerbations: a large adult population-based study. Allergy 69.12 (2014), 1673-80.

[A30] Fuhlbrigge A, Peden D, Apter AJ, Boushey HA, Camargo CA, et al. Asthma outcomes: Exacerbations. J Allergy Clin Immunol 129.3 (2012), S34-S48.

[A31] Tunceli O, Williams SA, Kern DM, Elhefni H, Pethick N, et al. Comparative effectiveness of budesonide-formoterol combination and fluticasone-salmeterol combination for asthma management: a United States retrospective database analysis. J Allergy Clin Immunol Pract 2.6 (2014), 719-26.

[A32] Tan CC, McDowell KM, Fenchel M, Szczesniak R, and Kercsmar CM. Spirometry use in children hospitalized with asthma. Pediatr. Pulmonol. 49.5 (2014), 451-7.

[A33] Nanchal R, Kumar G, Majumdar T, Taneja A, Patel J, et al. Utilization of mechanical ventilation for asthma exacerbations: analysis of a national database. Respir Care 59.5 (2014), 644-53.

[A34] Sumino K, O’Brian K, Bartle B, Au DH, Castro M, et al. Coexisting chronic conditions associated with mortality and morbidity in adult patients with asthma. J Asthma 51.3 (2014), 306-14.

[A35] Li L, Vollmer WM, Butler MG, Wu P, Kharbanda EO, et al. A comparison of confounding adjustment methods for assessment of asthma controller medication effectiveness. Am. J. Epidemiol. 179.5 (2014), 648-59.

[A36] Hagiwara M, Delea TE, and Stanford RH. Health-care utilization and costs with fluticasone propionate and fluticasone propionate/salmeterol in asthma patients at risk for exacerbations. Allergy Asthma Proc 35.1 (2014), 54-62. 
[A37] Ali AK, Hartzema AG, Winterstein AG, Segal R, Lu X, et al. Application of multicategory exposure marginal structural models to investigate the association between long-acting betaagonists and prescribing of oral corticosteroids for asthma exacerbations in the Clinical Practice Research Datalink. Value Health 18.2 (2015), 260-70.

[A38] Schatz M, Zeiger RS, Yang SJ, Chen W, Crawford W, et al. Change in asthma control over time: predictors and outcomes. J Allergy Clin Immunol Pract 2.1 (), 59-64.

[A39] Wu AC, Li L, Fung V, Kharbanda EO, Larkin EK, et al. Use of leukotriene receptor antagonists are associated with a similar risk of asthma exacerbations as inhaled corticosteroids. $J$ Allergy Clin Immunol Pract 2.5 (), 607-13.

[A40] Tse SM, Charland SL, Stanek E, Herrera V, Goldfarb S, et al. Statin use in asthmatics on inhaled corticosteroids is associated with decreased risk of emergency department visits. Curr Med Res Opin 30.4 (2014), 685-93.

[A41] Kim S, Kim J, Park SY, Um HY, Kim K, et al. Effect of pregnancy in asthma on health care use and perinatal outcomes. J Allergy Clin Immunol 136 (5 2015), 1215-23.e1-6.

[A42] Blais L, Kettani FZ, Forget A, Beauchesne MF, and Lemière C. Asthma exacerbations during the first trimester of pregnancy and congenital malformations: revisiting the association in a large representative cohort. Thorax 70.7 (2015), 647-52.

[A43] Keast SL, Thompson D, Farmer K, Smith M, Nesser N, et al. Impact of a prior authorization policy for montelukast on clinical outcomes for asthma and allergic rhinitis among children and adolescents in a state Medicaid program. J Manag Care Spec Pharm 20.6 (2014), 612-21.

[A44] Sullivan PW, Campbell JD, Ghushchyan VH, and Globe G. Outcomes before and after treatment escalation to Global Initiative for Asthma steps 4 and 5 in severe asthma. Ann. Allergy Asthma Immunol. 114.6 (2015), 462-9. 


\section{A.2 Published paper related to Chapter 2}




\title{
Defining asthma and assessing asthma outcomes using electronic health record data: a systematic scoping review
}

\author{
Mohammad A Al Sallakh, MD ${ }^{\bowtie 1, a}$, Eleftheria Vasileiou, MPH $^{2, a}$, Sarah E \\ Rodgers, $\mathrm{PhD}^{1, \mathrm{~b}}$, Ronan A Lyons, $\mathrm{MD}^{1, \mathrm{~b}}$, Aziz Sheikh, $\mathrm{MD}^{2, \mathrm{a}, \mathrm{b}}$ and Gwyneth A \\ Davies, $\mathrm{MD}^{1, \mathrm{a}}$ \\ ${ }^{1}$ Swansea University Medical School, Singleton Park, Swansea, SA2 8PP, UK \\ ${ }^{2}$ Usher Institute of Population Health Sciences and Informatics, The University of Edinburgh, Teviot Place, \\ Edinburgh, EH8 9AG, Scotland, UK \\ ${ }^{a}$ Asthma UK Centre for Applied Research \\ ${ }^{\mathrm{b}}$ The Farr Institute of Health Informatics Research
}

\begin{abstract}
Short Title
Defining and assessing asthma using EHR data
\end{abstract}

\section{Correspondence}

Mohammad A Al Sallakh, MD, MSc

Data Science Building, Swansea University, Singleton Park, Swansea, SA2 8PP, United Kingdom

Phone:

Email: M.A.AlSallakh@swansea.ac.uk

This is an author-submitted, peer-reviewed version of an article that has been accepted for publication in the European Respiratory Journal, prior to copy-editing, formatting and typesetting. This version of the article may not be duplicated or reproduced without prior permission from the copyright owner, the European Respiratory Society. The publisher is not responsible or liable for any errors or omissions in this version of the article or in any version derived from it by any other parties. The final, copy-edited, published article, which is the version of record, is available online (https://doi.org/10.1183/13993003.00204-2017) from the European Respiratory Journal without a subscription 18 months after the date of issue publication. 


\begin{abstract}
There is currently no consensus on approaches to defining asthma or assessing asthma outcomes using electronic health record (EHR)-derived data. We explored these approaches in the recent literature, and examined the clarity of reporting.

We systematically searched for asthma-related articles published between 1-1-2014 and 31-122015, extracted the algorithms used to identify asthma patients and assess severity, control and exacerbations, and examined how the validity of these outcomes was justified.

From 113 eligible articles, we found significant heterogeneity in the algorithms used to define asthma ( $n=66$ different algorithms), severity $(n=18)$, control $(n=9)$, and exacerbations $(n=24)$. For the majority of algorithms $(n=106)$, validity was not justified. In the remaining cases, approaches ranged from using algorithms validated in the same databases, to using non-validated algorithms that were based on clinical judgement or clinical guidelines. The implementation of these algorithms was sub-optimally described overall.

Although EHR-derived data are now widely used to study asthma, the approaches being used are significantly varied and are often underdescribed, rendering it difficult to assess the validity of studies and compare their findings. Given the substantial growth in this body of literature, it is crucial that scientific consensus is reached on the underlying definitions and algorithms.
\end{abstract}

Keywords: Algorithms; asthma; electronic health records; quality of reporting; reproducibility. 


\section{Introduction}

Asthma is in clinical practice a diagnosis based on the patient history, examination and objective tests [1]. It is however increasingly considered to represent a heterogeneous group of disorders with different phenotypes and endotypes [2]. The clinical definitions of asthma and its key outcomes, including disease severity, control, and attacks/exacerbations have been the subject of vigorous debate [3-8].

Particular challenges arise in the context of epidemiologic studies where validated operational definitions are needed $[9,10]$. These studies are, increasingly, being undertaken using electronic health record (EHR)-derived data, which adds a further layer of complexity as the use of valid and reliable approaches is essential in order to ensure the reproducibility of research findings [11].

In order to assess current approaches, we systematically interrogated the recent EHR-based asthma literature. Our specific objectives were to: i) describe the different methods of defining asthma and assessing disease severity, control and exacerbations in EHR-based studies; ii) investigate whether authors reported on the validity of those methods; and iii) assess their reporting practices. 


\section{Methods}

We conducted a systematic scoping review based on Arksey and O'Malley's five-stage framework, including identifying the research question, identifying relevant studies, study selection, data charting and collating, summarising and reporting the results [12]. The research questions were: (1) How were asthma and its key outcomes defined using EHR data in the recent literature? (2) How did authors report on the validity of their EHR-based algorithms? (3) How clearly were the EHR-related methods reported?

\section{Eligibility criteria and search strategy}

We searched PubMed using a broad query (Table E1) to retrieve asthma studies that used EHRderived data and were published between January 1, 2014 and December 31, 2015. The search query was iteratively improved by adding many variations and equivalents of the keywords "EHR" and "routinely collected data" as well as named data sources found in the literature. Only articles written in English were included.

\section{Study selection}

We excluded non-relevant articles by reviewing titles and abstracts, referring to the full-text when needed. We included only articles where asthma was a main finding. For the purpose of this review, we limited the concept of EHR-derived data to coded, objective, individual-level data that were generated as a by-product of routine health care.

\section{Data extraction and synthesis}

From each of the eligible articles, we extracted and summarised information from the full text and online supplements, including basic bibliography, setting (country) and design; names and types of EHR-derived data sources used; algorithms to identify asthma patients, assess disease severity, control, exacerbation; and how authors reported on algorithm validity. In this context, we referred to 'validation' as any attempt to assess the algorithm's concurrent or construct validity. We used the RECORD Statement's 13-items checklist to assess the clarity of reporting of other EHR-related aspects such as clinical code lists used in the algorithms, and the implications of using EHR data in asthma research. The RECORD Statement is a recently introduced extension to the STROBE Statement which helps improve the reporting of observational studies conducted using routinely collected data [13]. Table E2 describes the data extraction and charting tool. Article screening and data extraction were performed independently by two authors (MAS and EV) with a third author arbitrating (GAD).

\section{Role of the funding sources}

The funding sources had no role in study design; in the collection, analysis, and interpretation of data; in the writing of the report; or in the decision to submit the paper for publication. 


\section{Results}

We included 113 articles in the review. Figure 1 shows the study selection process. Most studies were conducted in the United States (US), Taiwan, and Canada (Table E3), and employed longitudinal designs (Table E4). The most commonly used data types were health insurance claims followed by medical record repositories and dispensing databases (Table E5).

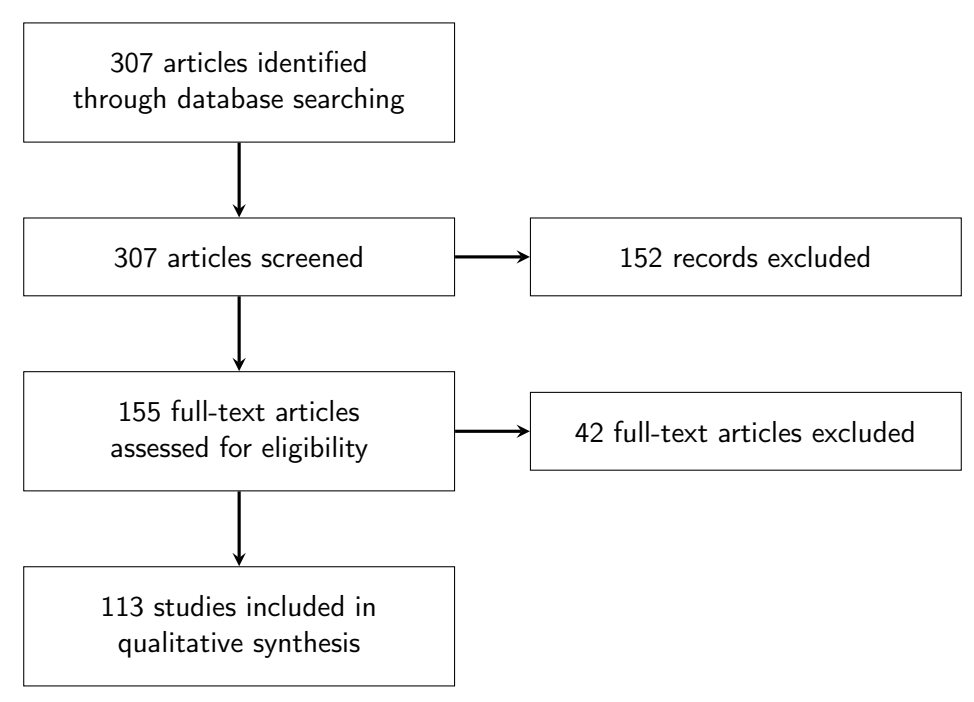

Figure 1: Flowchart for study selection in this scoping review.

\section{Defining asthma}

We identified 66 different algorithms to define asthma under seven diagnostic labels (Table E6).

'Persistent asthma' was defined over 12 and 24 months using the US Healthcare Effectiveness Data and Information Set (HEDIS) criteria [14], which involved assessing for any of the following asthma-related events: (1) emergency department (ED) visit, (2) hospitalisation, (3) outpatient visit and two asthma prescriptions, or (4) four asthma prescriptions [15-18]; by HEDIS criteria except "four asthma prescriptions" [19]; and by any asthma encounter (hospitalisation or ED visit) or using oral corticosteroids (OCS) for three or more days [20].

'Current asthma' was defined by any asthma encounter in the last three years [21].

'Current general practitioner (GP)-reported and diagnosed asthma' was defined as any asthma encounter in the last 12 months, and 'current GP-reported, diagnosed and treated asthma' as the same plus any asthma prescription in the same period [22].

Patients with treated asthma were otherwise required to have at least three dispensing events of asthma treatments in three different quarters of the year [23].

'Acute asthma' was defined using any asthma diagnosis codes in ED or inpatient data [24].

In the remaining studies, the label 'asthma' was defined using various algorithms, some of which were similar to those of the aforementioned more specific labels. 
The intervals over which asthma diagnostic/management and prescription codes where queried were specified in 31 and 8 studies, respectively. The positions of diagnostic codes in the encounter (i.e. primary or secondary) were specified in 37 studies.

We identified five approaches in these algorithms: requiring diagnostic/management events, prescription events, or both (Table E7). In addition, to exclude likely non-asthma patients, some studies applied additional non-asthma criteria to restrict the study population based on age (Table E8) and/or comorbidities (Table E9).

\section{Assessing asthma severity}

Eighteen studies used 20 different algorithms to assess asthma severity (Table E10), as binary (i.e. severe vs. non-severe asthma) [15, 23, 25-38] or ordinal variables (mild, moderate, and severe asthma [39]; or low, moderate, and high-risk asthma [40]). The algorithms were based on one or more of the following asthma-related variables: number and/or dosage of prescriptions - namely SABA, inhaled corticosteroids (ICS), OCS, and leukotriene receptor antagonist (LTRA) - and number of hospitalisations, ED and outpatient visits. Almost all algorithms (17) used prescriptions (either alone or with other variables), while one algorithm was based only on hospitalisations and ED visits [36]. The intervals over which asthma severity was assessed were three [29], six [38], 12 [15, 23, 28, 30-32, 34, 36, 37, 39, 40], 24 months [33, $35]$, or unclear [26, 27].

\section{Assessing asthma control}

Nine studies assessed asthma control using 11 algorithms, in 9 of which the interval was 12 months, in one 1-3 months, and in the remaining study this was unclear (Table E12). Uncontrolled asthma was defined by a minimum number/dose of SABA prescriptions [30, 31, 39, 41, 42]; any or short-course OCS prescriptions [30, 31, 41-44]; any hospitalisation or ED visit with either diagnosis of asthma [27, 30, 31, 41-43, 45] or — in already diagnosed asthma patients - diagnosis of status asthmaticus, pneumonia, dyspnoea, or respiratory insufficiency [30]; unscheduled outpatient visits for asthma or lower respiratory tract infections (LRTI) [31]; and GP consultations for LRTI requiring antibiotics in asthma patients [31]. Asthma impairment was defined based on the required SABA use, namely an average of more than two salbutamol puffs per day [31]. One study assessed asthma control based on number of OCS and SABA prescriptions per year (without giving any further details about the actual algorithm) [41].

\section{Defining exacerbations}

Twenty-four studies defined exacerbations using EHR-derived data (Table E11), as a dichotomous variable (absent vs. present) [16, 17, 23, 27, 30-32, 35, 37-39, 42-44, 46-54], or stratified into absent, moderate and severe [55]. Oral corticosteroid prescriptions were used as a marker for exacerbations in 17 studies, either alone [23, 30, 31, 35, 39, 42, 47, 48, 53] or with a concurrent asthma encounter (e.g., a GP, outpatient, or ED visit, or hospitalisation within five or seven days) $[16,17,32,37,38,46,52,54]$. In one study, exacerbations were defined by a minimum of six short-acting beta-2 agonist (SABA) prescriptions per year [47]. Other definitions included an outpatient code of 'asthma exacerbation' [52], asthma hospitalisation [23, 30, 32, 35, 37, 39, 43, 
Table 1: Practices of reporting or justifying the validity of algorithms to define and assess asthma using EHR-derived data.

\begin{tabular}{|c|c|c|c|c|c|}
\hline \multirow[t]{2}{*}{ Algorithm validity was justified by } & \multicolumn{5}{|c|}{ Number of algorithms } \\
\hline & $\begin{array}{l}\text { Identifying } \\
\text { asthma } \\
\text { pa- } \\
\text { tients }\end{array}$ & $\begin{array}{l}\text { Assessing } \\
\text { sever- } \\
\text { ity }\end{array}$ & $\begin{array}{l}\text { Assessing } \\
\text { control }\end{array}$ & $\begin{array}{l}\text { Defining } \\
\text { exacer- } \\
\text { bation }\end{array}$ & $\begin{array}{l}\text { Total } \\
\text { per } \\
\text { cate- } \\
\text { gory }\end{array}$ \\
\hline Validation of the same algorithm in the same database & 14 & 1 & 1 & 1 & 17 \\
\hline Validation of the same algorithm in different database(s) & 2 & 6 & 3 & 2 & 13 \\
\hline Validation of other diseases' algorithms in the same database & 2 & 0 & 0 & 0 & 2 \\
\hline Validation of other diseases' algorithms in different database(s) & 1 & 0 & 0 & 0 & 1 \\
\hline Being consistent with similar studies in the same database & 1 & 0 & 1 & 0 & 2 \\
\hline Being consistent with similar studies in different database(s) & 1 & 0 & 0 & 1 & 2 \\
\hline Validation or concordance analysis in the same study & 4 & 0 & 0 & 0 & 4 \\
\hline Being based on nationally developed algorithms & 3 & 0 & 0 & 2 & 5 \\
\hline Relying on the validity of database coding & 5 & 0 & 0 & 0 & 5 \\
\hline Being based on clinical guidelines & 0 & 3 & 0 & 0 & 3 \\
\hline Not justified & 76 & 8 & 4 & 18 & 106 \\
\hline
\end{tabular}

44, 46, 48, 50, 51, 53-55], asthma ED visit [16, 30-32, 35, 37, 38, 43, 44, 46, 48, 51-54], or hospitalisation with diagnosis of status asthmaticus, or - in already diagnosed asthma patients — diagnosis of pneumonia, dyspnoea, or respiratory insufficiency [30].

\section{Clarity of reporting}

Overall, the reporting of methodological aspects of using EHR-derived data was suboptimal. The majority of studies presented no information on the algorithms' validity. Among studies that reported on the validity, we identified 10 practices of reporting or justifying on the validity of algorithms (Table 1): (1) performing validation or concordance analysis in the same study against other measures based on different data sources (e.g., medical record review or patientreported measures); (2) referring to previous validation of similar algorithms in the same or (3) different databases; (4) referring to previous validation of similar algorithms for different diseases in the same or (5) different database (6); using algorithms 'consistent' with previous studies in the same or (7) different databases; (8) using nationally developed algorithms; (9) using algorithms based on clinical guidelines; (10) and relying on previous validation of the database content. Some studies did not provide clear algorithms for asthma severity or control, but only referred to their components [23, 35, 37, 38, 41].

Of the 113 reviewed studies, 40 studies used record-linkage, of which 17 mentioned it in the abstract, and 28 provided at least some explanation in the full text. The geographical region, time frame of data, and types or names of the data sources were mentioned in 83, 91, and 104 abstracts, respectively. Eighty-three studies reported their extent of access to the data sources. The intervals over which the algorithms were applied were often not reported. One hundred and eleven studies touched on the implications of using EHR data to study asthma. Of these, 64 and 63 studies discussed the risk of misclassification bias and unmeasured confounding, respectively. Six studies acknowledged the possible changes over time in data quality and coding practices and the entailing changes in case definition eligibility and accuracy. Five studies explained their data cleansing procedures. Finally, no study shared the programming codes of data preparation and analysis. 


\section{Discussion}

\section{Statement of principal findings}

This systematic analysis of the contemporaneous asthma literature has found evidence of considerable international activity in using EHR-derived data to study a variety of asthma populations and outcomes. Importantly, we also found wide variations in the approaches used with limited attention being paid to the validity of the underlying algorithms used and suboptimal reporting of studies. This poses a major challenge to the interpretation and reproducibility of this important, emerging body of research inquiry.

\section{Strengths and limitations}

To our knowledge, this is the first systematic exercise to investigate the quality of reporting on EHR-based studies, especially the validity of measures, in the context of asthma. In undertaking this work, we used robust approaches which involved two people independently selecting studies and undertaking data extraction. The findings may also apply to other chronic diseases. This review had no geographic limits, but it was confined to assessing the recent literature. Examining the most recent asthma literature is most likely to provide meaningful insights on current practices. A limitation is that we did not systematically check whether the references provided to support the claimed validity of algorithms in question actually provided sufficient evidence of validity. For example, differences might exist between the algorithms used in a given study and those previously validated.

\section{Interpretation in the light of previous studies}

Although EHR-derived data are convenient resources for research, they are originally collected for other purposes, and usually suffer from missing or incorrect data and potential biases [56-58]. In addition, EHR systems usually fail to capture complete and accurate clinical information at the point of care due to design limitations and inefficient use of these systems by clinicians to document clinical data $[59,60]$.

These issues impose challenges on their use to assess a complex and heterogeneous condition such as asthma. For example, asthma diagnosis codes, which are commonly used solely for patient identification, may be recorded after a trial or wrong diagnosis, and do not capture undiagnosed patients [61]. In addition, many EHR-derived databases often lack important variables, such as lung function, indication of dispensed medications, adherence to treatment, and lifestyle, which are vital for identifying and assessing asthma patients. These challenges are however not insurmountable. In this review, we found several techniques intended to improve algorithm accuracy such as age limitation, comorbidity exclusion, and diagnosis position restriction.

Ideally, algorithms should be validated in the databases in which they are used. However, this was often not the case. Instead, using algorithms with only reasonable face validity based on clinical guidelines or clinical judgement is a very common practice in EHR-based studies 
$[62,63]$. These approaches assume that clinical codes in the database accurately represent the patient's actual health care events [62].

Under-reporting on implementation details and methods' validity compromises transparency and reproducibility, a crucial issue in medical research. It has been previously found that in EHR-based studies, full lists of clinical codes were often not reported [64]. A recent, large-scale reproducibility exercise identified similar challenges due to suboptimal reporting of EHR-based studies, particularly sharing code lists and algorithms [65].

The significant methodological heterogeneity we found in EHR-based asthma assessment algorithms reflects, in addition to the content differences between the databases used, the lack of consensus on the clinical definitions in the first place despite continuous standardisation efforts $[5,6,66,67]$. The focus of our work was to examine asthma definitions and their validity specifically in the context of EHR, but this highlights the fundamental need to reach consensus on clinical asthma definitions and the appropriate validation of asthma diagnosis. For example, there is still an active debate on whether lung function is essential to establish asthma diagnosis $[7,8]$. A recent study also found significant variation in algorithms to assess asthma severity from health insurance data [68]. Unjustified inter-study variation in the operational definitions of the same clinical concepts creates challenges for comparability, meta-analysis and evidence synthesis. These issues have been raised for asthma [69] and other allergic conditions such as peanut allergy [70, 71] and anaphylaxis [72], where wide variations in findings were potentially attributed to inconsistent case definitions.

\section{Implications for policy, practice and research}

This review sheds light on the opportunities offered by the increasingly ubiquitous EHRs, but also highlights considerable heterogeneity and suboptimal reporting of EHR-based asthma assessment algorithms and the implications of these practices on comparability and reproducibility of studies.

Developing reliable algorithms to assess asthma outcomes using EHR data is a non-trivial challenge. In addition, standardising such algorithms across different populations may be impractical since databases differ in content, validity may not hold across different populations, and no best practice currently exists [68]. Similar challenges arise when comparing asthma epidemiology between multiple populations [73]. These methodologic issues, in addition to suboptimal reporting, should be considered when interpreting and synthesising evidence from geographically dispersed studies.

With the accelerating availability of EHR-derived data and their use to study asthma, we believe that consideration needs to be given to convening an international task force to work on the harmonisation of those algorithms under uniform and consistent clinical labels, while considering the differences between populations and databases. In addition, validation of these algorithms in the respective populations should be given a high priority. Furthermore, to allow more accurate assessment of asthma from EHR data, efforts are needed to improve the capture and coding of asthma-related data at the point of care [74] which requires more efficient EHR systems [59, 60]. In addition, emerging data sources such as patient-generated data and wearables need to be harnessed [75]. Finally, to improve the clarity of reporting on EHR-related methodological aspects, we strongly advocate the adoption of the RECORD Statement as an extension of the STROBE Statement by both authors and journal editors [13]. Optimal reporting should include complete code lists, detailed algorithms and validity assessment. Implications of using EHR- 
derived data to study a complex condition such as asthma should be clearly communicated to enable judgement of internal and external validity.

In summary, we have found that there is considerable international interest in exploiting EHRderived data to study asthma, but that there are considerable variations in the approaches used. These variations are compounded by sub-optimal reporting of methods, which makes it difficult to assess the reproducibility of research. Given the substantial investments taking place in EHRs globally, this body of work is likely to grow significantly in the coming years. It is therefore important that the asthma-interested research community works to place it on a solid footing in order to ensure the quality and reproducibility of this work. 


\section{Authors' contributions}

MAS, SER and GAD, AS, and RAL developed the concept and methods. MAS conducted the literature search. MAS and EV independently reviewed the studies with GAD arbitrating. All authors contributed to the development of methods, interpretation of findings, and manuscript writing, and critically reviewed and approved the final manuscript.

\section{Conflict of Interest Statement}

Aziz Sheikh reports grants from Asthma UK during the conduct of the study.

Support statement: This work was funded by Health and Care Research Wales and Abertawe Bro Morgannwg University Health Board. It was carried out with the support of the Asthma UK Centre for Applied Research [AUK-AC-2012-01]. We also acknowledge the support from The Farr Institute of Health Informatics Research. The Farr Institute is supported by a 10funder consortium: Arthritis Research UK, the British Heart Foundation, Cancer Research UK, the Economic and Social Research Council, the Engineering and Physical Sciences Research Council, the Medical Research Council, the National Institute of Health Research, the National Institute for Social Care and Health Research (Welsh Assembly Government), the Chief Scientist Office (Scottish Government Health Directorates), the Wellcome Trust, (MRC Grant Nos: CIPHER MR/K006525/1, Scotland MR/K007017/1).

\section{References}

[1] Global Initiative for Asthma. Global Strategy for Asthma Management and Prevention (2015 update). 2015.

[2] Wenzel SE. Asthma phenotypes: the evolution from clinical to molecular approaches. Nat Med 2012;18: 716725 .

[3] Hargreave FE and Nair P. The definition and diagnosis of asthma. Clin Exp Allergy 2009;39: 1652-1658.

[4] . A plea to abandon asthma as a disease concept. Lancet 2006;368: 705.

[5] Bousquet J, Mantzouranis E, Cruz AA, A1 t-Khaled N, Baena-Cagnani CE, Bleecker ER, et al. Uniform definition of asthma severity, control, and exacerbations: document presented for the World Health Organization Consultation on Severe Asthma. J Allergy Clin Immunol 2010;126: 926-938.

[6] Reddel HK, Bateman ED, Becker A, Boulet LP, Cruz AA, Drazen JM, et al. A summary of the new GINA strategy: a roadmap to asthma control. Eur Respir J 2015;46: 622-639.

[7] Akker ILv d., Zeijden $\mathrm{H}$ van der, and Verheij TJ. Is spirometry essential in diagnosing asthma? Yes. $\mathrm{Br} J$ Gen Pract 2016;66: 484-484.

[8] Levy ML. Is spirometry essential in diagnosing asthma? No. Br J Gen Pract 2016;66: 485-485.

[9] Toelle BG, Peat JK, Salome CM, Mellis CM, and Woolcock AJ. Toward a Definition of Asthma for Epidemiology. Am Rev Respir Dis 1992;146: 633-637.

[10] Pekkanen J and Pearce N. Defining asthma in epidemiological studies. Eur Respir J 1999;14: 951-957.

[11] Ioannidis JP. Why Most Published Research Findings Are False. PLoS Medicine 2005;2:e124.

[12] Arksey H and O'Malley L. Scoping studies: towards a methodological framework. Int J Soc Res Methodol 2005;8: 19-32.

[13] Benchimol EI, Smeeth L, Guttmann A, Harron K, Moher D, Petersen I, et al. The REporting of studies Conducted using Observational Routinely-collected health Data (RECORD) Statement. PLoS Med 2015;12:e1001885.

[14] Use of appropriate medications for people with asthma. HEDIS 2003. Vol. 2. Washington, DC: National Committee for Quality Assurance, 2003, 25-28.

[15] Wu CL, Andrews AL, Teufel RJ, and Basco WT. Demographic predictors of leukotriene antagonist monotherapy among children with persistent asthma. J. Pediatr. 2014;164:827-831.e1.

[16] Zeiger RS, Schatz M, Li Q, Chen W, Khatry DB, Gossage D, et al. High blood eosinophil count is a risk factor for future asthma exacerbations in adult persistent asthma. J Allergy Clin Immunol Pract 2014;2: 741-50.

[17] Schatz M, Zeiger RS, Yang SJ, Chen W, Crawford W, Sajjan S, et al. Change in asthma control over time: predictors and outcomes. J Allergy Clin Immunol Pract ;2: 59-64. 
[18] Jena AB, Ho O, Goldman DP, and Karaca-Mandic P. The Impact of the US Food and Drug Administration Chlorofluorocarbon Ban on Out-of-pocket Costs and Use of Albuterol Inhalers Among Individuals With Asthma. JAMA Intern Med 2015;175: 1171-9.

[19] McRoy L, Weech-Maldonado R, and Kilgore M. The relationship between direct to consumer advertising (DTCA) and asthma-related emergency department use among Medicaid-enrolled children. J Asthma 2014;51: 922-6.

[20] Wu AC, Butler MG, Li L, Fung V, Kharbanda EO, Larkin EK, et al. Primary adherence to controller medications for asthma is poor. Ann Am Thorac Soc 2015;12: 161-6.

[21] Tomasallo CD, Hanrahan LP, Tandias A, Chang TS, Cowan KJ, and Guilbert TW. Estimating Wisconsin asthma prevalence using clinical electronic health records and public health data. Am J Public Health 2014;104:e65-73.

[22] Mukherjee M, Gupta R, Farr A, Heaven M, Stoddart A, Nwaru BI, et al. Estimating the incidence, prevalence and true cost of asthma in the UK: secondary analysis of national stand-alone and linked databases in England, Northern Ireland, Scotland and Wales-a study protocol. BMJ Open 2014;4:e006647.

[23] Laforest L, Licaj I, Devouassoux G, Chatte G, Martin J, and Ganse EV. Asthma drug ratios and exacerbations: claims data from universal health coverage systems. Eur. Respir. J. 2014;43: 1378-86.

[24] Lemke LD, Lamerato LE, Xu X, Booza JC, Reiners JJ, Iii DMR, et al. Geospatial relationships of air pollution and acute asthma events across the Detroit-Windsor international border: study design and preliminary results. J Expo Sci Environ Epidemiol 2014;24: 346-57.

[25] Jian ZH, Huang JY, Lin FCF, Nfor ON, Jhang KM, Ku WY, et al. The use of corticosteroids in patients with COPD or asthma does not decrease lung squamous cell carcinoma. BMC Pulm Med 2015;15: 154.

[26] Garne E, Hansen AV, Morris J, Zaupper L, Addor MC, Barisic I, et al. Use of asthma medication during pregnancy and risk of specific congenital anomalies: A European case-malformed control study. J Allergy Clin Immunol 2015;136:1496-502.e1-7.

[27] Tan NC, Nadkarni NV, Lye WK, Sankari U, et al. Ten-year longitudinal study of factors influencing nocturnal asthma symptoms among Asian patients in primary care. NPJ Prim Care Respir Med 2015;25: 15064.

[28] Kenyon CC, Rubin DM, Zorc JJ, Mohamad Z, Faerber JA, and Feudtner C. Childhood Asthma Hospital Discharge Medication Fills and Risk of Subsequent Readmission. J. Pediatr. 2015;166: 1121-7.

[29] Rust G, Zhang S, Holloway K, and Tyler-Hill Y. Timing of emergency department visits for childhood asthma after initial inhaled corticosteroid use. Popul Health Manag 2015;18: 54-60.

[30] Bülow A von, Kriegbaum M, Backer V, and Porsbjerg C. The prevalence of severe asthma and low asthma control among Danish adults. J Allergy Clin Immunol Pract 2014;2: 759-67.

[31] Martin RJ, Price D, Roche N, Israel E, Aalderen WMC van, Grigg J, et al. Cost-effectiveness of initiating extrafine- or standard size-particle inhaled corticosteroid for asthma in two health-care systems: a retrospective matched cohort study. NPJ Prim Care Respir Med 2014;24: 14081.

[32] Schatz M, Meckley LM, Kim M, Stockwell BT, and Castro M. Asthma exacerbation rates in adults are unchanged over a 5-year period despite high-intensity therapy. J Allergy Clin Immunol Pract 2014;2:5704.e1.

[33] Capo-Ramos DE, Duran C, Simon AE, Akinbami LJ, and Schoendorf KC. Preventive asthma medication discontinuation among children enrolled in fee-for-service Medicaid. J Asthma 2014;51: 618-26.

[34] Nordlund B, Melén E, Schultz ES, Grönlund H, Hedlin G, and Kull I. Prevalence of severe childhood asthma according to the WHO. Respir Med 2014;108: 1234-7.

[35] Ismaila A, Corriveau D, Vaillancourt J, Parsons D, Stanford R, Su Z, et al. Impact of adherence to treatment with fluticasone propionate/salmeterol in asthma patients. Curr Med Res Opin 2014;30: 1417-25.

[36] Fung V, Graetz I, Galbraith A, Hamity C, Huang J, Vollmer WM, et al. Financial barriers to care among low-income children with asthma: health care reform implications. JAMA Pediatr 2014;168: 649-56.

[37] Dilokthornsakul P, Chaiyakunapruk N, Schumock GT, and Lee TA. Calendar time-specific propensity score analysis for observational data: a case study estimating the effectiveness of inhaled long-acting beta-agonist on asthma exacerbations. Pharmacoepidemiol Drug Saf 2014;23: 152-64.

[38] Adimadhyam S, Schumock GT, Walton S, Joo M, McKell J, and Lee TA. Risk of arrhythmias associated with ipratropium bromide in children, adolescents, and young adults with asthma: a nested case-control study. Pharmacotherapy 2014;34: 315-23.

[39] Blais L, Kettani FZ, and Forget A. Associations of maternal asthma severity and control with pregnancy complications. J Asthma 2014;51: 391-8.

[40] Chang J, Freed GL, Prosser LA, Patel I, Erickson SR, Bagozzi RP, et al. Comparisons of health care utilization outcomes in children with asthma enrolled in private insurance plans versus medicaid. $J$ Pediatr Health Care 2014;28: 71-9.

[41] Sullivan PW, Campbell JD, Ghushchyan VH, and Globe G. Outcomes before and after treatment escalation to Global Initiative for Asthma steps 4 and 5 in severe asthma. Ann. Allergy Asthma Immunol. 2015;114: 462-9.

[42] Ali AK, Hartzema AG, Winterstein AG, Segal R, Lu X, and Hendeles L. Application of multicategory exposure marginal structural models to investigate the association between long-acting beta-agonists and prescribing of oral corticosteroids for asthma exacerbations in the Clinical Practice Research Datalink. Value Health 2015;18: 260-70. 
[43] Wu AC, Li L, Fung V, Kharbanda EO, Larkin EK, Vollmer WM, et al. Use of leukotriene receptor antagonists are associated with a similar risk of asthma exacerbations as inhaled corticosteroids. $J$ Allergy Clin Immunol Pract ;2: 607-13.

[44] Tan CC, McDowell KM, Fenchel M, Szczesniak R, and Kercsmar CM. Spirometry use in children hospitalized with asthma. Pediatr. Pulmonol. 2014;49: 451-7.

[45] Keast SL, Thompson D, Farmer K, Smith M, Nesser N, and Harrison D. Impact of a prior authorization policy for montelukast on clinical outcomes for asthma and allergic rhinitis among children and adolescents in a state Medicaid program. J Manag Care Spec Pharm 2014;20: 612-21.

[46] Kim S, Kim J, Park SY, Um HY, Kim K, Kim Y, et al. Effect of pregnancy in asthma on health care use and perinatal outcomes. J Allergy Clin Immunol 2015;136:1215-23.e1-6.

[47] Confino-Cohen R, Brufman I, Goldberg A, and Feldman BS. Vitamin D, asthma prevalence and asthma exacerbations: a large adult population-based study. Allergy 2014;69: 1673-80.

[48] Tunceli O, Williams SA, Kern DM, Elhefni H, Pethick N, Wessman C, et al. Comparative effectiveness of budesonide-formoterol combination and fluticasone-salmeterol combination for asthma management: a United States retrospective database analysis. J Allergy Clin Immunol Pract 2014;2: 719-26.

[49] Bhattacharjee R, Choi BH, Gozal D, and Mokhlesi B. Association of adenotonsillectomy with asthma outcomes in children: a longitudinal database analysis. PLoS Med. 2014;11:e1001753.

[50] Nanchal R, Kumar G, Majumdar T, Taneja A, Patel J, Dagar G, et al. Utilization of mechanical ventilation for asthma exacerbations: analysis of a national database. Respir Care 2014;59: 644-53.

[51] Tse SM, Charland SL, Stanek E, Herrera V, Goldfarb S, Litonjua AA, et al. Statin use in asthmatics on inhaled corticosteroids is associated with decreased risk of emergency department visits. Curr Med Res Opin 2014;30: 685-93.

[52] Sumino K, O’Brian K, Bartle B, Au DH, Castro M, and Lee TA. Coexisting chronic conditions associated with mortality and morbidity in adult patients with asthma. J Asthma 2014;51: 306-14.

[53] Li L, Vollmer WM, Butler MG, Wu P, Kharbanda EO, and Wu AC. A comparison of confounding adjustment methods for assessment of asthma controller medication effectiveness. Am. J. Epidemiol. 2014;179: 648-59.

[54] Hagiwara M, Delea TE, and Stanford RH. Health-care utilization and costs with fluticasone propionate and fluticasone propionate/salmeterol in asthma patients at risk for exacerbations. Allergy Asthma Proc 2014;35: 54-62.

[55] Blais L, Kettani FZ, Forget A, Beauchesne MF, and Lemière C. Asthma exacerbations during the first trimester of pregnancy and congenital malformations: revisiting the association in a large representative cohort. Thorax 2015;70: 647-52.

[56] Schneeweiss S and Avorn J. A review of uses of health care utilization databases for epidemiologic research on therapeutics. J Clin Epidemiol 2005;58: 323-337.

[57] Jorm L. Routinely collected data as a strategic resource for research: priorities for methods and workforce. 2015;::

[58] Hemkens LG, Contopoulos-Ioannidis DG, and Ioannidis JPA. Routinely collected data and comparative effectiveness evidence: promises and limitations. Can Med Assoc J 2016;:.

[59] Sheikh A, Cornford T, Barber N, Avery A, Takian A, Lichtner V, et al. Implementation and adoption of nationwide electronic health records in secondary care in England: final qualitative results from prospective national evaluation in "early adopter" hospitals. BMJ 2011;343: d6054-d6054.

[60] Frenkel LD. Electronic health records-Applications for the allergist/immunologist: All that glitters is not gold. Allergy Asthma Proc 2016;37: 273-278.

[61] Huzel L, Roos LL, Anthonisen NR, and Manfreda J. Diagnosing asthma: the fit between survey and administrative database. Can Respir J 2002;9: 407-412.

[62] Manuel DG, Rosella LC, and Stukel TA. Importance of accurately identifying disease in studies using electronic health records. BMJ 2010;341: c4226.

[63] Shivade C, Raghavan P, Fosler-Lussier E, Embi PJ, Elhadad N, Johnson SB, et al. A review of approaches to identifying patient phenotype cohorts using electronic health records. J Am Med Inform Assoc 2014;21: 221-230.

[64] Springate DA, Kontopantelis E, Ashcroft DM, Olier I, Parisi R, Chamapiwa E, et al. ClinicalCodes: an online clinical codes repository to improve the validity and reproducibility of research using electronic medical records. PLoS One 2014;9:e99825.

[65] Wang SV, Verpillat P, Rassen JA, Patrick A, Garry EM, and Bartels DB. Transparency and Reproducibility of Observational Cohort Studies Using Large Healthcare Databases. Clin Pharmacol Ther 2016;99: 325332.

[66] Bel EH, Sousa A, Fleming L, Bush A, Chung KF, Versnel J, et al. Diagnosis and definition of severe refractory asthma: an international consensus statement from the Innovative Medicine Initiative (IMI). Thorax 2011;66: 910-917.

[67] Chung KF, Wenzel SE, Brozek JL, Bush A, Castro M, Sterk PJ, et al. International ERS/ATS guidelines on definition, evaluation and treatment of severe asthma. Eur Respir J 2014;43: 343-373.

[68] Jacob C, Haas JS, Bechtel B, Kardos P, and Braun S. Assessing asthma severity based on claims data: a systematic review. Eur J Health Econ 2016;: 
[69] Ford ES. The epidemiology of obesity and asthma. J Allergy Clin Immunol: In Practice 2005;115:897-909, quiz 910.

[70] Kotz D, Simpson CR, and Sheikh A. Incidence, prevalence, and trends of general practitioner-recorded diagnosis of peanut allergy in England, 2001 to 2005. J Allergy Clin Immunol 2011;127:623-630.e1.

[71] Custovic A and Nicolaou N. Peanut allergy: overestimated in epidemiology or underdiagnosed in primary care? J Allergy Clin Immunol: In Practice 2011;127: 631-632.

[72] Panesar S, Javad S, Silva Dd, Nwaru B, Hickstein L, Muraro A, et al. The epidemiology of anaphylaxis in Europe: a systematic review. Allergy 2013;68: 1353-1361.

[73] Nwaru BI, Mukherjee M, Gupta RP, Farr A, Heaven M, Stoddart A, et al. Challenges of harmonising data from UK national health surveys: a case study of attempts to estimate the UK prevalence of asthma. $J R$ Soc Med 2015;:

[74] Mukherjee M, Wyatt JC, Simpson CR, and Sheikh A. Usage of allergy codes in primary care electronic health records: a national evaluation in Scotland. Allergy 2016;:.

[75] Howie L, Hirsch B, Locklear T, and Abernethy AP. Assessing The Value Of Patient-Generated Data To Comparative Effectiveness Research. Health Aff (Millwood) 2014;33: 1220-1228. 


\section{Appendix B}

\section{Chapter 3 Appendix}

\section{B.1 Making sense of patient-reported currently treated asthma using routinely collected data}

Mohammad Al Sallakh, ${ }^{1}$ Sarah Rodgers, ${ }^{1}$ Ronan Lyons, ${ }^{1}$ Aziz Sheikh, ${ }^{2}$ Gwyneth Davies. ${ }^{1}$ P148 Making sense of patient-reported currently treated asthma using routinely collected data. Thorax 2016;71:A163-A164.

${ }^{1}$ Medical School, Swansea University, Swansea, UK; ${ }^{2}$ Usher Institute of Population Health Sciences and Informatics, The University of Edinburgh, Edinburgh, UK

An abstract presented at British Thoracic Society Winter Meeting 2016.

Background: Currently treated asthma (CTA) is commonly assessed in epidemiological studies and is typically self-reported.

Aims: To investigate how patient understanding of this label compared with objective measures from routinely collected data.

Methods: We obtained the valid CTA responses of individuals aged 16+ from the Welsh Health Survey 2014, who also had linked records in the GP dataset of the Secure Anonymised Information Linkage databank and complete GP registrations between 2009-2014. We queried their recent prescriptions and whether they had ever asthma diagnosis. We examined the concordance between self-reported CTA and each of 'ever prescriptions', 'ever diagnosis', and 'prescriptions in varying backward intervals from mid-2014', with the latter repeated by adding 'ever diagnosis'.

Results: Of 4,291 eligible people, $10.2 \%$ self-reported CTA, of these $11.2 \%$ and $22.4 \%$ had no prescriptions in the past 12 months and no recorded asthma diagnosis ever. For concordance between self-reported CTA and each of 'ever prescrip- 
tions' and 'ever diagnosis', Cohen's kappa was 0.42 and 0.68. For concordance between self-reported CTA and 'prescriptions in backward intervals', kappa was 0.76 for the 12-month interval but peaked to 0.77 at 9 -months. After adding 'ever diagnosis', the kappa became 0.78 for the 12-month measure (which represents the treated asthma criteria of the Quality of Outcomes Framework, QOF), and peaked to 0.79 at 18 -months.

Conclusions: In Wales, self-reported CTA agreed well with the QOF treated asthma criteria, but slightly better with 'any prescriptions in last 18 months and ever diagnosis'. However, the concordance remains suboptimal, demonstrating that objective measures from routinely collected data are preferred over self-reported CTA.

Funding: Health and Care Research Wales and ABMU Health Board. Supported by Asthma UK Centre for Applied Research (AUK-AC-2012-01) and the Farr Institute @ CIPHER. 


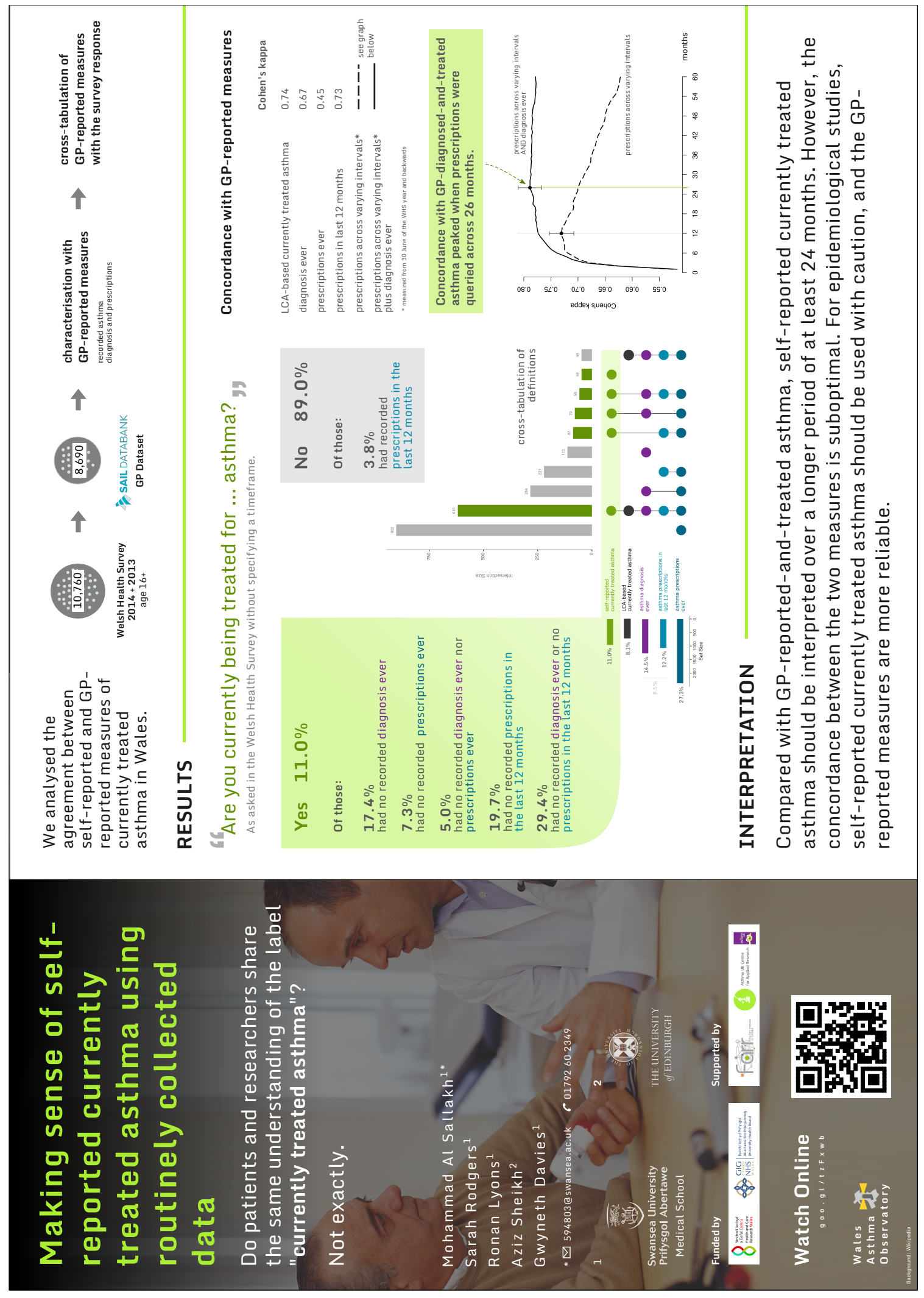




\section{B.2 Read Codes sets used to define the observed variables in the latent class analysis}

Table B.2.1: Read Codes sets used to define the observed variables in the latent class modelling in Chapter 3.

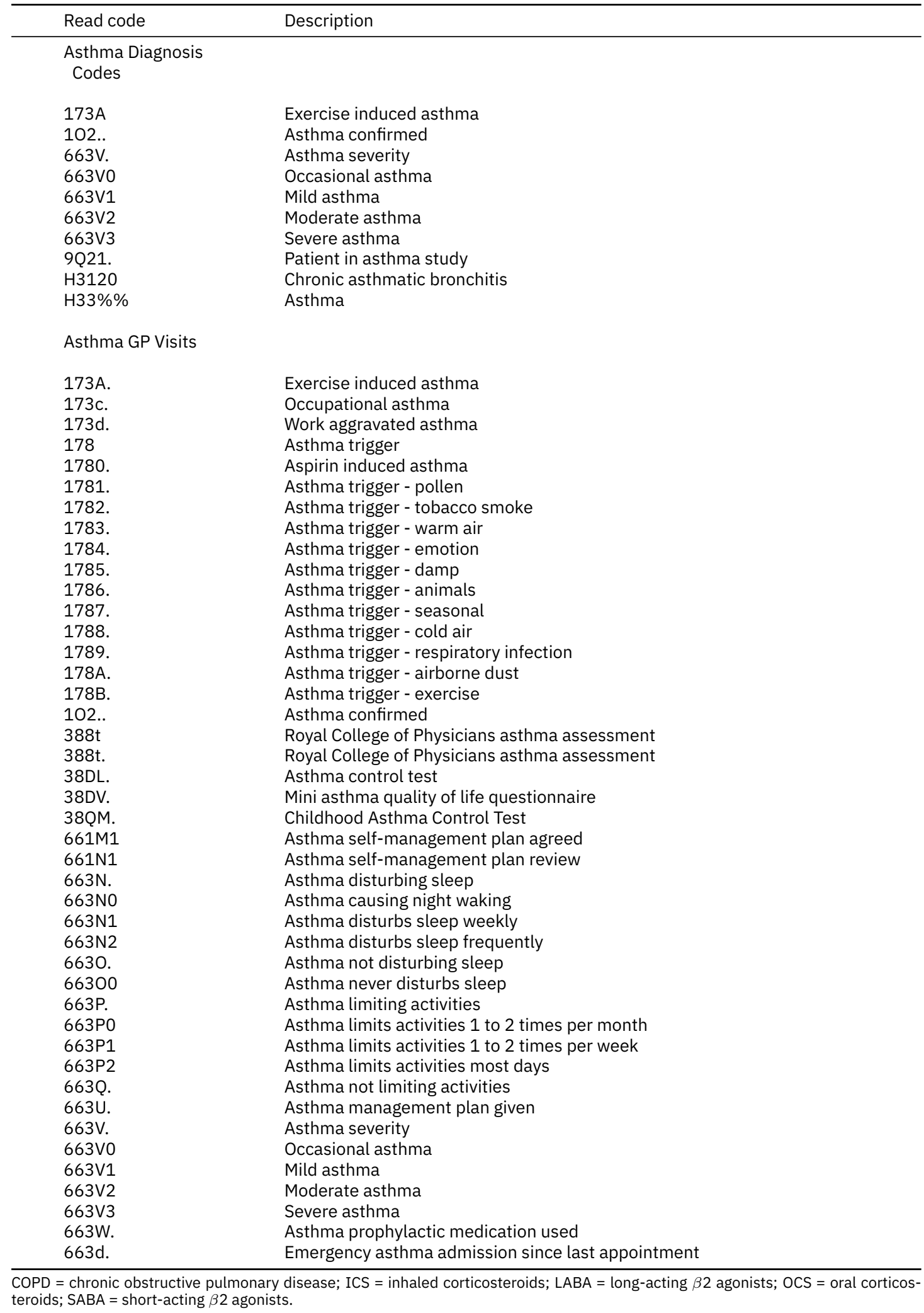


Table B.2.1: Read Codes sets used to define the observed variables in the latent class modelling in Chapter 3. (cont'd).

\begin{tabular}{|c|c|}
\hline Read code & Description \\
\hline $663 e$. & Asthma restricts exercise \\
\hline $663 e 0$ & Asthma sometimes restricts exercise \\
\hline $663 e 1$ & Asthma severely restricts exercise \\
\hline $663 f$. & Asthma never restricts exercise \\
\hline 663h. & Asthma - currently dormant \\
\hline 663j. & Asthma - currently active \\
\hline $663 \mathrm{~m}$. & Asthma accident and emergency attendance since last visit \\
\hline $663 n$. & Asthma treatment compliance satisfactory \\
\hline 663p. & Asthma treatment compliance unsatisfactory \\
\hline $663 q$. & Asthma daytime symptoms \\
\hline $663 r$. & Asthma causes night symptoms 1 to 2 times per month \\
\hline 663s. & Asthma never causes daytime symptoms \\
\hline $663 t$. & Asthma causes daytime symptoms 1 to 2 times per month \\
\hline $663 u$. & Asthma causes daytime symptoms 1 to 2 times per week \\
\hline $663 v$. & Asthma causes daytime symptoms most days \\
\hline $663 w$. & Asthma limits walking up hills or stairs \\
\hline $663 x$ & Asthma limits walking on the flat \\
\hline 663y. & Number of asthma exacerbations in past year \\
\hline $66 Y 5$. & Change in asthma management plan \\
\hline $66 Y 9$. & Step up change in asthma management plan \\
\hline 66YA. & Step down change in asthma management plan \\
\hline 66YC. & Absent from work or school due to asthma \\
\hline 66YE. & Asthma monitoring due \\
\hline 66YJ. & Asthma annual review \\
\hline 66YK. & Asthma follow-up \\
\hline 66YP. & Asthma night-time symptoms \\
\hline 66YQ. & Asthma monitoring by nurse \\
\hline 66YR. & Asthma monitoring by doctor \\
\hline $66 \mathrm{YZ}$. & Does not have asthma management plan \\
\hline 66Yp. & Asthma review using Royal College of Physicians three questions \\
\hline 66Yq. & Asthma causes night time symptoms 1 to 2 times per week \\
\hline $66 \mathrm{Yr}$. & Asthma causes symptoms most nights \\
\hline 66Ys. & Asthma never causes night symptoms \\
\hline $66 \mathrm{Yu}$. & Number of days absent from school due to asthma in past 6 months \\
\hline $679 J$. & Health education - asthma \\
\hline $679 J 0$ & Health education - asthma self management \\
\hline $679 J 1$ & Health education - structured asthma discussion \\
\hline $679 J 2$ & Health education - structured patient focused asthma discussion \\
\hline 8791. & Further asthma - drug prevent. \\
\hline 8793. & Asthma control step 0 \\
\hline 8794. & Asthma control step 1 \\
\hline 8795. & Asthma control step 2 \\
\hline 8796. & Asthma control step 3 \\
\hline 8797. & Asthma control step 4 \\
\hline 8798. & Asthma control step 5 \\
\hline 8B3j. & Asthma medication review \\
\hline 8СМА० & Patient has a written asthma personal action plan \\
\hline 8CRO. & Asthma clinical management plan \\
\hline $8 \mathrm{H} 2 \mathrm{P}$. & Emergency admission, asthma \\
\hline 8HTT. & Referral to asthma clinic \\
\hline 9N1d. & Seen in asthma clinic \\
\hline 9N1d0 & Seen in school asthma clinic \\
\hline 9NI8. & Asthma outreach clinic \\
\hline 9NNX. & Under care of asthma specialist nurse \\
\hline 90J.. & Asthma monitoring admin. \\
\hline $90 J 1$. & Attends asthma monitoring \\
\hline $90 J 2$. & Refuses asthma monitoring \\
\hline $90 J 3$. & Asthma monitor offer default \\
\hline $90 J 4$. & Asthma monitor 1st letter \\
\hline $90 J 5$. & Asthma monitor 2 nd letter \\
\hline $90 J 6$. & Asthma monitor 3rd letter \\
\hline $90 J 7$. & Asthma monitor verbal invite \\
\hline $90 J 8$. & Asthma monitor phone invite \\
\hline $90 J 9$. & Asthma monitoring deleted \\
\hline 90JA. & Asthma monitoring check done \\
\hline 90JB. & Asthma monitoring invitation SMS (short message service) text message \\
\hline 9OJC. & Asthma monitoring invitation email \\
\hline
\end{tabular}

COPD = chronic obstructive pulmonary disease; ICS = inhaled corticosteroids; LABA = long-acting $\beta 2$ agonists; OCS = oral corticosteroids; $\mathrm{SABA}=$ short-acting $\beta 2$ agonists. 
Table B.2.1: Read Codes sets used to define the observed variables in the latent class modelling in Chapter 3. (cont'd).

\begin{tabular}{|c|c|}
\hline Read code & Description \\
\hline $90 J z$. & Asthma monitoring admin.NOS \\
\hline 9Q21. & Patient in asthma study \\
\hline 9hA.. & Exception reporting: asthma quality indicators \\
\hline 9hA1. & Excepted from asthma quality indicators: Patient unsuitable \\
\hline SLF7. & Antiasthmatic poisoning \\
\hline SLF7z & Antiasthmatic poisoning NOS \\
\hline \multicolumn{2}{|c|}{ COPD Diagnosis Codes } \\
\hline H3... & Chronic obstructive pulmonary disease \\
\hline H31 & Chronic bronchitis \\
\hline H32 & Emphysema \\
\hline H36 & Mild chronic obstructive pulmonary disease \\
\hline H37 & Moderate chronic obstructive pulmonary disease \\
\hline H38 & Severe chronic obstructive pulmonary disease \\
\hline H39 & Very severe chronic obstructive pulmonary disease \\
\hline $\mathrm{H} 3 \mathrm{~A}$ & End stage chronic obstructive airways disease \\
\hline H3y & Other specified chronic obstructive airways disease \\
\hline$H 3 z$ & Chronic obstructive airways disease NOS \\
\hline H4640 & Chronic emphysema due to chemical fumes \\
\hline H4641 & Obliterative bronchiolitis due to chemical fumes \\
\hline H5832 & Eosinophilic bronchitis \\
\hline Hyu30 & {$[\mathrm{X}]$ Other emphysema } \\
\hline Hyu31 & [X]Other specified chronic obstructive pulmonary disease \\
\hline H3101 & Smokers' cough \\
\hline H31y0 & Chronic tracheitis \\
\hline \multicolumn{2}{|c|}{ COPD GP Visits } \\
\hline 66YL. & Chronic obstructive pulmonary disease follow-up \\
\hline 66YS. & Chronic obstructive pulmonary disease monitoring by nurse \\
\hline $66 \mathrm{YT}$. & Chronic obstructive pulmonary disease monitoring by doctor \\
\hline $66 Y B$ & Chronic obstructive pulmonary disease monitoring \\
\hline $66 \mathrm{YM}$ & Chronic obstructive pulmonary disease annual review \\
\hline $90 i$ & Chronic obstructive pulmonary disease monitoring administration \\
\hline \multicolumn{2}{|c|}{ SABA Inhalers } \\
\hline c11 & SALBUTAMOL [ORAL PREPARATIONS] \\
\hline c12 & SALBUTAMOL [PARENTERAL PREPARATIONS] \\
\hline c13 & SALBUTAMOL [INHALATION PREPRATIONS] \\
\hline c14 & TERBUTALINE SULPHATE [RESPIRATORY USE] \\
\hline c15 & FENOTEROL HYDROBROMIDE \\
\hline c1E & SALBUTAMOL [INHALATION PREPRATIONS 2] \\
\hline \multicolumn{2}{|l|}{ OCS } \\
\hline fe6 & PREDNISOLONE [ENDOCRINE] \\
\hline fe61. & PREDNISOLONE $1 \mathrm{mg}$ tablets \\
\hline fe62. & PREDNISOLONE 5mg tablets \\
\hline fe64. & *DELTA-PHORICOL $5 \mathrm{mg}$ tablets \\
\hline fe65. & DELTACORTRIL ENTERIC $2.5 \mathrm{mg}$ tablets \\
\hline fe66. & DELTACORTRIL ENTERIC 5mg tablets \\
\hline fe67. & *DELTALONE $1 \mathrm{mg}$ tablets \\
\hline fe68. & *DELTALONE $5 \mathrm{mg}$ tablets \\
\hline fe69. & *DELTASTAB 1mg tablets \\
\hline fe6a. & *DELTASTAB $5 \mathrm{mg}$ tablets \\
\hline fe6c. & *PRECORTISYL 1mg tablets \\
\hline fe6d. & *PRECORTISYL 5mg tablets \\
\hline fe6e. & PRECORTISYL FORTE $25 \mathrm{mg}$ tablets \\
\hline fe6f. & *PREDNESOL $5 \mathrm{mg}$ tablets \\
\hline fe6g. & *SINTISONE 5mg tablets \\
\hline fe6h. & PREDNISOLONE $2.5 \mathrm{mg}$ e/c tablets \\
\hline fe6i. & PREDNISOLONE $5 \mathrm{mg}$ e/c tablets \\
\hline fe6j. & PREDNISOLONE $5 \mathrm{mg}$ soluble tablets \\
\hline fe6k. & PREDNISOLONE 50mg tablets \\
\hline fe6l. & DILACORT $5 \mathrm{mg}$ gastro-resistant tablets \\
\hline
\end{tabular}

COPD = chronic obstructive pulmonary disease; ICS = inhaled corticosteroids; LABA = long-acting $\beta 2$ agonists; OCS = oral corticosteroids; $\mathrm{SABA}=$ short-acting $\beta 2$ agonists. 
Table B.2.1: Read Codes sets used to define the observed variables in the latent class modelling in Chapter 3. (cont'd).

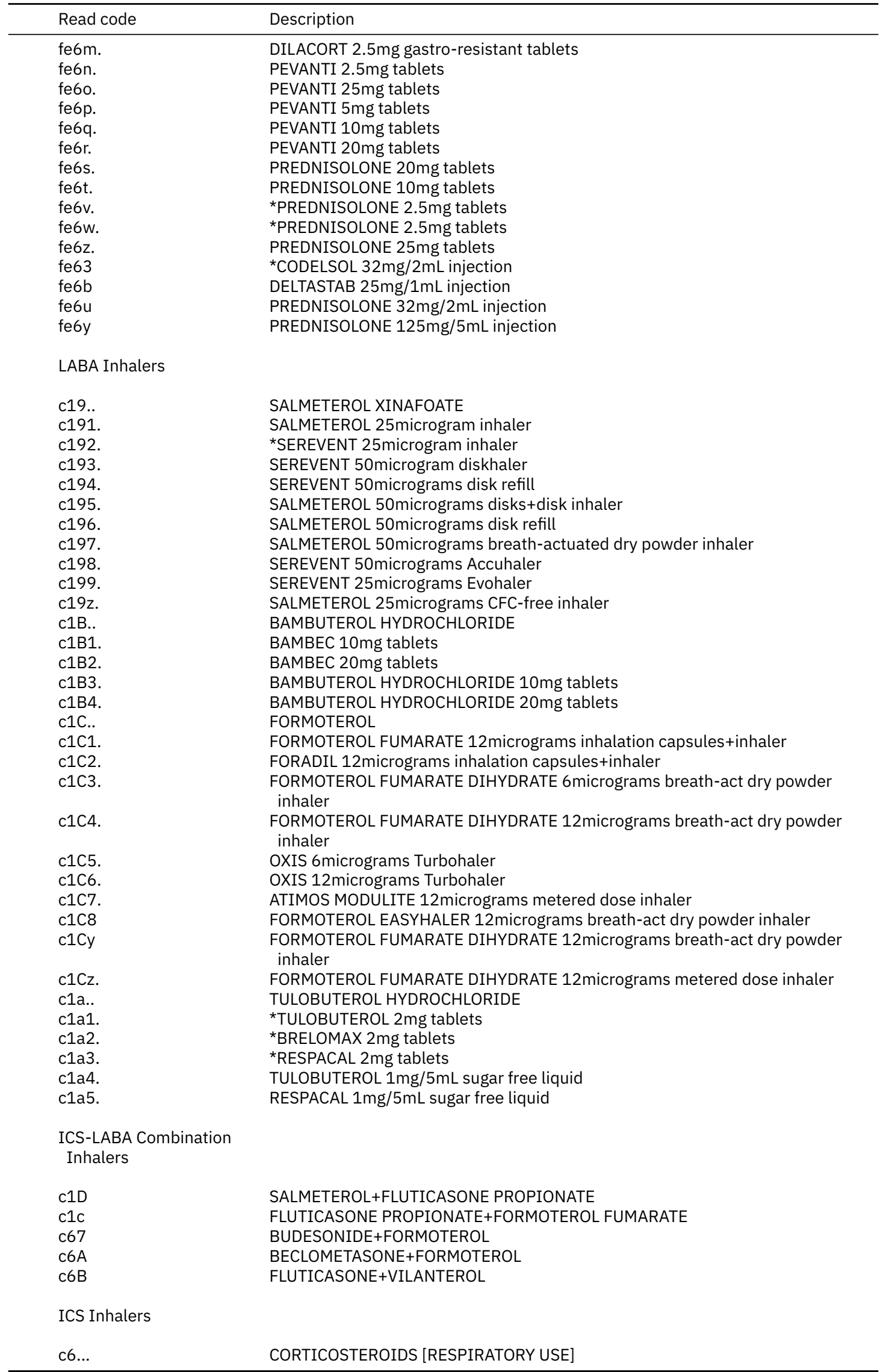

COPD = chronic obstructive pulmonary disease; ICS = inhaled corticosteroids; LABA = long-acting $\beta 2$ agonists; OCS = oral corticosteroids; $\mathrm{SABA}=$ short-acting $\beta 2$ agonists. 
Table B.2.1: Read Codes sets used to define the observed variables in the latent class modelling in Chapter 3. (cont'd).

\begin{tabular}{|c|c|}
\hline Read code & Description \\
\hline c61.. & BECLOMETASONE DIPROPIONATE [RESPIRATORY USE] \\
\hline c611. & BECLOFORTE 250microgram inhaler \\
\hline c612. & BECOTIDE-50 50microgram inhaler \\
\hline c613. & BECOTIDE 100micrograms rotacaps \\
\hline c614. & BECOTIDE 200micrograms rotacaps \\
\hline c615. & *BECOTIDE rotahaler device \\
\hline c616. & BECOTIDE 50micrograms/mL nebuliser solution \\
\hline c617. & BECOTIDE-100 100microgram inhaler \\
\hline c618. & *VOLUMATIC spacer device \\
\hline c619. & BECODISK 100 micrograms diskhaler $14 \times 8$ \\
\hline c61A. & BECLOMETASONE DIPROPIONATE 400micrograms disks+disk inhaler \\
\hline c61B. & BECLOMETASONE DIPROPIONATE 400micrograms disk refill \\
\hline c61C. & BECLOMETHASONE DIPROPIONATE 250micrograms inhaler+spacer device \\
\hline c61E. & $\begin{array}{l}\text { BECLOMETASONE DIPROPIONATE 250micrograms breath-actuated aerosol } \\
\text { inhaler }\end{array}$ \\
\hline c61F. & $\begin{array}{l}\text { BECLOMETASONE DIPROPIONATE 100micrograms breath-actuated aerosol } \\
\text { inhaler }\end{array}$ \\
\hline c61G. & *FILAIR 50micrograms inhaler \\
\hline $\mathrm{c} 61 \mathrm{H}$. & *FILAIR 100 micrograms inhaler \\
\hline c61J. & FILAIR FORTE 250micrograms inhaler \\
\hline c61K. & BECLAZONE 50micrograms inhaler \\
\hline c61L. & BECLAZONE 100micrograms inhaler \\
\hline c61M. & BECLAZONE 250micrograms inhaler \\
\hline $\mathrm{c} 61 \mathrm{~N}$. & BECLAZONE 50 EASI-BREATHE inhaler \\
\hline c610. & BECLAZONE 100 EASI-BREATHE inhaler \\
\hline c61P. & BECLAZONE 250 EASI-BREATHE inhaler \\
\hline c61Q. & BECLOFORTE INTEGRA 250micrograms inhaler+compact spacer \\
\hline c61R. & BECLOFORTE INTEGRA 250micrograms refill \\
\hline c61S. & BECLOMETHASONE DIPROPIONATE 250micrograms inhaler+compact spacer \\
\hline c61T. & BECLOMETHASONE DIPROPIONATE 250micrograms compact spacer refill \\
\hline c61U. & BECLOMETHASONE rotahaler device \\
\hline c61V. & $\begin{array}{l}\text { BECLOMETHASONE DIPROPIONATE 50micrograms vortex metered dose } \\
\text { inhaler }\end{array}$ \\
\hline c61W. & *BDP 50micrograms Spacehaler \\
\hline c61X. & $\begin{array}{l}\text { BECLOMETHASONE DIPROPIONATE 100micrograms vortex metered dose } \\
\text { inhaler }\end{array}$ \\
\hline c61Y. & *BDP 100micrograms Spacehaler \\
\hline c612. & $\begin{array}{l}\text { BECLOMETHASONE DIPROPIONATE 250micrograms vortex metered dose } \\
\text { inhaler }\end{array}$ \\
\hline c61a. & BECODISK 200micrograms diskhaler $14 \times 8$ \\
\hline c61b. & BECOTIDE 400micrograms rotacaps \\
\hline c61c. & BECODISK 100micrograms disk refill $14 \times 8$ \\
\hline c61d. & BECODISK 200micrograms disk refill 14x8 \\
\hline c61e. & BECODISK 400micrograms diskhaler 7x8 \\
\hline c61f. & BECODISK 400micrograms disk refill $7 \times 8$ \\
\hline c61g. & BECLOFORTE VM 250micrograms inhaler+volumatic \\
\hline c61h. & BECLOMETASONE DIPROPIONATE 400micrograms inhalation capsules \\
\hline c61i. & BECOTIDE-200 200microgram inhaler \\
\hline c61j. & ${ }^{*}$ AEROBEC 50microgram Autohaler \\
\hline c61k. & AEROBEC FORTE 250micrograms Autohaler \\
\hline c61l. & AEROBEC 100microgram Autohaler \\
\hline $\mathrm{c} 61 \mathrm{~m}$. & BECLOFORTE DISKHALER 400micrograms 14x8 \\
\hline c61n. & BECLOFORTE DISKS 400micrograms disk refill $14 \times 8$ \\
\hline c61p. & BECLOMETASONE DIPROPIONATE 100micrograms disks+disk inhaler \\
\hline c61q. & BECLOMETASONE DIPROPIONATE 200micrograms disks+disk inhaler \\
\hline c61r. & BECLOMETASONE DIPROPIONATE 100micrograms disk refill \\
\hline c61s. & BECLOMETASONE DIPROPIONATE 200micrograms disk refill \\
\hline c61u. & BECLOMETASONE DIPROPIONATE 200micrograms inhaler \\
\hline c61v. & BECLOMETASONE DIPROPIONATE 50micrograms inhaler \\
\hline c61w. & BECLOMETASONE DIPROPIONATE 100micrograms inhalation capsules \\
\hline c61x. & BECLOMETASONE DIPROPIONATE 200micrograms inhalation capsules \\
\hline c61y. & BECLOMETHASONE DIPROPIONATE 50micrograms $/ \mathrm{mL}$ nebuliser solution \\
\hline c61z. & BECLOMETASONE DIPROPIONATE 100micrograms inhaler \\
\hline c62.. & BECLOMETASONE COMPOUNDS \\
\hline c621. & *VENTIDE inhaler \\
\hline c622. & *VENTIDE Rotacaps \\
\hline c623. & *VENTIDE paediatric Rotacaps \\
\hline
\end{tabular}

COPD = chronic obstructive pulmonary disease; ICS = inhaled corticosteroids; LABA = long-acting $\beta 2$ agonists; OCS = oral corticosteroids; $\mathrm{SABA}=$ short-acting $\beta 2$ agonists. 
Table B.2.1: Read Codes sets used to define the observed variables in the latent class modelling in Chapter 3. (cont'd).

\begin{tabular}{|c|c|}
\hline Read code & Description \\
\hline c624. & *VENTIDE Rotahaler device \\
\hline c63.. & *BETAMETHASONE VALERATE \\
\hline c631. & ${ }^{\star}$ BEXTASOL 100microgram inhaler \\
\hline c63z. & BETAMETHASONE 100micrograms inhaler \\
\hline c64.. & BUDESONIDE [RESPIRATORY USE] \\
\hline c641. & PULMICORT 200micrograms inhaler 200dose \\
\hline c642. & PULMICORT 200micrograms refill 100dose \\
\hline c643. & PULMICORT 200micrograms refill 200dose \\
\hline c644. & PULMICORT LS 50micrograms inhaler \\
\hline c645. & PULMICORT LS 50micrograms refill \\
\hline c646. & ${ }^{\star}$ NEBUHALER spacer device \\
\hline c647. & PULMICORT 200microgram inhaler 100dose \\
\hline c649. & PULMICORT 400microgram Turbohaler 50dose \\
\hline c64A. & BUDESONIDE 200micrograms refill cannister \\
\hline c64B. & BUDESONIDE 50micrograms spacer inhaler \\
\hline c64C. & PULMICORT 200micrograms spacer inhaler \\
\hline c64D. & PULMICORT LS 50micrograms spacer inhaler \\
\hline c64E. & PULMICORT 200micrograms inhaler with NebuChamber \\
\hline c64F. & BUDESONIDE 200micrograms/dose dry powder cartridge refill \\
\hline c64G. & NOVOLIZER BUDESONIDE 200micrograms/dose dry powder cartridge refill \\
\hline $\mathrm{c} 64 \mathrm{H}$. & EASYHALER BUDESONIDE 100micrograms breath-actuated dry powder inhaler \\
\hline c64I. & EASYHALER BUDESONIDE 200micrograms breath-actuated dry powder inhaler \\
\hline c64J. & EASYHALER BUDESONIDE 400micrograms breath-actuated dry powder inhaler \\
\hline c64K. & PULMICORT 100micrograms CFC-free inhaler \\
\hline c64a. & PULMICORT 500micrograms Respules $2 \mathrm{~mL}$ unit \\
\hline c64b. & PULMICORT 1mg Respules 2mL unit \\
\hline c64c. & PULMICORT 100microgram Turbohaler 200dose \\
\hline c64d. & BUDESONIDE 100micrograms breath-actuated dry powder inhaler \\
\hline c64e. & BUDESONIDE 50micrograms refill cannister \\
\hline c64g. & BUDESONIDE 200micrograms breath-actuated dry powder inhaler \\
\hline c64h. & BUDESONIDE 400micrograms breath-actuated dry powder inhaler \\
\hline c64i. & BUDESONIDE 500micrograms/2mL nebuliser solution \\
\hline c64j. & BUDESONIDE $1 \mathrm{mg} / 2 \mathrm{~mL}$ nebuliser solution \\
\hline c64k. & *BUDESONIDE 200 Cyclocaps \\
\hline c64l. & *BUDESONIDE 400 Cyclocaps \\
\hline c64m. & BUDESONIDE 200micrograms inhalation capsules \\
\hline c64n. & BUDESONIDE 400micrograms inhalation capsules \\
\hline c64o. & BUDESONIDE 200micrograms inhaler with spacer device \\
\hline c64p. & $\begin{array}{l}\text { NOVOLIZER BUDESONIDE 200micrograms/dose dry powder cartridge and } \\
\text { refillable inhaler device }\end{array}$ \\
\hline c64u. & $\begin{array}{l}\text { BUDESONIDE 200micrograms/dose dry powder cartridge and refillable inhaler } \\
\text { device }\end{array}$ \\
\hline c64v. & BUDESONIDE 200micrograms inhaler \\
\hline c64x. & ${ }^{\star}$ BUDESONIDE refill 200 dose \\
\hline c64y. & BUDESONIDE 50micrograms inhaler \\
\hline c64z. & BUDESONIDE 200micrograms spacer inhaler \\
\hline c65.. & FLUTICASONE PROPIONATE [RESPIRATORY USE] \\
\hline c651. & FLIXOTIDE 50micrograms diskhaler \\
\hline c652. & FLIXOTIDE 100micrograms diskhaler \\
\hline c653. & FLIXOTIDE 250micrograms diskhaler \\
\hline c654. & FLUTICASONE PROPIONATE 50micrograms disks+disk inhaler \\
\hline c655. & FLUTICASONE PROPIONATE 100micrograms disks+disk inhaler \\
\hline c656. & FLUTICASONE PROPIONATE 250micrograms disks+disk inhaler \\
\hline c657. & FLIXOTIDE 50micrograms disk refill \\
\hline c658. & FLIXOTIDE 100micrograms disk refill \\
\hline c659. & FLIXOTIDE 250micrograms disk refill \\
\hline c65A. & FLUTICASONE PROPIONATE 50micrograms disk refill \\
\hline c65B. & FLUTICASONE PROPIONATE 100micrograms disk refill \\
\hline c65C. & FLUTICASONE PROPIONATE 250micrograms disk refill \\
\hline c65D. & FLIXOTIDE 25micrograms inhaler \\
\hline c65E. & FLIXOTIDE 50micrograms inhaler \\
\hline c65F. & FLIXOTIDE 125micrograms inhaler \\
\hline c65G. & FLUTICASONE PROPIONATE 25micrograms inhaler \\
\hline $\mathrm{c} 65 \mathrm{H}$. & FLUTICASONE PROPIONATE 50micrograms inhaler \\
\hline c65I. & FLUTICASONE PROPIONATE 125 micrograms inhaler \\
\hline c65K. & FLIXOTIDE 250micrograms inhaler \\
\hline c65L. & FLIXOTIDE 500micrograms diskhaler \\
\hline
\end{tabular}

COPD = chronic obstructive pulmonary disease; ICS = inhaled corticosteroids; LABA = long-acting $\beta 2$ agonists; OCS = oral corticosteroids; $\mathrm{SABA}=$ short-acting $\beta 2$ agonists. 
Table B.2.1: Read Codes sets used to define the observed variables in the latent class modelling in Chapter 3. (cont'd).

\begin{tabular}{|c|c|}
\hline Read code & Description \\
\hline c65M. & FLIXOTIDE 500micrograms disk refill \\
\hline $\mathrm{c} 65 \mathrm{~N}$. & FLUTICASONE PROPIONATE 500micrograms disks+disk inhaler \\
\hline c650. & FLUTICASONE PROPIONATE 500micrograms disk refill \\
\hline c65P. & $\begin{array}{l}\text { FLUTICASONE PROPIONATE 50micrograms breath-actuated dry powder } \\
\text { inhaler }\end{array}$ \\
\hline c65Q. & $\begin{array}{l}\text { FLUTICASONE PROPIONATE 100micrograms breath-actuated dry powder } \\
\text { inhaler }\end{array}$ \\
\hline c65R. & $\begin{array}{l}\text { FLUTICASONE PROPIONATE 250micrograms breath-actuated dry powder } \\
\text { inhaler }\end{array}$ \\
\hline c65S. & $\begin{array}{l}\text { FLUTICASONE PROPIONATE 500micrograms breath-actuated dry powder } \\
\text { inhaler }\end{array}$ \\
\hline c65T. & FLIXOTIDE 50micrograms Accuhaler \\
\hline c65U. & FLIXOTIDE 100micrograms Accuhaler \\
\hline c65V. & FLIXOTIDE 250micrograms Accuhaler \\
\hline c65W. & FLIXOTIDE 500micrograms Accuhaler \\
\hline c65X. & FLUTICASONE PROPIONATE $0.5 \mathrm{mg} / 2 \mathrm{~mL}$ nebulisation units \\
\hline c65Y. & FLUTICASONE PROPIONATE $2 \mathrm{mg} / 2 \mathrm{~mL}$ nebulisation units \\
\hline c65Z. & FLIXOTIDE $0.5 \mathrm{mg} / 2 \mathrm{~mL}$ Nebules \\
\hline c65a. & FLIXOTIDE $2 \mathrm{mg} / 2 \mathrm{~mL}$ Nebules \\
\hline c65b. & FLUTICASONE PROPIONATE 125 micrograms CFC-free inhaler \\
\hline c65c. & FLUTICASONE PROPIONATE 250micrograms CFC-free inhaler \\
\hline c65d. & FLIXOTIDE 125micrograms Evohaler \\
\hline c65e. & FLIXOTIDE 250micrograms Evohaler \\
\hline c65f. & FLUTICASONE PROPIONATE 50micrograms CFC-free inhaler \\
\hline c65g. & FLIXOTIDE 50micrograms Evohaler \\
\hline c66.. & BECLOMETASONE DIPROPIONATE [RESPIRATORY USE 2] \\
\hline c661. & *BDP 250micrograms Spacehaler \\
\hline c662. & BECOTIDE 50 EASI-BREATHE inhaler \\
\hline c663. & BECOTIDE 100 EASI-BREATHE inhaler \\
\hline c664. & BECLOFORTE EASI-BREATHE 250micrograms inhaler \\
\hline c665. & QVAR 50 inhaler \\
\hline c666. & QVAR 100 inhaler \\
\hline c667. & QVAR 50 Autohaler \\
\hline c668. & QVAR 100 Autohaler \\
\hline c669. & ${ }^{*}$ BECLAZONE 200 inhaler \\
\hline c66A. & $\begin{array}{l}\text { BECLOMETASONE DIPROPIONATE 50micrograms breath-actuated dry powder } \\
\text { inhaler }\end{array}$ \\
\hline c66B. & $\begin{array}{l}\text { BECLOMETASONE DIPROPIONATE 100micrograms breath-actuated dry } \\
\text { powder inhaler }\end{array}$ \\
\hline c66C. & $\begin{array}{l}\text { BECLOMETASONE DIPROPIONATE 250micrograms breath-actuated dry } \\
\text { powder inhaler }\end{array}$ \\
\hline c66D. & ASMABEC 50micrograms Clickhaler \\
\hline c66E. & ASMABEC 100micrograms Clickhaler \\
\hline c66F. & ASMABEC 250micrograms Clickhaler \\
\hline c66G. & $\begin{array}{l}\text { BECLOMETASONE DIPROPIONATE 400micrograms breath-actuated dry } \\
\text { powder inhaler }\end{array}$ \\
\hline $\mathrm{c} 66 \mathrm{H}$. & $\begin{array}{l}\text { BECLOMETASONE DIPROPIONATE 200micrograms breath-actuated dry } \\
\text { powder inhaler }\end{array}$ \\
\hline c66I. & $\begin{array}{l}\text { PULVINAL BECLOMETHASONE DIPROPIONATE 100micrograms } \\
\text { breath-actuated dry powder inhaler }\end{array}$ \\
\hline c66J. & $\begin{array}{l}\text { PULVINAL BECLOMETHASONE DIPROPIONATE 200micrograms } \\
\text { breath-actuated dry powder inhaler }\end{array}$ \\
\hline c66K. & $\begin{array}{l}\text { PULVINAL BECLOMETHASONE DIPROPIONATE 400micrograms } \\
\text { breath-actuated dry powder inhaler }\end{array}$ \\
\hline c66L. & ${ }^{*}$ BECLOMETASONE 100 cyclocaps \\
\hline c66M. & *BECLOMETASONE 200 cyclocaps \\
\hline $\mathrm{c} 66 \mathrm{~N}$. & *BECLOMETASONE 400 cyclocaps \\
\hline c66P. & BECODISK 100micrograms diskhaler $15 \times 8$ \\
\hline c66Q. & BECODISK 200micrograms diskhaler $15 \times 8$ \\
\hline c66R. & BECODISK 400 micrograms diskhaler $15 \times 8$ \\
\hline c66S. & BECODISK 100 micrograms disk refill $15 \times 8$ \\
\hline c66T. & BECODISK 200micrograms disk refill 15x8 \\
\hline c66U. & BECODISK 400 micrograms disk refill $15 \times 8$ \\
\hline c66V. & BECLOMETASONE DIPROPIONATE 50micrograms CFC-free inhaler \\
\hline c66W. & BECLOMETASONE DIPROPIONATE 100micrograms CFC-free inhaler \\
\hline c66x. & $\begin{array}{l}\text { BECLOMETASONE DIPROPIONATE 50micrograms CFC-free breath-actuated } \\
\text { aerosol inhaler }\end{array}$ \\
\hline
\end{tabular}


Table B.2.1: Read Codes sets used to define the observed variables in the latent class modelling in Chapter 3. (cont'd).

\begin{tabular}{|c|c|}
\hline Read code & Description \\
\hline c66Y. & $\begin{array}{l}\text { BECLOMETASONE DIPROPIONATE 100micrograms CFC-free breath-actuated } \\
\text { aerosol inhaler }\end{array}$ \\
\hline c66Z. & $\begin{array}{l}\text { QVAR EASI-BREATHE 50micrograms CFC-free breath-actuated dry powder } \\
\text { inhaler }\end{array}$ \\
\hline c66a. & $\begin{array}{l}\text { QVAR EASI-BREATHE 100micrograms CFC-free breath-actuated dry powder } \\
\text { inhaler }\end{array}$ \\
\hline c66c. & CLENIL MODULITE 50micrograms CFC-free inhaler \\
\hline c66d. & CLENIL MODULITE 100micrograms CFC-free inhaler \\
\hline c66e. & CLENIL MODULITE 200micrograms CFC-free inhaler \\
\hline c66f. & CLENIL MODULITE 250micrograms CFC-free inhaler \\
\hline c66g. & BECLOMETASONE DIPROPIONATE 200micrograms CFC-free inhaler \\
\hline c66h. & BECLOMETASONE DIPROPIONATE 250micrograms CFC-free inhaler \\
\hline c68.. & MOMETASONE [RESPIRATORY USE] \\
\hline c681. & MOMETASONE FUROATE 200micrograms breath-actuated dry powder inhaler \\
\hline c682. & MOMETASONE FUROATE 400micrograms breath-actuated dry powder inhaler \\
\hline c683. & ASMANEX TWISTHALER 200micrograms breath-actuated dry powder inhaler \\
\hline c684. & ASMANEX TWISTHALER 400micrograms breath-actuated dry powder inhaler \\
\hline c69.. & CICLESONIDE \\
\hline c691. & ALVESCO 160micrograms inhaler \\
\hline c692. & ALVESCO 80micrograms inhaler \\
\hline c69y. & CICLESONIDE 80micrograms inhaler \\
\hline c69z. & CICLESONIDE 160micrograms inhaler \\
\hline \multicolumn{2}{|c|}{ COPD-specific } \\
\hline \multicolumn{2}{|c|}{ Prescriptions } \\
\hline 8BMW. & Issue of chronic obstructive pulmonary disease rescue pack \\
\hline 81610 & Chronic obstructive pulmonary disease rescue pack not indicated \\
\hline 8IEZ. & Chronic obstructive pulmonary disease rescue pack declined \\
\hline a46.. & GLYCOPYRRONIUM BROMIDE [ANTISPASMODIC] \\
\hline a46z. & *GLYCOPYRRONIUM 2mg tablets \\
\hline c1b.. & INDACATEROL \\
\hline c1b1. & ONBREZ BREEZHALER 150micrograms inhalation capsules+inhaler \\
\hline c1b2. & INDACATEROL 150micrograms inhalation capsules+inhaler \\
\hline c1b3. & ONBREZ BREEZHALER 300micrograms inhalation capsules+inhaler \\
\hline c1b4. & INDACATEROL 300micrograms inhalation capsules+inhaler \\
\hline c1d.. & OLODATEROL \\
\hline c1d1. & STRIVERDI RESPIMAT 2.5micrograms inhaler \\
\hline $\mathrm{c} 1 \mathrm{~d} 2$ & OLODATEROL 2.5micrograms inhaler \\
\hline c1e.. & INDACATEROL+GLYCOPYRRONIUM \\
\hline c1e2. & INDACATEROL+GLYCOPYRRONIUM $85 \mathrm{mcg} / 43 \mathrm{mcg}$ inh powder caps+inh \\
\hline c3... & ANTICHOLINERGIC BRONCHODILATORS \\
\hline c31.. & IPRATROPIUM BROMIDE [1] \\
\hline c311. & ${ }^{*}$ ATROVENT 20micrograms inhaler \\
\hline c312. & ATROVENT 500microgram $/ 2 \mathrm{~mL}$ nebuliser solution \\
\hline c313. & ATROVENT FORTE 40microgram inhaler \\
\hline c314. & ATROVENT 250microgram/1mL nebuliser solution \\
\hline c315. & ATROVENT 20micrograms Autohaler \\
\hline c316. & STERI-NEB IPRATROPIUM 250micrograms/1mL nebulisation units \\
\hline c317. & STERI-NEB IPRATROPIUM 500micrograms $/ 2 \mathrm{~mL}$ nebulisation units \\
\hline c318. & ATROVENT 40micrograms Aerocaps refill pack \\
\hline c319. & ATROVENT 40micrograms Aerocaps+Aerohaler device \\
\hline c31A. & IPRATROPIUM BROMIDE 40mcg inhalation capsules \\
\hline c31B. & IPRATROPIUM BROMIDE 40mcg inhalation capsules+inhaler device \\
\hline c31C. & RESPONTIN 250micrograms/1mL Nebules \\
\hline c31D. & RESPONTIN 500micrograms/2mL Nebules \\
\hline c31E. & TROPIOVENT 250micrograms/1mL Steripoules \\
\hline c31F. & TROPIOVENT 500micrograms/2mL Steripoules \\
\hline c31G. & ATROVENT 20micrograms CFC-free inhaler \\
\hline c31t. & IPRATROPIUM BROMIDE 20micrograms CFC-free inhaler \\
\hline c31u. & IPRATROPIUM 20micrograms breath-actuated aerosol inhaler \\
\hline c31v. & IPRATROPIUM 250 micrograms/1mL nebuliser solution \\
\hline c31w. & IPRATROPIUM 500micrograms/2mL nebuliser solution \\
\hline c31x. & IPRATROPIUM 20micrograms inhaler \\
\hline c31y. & IPRATROPIUM 250micrograms/mL nebuliser solution \\
\hline c31z. & IPRATROPIUM 40microgram inhaler \\
\hline c32.. & OXITROPIUM BROMIDE \\
\hline
\end{tabular}

COPD = chronic obstructive pulmonary disease; ICS = inhaled corticosteroids; LABA = long-acting $\beta 2$ agonists; OCS = oral corticosteroids; $\mathrm{SABA}=$ short-acting $\beta 2$ agonists. 
Table B.2.1: Read Codes sets used to define the observed variables in the latent class modelling in Chapter 3. (cont'd).

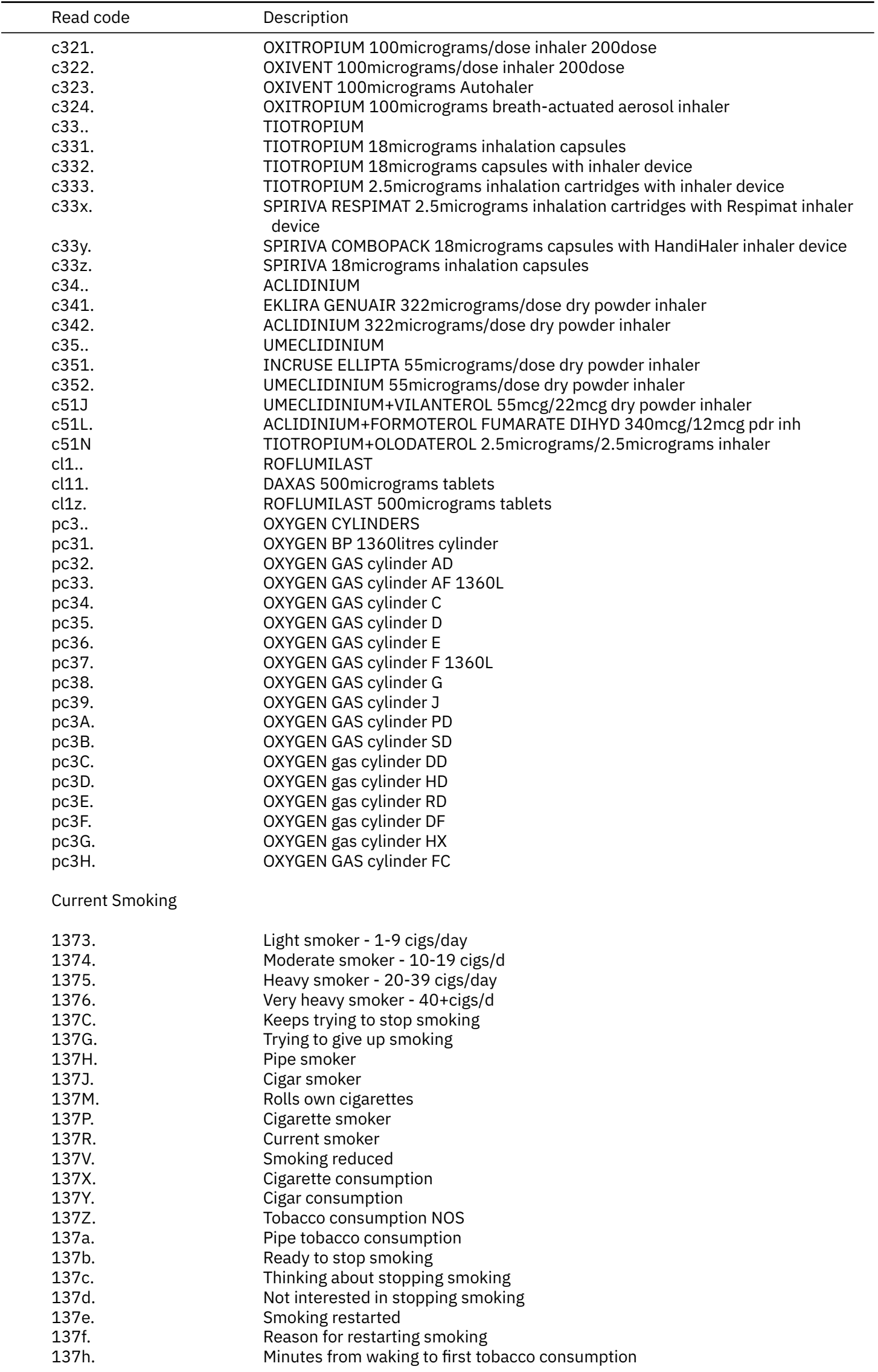

COPD = chronic obstructive pulmonary disease; ICS = inhaled corticosteroids; LABA = long-acting $\beta 2$ agonists; OCS = oral corticosteroids; $\mathrm{SABA}=$ short-acting $\beta 2$ agonists. 
Table B.2.1: Read Codes sets used to define the observed variables in the latent class modelling in Chapter 3. (cont'd).

\begin{tabular}{|c|c|}
\hline Read code & Description \\
\hline $137 \mathrm{~m}$. & Failed attempt to stop smoking \\
\hline 1370. & Waterpipe tobacco consumption \\
\hline \multicolumn{2}{|l|}{ Ex-smoking } \\
\hline 1378. & Ex-light smoker (1-9/day) \\
\hline 1379. & Ex-moderate smoker (10-19/day) \\
\hline 137A. & Ex-heavy smoker (20-39/day) \\
\hline 137B. & Ex-very heavy smoker (40+/day) \\
\hline $137 \mathrm{~F}$. & Ex-smoker - amount unknown \\
\hline 137K. & Stopped smoking \\
\hline $137 N$. & Ex pipe smoker \\
\hline 1370. & Ex cigar smoker \\
\hline 137S. & Ex smoker \\
\hline 137T. & Date ceased smoking \\
\hline 137j. & Ex-cigarette smoker \\
\hline $137 l$. & Ex roll-up cigarette smoker \\
\hline
\end{tabular}

COPD = chronic obstructive pulmonary disease; ICS = inhaled corticosteroids; LABA = long-acting $\beta 2$ agonists; OCS = oral corticosteroids; $\mathrm{SABA}=$ short-acting $\beta 2$ agonists. 


\section{B.3 Item-response probabilities for the competing latent class models}

The following diagrams shows item-response probabilities in the classes of each of the competing models. The names and levels of the observed variables are shown on the left-most column in each diagram. Model diagnostics are shown below each diagram and includes Akaike Information Criterion (AIC), Bayesian Information Criterion (BIC), likelihood ratio chi-squared statistic (Gsq), Pearson's Chi-square goodness of fit statistic (Chisq), maximum log-likelihood value (llik), number of iterations needed (numiter), number of individuals included in the modelling ( $N$ ), and number of estimated parameters (i.e. the number of degrees of freedom used; npar). 


\section{\#classes: 1}

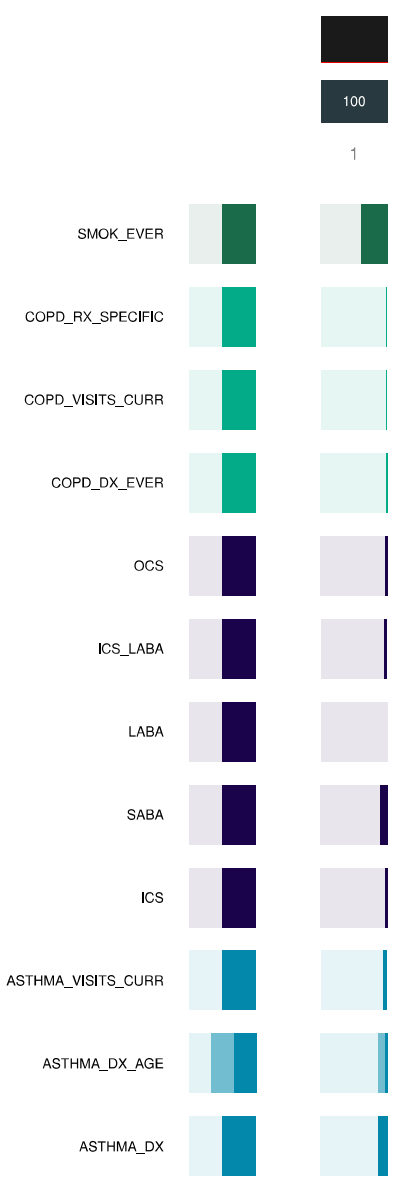

$\mathrm{AIC}=297943.83, \mathrm{BIC}=298058.48, \mathrm{Gsq}=109287.92$, Chisq $=$ 139774016756.94, llik $=-148958.91$, numiter $=1 / 3000, N=$ $50000 / 50000$, npar $=13$ 


\section{\#classes: 2}

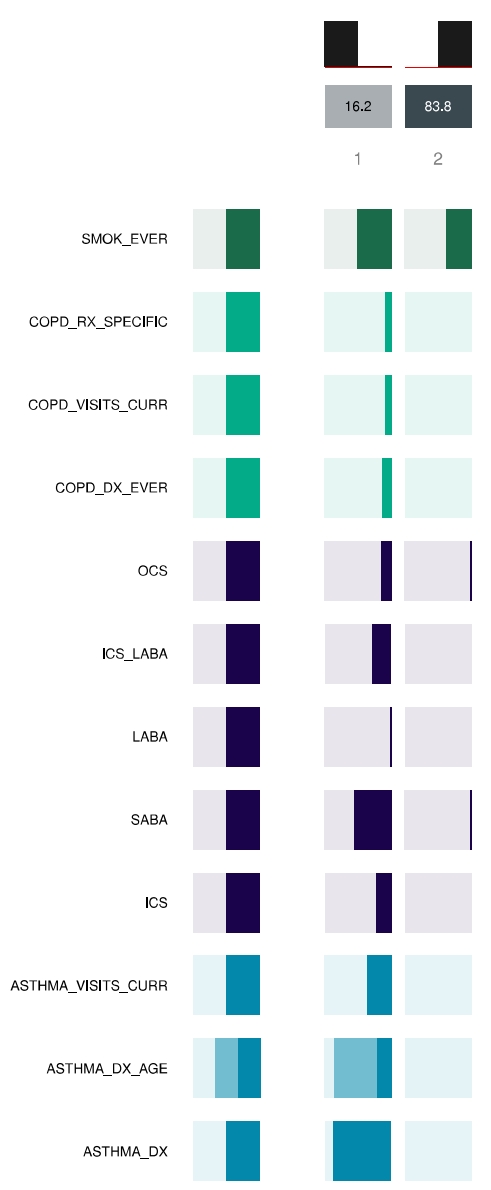

$\mathrm{AIC}=219636.16, \mathrm{BIC}=219874.3, \mathrm{Gsq}=30952.25$, Chisq $=$ 7159142.63, llik $=-109791.08$, numiter $=19 / 3000, \mathrm{~N}=$ $50000 / 50000$, npar $=27$ 
\#classes: 3

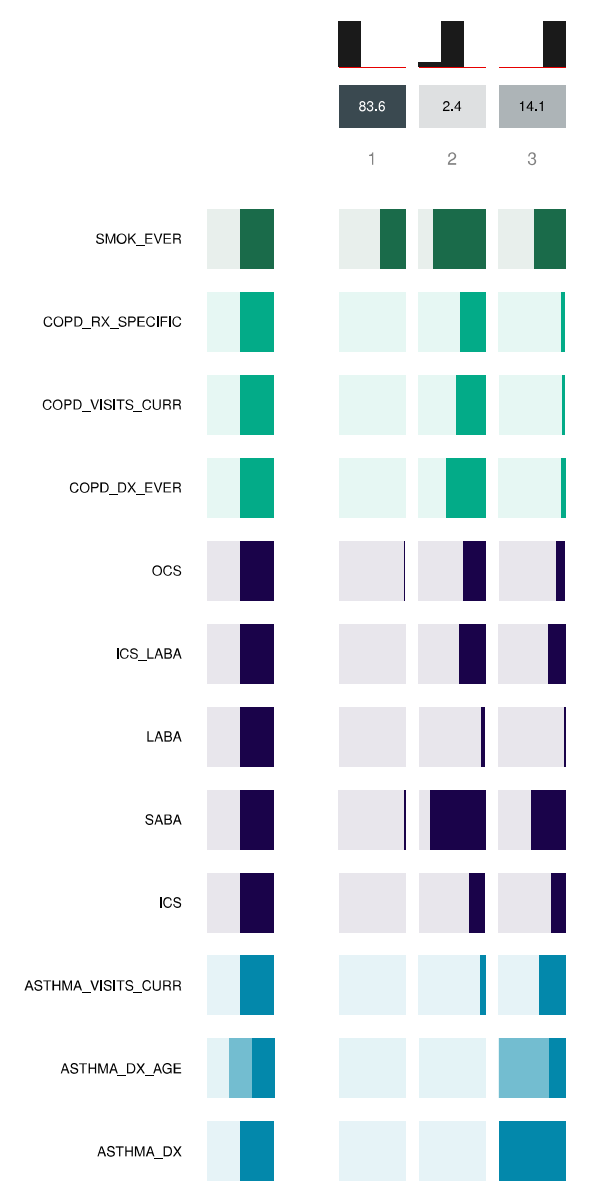

$\mathrm{AIC}=207915.8, \mathrm{BIC}=208277.41, \mathrm{Gsq}=19203.89$, Chisq $=$ 3695583.03, llik $=-103916.9$, numiter $=258 / 3000, N=$ $50000 / 50000, \mathrm{npar}=41$ 
\#classes: 4

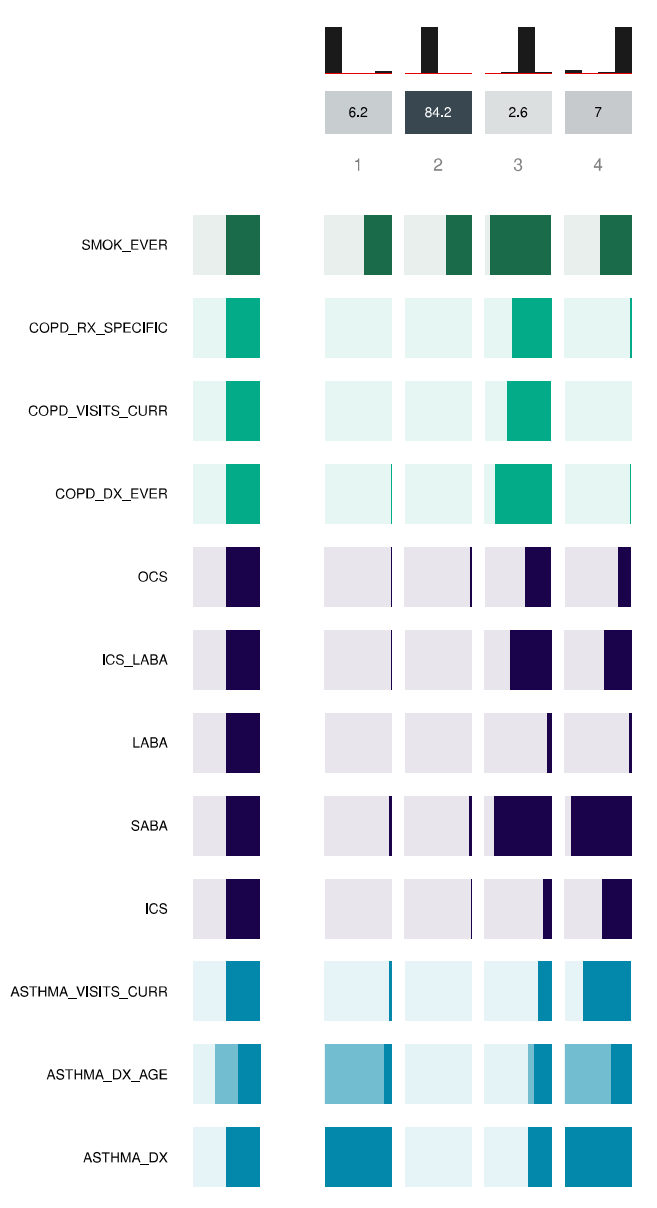

$\mathrm{AIC}=197295.36, \mathrm{BIC}=197780.45, \mathrm{Gsq}=8555.45$, Chisq $=$ 66993.38, llik $=-98592.68$, numiter $=53 / 3000, N=$ $50000 / 50000, \mathrm{npar}=55$ 
\#classes: 5

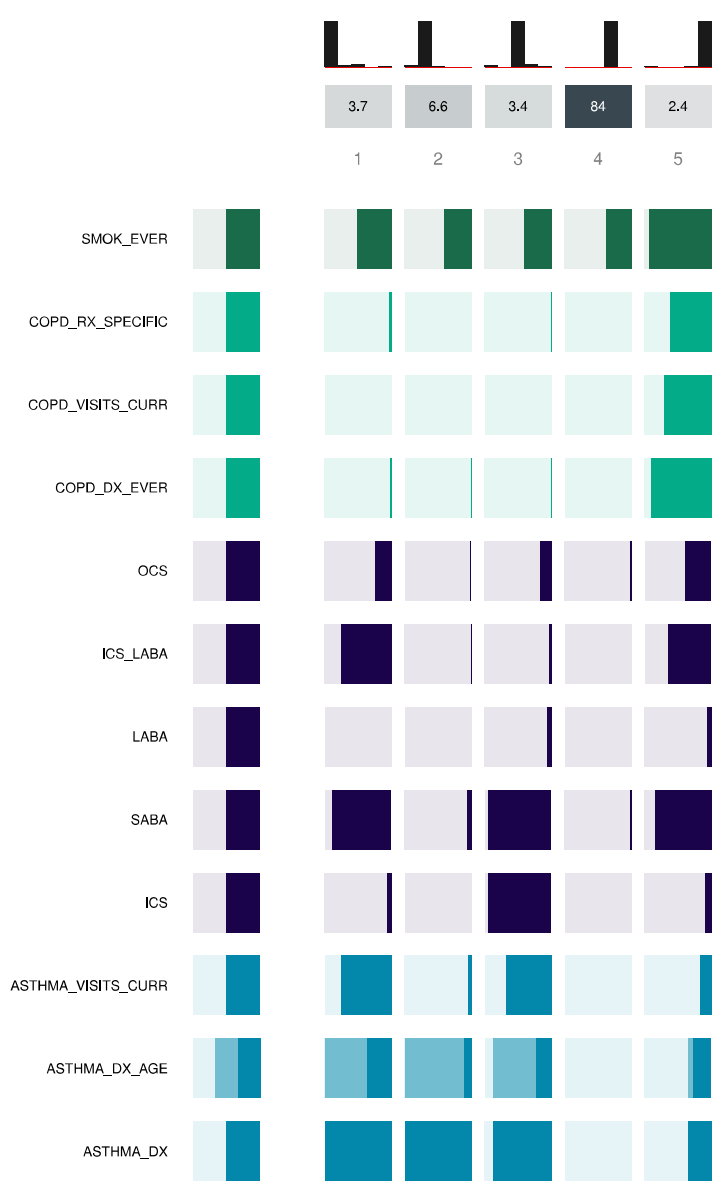

$\mathrm{AIC}=195593.31, \mathrm{BIC}=196201.88, \mathrm{Gsq}=6825.4$, Chisq $=$ 24435.3, llik $=-97727.66$, numiter $=76 / 3000, N=$ $50000 / 50000, \mathrm{npar}=69$ 
\#classes: 6

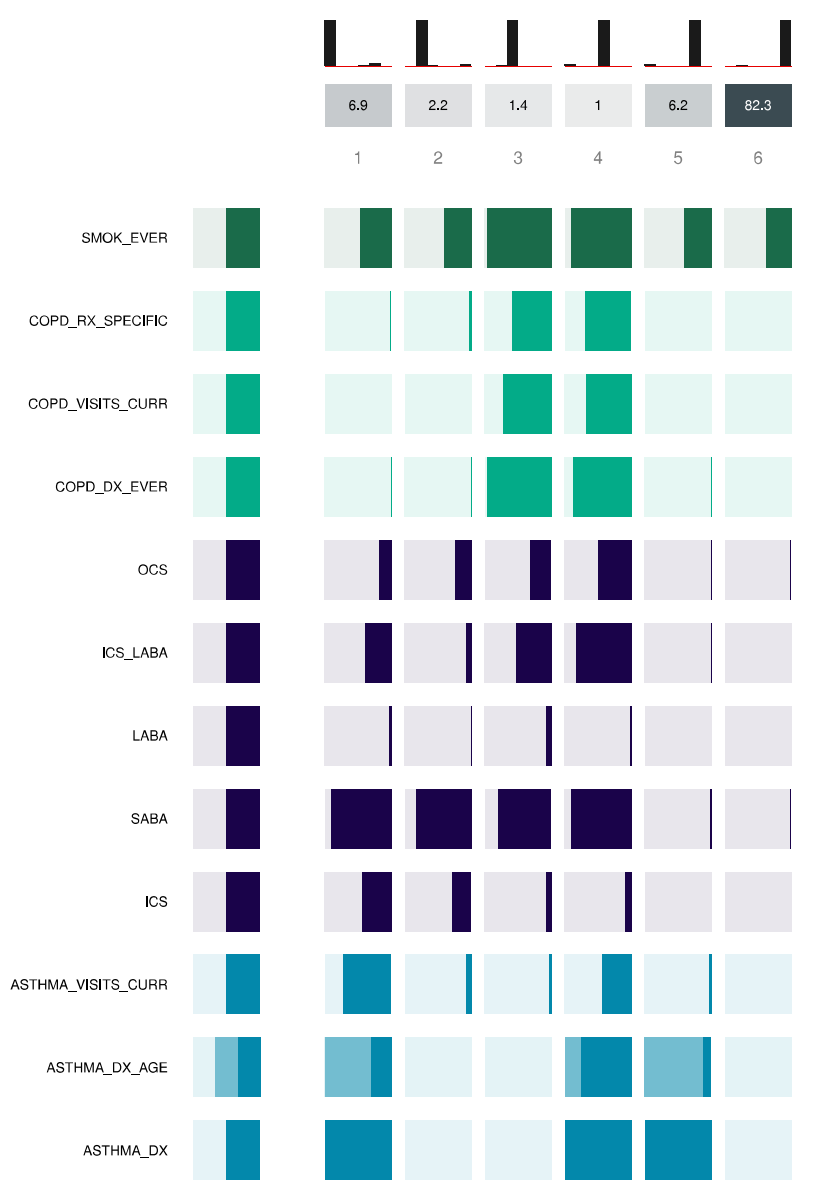

$\mathrm{AIC}=192656.15, \mathrm{BIC}=193388.19, \mathrm{Gsq}=3860.24$, Chisq $=$ 12060.83, llik $=-96245.08$, numiter $=202 / 3000, N=$ $50000 / 50000$, npar $=83$ 


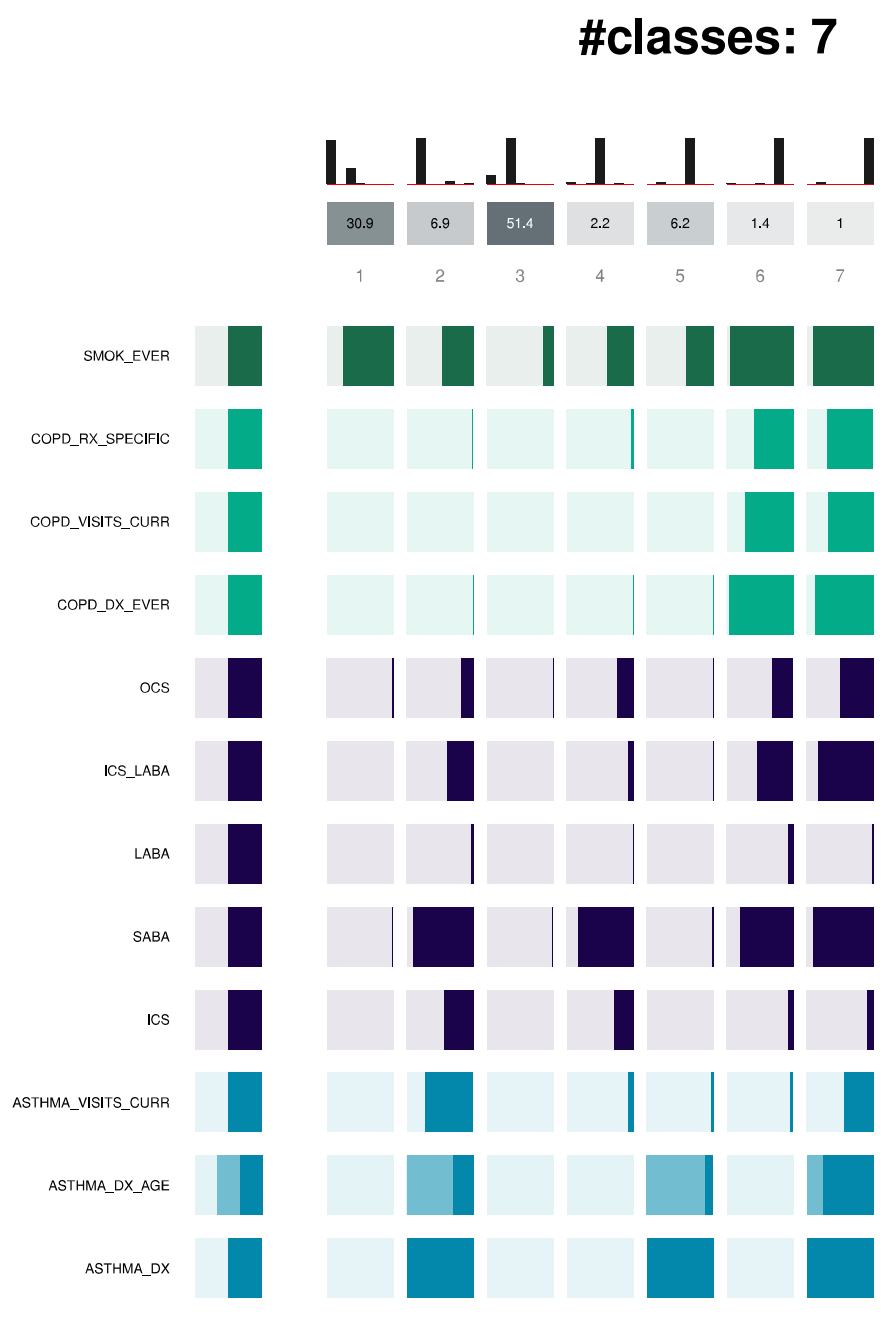

$A I C=192592.52, \mathrm{BIC}=193448.03, \mathrm{Gsq}=3768.61$, Chisq $=$ 11973.55, llik $=-96199.26$, numiter $=3000 / 3000, \mathrm{~N}=$ 50000/50000, npar $=97$ 
\#classes: 8

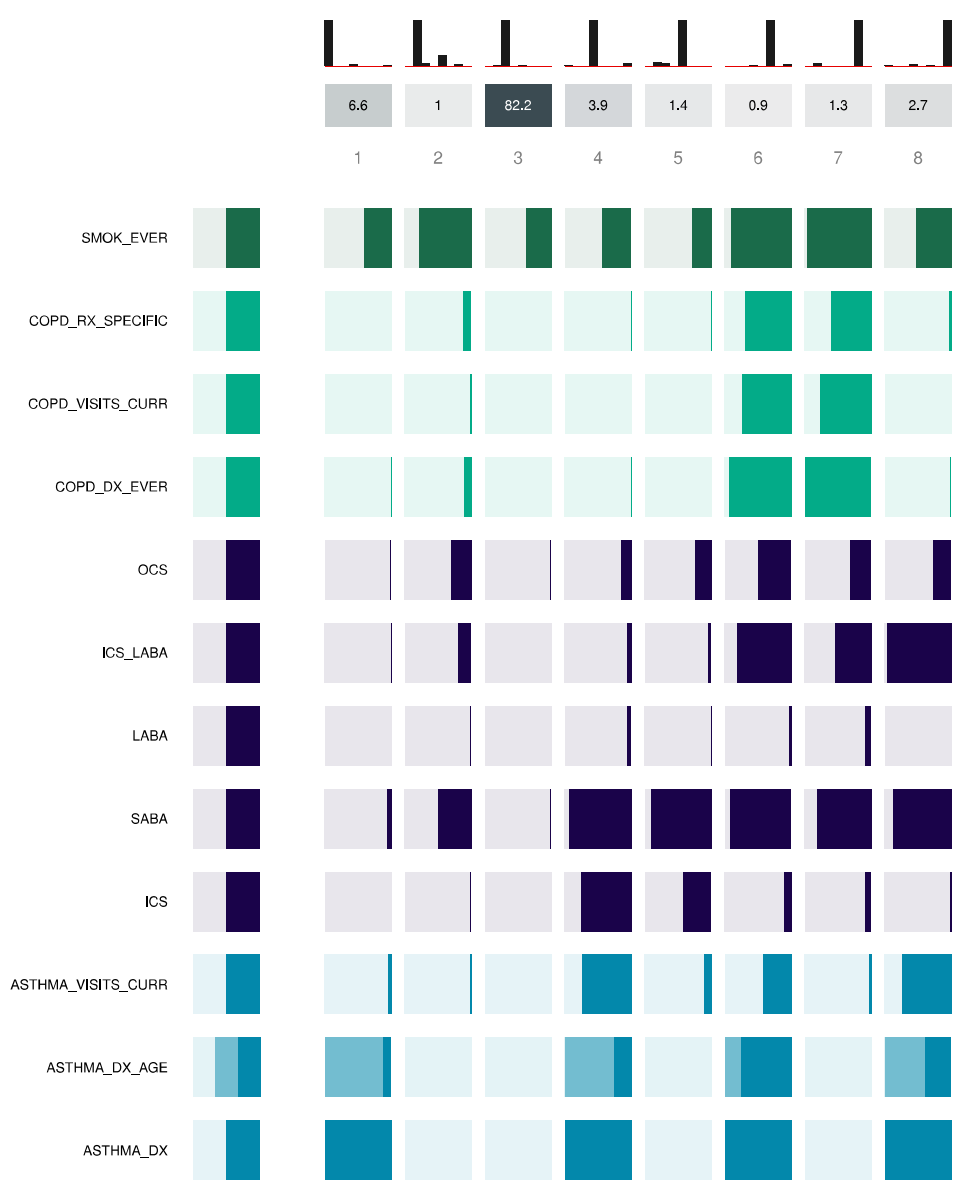

$\mathrm{AIC}=190965.89, \mathrm{BIC}=191944.88, \mathrm{Gsq}=2113.98, \mathrm{Chisq}=$ 9427.48, llik $=-95371.94$, numiter $=1836 / 3000, N=$ $50000 / 50000$, npar $=111$ 
\#classes: 9

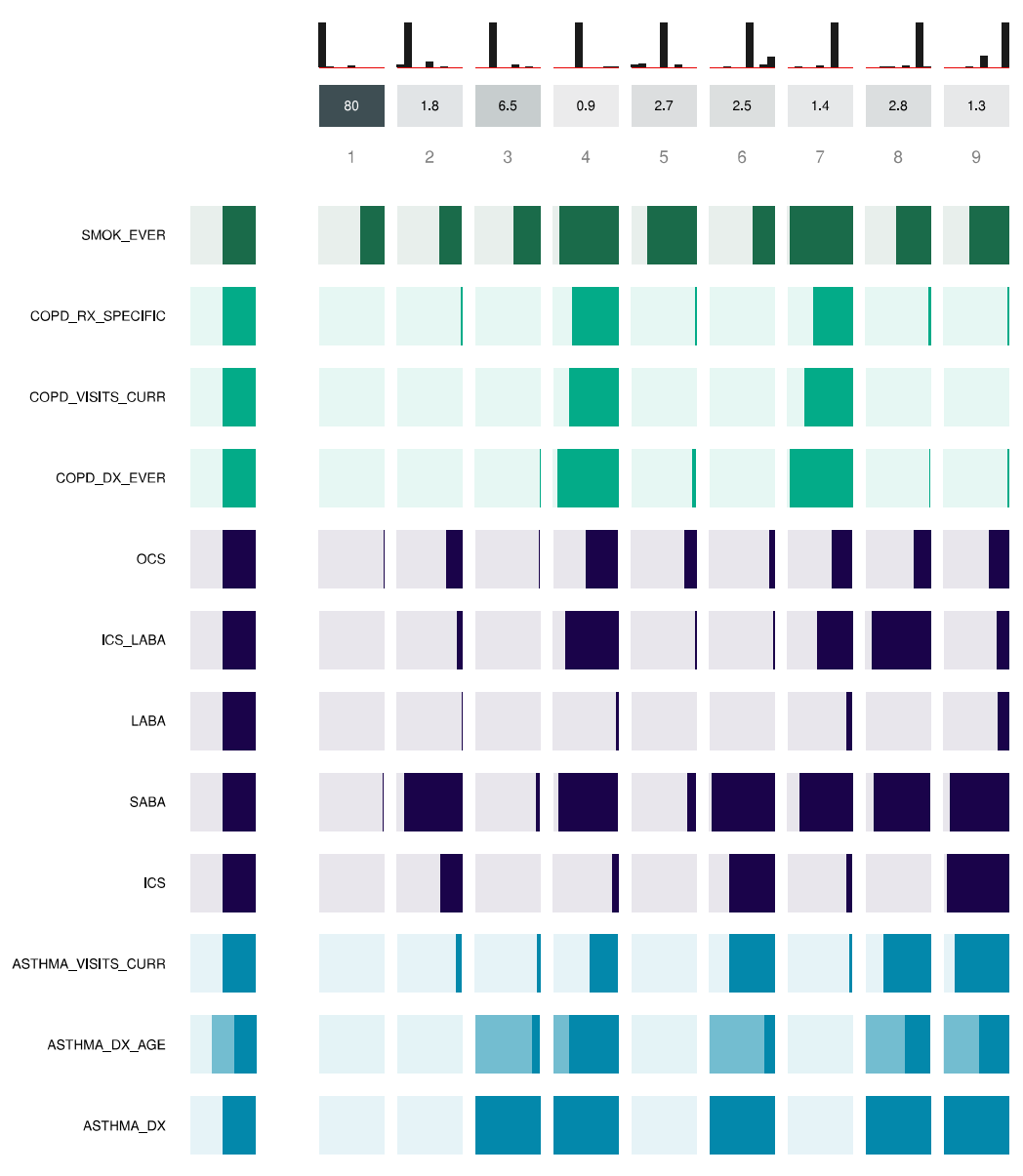

$\mathrm{AIC}=190829.52, \mathrm{BIC}=191931.99, \mathrm{Gsq}=1949.61, \mathrm{Chisq}=$ 7716.2, llik $=-95289.76$, numiter $=3000 / 3000, \mathrm{~N}=$ $50000 / 50000$, npar $=125$ 
\#classes: 10

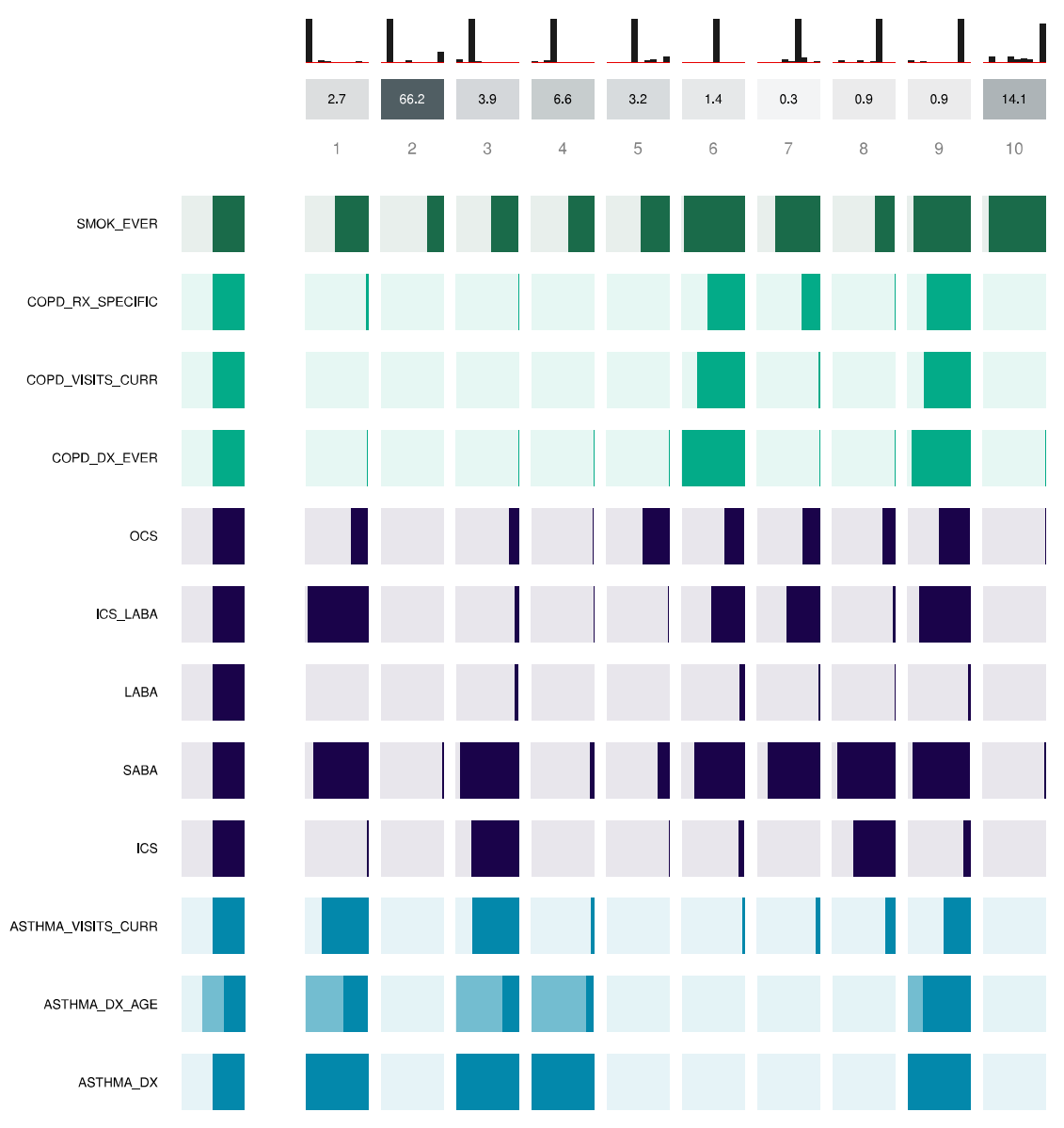

$\mathrm{AIC}=190899.72, \mathrm{BIC}=192125.67, \mathrm{Gsq}=1991.81, \mathrm{Chisq}=$ 8809.87, llik $=-95310.86$, numiter $=3000 / 3000, \mathrm{~N}=$ $50000 / 50000$, npar $=139$ 
\#classes: 11

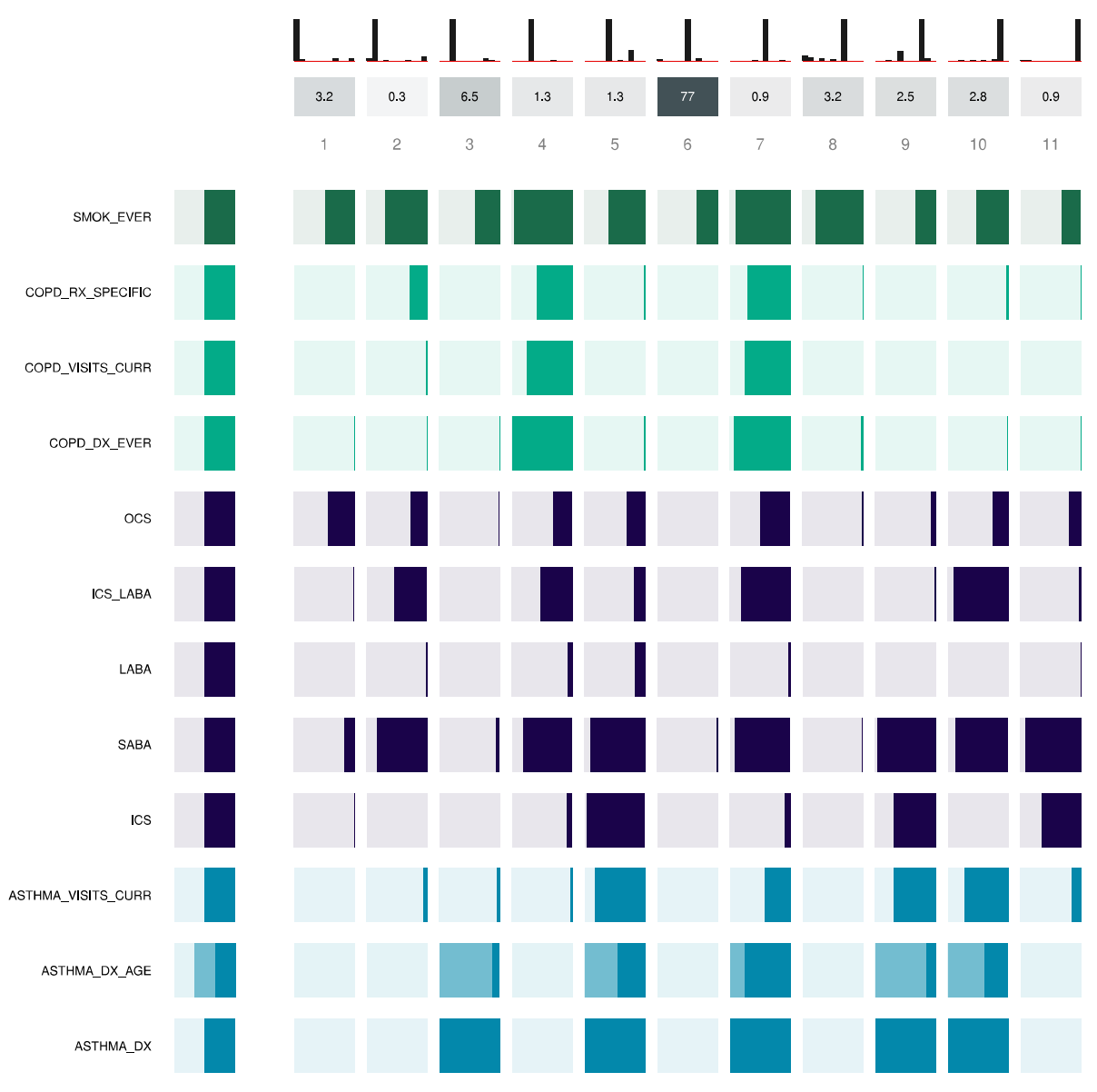

$\mathrm{AIC}=190744.87, \mathrm{BIC}=192094.3, \mathrm{Gsq}=1808.96$, Chisq $=$ 7230.18, llik $=-95219.44$, numiter $=3000 / 3000, N=$ $50000 / 50000$, npar $=153$ 


\section{\#classes: 12}

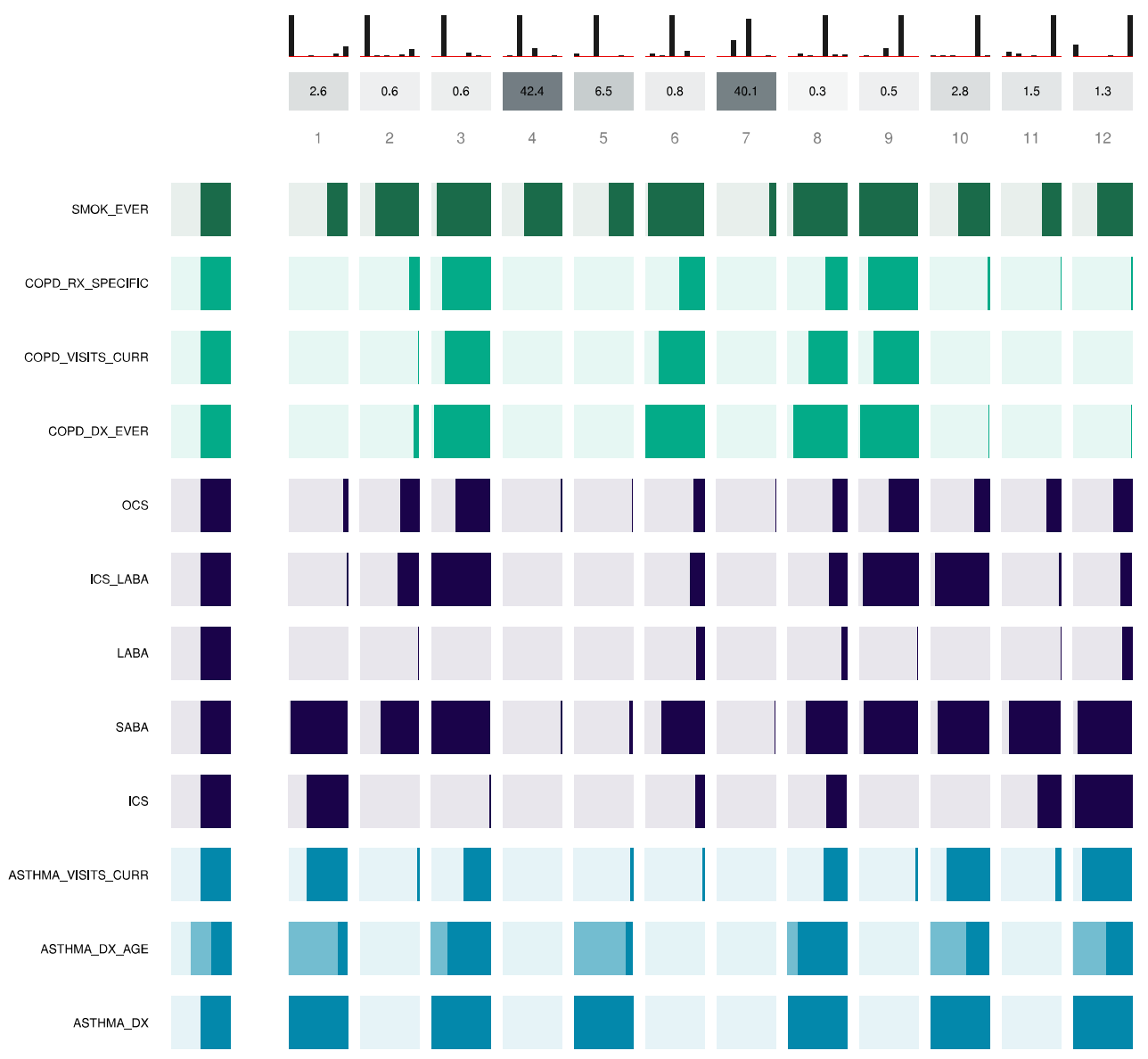

$\mathrm{AIC}=190461.81, \mathrm{BIC}=191934.71, \mathrm{Gsq}=1497.9$, Chisq $=$ 3916.75 , llik $=-95063.9$, numiter $=3000 / 3000, N=$ $50000 / 50000$, npar $=167$ 


\section{B.4 Related study protocol: identifying patients with asthma-COPD overlap syndrome using latent class analysis of electronic health record data}

The following study protocol is related to the work presented in Chapter 3 and is focused on identifying people with asthma-COPD overlap syndrome using latent class analysis of electronic health record data. It has been published in npj Primary Care Respiratory Medicine (DOI: 10.1038/s41533-018-0088-4, https://www.nature.com/articles/s41533-018-0088-4). 


\section{Identifying patients with asthma-chronic obstructive pulmonary disease overlap syndrome using latent class analysis of electronic health record data: a study protocol}

Mohammad A A Sallakh, MD ${ }^{\text {B1,a }}$, Sarah E Rodgers, PhD ${ }^{1, b}$, Ronan A Lyons, MD ${ }^{1, b}$, Aziz Sheikh, MD $D^{2, a, b}$ and Gwyneth A Davies, MD ${ }^{1, a}$

${ }^{1}$ Swansea University Medical School, Singleton Park, Swansea, SA2 8PP, UK

${ }^{2}$ Usher Institute of Population Health Sciences and Informatics, The University of Edinburgh

${ }^{a}$ Asthma UK Centre for Applied Research

${ }^{\mathrm{b}}$ The Farr Institute of Health Informatics Research

Correspondence

Mohammad A Al Sallakh, MD, MSc

Data Science Building, Swansea University, Singleton Park, Swansea, SA2 8PP, United Kingdom

Phone:

Email: M.A.Alsallakh@swansea.ac.uk 


\section{Introduction}

Asthma and chronic obstructive pulmonary disease (COPD) are two common different clinical diagnoses with overlapping clinical features. Global Initiative for Asthma (GINA) defined asthma based on variable respiratory symptoms and expiratory airflow limitation. ${ }^{1}$ On the other hand, the Global Initiative for Chronic Obstructive Lung Disease (GOLD) defined COPD based on persistent respiratory symptoms and airflow limitations. ${ }^{2}$ While asthma affects people from the early school age, COPD mainly affects those aged over 40 years with a smoking history. Clinically, the differentiation between the two diseases and identifying their overlap in those older people can be challenging. ${ }^{1}$ Co-existence of clinical features of both conditions along with persistent airflow limitation has been recently recognized by a joint committee publication between GOLD and GINA as the asthma-COPD overlap syndrome (ACOS). ${ }^{3}$

However, there are currently no universally agreed consensus clinical definitions for the diagnosis of asthma, ${ }^{4-9}$ COPD, ${ }^{10,11}$ and ACOS. ${ }^{12-15}$ Subsequently, the prevalence of these three conditions is highly dependent on the different available case definitions and data sources.16-20

In studies conducted using electronic health records (EHR), identifying patient groups is further complicated by the limitations of these data, such as missing data and coding errors. ${ }^{21-23}$ Despite the lack of consensus clinical definitions, we expect EHR data of people with "ACOS" to be systematically different from those with "asthma only" or "COPD only". Case definitions aiming to differentiate between those patient groups based solely on clinical knowledge or face validity may be inaccurate, and validating them with traditional methods, e.g., review of full patient records, is time-consuming and labour-intensive. Clustering methods overcome these challenges by automatically identifying subgroups in the population that best explain the patterns in high-dimensional EHR data, without an a priori hypothesis about those subgroups and their labels. ${ }^{24}$ Latent class analysis (LCA) is such a 
method that can probabilistically identify patients with asthma and/or COPD using the available recorded data.

\section{Aims}

We plan to develop an LCA model to identify and characterise patients with asthma, COPD and ACOS in Wales. Based on this LCA model, we will derive a classification algorithm, and compare its performance with commonly used objective and self-reported case definitions for asthma and COPD.

\section{Methods}

We will use primary care data on asthma and COPD recorded in or before 2014 for a sample of the Welsh population to find, using LCA, clinically meaningful classes (i.e. clusters) related to the two conditions in that year. We will follow the STROBE ${ }^{25}$ and RECORD Statements ${ }^{26}$ in reporting the full study.

\section{Data sources}

We will use the following two de-identified datasets from the Secure Anonymised Information

Linkage (SAIL) Databank in Wales: ${ }^{27,28}$

- The Welsh Demographic Service (WDS) which contains demographic and administrative information for the National Health Services (NHS) patient in Wales.

- The General Practitioner (GP) dataset which contains primary care events, such as diagnoses, clinical findings, prescriptions codified in Read codes by general practitioners.

At the time of writing of this protocol, the most recent extract of the GP dataset was in March 2017, covering about $80 \%$ of GP surgeries in Wales. 


\section{Patient population}

The study sample will be randomly selected from the total population of Wales within the SAIL Databank in 2014. The sampling will be stratified by general practices to improve their representativeness. We will determine the sample size based on the computational capacity in the SAIL Databank which will be available for this study. The sampling frame will include all individuals who were aged at least 40 years on 1-1-2014.

\section{Latent class modelling}

LCA is a finite mixture modelling method that aims to divide a sample into classes or clusters related to a set of observed variables. ${ }^{24,29}$ LCA assumes that the patterns in these observed variables can be explained by, in addition to measurement errors, a hidden categorical variable that divides the sample into a pre-defined number of distinct classes.

In our study, we will construct observed variables from asthma- and COPD-related events recorded in the GP Dataset. The construction of observed variables will be based on their usefulness, from a clinical perspective, for identifying and distinguishing between patients with asthma and/or COPD. These variables will include diagnosis, GP visits, and prescriptions related to asthma and COPD, as well as history of allergy (including atopic eczema/dermatitis, food allergy, allergic rhinitis, and anaphylaxis) and smoking history (see Table 1). GP visits and prescriptions will be queried during 2014, while the other events will be queried in or any time before 2014 .

Model parameters include proportions of the latent classes, and probabilities of observing the levels of observed variables in each latent class, a.k.a item-response probabilities. Parameters are estimated by the expectation-maximisation (EM) algorithm, which iteratively searches for maximum-likelihood parameter values for which the data are more likely to be observed. ${ }^{30}$ Based on observed characteristics, each individual is assigned 
membership probability in each latent class, ${ }^{29}$ and is finally assigned to the latent class of maximum membership probability. ${ }^{31}$

We will begin the modelling for two latent classes and will then iteratively increase the numbers of latent classes. Model selection will be based on model diagnostics and interpretability.

We will look for a model for which the Bayesian Information Criterion (BIC) $)^{32,33}$ is ideally minimum, or becomes 'stabilised', indicating no significant improvement in information gain beyond a certain number of classes. In addition, the selected model should be clinically relevant; we will use the estimated item-response probabilities to assign labels consistent with "asthma", "COPD", "both" (ACOS), and "none" to the latent classes. We will use class shares as prevalence estimates for these clinical labels among the age groups of 40 and over in 2014.

LCA modelling will be performed using the R package poLCA (version 1.4.1, 2014). ${ }^{34}$

\section{Derivation of a classification algorithm}

Based on the LCA model, we will derive a classification algorithm to identify patients with asthma, COPD and ACOS according to their characteristics. To do so, we will perform recursive partitioning ${ }^{35}$ using the assigned latent classes as labels and the aforementioned observed variables as predictors. We will use the R package rpart (version 4.1-11, 2017) ${ }^{36}$ for this purpose.

\section{Comparison with other case definitions}

We will compare the LCA model and the derived classification algorithm with other objective and self-reported measures. As objective measures, we will use definitions used in the Quality of Outcomes Framework (QOF) 2014-2015 indicators for 'treated asthma' (AST001) and 'COPD' (COPDO01). ${ }^{37}$ From the Welsh Health Survey (WHS) 2014, ${ }^{38}$ we will use self- 
reported responses on current treatment of 'asthma', 'emphysema', and 'spells of bronchitis that have lasted over 3 years', with any of the latter two representing currently-treated COPD. We will treat invalid and missing responses as negative responses. We will perform the comparisons in the group of the WHS 2014 participants who were aged 40 years or over on 1-1-2014, and whose responses where successfully linked to the SAIL Databank. We will calculate diagnostic accuracy measures of the LCA model and the classification algorithm against each of the above case definitions and vice versa.

\section{Ethics, timeline and dissemination}

We obtained an approval to use the SAIL Databank from the Information Governance Review Panel. NHS Research Ethics Committee approval for this study is not required because we will only use anonymised data. The data extraction and statistical analysis will be performed between March and May 2018. The full paper will be submitted for publication in a respiratory care-related peer-reviewed journal in due course.

\section{Discussion}

While the interest in ACOS is growing, there is no consensus definition for this emerging and debated concept, ${ }^{39}$ leading to wide variations in prevalence and impaired comparability between studies. With the increasing use of EHR data to study asthma and COPD, it is important to develop operational definitions for ACOS based on such data. In this study, we will perform LCA on recorded events of diagnosis, prescriptions, and healthcare utilisation for asthma and COPD in routinely collected primary care data. By including observed variables for asthma and COPD in the same model, we will be able to identify patients with either or both conditions (i.e. ACOS).

An inherent limitation of routinely collected EHR data is the lack of vital pieces of information that are often used to make diagnoses at the point of care. Unlike diagnosis and prescriptions which are generally well coded, important diagnostic tests such as lung 
function and peripheral eosinophil count are often poorly and inconsistently recorded in primary care datasets. These missing data would have been potentially useful for improving the accuracy of our model. However, it is often difficult to assess data missingness in eventbased databases. The GP Dataset in the SAIL Databank is a long-format dataset, in which each row contains a dated code representing a single primary care event. The presence of a code usually indicates that the corresponding event occurred. However, when a code is absent, it is often impossible to ascertain whether the event did not occur or whether it was simply not recorded or coded. This is a particular challenge for events that are known to be poorly recorded. Therefore, since the quality of observed variables is essential in LCA, we will only include variables that are thought to be of reasonable quality in the SAIL Databank. In interpreting the results, we will consider the limitations of EHR-derived data such as the possibility of missing or incorrect codes and the changes in coding practices over time.

LCA itself has limitations. The construction of observed variables, model selection and interpretation involves a level of subjectivity. The model's interpretation and usefulness depends largely on the choice and structure of observed variables. In our LCA modelling, the clinical meaning of the latent classes will be based on surrogate variables, such as diagnosis, GP visits, and prescriptions, rather than on more direct disease markers such as clinical and laboratory findings. Nevertheless, we hypothesise that LCA of these surrogate variables can reasonably distinguish between patients with asthma, COPD, and ACOS. This will also provide an opportunity to assess how clustering based on these surrogate variables will perform compared with that based on disease markers. ${ }^{40-47}$ Comparing our LCA model and classification algorithm against other objective and self-reported measures will provide useful information about their validity and performance. 
Contributions: All authors contributed to refinement of the study protocol and manuscript writing and critically reviewed and approved the final manuscript. Mohammad A Al Sallakh developed the statistical methods.

Funding and Support: This work is funded by Health and Care Research Wales and Abertawe Bro Morgannwg University Health Board. It is carried out with the support of the Asthma UK Centre for Applied Research [AUK-AC-2012-01]. We also acknowledge the support from The Farr Institute of Health Informatics Research. The Farr Institute is supported by a 10-funder consortium: Arthritis Research UK, the British Heart Foundation, Cancer Research UK, the Economic and Social Research Council, the Engineering and Physical Sciences Research Council, the Medical Research Council, the National Institute of Health Research, the National Institute for Social Care and Health Research (Welsh Assembly Government), the Chief Scientist Office (Scottish Government Health Directorates), the Wellcome Trust, (MRC Grant Nos: CIPHER MR/K006525/1, Scotland MR/K007017/1).

Conflict of Interest: AS is the Editor-in-Chief of npj PCRM. The remaining authors declare no conflict of interest. 


\section{References}

1. Global Initiative for Asthma. Global Strategy for Asthma Management and Prevention (2017 update) (2017).

2. Global Initiative for Chronic Obstructive Lung Disease. Global strategy for prevention, diagnosis and management of COPD (2018).

3. Global Initiative for Asthma and Global Initiative for Chronic Obstructive Lung Disease. Diagnosis of diseases of chronic airflow limitation: asthma, COPD and asthma-COPD overlap syndrome (ACOS).

(2015).

4. Hargreave FE \& Nair P. The definition and diagnosis of asthma. Clin Exp Allergy 39, 1652-1658 (2009).

5. A plea to abandon asthma as a disease concept. Lancet 368, 705 (2006).

6. Bousquet J et al. Uniform definition of asthma severity, control, and exacerbations: document presented for the World Health Organization Consultation on Severe Asthma. J Allergy Clin Immunol 126, 926-938 (2010).

7. Reddel HK et al. A summary of the new GINA strategy: a roadmap to asthma control. Eur Respir J 46, 622639 (2015).

8. van den Akker IL, van der Zeijden H \& Verheij TJ. Is spirometry essential in diagnosing asthma? Yes. $\mathrm{Br} J$ Gen Pract 66, 484-484 (2016).

9. Levy ML. Is spirometry essential in diagnosing asthma? No. Br J Gen Pract 66, 485-485 (2016).

10. Brusasco V. Spirometric definition of COPD: exercise in futility or factual debate? Thorax $67,569-570$ (2012).

11. Vestbo J. COPD: Definition and Phenotypes. Clin Chest Med 35, 1-6 (2014).

12. Bateman ED, Reddel HK, van Zyl-Smit RN \& Agusti A. The asthma-COPD overlap syndrome: towards a revised taxonomy of chronic airways diseases? Lancet Respir Med 3, 719-728 (2015).

13. Bujarski S, Parulekar AD, Sharafkhaneh A \& Hanania NA. The asthma COPD overlap syndrome (ACOS). Curr Allergy Asthma Rep 15, 509 (2015).

14. McDonald VM \& Gibson PG. To define is to limit: perspectives on asthma-COPD overlap syndrome and personalised medicine. Eur Respir J 49, 1700336 (2017).

15. Miravitlles M. Diagnosis of asthma-COPD overlap: the five commandments. Eur Respir J 49, 1700506 (2017).

16. Ford ES. The epidemiology of obesity and asthma. J Allergy Clin Immunol Pract 115, 897-909, quiz 910 (2005).

17. Mukherjee $\mathrm{M}$ et al. The epidemiology, healthcare and societal burden and costs of asthma in the UK and its member nations: analyses of standalone and linked national databases. BMC Med 14 (2016). 
18. Bonten TN et al. Defining asthma-COPD overlap syndrome: a population-based study. Eur Respir J 49, 1602008 (2017).

19. Halbert R, Isonaka S, George D \& Iqbal A. Interpreting COPD Prevalence Estimates. Chest 123, 1684- 1692 (2003).

20. Viegi G et al. Definition, epidemiology and natural history of COPD. Eur Respir J 30, 993-1013 (2007).

21. Schneeweiss $S \&$ Avorn J. A review of uses of health care utilization databases for epidemiologic research on therapeutics. J Clin Epidemiol 58, 323-337 (2005).

22. Jorm L. Routinely collected data as a strategic resource for research: priorities for methods and workforce.

Public Health Research \& Practice 25 (2015).

23. Al Sallakh MA et al. Defining asthma and assessing asthma outcomes using electronic health record data: a systematic scoping review. Eur Respir $J 49$ (2017).

24. Howard R, Rattray M, Prosperi M \& Custovic A. Distinguishing Asthma Phenotypes Using Machine Learning Approaches. Curr Allergy Asthma Rep 15, 38 (2015).

25. von Elm E et al. The Strengthening the Reporting of Observational Studies in Epidemiology (STROBE) statement: guidelines for reporting observational studies. J Clin Epidemiol 61, 344-349 (2008).

26. Benchimol El et al. The REporting of studies Conducted using Observational Routinely-collected health Data (RECORD) Statement. PLoS Med 12, e1001885 (2015).

27. Lyons RA et al. The SAIL databank: linking multiple health and social care datasets. BMC Med Inform Decis Mak 9, 3 (2009).

28. Ford DV et al. The SAIL Databank: building a national architecture for e-health research and evaluation. BMC Health Serv Res 9, 157 (2009).

29. Linda M. Collins STL. Latent Class and Latent Transition Analysis: With Applications in the Social, Behavioral, and Health Sciences. 285 Seiten. ISBN: 0470228393 (John Wiley \& Sons Inc, 2010).

30. Dempster AP, Laird NM \& Rubin DB. Maximum likelihood from incomplete data via the EM algorithm. $J R$ Stat Soc Series B Stat Methodol, 1-38 (1977).

31. McLachlan G \& Peel D. Finite Mixture Models 1st ed. ISBN: 9780471006268 (Wiley-Interscience, 2000).

32. Schwarz G. Estimating the dimension of a model. Ann Stat 6, 461-464 (1978).

33. Nylund KL, Asparouhov T \& Muthén BO. Deciding on the number of classes in latent class analysis and growth mixture modeling: A Monte Carlo simulation study. Struct Equ Modeling 14, 535-569 (2007).

34. Linzer DA \& Lewis JB. poLCA: An R package for polytomous variable latent class analysis. J Stat Softw 42, 1-29 (2011).

35. Strobl C, Malley J \& Tutz G. An introduction to recursive partitioning: rationale, application, and characteristics of classification and regression trees, bagging, and random forests. Psychol Methods 14, 323-348 (2009). 
36. Therneau TM \& Atkinson EJ. An Introduction to Recursive Partitioning Using the RPART Routines (2015).

37. General Medical Services Contract: Quality and Outcomes Framework Statistics for Wales, 2014-15. Report. 2015.

38. Welsh Assembly Government. Welsh Health Survey 2014: Health status, illnesses, and other conditions (2015).

39. Rodrigo GJ, Neffen H \& Plaza V. Asthma-chronic obstructive pulmonary disease overlap syndrome: a controversial concept. Curr Opin Allergy Clin Immunol 17, 36-41 (2017).

40. Haldar P et al. Cluster analysis and clinical asthma phenotypes. Am J Respir Crit Care Med 178, 218-224 (2008).

41. Moore WC et al. Identification of asthma phenotypes using cluster analysis in the Severe Asthma Research Program. Am J Respir Crit Care Med 181, 315-323 (2010).

42. Garcia-Aymerich J et al. Phenotyping asthma, rhinitis and eczema in MeDALL population-based birth cohorts: an allergic comorbidity cluster. Allergy (2015).

43. Weatherall $\mathrm{M}$ et al. Distinct clinical phenotypes of airways disease defined by cluster analysis. Eur Respir J 34, 812-818 (2009).

44. Mäkikyrö EMS, Jaakkola MS \& Jaakkola JJK. Subtypes of asthma based on asthma control and severity: a latent class analysis. Respir Res 18 (2017).

45. Weinmayr $\mathrm{G}$ et al. Asthma phenotypes identified by latent class analysis in the ISAAC phase II Spain study. Clin Exp Allergy 43, 223-232 (2013).

46. Burgel PR et al. Clinical COPD phenotypes: a novel approach using principal component and cluster analyses. Eur Respir J 36, 531-539 (2010).

47. Ghebre MA et al. Biological clustering supports both Dutch and British hypotheses of asthma and chronic obstructive pulmonary disease. J Allergy Clin Immunol 135, 63-72.e10 (2015). 


\section{Appendix C}

\section{Chapter 4 Appendix}

C.1 SAIL Databank Datasets used in the Observatory 
Table C.1.1: Data fields of the datasets that were used for the development of the Wales Asthma Observatory. These metadata a the SAIL Databank in 2018.

\begin{tabular}{|c|c|c|c|c|c|c|}
\hline Column Name & Friendly Name & Type & $\begin{array}{l}\text { NULL } \\
\text { Count }\end{array}$ & NULL \% & $\begin{array}{l}\text { Distinct } \\
\text { Values }\end{array}$ & Description \\
\hline \multicolumn{7}{|c|}{ Welsh Demographic Service (WDS) - AR_PERS (Administrative Register - Persons) } \\
\hline pers_id_e & Encrypted Person Id & integer & 0 & 0 & 5218464 & $\begin{array}{l}\text { An encrypted unique identifier f } \\
\text { Demographic Service. }\end{array}$ \\
\hline alf_e & $\begin{array}{l}\text { Encrypted Anonymised } \\
\text { Linking Field }\end{array}$ & bigint & 0 & 0 & 5218464 & $\begin{array}{l}\text { The Anonymised Linking Field, } \\
\text { database, is derived from the p } \\
\text { encryption occurs in NWIS and } \\
\text { supplied in the data extract the }\end{array}$ \\
\hline wob & Week of Birth & date & 0 & 0 & 6880 & $\begin{array}{l}\text { The date of the Monday that occ } \\
\text { to this data item is limited, how } \\
\text { events. }\end{array}$ \\
\hline dod & Date of Death & date & 4394400 & 84.21 & 10079 & The date of death for the individ \\
\hline gndr_cd & $\begin{array}{l}\text { Gender code (also } \\
\text { known as sex) }\end{array}$ & character & 0 & 0 & 3 & This is the sex (gender) of perso \\
\hline avail_from_dt & Available from date & date & 0 & 0 & 1 & Date when the data made availa \\
\hline
\end{tabular}


Table C.1.1: SAIL Databank Datasets used in the Observatory (cont'd).

\begin{tabular}{|c|c|c|c|c|c|c|}
\hline Column Name & Friendly Name & Type & $\begin{array}{l}\text { NULL } \\
\text { Count }\end{array}$ & NULL \% & $\begin{array}{l}\text { Distinct } \\
\text { Values }\end{array}$ & Description \\
\hline \multicolumn{7}{|c|}{ Welsh Demographic Service (WDS) - AR_PERS_ADD (Administrative Register - Addresses) } \\
\hline pers_id_e & Encrypted Person Id & integer & 0 & 0 & 5240178 & $\begin{array}{l}\text { An encrypted unique identifier ff } \\
\text { Demographic Service. }\end{array}$ \\
\hline ralf_e & $\begin{array}{l}\text { Encrypted Residential } \\
\text { Anonymous Linking } \\
\text { Field }\end{array}$ & bigint & 1242454 & 8.79 & 1332673 & $\begin{array}{l}\text { The encrypted Residential Anony } \\
\text { derived from the persons posta } \\
\text { described in the paper "Resider } \\
\text { novel information infrastructur } \\
\text { environment and individuals he }\end{array}$ \\
\hline ralf_sts_cd & $\begin{array}{l}\text { Residential Anonymous } \\
\text { Linking } \\
\text { Field_status_code }\end{array}$ & character & 0 & 0 & 3 & The status code generated when \\
\hline uprn_qas_match_cd & $\begin{array}{l}\text { UPRN quality match } \\
\text { code }\end{array}$ & character & 134288 & 0.95 & 1260 & The UPRN (Unique Property Ref \\
\hline lsoa_cd & $\begin{array}{l}\text { Local Super Output } \\
\text { Area code }\end{array}$ & character & 1228010 & 8.69 & 1896 & The Local Super Output Area of \\
\hline row_sts & Row status & character & 0 & 0 & 2 & Row status code. \\
\hline from_dt & From date & date & 0 & 0 & 28413 & From date. \\
\hline to_dt & To date & date & 0 & 0 & 10428 & To date. \\
\hline avail_from_dt & Available from date & date & 0 & 0 & 1 & Date when the data made availa \\
\hline \multicolumn{7}{|c|}{ Welsh Demographic Service (WDS) - AR_PERS_GP (Administrative Register - GP registration } \\
\hline pers_id_e & Encrypted Person Id & integer & 0 & 0 & 5240179 & $\begin{array}{l}\text { An encrypted unique identifier f } \\
\text { Demographic Service. }\end{array}$ \\
\hline prac_cd_e & $\begin{array}{l}\text { Encrypted GP practice } \\
\text { code }\end{array}$ & integer & 177360 & 1.25 & 663 & The encrypted GP practice code \\
\hline row_sts & Row status & character & 0 & 0 & 2 & Row status code. \\
\hline
\end{tabular}


Table C.1.1: SAIL Databank Datasets used in the Observatory (cont'd).

\begin{tabular}{|c|c|c|c|c|c|c|}
\hline Column Name & Friendly Name & Type & $\begin{array}{l}\text { NULL } \\
\text { Count }\end{array}$ & NULL \% & $\begin{array}{l}\text { Distinct } \\
\text { Values }\end{array}$ & Description \\
\hline from_dt & From date & date & 0 & 0 & 30723 & From date. \\
\hline to_dt & To date & date & 0 & 0 & 12754 & To date. \\
\hline avail_from_dt & Available from date & date & 0 & 0 & 1 & Date when the data made availa \\
\hline
\end{tabular}


Table C.1.1: SAIL Databank Datasets used in the Observatory (cont'd).

\begin{tabular}{|c|c|c|c|c|c|c|}
\hline Column Name & Friendly Name & Type & $\begin{array}{l}\text { NULL } \\
\text { Count }\end{array}$ & NULL \% & $\begin{array}{l}\text { Distinct } \\
\text { Values }\end{array}$ & Description \\
\hline \multicolumn{7}{|c|}{ Welsh Longitudinal General Practice (WLGP) } \\
\hline alf_e & $\begin{array}{l}\text { Encrypted Anonymised } \\
\text { Linking Field }\end{array}$ & bigint & 3462255 & 0.15 & 4016617 & $\begin{array}{l}\text { The Anonymised Linking Field, } \\
\text { database, is derived from the p } \\
\text { encryption occurs in NWIS and } \\
\text { supplied in the data extract the }\end{array}$ \\
\hline alf_sts_cd & ALF status code & character & 0 & 0 & 5 & Status code assigned when deriv \\
\hline alf_mtch_pct & ALF match percentage & decimal & 2367210983 & 99.68 & 90859 & $\begin{array}{l}\text { Match percentage assigned whe } \\
\text { Field. }\end{array}$ \\
\hline prac_cd_e & $\begin{array}{l}\text { Encrypted GP practice } \\
\text { code }\end{array}$ & integer & 0 & 0 & 340 & The encrypted GP practice code \\
\hline local_num_e & NA & bigint & 0 & 0 & 274452 & NA \\
\hline gndr_cd & $\begin{array}{l}\text { Gender code (also } \\
\text { known as sex) }\end{array}$ & character & 0 & 0 & 3 & This is the sex (gender) of perso \\
\hline wob & Week of Birth & date & 0 & 0 & 6857 & $\begin{array}{l}\text { The date of the Monday that occ } \\
\text { to this data item is limited, how } \\
\text { events. }\end{array}$ \\
\hline lsoa_cd & $\begin{array}{l}\text { Local Super Output } \\
\text { Area code }\end{array}$ & character & 18212565 & 0.77 & 6606 & The Local Super Output Area of \\
\hline reg_cat_cd & $\begin{array}{l}\text { Registration category } \\
\text { code }\end{array}$ & character & 0 & 0 & 36 & This denotes the registration sta \\
\hline event_dt & Event date & date & 0 & 0 & 52003 & The date the event occurred. \\
\hline event_yr & Event year & smallint & 0 & 0 & 273 & The year the event occurred. \\
\hline event_cd_vrs & Event code version & character & 0 & 0 & 2 & $\begin{array}{l}\text { This denotes the coding classific } \\
\text { collated from different GP prac } \\
\text { are a variety of coding classific } \\
\text { use Read Code version } 2 \text {. }\end{array}$ \\
\hline
\end{tabular}


Table C.1.1: SAIL Databank Datasets used in the Observatory (cont'd).

\begin{tabular}{|c|c|c|c|c|c|c|}
\hline Column Name & Friendly Name & Type & $\begin{array}{l}\text { NULL } \\
\text { Count }\end{array}$ & NULL \% & $\begin{array}{l}\text { Distinct } \\
\text { Values }\end{array}$ & Description \\
\hline event_cd & Event code & character & 0 & 0 & 267925 & $\begin{array}{l}\text { This code documents clinical inf } \\
\text { majority of practices this will b } \\
\text { description denoting a sign, syr }\end{array}$ \\
\hline event_val & Event value & decimal & 1048003348 & 44.13 & 118407 & $\begin{array}{l}\text { This value is associated to the } \mathrm{E} \\
\text { pressure or the number of tabl }\end{array}$ \\
\hline episode & Episode & character & 0 & 0 & 6 & This denotes the type of episode \\
\hline sequence & Sequence & integer & 0 & 0 & 108853875 & $\begin{array}{l}\text { This numeric sequence denotes } \\
\text { event. For example if a blood p } \\
\text { within an appointment. }\end{array}$ \\
\hline
\end{tabular}


Table C.1.1: SAIL Databank Datasets used in the Observatory (cont'd).

\begin{tabular}{|c|c|c|c|c|c|c|}
\hline Column Name & Friendly Name & Type & $\begin{array}{l}\text { NULL } \\
\text { Count }\end{array}$ & NULL \% & $\begin{array}{l}\text { Distinct } \\
\text { Values }\end{array}$ & Description \\
\hline \multicolumn{7}{|c|}{ Patient Episode Database for Wales (PEDW) - SPELL } \\
\hline prov_unit_cd & Provider unit code & character & 0 & 0 & 662 & $\begin{array}{l}\text { This is the organisation code of } \\
\text { identifies the health care provic } \\
\text { treatment of the patient. }\end{array}$ \\
\hline spell_num_e & Encrypted spell number & integer & $<5$ & 0 & 18555146 & $\begin{array}{l}\text { A number (alphanumeric) to pro } \\
\text { provider spell for a health care }\end{array}$ \\
\hline gndr_cd & $\begin{array}{l}\text { Gender code (also } \\
\text { known as sex) }\end{array}$ & character & 0 & 0 & 7 & This is the sex (gender) of perso \\
\hline res_dha_cd & $\begin{array}{l}\text { District health } \\
\text { authority of residence } \\
\text { code }\end{array}$ & character & 30526 & 0.16 & 1276 & The District Health Authority in \\
\hline admis_yr & Admission year & character & 72 & 0 & 104 & The year of the beginning of a $\mathrm{h}$ \\
\hline admis_dt & Admission date & date & 72 & 0 & 10242 & $\begin{array}{l}\text { This is the beginning of a hospit } \\
\text { consultant has assumed respon } \\
\text { the patient. This may be before } \\
\text { completed and the patient is tre }\end{array}$ \\
\hline fin_admis_yr & $\begin{array}{l}\text { Financial admission } \\
\text { year }\end{array}$ & character & 72 & 0 & 102 & $\begin{array}{l}\text { The financial year of the beginni } \\
\text { admission. }\end{array}$ \\
\hline admis_mthd_cd & Admission method code & character & 974 & 0.01 & 21 & This is the method of admission \\
\hline admis_source_cd & Admission source code & character & 8550 & 0.04 & 49 & This is the source of admission $t$ \\
\hline $\begin{array}{l}\text { intended_manage- } \\
\text { ment_cd }\end{array}$ & $\begin{array}{l}\text { Intended management } \\
\text { code }\end{array}$ & character & 1930 & 0.01 & 8 & $\begin{array}{l}\text { The intended pattern of bed use } \\
\text { made to admit, and only applies } \\
\text { This categorization describes } \\
\text { Occasionally the patients treatr } \\
\text { patient admitted as a day case } \\
\text { in overnight. Therefore another } \\
\text { describe what actually happens }\end{array}$ \\
\hline
\end{tabular}


Table C.1.1: SAIL Databank Datasets used in the Observatory (cont'd).

\begin{tabular}{|c|c|c|c|c|c|c|}
\hline Column Name & Friendly Name & Type & $\begin{array}{l}\text { NULL } \\
\text { Count }\end{array}$ & NULL \% & $\begin{array}{l}\text { Distinct } \\
\text { Values }\end{array}$ & Description \\
\hline disch_yr & Discharge year & character & 21984 & 0.11 & 31 & $\begin{array}{l}\text { The year of discharge from the } \mathrm{F} \\
\text { patient dies or is discharged frc } \\
\text { bed(s) within a single hospital } \\
\text { consultant episode of care and }\end{array}$ \\
\hline disch_dt & Discharge date & date & 21983 & 0.11 & 9481 & $\begin{array}{l}\text { Date of discharge from the Hosp } \\
\text { dies or is discharged from a cor } \\
\text { within a single hospital provide } \\
\text { consultant episode of care and }\end{array}$ \\
\hline fin_disch_yr & $\begin{array}{l}\text { Financial discharge } \\
\text { year }\end{array}$ & character & 21984 & 0.11 & 30 & $\begin{array}{l}\text { The financial year of the end of } \\
\text { discharge. }\end{array}$ \\
\hline disch_mthd_cd & Discharge method code & character & 0 & 0 & 8 & This is the method of discharge \\
\hline disch_destination_cd & $\begin{array}{l}\text { Discharge destination } \\
\text { code }\end{array}$ & character & 30548 & 0.16 & 75 & $\begin{array}{l}\text { The classification of where a pat } \\
\text { spell, or a note that the patient }\end{array}$ \\
\hline dur_elect_wait & $\begin{array}{l}\text { Duration of elective } \\
\text { wait }\end{array}$ & decimal & 9064942 & 46.83 & 3271 & $\begin{array}{l}\text { This is the waiting time from the } \\
\text { where the treatment actually ta }\end{array}$ \\
\hline pat_class_cd & $\begin{array}{l}\text { Patient classification } \\
\text { code }\end{array}$ & character & 5833 & 0.03 & 9 & $\begin{array}{l}\text { A coded classification of Patients } \\
\text { Provider Spell. }\end{array}$ \\
\hline spell_dur & Spell duration & integer & 22093 & 0.11 & 3163 & $\begin{array}{l}\text { The period of time in days betwe } \\
\text { discharge date of the provider }\end{array}$ \\
\hline admis_spec_cd & $\begin{array}{l}\text { Admission speciality } \\
\text { code }\end{array}$ & character & 0 & 0 & 145 & $\begin{array}{l}\text { This is the specialty under which } \\
\text { either be the same as the speci } \\
\text { specialty or a different specialty } \\
\text { specialty function. Note that bo } \\
\text { specialty function should be ba }\end{array}$ \\
\hline disch_spec_cd & $\begin{array}{l}\text { Discharge speciality } \\
\text { code }\end{array}$ & character & 0 & 0 & 146 & $\begin{array}{l}\text { This is the specialty under which } \\
\text { either be the same as the speci } \\
\text { specialty or a different specialty } \\
\text { specialty function. Note that bo } \\
\text { specialty function should be ba }\end{array}$ \\
\hline ua_cd & Unitary Authority code & character & 62105 & 0.32 & 196 & The unitary authority in which tl \\
\hline
\end{tabular}


Table C.1.1: SAIL Databank Datasets used in the Observatory (cont'd).

\begin{tabular}{|c|c|c|c|c|c|c|}
\hline Column Name & Friendly Name & Type & $\begin{array}{l}\text { NULL } \\
\text { Count }\end{array}$ & NULL \% & $\begin{array}{l}\text { Distinct } \\
\text { Values }\end{array}$ & Description \\
\hline res_ward_cd & Ward code of residence & character & 86052 & 0.44 & 320 & The electoral ward in which the \\
\hline reg_gp_cd_e & $\begin{array}{l}\text { Encrypted registered } \\
\text { GP code }\end{array}$ & integer & 22083 & 0.11 & 42463 & The encrypted unique GP Practi \\
\hline reg_gp_prac_cd_e & $\begin{array}{l}\text { Encrypted registered } \\
\text { GP practice code }\end{array}$ & integer & 1239686 & 6.4 & 10192 & The encrypted unique GP Practi \\
\hline ref_cd_e & $\begin{array}{l}\text { Encrypted referrer } \\
\text { code }\end{array}$ & integer & 897630 & 4.64 & 47174 & $\begin{array}{l}\text { This is the nationally recognized } \\
\text { may be a General Medical Prac } \\
\text { (GDP), Consultant or Independ } \\
\text { GDP, Consultant or Independen }\end{array}$ \\
\hline ref_org_cd & $\begin{array}{l}\text { Referring organisation } \\
\text { code }\end{array}$ & character & 1054418 & 5.45 & 11670 & The code of the referring organi \\
\hline admis_dec_dt & $\begin{array}{l}\text { Admission decision } \\
\text { date }\end{array}$ & date & 4919073 & 25.41 & 10071 & This is the date upon which the \\
\hline local_health_grp_cd & Local health group code & integer & 19357775 & 100 & 0 & The local health group associate \\
\hline curr_prov_unit_cd & $\begin{array}{l}\text { Current provider unit } \\
\text { code }\end{array}$ & character & 0 & 0 & 333 & $\begin{array}{l}\text { The current organisation code o } \\
\text { identifies the health care provi } \\
\text { treatment of the patient. To en } \\
\text { years, a current field can be us } \\
\text { version/name. }\end{array}$ \\
\hline curr_res_dha_cd & $\begin{array}{l}\text { Current district health } \\
\text { authority of residence } \\
\text { code }\end{array}$ & character & 199 & 0 & 312 & $\begin{array}{l}\text { The current District Health Auth } \\
\text { longtiduninal analysis over a p } \\
\text { map previously used codes to }\end{array}$ \\
\hline $\begin{array}{l}\text { curr_lo- } \\
\text { cal_health_grp_cd }\end{array}$ & $\begin{array}{l}\text { Current local health } \\
\text { group code }\end{array}$ & integer & 19357775 & 100 & 0 & $\begin{array}{l}\text { The current local health group } \\
\text { enable longtiduninal analysis o } \\
\text { used to map previously used co }\end{array}$ \\
\hline curr_ua_cd & $\begin{array}{l}\text { Current Unitary } \\
\text { Authority code }\end{array}$ & character & 62105 & 0.32 & 196 & $\begin{array}{l}\text { The current unitary authority in } \\
\text { longtiduninal analysis over a p } \\
\text { map previously used codes to }\end{array}$ \\
\hline
\end{tabular}


Table C.1.1: SAIL Databank Datasets used in the Observatory (cont'd).

\begin{tabular}{|c|c|c|c|c|c|c|}
\hline Column Name & Friendly Name & Type & $\begin{array}{l}\text { NULL } \\
\text { Count }\end{array}$ & NULL \% & $\begin{array}{l}\text { Distinct } \\
\text { Values }\end{array}$ & Description \\
\hline curr_res_ward_cd & $\begin{array}{l}\text { Current ward residence } \\
\text { code }\end{array}$ & character & 86052 & 0.44 & 320 & $\begin{array}{l}\text { The current electoral ward in wl } \\
\text { longtiduninal analysis over a pe } \\
\text { map previously used codes to c }\end{array}$ \\
\hline pat_id_e & $\begin{array}{l}\text { Encrypted patient } \\
\text { identifier }\end{array}$ & integer & 0 & 0 & 3923115 & An encrypted unique Patient Ide \\
\hline alf_e & $\begin{array}{l}\text { Encrypted Anonymised } \\
\text { Linking Field }\end{array}$ & bigint & 1026066 & 5.3 & 3228910 & $\begin{array}{l}\text { The Anonymised Linking Field, } \\
\text { database, is derived from the p } \\
\text { encryption occurs in NWIS and } \\
\text { supplied in the data extract the }\end{array}$ \\
\hline case_rec_num_e & $\begin{array}{l}\text { Encrypted casenote } \\
\text { record number }\end{array}$ & integer & 3211 & 0.02 & 4964329 & $\begin{array}{l}\text { This is the case record number. } \\
\text { health care provider. }\end{array}$ \\
\hline alf_sts_cd & ALF status code & character & 0 & 0 & 5 & Status code assigned when deri \\
\hline alf_mtch_pct & ALF match percentage & decimal & 19057851 & 98.45 & 36332 & $\begin{array}{l}\text { Match percentage assigned whe } \\
\text { Field. }\end{array}$ \\
\hline hrg_localpayment_cd & $\begin{array}{l}\text { HRG local payment } \\
\text { code }\end{array}$ & varchar & 11598773 & 59.92 & 1918 & The Healthcare Resource Group \\
\hline hrg_localpayment_desc & $\begin{array}{l}\text { HRG local payment } \\
\text { description }\end{array}$ & varchar & 0 & 0 & 3079 & $\begin{array}{l}\text { The Healthcare Resource Group } \\
\text { spell. }\end{array}$ \\
\hline hrg_referencecost_cd & $\begin{array}{l}\text { HRG reference cost } \\
\text { code }\end{array}$ & varchar & 11601219 & 59.93 & 4439 & The Healthcare Resource Group \\
\hline hrg_referencecost_desc & $\begin{array}{l}\text { HRG reference cost } \\
\text { description }\end{array}$ & varchar & 0 & 0 & 6152 & $\begin{array}{l}\text { The Healthcare Resource Group } \\
\text { overall spell }\end{array}$ \\
\hline hrg_v31_cd & HRG version 31 code & varchar & 5887305 & 30.41 & 572 & Healthcare Resource Group cod \\
\hline hrg_v31_desc & $\begin{array}{l}\text { HRG version } 31 \\
\text { description }\end{array}$ & varchar & 0 & 0 & 572 & $\begin{array}{l}\text { Healthcare Resource Group cod } \\
\text { codes (v3.1) }\end{array}$ \\
\hline hrg_v35_cd & HRG version 35 code & varchar & 5886058 & 30.41 & 610 & Healthcare Resource Group cod \\
\hline
\end{tabular}


Table C.1.1: SAIL Databank Datasets used in the Observatory (cont'd).

\begin{tabular}{|c|c|c|c|c|c|c|}
\hline Column Name & Friendly Name & Type & $\begin{array}{l}\text { NULL } \\
\text { Count }\end{array}$ & NULL \% & $\begin{array}{l}\text { Distinct } \\
\text { Values }\end{array}$ & Description \\
\hline hrg_v35_desc & $\begin{array}{l}\text { HRG version } 31 \\
\text { description }\end{array}$ & varchar & 0 & 0 & 609 & $\begin{array}{l}\text { Healthcare Resource Group cod } \\
\text { codes (v3.5) }\end{array}$ \\
\hline lsoa_cd_2001 & $\begin{array}{l}\text { Local Super Output } \\
\text { Area code } 2001\end{array}$ & character & 92527 & 0.48 & 34261 & The Local Super Output Area of \\
\hline avail_from_dt & Available from date & date & 0 & 0 & 1 & Date when the data made availa \\
\hline \multicolumn{7}{|c|}{ Patient Episode Database for Wales (PEDW) - EPISODE } \\
\hline prov_unit_cd & Provider unit code & character & 0 & 0 & 663 & $\begin{array}{l}\text { This is the organisation code of } \\
\text { identifies the health care provic } \\
\text { treatment of the patient. }\end{array}$ \\
\hline spell_num_e & Encrypted spell number & integer & $<5$ & 0 & 18555146 & $\begin{array}{l}\text { A number (alphanumeric) to pro } \\
\text { provider spell for a health care }\end{array}$ \\
\hline epi_num & Episode number & character & 0 & 0 & 99 & $\begin{array}{l}\text { A number used to identify episo } \\
\text { each consultant episode in a ho }\end{array}$ \\
\hline epi_str_yr & Episode start year & character & 573 & 0 & 101 & $\begin{array}{l}\text { The year of the start of a stay, ar } \\
\text { period of time. }\end{array}$ \\
\hline epi_str_dt & Episode start date & date & 573 & 0 & 10084 & $\begin{array}{l}\text { This is the start date of a stay, ar } \\
\text { period of time. }\end{array}$ \\
\hline epi_end_yr & Episode end year & character & 0 & 0 & 29 & $\begin{array}{l}\text { The year of the end of a stay, an } \\
\text { period of time. }\end{array}$ \\
\hline epi_end_dt & Episode end date & date & 0 & 0 & 9477 & $\begin{array}{l}\text { This is the end of a stay, an episc } \\
\text { time. }\end{array}$ \\
\hline prov_site_cd & Provider site code & character & 0 & 0 & 1949 & $\begin{array}{l}\text { This is the organisation code of } \\
\text { identifies the health care provic } \\
\text { treatment of the patient. }\end{array}$ \\
\hline age_epi_str_yr & Age at start of episode & integer & 3198 & 0.01 & 196 & The age of patient at start date 0 \\
\hline age_epi_str_under1 & $\begin{array}{l}\text { Age at start of episode } \\
\text { for under } 1 \mathrm{~s}\end{array}$ & integer & 21632323 & 99.3 & 182 & $\begin{array}{l}\text { Is the age of patient at start date } \\
\text { episode. }\end{array}$ \\
\hline
\end{tabular}


Table C.1.1: SAIL Databank Datasets used in the Observatory (cont'd).

\begin{tabular}{|c|c|c|c|c|c|c|}
\hline Column Name & Friendly Name & Type & $\begin{array}{l}\text { NULL } \\
\text { Count }\end{array}$ & NULL \% & $\begin{array}{l}\text { Distinct } \\
\text { Values }\end{array}$ & Description \\
\hline con_spec_main_cd & $\begin{array}{l}\text { Consultant main } \\
\text { speciality code }\end{array}$ & character & 7507 & 0.03 & 162 & $\begin{array}{l}\text { A unique code identifying each } \\
\text { Colleges.Specialties are divisio } \\
\text { body systems (dermatology), ac } \\
\text { medicine), clinical function (rh } \\
\text { combinations of these factors. } \\
\text { Colleges and Faculties should b } \\
\text { and Specialist Medical Practice } \\
2003 \text { and European Primary an } \\
1998 \text {. }\end{array}$ \\
\hline con_spec_cd_of_treat & $\begin{array}{l}\text { Consultant treatment } \\
\text { speciality code }\end{array}$ & character & 0 & 0 & 148 & $\begin{array}{l}\text { This is the specialty under which } \\
\text { either be the same as the speci } \\
\text { specialty or a different specialt } \\
\text { specialty function. Note that bo } \\
\text { specialty function should be ba }\end{array}$ \\
\hline epi_dur & Episode duration & decimal & 645 & 0 & 2879 & $\begin{array}{l}\text { The period of time in days betwe } \\
\text { the End Date of Consultant Epi } \\
\text { in days between the Start Date } \\
\text { period for unfinished episodes. }\end{array}$ \\
\hline diag_cd_123 & $\begin{array}{l}\text { Diagnosis code (3 } \\
\text { digits) }\end{array}$ & varchar & 689333 & 3.16 & 2415 & The first 3 digits of the Diagnost \\
\hline diag_cd_4 & $\begin{array}{l}\text { Diagnosis code (4th } \\
\text { digit) }\end{array}$ & varchar & 693337 & 3.18 & 13 & The 4 th digit of the Diagnostic c \\
\hline diag_cd_1234 & $\begin{array}{l}\text { Diagnosis code (4 } \\
\text { digits) }\end{array}$ & varchar & 693337 & 3.18 & 10963 & The first 4 digits of the Diagnost \\
\hline oper_cd_123 & $\begin{array}{l}\text { Operation code ( } 3 \\
\text { digits) }\end{array}$ & varchar & 9834137 & 45.14 & 1449 & The first 3 digits of the Procedur \\
\hline oper_cd_4 & $\begin{array}{l}\text { Operation code (4th } \\
\text { digits) }\end{array}$ & varchar & 9847548 & 45.21 & 17 & The 4th digit of the Procedure $\mathrm{c}$ \\
\hline oper_cd & Operation code & varchar & 9847548 & 45.21 & 7869 & The full Procedure code availabl \\
\hline
\end{tabular}


Table C.1.1: SAIL Databank Datasets used in the Observatory (cont'd).

\begin{tabular}{|c|c|c|c|c|c|c|}
\hline Column Name & Friendly Name & Type & $\begin{array}{l}\text { NULL } \\
\text { Count }\end{array}$ & NULL \% & $\begin{array}{l}\text { Distinct } \\
\text { Values }\end{array}$ & Description \\
\hline curr_prov_unit_cd & $\begin{array}{l}\text { Current provider unit } \\
\text { code }\end{array}$ & varchar & 0 & 0 & 333 & $\begin{array}{l}\text { The current organisation code o } \\
\text { identifies the health care provic } \\
\text { treatment of the patient. To en } \\
\text { years, a current field can be us } \\
\text { version/name. }\end{array}$ \\
\hline curr_prov_site_cd & $\begin{array}{l}\text { Current provider site } \\
\text { code }\end{array}$ & varchar & 0 & 0 & 1291 & $\begin{array}{l}\text { The current organisation code o } \\
\text { identifies the health care provic } \\
\text { treatment of the patient. To en } \\
\text { years, a current field can be us } \\
\text { version/name. }\end{array}$ \\
\hline fin_epi_end_yr & $\begin{array}{l}\text { Financial episode end } \\
\text { year }\end{array}$ & character & 0 & 0 & 28 & The financial year of the end of \\
\hline hsw_first_epi_in_spell & $\begin{array}{l}\text { HSW first episode in } \\
\text { spell }\end{array}$ & integer & 21784142 & 100 & 0 & Flag to show if an episode is firs \\
\hline site_cd_of_treat & Site code of treatment & character & 3348076 & 15.37 & 1885 & \\
\hline gmc_con_cd_e & $\begin{array}{l}\text { Encrypted GMC } \\
\text { consultant code }\end{array}$ & integer & 519033 & 2.38 & 36175 & $\begin{array}{l}\text { Nationally agreed form for const } \\
\text { General Medical Council (GMC } \\
\text { Consultant or locum Consultan } \\
\text { Registration Number will be us }\end{array}$ \\
\hline ua_cd & Unitary Authority code & character & 70916 & 0.33 & 196 & The unitary authority in which tl \\
\hline hrg_localpayment_cd & $\begin{array}{l}\text { HRG local payment } \\
\text { code }\end{array}$ & varchar & 12752672 & 58.54 & 4441 & The Healthcare Resource Group \\
\hline hrg_localpayment_desc & $\begin{array}{l}\text { HRG local payment } \\
\text { description }\end{array}$ & varchar & 0 & 0 & 6155 & $\begin{array}{l}\text { The Healthcare Resource Group } \\
\text { spell. }\end{array}$ \\
\hline hrg_referencecost_cd & $\begin{array}{l}\text { HRG reference cost } \\
\text { code }\end{array}$ & varchar & 12702016 & 58.31 & 1920 & The Healthcare Resource Group \\
\hline hrg_referencecost_desc & $\begin{array}{l}\text { HRG reference cost } \\
\text { description }\end{array}$ & varchar & 0 & 0 & 3083 & $\begin{array}{l}\text { The Healthcare Resource Group } \\
\text { overall spell }\end{array}$ \\
\hline hrg_v31_cd & HRG version 31 code & varchar & 6604546 & 30.32 & 572 & Healthcare Resource Group cod \\
\hline
\end{tabular}


Table C.1.1: SAIL Databank Datasets used in the Observatory (cont'd).

\begin{tabular}{|c|c|c|c|c|c|c|}
\hline Column Name & Friendly Name & Type & $\begin{array}{l}\text { NULL } \\
\text { Count }\end{array}$ & NULL \% & $\begin{array}{l}\text { Distinct } \\
\text { Values }\end{array}$ & Description \\
\hline hrg_v31_desc & $\begin{array}{l}\text { HRG version } 31 \\
\text { description }\end{array}$ & varchar & 0 & 0 & 572 & $\begin{array}{l}\text { Healthcare Resource Group cod } \\
\text { codes (v3.1) }\end{array}$ \\
\hline hrg_v35_cd & HRG version 35 code & varchar & 6603156 & 30.31 & 610 & Healthcare Resource Group cod \\
\hline hrg_v35_desc & $\begin{array}{l}\text { HRG version } 31 \\
\text { description }\end{array}$ & varchar & 0 & 0 & 609 & $\begin{array}{l}\text { Healthcare Resource Group cod } \\
\text { codes (v3.5) }\end{array}$ \\
\hline avail_from_dt & Available from date & date & 0 & 0 & 1 & Date when the data made availa \\
\hline \multicolumn{7}{|c|}{ Patient Episode Database for Wales (PEDW) - DIAG } \\
\hline prov_unit_cd & Provider unit code & character & 0 & 0 & 642 & $\begin{array}{l}\text { This is the organisation code of } \\
\text { identifies the health care provic } \\
\text { treatment of the patient. }\end{array}$ \\
\hline spell_num_e & Encrypted spell number & integer & $<5$ & 0 & 18129197 & $\begin{array}{l}\text { A number (alphanumeric) to pro } \\
\text { provider spell for a health care }\end{array}$ \\
\hline epi_num & Episode number & character & 0 & 0 & 99 & $\begin{array}{l}\text { A number used to identify episo } \\
\text { each consultant episode in a ho }\end{array}$ \\
\hline diag_num & Diagnosis number & integer & 0 & 0 & 14 & $\begin{array}{l}\text { A number used to identify the pc } \\
1 \text { relates to the primary ICD Di } \\
\text { ICD diagnositic codes. }\end{array}$ \\
\hline diag_cd_123 & $\begin{array}{l}\text { Diagnosis code ( } 3 \\
\text { digits) }\end{array}$ & character & 0 & 0 & 2668 & The first 3 digits of the Diagnost \\
\hline diag_cd_4 & $\begin{array}{l}\text { Diagnosis code (4th } \\
\text { digit) }\end{array}$ & character & 81534 & 0.11 & 33 & The 4 th digit of the Diagnostic c \\
\hline diag_cd_56 & $\begin{array}{l}\text { Diagnosis code (5th } \\
\text { and 6th digits) }\end{array}$ & character & 65323041 & 91.52 & 262 & The 5th and 6th digit of the Diag \\
\hline diag_cd_1234 & $\begin{array}{l}\text { Diagnosis code (4 } \\
\text { digits) }\end{array}$ & character & 0 & 0 & 15420 & The first 4 digits of the Diagnost \\
\hline diag_cd & Diagnosis code & character & 0 & 0 & 40086 & The full Diagnostic code availabl \\
\hline
\end{tabular}


Table C.1.1: SAIL Databank Datasets used in the Observatory (cont'd).

\begin{tabular}{|c|c|c|c|c|c|c|}
\hline Column Name & Friendly Name & Type & $\begin{array}{l}\text { NULL } \\
\text { Count }\end{array}$ & NULL \% & $\begin{array}{l}\text { Distinct } \\
\text { Values }\end{array}$ & Description \\
\hline avail_from_dt & Available from date & date & 0 & 0 & 1 & Date when the data made availa \\
\hline \multicolumn{7}{|c|}{ Emergency Department Data Set (EDDS) } \\
\hline record_id & Record ID & character & 8506326 & 100 & 0 & This is the field to identify the ty \\
\hline prov_unit_cd & Provider unit code & character & 0 & 0 & 14 & $\begin{array}{l}\text { This is the organisation code of } \\
\text { identifies the health care provic } \\
\text { treatment of the patient. }\end{array}$ \\
\hline prov_site_cd & Provider site code & character & 0 & 0 & 23 & $\begin{array}{l}\text { This is the organisation code of } \\
\text { identifies the health care provic } \\
\text { treatment of the patient. }\end{array}$ \\
\hline admin_arr_dt & $\begin{array}{l}\text { Administrative arrival } \\
\text { date }\end{array}$ & date & 0 & 0 & 3327 & $\begin{array}{l}\text { Accident and Emergency Attend } \\
\text { Accident and Emergency recep } \\
\text { has arrived and needs to be see } \\
\text { Department. Notification could } \\
\text { themselves, or a person accomp }\end{array}$ \\
\hline admin_arr_tm & $\begin{array}{l}\text { Administrative arrival } \\
\text { time }\end{array}$ & time & 0 & 0 & 1440 & $\begin{array}{l}\text { Accident and Emergency Attend } \\
\text { Accident and Emergency recep } \\
\text { has arrived and needs to be se } \\
\text { Department. Notification could } \\
\text { themselves, or a person accom }\end{array}$ \\
\hline alf_e & $\begin{array}{l}\text { Encrypted Anonymised } \\
\text { Linking Field }\end{array}$ & bigint & 272963 & 3.21 & 2537941 & $\begin{array}{l}\text { The Anonymised Linking Field, } \\
\text { database, is derived from the p } \\
\text { encryption occurs in NWIS and } \\
\text { supplied in the data extract the }\end{array}$ \\
\hline nhs_no_ind & NA & character & 636636 & 7.48 & 30 & NA \\
\hline prac_cd_e & $\begin{array}{l}\text { Encrypted GP practice } \\
\text { code }\end{array}$ & integer & 189972 & 2.23 & 12840 & The encrypted GP practice code \\
\hline dob_year & Year of Date of Birth & character & 1168 & 0.01 & 128 & The year of the Date of birth of \\
\hline
\end{tabular}


Table C.1.1: SAIL Databank Datasets used in the Observatory (cont'd).

\begin{tabular}{|c|c|c|c|c|c|c|}
\hline Column Name & Friendly Name & Type & $\begin{array}{l}\text { NULL } \\
\text { Count }\end{array}$ & NULL \% & $\begin{array}{l}\text { Distinct } \\
\text { Values }\end{array}$ & Description \\
\hline age & $\begin{array}{l}\text { Age at start of } \\
\text { admission }\end{array}$ & smallint & 1168 & 0.01 & 143 & The age of patient at start date \\
\hline sex & $\begin{array}{l}\text { Sex (also known as } \\
\text { gender code) }\end{array}$ & character & 414 & 0 & 7 & This is the sex (gender) of perso \\
\hline lsoa_cd & $\begin{array}{l}\text { Local Super Output } \\
\text { Area code }\end{array}$ & character & 211271 & 2.48 & 35994 & The Local Super Output Area of \\
\hline ref_cd_e & NA & bigint & 472888 & 5.56 & 23123 & NA \\
\hline arrival_mode & Mode of arrival & character & 101884 & 1.2 & 18 & $\begin{array}{l}\text { The principal means by which a } \\
\text { department. }\end{array}$ \\
\hline amb_incid_no_e & $\begin{array}{l}\text { Encrypted ambulance } \\
\text { incident number }\end{array}$ & integer & 7357724 & 86.5 & 1059762 & $\begin{array}{l}\text { When a patient arrives by ambul } \\
\text { journey, allocated by the Ambul }\end{array}$ \\
\hline site_cd_of_treat & Site code of treatment & character & 0 & 0 & 51 & \\
\hline health_event_dt & Health event date & date & 1784422 & 20.98 & 4266 & $\begin{array}{l}\text { Date of the incident / acute med } \\
\text { Emergency Department Attend }\end{array}$ \\
\hline health_event_tm & Health event time & time & 2909289 & 34.2 & 1440 & $\begin{array}{l}\text { This is the time of the incident / } \\
\text { and Emergency Department At }\end{array}$ \\
\hline attend_group & Attendance group & character & 218653 & 2.57 & 25 & A general reason for an Acciden \\
\hline attend_category & Attendance Category & character & 6751 & 0.08 & 6 & $\begin{array}{l}\text { Accident and Emergency Attend } \\
\text { patient is making a first or follo } \\
\text { Emergency Department. }\end{array}$ \\
\hline diag_cd_1 & Diagnosis Type code 1 & character & 943809 & 11.1 & 756 & $\begin{array}{l}\text { A broad list of diagnosis types, } \\
\text { Emergency Department Attend }\end{array}$ \\
\hline diag_cd_2 & Diagnosis Type code 2 & character & 6706626 & 78.84 & 670 & $\begin{array}{l}\text { A broad list of diagnosis types, } \\
\text { Emergency Department Attend }\end{array}$ \\
\hline diag_cd_3 & Diagnosis Type code 3 & character & 6987207 & 82.14 & 459 & $\begin{array}{l}\text { A broad list of diagnosis types, } \\
\text { Emergency Department Attend }\end{array}$ \\
\hline
\end{tabular}


Table C.1.1: SAIL Databank Datasets used in the Observatory (cont'd).

\begin{tabular}{|c|c|c|c|c|c|c|}
\hline Column Name & Friendly Name & Type & $\begin{array}{l}\text { NULL } \\
\text { Count }\end{array}$ & NULL \% & $\begin{array}{l}\text { Distinct } \\
\text { Values }\end{array}$ & Description \\
\hline diag_cd_4 & Diagnosis Type code 4 & character & 7022431 & 82.56 & 270 & The 4 th digit of the Diagnostic \\
\hline diag_cd_5 & Diagnosis Type code 5 & character & 7027918 & 82.62 & 151 & $\begin{array}{l}\text { A broad list of diagnosis types, } \\
\text { Emergency Department Attend }\end{array}$ \\
\hline diag_cd_6 & Diagnosis Type code 6 & character & 7029211 & 82.64 & 98 & $\begin{array}{l}\text { A broad list of diagnosis types, } \\
\text { Emergency Department Attend }\end{array}$ \\
\hline anat_area_cd_1 & Anatomical area code 1 & character & 1125400 & 13.23 & 39 & A list of parts of the human body \\
\hline anat_area_cd_2 & Anatomical area code 2 & character & 6884335 & 80.93 & 40 & A list of parts of the human body \\
\hline anat_area_cd_3 & Anatomical area code 3 & character & 7005530 & 82.36 & 39 & A list of parts of the human body \\
\hline anat_area_cd_4 & Anatomical area code 4 & character & 7024604 & 82.58 & 39 & A list of parts of the human body \\
\hline anat_area_cd_5 & Anatomical area code 5 & character & 7028269 & 82.62 & 36 & A list of parts of the human body \\
\hline anat_area_cd_6 & Anatomical area code 6 & character & 7029132 & 82.63 & 34 & A list of parts of the human body \\
\hline side_cd_1 & Anatomical side code 1 & character & 3067296 & 36.06 & 9 & An indication of the side of the $h$ \\
\hline side_cd_2 & Anatomical side code 2 & character & 8149792 & 95.81 & 9 & An indication of the side of the $h$ \\
\hline side_cd_3 & Anatomical side code 3 & character & 8269796 & 97.22 & 10 & An indication of the side of the $h$ \\
\hline side_cd_4 & Anatomical side code 4 & character & 8290114 & 97.46 & 9 & An indication of the side of the $h$ \\
\hline side_cd_5 & Anatomical side code 5 & character & 8294677 & 97.51 & 8 & An indication of the side of the $h$ \\
\hline side_cd_6 & Anatomical side code 6 & character & 8295877 & 97.53 & 6 & An indication of the side of the $h$ \\
\hline treat_cd_1 & Treatment code 1 & character & 1383221 & 16.26 & 17 & $\begin{array}{l}\text { A broad list of types of treatmen } \\
\text { patient as a result of an Accide }\end{array}$ \\
\hline
\end{tabular}


Table C.1.1: SAIL Databank Datasets used in the Observatory (cont'd).

\begin{tabular}{|c|c|c|c|c|c|c|}
\hline Column Name & Friendly Name & Type & $\begin{array}{l}\text { NULL } \\
\text { Count }\end{array}$ & NULL \% & $\begin{array}{l}\text { Distinct } \\
\text { Values }\end{array}$ & Description \\
\hline treat_cd_2 & Treatment code 2 & character & 6609855 & 77.71 & 17 & $\begin{array}{l}\text { A broad list of types of treatmen } \\
\text { patient as a result of an Accide }\end{array}$ \\
\hline treat_cd_3 & Treatment code 3 & character & 7008801 & 82.4 & 17 & $\begin{array}{l}\text { A broad list of types of treatmen } \\
\text { patient as a result of an Accide }\end{array}$ \\
\hline treat_cd_4 & Treatment code 4 & character & 7164431 & 84.22 & 17 & $\begin{array}{l}\text { A broad list of types of treatmen } \\
\text { patient as a result of an Accide }\end{array}$ \\
\hline treat_cd_5 & Treatment code 5 & character & 7222299 & 84.91 & 17 & $\begin{array}{l}\text { A broad list of types of treatmen } \\
\text { patient as a result of an Accide }\end{array}$ \\
\hline treat_cd_6 & Treatment code 6 & character & 7238663 & 85.1 & 17 & $\begin{array}{l}\text { A broad list of types of treatmen } \\
\text { patient as a result of an Accide }\end{array}$ \\
\hline invest_cd_1 & Investigation code 1 & character & 1561708 & 18.36 & 14 & $\begin{array}{l}\text { A broad list of types of investiga } \\
\text { diagnosis during and Accident }\end{array}$ \\
\hline invest_cd_2 & Investigation code 2 & character & 6873257 & 80.8 & 11 & $\begin{array}{l}\text { A broad list of types of investiga } \\
\text { diagnosis during and Accident }\end{array}$ \\
\hline invest_cd_3 & Investigation code 3 & character & 7161646 & 84.19 & 11 & $\begin{array}{l}\text { A broad list of types of investiga } \\
\text { diagnosis during and Accident }\end{array}$ \\
\hline invest_cd_4 & Investigation code 4 & character & 7373525 & 86.68 & 11 & $\begin{array}{l}\text { A broad list of types of investiga } \\
\text { diagnosis during and Accident }\end{array}$ \\
\hline invest_cd_5 & Investigation code 5 & character & 7501448 & 88.19 & 11 & $\begin{array}{l}\text { A broad list of types of investiga } \\
\text { diagnosis during and Accident }\end{array}$ \\
\hline invest_cd_6 & Investigation code 6 & character & 7518976 & 88.39 & 10 & $\begin{array}{l}\text { A broad list of types of investiga } \\
\text { diagnosis during and Accident }\end{array}$ \\
\hline admin_end_dt & Administrative end date & date & 61624 & 0.72 & 3329 & This is the date that the patients \\
\hline admin_end_tm & Administrative end time & time & 82466 & 0.97 & 1440 & This is the time that the patients \\
\hline add_details & $\begin{array}{l}\text { Additional incident } \\
\text { details }\end{array}$ & varchar & 8506326 & 100 & 0 & $\begin{array}{l}\text { A record of any additional detail } \\
\text { Emergency Department Attend } \\
\text { the dataset. }\end{array}$ \\
\hline
\end{tabular}


Table C.1.1: SAIL Databank Datasets used in the Observatory (cont'd).

\begin{tabular}{|c|c|c|c|c|c|c|}
\hline Column Name & Friendly Name & Type & $\begin{array}{l}\text { NULL } \\
\text { Count }\end{array}$ & NULL \% & $\begin{array}{l}\text { Distinct } \\
\text { Values }\end{array}$ & Description \\
\hline discharge & Outcome of attendance & character & 161927 & 1.9 & 27 & $\begin{array}{l}\text { This records the outcome of the } \\
\text { Attendance. }\end{array}$ \\
\hline location_type & Location place type & character & 338871 & 3.98 & 69 & $\begin{array}{l}\text { The type of place where the phy: } \\
\text { the Accident and Emergency D }\end{array}$ \\
\hline road_user & Road user & character & 767921 & 9.03 & 15 & This is the nature of the patients \\
\hline presenting_complaint & Presenting complaint & varchar & 8506326 & 100 & 0 & $\begin{array}{l}\text { This is the presenting complaint } \\
\text { and Emergency Department. }\end{array}$ \\
\hline mech_of_inj & Mechanism of injury & character & 564890 & 6.64 & 180 & The mechanics of how the physi \\
\hline activity & Activity at time of injury & character & 666342 & 7.83 & 18 & $\begin{array}{l}\text { What the patient was doing at th } \\
\text { picture of how the physical or }\end{array}$ \\
\hline sport & Sporting activity & character & 1088994 & 12.8 & 37 & $\begin{array}{l}\text { The sport in which the patient w } \\
\text { the Accident and Emergency D }\end{array}$ \\
\hline crn_pseud_e & $\begin{array}{l}\text { Encrypted } \\
\text { Pseudonimised Case } \\
\text { Record Number }\end{array}$ & integer & 0 & 0 & 3221753 & $\begin{array}{l}\text { This is the case record number. } \\
\text { health care provider. }\end{array}$ \\
\hline alf_sts_cd & ALF status code & character & 0 & 0 & 5 & Status code assigned when deriv \\
\hline alf_mtch_pct & ALF match percentage & decimal & 8399224 & 98.74 & 27110 & $\begin{array}{l}\text { Match percentage assigned whe } \\
\text { Field. }\end{array}$ \\
\hline alcohol_ind & Alcohol indicator & character & 405798 & 4.77 & 29 & $\begin{array}{l}\text { In the clinical opinion of the Em } \\
\text { alcohol in the presenting patien }\end{array}$ \\
\hline avail_from_dt & Available from date & date & 0 & 0 & 1 & Date when the data made availa \\
\hline batch_num & Batch number & smallint & 0 & 0 & 1 & $\begin{array}{l}\text { The batch number is a sequentia } \\
\text { SAIL. }\end{array}$ \\
\hline triage_cat & Triage category & character & 371878 & 4.37 & 16 & $\begin{array}{l}\text { The triage category is assigned } \\
\text { by medical or nursing staff in a }\end{array}$ \\
\hline
\end{tabular}


Table C.1.1: SAIL Databank Datasets used in the Observatory (cont'd).

\begin{tabular}{|c|c|c|c|c|c|c|}
\hline Column Name & Friendly Name & Type & $\begin{array}{l}\text { NULL } \\
\text { Count }\end{array}$ & NULL \% & $\begin{array}{l}\text { Distinct } \\
\text { Values }\end{array}$ & Description \\
\hline \multicolumn{7}{|c|}{ Outpatient Dataset (OPD) - OUTPATIENTS } \\
\hline prov_unit_cd & Provider unit code & character & 0 & 0 & 237 & $\begin{array}{l}\text { This is the organisation code of } \\
\text { identifies the health care provic } \\
\text { treatment of the patient. }\end{array}$ \\
\hline prov_site_cd & Provider site code & character & 0 & 0 & 679 & $\begin{array}{l}\text { This is the organisation code of } \\
\text { identifies the health care provic } \\
\text { treatment of the patient. }\end{array}$ \\
\hline alf_e & $\begin{array}{l}\text { Encrypted Anonymised } \\
\text { Linking Field }\end{array}$ & bigint & 484178 & 0.93 & 3187662 & $\begin{array}{l}\text { The Anonymised Linking Field, } \\
\text { database, is derived from the p } \\
\text { encryption occurs in NWIS and } \\
\text { supplied in the data extract the }\end{array}$ \\
\hline gndr_cd & $\begin{array}{l}\text { Gender code (also } \\
\text { known as sex) }\end{array}$ & character & 3400 & 0.01 & 7 & This is the sex (gender) of perso \\
\hline age_at_appt & Age at appointment & integer & 22742 & 0.04 & 156 & The age of the individual at the $t$ \\
\hline reg_prac_cd_e & $\begin{array}{l}\text { Encrypted registered } \\
\text { GP practice code }\end{array}$ & integer & 49357 & 0.09 & 9026 & The encrypted unique GP Practi \\
\hline ref_cd_e & $\begin{array}{l}\text { Encrypted referrer } \\
\text { code }\end{array}$ & integer & 2713322 & 5.19 & 42737 & $\begin{array}{l}\text { This is the nationally recognized } \\
\text { may be a General Medical Prac } \\
\text { (GDP), Consultant or Independ } \\
\text { GDP, Consultant or Independen }\end{array}$ \\
\hline case_rec_num_e & $\begin{array}{l}\text { Encrypted casenote } \\
\text { record number }\end{array}$ & integer & 0 & 0 & 4796488 & $\begin{array}{l}\text { This is the case record number. } \\
\text { health care provider. }\end{array}$ \\
\hline ref_dt & Date of patient referral & date & 10036898 & 19.19 & 10093 & $\begin{array}{l}\text { This is the date on which the pat } \\
\text { point should be the date of refe } \\
\text { date the letter was received in } \\
\text { specify a date of referral, the da } \\
\text { be used. }\end{array}$ \\
\hline
\end{tabular}


Table C.1.1: SAIL Databank Datasets used in the Observatory (cont'd).

\begin{tabular}{|c|c|c|c|c|c|c|}
\hline Column Name & Friendly Name & Type & $\begin{array}{l}\text { NULL } \\
\text { Count }\end{array}$ & NULL \% & $\begin{array}{l}\text { Distinct } \\
\text { Values }\end{array}$ & Description \\
\hline clinical_ref_dt & Clinical referral date & date & 9377699 & 17.93 & 9864 & $\begin{array}{l}\text { The Clinical Referral Date (CRD } \\
\text { start of a period of waiting eith } \\
\text { episode of treatment such as e } \\
\text { lists used for booking patients, } \\
\text { circumstances. It is not used to }\end{array}$ \\
\hline waiting_list_dt & Waiting list date & date & 24015264 & 45.92 & 8635 & $\begin{array}{l}\text { The Waiting List Date is set initi } \\
\text { Date. It is used to calculate wa } \\
\text { Health Board/Trust performan } \\
\text { performance targets. It is not } \\
\text { booking or to order inpatient o } \\
\text { surgery. There are a number of } \\
\text { These include rescheduling an } \\
\text { reinstatement to a waiting list } \\
\text { chosen to remain with a consul } \\
\text { different consultant. }\end{array}$ \\
\hline priority_type_cd & $\begin{array}{l}\text { Priority type new } \\
\text { patients }\end{array}$ & character & 31340596 & 59.92 & 5 & $\begin{array}{l}\text { This is the priority of a request } \\
\text { attendances only, that is, wher } \\
\text { services to be provided by a Co } \\
\text { Consultant.For a Follow Up Att } \\
\text { Attendance, Priority Type must }\end{array}$ \\
\hline source_of_ref_cd & $\begin{array}{l}\text { Source of referal } \\
\text { outpatients code }\end{array}$ & character & 326558 & 0.62 & 35 & $\begin{array}{l}\text { This is a classification which is } \\
\text { Outpatient Episode or Outpatie }\end{array}$ \\
\hline con_spec_main_cd & $\begin{array}{l}\text { Consultant main } \\
\text { speciality code }\end{array}$ & character & 41308 & 0.08 & 163 & $\begin{array}{l}\text { A unique code identifying each } \\
\text { Colleges.Specialties are divisio } \\
\text { body systems (dermatology), a } \\
\text { medicine), clinical function (rh } \\
\text { combinations of these factors. } \\
\text { Colleges and Faculties should } \\
\text { and Specialist Medical Practice } \\
2003 \text { and European Primary an } \\
1998 \text {. }\end{array}$ \\
\hline con_spec_cd_of_treat & $\begin{array}{l}\text { Consultant treatment } \\
\text { speciality code }\end{array}$ & character & 19168 & 0.04 & 186 & $\begin{array}{l}\text { This is the specialty under whic } \\
\text { either be the same as the speci } \\
\text { specialty or a different specialt } \\
\text { specialty function. Note that b } \\
\text { specialty function should be ba }\end{array}$ \\
\hline
\end{tabular}


Table C.1.1: SAIL Databank Datasets used in the Observatory (cont'd).

\begin{tabular}{|c|c|c|c|c|c|c|}
\hline Column Name & Friendly Name & Type & $\begin{array}{l}\text { NULL } \\
\text { Count }\end{array}$ & NULL \% & $\begin{array}{l}\text { Distinct } \\
\text { Values }\end{array}$ & Description \\
\hline local_spec_cd & Local sub specialty & character & 8737323 & 16.71 & 358 & $\begin{array}{l}\text { This is a locally or nationally def } \\
\text { specialty boundaries. }\end{array}$ \\
\hline clinic_purpose_cd & Clinic Purpose code & character & 7355159 & 14.06 & 46859 & $\begin{array}{l}\text { This is the function of an outpat } \\
\text { national classification of functic } \\
\text { function titles must be decided } \\
\text { shown, such as: a. Obstetric an }\end{array}$ \\
\hline gmc_con_cd_e & $\begin{array}{l}\text { Encrypted GMC } \\
\text { consultant code }\end{array}$ & integer & 225378 & 0.43 & 24479 & $\begin{array}{l}\text { Nationally agreed form for cons } \\
\text { General Medical Council (GMC } \\
\text { Consultant or locum Consultan } \\
\text { Registration Number will be us }\end{array}$ \\
\hline att_id_e & $\begin{array}{l}\text { Encrypted attendance } \\
\text { identifier }\end{array}$ & integer & 0 & 0 & 43721493 & $\begin{array}{l}\text { A sequential number or time of } \\
\text { appointment to be uniquely ide }\end{array}$ \\
\hline admin_cat_cd & $\begin{array}{l}\text { Administrative } \\
\text { category code }\end{array}$ & character & 1859 & 0 & 17 & $\begin{array}{l}\text { This is to indicate whether the } \mathrm{p} \\
\text { patient etc. }\end{array}$ \\
\hline loc_type_cd & Location type code & character & 1464101 & 2.8 & 26 & This is a classification of location \\
\hline site_cd_of_treat & Site code of treatment & character & 21452 & 0.04 & 1928 & \\
\hline med_staff_type_cd & $\begin{array}{l}\text { Medical staff type } \\
\text { seeing patient code }\end{array}$ & character & 1531441 & 2.93 & 11 & $\begin{array}{l}\text { A classification of the type of me } \\
\text { Outpatient attendance. }\end{array}$ \\
\hline attend_dt & Attendance date & date & 0 & 0 & 5230 & This is the date of an attendance \\
\hline first_attend_cd & $\begin{array}{l}\text { First Attendance } \\
\text { Category }\end{array}$ & character & 8262 & 0.02 & 5 & $\begin{array}{l}\text { The first attendance is the start } \\
\text { attendance in a series with the } \\
\text { following a referral. }\end{array}$ \\
\hline attend_cd & Attendance code & character & 61143 & 0.12 & 13 & $\begin{array}{l}\text { This indicates whether a person } \\
\text { patient did not attend, it also in } \\
\text { given. }\end{array}$ \\
\hline outcome_cd & Outcome of attendance & character & 3388267 & 6.48 & 14 & This records the outcome of the \\
\hline
\end{tabular}


Table C.1.1: SAIL Databank Datasets used in the Observatory (cont'd).

\begin{tabular}{|c|c|c|c|c|c|c|}
\hline Column Name & Friendly Name & Type & $\begin{array}{l}\text { NULL } \\
\text { Count }\end{array}$ & NULL \% & $\begin{array}{l}\text { Distinct } \\
\text { Values }\end{array}$ & Description \\
\hline last_dna_cancel_dt & $\begin{array}{l}\text { Last DNA or patient } \\
\text { cancelled date }\end{array}$ & date & 48882640 & 93.46 & 7572 & $\begin{array}{l}\text { This derived item should only be } \\
\text { treatment }\end{array}$ \\
\hline oper_sts_flg & Operation status flag & character & 1704072 & 3.26 & 11 & $\begin{array}{l}\text { Operation status should be used } \\
\text { knowledge regarding the opera }\end{array}$ \\
\hline oper_cd_123 & $\begin{array}{l}\text { Operation code (3 } \\
\text { digits) }\end{array}$ & character & 49048246 & 93.78 & 943 & The first 3 digits of the Procedur \\
\hline oper_cd_4 & $\begin{array}{l}\text { Operation code ( } 4 \text { th } \\
\text { digits) }\end{array}$ & character & 49150721 & 93.97 & 34 & The 4 th digit of the Procedure ce \\
\hline lsoa_cd & $\begin{array}{l}\text { Local Super Output } \\
\text { Area code }\end{array}$ & character & 231313 & 0.44 & 25100 & The Local Super Output Area of \\
\hline alf_sts_cd & ALF status code & character & 0 & 0 & 5 & Status code assigned when deri \\
\hline alf_mtch_pct & ALF match percentage & decimal & 52135801 & 99.68 & 33811 & $\begin{array}{l}\text { Match percentage assigned whe } \\
\text { Field. }\end{array}$ \\
\hline avail_from_dt & Available from date & date & 0 & 0 & 1 & Date when the data made availa \\
\hline \multicolumn{7}{|c|}{ Annual District Death Extract (ADDE) } \\
\hline alf_e & & bigint & 35345 & 5.12 & 655547 & \\
\hline alf_sts_cd & & character & 0 & 0 & 2 & \\
\hline $\begin{array}{l}\text { death_annual- } \\
\text { record_ind_cd }\end{array}$ & & character & 37857 & 5.48 & 1 & \\
\hline count_death & & integer & 0 & 0 & 1 & \\
\hline death_dt & & date & 14 & 0 & 7949 & \\
\hline death_dt_valid & & varchar & 0 & 0 & 2 & \\
\hline
\end{tabular}


Table C.1.1: SAIL Databank Datasets used in the Observatory (cont'd).

\begin{tabular}{|c|c|c|c|c|c|c|}
\hline Column Name & Friendly Name & Type & $\begin{array}{l}\text { NULL } \\
\text { Count }\end{array}$ & NULL \% & $\begin{array}{l}\text { Distinct } \\
\text { Values }\end{array}$ & Description \\
\hline death_reg_dt & & timestamp & 0 & 0 & 5706 & \\
\hline death_reg_dt_valid & & varchar & 0 & 0 & 1 & \\
\hline neonatal_ind_flg & & integer & 0 & 0 & 3 & \\
\hline dec_urbanrural_cd & & character & $<5$ & 0 & 8 & \\
\hline $\begin{array}{l}\text { dec_stats_curr_cen- } \\
\text { sus_lsoa_cd }\end{array}$ & & varchar & 18 & 0 & 1909 & \\
\hline $\begin{array}{l}\text { dec_stats_curr_cen- } \\
\text { sus_la_ç্d }\end{array}$ & & varchar & 18 & 0 & 22 & \\
\hline $\begin{array}{l}\text { dec_stats_curr_cen- } \\
\text { sus_la_previous_cd }\end{array}$ & & varchar & 18 & 0 & 22 & \\
\hline $\begin{array}{l}\text { dec_stats_curr_cen- } \\
\text { sus_country_cd }\end{array}$ & & varchar & 18 & 0 & 1 & \\
\hline $\begin{array}{l}\text { dec_stats_curr_cen- } \\
\text { sus_health_org_cd }\end{array}$ & & varchar & 18 & 0 & 7 & \\
\hline $\begin{array}{l}\text { dec_stats_curr_cen- } \\
\text { sus_health_org_previ- } \\
\text { ous_cd }\end{array}$ & & varchar & 18 & 0 & 7 & \\
\hline $\begin{array}{l}\text { dec_stats_prev_cen- } \\
\text { sus_lsoa_cd }\end{array}$ & & varchar & 76 & 0.01 & 1896 & \\
\hline $\begin{array}{l}\text { dec_stats_prev_cen- } \\
\text { sus_la_cd }\end{array}$ & & varchar & 76 & 0.01 & 22 & \\
\hline $\begin{array}{l}\text { dec_stats_prev_cen- } \\
\text { sus_la_prev_cd }\end{array}$ & & varchar & 76 & 0.01 & 7 & \\
\hline $\begin{array}{l}\text { dec_stats_prev_cen- } \\
\text { sus_country_cd }\end{array}$ & & varchar & 76 & 0.01 & 1 & \\
\hline
\end{tabular}


Table C.1.1: SAIL Databank Datasets used in the Observatory (cont'd).

\begin{tabular}{|c|c|c|c|c|c|c|}
\hline Column Name & Friendly Name & Type & $\begin{array}{l}\text { NULL } \\
\text { Count }\end{array}$ & NULL \% & $\begin{array}{l}\text { Distinct } \\
\text { Values }\end{array}$ & Description \\
\hline $\begin{array}{l}\text { dec_stats_prev_cen- } \\
\text { sus_health_org_cd }\end{array}$ & & varchar & 76 & 0.01 & 22 & \\
\hline $\begin{array}{l}\text { dec_stats_prev_cen- } \\
\text { sus_health_org_prev_cd }\end{array}$ & & character & 76 & 0.01 & 7 & \\
\hline dec_sha_cd & & varchar & 0 & 0 & 1 & \\
\hline dec_health_org_cd & & varchar & 0 & 0 & 7 & \\
\hline dec_sex_cd & & character & 0 & 0 & 2 & \\
\hline $\begin{array}{l}\text { deathcause_diag_un- } \\
\text { derlying_cd }\end{array}$ & & varchar & 2160 & 0.31 & 4205 & \\
\hline deathcause_diag_1_cd & & varchar & $<5$ & 0 & 2997 & \\
\hline deathcause_diag_2_cd & & varchar & 167660 & 24.27 & 4714 & \\
\hline deathcause_diag_3_cd & & varchar & 416684 & 60.31 & 4292 & \\
\hline deathcause_diag_4_cd & & varchar & 567539 & 82.15 & 3623 & \\
\hline deathcause_diag_5_cd & & varchar & 640374 & 92.69 & 2683 & \\
\hline deathcause_diag_6_cd & & varchar & 671192 & 97.15 & 1879 & \\
\hline deathcause_diag_7_cd & & varchar & 683353 & 98.91 & 1245 & \\
\hline deathcause_diag_8_cd & & varchar & 687856 & 99.56 & 750 & \\
\hline $\begin{array}{l}\text { death_communal_es- } \\
\text { tablishment_cd_e }\end{array}$ & & bigint & 0 & 0 & 2663 & \\
\hline death_urbanrural_cd & & character & 145133 & 21.01 & 8 & \\
\hline
\end{tabular}


Table C.1.1: SAIL Databank Datasets used in the Observatory (cont'd).

\begin{tabular}{|c|c|c|c|c|c|c|}
\hline Column Name & Friendly Name & Type & $\begin{array}{l}\text { NULL } \\
\text { Count }\end{array}$ & NULL \% & $\begin{array}{l}\text { Distinct } \\
\text { Values }\end{array}$ & Description \\
\hline $\begin{array}{l}\text { deathstats_curr_cen- } \\
\text { sus_lsoa_cd }\end{array}$ & & varchar & 145135 & 21.01 & 4215 & \\
\hline $\begin{array}{l}\text { deathstats_curr_cen- } \\
\text { sus_la_cd }\end{array}$ & & varchar & 145135 & 21.01 & 340 & \\
\hline $\begin{array}{l}\text { deathstats_curr_cen- } \\
\text { sus_la_prev_cd }\end{array}$ & & varchar & 145270 & 21.03 & 333 & \\
\hline $\begin{array}{l}\text { deathstats_curr_cen- } \\
\text { sus_country_cd }\end{array}$ & & varchar & 145135 & 21.01 & 2 & \\
\hline $\begin{array}{l}\text { deathstats_curr_cen- } \\
\text { sus_health_org_cd }\end{array}$ & & varchar & 145135 & 21.01 & 157 & \\
\hline $\begin{array}{l}\text { deathstats_curr_cen- } \\
\text { sus_health_org_prev_cd }\end{array}$ & & character & 165362 & 23.93 & 7 & \\
\hline $\begin{array}{l}\text { deathstats_prev_cen- } \\
\text { sus_lsoa_cd }\end{array}$ & & varchar & 145137 & 21.01 & 4198 & \\
\hline $\begin{array}{l}\text { deathstats_prev_cen- } \\
\text { sus_la_cd }\end{array}$ & & varchar & 145137 & 21.01 & 340 & \\
\hline $\begin{array}{l}\text { deathstats_prev_cen- } \\
\text { sus_la_prev_cd }\end{array}$ & & varchar & 145137 & 21.01 & 157 & \\
\hline $\begin{array}{l}\text { deathstats_prev_cen- } \\
\text { sus_country_cd }\end{array}$ & & varchar & 145137 & 21.01 & 2 & \\
\hline $\begin{array}{l}\text { deathstats_prev_cen- } \\
\text { sus_health_org_cd }\end{array}$ & & varchar & 145137 & 21.01 & 340 & \\
\hline $\begin{array}{l}\text { deathstats_prev_cen- } \\
\text { sus_health_org_prev_cd }\end{array}$ & & varchar & 165362 & 23.93 & 7 & \\
\hline death_ccg_cd & & varchar & 670674 & 97.07 & 209 & \\
\hline $\begin{array}{l}\text { death_countyanddis- } \\
\text { trict_cd }\end{array}$ & & varchar & 145156 & 21.01 & 172 & \\
\hline
\end{tabular}


Table C.1.1: SAIL Databank Datasets used in the Observatory (cont'd).

\begin{tabular}{|c|c|c|c|c|c|}
\hline Friendly Name & Type & $\begin{array}{l}\text { NULL } \\
\text { Count }\end{array}$ & NULL \% & $\begin{array}{l}\text { Distinct } \\
\text { Values }\end{array}$ & Description \\
\hline death_health_org_cd & varchar & 145156 & 21.01 & 157 & \\
\hline $\begin{array}{l}\text { death_nhs_establish- } \\
\text { ment_ind_cd }\end{array}$ & varchar & 160663 & 23.25 & 2 & \\
\hline $\begin{array}{l}\text { death_establish- } \\
\text { ment_type_cd }\end{array}$ & varchar & 160663 & 23.25 & 49 & \\
\hline $\begin{array}{l}\text { death_postcode_impu- } \\
\text { tation_ind_cd }\end{array}$ & varchar & 690891 & 100 & 1 & \\
\hline dec_age & varchar & 0 & 0 & 204 & \\
\hline dec_age_unit_cd & varchar & 0 & 0 & 4 & \\
\hline dec_occ_type_cd & varchar & 6 & 0 & 5 & \\
\hline $\begin{array}{l}\text { dec_or_mother_socioe- } \\
\text { conomic_class_cd }\end{array}$ & varchar & 447238 & 64.73 & 49 & \\
\hline $\begin{array}{l}\text { dec_husband_or_fa- } \\
\text { ther_socioeco- } \\
\text { nomic_class_cd }\end{array}$ & varchar & 578935 & 83.8 & 48 & \\
\hline dec_or_mother_occ_class_cd & varchar & 447220 & 64.73 & 944 & \\
\hline $\begin{array}{l}\text { dec_husband_or_fa- } \\
\text { ther_occ_class_cd }\end{array}$ & varchar & 578930 & 83.79 & 904 & \\
\hline $\begin{array}{l}\text { dec_or_mother_re- } \\
\text { tired_ind_cd }\end{array}$ & varchar & 618493 & 89.52 & 1 & \\
\hline $\begin{array}{l}\text { dec_husband_or_fa- } \\
\text { ther_retired_ind̄_cd }\end{array}$ & varchar & 663323 & 96.01 & 1 & \\
\hline dec birthcountry cd & varchar & 21 & 0 & 330 & \\
\hline
\end{tabular}


Table C.1.1: SAIL Databank Datasets used in the Observatory (cont'd).

\begin{tabular}{|c|c|c|c|c|c|}
\hline Friendly Name & Type & $\begin{array}{l}\text { NULL } \\
\text { Count }\end{array}$ & NULL \% & $\begin{array}{l}\text { Distinct } \\
\text { Values }\end{array}$ & Description \\
\hline $\begin{array}{l}\text { dec_governmentof- } \\
\text { fice_region_cd }\end{array}$ & varchar & 0 & 0 & 1 & \\
\hline $\begin{array}{l}\text { dec_countyanddis- } \\
\text { trict_cd }\end{array}$ & varchar & 0 & 0 & 22 & \\
\hline dec_county_cd & varchar & 0 & 0 & 2 & \\
\hline dec_countydistrict_cd & varchar & 0 & 0 & 22 & \\
\hline dec_ccg_cd & varchar & 690892 & 100 & 0 & \\
\hline dec_lsoa_cd & varchar & 0 & 0 & 1909 & \\
\hline dec_ward_cd & varchar & 0 & 0 & 148 & \\
\hline $\begin{array}{l}\text { death- } \\
\text { cause_diag_sec_cause_cd }\end{array}$ & varchar & 665526 & 96.33 & 658 & \\
\hline $\begin{array}{l}\text { deathcause_row- } \\
\text { pos_1_cd }\end{array}$ & varchar & 95743 & 13.86 & 8 & \\
\hline $\begin{array}{l}\text { deathcause_row- } \\
\text { pos_2_cd }\end{array}$ & varchar & 253788 & 36.73 & 10 & \\
\hline $\begin{array}{l}\text { deathcause_row- } \\
\text { pos_3_cd }\end{array}$ & varchar & 479168 & 69.35 & 10 & \\
\hline $\begin{array}{l}\text { deathcause_row- } \\
\text { pos_4_cd }\end{array}$ & varchar & 609554 & 88.23 & 10 & \\
\hline $\begin{array}{l}\text { deathcause_row- } \\
\text { pos_5_cd }\end{array}$ & varchar & 663883 & 96.09 & 8 & \\
\hline $\begin{array}{l}\text { deathcause_row- } \\
\text { pos_6_cd }\end{array}$ & varchar & 682433 & 98.78 & 9 & \\
\hline
\end{tabular}


Table C.1.1: SAIL Databank Datasets used in the Observatory (cont'd).

\begin{tabular}{|c|c|c|c|c|c|c|}
\hline Column Name & Friendly Name & Type & $\begin{array}{l}\text { NULL } \\
\text { Count }\end{array}$ & NULL \% & $\begin{array}{l}\text { Distinct } \\
\text { Values }\end{array}$ & Description \\
\hline $\begin{array}{l}\text { deathcause_row- } \\
\text { pos_7_cd }\end{array}$ & & varchar & 688336 & 99.63 & 9 & \\
\hline $\begin{array}{l}\text { deathcause_row- } \\
\text { pos_8_cd }\end{array}$ & & varchar & 690079 & 99.88 & 7 & \\
\hline avail_from_dt & & date & 0 & 0 & 1 & \\
\hline
\end{tabular}


Table C.1.2: Frequency of events and number of patients in calendar year for each of the SAIL datasets used in the Wales Asthma Observatory.

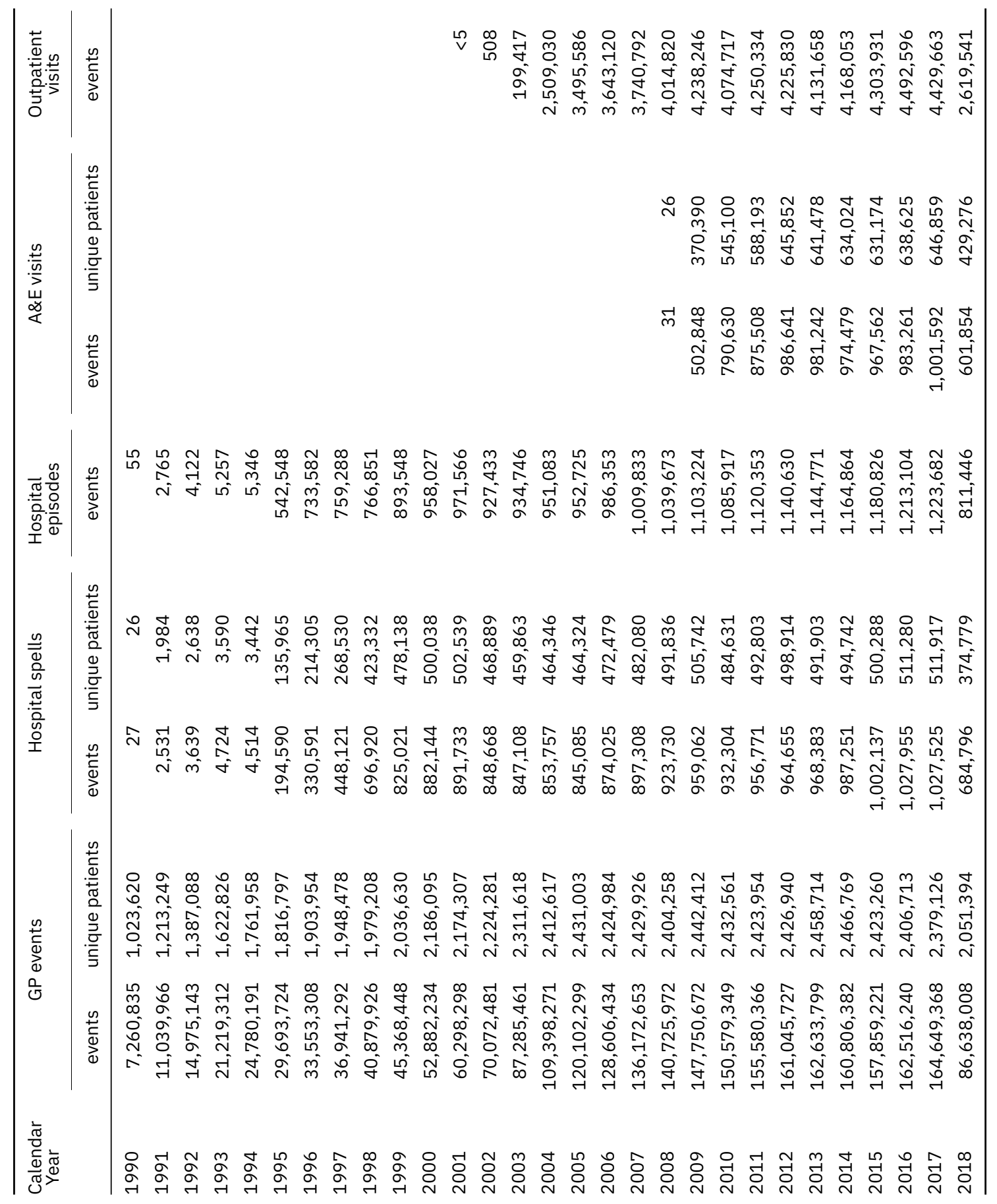



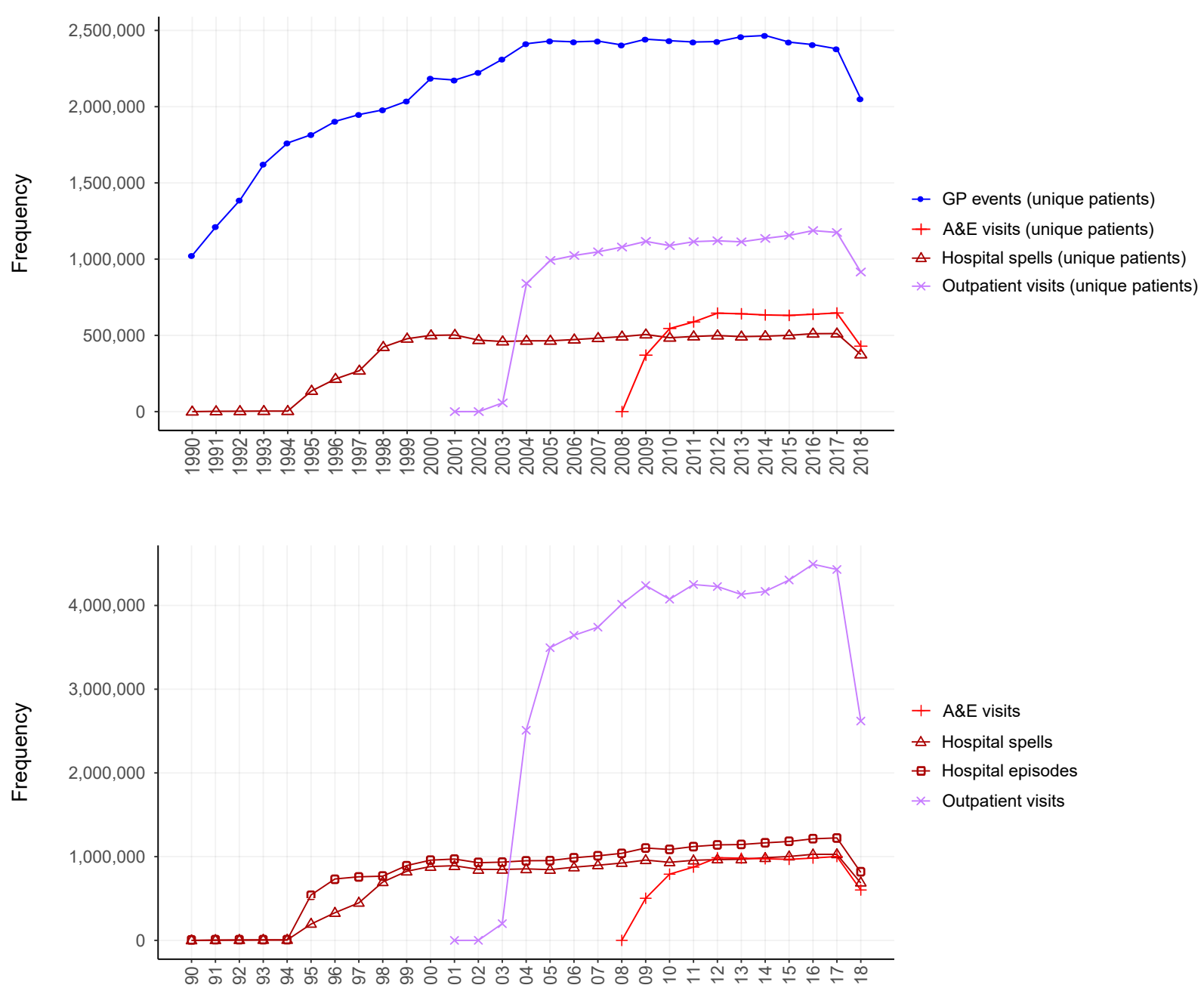

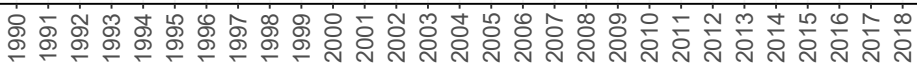

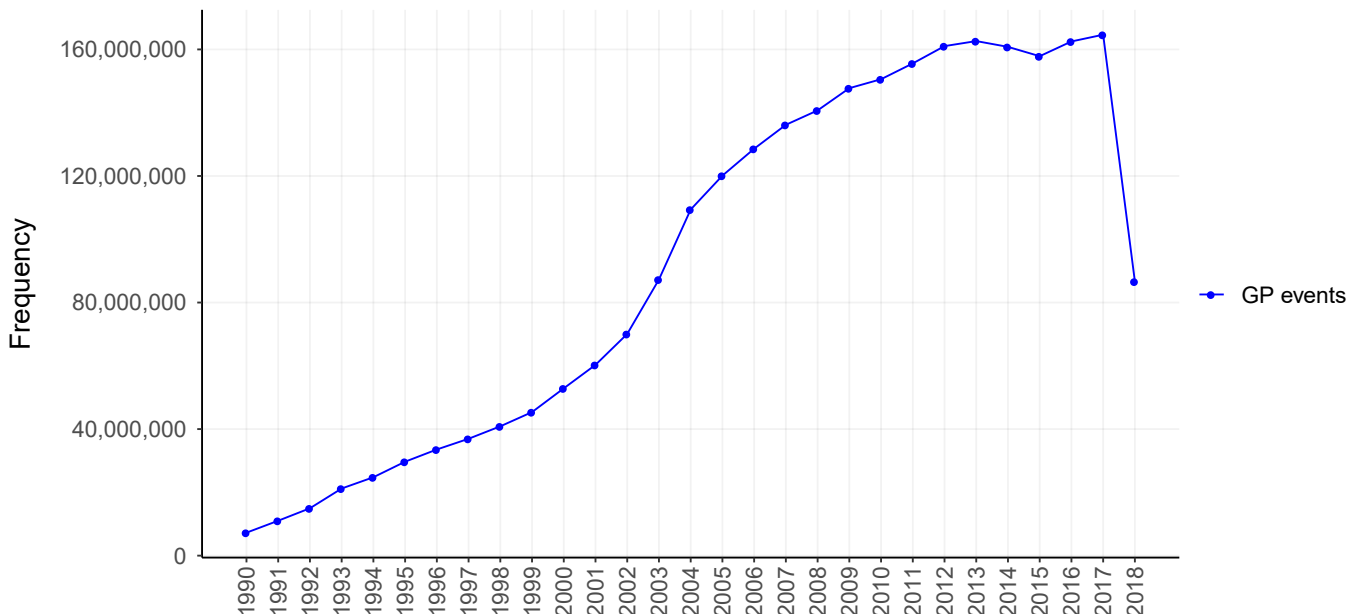

Figure C.1.1: Frequency of events and number of patients in calendar years for each of the SAIL datasets used in the Wales Asthma Observatory. 


\section{C.2 SAIL IGRP approval letter}

10 April 2015

Dr Gwyneth Davies

College of Medicine

ILS1

Swansea University

Singleton Park,

Swansea, SA2 8PP
Dear Gwyneth

Re: Wales Asthma Observatory

Your proposal to use the SAIL databank has been assessed by the SAIL Collaboration Review System (CRS). The CRS consists of the SAIL Management Team and the Information Governance Review Panel (IGRP). The membership of the IGRP is comprised of senior representatives from:

- British Medical Association (BMA)

- National Research Ethics Service (NRES)

- Public Health Wales

- NHS Wales Informatics Service (NWIS)

- Involving People

After careful consideration the proposal has been given approval to commence with analysis.

The project has been given a SAIL project number of 0317 .

Creation of project specific data view

Work will now commence on the creation of the project specific data view. The analyst working on this will be Mohammad Al Sallakh and they will be in contact with you to confirm your data specification.

Publication statement

All publications must acknowledge the use of SAIL data.

Yours sincerely

Cynthia McNerney

Information Governance Coordinator

\section{SAIL DATABANK}

Institute of Life Science 2

College of Medicine

Swansea University

Singleton Park

Swansea

SA2 8PP

SAILDatabank@swansea.ac.uk

www.SAILDatabank.com

Figure C.2.1: Approval letter by the SAIL Databank IGRP panel for using SAIL data in the development of the Wales Asthma Observatory. 


\section{C.3 A tool for automatic characterisation of cohorts using primary care data.}

The following abstract has been presented at the Informatics for Health 2017 congress, 24-26 April 2017 in Manchester, UK [476, page 164].

\section{Abstract no. 594 A tool to improve the efficiency and reproducibility of research using electronic health record databases}

Mohammad Al Sallakh and Gwyneth Davies, Swansea University Medical School, Swansea

Sarah Rodgers, Farr Institute, CIPHER, Swansea

Ronan Lyons, Farr Institute, CIPHER, Swansea

Aziz Sheikh, Usher Institute of Population Health Sciences and Informatics, University of Edinburgh, Edinburgh

Introduction: Interrogation of routine electronic health record (EHR) databases often involves repetitive programming tasks, such as manually constructing and modifying complex database queries, requiring significant time from an experienced data analyst. The objective was to develop a tool to automate the selection and characterisation of cohorts from primary care databases to be used by data analysts and researchers.

Methods: We identified a set of common elementary approaches to query clinical variables from the primary care database of the Secure Anonymised Information Linkage databank. We then designed an easy-to-use web-based user interface to allow using combinations of these approaches as 'building blocks' for querying more complex variables. We created an R programme to automatically generate and execute the corresponding Structured Query Language (SQL) queries.

Results: The developed prototype allows researchers to query clinical information from primary care databases based on the following elementary variable types: (1) count of events of interest (e.g. asthma prescriptions) or their distinct dates (2) the code or date of the earliest or latest event of interest (e.g. type of the earliest smoking cessation prescription) (3) the code or date of the event of maximum or minimum value (e.g., maximum BMI recording ever) and (4) count of events of interest having complex temporal constraints with other events (e.g., 
count of asthma doctor visits with oral steroid prescriptions within one week). Researchers may choose fixed, dynamic, or individualised query intervals. Algorithms are saved on a web server as versioned and shareable objects. The prototype integrates with a Read Codes dictionary and a shareable codeset repository allowing researchers to keep a record of codes used for reporting transparency.

Discussion: The developed prototype provides a scalable, versatile solution for the implementation of complex cohort selection and characterisation algorithms using primary care databases. The automatic generation of SQL queries reduces human errors and should enable rapid and scalable implementation of these algorithms, which has the potential to improve research efficiency and reproducibility. In addition, the graphical user interface allows researchers with no programming skills to interrogate the data. The tool is under active development to improve the functionality and usability, and we look forward to testing it in other databases and assessing its suitability in different research contexts. We plan to make this tool available under an open source licence. 


\section{(TD A tool for automatic characterisation of cohorts using primary care data}

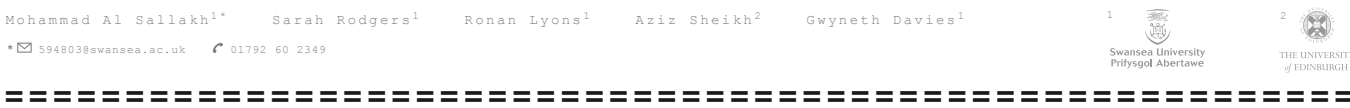

Interrogation of EHR databases for research is often an unstreamlined, poorly documented process that hinders transparency and reproducibility.
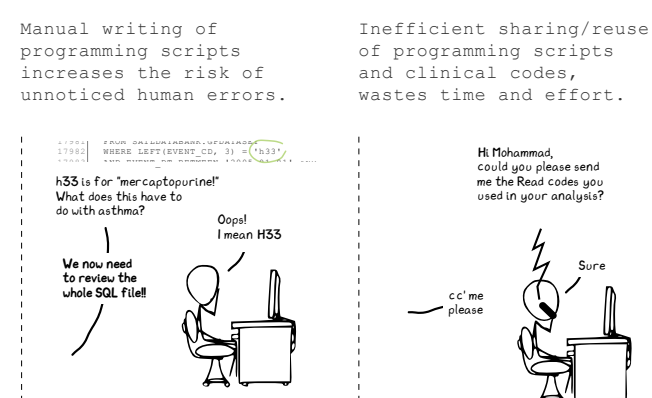
wastes time and effort. data.

Analysts spend significant

time on repetitive work, which could be otherwise automated.
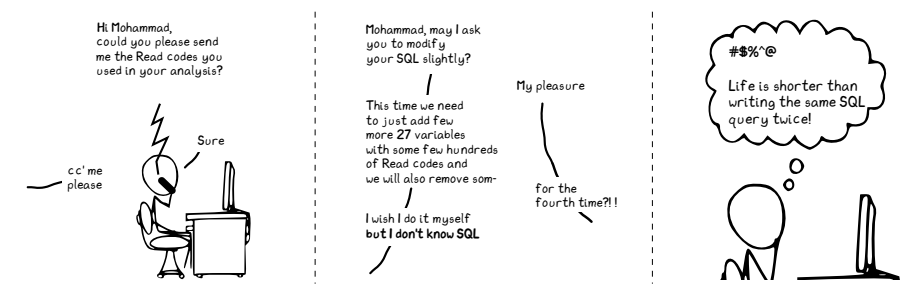

\section{-- So, what is GP-ACT?}

It is a platform that allows researchers and analysts to query complex clinical information from primary care databases based on common elementary variable types. Users can maintain and share clinical codes and phenotyping algorithms as versioned and cite-able objects on a web server, and can run automatically-generated SQL queries against the database.

Currently supported variable types:

(1) Count of events of interest or their distinct dates

(2) Code or date of the earliest or latest event of interest

(3) Code or date of the event of maximum or minimum value

(4) Count of events of interest having temporal constraints with other events

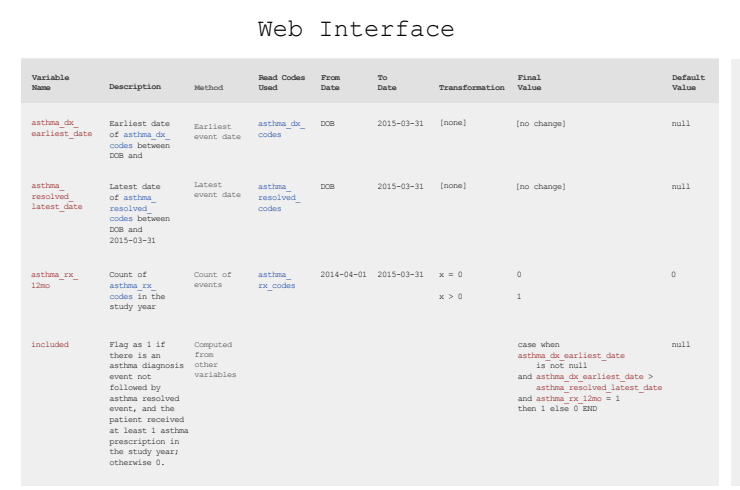

Web Server

SAIL DATABANK
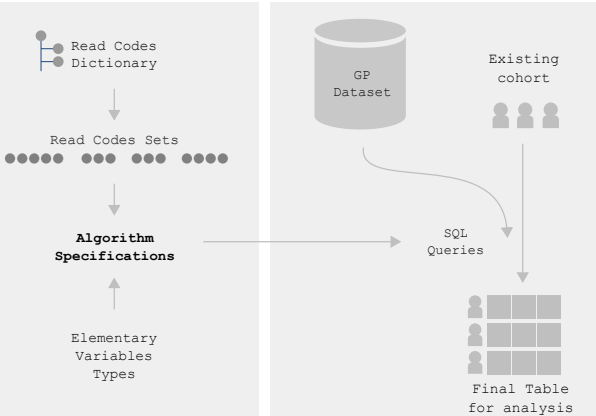

-- How does it help?

GP-ACT provides a scalable, versatile solution for implementing cohort characterisation algorithms.

Automatic SQL queries generation -> less human errors, rapid implementation, improved research efficiency, and direct involvement of non-technical researchers

Sharable code sets and algorithms -> better research transparency and reproducibility

funded by $8=$ supported by oforitr.

Figure C.3.1: A poster presented at the Informatics for Health 2017 congress in Manchester, United Kingdom. 


\section{C.4 Read codes used in the assessment of data qual- ity in Chapter 4}

Table C.4.1: Asthma diagnosis Read codes.

\begin{tabular}{ll}
\hline Read code & Description \\
\hline Asthma diagnosis codes & \\
173A. & Exercise induced asthma \\
H3120 & Chronic asthmatic bronchitis \\
H33.. & Asthma \\
H330. & Extrinsic (atopic) asthma \\
H3300 & Extrinsic asthma without status asthmaticus \\
H3301 & Extrinsic asthma with status asthmaticus \\
H330z & Extrinsic asthma NOS \\
H331. & Intrinsic asthma \\
H3310 & Intrinsic asthma without status asthmaticus \\
H3311 & Intrinsic asthma with status asthmaticus \\
H331z & Intrinsic asthma NOS \\
H332. & Mixed asthma \\
H333. & Acute exacerbation of asthma \\
H334. & Brittle asthma \\
H335. & Chronic asthma with fixed airflow obstruction \\
H33z. & Asthma unspecified \\
H33zO & Status asthmaticus NOS \\
H33z1 & Asthma attack \\
H33z2 & Late-onset asthma \\
H33zz & Asthma NOS \\
H3B.. & Asthma-chronic obstructive pulmonary disease overlap syndrome \\
Asthma resolved codes & \\
21262 & Asthma resolved \\
$212 G$. & Asthma resolved \\
\hline & \\
\hline
\end{tabular}


Table C.4.3: Asthma-related event groups chosen for the coding quality analysis.

\begin{tabular}{|c|c|}
\hline Read code & Description \\
\hline \multicolumn{2}{|c|}{ Asthma triggers } \\
\hline 178. & Asthma trigger \\
\hline 1780. & Aspirin inducted asthma \\
\hline 1781. & Asthma trigger - pollen \\
\hline 1782. & Asthma trigger - tobacco smoke \\
\hline 1783. & Asthma trigger - warm air \\
\hline 1784. & Asthma trigger - emotion \\
\hline 1785. & Asthma trigger - damp \\
\hline 1786. & Asthma trigger - animals \\
\hline 1787. & Asthma trigger - seasonal \\
\hline 1788. & Asthma trigger - cold air \\
\hline 1789. & Asthma trigger - respiratory infections \\
\hline $178 A$. & Asthma trigger - airborne dust \\
\hline $178 \mathrm{~B}$. & Asthma trigger - exercise \\
\hline \multicolumn{2}{|c|}{ Asthma severity } \\
\hline $663 V 1$ & Mild asthma \\
\hline $663 V 2$ & Moderator asthma \\
\hline $663 V 3$ & Severe asthma \\
\hline \multicolumn{2}{|c|}{ Asthma control steps } \\
\hline 8793. & Asthma control step 0 \\
\hline 8793. & Asthma control step 1 \\
\hline 8793. & Asthma control step 2 \\
\hline 8793. & Asthma control step 3 \\
\hline 8793. & Asthma control step 4 \\
\hline 8793. & Asthma control step 5 \\
\hline \multicolumn{2}{|c|}{ Spirometry } \\
\hline 33G1. & Spirometry reversibility positive \\
\hline $33 \mathrm{H} 1$. & Positive reversibility test to salbutamol \\
\hline $33 \mathrm{I} 1$. & Positive reversibility test to ipratropium bromide \\
\hline $33 J 1$. & $\begin{array}{l}\text { Positive reversibility test to a combination of salbutamol and } \\
\text { ipratropium bromide }\end{array}$ \\
\hline $33 \mathrm{~K} 1$. & Positive reversibility test to corticosteroids \\
\hline $663 \mathrm{~J}$. & Airways obstruction reversible \\
\hline 745D4 & Post bronchodilator spirometry \\
\hline $8 \mathrm{HRC}$. & Referral for spirometry \\
\hline \multicolumn{2}{|c|}{ Serum eosinophil count } \\
\hline $42 \mathrm{~K} .$. & Eosinophil count \\
\hline $42 \mathrm{~K} 1$. & Eosinophil count normal \\
\hline $42 \mathrm{~K} 3$. & Eosinophil count raised \\
\hline $42 \mathrm{KZ}$. & Eosinophil count NOS (not otherwise specified) \\
\hline $42 b 9$. & Percentage eosinophil \\
\hline \multicolumn{2}{|c|}{ Serum total IgE } \\
\hline $43 J 7$. & IgE \\
\hline 43Jw. & Total immunoglobulin E level \\
\hline
\end{tabular}




\section{C.5 Density distributions of lung function test val- ues}

Figure C.5.1: Beanplots showing density distributions for lung function event values.
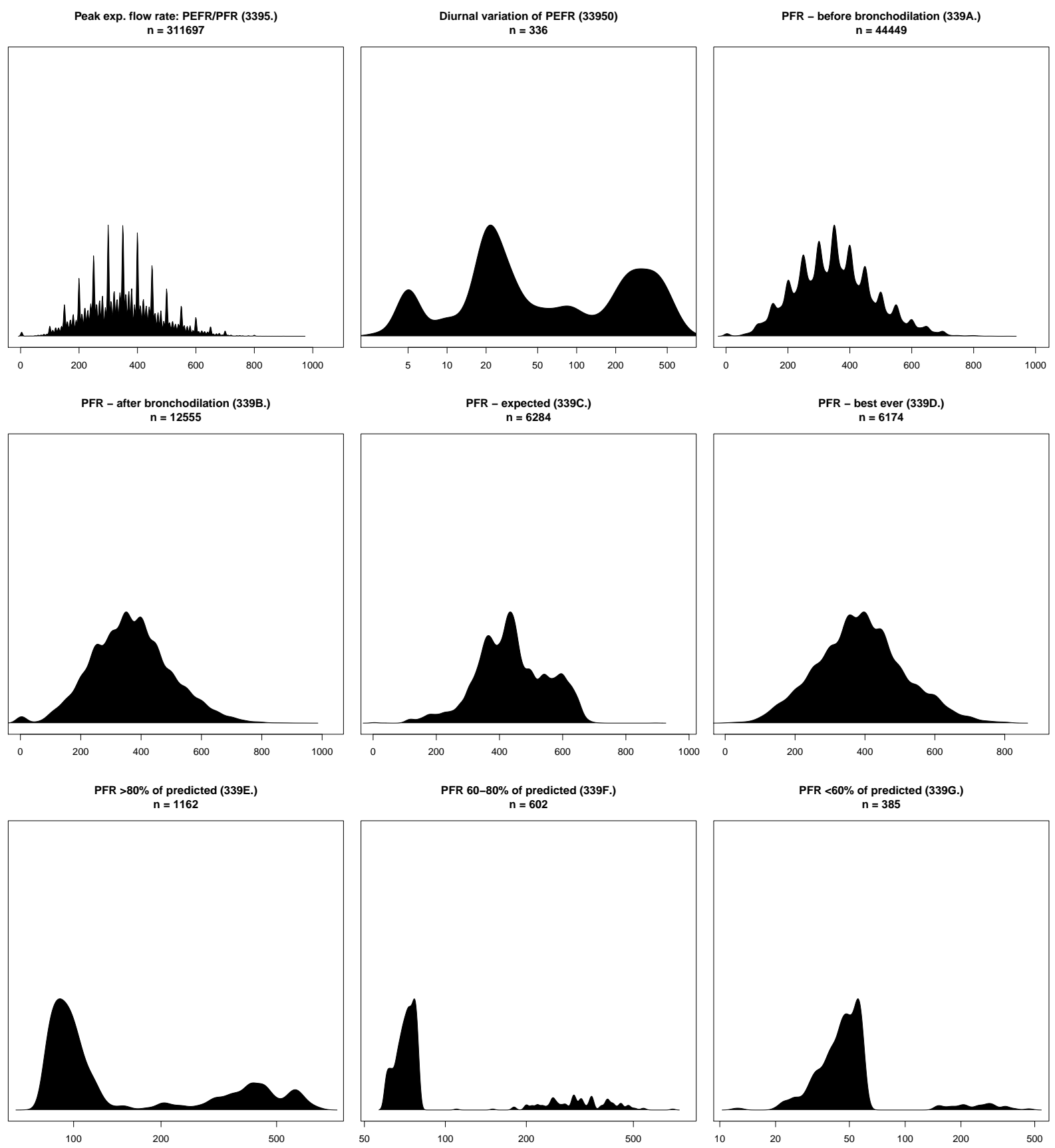

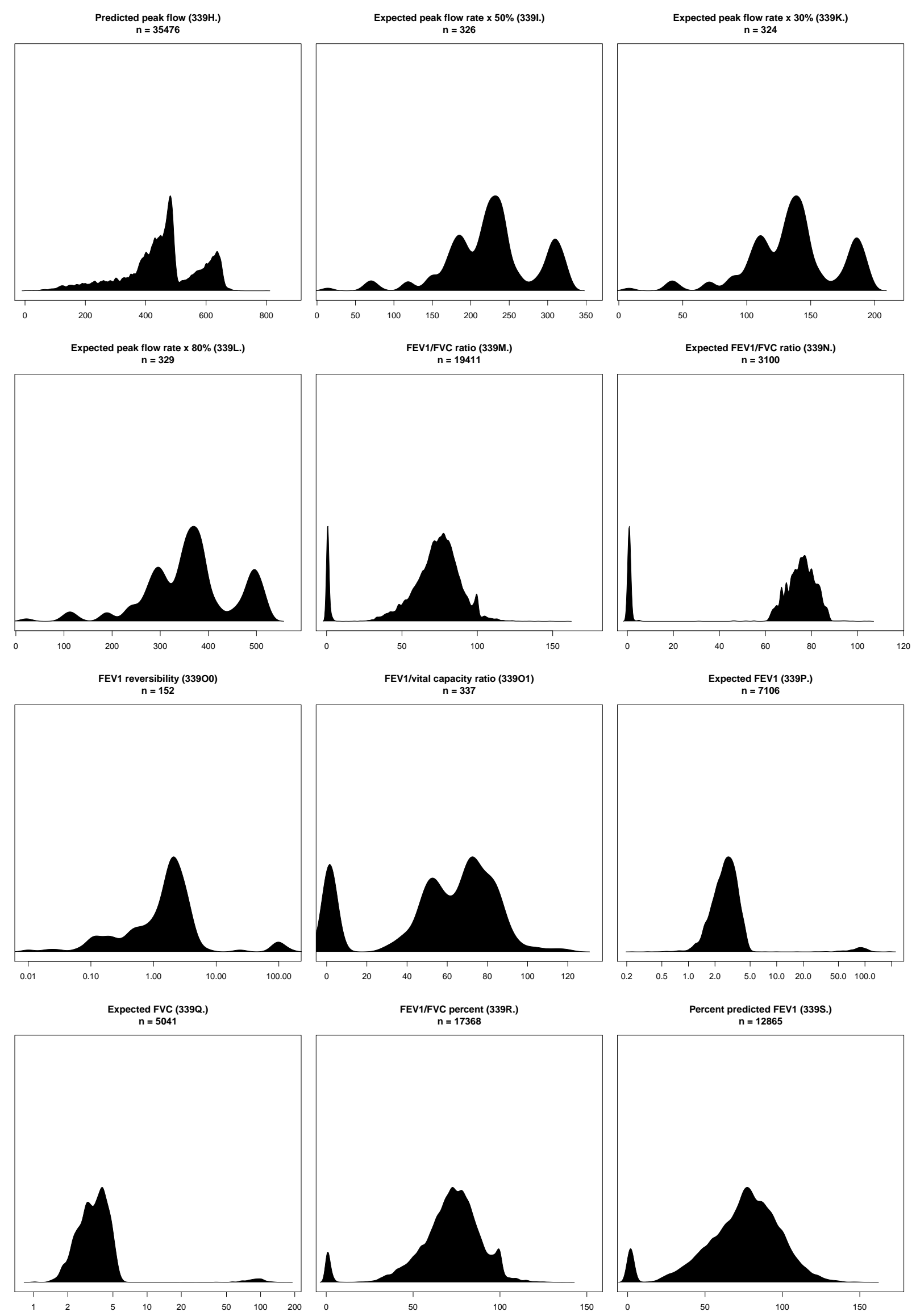

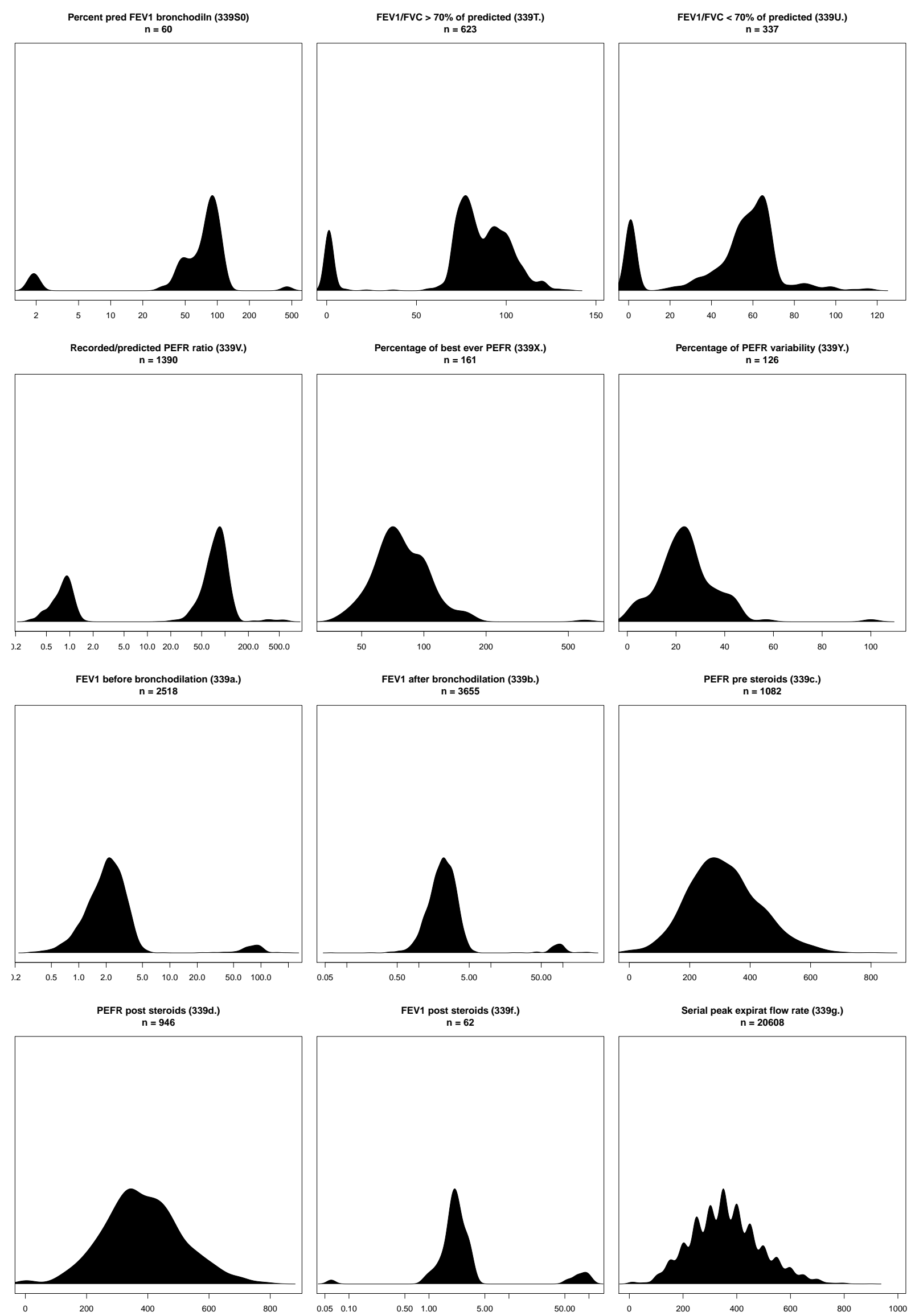

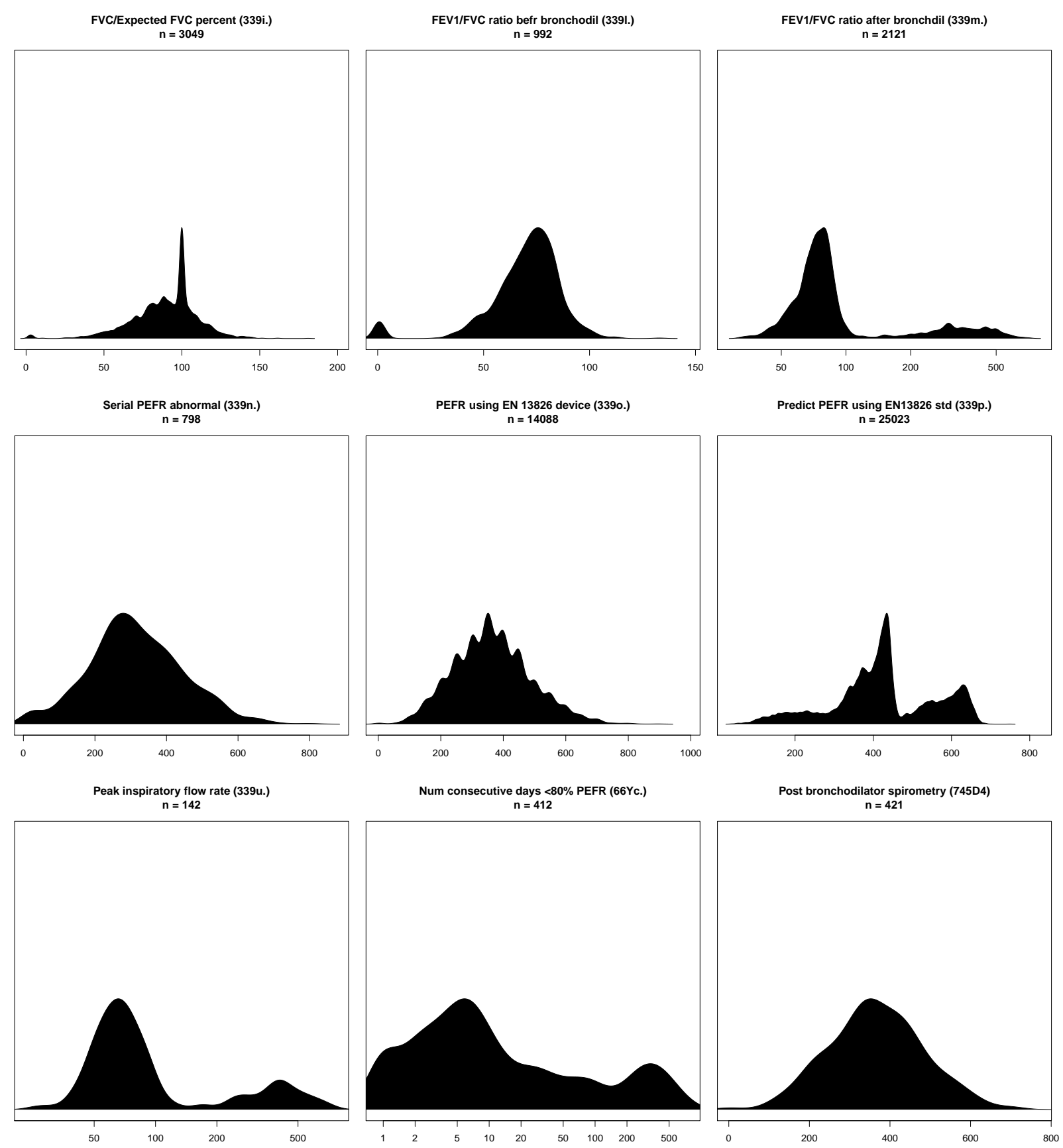
Appendix D

\section{Chapter 5 Appendix}




\section{D.1 Meeting abstract}

I presented the following abstract about the findings in Chapter 5 at The Lancet's Public Health Science Conference 2017 in London and the European Respiratory Society International Congress 2017 in Milan. 
Socioeconomic deprivation and inequalities in asthma care in Wales

Mohammad A Al Sallakh, Sarah E Rodgers, Ronan A Lyons, Aziz Sheikh, Gwyneth A Davies

Abstract

Background Area-based deprivation indices are widely used to study health inequalities. We explored whether inequality exists for asthma care across socioeconomic deprivation levels in Wales.
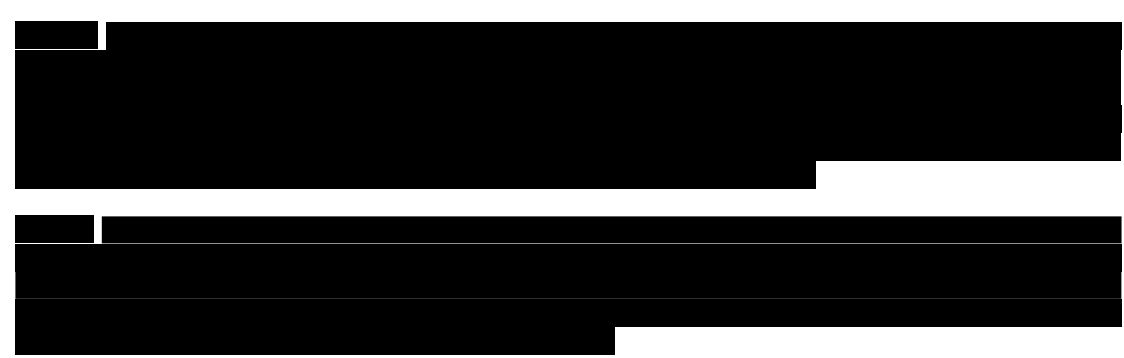

1
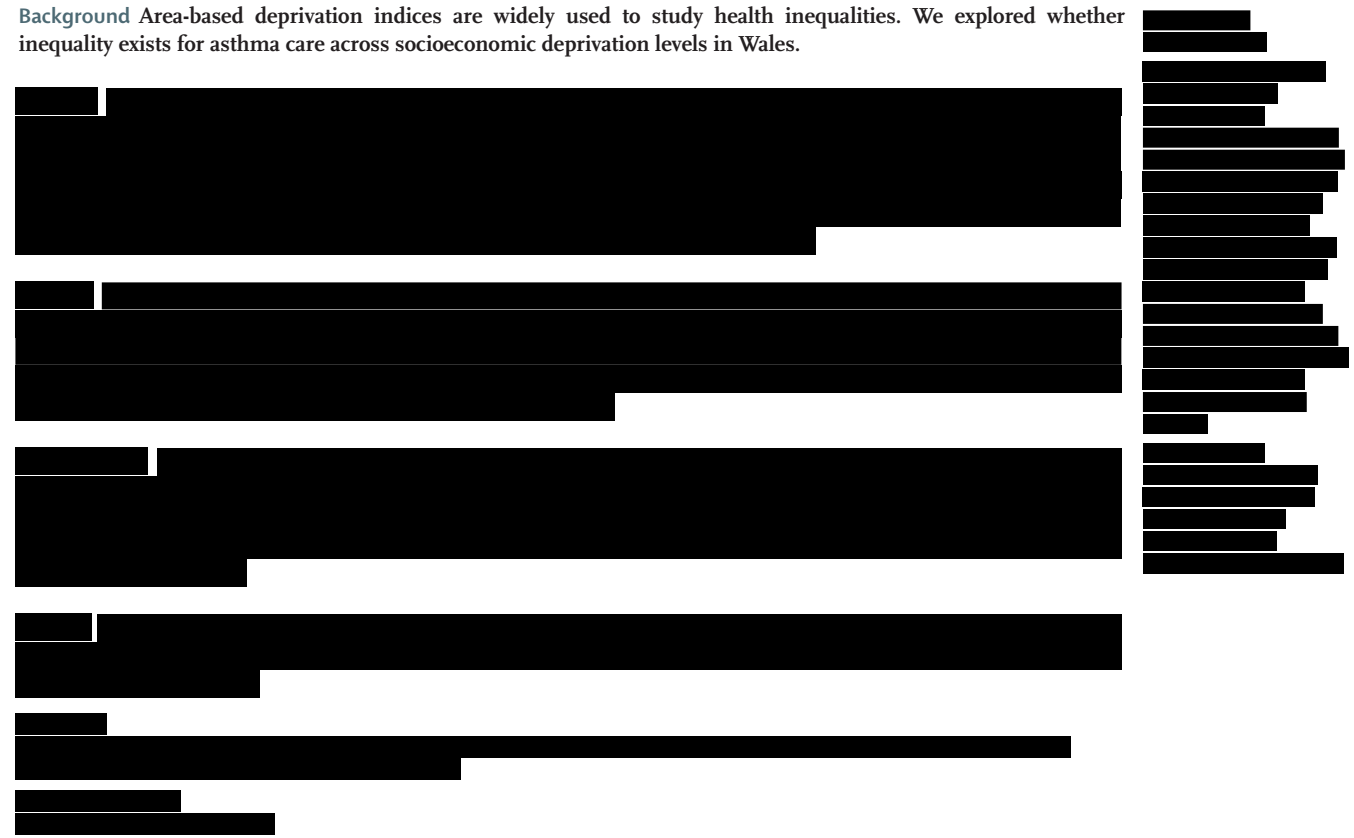


\section{Appendix E}

\section{Clinical codes}

These codes were used in asthma case definitions and outcome variables in Chapter 4 and Chapter 5.

\begin{tabular}{|c|c|}
\hline Code & Description \\
\hline \multicolumn{2}{|c|}{ Asthma GP Visits (Read codes) } \\
\hline $173 c$. & Occupational asthma \\
\hline $173 d$. & Work aggravated asthma \\
\hline 178. & Asthma trigger \\
\hline 1780. & Aspirin induced asthma \\
\hline 1781. & Asthma trigger - pollen \\
\hline 1782. & Asthma trigger - tobacco smoke \\
\hline 1783. & Asthma trigger - warm air \\
\hline 1784. & Asthma trigger - emotion \\
\hline 1785. & Asthma trigger - damp \\
\hline 1786. & Asthma trigger - animals \\
\hline 1787. & Asthma trigger - seasonal \\
\hline 1788. & Asthma trigger - cold air \\
\hline 1789. & Asthma trigger - respiratory infection \\
\hline $178 \mathrm{~A}$. & Asthma trigger - airborne dust \\
\hline 178B. & Asthma trigger - exercise \\
\hline 102.. & Asthma confirmed \\
\hline $388 \mathrm{t}$. & Royal College of Physicians asthma assessment \\
\hline 38DL. & Asthma control test \\
\hline 38DV. & Mini asthma quality of life questionnaire \\
\hline 38QM. & Childhood Asthma Control Test \\
\hline $661 \mathrm{M} 1$ & Asthma self-management plan agreed \\
\hline $661 \mathrm{N1}$ & Asthma self-management plan review \\
\hline $663 d$. & Emergency asthma admission since last appointment \\
\hline $663 \mathrm{e}$. & Asthma restricts exercise \\
\hline $663 \mathrm{e} 0$ & Asthma sometimes restricts exercise \\
\hline $663 \mathrm{e} 1$ & Asthma severely restricts exercise \\
\hline $663 f$. & Asthma never restricts exercise \\
\hline $663 \mathrm{~h}$. & Asthma - currently dormant \\
\hline $663 \mathrm{j}$. & Asthma - currently active \\
\hline $663 \mathrm{~m}$. & Asthma accident and emergency attendance since last visit \\
\hline $663 n$. & Asthma treatment compliance satisfactory \\
\hline $663 N$. & Asthma disturbing sleep \\
\hline $663 N 0$ & Asthma causing night waking \\
\hline $663 N 1$ & Asthma disturbs sleep weekly \\
\hline $663 N 2$ & Asthma disturbs sleep frequently \\
\hline 6630. & Asthma not disturbing sleep \\
\hline 66300 & Asthma never disturbs sleep \\
\hline 663p. & Asthma treatment compliance unsatisfactory \\
\hline 663P. & Asthma limiting activities \\
\hline 663P0 & Asthma limits activities 1 to 2 times per month \\
\hline $663 \mathrm{P} 1$ & Asthma limits activities 1 to 2 times per week \\
\hline 663P2 & Asthma limits activities most days \\
\hline
\end{tabular}


Table E.1: (cont'd).

\begin{tabular}{|c|c|}
\hline Code & Description \\
\hline $663 q$. & Asthma daytime symptoms \\
\hline $663 Q$. & Asthma not limiting activities \\
\hline $663 r$. & Asthma causes night symptoms 1 to 2 times per month \\
\hline 663s. & Asthma never causes daytime symptoms \\
\hline $663 t$. & Asthma causes daytime symptoms 1 to 2 times per month \\
\hline $663 u$. & Asthma causes daytime symptoms 1 to 2 times per week \\
\hline $663 U$. & Asthma management plan given \\
\hline $663 v$. & Asthma causes daytime symptoms most days \\
\hline 663V. & Asthma severity \\
\hline 663V0 & Occasional asthma \\
\hline $663 \mathrm{~V} 1$ & Mild asthma \\
\hline $663 V 2$ & Moderate asthma \\
\hline $663 V 3$ & Severe asthma \\
\hline $663 w$. & Asthma limits walking up hills or stairs \\
\hline 663W. & Asthma prophylactic medication used \\
\hline $663 x$ & Asthma limits walking on the flat \\
\hline 663y. & Number of asthma exacerbations in past year \\
\hline $66 Y 5$. & Change in asthma management plan \\
\hline $66 Y 9$. & Step up change in asthma management plan \\
\hline 66YA. & Step down change in asthma management plan \\
\hline 66YC. & Absent from work or school due to asthma \\
\hline 66YE. & Asthma monitoring due \\
\hline $66 Y J$. & Asthma annual review \\
\hline 66YK. & Asthma follow-up \\
\hline 66Yp. & Asthma review using Royal College of Physicians three questions \\
\hline 66YP. & Asthma night-time symptoms \\
\hline 66YQ. & Asthma monitoring by nurse \\
\hline $66 Y q .$. & Asthma causes night time symptoms 1 to 2 times per week \\
\hline $66 Y r$. & Asthma causes symptoms most nights \\
\hline 66YR. & Asthma monitoring by doctor \\
\hline $66 Y s$. & Asthma never causes night symptoms \\
\hline $66 Y u$. & Number of days absent from school due to asthma in past 6 months \\
\hline $66 Y Z$. & Does not have asthma management plan \\
\hline $679 J$. & Health education - asthma \\
\hline $679 J 0$ & Health education - asthma self management \\
\hline $679 J 1$ & Health education - structured asthma discussion \\
\hline $679 J 2$ & Health education - structured patient focused asthma discussion \\
\hline 8791. & Further asthma - drug prevent. \\
\hline 8793. & Asthma control step 0 \\
\hline 8794. & Asthma control step 1 \\
\hline 8795. & Asthma control step 2 \\
\hline 8796. & Asthma control step 3 \\
\hline 8797. & Asthma control step 4 \\
\hline 8798. & Asthma control step 5 \\
\hline 8B3j. & Asthma medication review \\
\hline 8CMA0 & Patient has a written asthma personal action plan \\
\hline 8CRO. & Asthma clinical management plan \\
\hline $8 \mathrm{H} 2 \mathrm{P}$. & Emergency admission, asthma \\
\hline 8HTT. & Referral to asthma clinic \\
\hline 9hA.. & Exception reporting: asthma quality indicators \\
\hline 9hA1. & Excepted from asthma quality indicators: Patient unsuitable \\
\hline 9N1d. & Seen in asthma clinic \\
\hline 9N1d0 & Seen in school asthma clinic \\
\hline 9NI8. & Asthma outreach clinic \\
\hline 9NNX. & Under care of asthma specialist nurse \\
\hline $90 J .$. & Asthma monitoring admin. \\
\hline $90 J 1$. & Attends asthma monitoring \\
\hline $90 J 2$. & Refuses asthma monitoring \\
\hline $90 J 3$. & Asthma monitor offer default \\
\hline $90 J 4$. & Asthma monitor 1st letter \\
\hline $90 J 5$. & Asthma monitor 2 nd letter \\
\hline $90 J 6$. & Asthma monitor 3rd letter \\
\hline $90 J 7$. & Asthma monitor verbal invite \\
\hline $90 J 8$. & Asthma monitor phone invite \\
\hline $90 J 9$. & Asthma monitoring deleted \\
\hline 90JA. & Asthma monitoring check done \\
\hline 90JB. & Asthma monitoring invitation SMS (short message service) text message \\
\hline 90JC. & Asthma monitoring invitation email \\
\hline $90 J Z$. & Asthma monitoring admin.NOS \\
\hline
\end{tabular}


Table E.1: (cont'd).

\begin{tabular}{|c|c|}
\hline Code & Description \\
\hline 9Q21. & Patient in asthma study \\
\hline SLF7. & Antiasthmatic poisoning \\
\hline SLF7z & Antiasthmatic poisoning NOS \\
\hline \multicolumn{2}{|c|}{ Asthma Reviews } \\
\hline $66 Y J$. & Asthma annual review \\
\hline 66YK. & Asthma follow-up \\
\hline 66Yp. & Asthma review using Royal College of Physicians three questions \\
\hline 66YQ. & Asthma monitoring by nurse \\
\hline 8B3j. & Asthma medication review \\
\hline $90 J A$. & Asthma monitoring check done \\
\hline \multicolumn{2}{|c|}{ Asthma Emergency Department Visits (A\&E code) } \\
\hline $14 \mathrm{~A}$ & Asthma \\
\hline \multicolumn{2}{|c|}{ Asthma Hospitalisations (ICD-10 codes) } \\
\hline J45 & Asthma \\
\hline $\mathrm{J} 45.0$ & Predominantly allergic asthma \\
\hline J45.1 & Nonallergic asthma \\
\hline J45.8 & Mixed asthma \\
\hline J45.9 & Asthma, unspecified \\
\hline $\mathrm{J} 46$ & Status asthmaticus \\
\hline \multicolumn{2}{|c|}{ Asthma Prescriptions (Read codes) } \\
\hline \multicolumn{2}{|l|}{ SABA } \\
\hline c11\%\% & SALBUTAMOL [ORAL PREPARATIONS] \\
\hline c12\%\% & SALBUTAMOL [PARENTERAL PREPARATIONS] \\
\hline c13\%\% & SALBUTAMOL [INHALATION PREPRATIONS] \\
\hline c14\%\% & TERBUTALINE SULPHATE [RESPIRATORY USE] \\
\hline c15\%\% & FENOTEROL HYDROBROMIDE \\
\hline c1E\% \% & SALBUTAMOL [INHALATION PREPRATIONS 2] \\
\hline \multicolumn{2}{|l|}{ ICS } \\
\hline c615. & *BECOTIDE rotahaler device \\
\hline c616. & BECOTIDE 50micrograms/mL nebuliser solution \\
\hline c617. & BECOTIDE-100 100microgram inhaler \\
\hline c618. & *VOLUMATIC spacer device \\
\hline c619. & BECODISK 100micrograms diskhaler 14x8 \\
\hline c61A. & BECLOMETASONE DIPROPIONATE 400micrograms disks+disk inhaler \\
\hline c61B. & BECLOMETASONE DIPROPIONATE 400micrograms disk refill \\
\hline c61C. & BECLOMETHASONE DIPROPIONATE 250micrograms inhaler+spacer device \\
\hline c61E. & $\begin{array}{l}\text { BECLOMETASONE DIPROPIONATE 250micrograms breath-actuated aerosol } \\
\text { inhaler }\end{array}$ \\
\hline c61F. & $\begin{array}{l}\text { BECLOMETASONE DIPROPIONATE 100micrograms breath-actuated aerosol } \\
\text { inhaler }\end{array}$ \\
\hline c61G. & *FILAIR 50micrograms inhaler \\
\hline $\mathrm{c} 61 \mathrm{H}$. & *FILAIR 100micrograms inhaler \\
\hline c61J. & FILAIR FORTE 250micrograms inhaler \\
\hline c61K. & BECLAZONE 50micrograms inhaler \\
\hline c61L. & BECLAZONE 100micrograms inhaler \\
\hline c61M. & BECLAZONE 250micrograms inhaler \\
\hline c61N. & BECLAZONE 50 EASI-BREATHE inhaler \\
\hline c610. & BECLAZONE 100 EASI-BREATHE inhaler \\
\hline c61P. & BECLAZONE 250 EASI-BREATHE inhaler \\
\hline c61Q. & BECLOFORTE INTEGRA 250micrograms inhaler+compact spacer \\
\hline c61R. & BECLOFORTE INTEGRA 250micrograms refill \\
\hline c61S. & BECLOMETHASONE DIPROPIONATE 250micrograms inhaler+compact spacer \\
\hline c61T. & BECLOMETHASONE DIPROPIONATE 250micrograms compact spacer refill \\
\hline c61U. & BECLOMETHASONE rotahaler device \\
\hline c61V. & $\begin{array}{l}\text { BECLOMETHASONE DIPROPIONATE 50micrograms vortex metered dose } \\
\text { inhaler }\end{array}$ \\
\hline c61W. & *BDP 50micrograms Spacehaler \\
\hline
\end{tabular}


Table E.1: (cont'd).

\begin{tabular}{|c|c|}
\hline Code & Description \\
\hline c61X. & $\begin{array}{l}\text { BECLOMETHASONE DIPROPIONATE 100micrograms vortex metered dose } \\
\text { inhaler }\end{array}$ \\
\hline c61Y. & *BDP 100micrograms Spacehaler \\
\hline c61Z. & $\begin{array}{l}\text { BECLOMETHASONE DIPROPIONATE 250micrograms vortex metered dose } \\
\text { inhaler }\end{array}$ \\
\hline c61a. & BECODISK 200micrograms diskhaler $14 \times 8$ \\
\hline c61b. & BECOTIDE 400micrograms rotacaps \\
\hline c61c. & BECODISK 100 micrograms disk refill $14 \times 8$ \\
\hline c61d. & BECODISK 200 micrograms disk refill 14x8 \\
\hline c61e. & BECODISK 400micrograms diskhaler 7x8 \\
\hline c61f. & BECODISK 400micrograms disk refill $7 \times 8$ \\
\hline c61g. & BECLOFORTE VM 250micrograms inhaler+volumatic \\
\hline c61h. & BECLOMETASONE DIPROPIONATE 400 micrograms inhalation capsules \\
\hline c61i. & BECOTIDE-200 200microgram inhaler \\
\hline c61j. & ${ }^{\star}$ AEROBEC 50microgram Autohaler \\
\hline c61k. & AEROBEC FORTE 250 micrograms Autohaler \\
\hline c61l. & AEROBEC 100microgram Autohaler \\
\hline $\mathrm{c} 61 \mathrm{~m}$. & BECLOFORTE DISKHALER 400 micrograms $14 \times 8$ \\
\hline c61n. & BECLOFORTE DISKS 400micrograms disk refill 14x8 \\
\hline c61p. & BECLOMETASONE DIPROPIONATE 100micrograms disks+disk inhaler \\
\hline c61q. & BECLOMETASONE DIPROPIONATE 200micrograms disks+disk inhaler \\
\hline c61r. & BECLOMETASONE DIPROPIONATE 100 micrograms disk refill \\
\hline c61s. & BECLOMETASONE DIPROPIONATE 200micrograms disk refill \\
\hline c61u. & BECLOMETASONE DIPROPIONATE 200micrograms inhaler \\
\hline c61v. & BECLOMETASONE DIPROPIONATE 50micrograms inhaler \\
\hline c61w. & BECLOMETASONE DIPROPIONATE 100micrograms inhalation capsules \\
\hline c61x. & BECLOMETASONE DIPROPIONATE 200 micrograms inhalation capsules \\
\hline c61y. & BECLOMETHASONE DIPROPIONATE 50 micrograms $/ \mathrm{mL}$ nebuliser solution \\
\hline c61z. & BECLOMETASONE DIPROPIONATE 100 micrograms inhaler \\
\hline c62.. & BECLOMETASONE COMPOUNDS \\
\hline c621. & *VENTIDE inhaler \\
\hline c622. & *VENTIDE Rotacaps \\
\hline c623. & *VENTIDE paediatric Rotacaps \\
\hline c624. & *VENTIDE Rotahaler device \\
\hline c63.. & *BETAMETHASONE VALERATE \\
\hline c631. & *BEXTASOL 100 microgram inhaler \\
\hline c63z. & BETAMETHASONE 100micrograms inhaler \\
\hline c64.. & BUDESONIDE [RESPIRATORY USE] \\
\hline c641. & PULMICORT 200micrograms inhaler 200dose \\
\hline c642. & PULMICORT 200micrograms refill 100dose \\
\hline c643. & PULMICORT 200 micrograms refill 200 dose \\
\hline c644. & PULMICORT LS 50micrograms inhaler \\
\hline c645. & PULMICORT LS 50micrograms refill \\
\hline c646. & *NEBUHALER spacer device \\
\hline c647. & PULMICORT 200microgram inhaler 100dose \\
\hline c649. & PULMICORT 400microgram Turbohaler 50dose \\
\hline c64A. & BUDESONIDE 200micrograms refill cannister \\
\hline c64B. & BUDESONIDE 50 micrograms spacer inhaler \\
\hline c64C. & PULMICORT 200micrograms spacer inhaler \\
\hline c64D. & PULMICORT LS 50micrograms spacer inhaler \\
\hline c64E. & PULMICORT 200micrograms inhaler with NebuChamber \\
\hline c64F. & BUDESONIDE 200micrograms/dose dry powder cartridge refill \\
\hline c64G. & NOVOLIZER BUDESONIDE 200 micrograms/dose dry powder cartridge refill \\
\hline $\mathrm{c} 64 \mathrm{H}$. & EASYHALER BUDESONIDE $100 \mathrm{micrograms}$ breath-actuated dry powder inhaler \\
\hline c64I. & EASYHALER BUDESONIDE 200micrograms breath-actuated dry powder inhaler \\
\hline c64J. & EASYHALER BUDESONIDE 400micrograms breath-actuated dry powder inhaler \\
\hline c64K. & PULMICORT 100micrograms CFC-free inhaler \\
\hline c64a. & PULMICORT 500micrograms Respules $2 \mathrm{~mL}$ unit \\
\hline c64b. & PULMICORT 1mg Respules $2 \mathrm{~mL}$ unit \\
\hline c64c. & PULMICORT 100microgram Turbohaler 200dose \\
\hline c64d. & BUDESONIDE 100micrograms breath-actuated dry powder inhaler \\
\hline c64e. & BUDESONIDE 50 micrograms refill cannister \\
\hline c64g. & BUDESONIDE 200micrograms breath-actuated dry powder inhaler \\
\hline c64h. & BUDESONIDE 400micrograms breath-actuated dry powder inhaler \\
\hline c64i. & BUDESONIDE 500 micrograms $/ 2 \mathrm{~mL}$ nebuliser solution \\
\hline c64j. & BUDESONIDE $1 \mathrm{mg} / 2 \mathrm{~mL}$ nebuliser solution \\
\hline $\mathrm{c} 64 \mathrm{k}$. & *BUDESONIDE 200 Cyclocaps \\
\hline c64l. & *BUDESONIDE 400 Cyclocaps \\
\hline c64m. & BUDESONIDE 200micrograms inhalation capsules \\
\hline
\end{tabular}


Table E.1: (cont'd).

\begin{tabular}{|c|c|}
\hline Code & Description \\
\hline c64n. & BUDESONIDE 400micrograms inhalation capsules \\
\hline c64o. & BUDESONIDE 200micrograms inhaler with spacer device \\
\hline c64p. & $\begin{array}{l}\text { NOVOLIZER BUDESONIDE 200micrograms/dose dry powder cartridge and } \\
\text { refillable inhaler device }\end{array}$ \\
\hline c64u. & $\begin{array}{l}\text { BUDESONIDE 200micrograms/dose dry powder cartridge and refillable inhaler } \\
\text { device }\end{array}$ \\
\hline c64v. & BUDESONIDE 200micrograms inhaler \\
\hline c64x. & ${ }^{*}$ BUDESONIDE refill 200dose \\
\hline c64y. & BUDESONIDE 50micrograms inhaler \\
\hline c64z. & BUDESONIDE 200micrograms spacer inhaler \\
\hline c65.. & FLUTICASONE PROPIONATE [RESPIRATORY USE] \\
\hline c651. & FLIXOTIDE 50micrograms diskhaler \\
\hline c652. & FLIXOTIDE 100micrograms diskhaler \\
\hline c653. & FLIXOTIDE 250micrograms diskhaler \\
\hline c654. & FLUTICASONE PROPIONATE 50micrograms disks+disk inhaler \\
\hline c655. & FLUTICASONE PROPIONATE 100micrograms disks+disk inhaler \\
\hline c656. & FLUTICASONE PROPIONATE 250micrograms disks+disk inhaler \\
\hline c657. & FLIXOTIDE 50micrograms disk refill \\
\hline c658. & FLIXOTIDE 100micrograms disk refill \\
\hline c659. & FLIXOTIDE 250micrograms disk refill \\
\hline c65A. & FLUTICASONE PROPIONATE 50micrograms disk refill \\
\hline c65B. & FLUTICASONE PROPIONATE 100micrograms disk refill \\
\hline c65C. & FLUTICASONE PROPIONATE 250micrograms disk refill \\
\hline c65D. & FLIXOTIDE 25micrograms inhaler \\
\hline c65E. & FLIXOTIDE 50micrograms inhaler \\
\hline c65F. & FLIXOTIDE 125micrograms inhaler \\
\hline c65G. & FLUTICASONE PROPIONATE 25micrograms inhaler \\
\hline $\mathrm{c} 65 \mathrm{H}$. & FLUTICASONE PROPIONATE 50micrograms inhaler \\
\hline c65I. & FLUTICASONE PROPIONATE 125micrograms inhaler \\
\hline c65K. & FLIXOTIDE 250micrograms inhaler \\
\hline c65L. & FLIXOTIDE 500micrograms diskhaler \\
\hline c65M. & FLIXOTIDE 500micrograms disk refill \\
\hline c65N. & FLUTICASONE PROPIONATE 500micrograms disks+disk inhaler \\
\hline c650. & FLUTICASONE PROPIONATE 500micrograms disk refill \\
\hline c65P. & $\begin{array}{l}\text { FLUTICASONE PROPIONATE 50micrograms breath-actuated dry powder } \\
\text { inhaler }\end{array}$ \\
\hline c65Q. & $\begin{array}{l}\text { FLUTICASONE PROPIONATE 100micrograms breath-actuated dry powder } \\
\text { inhaler }\end{array}$ \\
\hline c65R. & $\begin{array}{l}\text { FLUTICASONE PROPIONATE 250micrograms breath-actuated dry powder } \\
\text { inhaler }\end{array}$ \\
\hline c65S. & $\begin{array}{l}\text { FLUTICASONE PROPIONATE 500micrograms breath-actuated dry powder } \\
\text { inhaler }\end{array}$ \\
\hline c65T. & FLIXOTIDE 50micrograms Accuhaler \\
\hline c65U. & FLIXOTIDE 100micrograms Accuhaler \\
\hline c65V. & FLIXOTIDE 250micrograms Accuhaler \\
\hline c65W. & FLIXOTIDE 500micrograms Accuhaler \\
\hline c65X. & FLUTICASONE PROPIONATE $0.5 \mathrm{mg} / 2 \mathrm{~mL}$ nebulisation units \\
\hline c65Y. & FLUTICASONE PROPIONATE $2 \mathrm{mg} / 2 \mathrm{~mL}$ nebulisation units \\
\hline c65Z. & FLIXOTIDE $0.5 \mathrm{mg} / 2 \mathrm{~mL}$ Nebules \\
\hline c65a. & FLIXOTIDE $2 \mathrm{mg} / 2 \mathrm{~mL}$ Nebules \\
\hline c65b. & FLUTICASONE PROPIONATE 125micrograms CFC-free inhaler \\
\hline c65c. & FLUTICASONE PROPIONATE 250micrograms CFC-free inhaler \\
\hline c65d. & FLIXOTIDE 125micrograms Evohaler \\
\hline c65e. & FLIXOTIDE 250micrograms Evohaler \\
\hline c65f. & FLUTICASONE PROPIONATE 50micrograms CFC-free inhaler \\
\hline c65g. & FLIXOTIDE 50micrograms Evohaler \\
\hline c66.. & BECLOMETASONE DIPROPIONATE [RESPIRATORY USE 2] \\
\hline c661. & ${ }^{\star B D P}$ 250micrograms Spacehaler \\
\hline c662. & BECOTIDE 50 EASI-BREATHE inhaler \\
\hline c663. & BECOTIDE 100 EASI-BREATHE inhaler \\
\hline c664. & BECLOFORTE EASI-BREATHE 250micrograms inhaler \\
\hline c665. & QVAR 50 inhaler \\
\hline c666. & QVAR 100 inhaler \\
\hline c667. & QVAR 50 Autohaler \\
\hline c668. & QVAR 100 Autohaler \\
\hline c669. & ${ }^{*}$ BECLAZONE 200 inhaler \\
\hline c66A. & $\begin{array}{l}\text { BECLOMETASONE DIPROPIONATE 50micrograms breath-actuated dry powder } \\
\text { inhaler }\end{array}$ \\
\hline
\end{tabular}


Table E.1: (cont'd).

\begin{tabular}{|c|c|}
\hline Code & Description \\
\hline c66B. & $\begin{array}{l}\text { BECLOMETASONE DIPROPIONATE 100micrograms breath-actuated dry } \\
\text { powder inhaler }\end{array}$ \\
\hline c66C. & $\begin{array}{l}\text { BECLOMETASONE DIPROPIONATE 250micrograms breath-actuated dry } \\
\text { powder inhaler }\end{array}$ \\
\hline c66D. & ASMABEC 50micrograms Clickhaler \\
\hline c66E. & ASMABEC 100micrograms Clickhaler \\
\hline c66F. & ASMABEC 250micrograms Clickhaler \\
\hline c66G. & $\begin{array}{l}\text { BECLOMETASONE DIPROPIONATE 400micrograms breath-actuated dry } \\
\text { powder inhaler }\end{array}$ \\
\hline $\mathrm{c} 66 \mathrm{H}$. & $\begin{array}{l}\text { BECLOMETASONE DIPROPIONATE 200micrograms breath-actuated dry } \\
\text { powder inhaler }\end{array}$ \\
\hline c66I. & $\begin{array}{l}\text { PULVINAL BECLOMETHASONE DIPROPIONATE 100micrograms } \\
\text { breath-actuated dry powder inhaler }\end{array}$ \\
\hline c66J. & $\begin{array}{l}\text { PULVINAL BECLOMETHASONE DIPROPIONATE 200micrograms } \\
\text { breath-actuated dry powder inhaler }\end{array}$ \\
\hline c66K. & $\begin{array}{l}\text { PULVINAL BECLOMETHASONE DIPROPIONATE 400micrograms } \\
\text { breath-actuated dry powder inhaler }\end{array}$ \\
\hline c66L. & *BECLOMETASONE 100 cyclocaps \\
\hline c66M. & *BECLOMETASONE 200 cyclocaps \\
\hline c66N. & ${ }^{*}$ BECLOMETASONE 400 cyclocaps \\
\hline c66P. & BECODISK 100micrograms diskhaler $15 \times 8$ \\
\hline c66Q. & BECODISK 200micrograms diskhaler 15x8 \\
\hline c66R. & BECODISK 400micrograms diskhaler $15 \times 8$ \\
\hline c66S. & BECODISK 100micrograms disk refill 15x8 \\
\hline c66T. & BECODISK 200 micrograms disk refill 15x8 \\
\hline c66U. & BECODISK 400micrograms disk refill 15x8 \\
\hline c66V. & BECLOMETASONE DIPROPIONATE 50micrograms CFC-free inhaler \\
\hline c66W. & BECLOMETASONE DIPROPIONATE 100micrograms CFC-free inhaler \\
\hline c66X. & $\begin{array}{l}\text { BECLOMETASONE DIPROPIONATE 50micrograms CFC-free breath-actuated } \\
\text { aerosol inhaler }\end{array}$ \\
\hline c66Y. & $\begin{array}{l}\text { BECLOMETASONE DIPROPIONATE 100micrograms CFC-free breath-actuated } \\
\text { aerosol inhaler }\end{array}$ \\
\hline c66Z. & $\begin{array}{l}\text { QVAR EASI-BREATHE 50micrograms CFC-free breath-actuated dry powder } \\
\text { inhaler }\end{array}$ \\
\hline c66a. & $\begin{array}{l}\text { QVAR EASI-BREATHE 100micrograms CFC-free breath-actuated dry powder } \\
\text { inhaler }\end{array}$ \\
\hline c66c. & CLENIL MODULITE 50micrograms CFC-free inhaler \\
\hline c66d. & CLENIL MODULITE 100micrograms CFC-free inhaler \\
\hline c66e. & CLENIL MODULITE 200micrograms CFC-free inhaler \\
\hline c66f. & CLENIL MODULITE 250micrograms CFC-free inhaler \\
\hline c66g. & BECLOMETASONE DIPROPIONATE 200micrograms CFC-free inhaler \\
\hline c66h. & BECLOMETASONE DIPROPIONATE 250micrograms CFC-free inhaler \\
\hline c68.. & MOMETASONE [RESPIRATORY USE] \\
\hline c681. & MOMETASONE FUROATE 200micrograms breath-actuated dry powder inhaler \\
\hline c682. & MOMETASONE FUROATE 400 micrograms breath-actuated dry powder inhaler \\
\hline c683. & ASMANEX TWISTHALER 200micrograms breath-actuated dry powder inhaler \\
\hline c684. & ASMANEX TWISTHALER 400micrograms breath-actuated dry powder inhaler \\
\hline c69.. & CICLESONIDE \\
\hline c691. & ALVESCO 160micrograms inhaler \\
\hline c692. & ALVESCO 80micrograms inhaler \\
\hline c69y. & CICLESONIDE 80micrograms inhaler \\
\hline c69z. & CICLESONIDE 160micrograms inhaler \\
\hline \multicolumn{2}{|c|}{ ICS-LABA } \\
\hline c1D.. & SALMETEROL+FLUTICASONE PROPIONATE \\
\hline c1D1. & SERETIDE 100 Accuhaler \\
\hline c1D2. & SERETIDE 250 Accuhaler \\
\hline c1D3. & SERETIDE 500 Accuhaler \\
\hline c1D4. & SERETIDE 50 Evohaler \\
\hline c1D5. & SERETIDE 125 Evohaler \\
\hline c1D6. & SERETIDE 250 Evohaler \\
\hline c1D7. & SIRDUPLA 25micrograms/125micrograms inhaler \\
\hline c1D8. & SIRDUPLA 25micrograms/250micrograms inhaler \\
\hline c1Du. & $\begin{array}{l}\text { SALMETEROL+FLUTICASONE PROPIONATE 25micrograms/50micrograms } \\
\text { CFC-free inhaler }\end{array}$ \\
\hline c1Dv. & $\begin{array}{l}\text { SALMETEROL+FLUTICASONE PROPIONATE 25micrograms/125micrograms } \\
\text { CFC-free inhaler }\end{array}$ \\
\hline
\end{tabular}


Table E.1: (cont'd).

\begin{tabular}{|c|c|}
\hline Code & Description \\
\hline c1Dw. & $\begin{array}{l}\text { SALMETEROL+FLUTICASONE PROPIONATE 25micrograms/250micrograms } \\
\text { CFC-free inhaler }\end{array}$ \\
\hline c1Dx. & $\begin{array}{l}\text { SALMETEROL+FLUTICASONE PROPIONATE 50micrograms/100micrograms } \\
\text { breath-actuated dry powder inhaler }\end{array}$ \\
\hline c1Dy. & $\begin{array}{l}\text { SALMETEROL+FLUTICASONE PROPIONATE 50micrograms/250micrograms } \\
\text { breath-actuated dry powder inhaler }\end{array}$ \\
\hline c1Dz. & $\begin{array}{l}\text { SALMETEROL+FLUTICASONE PROPIONATE 50micrograms/500micrograms } \\
\text { breath-actuated dry powder inhaler }\end{array}$ \\
\hline c1c.. & FLUTICASONE PROPIONATE+FORMOTEROL FUMARATE \\
\hline c1c1. & FLUTIFORM 50micrograms/5micrograms inhaler \\
\hline c1c2. & FLUTIFORM 125micrograms/5micrograms inhaler \\
\hline c1c3. & FLUTIFORM 250micrograms/10micrograms inhaler \\
\hline c1cx. & FLUTICASONE PROPIONATE+FORMOTEROL FUMARATE $250 \mathrm{mcg} / 10 \mathrm{mcg}$ inh \\
\hline c1cy. & FLUTICASONE PROPIONATE+FORMOTEROL FUMARATE $125 \mathrm{mcg} / 5 \mathrm{mcg}$ inh \\
\hline c1cz. & FLUTICASONE PROPIONATE+FORMOTEROL FUMARATE $50 \mathrm{mcg} / 5 \mathrm{mcg}$ inh \\
\hline c67.. & BUDESONIDE+FORMOTEROL \\
\hline c671. & SYMBICORT 100/6 Turbohaler \\
\hline c672. & SYMBICORT 200/6 Turbohaler \\
\hline c673. & SYMBICORT 400/12 Turbohaler \\
\hline c674. & DUORESP SPIROMAX 160mcg/4.5mcg breath-act dry powder inhaler \\
\hline c675. & DUORESP SPIROMAX 320mcg/9mcg breath-act dry powder inhaler \\
\hline c67x. & $\begin{array}{l}\text { BUDESONIDE+FORMOTEROL FUMARATE DIHYDRATE } \\
\text { 400micrograms/12micrograms breath-actuated dry powder inhaler }\end{array}$ \\
\hline c67y. & $\begin{array}{l}\text { BUDESONIDE+FORMOTEROL FUMARATE DIHYDRATE } \\
\text { 200micrograms/6micrograms breath-actuated dry powder inhaler }\end{array}$ \\
\hline c67z. & $\begin{array}{l}\text { BUDESONIDE+FORMOTEROL FUMARATE DIHYDRATE } \\
\text { 100micrograms/6micrograms breath-actuated dry powder inhaler }\end{array}$ \\
\hline c6A.. & BECLOMETASONE+FORMOTEROL \\
\hline c6A1. & FOSTAIR 100micrograms/6micrograms inhaler \\
\hline c6A2. & FOSTAIR NEXTHALER 100micrograms/6micrograms powder inhaler \\
\hline c6Ay. & BECLOMET DIPROP+FORMOTERL FUMARATE DIHYD $100 \mathrm{mcg} / 6 \mathrm{mcg} \mathrm{pdr}$ inh \\
\hline c6Az. & $\begin{array}{l}\text { BECLOMETASONE DIPROPIONATE+FORMETEROL FUMARATE DIHYDRATE } \\
\text { 100micrograms/6micrograms inhaler }\end{array}$ \\
\hline c6B.. & FLUTICASONE+VILANTEROL \\
\hline c6B1. & RELVAR ELLIPTA 184micrograms/22micrograms inhaler \\
\hline c6B2. & FLUTICASONE FUROATE+VILANTEROL $184 \mathrm{mcg} / 22 \mathrm{mcg}$ dry pdr inhaler \\
\hline c6B3. & RELVAR ELLIPTA 92micrograms/22micrograms inhaler \\
\hline c6B4. & FLUTICASONE FUROATE+VILANTEROL $92 \mathrm{mcg} / 22 \mathrm{mcg}$ dry pdr inhaler \\
\hline \multicolumn{2}{|c|}{ Theophyllines } \\
\hline c41.. & AMINOPHYLLINE \\
\hline c411. & AMINOPHYLLINE 100mg tablets \\
\hline c412. & AMINOPHYLLINE 250mg/10mL injection \\
\hline c413. & AMINOPHYLLINE 500mg/2mL injection \\
\hline c414. & AMINOPHYLLINE 50mg suppositories \\
\hline c415. & AMINOPHYLLINE 100mg suppositories \\
\hline c416. & AMINOPHYLLINE 150mg suppositories \\
\hline c417. & AMINOPHYLLINE 180mg suppositories \\
\hline c418. & AMINOPHYLLINE $360 \mathrm{mg}$ suppositories \\
\hline c419. & *THEODROX tablets \\
\hline c41A. & *NORPHYLLIN 100mg tablets \\
\hline c41B. & NORPHYLLIN SR 225mg m/r tablets \\
\hline c41C. & NORPHYLLIN SR 350mg $\mathrm{m} / \mathrm{r}$ tablets \\
\hline c41a. & PHYLLOCONTIN CONTINUS 225mg $\mathrm{m} / \mathrm{r}$ tablets \\
\hline c41b. & PHYLLOCONTIN FORTE 350mg m/r tablets \\
\hline c41c. & PHYLLOCONTIN PAEDIATRIC $100 \mathrm{mg} \mathrm{m} / \mathrm{r}$ tablets \\
\hline c41d. & AMINOPHYLLINE $225 \mathrm{mg} \mathrm{m} / \mathrm{r}$ tablets \\
\hline c41e. & *PECRAM $225 \mathrm{mg} \mathrm{m} / \mathrm{r}$ tablets \\
\hline c41f. & AMINOPHYLLINE $350 \mathrm{mg} \mathrm{m} / \mathrm{r}$ tablets \\
\hline c41g. & AMINOPHYLLINE $100 \mathrm{mg} \mathrm{m} / \mathrm{r}$ tablets \\
\hline c41h. & ${ }^{\star}$ AMNIVENT $225 \mathrm{mg} \mathrm{m} / \mathrm{r}$ tablets \\
\hline c41i. & ${ }^{\star}$ AMNIVENT 350mg $\mathrm{m} / \mathrm{r}$ tablets \\
\hline c41j. & MIN-I-JET AMINOPHYLLINE 250mg/10mL injection \\
\hline c41k. & AMINOPHYLLINE 250mg/10mL prefilled syringe \\
\hline c41m. & AMINOPHYLLINE HYDRATE $225 \mathrm{mg} \mathrm{m} / \mathrm{r}$ tablets \\
\hline c43.. & THEOPHYLLINE \\
\hline c431. & *BIOPHYLLINE $125 \mathrm{mg} / 5 \mathrm{~mL}$ syrup \\
\hline c432. & *NUELIN $125 \mathrm{mg}$ tablets \\
\hline
\end{tabular}


Table E.1: (cont'd).

\begin{tabular}{|c|c|}
\hline Code & Description \\
\hline c433. & *NUELIN 60mg/5mL liquid \\
\hline c434. & ${ }^{*}$ LASMA 300mg $\mathrm{m} / \mathrm{r}$ tablets \\
\hline c435. & NUELIN SA $175 \mathrm{mg} \mathrm{m} / \mathrm{r}$ tablets \\
\hline c436. & NUELIN SA-250 $250 \mathrm{mg} \mathrm{m} / \mathrm{r}$ tablets \\
\hline c437. & ${ }^{*}$ PRO-VENT $300 \mathrm{mg} \mathrm{m} / \mathrm{r}$ capsules \\
\hline c438. & SLO-PHYLLIN $60 \mathrm{mg} \mathrm{m} / \mathrm{r}$ capsules \\
\hline c439. & SLO-PHYLLIN $125 \mathrm{mg} \mathrm{m} / \mathrm{r}$ capsules \\
\hline c43A. & THEOPHYLLINE $200 \mathrm{mg} / 10 \mathrm{~mL}$ injection \\
\hline c43B. & THEOPHYLLINE $10 \mathrm{mg} / 5 \mathrm{~mL}$ sugar free solution \\
\hline c43a. & SLO-PHYLLIN 250mg m/r capsules \\
\hline c43b. & ${ }^{*}$ THEO-DUR $200 \mathrm{mg} \mathrm{m} / \mathrm{r}$ tablets \\
\hline c43c. & *THEO-DUR $300 \mathrm{mg} \mathrm{m} / \mathrm{r}$ tablets \\
\hline$c 43 d$. & *THEOGRAD $350 \mathrm{mg} \mathrm{m} / \mathrm{r}$ tablets \\
\hline c43e. & UNIPHYLLIN CONTINUS $400 \mathrm{mg} \mathrm{m} / \mathrm{r}$ tablets \\
\hline c43f. & UNIPHYLLIN CONTINUS $200 \mathrm{mg} \mathrm{m} / \mathrm{r}$ tablets \\
\hline c43g. & LABOPHYLLINE 200mg/10mL injection \\
\hline $\mathrm{c} 43 \mathrm{~h}$. & UNIPHYLLIN CONTINUS $300 \mathrm{mg} \mathrm{m} / \mathrm{r}$ tablets \\
\hline c43i. & ${ }^{*}$ BIOPHYLLINE $350 \mathrm{mg} \mathrm{m} / \mathrm{r}$ tablets \\
\hline c43j. & ${ }^{*}$ BIOPHYLLINE $500 \mathrm{mg} \mathrm{m} / \mathrm{r}$ tablets \\
\hline $\mathrm{c} 43 \mathrm{k}$. & THEOPHYLLINE $500 \mathrm{mg} \mathrm{m} / \mathrm{r}$ tablets \\
\hline $\mathrm{c} 43 \mathrm{~m}$. & ${ }^{*}$ THEOPHYLLINE $125 \mathrm{mg} / 5 \mathrm{~mL}$ syrup \\
\hline c43n. & ${ }^{*}$ THEOPHYLLINE $125 \mathrm{mg}$ tablets \\
\hline c43o. & ${ }^{*}$ THEOPHYLLINE $60 \mathrm{mg} / 5 \mathrm{~mL}$ liquid \\
\hline c43p. & THEOPHYLLINE $175 \mathrm{mg} \mathrm{m} / \mathrm{r}$ tablets \\
\hline c43q. & THEOPHYLLINE $250 \mathrm{mg} \mathrm{m} / \mathrm{r}$ tablets \\
\hline c43r. & THEOPHYLLINE $300 \mathrm{mg} \mathrm{m} / \mathrm{r}$ capsules \\
\hline c43s. & THEOPHYLLINE $60 \mathrm{mg} \mathrm{m} / \mathrm{r}$ capsules \\
\hline c43t. & THEOPHYLLINE $125 \mathrm{mg} \mathrm{m} / \mathrm{r}$ capsules \\
\hline c43u. & THEOPHYLLINE $250 \mathrm{mg} \mathrm{m} / \mathrm{r}$ capsules \\
\hline c43v. & THEOPHYLLINE 200mg m/r tablets \\
\hline$c 43 w$. & THEOPHYLLINE $300 \mathrm{mg} \mathrm{m} / \mathrm{r}$ tablets \\
\hline$c 43 x$ & THEOPHYLLINE $350 \mathrm{mg} \mathrm{m} / \mathrm{r}$ tablets \\
\hline c43y. & THEOPHYLLINE $400 \mathrm{mg} \mathrm{m} / \mathrm{r}$ tablets \\
\hline c43z. & ${ }^{*}$ THEOPHYLLINE 200mg tablets \\
\hline \multicolumn{2}{|l|}{ LTRA } \\
\hline cA... & LEUKOTRIENE RECEPTOR ANTAGONIST \\
\hline cA1.. & MONTELUKAST \\
\hline cA11. & MONTELUKAST 10mg tablets \\
\hline cA12. & MONTELUKAST $5 \mathrm{mg}$ chewable tablets \\
\hline cA13. & SINGULAIR $10 \mathrm{mg}$ tablets \\
\hline cA14. & SINGULAIR PAEDIATRIC $5 \mathrm{mg}$ chewable tablets \\
\hline cA15. & SINGULAIR PAEDIATRIC $4 \mathrm{mg}$ chewable tablets \\
\hline cA16. & SINGULAIR PAEDIATRIC $4 \mathrm{mg} / \mathrm{sachet}$ granules \\
\hline cA1y. & MONTELUKAST 4mg/sachet granules \\
\hline cA1z. & MONTELUKAST 4mg chewable tablets \\
\hline cA2.. & ZAFIRLUKAST \\
\hline cA21. & ZAFIRLUKAST $20 \mathrm{mg}$ tablets \\
\hline cA22. & ACCOLATE $20 \mathrm{mg}$ tablets \\
\hline \multicolumn{2}{|l|}{ ocs } \\
\hline fe61 & PREDNISOLONE $1 \mathrm{mg}$ tablets \\
\hline fe62 & PREDNISOLONE $5 \mathrm{mg}$ tablets \\
\hline fe66 & DELTACORTRIL ENTERIC $5 \mathrm{mg}$ tablets \\
\hline fe6i & PREDNISOLONE $5 \mathrm{mg}$ e/c tablets \\
\hline fe6j & PREDNISOLONE 5mg soluble tablets \\
\hline fe6k & PREDNISOLONE 50mg tablets \\
\hline fe6z & PREDNISOLONE 25mg tablets \\
\hline
\end{tabular}

